\title{
Bulk Vitrification Performance Enhancement: Refractory Lining Protection Against Molten Salt Penetration
}
P Hrma
C P Rodriguez
L M Bagaasen
S T Yokuda
M J Schweiger
$\mathrm{J}$ Matyas
M B Evans
W C Buchmiller
B T Smith
A B Gallegos
B M Arrigoni
A Fluegel
D-S Kim

July 2007

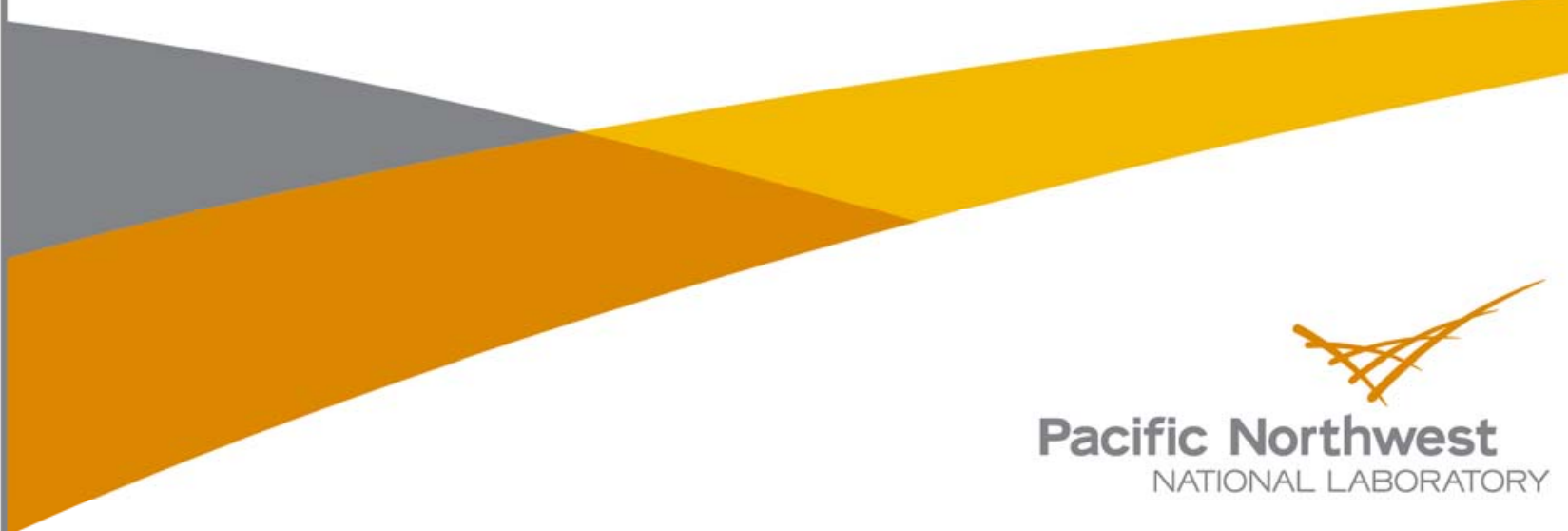




\title{
DISCLAIMER
}

This report was prepared as an account of work sponsored by an agency of the United States Government. Neither the United States Government nor any agency thereof, nor Battelle Memorial Institute, nor any of their employees, makes any warranty, express or implied, or assumes any legal liability or responsibility for the accuracy, completeness, or usefulness of any information, apparatus, product, or process disclosed, or represents that its use would not infringe privately owned rights. Reference herein to any specific commercial product, process, or service by trade name, trademark, manufacturer, or otherwise does not necessarily constitute or imply its endorsement, recommendation, or favoring by the United States Government or any agency thereof, or Battelle Memorial Institute. The views and opinions of authors expressed herein do not necessarily state or reflect those of the United States Government or any agency thereof.

\author{
PACIFIC NORTHWEST NATIONAL LABORATORY \\ operated by \\ BATTELLE \\ for the \\ UNITED STATES DEPARTMENT OF ENERGY \\ under Contract DE-ACO5-76RL01830
}

Printed in the United States of America
Available to DOE and DOE contractors from the
Office of Scientific and Technical Information,
P.O. Box 62, Oak Ridge, TN 37831-0062;
ph: (865) 576-8401
fax: (865) 5765728
email: reports@adonis.osti.gov

\author{
Available to the public from the National Technical Information Service, \\ U.S. Department of Commerce, 5285 Port Royal Rd., Springfield, VA 22161 \\ ph: (800) 553-6847 \\ fax: (703) 605-6900 \\ email: orders@nits.fedworld.gov \\ online ordering: http://www.ntis.gov/ordering.htm
}




\section{Bulk Vitrification Performance Enhancement: Refractory Lining Protection Against Molten Salt Penetration}
P. Hrma
C. P. Rodriguez
L. M. Bagaasen
S. T. Yokuda
M. J. Schweiger
J. Matyas
M. B. Evans
W. C. Buchmiller
B. T. Smith
A. B. Gallegos
B. M. Arrigoni
A. Fluegel

July 2007

Prepared for the U.S. Department of Energy

under Contract DE-AC05-76RL01830

Pacific Northwest National Laboratory

Richland, Washington 99352 


\begin{abstract}
Bulk vitrification (BV) is a process that heats a feed material that consists of glass-forming solids and dried low-activity waste (LAW) in a disposable refractory-lined metal box using electrical power supplied through carbon electrodes. The feed is heated to the point that the LAW decomposes and combines with the solids to generate a vitreous waste form. This study supports the BV design and operations by exploring various methods aimed at reducing the quantities of soluble Tc in the castable refractory block (CRB) portion of the refractory lining, which limits the effectiveness of the final waste form. The CRB has sufficient open porosity to allow penetration of the low viscosity molten ionic salt (MIS) that forms when the LAW waste salts melt. This penetration of MIS is the primary mechanism that allows Tc in a soluble form to migrate into the CRB.
\end{abstract}

The MIS formed in significant quantities at temperatures above $300^{\circ} \mathrm{C}$, remained stable until roughly $550^{\circ} \mathrm{C}$ where it began to thermally decompose, and was completely decomposed by $800^{\circ} \mathrm{C}$. The estimated volume fraction of MIS in the feed was greater than 40\%, and the CRB material contained 11 to $15 \%$ open porosity, a combination allowing a large quantity of MIS to migrate through the feed and penetrate the open porosity of the CRB. If the MIS is decomposed at temperatures below $300^{\circ} \mathrm{C}$ or can be contained in the feed until it fully decomposes by $800^{\circ} \mathrm{C}$, MIS migration into the CRB can be avoided.

Laboratory and crucible-scale experiments showed that a variety of methods can decrease MIS penetration into the CRB individually or in combination. Modification of the CRB to block MIS penetration was not deemed practical as a method to prevent the large quantities of MIS penetration seen in the full-scale (FS)-38C test but may be useful to reduce the impacts of the lower levels of MIS penetration. Modification of the BV feed materials to better contain the MIS proved to be more successful.

A series of qualitative and quantitative crucible tests were developed that allowed screening of feed modifications that might be used to reduce MIS penetration. These tests showed that increasing the specific surface area of the soil (used as the primary glass-forming solid in the baseline process) by grinding stopped MIS penetration nearly entirely for feeds that contained waste simulants with lower quantities of nitrate salts. Grinding soil significantly reduced MIS penetration in feeds with higher nitrate quantities, but it was necessary to add carbohydrates (sucrose or cellulose) to destroy a portion of the nitrate at low temperatures to reach the same low levels of MIS penetration seen for the lower nitrate feeds.

Developing feeds to reduce MIS penetration in FS BV applications resulted in two additional refinements. Soil-grinding to the necessary levels proved to be difficult and expensive, so the fine soil was replaced with readily available fine-grained glass-forming minerals (GFMs). Cellulose was shown to have less impact on dryer operation and was chosen as the carbohydrate source to use in subsequent engineeringand FS tests. 



\section{Summary}

Bulk vitrification (BV) is a process that heats a feed material that consists of glass-forming solids and dried low-activity waste (LAW) in a disposable refractory-lined metal box using electrical power supplied through carbon electrodes. The feed is heated to the point that the LAW decomposes and combines with the solids to generate a vitreous waste form. This study supports the BV design and operations by exploring various methods aimed at reducing the quantities of soluble Tc in the castable refractory block (CRB) portion of the refractory lining, which limits the effectiveness of the final waste form. The CRB has sufficient open porosity to allow penetration of the low-viscosity molten ionic salt (MIS) that forms when the LAW waste salts melt. This penetration of MIS is the primary mechanism that allows Tc in a soluble form to migrate into the CRB.

Scoping tests from earlier studies (Hrma 2005a) helped eliminate methods that showed little promise, while other tests appeared inconclusive. However, the understanding of BV mechanisms and melt interaction with refractory materials gained from these earlier studies led to several new ideas that merited further exploration. The exploratory study described in this report was based on previous work, ideas that emerged during experimental studies, new ideas put forward during early brainstorming sessions, and new ideas that emerged during the work itself.

The importance of MIS penetration to the BV process is unique, and standard tests are not available to readily measure the amount of MIS penetration. This exploratory study needed to develop meaningful measurement techniques in parallel with screening and down-selecting potential mitigation methods. Detailed information on various measurement methods and early down selection are included in the appendixes. The main body of the report describes the CRB and glass-feed compositions in Section 4; reviews important MIS, feed, and CRB properties in Section 5; summarizes the less successful CRB treatment methods in Section 6; discusses the positive impacts of increasing the specific surface area of feed solids in Section 7; describes the positive impacts of carbohydrate addition that were necessary to reduce MIS penetration from feeds containing S-109 waste simulant in Section 8; and finishes with a summary of the additional feed modifications that were necessary for large-scale applications in Section 9. The overall conclusions from Sections 5 to 9 are given below.

Measurements and calculations of the MIS, CRB, and feed properties were performed to improve the understanding of MIS penetration. The results of these measurements are:

- The volume fraction of MIS in six-tank composite feeds before decomposing was $41.6 \%$. Considering that $\mathrm{B}_{2} \mathrm{O}_{3}$ can combine with MIS to form a common liquid phase, the volume fraction of liquid phase in the feed at temperatures above $450^{\circ} \mathrm{C}$ could be as high as $46 \%$.

- Although the CRB material (Viborcast 60PC) is dense by refractory standards, it still contains 11 to $15 \%$ open porosity.

- The results of evolved gas analyses, thermal gravimetric analysis, differential thermal analysis, and hot-stage microscopy show that the waste ingredients undergo minor reactions with organic carbon in the feed temperatures as low as $150^{\circ} \mathrm{C}$ but remain essentially unchanged to temperatures of about $300^{\circ} \mathrm{C}$. At temperatures above $300^{\circ} \mathrm{C}$, the waste materials begin to form a single molten MIS 
phase. This MIS remains stable until roughly $550^{\circ} \mathrm{C}$ where it begins to thermally decompose with the evolution of gas. By $800^{\circ} \mathrm{C}$, the thermal decomposition of the nitrate salts is complete.

Modification of the CRB was not deemed practical as a method to prevent the large quantities of MIS penetration seen in FS-38C. However, these methods may be useful to reduce the impacts of the lower levels of MIS penetration. The conclusions from the methods attempted in this study are given below:

\section{Sacrificial Layer at CRB-Feed Contact Surface}

- Crucible tests showed that a sacrificial layer could be made by adding 20 to 50 mass $\% \mathrm{Na}_{2} \mathrm{SiO}_{3}$ (NS) to the 60PC material. Adding 25 mass\% NS appeared to give the best results in that the material stayed intact to high enough temperatures to be in place during the MIS attack but completely dissolved in the glass melt when heated to $1200^{\circ} \mathrm{C}$.

- Manufacturability tests indicated that a large composite sample of 60PC with a sacrificial layer made from 75\% 60PC and 25 mass\% NS could be fabricated. This composite had good structural integrity as-prepared and after cooling from a heat-treatment to $1200^{\circ} \mathrm{C}$. This qualitative test indicated that this type of composite could be prepared at the larger scales required for BV.

Treating the CRB with a Lyophobic Material (Carbon)

- Of the various carbon sources added to 60PC, the best results were obtained by adding 18 mass\% of graphite. This $18 \%$ addition of ground-graphite decreased the depth of MIS penetration into a CRB sample from $10 \mathrm{~mm}$ to less than $6 \mathrm{~mm}$.

- Manufacturability tests indicated that a large composite sample of 60PC with an inner layer of 60PC containing 17.4 mass\% of graphite could be fabricated. The altered layer formed a porous solid with fine cracks running through the material. It appeared to bond well to the layer, but it crumbled under pressure, showing that this type of composite might not be durable enough for $\mathrm{BV}$ applications. No attempt was made to measure the impact of carbon addition on the electrical conductivity of the modified CRB or determine whether graphite in the CRB can resist the environment above the melt line.

\section{Protecting the CRB Surface with alumina-zirconia-silica (AZS) Tiles}

- Room-temperature dye-penetration tests and molten-salt dye-penetration tests showed that AZS tiles could be effectively bonded to CRB materials and that there was no preferential penetration of the dye or molten salt through the AZS-CRB interface.

- The AZS porosity was sufficiently open to allow penetration of molten salt into the tile. Thus, while the interface was well bonded, both molten salt and ink penetrated into AZS tiles as easily as in to the CRB, indicating that the tiles would supply insufficient protection against MIS penetration. 
Immobilizing Contaminants in the Upper Portion of the CRB with Higher Temperatures

- Analyses of the CRB from a partially completed full-scale test (FS-38B) showed that condensation on the upper portions did not lead to significant Re migration into the CRB. As a result, efforts in this area were reduced to a series of scoping tests.

- Both pH and electrical conductivity of leachates from heat-treated mixtures of CRB and simulated condensates decreased as the temperature of the heat-treatment increased, showing a progressive incorporation of the condensate into a durable glass phase that the condensate formed with the CRB bonding cement.

Immobilizing the MIS in the BV feeds by increasing the surface area of the feed solids proved to be a more effective way to reduce MIS penetration. The conclusions from the early portions of the MIS penetration studies are given below. All the initial studies were performed using six-tank composite waste-feed simulant.

- Dissolving $\mathrm{B}_{2} \mathrm{O}_{3}$ in aqueous LAW before blending it with other feed materials did not decrease MIS mobility. Preliminary tests showed that $\mathrm{B}_{2} \mathrm{O}_{3}$ substantially increased the liquidus temperature of MIS. However, MIS penetration tests showed extensive penetration even when $\mathrm{B}_{2} \mathrm{O}_{3}$ was added at twice the concentration used in the current baseline glass formulation.

- Silica crucibles were effective model refractories and had several advantages over CRB tests. The silica crucibles were more uniform in composition, structure, and porosity; and MIS penetration areas had a distinct color and a sharp boundary, allowing unambiguous assessment of MIS penetration levels.

- Tests of MIS penetration from feed into silica crucibles showed that significant penetration starts as low as $350^{\circ} \mathrm{C}$ and increases as the temperature increases to $500^{\circ} \mathrm{C}$. At higher temperatures, MIS decomposition becomes more extensive. At $800^{\circ} \mathrm{C}$, the MIS is fully decomposed, and little additional penetration occurs.

- MIS penetration has no visual effect on the $60 \mathrm{PC}$ material at temperatures up to $750^{\circ} \mathrm{C}$. At temperatures of $1200^{\circ} \mathrm{C}$ or higher, the penetrated MIS causes significant swelling of the CRB and can lead to crack formation at an interface between an MIS-saturated region and a dry portion of the CRB.

- Crucible experiments indicated that the atypical nature of the dry-blended feed used for the FS-38C test did not contribute to the extensive MIS penetration. Dryer-prepared feed would have produced similar results.

- A partial replacement of soil with fine-grain materials, including silica powder ( $<75 \mu \mathrm{m})$, kaolin clay, bentonite clay, diatomaceous earth, and fly ash, did not significantly reduce MIS migration. The best initial candidate was a 10 -wt $\%$ addition of diatomaceous earth, but increasing the quantity of this material from 10 to 15, 20, and 25 wt\% led to a progressively worse crucible degradation. 
- $\quad$ CRB and silica crucible tests showed that grinding the Hanford soil reduced MIS penetration depths. The degree of reduction was a function of the extent of grinding and the fraction of ground material added. These results indicated that the reduction in MIS migration was a function of the specific surface area of feed solids and that sufficiently high surface areas in the feed solids could eliminate MIS penetration.

- Tests with CRB rods showed that the extent of MIS penetration at $550^{\circ} \mathrm{C}$ continued to increase for periods up to 24 hours for feeds prepared with as-received Hanford soil. Ground soils showed no increased MIS penetration for time periods as long as 24 hours, indicating that high-surfacearea materials would prevent MIS penetration in FS melts.

The early FS work was conducted with feeds prepared with the six-tank composite simulant to tie back to the earlier Tc-spiked ES tests that also used six-tank composite feeds. However, because the Demonstration Bulk Vitrification System would be treating waste from S-109, a decision was made to shift to the S-109 simulant for the FS-38C test. Since six-tank simulant was used in all work described in previous sections of this report, additional tests were designed to obtain data on the impact of soil grinding on S-109 feed. The MIS migration behavior was different for the S-109 simulant. The conclusions from the S-109 MIS migration studies are given below.

- The S-109 simulant showed significantly higher MIS penetration levels than the six-tank composite. The main difference in the compositions is that S-109 has a higher content of nitrates (281 versus $158 \mathrm{~g} / \mathrm{kg} \mathrm{NaNO}_{3}$ ) and a lower content of organic components ( 0.8 versus $8.7 \mathrm{~g} / \mathrm{kg}$ $\mathrm{CH}_{3} \mathrm{COONa}$ ). The S-109 simulant also contains a significantly lower concentration of $\mathrm{Na}_{2} \mathrm{CO}_{3}$ and $\mathrm{NaOH}$ ( 0 versus $41 \mathrm{~g} / \mathrm{kg} \mathrm{Na}_{2} \mathrm{CO}_{3}$ and 6 versus $41 \mathrm{~g} / \mathrm{kg} \mathrm{NaOH}$ ). All these differences are expected to play a role in the different MIS penetration behavior, but the degree of impact for the various factors were not investigated in this study.

- The soil grinding operations showed little impact on MIS migration when grinding times were increased from 2 to 5 minutes. This indicated that soil grinding as the sole MIS migration mitigation might have practical limits.

- The MIS penetration behavior for the S-109 simulant was similar to that seen for the six-tank composite. Little penetration occurred at a temperature of $300^{\circ} \mathrm{C}$, but significant penetration occurred at temperatures of 400 and $500^{\circ} \mathrm{C}$.

- The ground soil reduced MIS penetration for the S-109 simulant by 75\% but did not reduce penetration to the low levels seen for the six-tank composite feed. This indicated that soil grinding alone would not be enough to reduce MIS penetration to the desired levels.

- Adding diatomaceous earth and high-surface-area bentonite clay to ground soil did not significantly reduce the MIS penetration levels.

- Adding organic carbon in the form of sucrose significantly reduced the level of MIS penetration. Mass-loss calculations confirmed that this was the result of nitrate destruction at low enough temperatures to prevent MIS migration. Sugar additions were effective in both as-received soil and in ground soil. 
- Combining the effects of increased surface area in the feed solids and the effects of sucrose additions indicated that a family of solutions where MIS penetration could be essentially eliminated existed. However, practical limits for soil grinding and sugar-addition levels narrowed the range of possible solutions.

The early laboratory work established that MIS migration could be controlled through combinations of high-surface-area solid materials and the addition of carbon supplied as sucrose. The next phase of the study used this laboratory result to explore its practical implementation for FS operations. The conclusions from the application phase of this study are given below.

- Problems associated with drying the bulk vitrification feed with sucrose additions required investigation of alternate forms of carbon. Alpha cellulose, which performed better in dryer tests, was shown to be as effective as sucrose in the reduction of MIS migration. Sucrose, cellulose, or combinations of the two produced essentially the same results. The reduction in MIS penetration was a linear function of the amount of carbon added to the feed.

- Small silica crucible tests showed that MIS penetration in feeds with as-received Hanford soil could be reduced to low levels with carbon additions corresponding to an approximately 1.2 carbon-to-nitrogen $(\mathrm{C}: \mathrm{N})$ ratio. Feeds with ground soils only required a carbon addition of 0.75 $\mathrm{C}: \mathrm{N}$ ratio to achieve low MIS penetration levels. The C:N ratio expresses the number of moles of $\mathrm{C}$ from carbohydrates (sucrose, cellulose) added per one mole of $\mathrm{N}$ in nitrates and nitrites in MIS.

- Studies conducted by Praxair under the direction of Pacific Northwest National Laboratory indicated that grinding Hanford soil to levels equivalent to that obtained in the laboratory grinding operations would be an expensive operation that would reduce the benefits of using Hanford soil as an inexpensive feedstock for bulk vitrification melting operations.

- GFMs similar to those used by the Waste Treatment Plant did not significantly reduce MIS migration until the -200 mesh silica material was replaced with silica that had a particle size of $<5 \mu$.

- The use of GFMs would allow for higher waste loading by replacing the sodium and potassium flux materials in the soil with waste sodium. A rough-cost estimate showed that the increased waste loading would essentially balance the cost of purchasing and shipping the GFMs.

- The BET-measured surface area of the GFMs with the fine silica $(<5 \mu)$ was only half the value of the as-received Hanford soil. If confirmed, this result indicates that reducing the surface area in a specific type of feed solid will tend to reduce MIS penetration but that it is not possible to predict MIS penetration reduction only from measurements of the surface areas of feed solids. The reasons for the difference in the performance of the GFMs versus the Hanford soil were not investigated further in this study.

- Feeds without carbohydrate additions resulted in a homogeneous coloration of the crucible internal surface. Carbohydrate-containing feeds left a spotted crucible surface. The spotting 
effect tended to be more pronounced for coarser feed solids. The spotting effect was probably associated with non-uniform distribution of carbohydrates in granulated feeds.

- Feeds with higher C:N carbohydrate additions tended to produce foam in crucibles and smoke at high enough rates to be noticeable outside the treatment furnace. Although higher C:N carbohydrate additions further reduced MIS penetration, levels greater than 0.75 are not recommended due to the rapid feed reactions.

- Small silica crucible tests indicated that carbohydrate additions resulted in temperature spikes in the feed. These temperature spikes were initiated at the outer portion of the crucible at an average temperature of $246^{\circ} \mathrm{C}$ and resulted in an average maximum temperature of $467^{\circ} \mathrm{C}$. The initiation temperature and maximum temperature were independent of carbohydrate levels. However, propagation of the reaction to the center of the crucible appeared to happen faster with higher carbohydrate additions. This thermal behavior indicated that, as desired, the carbohydrate reactions started and destroyed a portion of the nitrates before a significant quantity of MIS formed. The average maximum temperature of the feed indicates that the energy given off in the nitrate-destruction reaction and retained in the feed was not excessive and was only sufficient to raise the temperature of the feed to the furnace temperature.

- A side benefit to the presence of carbohydrates in feed is accelerated conversion reactions that proceed more uniformly within the feed volume.

The last set of confirmatory tests was conducted in large CRB crucibles. The conclusions from those tests are given below:

- The large CRB crucible tests proved to be an effective way to test possible solutions at a scale that better represented the cold-cap thicknesses that might be expected in the FS BV melter. The large CRB tests generally confirmed the results obtained in the silica crucible tests.

- Three baseline tests were conducted with feeds containing as-received soil and S-109 simulant. These tests showed that more than $40 \%$ of the MIS migrated to the wall of the CRB crucible, and a significant portion passed through the wall. A CRB crucible with a cemented seam performed no worse than an as-received crucible, indicating that the seams in the FS CRB are not the weak points for MIS penetration. A comparison of the feed prepared with the liquid simulant and the simulated FS-38C feed showed that the MIS penetration was not simply a result of using a nonprototypic dry blended feed in FS-38C and that dryer prepared feed using as-received Hanford soil would also show significant MIS migration. The baseline results demonstrated the high mobility of MIS and demonstrated that the MIS would travel at least several inches if not further.

- Limited large CRB crucible tests indicated that using only carbohydrate additions with asreceived soil to reduce MIS migration was not as effective at the larger CRB crucible scale. Total migrated MIS dropped to 10.3 mass\% in the small silica crucible when sucrose was added at a $\mathrm{C}: \mathrm{N}$ ratio of 0.63 to a feed with as-received soil, but the same sucrose addition allowed 31.8 mass $\%$ of the MIS to penetrate into the large CRB crucible wall. 
- Large CRB crucible tests confirmed that adding fine-grained materials improved the effectiveness of carbohydrate additions to reduce MIS penetration. An addition of cellulose at a C:N of 0.75 and the use of fine-grain GFMs in place of soil resulted in a decrease of migrated MIS to an average value of $6.8 \%$ (i.e., $6.8 \%$ of MIS initially present in feed migrated to the crucible compared with $44 \%$ from feed containing as-received soil).

- Thermocouples placed in multiple locations in a large CRB crucible melt showed similar temperature spikes to those seen in the silica crucible tests. These spikes were roughly the same magnitude as those seen in the silica crucibles, but the center portion of the feed showed a significant delay of 15 to 30 minutes. After the outer portions reacted, the center portions of the feed heated to $100^{\circ} \mathrm{C}$ but plateaued at this temperature for 15 minutes before reacting further. This temperature behavior indicates that the outer portions of the feed dry out and react but that the propagation of the reaction to nearby areas is delayed by the energy requirements necessary to drive off the residual moisture in the feed and heat the solids in those locations. These results indicate that runaway reactions of large beds of cellulose-containing feed are unlikely.

The results of this extensive study lead to several recommendations related to future activities:

- Near term ES and FS tests should focus on formulations that include the use of fine-grained GFMs and alpha-cellulose. The GFMs should include silica sources that have a particle size of $<5 \mu \mathrm{m}$ and an addition of cellulose at a C:N of 0.75 .

- Investigations should be conducted to find less expensive sources of suitable fine silica and cellulose material. These two materials account for the majority of the formulation modification costs that were necessary to reduce the MIS migration.

- Investigations should be conducted to take advantage of the flexibility supplied by the shift from Hanford soil to GFMs and produce improved glass waste forms. Potential improvements that might be realized include higher waste loadings, reduced metal precipitation, better sulfate tolerance, and improved processing rates.

- Investigations should be conducted to better understand why the surface area of the GFMs and Hanford soil appear to have different MIS migration reduction abilities. The improved understanding might lead to better formulations with reduced costs.

- Investigations should be conducted to better understand the difference in the MIS penetration behavior of the six-tank composite and the S-109 simulants. These investigations would help ensure that the S-109 simulant represents the most difficult wastes from a MIS mitigation standpoint and might suggest tank blending schemes or additions that could be made that would reduce MIS migration.

- Further investigations of the cellulose/nitrate/nitrite waste reactions should be conducted to ensure that the exothermic reactions necessary to prevent MIS migration are properly controlled in other portions of the BV process to address any safety concerns. 
- If the small levels of MIS penetration seen with the GFM/cellulose glass formulations prove to be a concern, modifying the inner layer of the CRB with sodium silicate appeared to be an effective way to create a sacrificial layer on the inner surface that might help return any penetrated Tc back to the glass melt. ES tests would be the next step in a process to see if this method could further reduce the levels of Tc that remain in the CRB after melting. 


\section{Acronyms}

AZS alumina-zirconia-silica

BET Brunauer, Emmett, and Teller (a method for measuring surface areas)

BV bulk vitrification

CRB castable refractory block

DTA differential thermal analysis

EDS energy dispersive spectroscopy

ES engineering scale

ESF engineering-scale feed

FS full scale

GFM glass-forming mineral

LAW low-activity waste

LOI loss-on-ignition

MIS molten ionic salt

NS $\quad \mathrm{Na}_{2} \mathrm{SiO}_{3}$ (sodium metasilicate)

PSD Particle Size Distribution

SEM scanning electron microscopy

TC thermocouple

TGA thermal gravimetric analysis

WTP Waste Treatment Plant 



\section{Contents}

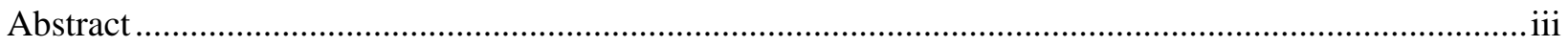

Summary

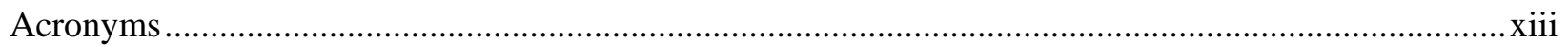

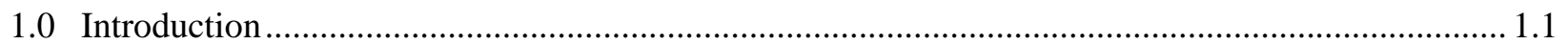

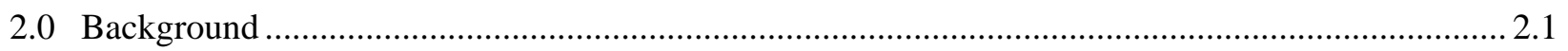

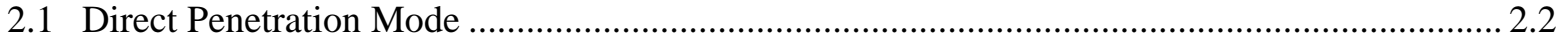

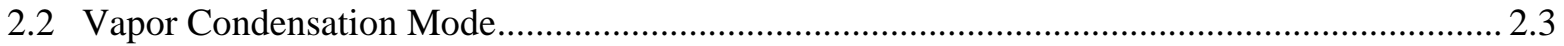

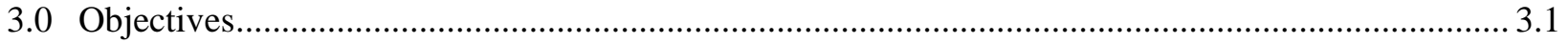

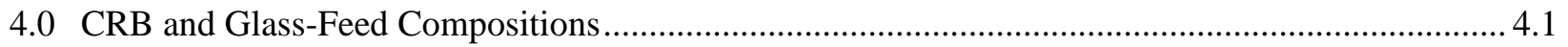

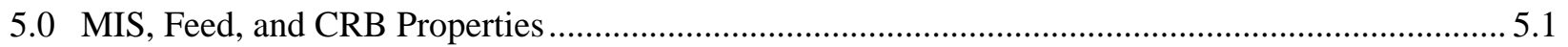

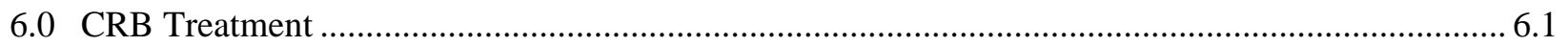

6.1 Creating a Sacrificial Inner Layer on CRB …....................................................................... 6.1

6.2 Creating an Inner CRB Layer that is less Susceptible to MIS Penetration ............................... 6.2

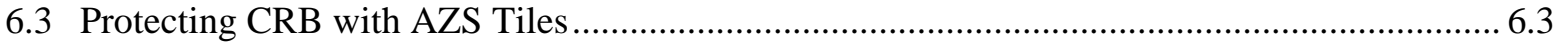

6.4 Converting Tc/Re into Insoluble Form in the Upper CRB...................................................... 6.3

7.0 MIS Immobilization by Increasing Specific Surface Area of Feed Solids ..................................... 7.1

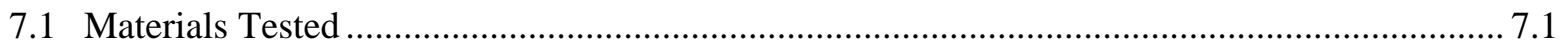

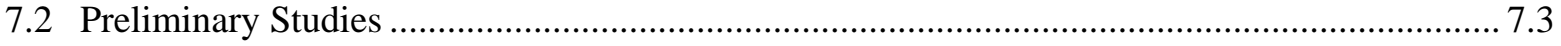

7.3 Effect of Temperature and Time on MIS Penetration ................................................................. 7.5

7.4 Effect of Fine-Grain Materials on MIS Penetration .................................................................. 7.6

7.5 Effect of Ground Soil Fraction on MIS Penetration from Six-Tank Composite Feed into

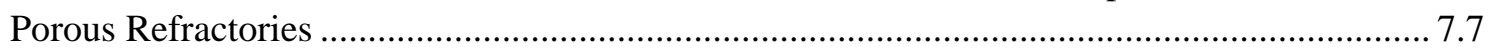

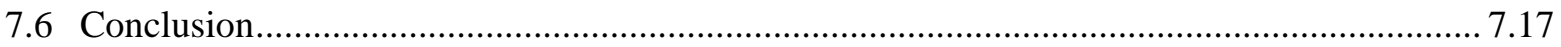

8.0 Study of MIS Penetration from Feeds Containing S-109 LAW Simulant ........................................ 8.1

9.0 Feed Development for Large-Scale Applications ........................................................................ 9.1

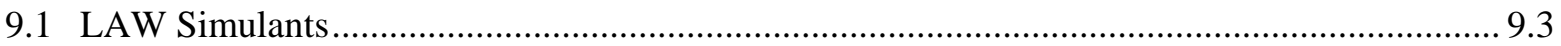

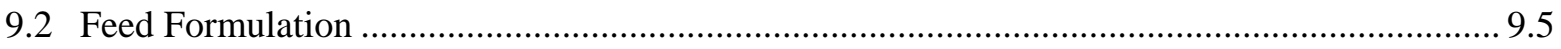


9.3 MIS Migration: Experiments with Silica Crucibles .......................................................... 9.9

9.4 MIS Migration: Experiments with Large Vibrocast Crucibles …........................................ 9.26

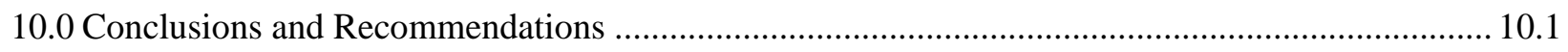

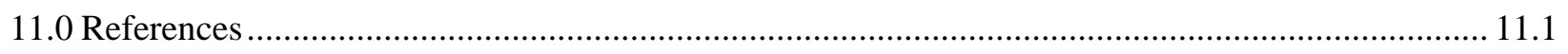

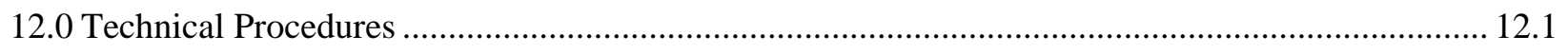

Appendix A: CRB Inner-Surface Treatment ................................................................................. A.1

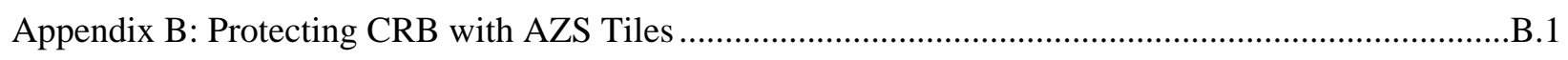

Appendix C: Converting Tc/Re into Insoluble Form in the Upper CRB ..............................................1

Appendix D: MIS Immobilization by Increasing Specific Surface Area of Feed Solids:

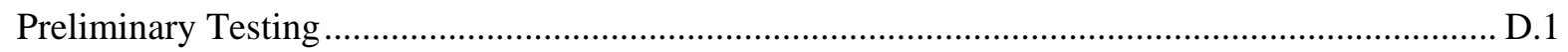

Appendix E: Effect of Time, Temperature, and Particle Size on MIS Penetration into Porous

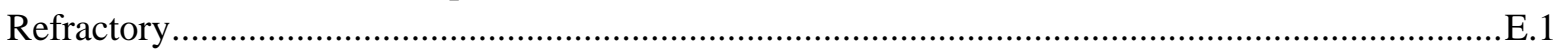

Appendix F: MIS Penetration into Porous Refractory from Feeds Containing Fine-Grain Materials........F.1

Appendix G: Room-Temperature Physical Modeling …................................................................... G.1

Appendix H: Effect of Ground Soil Fraction on MIS Penetration into Porous Refractory ...................... H.1

Appendix I: Impact of Soil Grinding on the Particle Size Distribution and the Specific Surface Area ...... I.1

Appendix J: Effect of Temperature on MIS Migration for Feeds with S-109 Simulant.............................1

Appendix K: Effects of Soil Grinding, Fine-Grained Materials, and Carbohydrate Addition on MIS

Migration for Feeds with S-109 Simulant................................................................................ K.1

Appendix L: Experiments with Ground Soil and Sucrose Addition .....................................................1

Appendix M: Temperature Evolution Data ....................................................................................... 


\section{Figures}

5.1. Evolved Gases from and Relative Volume of BV Feed [Hrma 2005a] ........................................5.3

5.2. DTA-TGA of $\mathrm{B}_{2} \mathrm{O}_{3}$ Predissolved in LAW Simulant Solution

5.3. Hot Stage Microscopy Images of LAW (six tank composite sans $\mathrm{Al}\left(\mathrm{NO}_{3}\right)_{3} \cdot 9 \mathrm{H}_{2} \mathrm{O}$ and $\mathrm{NaOH}$ ) and the Six-Tank Composite Baseline Feed; Numbers Under the Images Indicate Temperature in ${ }^{\circ} \mathrm{C}$ (the rate of heating was $5^{\circ} \mathrm{C} / \mathrm{min}$, and the crucible diameter was $6 \mathrm{~mm}$ )

6.1. Crucible Section Showing the Destruction of the NS-Containing Layer and Partly Corroded Vibrocast Compact and Specimen (the assembly was heat-treated for 4 hours at $1200^{\circ} \mathrm{C}$ ).

6.2. Concentration Profile by EDS of Compacted Vibrocast Mixed with 18 mass\% of Graphite

6.3. Effects of Temperature (top) and Condensate Composition (bottom) on the Leachate $\mathrm{pH}$

7.1. Dimensions of a Randomly Selected Medium-Sized Silica Crucible; a CRB Rod Is Adjusted by a Pt Wire in the Middle of the Feed

7.2. Comparison of MIS Penetration to Silica Crucibles and CRB Rod from Baseline Feed and 38B Feed

7.3. Mass of MIS Soaked into Crucible Wall Versus Temperature

7.4. Sections Through Medium Crucibles with Feeds Containing Fine-Grain Ingredients Heated at $5^{\circ} \mathrm{C} / \mathrm{min}$ to $1200^{\circ} \mathrm{C}$ for 1 -Hour Dwell

7.5. Sections Through Crucibles and Vibrocast Rods Exposed to Feed with 0 to 25 Mass\% of Diatomaceous Earth

7.6. CRB Specimens Exposed to Feed with Ground Soil Heated to $800^{\circ} \mathrm{C}$; Sample Scan Went Through the Middle of Sample from Left to Right (left) and from Bottom to Top (right)

7.7. MIS Migration Depth Versus Fraction of Soil Ground.

7.8. A Typical Assembly of CRB Rod and ESF (original feed top and ground feed bottom) in a Pt Crucible Before (left) and After (right) Heat-Treatment at $550^{\circ} \mathrm{C}$ for 1 Hour

7.9. Micrographs of Thin Sections of CRB Samples Heat-Treated at Various Times and Temperatures Before (right image) and After (left image) Heat-Treatment in Baseline Feed........ 7.11

7.10. Cross-Section Through a CRB Rod Heat-Treated in Original (baseline) Feed at $550^{\circ} \mathrm{C}$ for 1 Hour and then at $1200^{\circ} \mathrm{C}$ for 1 Hour ....

7.11. Comparison of Microstructure of CRB Samples Heat-Treated With (left and right) and Without (center) the Presence of Feed

7.12. A Different Image of the Sample Shown in 7.11 (heat-treated in feed to $550^{\circ} \mathrm{C}$ for 1 hour and then at $1200^{\circ} \mathrm{C}$ for 1 hour).

7.13. Three CRB Rods After Exposure to Ground Feed at $550^{\circ} \mathrm{C}$ and Subsequently Heated at $1200^{\circ} \mathrm{C}$; the Sections Are on the Right

7.14. Effect of $550^{\circ} \mathrm{C}$ Heat-Treatment Time on MIS Penetration to CRB from Original and Ground Feed; the Lines Represent a Square-Root Fit. 


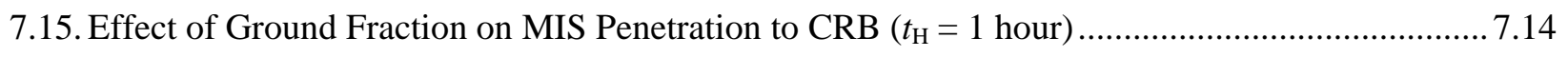

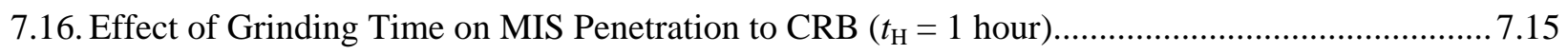

7.17. Feed Density as a Function of Ground Fraction.......................................................................... 7.15

7.18. Particle Size Distribution of Original and Ground (for 120 s) Feed, Untreated, and

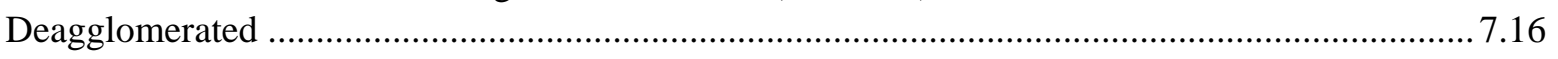

7.19. Particle Size Distribution of Original (as-received) and Ground (for 120 s) Feed, Untreated

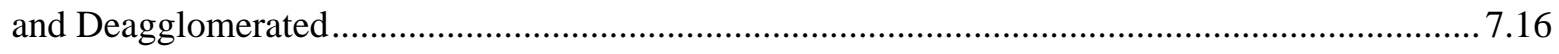

7.20. Effect of Specific Surface Area on MIS Penetration to CRB $\left(t_{\mathrm{H}}=1\right.$ hour $)$................................. 7.17

8.1. Comparison of PSD of Hanford Soil As-Received and Ground for 2 and 5 Minutes; Samples were Deagglomerated

8.2. Comparison of Specific Surface Area of Hanford Soil Measured with BET Method with the Area Calculated from the Deagglomerated PSD ....................................................................... 8.4

8.3. Effect of Temperature on MIS Penetration to Silica Crucible from Feed with S-109 Simulant....... 8.5

8.4. Mass Loss of Feed (with S-109 simulant and as-received or ground soil) Versus Temperature .......8.6

8.5. Effect of Additions of Diatomaceous Earth (DE) and Bentonite Addition on MIS Migration into Silica Crucible from Feed with 2-Min Ground Soil (left) and on Unreacted MIS Fraction in Feed at $500^{\circ} \mathrm{C}$ (right).....

8.6. Effect of Organic Addition on MIS Migration into Silica Crucible from Feed with 2-Min Ground Soil (left) and on Unreacted MIS Fraction in Feed at $500^{\circ} \mathrm{C}$ (right).

8.7. Mass Balances for MIS at $500^{\circ} \mathrm{C}$ in Various Feeds Containing As-Received Soil (AR) and Ground Soil (for 2 and 5 min).

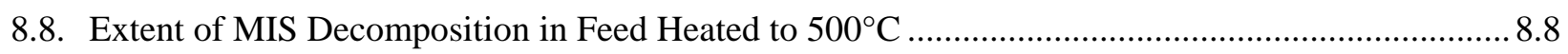

8.9. MIS Present in Crucible Walls at $500^{\circ} \mathrm{C}$, Fraction of Total Estimated from $1000^{\circ} \mathrm{C}$ Data 8.9

8.10. Effect of Sucrose Addition (in stoichiometric \%) on the Fraction of MIS Lost from Feed with Soil As-Received (5.74 $\mathrm{m}^{2} / \mathrm{g}$ specific surface area) and Ground $\left(9.75 \mathrm{~m}^{2} / \mathrm{g}\right.$ specific surface area) to Crucible Walls ( $\mathrm{G}$ granular sucrose added to dry feed, $\mathrm{P}$ powdered sucrose, AR as-received soil)

8.11. Effect of Soil Specific Surface Area (by BET) on the Fraction of MIS Lost from Feed to Crucible Walls (no sucrose has been added to feed).

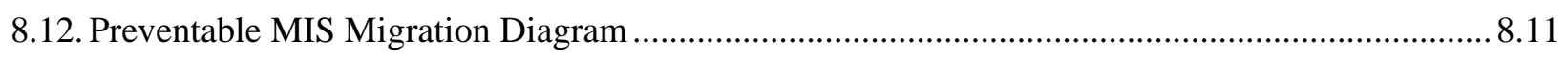

9.1. Vibrocast Crucible Before Heat-Treatment, Empty, and Filled with Feed .................................... 9.2

9.2. Mass Fraction of Silica-Crucible-Migrated MIS from Feed with As-Received Soil Versus Carbohydrate Addition

9.3. Mass Fraction of Silica-Crucible-Migrated MIS from Feed with Ground Soil Versus Carbohydrate Addition.

9.4. Mass Fraction of Silica-Crucible-Migrated MIS from Feed with Praxair Ground Soil Versus Carbohydrate Addition 
9.5. Mass Fraction of Silica-Crucible-Migrated MIS from Feed with GFMs Versus Cellulose Addition (see Table 9.11 notes for legend acronyms).

9.6. Mass Fraction of Silica-Crucible-Migrated MIS from Feed with GFMs Versus Cellulose Addition of C:N > 0.5 (see Table 9.11 notes for legend acronyms).....

9.7. Mass Fraction of Silica-Crucible-Migrated MIS from Feed with Zero Carbohydrate Addition.....9.15

9.8. Migrated MIS Fraction, Estimated Versus Measured (full points: data used for calculation;

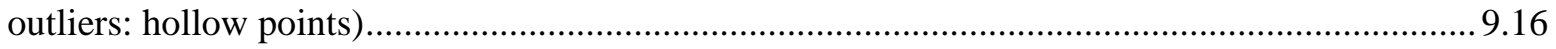

9.9. Preventable MIS Migration Diagram ……................................................................................. 9.17

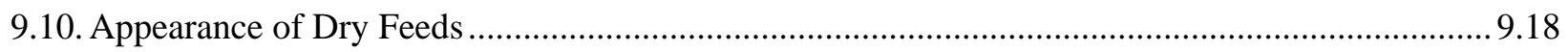

9.11. Appearance of Feeds as Removed from Crucibles (PSC Praxair Seattle Company, AR as

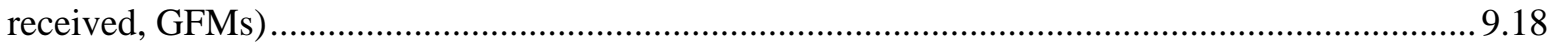

9.12. Appearance of Crucibles After Feeds Were Removed...................................................................18

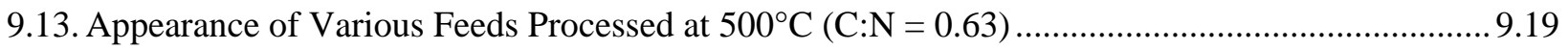

9.14. Feeds from As-Received Soil with Various Additions of Carbohydrates Processed at $500^{\circ} \mathrm{C}$ (top: all carbon from cellulose; bottom: 0.13 carbon from sucrose, the rest from cellulose).......... 9.19

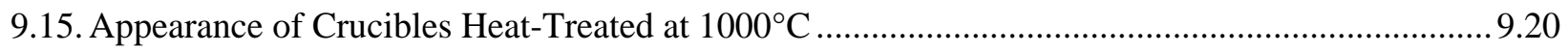

9.16. Appearance of Crucibles Heat-Treated at $1000^{\circ} \mathrm{C}$ (carbohydrate was added to the feeds removed at $500^{\circ} \mathrm{C}$ )

9.17. Appearance of Crucibles Heat-Treated at $1000^{\circ} \mathrm{C}$ (feeds were removed at $500^{\circ} \mathrm{C}$; no carbohydrate was added).

9.18. Appearance of Crucibles Heat-Treated at $1000^{\circ} \mathrm{C}$ (feeds with as-received soil were removed at $\left.500^{\circ} \mathrm{C}\right)$......

9.19. Appearance of Crucibles Heat-Treated at $1000^{\circ} \mathrm{C}$ (carbohydrate was added to GFM-

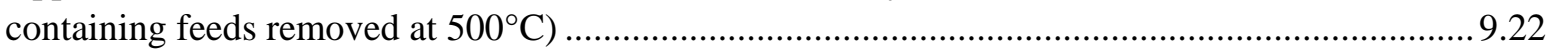

9.20. Thermocouple Placement in Silica Crucible ..............................................................................2. 9.23

9.21. Feed Temperature Response to Furnace Temperature Increasing at $5^{\circ} \mathrm{C} / \mathrm{min}$ (see Appendix M; AR as received soil, PSC soil ground at Praxair, GFMs, M feed body center, $\mathrm{T}$ feed top surface; numbers after the feed acronym represent the C:N ratio)

9.22. Reaction Starting Temperature in Feed Center as a Function of Carbohydrate Addition...............9.26

9.23. Crucible with a Joint Before and After the Heat-Treatments ....................................................... 9.27

9.24. Feeds Removed from Crucible after 20 Hours at $500^{\circ} \mathrm{C}$ (38-C feed left and Praxair feed right) ... 9.27

9.25. Crucibles with Feed after $500^{\circ} \mathrm{C}$ Heat-Treatment; from Left to Right: Feed with Sucrose, Feed with GFMs (dryer I), and Feed with Praxair-Ground Soil 9.27

9.26. Vibrocast Crucibles after $1000^{\circ} \mathrm{C}$ Heat-Treatment 9.28

9.27. Mass Fraction of Silica-Crucible-Migrated MIS from Feed with Soil Versus Carbohydrate Addition, Including Vibrocast (CRB) Crucible Data 
9.28. Mass Fraction of Silica-Crucible-Migrated MIS from Feed with GFMs Versus

Carbohydrate Addition, Including Vibrocast (CRB) Crucible Data

9.29. Migrated MIS Fraction, Vibrocast Versus Silica Crucibles ............................................................ 9.31

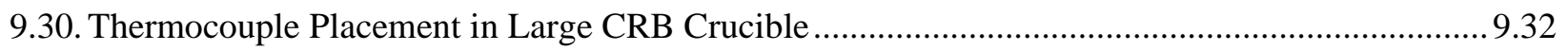

9.31. Temperature Field Evolution of CRB Crucible Feed................................................................ 9.34

9.32. The Initial Stage of Temperature Field Evolution of CRB Crucible Feed .......................................34

9.33. Temperature Field Evolution of CRB Crucible Feed During the Heat-Up Period.......................... 9.36

9.34. Temperature Field Evolution of CRB Crucible Feed During the Constant Furnace

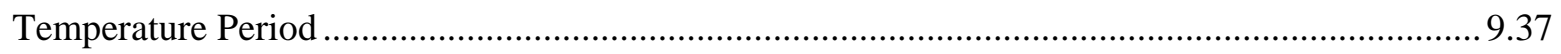

A.1. Mixtures of Vibrocast with NS Heat-Treated at $1200^{\circ} \mathrm{C}$ for 2 Hours........................................... A.2

A.2. Vibrocast with 50 Mass\% NS Heat-Treated at $1200^{\circ} \mathrm{C}$ for 2 Hours............................................. A.2

A.3. LOI of NS as a Function of Temperature and NS Content (in mass fractions)............................... A.3

A.4. Vibrocast with 50 Mass\% of NS (top) and with 20 Mass\% of NS (bottom) After Heating at $5^{\circ} \mathrm{C} / \mathrm{min}$ to the Following Temperatures (from left to right): 800, 900, 1000, 1100, 1200, 1300,

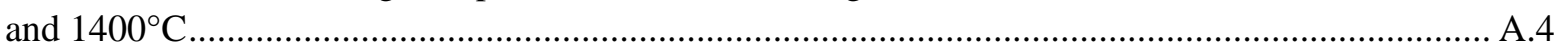

A.5. Fractured Vibrocast with 20-Mass $\%$ of NS Heated to $1300^{\circ} \mathrm{C}$ (left) $1400^{\circ} \mathrm{C}$ (right) ..................... A.4

A.6. Fractured Vibrocast with 50-Mass\% of NS Heated to (from left to right) $1000^{\circ} \mathrm{C}, 1100^{\circ} \mathrm{C}$, $1200^{\circ} \mathrm{C}, 1300^{\circ} \mathrm{C}$, and $1400^{\circ} \mathrm{C}$

A.7. Detail of Fractured Vibrocast with 50 -mass\% of NS Heated to $1300^{\circ} \mathrm{C}$ (left) and $1400^{\circ} \mathrm{C}$ (right)

A.8. CRB Specimen, as Cut (left) and After 1 Hour at $1200^{\circ} \mathrm{C}$ (right) .....

A.9. ESF Melted over Compact Vibrocast (left), CRB Specimen (middle), and Compact Vibrocast with $20 \%$ of NS (right) $1300^{\circ} \mathrm{C}, 1 \mathrm{Hr}$.....

A.10. Alumina Crucibles with Compact Vibrocast (left), CRB Specimen (middle), and Compact Vibrocast with 20\% of NS (right); the Refractories Were Covered with ESF and Heat-Treated; Sections Through the Crucibles Are in the Bottom Row of Images $1200^{\circ} \mathrm{C}, 1 \mathrm{Hr}$.

A.11. Alumina Crucibles with Compact Vibrocast (left), CRB Specimen (second from left), Compact Vibrocast with 20\% of NS (second from right), and Compact Vibrocast with $30 \%$ of NS (right); the Refractories Were Covered with ESF and Heat-Treated at $1200^{\circ} \mathrm{C}$ for 1 Hour; the Rows Show (from above down) Raw Refractory Materials, Melts, Sections Through Crucibles, and Details of Refractory Materials After Heat-Treatment

A.12. Preparation of CRB Assembly: CRB Specimen, Specimen and Vibrocast Compact, and Vibrocast with 25 mass\% NS on Top ..... 
A.13. Crucible Section Showing the Destruction of the NS-Containing Layer and Partly Corroded

Vibrocast Compact and Specimen (the assembly was heat-treated for 4 hours at $1200^{\circ} \mathrm{C}$ )

A.14. Details of the Refractory-Melt Assembly Heat-Treated for 4 Hours at $1200^{\circ} \mathrm{C}$

A.15. From Left to Right: Crucible Containing Vibrocast with 25 Mass\% of NS Dried at $200^{\circ} \mathrm{C}$; Same Crucible with ESF Heated to $500^{\circ} \mathrm{C}$; Same Crucible After Feed Removal and Heating to After $1200^{\circ} \mathrm{C}$

A.16. Vertical Section Through the Crucible Shown in A.15, Right.

A.17. Interface Between Vibrocast and Vibrocast-NS (25\%) Mixture.

A.18. CRB Soaked with Caramel (top left) and with Parowax (top right); Bottom Pictures Show Specimens Sectioned After Heat-Treatment in Baseline Feed

A.19. Sections Through Crucibles with CRB Mixed with 20 Mass\% Carbonaceous Material at the Bottom and Glass on the Top.....

A.20. Concentration Profiles by EDS of Compacted Vibrocast Mixed with 22.4 Mass\% of Carbon Flakes (left) and 18.0 Mass\% Graphite (right).....

A.21. Alumina Crucibles with Compacted CRB-Lampblack and CRB-Graphite Mixtures and Glass Melted at $1200^{\circ} \mathrm{C}$ for 1 Hour.

A.22. Double Slab with Lampblack After $200^{\circ} \mathrm{C}$ (above left) and $1200^{\circ} \mathrm{C}$ (below left) HeatTreatment; the Slabs with Graphite Are on the Right

B.1. Thermal Expansion of Greystone Tile, AZS, and Vibrocast 60PC Specimen (specimens heated and cooled down at $2^{\circ} \mathrm{C} / \mathrm{min}$ ) (Hrma et al. 2005a)

B.2. Fractured CRB-AZS Composite After 5-min (left) and 2-hour (right) Sulfate-Chromate Dye Penetration Test.

B.3. Fractured CRB-AZS Composite After Room-Temperature Dye-Penetration Test

B.4. Fractured CRB-AZS Composite After Room-Temperature Dye-Penetration Test, Bottom View from Delaminated Interface.

C.1. Distribution of $\mathrm{Cl}$ in the Upper CRB (38B).

C.2. Distribution of $\mathrm{S}$ in the Upper CRB (38B).

C.3. Distribution of $\mathrm{Na}$ in the Upper CRB (38B)

C.4. Effects of Temperature (left) and Condensate Composition (right) on the Leachate $\mathrm{pH}$

C.5. Effects of Temperature on the Leachate Electrical Conductivity (left); Correlation Between $\mathrm{pH}$ and Electrical Conductivity (right). 
C.6. Vibrocast Original (left) and Heat-Treated at $1300^{\circ} \mathrm{C}$ for 2 Hours (right)

C.7. Vibrocast Containing $\mathrm{Na}_{2} \mathrm{O}$ (left) $3 \mathrm{Na}_{2} \mathrm{O} \cdot \mathrm{B}_{2} \mathrm{O}_{3}$ (middle) and $\mathrm{Na}_{2} \mathrm{O} \cdot \mathrm{B}_{2} \mathrm{O}_{3}$ (right) and HeatTreated at $1300^{\circ} \mathrm{C}$ for 2 Hours

D.1. Fine-Grain Materials Overlaid with Dry LAW and Heated to $550^{\circ} \mathrm{C}$ and $1050^{\circ} \mathrm{C}$

D.2. Fine-Grain Materials Blended with Liquid LAW, Dried at $103^{\circ} \mathrm{C}$, and then Heated to Various Temperatures.

D.3. Silica Crucible with LAW Simulant Heated to $450^{\circ} \mathrm{C}$ : Top View (left) and Side View (right)....... D.5

D.4. Sample Appearance after Heat-Treatments at Different Temperatures: Soil Mixed with Wet LAW (left) and Soil Overlaid with Dry LAW (right) ............................................................... D.6

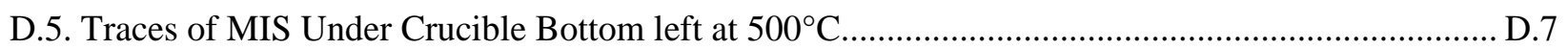

D.6. Cross-Section of Silica Crucible with Soil Overlaid with Dry LAY and Heated to $1050^{\circ} \mathrm{C}$............ D.7

D.7. Cross-Section of Silica Crucible with Soil Mixed with Liquid LAW and Heated to $1300^{\circ} \mathrm{C}$.......... D.8

D.8. Dimensions of Randomly Selected Silica Crucibles: a Small Crucible (left) and a Medium Crucible with a CRB Rod Adjusted by a Pt Wire (right) .............................................................. D.9

D.9. Silica Crucible with a CRB Rod and Feed.......................................................................... D.10

D.10. Baseline Feed Molten at $1200^{\circ} \mathrm{C}$ in Small Crucible .......................................................... D.10

D.11. Cross-Section Through the Medium Crucible with Baseline Feed Heated to $1200^{\circ} \mathrm{C} \ldots \ldots \ldots \ldots \ldots . . . . . .11$

D.12. The Bottom Part of the Sectioned CRB Rod as Removed from Baseline Melt ......................... D.11

D.13. AMEC 38B Feed Molten at $1200^{\circ} \mathrm{C}$ in Small Crucible .......................................................... D.12

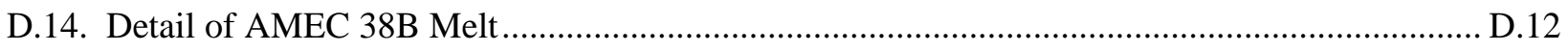

D.15. Cross-Section Through the Medium Crucible with AMEC 38B Feed Molten at $1200^{\circ}$ C ........... D.12

D.16. CRB Rod, as Removed from AMEC 38B Melt and Sectioned …........................................... D.13

D.17. Melt with $\mathrm{B}_{2} \mathrm{O}_{3}$ Premixed with LAW: Small Crucible (left), Medium Crucible (right) ............. D.15

D.18. CRB Rod as Removed from Feed with $\mathrm{B}_{2} \mathrm{O}_{3}$ Dissolved in LAW and Sectioned ...................... D.16

D.19. Melt wit $\mathrm{B}_{2} \mathrm{O}_{3}$ Premixed with LAW: Small Crucible ....................................................... D.16

D.20. Melt wit $\mathrm{B}_{2} \mathrm{O}_{3}$ Premixed with LAW: Medium Crucible ...................................................... D.17

D.21. Section Through CRB Rod Removed from Feed with $\mathrm{B}_{2} \mathrm{O}_{3}$ Dissolved in LAW ...................... D.17 


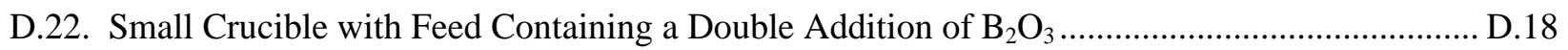

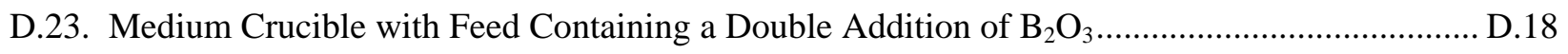

D.24. CRB Rod Immersed in Feed Containing a Double Addition of $\mathrm{B}_{2} \mathrm{O}_{3}$.................................. D.19

D.25. Small Crucible with Feed Containing a Double Addition of $\mathrm{B}_{2} \mathrm{O}_{3}$ Heated to $500^{\circ} \mathrm{C} \ldots \ldots \ldots \ldots \ldots \ldots . . . . . .19$

D.26. Small Crucible Heated to $1200^{\circ} \mathrm{C}$ with Feed Removed (see D.25) ....................................... D.20

D.27. Small Crucible Heated to $1200^{\circ} \mathrm{C}$ with Feed Removed (see D.26) ........................................ D.20

E.1. Feed/Melt Appearance After Heat-Treatment at Different Temperatures; Fracture Surfaces

Through CRB Specimens (after a 4 to $5 \mathrm{~mm}$ cut with a dry saw) .................................................... 1

E.2. CRB Specimens Exposed to Baseline Feed Heated to $550^{\circ} \mathrm{C}$ (top), $800^{\circ} \mathrm{C}$ (middle), and $1300^{\circ} \mathrm{C}$ (bottom): EDS Analysis of the Sample Surface; the Sample Scan Went Through the Middle of the Sample from the Left Side to the Right Side (left) and from the Bottom

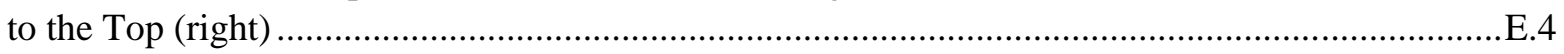

E.3. Hot Stage Microscopy Images of the Baseline Feed (left) and the 38-B Feed (right) with a CRB Sample; Numbers Under the Images Indicate Temperature in ${ }^{\circ} \mathrm{C}$ (the rate of heating was $5^{\circ} \mathrm{C} / \mathrm{min}$, and the crucible diameter was $6 \mathrm{~mm}$ )......

E.4. Small Silica Crucible with Baseline Feed Heated at $5^{\circ} \mathrm{C}$ to the Temperatures Indicated (in ${ }^{\circ} \mathrm{C}$ ) with No Hold and 1-Hour Hold: Views from Two Opposite Sides

E.5. Small Silica Crucibles with Baseline Feed; Heat-Treatment Temperature Decreases from Left $\left(500^{\circ} \mathrm{C}\right)$ to Right $\left(350^{\circ} \mathrm{C}\right)$; from Top to Bottom: Feed After Heat-Treatments, Empty Crucibles After Feed Removal and Heating to $1200^{\circ} \mathrm{C}$ (top view, side view, and sections).....

E.6. Mass (left) and Penetration Depth (right) of MIS Migrated into Crucible Wall Versus Temperature

E.7. Mass Density of MIS Migrated into Crucible Wall Versus Temperature.

F.1. From the Left to Right: Fly Ash, Kaolin, Bentonite, Diatomaceous Earth, and Hanford Soil As-Received (above) and After $30 \mathrm{~min}$ at $1200^{\circ} \mathrm{C}$ (below).....

F.2. Melts from Feed with Diatomaceous Earth and the Appearance of Sectioned Specimens; Left to Right: $800^{\circ} \mathrm{C}, 1000^{\circ} \mathrm{C}$, and $1300^{\circ} \mathrm{C}$

F.3. CRB Specimens Exposed to Feed with Diatomaceous Earth Heated to $800^{\circ} \mathrm{C}$ (top) and $1300^{\circ} \mathrm{C}$ (bottom): EDS Analysis of the Sample Surface.

F.4. Summary of MIS Penetration Tests F.5

F.5. Small Crucibles with Feed Containing 0 to 25 Mass\% of Diatomaceous Earth F.8 
F.6. Sections Through Crucibles and Vibrocast Rods Exposed to Feed with 0 to 25 Mass\% of Diatomaceous Earth

G.1. Ink-Colored Water Penetration into Vibrocast Samples from Various Wet Solids; from Left to Right: Hanford Soil (4 hours), Silica Sand (3 and 6 hours), and Ground Silica (3 and 6 hours)......

H.1. Melts from Feed with Ground Soil and the Appearance of Sectioned Specimens; Left to Right: $800^{\circ} \mathrm{C}, 1000^{\circ} \mathrm{C}$, and $1300^{\circ} \mathrm{C}$

H.2. CRB Specimens Exposed to Feed with Ground Soil Heated to $800^{\circ} \mathrm{C}$ (top) and $1300^{\circ} \mathrm{C}$ (bottom)

H.3. Small Crucibles with Baseline Feed Composition and 0 to $100 \%$ Soil Ground

H.4. Sections Through Crucibles and Vibrocast Rods Exposed to Feed of Baseline Composition and 0 to $100 \%$ Soil Ground.

H.5. MIS Penetration Depth Versus Fraction of Soil Ground (S - small crucible, L - medium crucible, $\mathrm{W}$ - wall, $\mathrm{B}$ - bottom).

H.6. Effect of $550^{\circ} \mathrm{C}$ Heat-Treatment Time on MIS Penetration to CRB from Original and Ground Feed

H.7. Effect of $550^{\circ} \mathrm{C}$ Heat-Treatment Time on MIS Penetration to CRB from Original and Ground Feed: Exponential Fit (left) and Square-Root Fit (right)

H.8. Mass Loss of Feed (original and ground) Versus Time at $550^{\circ} \mathrm{C}$ (left) and $1200^{\circ} \mathrm{C}$-net Mass Loss of MIS in CRB Versus Mass Loss of Feed (right)

H.9. Net Mass Gain Versus Temperature

H.10. Mass Loss of Feed at $550^{\circ} \mathrm{C}$ Versus Ground Fraction

H.11. Effect of Ground Fraction on MIS Penetration to CRB $\left(t_{\mathrm{H}}=1\right.$ hour $)$

H.12. Effect of Grinding Time on MIS Penetration to CRB $\left(t_{\mathrm{H}}=1\right.$ hour)

H.13. Feed Density as a Function of Ground Fraction H.16

H.14. PSD of Original and Ground (for 120 seconds) Feed, Untreated and Deagglomerated H.17

H.15. PSD of Original (as-received) and Ground (for 120 seconds) Feed, Untreated and Deagglomerated

H.16. Effect of Specific Surface Area on MIS Penetration to CRB $\left(t_{\mathrm{H}}=1\right.$ hour $)$.

I.1. Repeated Measurements of PSD of Hanford Soil As-Received (top), Ground for 2 Minutes (middle), and Ground for 5 Minutes (bottom); D Deagglomerated Samples, N Original Samples (not deagglomerated)..... 
I.2. Averaged Measurements of PSD of Hanford Soil As-Received (top), Ground for 2 Minutes (middle), and Ground for 5 Minutes (bottom); D Deagglomerated Samples, N Original Samples (not deagglomerated).

I.3. Comparison of PSD of Hanford Soil As-Received and Ground for 2 and 5 Minutes;

D Deagglomerated Samples, N Original Samples (not deagglomerated)

I.4. Specific Surface Area of Hanford Soil Calculated from the PSD as a Function of Grinding Time Based on Original (N) and Deagglomerated Samples (D)

I.5. Comparison of Specific Surface Area of Hanford Soil Measured with BET and Langmuir Methods.

I.6. Comparison of Specific Surface Area of Hanford Soil Measured with BET Method with the Area Calculated from the PSD; D Deagglomerated Samples, N Original Samples (not deagglomerated).....

J.1. Effect of Temperature on MIS Penetration to CRB from Feed with S-109 Simulant

J.2. Sections Through Silica Crucibles in Which Feed Containing S-109 LAW Simulant and Soil (as-received and 2-min ground) Was Heated for 30 Minutes at Temperatures of $300^{\circ} \mathrm{C}, 400^{\circ} \mathrm{C}$, and $500^{\circ} \mathrm{C}$

J.3. Silica Crucibles with Feed Containing S-109 LAW Simulant and Soil (as-received and 2-min ground) Before and After 30-Minute Heat-Treatment at $400^{\circ} \mathrm{C}$

J.4. Appearance of Silica Crucibles in Which Feed Containing S-109 LAW Simulant and Soil (as-received and 2-min ground) Was Heated for 30 Minutes at Temperatures of $300^{\circ} \mathrm{C}$, $400^{\circ} \mathrm{C}$, and $500^{\circ} \mathrm{C}$

K.1. MIS Penetration into Silica Crucible; Feeds Were Prepared from Hanford Soil Ground for 2-Minutes and Two Types of LAW Simulant.

K.2. MIS Penetration into Silica Crucible from Feeds with s-109 Simulant; Hanford Soil Was Ground for 0, 2, and 5 min

K.3. Effect of 5-Mass\% Bentonite and 5-Mass\% Diatomaceous Earth Additions on MIS Penetration into Silica Crucible from Feeds with s-109 Simulant and 2-Minute Ground Hanford Soil

K.4. Effect of Diatomaceous Earth Addition (in mass\%) on MIS Penetration into Silica Crucible from Feeds with s-109 Simulant and 2-Minute Ground Hanford Soil....

K.5. Effect of Formic Acid Addition on MIS Penetration into Silica Crucible from Feeds with s-109 Simulant and 2-Minute Ground Hanford Soil

K.6. Effect of Sucrose Addition (in \% of the stoichiometric amount) on MIS Penetration into Silica Crucible from Feeds with s-109 Simulant and 2-Minute Ground Hanford Soil K.4 
K.7. Effect of Sucrose Addition (in \% of the stoichiometric amount) on MIS Penetration into Silica Crucible from Feeds with s-109 Simulant and As-Received Hanford Soil K.4

K.8. Effect of Diatomaceous Earth and Bentonite Additions on MIS Migration into Silica Crucible ..... K.6

K.9. Effect of Diatomaceous Earth and Bentonite Additions on MIS Decomposition in Feed. K.6

K.10. Effect of Sucrose and Formic Acid Additions on MIS Migration into Silica Crucible K.7

K.11. Effect of Sucrose and Formic Acid Additions on MIS Decomposition in Feed K.7

K.12. Effect of the Specific Surface Area of Soil on MIS Migration into Silica Crucible K.8

K.13. Mass Balances for MIS in Various Feeds K.12

K.14. Decomposition of MIS that Remained at $500^{\circ} \mathrm{C}$ K.13

K.15. MIS Present in Crucible Walls at $500^{\circ} \mathrm{C}$, Fraction of Total Estimated from $1000^{\circ} \mathrm{C}$ Data K.16

L.1. Effect of Soil Specific Surface Area (by BET) on the Fraction of MIS Lost from Feed to Crucible Walls (no sucrose has been added to feed)

L.2. Effect of Sucrose Addition (in stoichiometric \%) on the Fraction of MIS Lost from Feed with Soil As-Received (5.74 $\mathrm{m}^{2} / \mathrm{g}$ specific surface area) and Ground $\left(9.75 \mathrm{~m}^{2} / \mathrm{g}\right.$ specific surface area) to Crucible Walls ( $G$ granular sucrose added to dry feed, $P$ powdered sucrose added to dry feed; sucrose was dissolved in liquid LAW simulant for all other data points)

L.3. Calculated Versus Measured Fraction of MIS Migrated into Crucible Walls

L.4. Preventable MIS Migration Diagram

L.5. Effect of Carbohydrate Addition (as C/N ratio) on the Fraction of MIS Remaining in Feed (the horizontal line represents complete decomposition)

L.6. Effect of Specific Surface Area of Soil on the Fraction of MIS Remaining in Feed (the horizontal line represents complete decomposition)...... 


\section{Tables}

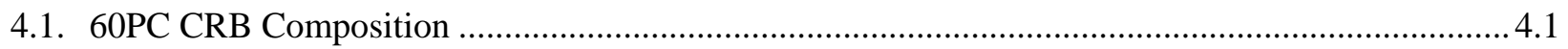

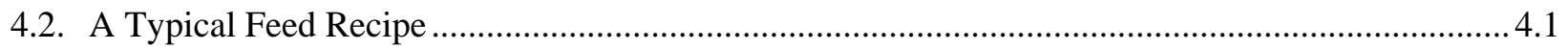

4.3. Six-Tank Composite LAW Simulant Composition..................................................................... 4.2

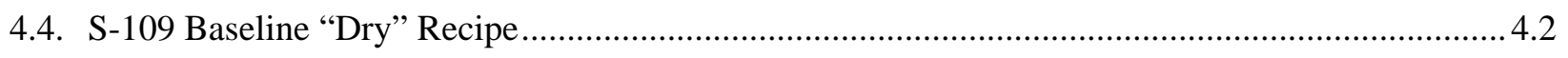

4.5. Mass and Volume Fractions of Feed Components (see 4.2) ….................................................... 4.3

5.1. LOI and Density of CRB Rods ${ }^{(\mathrm{a})}$ Heat-Treated to $550^{\circ} \mathrm{C}$ and $1200^{\circ} \mathrm{C}$......................................... 5.1

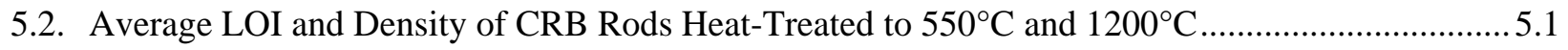

5.3. CRB Porosity (volume fraction), As-Received and Heat-Treated at $1200^{\circ} \mathrm{C}$ for 1 Hour ................. 5.2

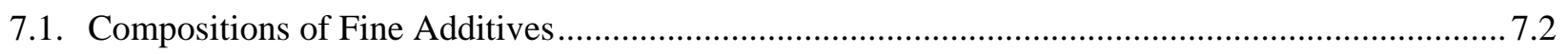

8.1. Comparison of S-109 and Six-Tank LAW Simulants (in mass fractions of components)................8.2

8.2. Mass Fractions of Glass Components from Feed Ingredients ......................................................... 8.2

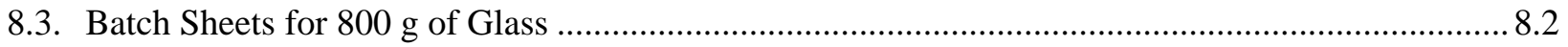

8.4. Specific Surface Area Measured with BET and Langmuir Methods and Calculated from Deagglomerated PSD for As-Received and Ground Soils ....................................................... 8.3

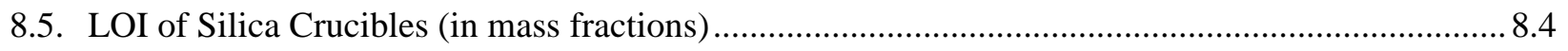

8.6. Feed Mass Loss (in mass fractions) as a Function of Temperature ................................................. 8.5

8.7. Specific Surface Area of Soil Measured by the BET Method......................................................... 8.10

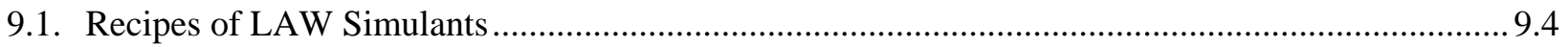

9.2. Content of Glass Components in LAW Simulants........................................................................ 9.4

9.3. Content of nitrates and nitrites in LAW simulants ....................................................................... 9.5

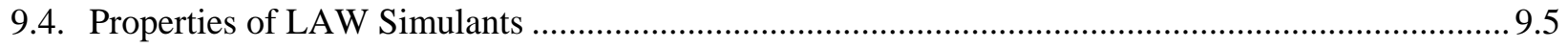

9.5. Compositions of Minerals (in mass fractions) ............................................................................... 9.6

9.6. Recipes for Feeds from Minerals and S-109 LAW Simulant (new) To Make $100 \mathrm{~g}$ of Glass..........9.7

9.7. Compositions (in mass fractions) of Glasses Made from Minerals and S-109 LAW Simulant........9.7

9.8. Cost Comparison Between GFM Feeds with Low (Feed B) and High (Feed C) Waste Loading.....9.8

9.9. Fractions of Crucible-Migrated MIS: Feeds with Soil (as received or ground at PNNL) and

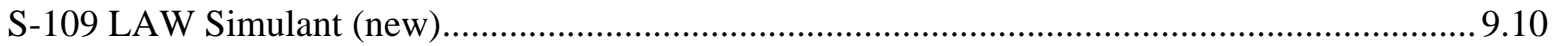

9.10. Fractions of Crucible-Migrated MIS: Feeds with Soil (ground at Praxair) and S-109 LAW Simulant (new).

9.11. Fractions of Crucible-Migrated MIS: Feeds with Minerals, S-109 LAW Simulant (new), and Cellulose Addition

9.12. Specific Surface Area of Minerals by BET and Particle Size Distribution (PSD)..... 
9.13. MIS Migration Linear Regression Coefficients

9.14. Temperature Development (in ${ }^{\circ} \mathrm{C}$ ) in the Center Point of Feeds ................................................ 9.25

9.15. Temperature Development (in ${ }^{\circ} \mathrm{C}$ ) on a Surface Point of Feeds .................................................. 9.25

9.16. Masses of Vibrocast Crucibles and Feeds in kg........................................................................... 9.28

9.17. Fractions of Crucible-Migrated MIS: Baseline Feeds.................................................................29.29

9.18. Fractions of Crucible-Migrated MIS: Feeds with Carbohydrates ................................................ 9.30

9.19. Thermocouple Placement ( $r$ is the distance from crucible axis, and $z$ is the distance from

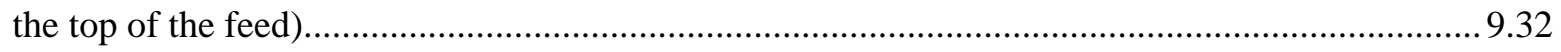

9.20. Temperature Distribution Evolution in a CRB Crucible Feed .................................................... 9.33

A.1. LOI of Vibrocast-NS Mixtures as a Function of Temperature and NS Content (in mass fractions)

A.2. Masses in g of CRB with CAs, Feed, Corresponding Glass (79.5 mass\% of feed), Crucible Content after $1200^{\circ} \mathrm{C}$ Heat-Treatment, and Losses.

A.3. Masses in g of CRB with CA, Feed, Corresponding Glass (76.5 mass\% of feed), Crucible Content after $1200^{\circ} \mathrm{C}$ Heat-Treatment, and Losses................................................................ A.16

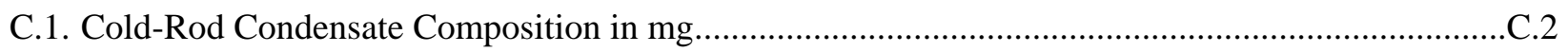

C.2. A Possible Condensate Composition Based on Cold-Rod Data ......................................................2

C.3. Condensate Composition and Condensate Addition Based on SEM-EDS Data.................................4

C.4. Compositions in at\% of Cement Areas of CRB Samples Exposed to Simulated Condensate and Reported in Hrma et al. (2005), Appendix B ........................................................................

C.5. Compositions in at\% of Cement Areas of CRB Samples Exposed to Simulated Condensate and Reported in Hrma et al. (2005), Appendix B

C.6. Composition in at\% of Vibrocast, Cement Portion of CRB (average from data listed in C.4) and its Mixtures with Condensate Simulants

C.7. Hypothetical Composition in at\% of Glass Phase from Cement Portion of CRB Fused with Condensate Simulants

D.1. Dry LAW Overlaid on Fines: Scoping Test Results ............................................................ D. 1

D.2. Liquid LAW Blended with Fines: Scoping Test Results .......................................................... D.3

D.3. Blending $\mathrm{B}_{2} \mathrm{O}_{3}$ with Liquid LAW Simulant....................................................................... D.14

E.1. LOI of Baseline Feed Heated at $5^{\circ} \mathrm{C} / \mathrm{Min}$ to 550 and $800^{\circ} \mathrm{C}$ and Held for 0 and 1 Hour ...................7 
E.2. Small Crucible Experiment: Measured Data.............................................................................

E.3. Small Crucible Experiment: Definitions, Symbols, Values, and Units...........................................9

E.4. Small Crucible Experiment: Calculated Data ................................................................................

E.5. Gas Release from MIS Components (normalized to $800^{\circ} \mathrm{C}$ ) …...................................................10

E.6. Small Crucible Dimensions................................................................................................ 11

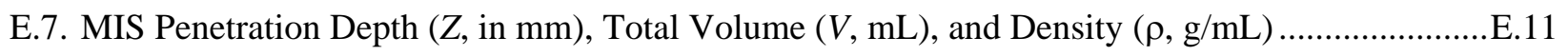

F.1. Fractions of Glass Originating from Feed Ingredients.................................................................1

F.2. Compositions (in mass fractions) of Glasses with Batch Ingredients Listed in F.1........................F.1

F.3. LOI of Hanford Soil and Fine-Grain Materials...........................................................................2

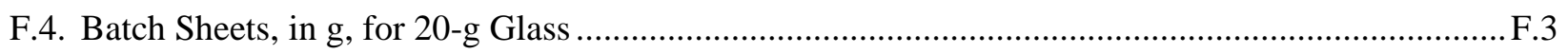

F.5. Masses of Batched Materials, Feeds, and Losses on Heating (in g, except for mass fractions) ........F.7

G.1. Viscosity and Surface Tension of Liquid $\mathrm{NaNO}_{3}$ and $\mathrm{H}_{2} \mathrm{O}$........................................................ G.1

H.1. Data Obtained from CRB Rods Exposed to Original and Ground Feeds ..................................... H.6

H.2. Summary of Averaged Gains/Losses Resulting from Heat-Treatments of CRB Rods Exposed to Original and Ground Feeds ................................................................................................. H.7

H.3. Effect of $550^{\circ} \mathrm{C}$ Heat-Treatment Time on MIS Penetration to CRB from Original Feed................ H.8

H.4. Effect of $550^{\circ} \mathrm{C}$ Heat-Treatment Time on MIS Penetration to CRB from Ground Feed.................. H.8

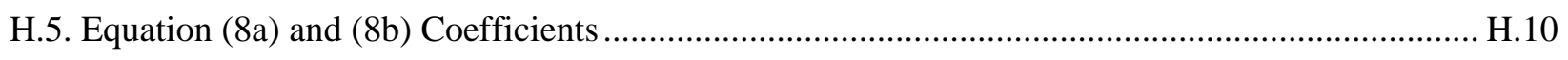

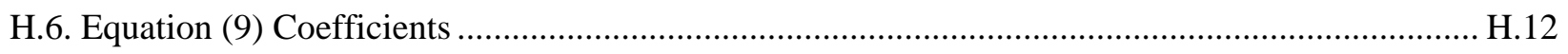

H.7. Effect of Temperature on 1-Hour MIS Penetration into CRB Sample ........................................ H.12

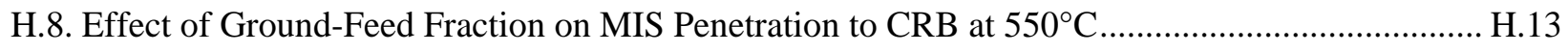

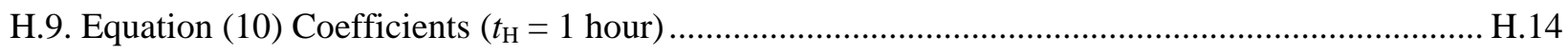

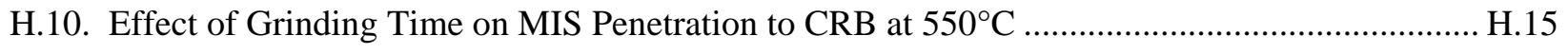

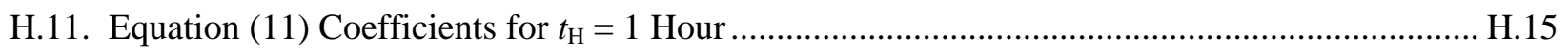

H.12. Feed Density as a Function of Ground Fraction ................................................................ H.16 
H.13. Specific Surface Area as a Function of Feed Grinding Time.

I.1. Specific Surface Area (a) of Hanford Soil Calculated from the PSD as a Function of Grinding Time ( $t$ ) Based on Original (N) and Deagglomerated Samples (D) I.4

I.2. Specific Surface Area of Hanford Soil Measured with BET and Langmuir Methods .5

J.1. Effect of Temperature on MIS Penetration to Silica Crucible from Feed with S-109 Simulant and As-Received Soil.

J.2. Effect of Temperature on MIS Penetration to Silica Crucible from Feed with S-109 Simulant and 2-Minute Ground Soil

K.1. Batch Sheets for Feeds Containing S-109 Simulant K.1

K.2. Effect of Grinding Time on MIS Penetration into Silica Crucibles: Basic Data. K.4

K.3. Effect of Diatomaceous Earth and Bentonite Additions on MIS Penetration into Silica

Crucibles from Feeds with 2-Minute Ground Soil: Basic Data

K.4. Effect of Sucrose and Formic Acid Additions on MIS Penetration into Silica Crucibles from Feeds: Basic Data.

K.5. Effect of Grinding Time on MIS Penetration into Silica Crucibles: MIS Migration and Reaction

K.6. Effect of Diatomaceous Earth and Bentonite Additions on MIS Penetration into Silica Crucibles: MIS Migration and Reaction

K.7. Effect of Sucrose and Formic Acid Additions on MIS Penetration into Silica Crucibles: MIS Migration and Reaction.

K.8. Effect of Grinding Time on MIS Penetration into Silica Crucibles: MIS Migration and Reaction (in mass fractions).

K.9. Effect of Diatomaceous Earth and Bentonite Additions on MIS Penetration into Silica Crucibles: MIS Migration and Reaction (in mass fractions).

K.10. Effect of Sucrose and Formic Acid Additions on MIS Penetration into Silica Crucibles: MIS Migration and Reaction (in mass fractions).

K.11. Effect of Grinding Time on MIS Penetration into Silica Crucibles

K.12. Effect of Diatomaceous Earth and Bentonite Additions on MIS Penetration into Silica Crucibles (soil 2-min ground)

K.13. Effect of Sucrose and Formic Acid Additions on MIS Penetration into Silica Crucibles K.15

L.1. Specific Surface Area of Soil Measured by the BET Method L.1 
L.2. Effect of Grinding and Sucrose Addition on MIS Penetration into Silica Crucibles: Basic Data .....L.2

L.3. Effect of Grinding and Sucrose Addition on MIS Penetration into Silica Crucibles MIS

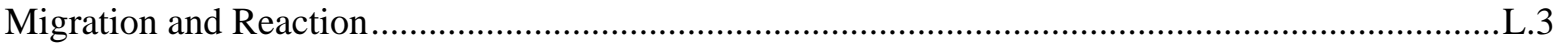

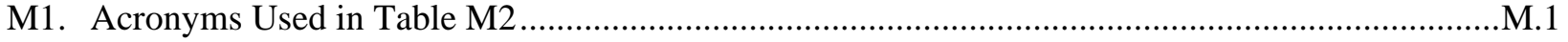

M2. Temperature Evolution Data (temperatures are in ${ }^{\circ} \mathrm{C}$ ) .............................................................1 


\subsection{Introduction}

This study supports the bulk vitrification (BV) design and operation by exploring various methods aimed at reducing the quantities of soluble Tc in the castable refractory lining referred to in this report as castable refractory block (CRB). Various scoping tests for performance enhancement were conduced in FY04/FY05 (Kim 2005, Hrma 2005a). The results from previous testing helped eliminate methods that showed little promise, such as coating the CRB with a thin layer of inorganic glazes. Results from other tests, such as protecting the CRB with ceramic tiles, appeared inconclusive. However, studies of the mechanism of BV and melt interaction with refractory materials led to several new ideas that merited further exploration.

This exploratory study was based on previous work (CRB surface treatment with fluxes, aluminazirconia-silica [AZS] tiles), ideas that emerged during recent experimental studies (molten salt absorbing additives), new ideas put forward during brainstorming sessions (adding carbon to areas where the feed contacted the CRB), and new ideas that emerged during the work itself (soil grinding, organic carbon addition to feed, replacing soil with a mixture of minerals).

Although thin layers of inorganic glazes failed to prevent the penetration of Re, the Tc nonradioactive surrogate, into the CRB (Hrma et al. 2005a), other CRB surface-treatment methods have been conceived that may be effective and merit further testing. Two methods have been tried: creating sacrificial layers that would fuse with glass, thus returning the migrated Tc, and filling the CRB pores with lyophobic material (carbon) to make the CRB less wettable by molten salts. In addition to altering the glass-contact layer of the CRB itself, tiling the CRB was also tested. Previous studies of protective tiles were incomplete because the most promising AZS fused-cast tiles were not obtained in time. This report includes a follow up study that tested AZS tiles as protective barriers for the CRB.

An alternative way of preventing the Tc-bearing molten ionic salt (MIS) from penetrating into the CRB is decreasing MIS mobility within the BV feed. The volume fraction of the early melt in the feed (MIS + $\mathrm{B}_{2} \mathrm{O}_{3}$ ) is nearly as large as the volume-fraction solids (soil $+\mathrm{ZrO}_{2}$ ). Since the viscosity of the early melt is as low as room-temperature water, a part of the MIS can be highly mobile, depending on the scale of mixing and the specific surface of the solids in feed that holds a portion of MIS by capillary forces. Two methods were used to decrease the content of freely mobile MIS within the feed. One method was increasing the surface area of the feed solids. This was achieved by adding fine-grained materials as feed constituents or grinding the existing constituents (soil). The other method was based on decreasing the volume of MIS before it was capable of migration. This was achieved by adding carbohydrates (sugar and cellulose) to the feed. Carbohydrates react with sodium nitrates and nitrites, which constitute a major fraction of MIS, at temperatures lower than $300^{\circ} \mathrm{C}$.

Experiments conducted showed that a variety of methods can decrease MIS penetration into the CRB individually or in combination. Manipulating the CRB is a possibility limited to feeds and charging methods that expose the CRB to only a mild attack by the MIS. Increasing the specific surface of the soil stopped MIS penetration nearly entirely in small crucible experiments for a specific feed. Adding carbohydrates was also successful. Fine-grained feed with carbohydrate appeared most promising. Finally, to avoid expensive soil-grinding, feeds were tested in which soil was replaced with readily available fine-grained glass-forming minerals (GFMs). 


\subsection{Background}

Previous work (Hrma et al. 2005a, 2005b, pp. 233-250) showed how a substantial portion of a well dispersed but very minute component ( $\sim 90 \mathrm{mg} \mathrm{Re}_{2} \mathrm{O}_{7} / \mathrm{kg}$ dry feed) can segregate from a large volume of feed and migrate into the porous refractory wall that is contacted by that feed for a relatively short time at a relatively low temperature. These findings are summarized in this section.

The BV melter feed used in FY04-FY06 testing was a mixture of low-activity waste (LAW) simulant, aluminosilicate soil, and additives $\left(\mathrm{ZrO}_{2}\right.$ and $\left.\mathrm{B}_{2} \mathrm{O}_{3}\right)$. The LAW is a mixture of salts. As the feed temperature increased, LAW melts, forming a single molten salt phase that incorporates some $\mathrm{B}_{2} \mathrm{O}_{3}$. Molten salts can first appear at a temperature as low as $150^{\circ} \mathrm{C}$ and begin to penetrate the $\mathrm{CRB}$ at $300^{\circ} \mathrm{C}$. It wets the refractory components of the feed, spreading over and bridging solid grains and filling some of the space between them. As the temperature increases to $650^{\circ} \mathrm{C}$, the nitrate and nitrite salts ( 66 mass $\%$ of six-tank composite LAW simulant) are nearly fully decomposed. The $\mathrm{Na}_{2} \mathrm{O}$ from the nitrate decomposition reacts with refractory grains in the feed (soil, $\mathrm{ZrO}_{2}$, or other GFMs) to form viscous glassforming liquid.

Technetium can penetrate into the CRB in three ways: direct penetration of molten LAW from the feed, condensation of vapors on the upper CRB (above the feed line), and penetration of molten sulfate mix that accumulated on the glass melt surface. This report addresses mainly the direct MIS penetration modes and briefly deals with the condensation mode that appears less significant. The effect of segregated sulfate on the CRB may be addressed in the future if sulfate segregation is found to be a problem. 


\subsection{Direct Penetration Mode}

At the interface between the melter feed and the CRB, the molten salt from LAW, containing Tc or Re, its nonradiactive surrogate, migrates into the CRB open pores. The penetration proceeds by capillarity in the same manner as a sheet of filter paper absorbs moisture when in contact with moist sand. Penetration is facilitated by the low viscosity of molten salts. ${ }^{\text {(a) }}$

The amount of the MIS that migrates into the CRB is limited by the availability of freely moving MIS in the feed, by the interaction of MIS with the CRB, and by the time of contact between the feed and CRB before the fraction of MIS in the feed is decreased by feed reactions.

When the nitrates from MIS that migrated to CRB decompose, $\mathrm{NO}_{\mathrm{x}}$ gas escapes through the CRB pores, while sulfates, chlorides, borates, and chromates are left behind together with pertechnetates and perrhenates. At temperatures above $\sim 1000^{\circ} \mathrm{C}, \mathrm{Na}_{2} \mathrm{O}$ from the $\mathrm{NaNO}_{3}$ decomposition forms a viscous melt with the fine-grained (cementicious) refractory components. A large portion of pertechnetate and perrhenates from the molten salt dissolves in this glass phase and becomes immobilized, but a small residue remains in CRB in a water-soluble form that reduces the overall effectiveness of the BV waste form.

The transport of $\mathrm{Tc} / \mathrm{Re}$ into the $\mathrm{CRB}$ can be decreased by preventing the entrance of MIS into the CRB pores. Once Tc/Re enters into the CRB, it can be immobilized by converting it into a component of a durable glassy phase. These options are addressed in this report.

(a) The rate of penetration of molten salt into refractory pores may be estimated by connecting Darcy's law written in the form $\Delta P / L=\eta L / K t$ with the Young-Laplace equation, $\Delta P=2 \sigma / r$, where $\Delta P$ is the capillary pressure, $L$ is the meniscus distance from the refractory-melt interface, $\eta$ is the viscosity, $r$ is the pore radius, $K$ is the permeability, $t$ is the time, and $\sigma$ is the surface tension. Eliminating $\Delta P$ from these two equations and rearranging the result, we obtain

$$
L=\left(\frac{2 K \sigma}{r \eta}\right)^{1 / 2} t^{1 / 2}
$$

This equation can be extended to evaluate the relative significance of hydrostatic pressure. Assuming that the MIS in feed is continuous, the hydrostatic pressure is $\Delta p_{\mathrm{h}}=\rho g h$, where $\rho$ is the MIS density, $g$ the gravity constant, and $h$ the thickness of the feed layer. At the bottom of a 0.1 -m-thick layer of feed, $\Delta p_{\mathrm{h}}$ is approximately $1 \mathrm{kPa}$, a value at least 3 orders of magnitude smaller than the capillary pressure, considering that the pore size in the refractory cement of the CRB is $<0.1 \mu \mathrm{m}$, and $\sigma$ is approximately $0.1 \mathrm{~N} / \mathrm{m}$.

According to the above equation, the depth of MIS penetration increases with the square root of time. The time available for penetration is limited by the rate of temperature increase. Once the temperature is high enough, the nitrites and nitrates decompose, producing large volumes of gas, and thus change the dynamics of the process. However, processing experience indicates that there is sufficient time for significant MIS penetration. 


\subsection{Vapor Condensation Mode}

At the glass melt-surface, sulfates and chlorides gradually evaporate. The vapors of molten salts combine with volatile components from glass (such as sodium borate). As reported by Gibson (1993a, 1993b) and Darab and Smith (1996), Tc and Re compounds, such as alkali pertechnetates and perrhenates, are volatile at relatively low temperatures. Vapors condense on colder surfaces, such as the box lid and the upper portions of the CRB. Vapor condensation may occur also on a hot refractory surface because of capillary forces (i.e., capillary condensation caused by reduced equilibrium vapor pressure above surfaces of negative curvature) and by chemical affinity if the condensate reacts with the CRB components. If these condensates accumulate on the CRB in sufficient quantities, molten salt may penetrate, by capillary suction, through open pores of the castable refractory into the interior of the CRB. Volatile salts can also penetrate into the upper portions of the CRB by gaseous diffusion and convection. Analyses of the CRB from a partially completed full-scale test (FS-38B) allowed an evaluation of this potential mechanism. These analyses (Cooley et al. 2006) showed that condensation on the upper portions did not lead to significant Re migration into the CRB. 


\subsection{Objectives}

The objective of this study was to investigate methods to reduce the amount of Tc (or Re, its nonradiactive surrogate) migration into the CRB and select those that have a high probability of success. The results will be used to identify methods to reduce Tc (Re) migration in future engineering and FS tests. 


\subsection{CRB and Glass-Feed Compositions}

The standard CRB material used in these studies was vibrocast 60PC. Table 4.1 shows the composition of this material. Vibrocast 60PC is a 60-percent alumina, mullite-based, low-cement castable designed to provide minimum porosity and low thermal expansion with maximum density and high fire resistance. The material was chosen primarily for its capability to withstand severe high-temperature corrosive and erosive environments. Although the vibrocast material is dense by castable refractory standards, the material is still porous and has a high relative surface area. The mullite, silica, and corundum particles are bonded with refractory cement that resists attack by molten glass, but readily absorbs low-viscosity molten salts.

Table 4.1. Vibrocast 60PC CRB Composition

\begin{tabular}{||l|c|c|c|c|c|c||}
\hline Component & $\mathbf{A l}_{2} \mathbf{O}_{3}$ & $\mathbf{S i O}_{2}$ & $\mathbf{F e}_{2} \mathbf{O}_{3}$ & $\mathbf{T i O}_{2}$ & $\mathbf{C a O}$ & Other \\
\hline Mass fraction & 0.608 & 0.345 & 0.001 & 0.018 & 0.016 & 0.003 \\
\hline \hline
\end{tabular}

The standard glass-feed composition used in this study was based on a mixture of glass formers with a six-tank composite waste simulant as defined in Table 4.2 and Table 4.3. However, a number of experiments were also performed with a Hanford S-109 tank waste simulant (see Table 4.4 and Section 8 for further details) that was used in the AMEC FS-38C test and was intended to simulate the S-109 tank waste that is planned for use in the initial radioactive BV testing. Experiments were also performed with other types of feed, such as no-iron glass feed and feeds containing GFMs.

Table 4.2. A Typical Feed Recipe ${ }^{(a)}$

\begin{tabular}{||l|c||}
\hline \hline Component & Mass Fraction \\
\hline Dry six-tank simulant ${ }^{(\mathrm{b})}$ & 0.3544 \\
\hline $\mathrm{HRTS}^{(\mathrm{c})}$ Soil & 0.5503 \\
\hline $\mathrm{ZrO}_{2}$ & 0.0556 \\
\hline $\mathrm{B}_{2} \mathrm{O}_{3}$ & 0.0397 \\
\hline (a) This recipe was used as the "baseline feed” \\
for feeds containing the six-tank composite. \\
(b) $\mathrm{NaOH}$ and $\mathrm{Al}\left(\mathrm{NO}_{3}\right)_{3} \cdot 9 \mathrm{H}_{2} \mathrm{O}$ may be added \\
separately from the waste simulant. \\
(c) Soil from AMEC's Horn Rapids Test Site, \\
Richland, Washington. \\
\hline
\end{tabular}


Table 4.3. Six-Tank Composite LAW Simulant Composition

\begin{tabular}{||l|c|c|l|c|c||}
\hline \multicolumn{1}{|c|}{ Reagent } & $\begin{array}{c}\text { Concentration } \\
\text { g/L }\end{array}$ & $\begin{array}{c}\text { Mass } \\
\text { Fraction }\end{array}$ & \multicolumn{1}{c|}{ Reagent } & $\begin{array}{c}\text { Concentration } \\
\text { g/L }\end{array}$ & $\begin{array}{c}\text { Mass } \\
\text { Fraction }\end{array}$ \\
\hline $\mathrm{CH}_{3} \mathrm{COONa}$ & 10.79 & 0.0284 & $\mathrm{NaCl}$ & 2.56 & 0.0067 \\
\hline $\mathrm{KNO}_{3}$ & 1.25 & 0.0033 & $\mathrm{NaF}$ & 1.33 & 0.0035 \\
\hline $\mathrm{Na}_{2} \mathrm{C}_{2} \mathrm{O}_{4}$ & 1.58 & 0.0042 & $\mathrm{NaNO}_{2}$ & 29.26 & 0.0770 \\
\hline $\mathrm{Na}_{2} \mathrm{CO}_{3}$ & 50.35 & 0.1325 & $\mathrm{NaNO}_{3}$ & 196.11 & 0.5163 \\
\hline $\mathrm{Na}_{2} \mathrm{CrO}_{4}$ & 1.68 & 0.0044 & $\mathrm{NaOH}$ & 29.58 & 0.0779 \\
\hline $\mathrm{Na}_{2} \mathrm{SO}_{4}$ & 12.78 & 0.0336 & $\mathrm{Al}_{4}\left(\mathrm{NO}_{3}\right)_{3} \cdot 9 \mathrm{H}_{2} \mathrm{O}$ & 23.9 & 0.0629 \\
\hline $\mathrm{Na}_{3} \mathrm{PO}_{4} \cdot 12 \mathrm{H}_{2} \mathrm{O}$ & 18.7 & 0.0492 & $\mathrm{CsNO}_{3}$ & $1.0 \mathrm{E}-05$ & 0.0000 \\
\hline $\mathrm{H}_{2} \mathrm{O}$ & 860 & & \multicolumn{2}{c|}{379.87} \\
\hline Total solids & & & \multicolumn{2}{c|}{172.41} \\
\hline Total glass components & \\
\hline \multicolumn{2}{|l}{ Density, g/mL } \\
\hline
\end{tabular}

Table 4.4. S-109 Baseline "Dry" Recipe

\begin{tabular}{||l|c||}
\hline \multicolumn{1}{|c|}{ Reagent } & $\begin{array}{c}\text { Mass } \\
\text { Fraction }\end{array}$ \\
\hline $\mathrm{Al}(\mathrm{OH})_{3}$ & 0.0021 \\
\hline $\mathrm{NaCl}$ & 0.0018 \\
\hline $\mathrm{Na}_{2} \mathrm{CrO}_{4} \cdot 4 \mathrm{H}_{2} \mathrm{O}$ & 0.0079 \\
\hline $\mathrm{KNO}_{3}$ & 0.0010 \\
\hline $\mathrm{NaNO}_{2}$ & 0.0103 \\
\hline $\mathrm{NaNO}_{3}$ & 0.8428 \\
\hline $\mathrm{NaOH}_{3}$ & 0.0186 \\
\hline $\mathrm{Na}_{3} \mathrm{PO}_{4} \cdot 12 \mathrm{H}_{2} \mathrm{O}$ & 0.0501 \\
\hline $\mathrm{Na}_{2} \mathrm{SO}_{4}$ & 0.0126 \\
\hline $\mathrm{Na}_{2} \mathrm{CO}_{3} \cdot \mathrm{H}_{2} \mathrm{O}$ & 0.0505 \\
\hline $\mathrm{NaCH}_{3} \mathrm{COO}$ & 0.0024 \\
\hline
\end{tabular}

Table 4.5 lists the liquid density of the MIS (from six-tank composite simulant), the particle densities of the other components of the glass feed, and the calculated volume fractions for the feed composition summarized in Table 4.2. The volume fraction of MIS before decomposing was $41.6 \%$. Considering that $\mathrm{B}_{2} \mathrm{O}_{3}$ can combine with MIS to form a common liquid phase, the volume fraction of liquid phase in the feed at temperatures above $450^{\circ} \mathrm{C}$ could be as high as $46 \%$. This high-liquid-volume fraction could lead to segregation of the flux components from the refractory components in the feed by migration and drainage. Though the resulting glass is likely to be homogeneous because of strong convection currents during melting, low-viscosity melt can migrate towards and penetrate into the porous CRB material. 
Table 4.5. Mass and Volume Fractions of Feed Components (see Table 4.2)

\begin{tabular}{||l|c|c|r||}
\hline & $\begin{array}{c}\text { Mass } \\
\text { Fraction }\end{array}$ & $\begin{array}{c}\text { Density, } \\
\text { g/mL }\end{array}$ & $\begin{array}{c}\text { Volume } \\
\text { Fraction }\end{array}$ \\
\hline $\mathrm{MIS}$ & 0.3544 & 2.205 & 0.4164 \\
\hline $\mathrm{B}_{2} \mathrm{O}_{3}$ & 0.0397 & 2.55 & 0.0403 \\
\hline $\mathrm{Soil}$ & 0.5503 & 2.753 & 0.5179 \\
\hline $\mathrm{ZrO}_{2}$ & 0.0556 & 5.68 & 0.0254 \\
\hline
\end{tabular}




\subsection{MIS, Feed, and CRB Properties}

Table 5.1 and Table 5.2 summarize loss-on-ignition (LOI) data for several CRB samples. Table 5.1 shows that the $\mathrm{LOI}$ at $550^{\circ} \mathrm{C}$ was $\sim 2$ mass $\%$ and did not increase with the heat-treatment time at $550^{\circ} \mathrm{C}$. Table 5.2 shows that the LOI only slightly increased with further heating to $1200^{\circ} \mathrm{C}$. The bulk density of the CRB was $2.50 \mathrm{~g} / \mathrm{cm}^{3}$ before heating and decreased to $2.45 \mathrm{~g} / \mathrm{cm}^{3}$ after heating to $550^{\circ} \mathrm{C}$. There was little additional change in the density after heating to $1200^{\circ} \mathrm{C}$.

Table 5.1. LOI and Density of CRB Rods ${ }^{(a)}$ Heat-Treated to $550^{\circ} \mathrm{C}$ and $1200^{\circ} \mathrm{C}$

\begin{tabular}{|c|c|c|c|c|c|c|c|}
\hline Hold time at $550^{\circ} \mathrm{C}, \mathrm{h}^{(\mathrm{b})}$ & $\overline{\mathbf{0}}$ & 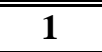 & 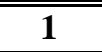 & $\overline{8}$ & 8 & 24 & 24 \\
\hline Mass before heating, $\mathrm{g}$ & 45.78 & 51.71 & 48.25 & 51.45 & 51.37 & 49.57 & 51.34 \\
\hline Mass after heating to $550^{\circ} \mathrm{C}, \mathrm{g}$ & 44.92 & 50.60 & 47.37 & 50.49 & 50.39 & 48.55 & 50.41 \\
\hline Mass after 1-hour heating to $1200^{\circ} \mathrm{C}$, g & 44.79 & 50.48 & 47.34 & 50.34 & 50.23 & 48.50 & 50.26 \\
\hline Fractional loss at $550^{\circ} \mathrm{C}$ & 0.019 & 0.021 & 0.018 & 0.019 & 0.019 & 0.021 & 0.018 \\
\hline Fractional mass loss at $1200^{\circ} \mathrm{C}$ & 0.022 & 0.024 & 0.019 & 0.022 & 0.022 & 0.022 & 0.021 \\
\hline Volume, mL & & 20.34 & 19.57 & & & & \\
\hline Density before heating, $\mathrm{g} / \mathrm{mL}$ & & 2.542 & 2.465 & & & & \\
\hline Density after heating to $550^{\circ} \mathrm{C}, \mathrm{g} / \mathrm{mL}$ & & 2.487 & 2.420 & & & & \\
\hline Density after heating to $1200^{\circ} \mathrm{C}, \mathrm{g} / \mathrm{mL}$ & & 2.481 & 2.419 & & & & \\
\hline
\end{tabular}

Table 5.2. Average LOI and Density of CRB Rods Heat-Treated to $550^{\circ} \mathrm{C}$ and $1200^{\circ} \mathrm{C}$

\begin{tabular}{||l|r|c||}
\hline & Average & SD \\
\hline Fractional loss at $550^{\circ} \mathrm{C}$ & 0.019 & 0.0017 \\
\hline Fractional mass loss at $1200^{\circ} \mathrm{C}$ & 0.022 & 0.0016 \\
\hline Density before heating, $\mathrm{g} / \mathrm{mL}$ & 2.504 & 0.054 \\
\hline Density after heating to $550^{\circ} \mathrm{C}, \mathrm{g} / \mathrm{mL}$ & 2.454 & 0.047 \\
\hline Density after heating to $1200^{\circ} \mathrm{C}, \mathrm{g} / \mathrm{mL}$ & 2.450 & 0.044 \\
\hline
\end{tabular}

The CRB bulk density was also estimated by measuring the mass and dimensions of CRB rods used for testing (CRB rods $\sim 120 \mathrm{~mm}$ long and 15- to $19 \mathrm{~mm}$ wide were cut from a CRB monolith). The average CRB density for these samples was $2.579 \pm 0.076 \mathrm{~g} / \mathrm{mL}$. The high standard deviation was partly due to the variability of the material, but was mainly caused by the inaccuracy of the rod-volume assessment, which was based on a single measurement of each of the rod dimensions that ignored the slight variations of cross-sectional areas of the rods along their length.

In an independent study, the bulk density of the CRB was measured by determining the mass and volume of cast cylindrical samples ( $40.3 \mathrm{~mm}$ in diameter and $23.5 \mathrm{~mm}$ in height). The average result was $2.456 \pm 0.021 \mathrm{~g} / \mathrm{mL}$, which had a better overall precision but was lower than the value obtained for the CRB rods. This difference indicates that the bulk density may vary from batch to batch and/or be dependent on the casting method. The bulk density of these cylindrical samples after being heat-treated for 1 hour at $1200^{\circ} \mathrm{C}$ was $2.443 \pm 0.006 \mathrm{~g} / \mathrm{mL}$. 
A gas pycnometer (operating on the basis of measuring the He displacement volume) was used to determine the particle densities of the six-tank composite MIS and CRB. The particle density of the MIS (prepared by melting at $450^{\circ} \mathrm{C}$ ) was $2.2050 \pm 0.0004 \mathrm{~g} / \mathrm{mL}$ (this value was used in Table 4.5), and the particle density of the CRB was $3.145 \pm 0.0020 \mathrm{~g} / \mathrm{mL}$.

The total porosity of CRB was calculated from the particle-density and the bulk-density data. The open porosity was measured by weighing CRB samples soaked in water under vacuum. Table 5.3 summarizes the results.

Table 5.3. CRB Porosity (volume fraction), As-Received and Heat-Treated at $1200^{\circ} \mathrm{C}$ for 1 Hour

\begin{tabular}{||r|c|c|c|c||}
\hline & \multicolumn{2}{|c|}{ Total Porosity } & \multicolumn{2}{c|}{ Open Porosity } \\
\hline & Ave. & St. dev. & Ave. & St. dev. \\
\hline As-received & 0.217 & 0.003 & 0.158 & 0.004 \\
\hline $1200^{\circ} \mathrm{C}$-heat-treated & 0.225 & 0.005 & 0.151 & 0.002 \\
\hline \hline
\end{tabular}

In an independent measurement, the CRB open porosity was obtained by determining the amount of water absorbed by a rectangular rod with a volume of $18.3 \mathrm{~mL}$. This measurement resulted in an open porosity of 0.1106 , indicating a significant variability in the porosity. The closed porosity ( $6 \%$ ) was confined to pores inside the mineral particles.

The evolved gas analysis of a BV feed (Figure 5.1) shows that the reaction between the organic and nitric components of LAW begins to evolve gases at temperatures as low as $150^{\circ} \mathrm{C}$, continuing with several overlapping peaks between $300^{\circ} \mathrm{C}$ and $650^{\circ} \mathrm{C}$. The major nitrate decomposition reaction occurs between $550^{\circ} \mathrm{C}$ and $800^{\circ} \mathrm{C}$. A small fraction of nitrate may survive in the form of sodium aluminosilicate nitrate (sodalite). During the melting process, 45.4 mass\% of dry LAW (six-tank composite simulant) becomes glass; the rest, 54.6 mass\%, escapes in the form of gas (mainly $\mathrm{NO}_{\mathrm{x}}$ and $\mathrm{CO}_{\mathrm{x}}$-note that $\mathrm{NO}_{2}$ has not been detected; it probably decomposes into $\mathrm{NO}$ and $\mathrm{O}_{2}$ ).

Simulant LOI was evaluated from various experiments, and the data are reported throughout this report. The simulant mass changes based on DTA-TGA are shown in Figure 5.2. The decomposition of nitrate is complete at $772^{\circ} \mathrm{C}$. The LOI at $800^{\circ} \mathrm{C}$ is 47.2 mass $\%$, a value close to the theoretical value of 49.1 mass\% (based on the 54.6-mass\% LOI of the dry LAW and on the assumption of no retention of water by $\mathrm{B}_{2} \mathrm{O}_{3}$ at a temperature $>200^{\circ} \mathrm{C}$ ).

Discrepancies between evolved gas analysis and DTA-TGA nitrate reaction temperatures can be attributed to the larger sample size used in the evolved-gas analysis. Samples of a large size cannot be heated uniformly. The temperature gradients that arise during heating result in non-uniform reaction progress throughout the sample volume. Samples that are very small are influenced by the proximity of container walls and the atmosphere. This affects solid-gas reactions, reactions that are controlled by nucleation, and reactions in the presence of liquid. 


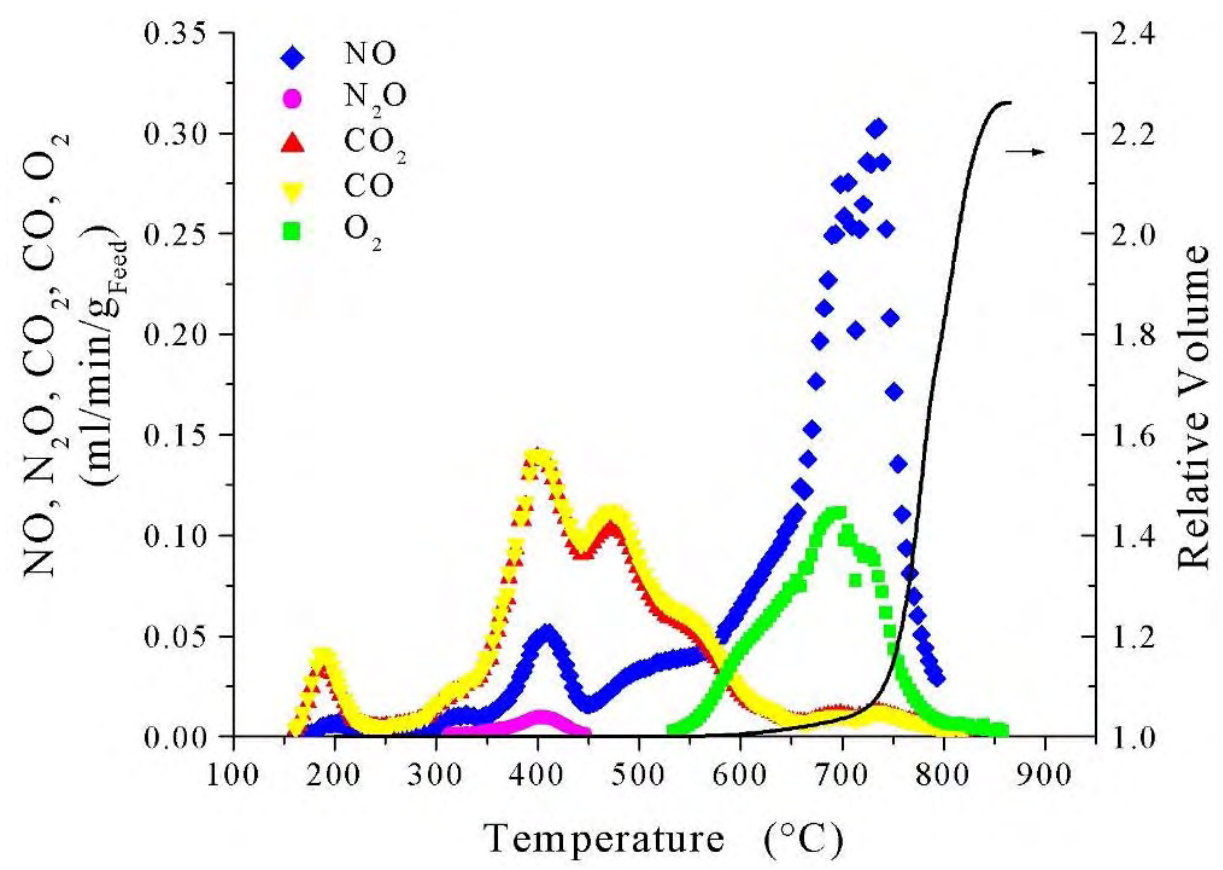

Figure 5.1. Evolved Gases from and Relative Volume of BV Feed [Hrma 2005a]

Sample: 6 tank simulant+B2O3 Size: $42.4796 \mathrm{mg}$

TGA-DTA

File: C:ITAIDatalSDTIJarrodl6 tank sim. + B2O3

Run Date: 10-Mar-2006 10:24

Instrument: 2960 SDT V3.0F

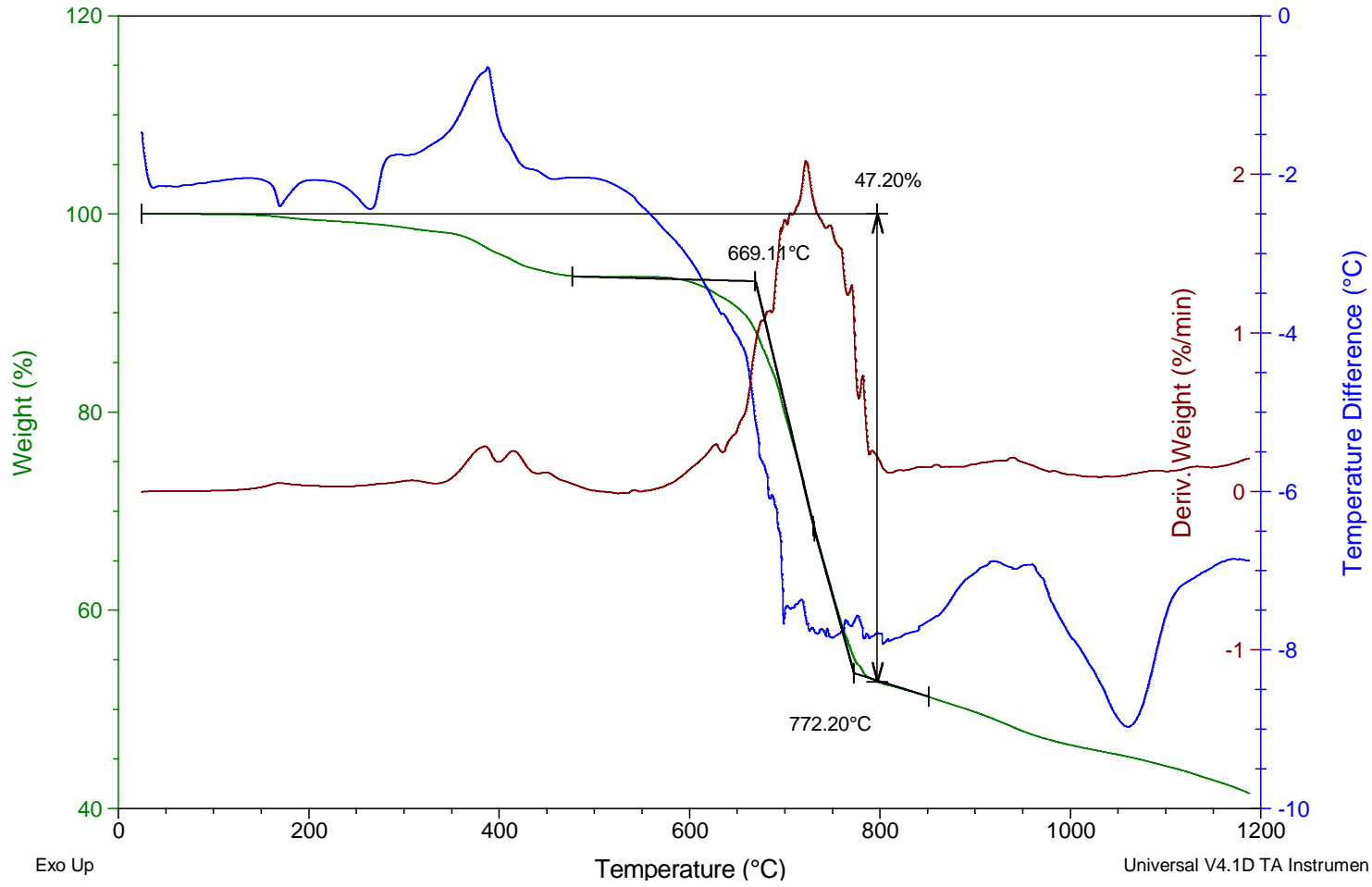

Figure 5.2. DTA-TGA of $\mathrm{B}_{2} \mathrm{O}_{3}$ Predissolved in LAW Simulant Solution 
Hot-stage microscopy was used to further investigate the nature of early feed melting reactions.

Figure 5.3 displays, in a side-by-side comparison, the response of simulated LAW and the baseline sixtank composite feed to heating. The feed was prepared by mixing solid feed additives with liquid LAW simulant and drying. The simulated LAW is not a dried-liquid LAW simulant, but a mixture of solid chemicals. The main component is $\mathrm{NaNO}_{3}$ in the form of spherical particles. The red crystal is $\mathrm{Na}_{2} \mathrm{CrO}_{4}$, which is yellow at room temperature but turns orange and then red on heating to $325^{\circ} \mathrm{C}$. The dry mix contains all the chemicals listed in Table 4.3 except $\mathrm{Al}\left(\mathrm{NO}_{3}\right)_{3} \cdot 9 \mathrm{H}_{2} \mathrm{O}$ and $\mathrm{NaOH}$.

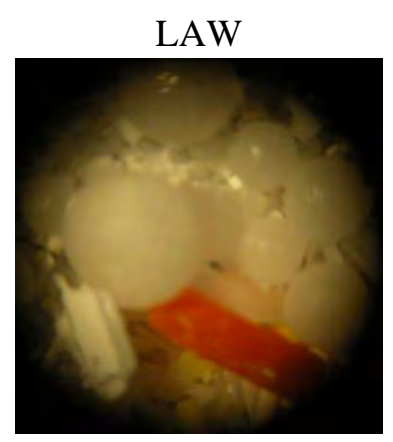

325

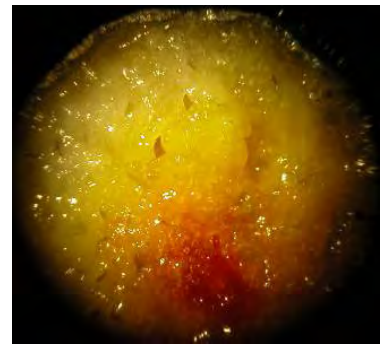

500

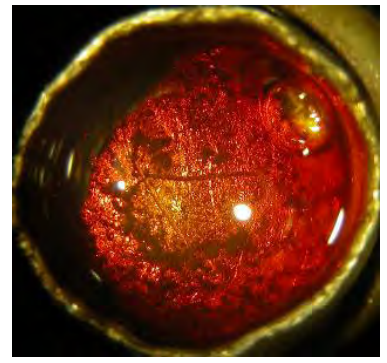

700

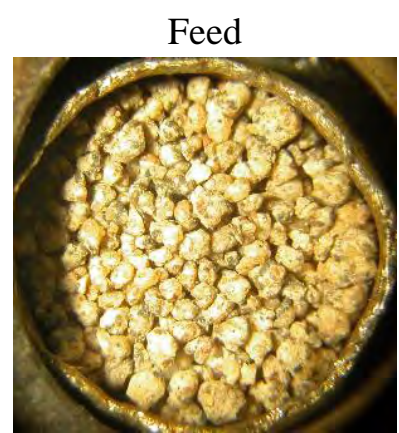

100

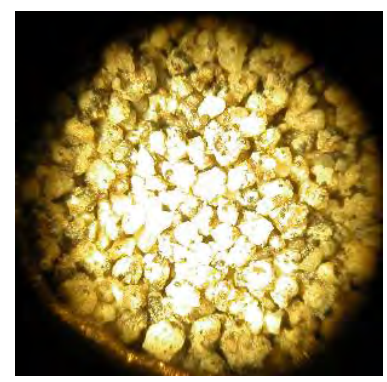

500

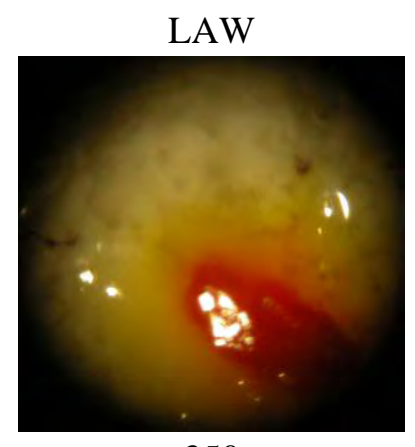

350

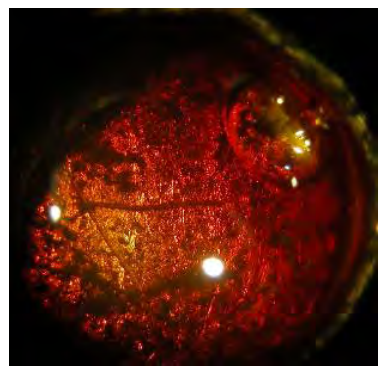

600

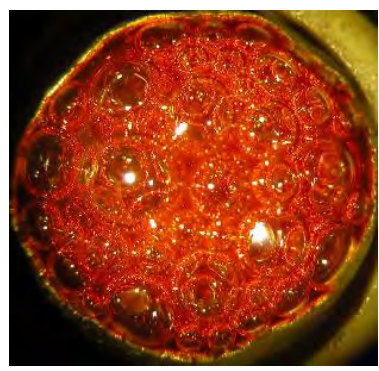

760

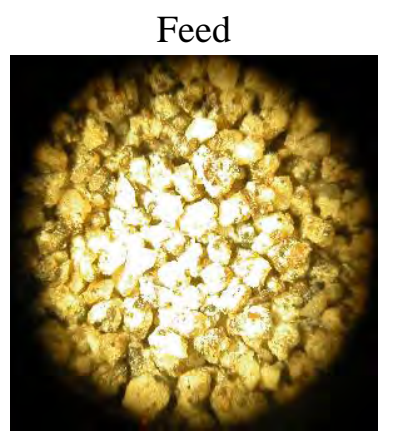

350

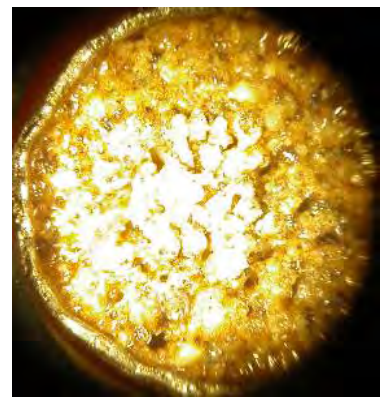

750

Figure 5.3. Hot Stage Microscopy Images of LAW (six tank composite sans $\mathrm{Al}\left(\mathrm{NO}_{3}\right)_{3} \cdot 9 \mathrm{H}_{2} \mathrm{O}$ and $\mathrm{NaOH})$ and the Six-Tank Composite Baseline Feed; Numbers Under the Images Indicate Temperature in ${ }^{\circ} \mathrm{C}$ (the rate of heating was $5^{\circ} \mathrm{C} / \mathrm{min}$, and the crucible diameter was 6 mm) 
LAW

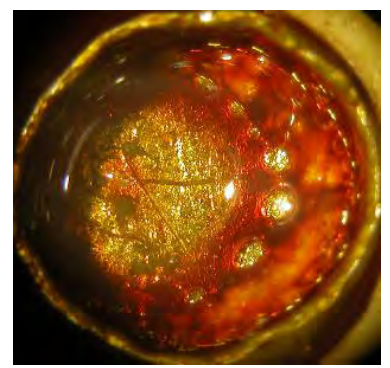

852

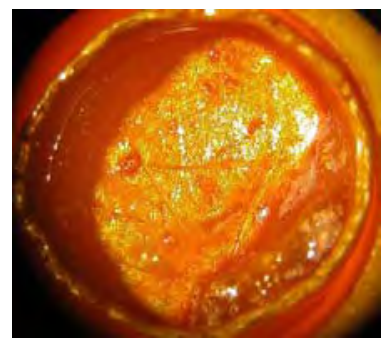

1000

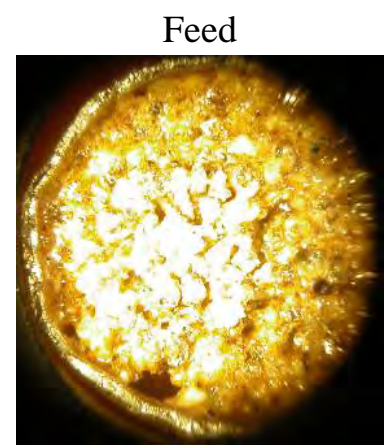

850

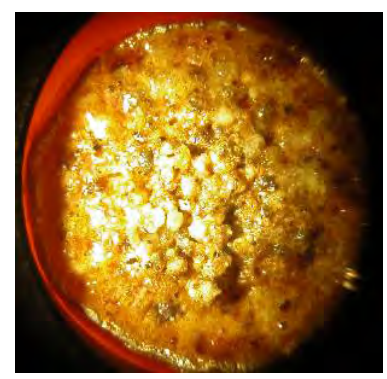

1000
LAW

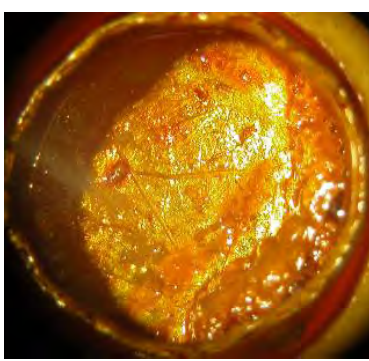

940
Feed

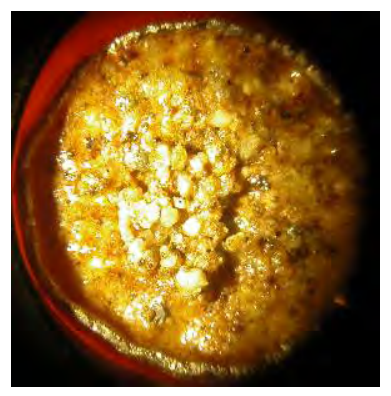

950

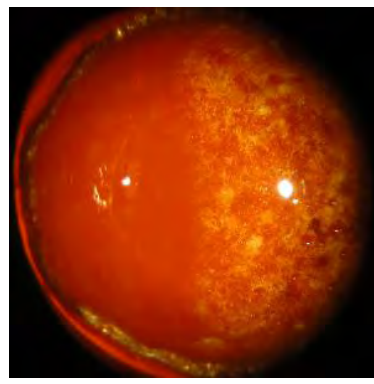

1150

Figure 5.3 (Contd)

The most striking feature of the test was that the feed did not exhibit visible changes while the LAW undergoes melting at $350^{\circ} \mathrm{C}$ and vigorous bubbling at $750^{\circ} \mathrm{C}$. The only visible change in the feed was the sintering that occurred at $750^{\circ} \mathrm{C}$ and the melting that began at $1000^{\circ} \mathrm{C}$. 


\subsection{CRB Treatment}

This study focused on two approaches with respect to the prevention of MIS penetration (hence, ultimately, Tc penetration) into the CRB. The first consisted of various modifications of the CRB itself, and the second attempted to immobilize the MIS inside the feed. The CRB was modified by 1) creating a sacrificial layer at the CRB-feed contact surface, 2) treating the CRB with a lyophobic material (carbon), 3) protecting the CRB surface with AZS tiles, and 4) immobilizing vapors from feed and molten glass in the upper portion of the CRB with higher temperatures at the end of the BV process. Immobilization of MIS in the feed was attempted by increasing the MIS viscosity and by increasing the surface area of those feed components that remained solid in the temperature interval where MIS exists. This section reviews the results of experiments with various CRB treatments. Only a brief description is provided for the CRB treatments because none of the methods developed is deemed practicable at present, though some of the ideas tested and results obtained may become useful in the future. Further details for the CRB treatments are reported in Appendices A through C. All testing was done with pre-prepared engineering-scale feed (ESF) containing six-tank composite LAW simulant. This feed was prepared by mixing solids into liquid simulant and pan drying in a manner that simulates dryer operations. The ESF composition was the same as that listed in Table 4.2. The results of studies of MIS immobilization in the feed are described in Sections 7 and 8 .

\subsection{Creating a Sacrificial Inner Layer on CRB}

Under certain circumstances, the depth of MIS penetration into the CRB does not exceed several millimeters. These circumstances have not been precisely identified, but are probably associated with feeds containing low fractions of free-moving MIS and limited contact times of the feed with CRB. Feeds with a low content of nitrates and a high content of organics charged at a slow rate into the BV melter produced a significant but limited migration of MIS into the CRB. If the MIS-affected layer of the CRB can be removed and returned to the glass melt, soluble Tc would be eliminated from the refractory lining. This can be done by making the inner layer of the CRB less refractory so the layer can withstand the attack by molten salt at temperatures below $750^{\circ} \mathrm{C}$, but not the attack by molten glass at temperatures above $1000^{\circ} \mathrm{C}$. Such a sacrificial layer can be produced by adding fluxes to the CRB.

Tests were performed with additions of 20 to 50 mass $\%$ of sodium metasilicate, $\mathrm{Na}_{2} \mathrm{SiO}_{3}$ (NS), to Vibrocast 60PC. Mixtures were compacted into alumina crucibles, covered with six-tank composite feed, and ramp-heated at $5^{\circ} \mathrm{C} / \mathrm{min}$ to $1200^{\circ} \mathrm{C}$ with a 1 -hour hold. The optimum fraction was 25 mass $\%$ of NS (see Appendix A, Section A.1). Figure 6.1 shows a section through the crucible in which an $~ 6-m m$ layer of containing $25 \%$ of NS was rammed on an $\sim 20$-mm-thick layer of pure Vibrocast 60PC and covered with feed. The assembly was held for 4 hours at $1200^{\circ} \mathrm{C}$. As Figure 6.1 demonstrates, the NS-containing layer was gone, leaving only undissolved large grains of the 60PC material that floated to the top of the glass melt where they remained with segregated yellow sulfate.

To check manufacturability, a larger area $(100 \times 90-\mathrm{mm})$ composite sample that had a 15 - to 20 -mm-thick layer of Vibrocast 60PC material bonded to a 10- to 15-mm-thick layer of vibrocast material with 25 mass $\%$ NS was prepared and heated at $5^{\circ} \mathrm{C} / \mathrm{min}$ to $1200^{\circ} \mathrm{C}$. After cooling, the composite showed no tendency to crack or separate at the interface between vibrocast and viborcast-NS composite even when struck with a hammer. 

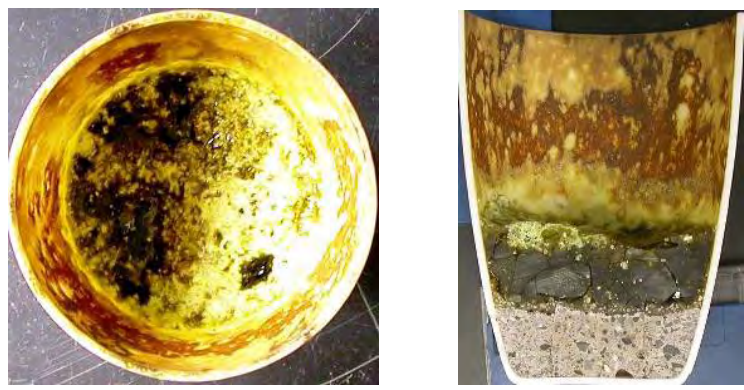

Figure 6.1. Crucible Section Showing the Destruction of the NS-Containing Layer and Partly Corroded Vibrocast Compact and Specimen (the assembly was heat-treated for $\mathbf{4}$ hours at $1200^{\circ} \mathrm{C}$ )

This qualitative test indicated that this type of composite could be prepared at the larger scales required for BV.

\subsection{Creating an Inner CRB Layer that is less Susceptible to MIS Penetration}

Because carbon is not readily wetted by oxy-ionic salts, experiments were conducted to see if filling the porosity of the inner layer of CRB material with carbonaceous material could make the CRB more lyophobic with respect to molten salts. After unsuccessful experiments with saturating the CRB with molten sugar and molten wax (because of the difficulty of impregnating CRB with these melts under vacuum, see Appendix A, Section A.2), tests were performed with vibrocast 60PC mixed with various carbonaceous materials (see Appendix A, Section A.2).

Samples were compacted into alumina crucibles, covered with six-tank composite feed, and heat-treated at $1200^{\circ} \mathrm{C}$ for 1 hour. The best results were obtained with the addition of 18 mass $\%$ of graphite. A scanning electron microscopy-energy dispersive spectroscopy (SEM-EDS) scan, shown in Figure 6.2, through the middle of the sample from the top to the bottom shows that an $18 \%$ addition of groundgraphite decreased the amount of MIS that penetrated into the CRB. MIS penetration was not totally prevented, but it was reduced to $6 \mathrm{~mm}$ compared to full penetration throughout a CRB sample with no carbon addition.

The manufacturability of the CRB with an inner layer of vibrocast containing 17.4 mass\% of graphite was tested by making a composite slab. The size of the slab, the layer thickness, and the procedure followed were similar as in the test performed with the NS-vibrocast mixture. The altered layer formed a porous solid with fine cracks running through the material. It appeared to bond well to the vibrocast layer, but it crumbled under pressure, showing that this type of composite might not be durable enough for BV applications. No attempt was made to measure the impact of carbon addition on the electrical conductivity of the modified CRB or determine whether graphite in the CRB can resist the environment above the melt line. If the graphite addition to CRB is considered in the future, these effects will need to be addressed. 


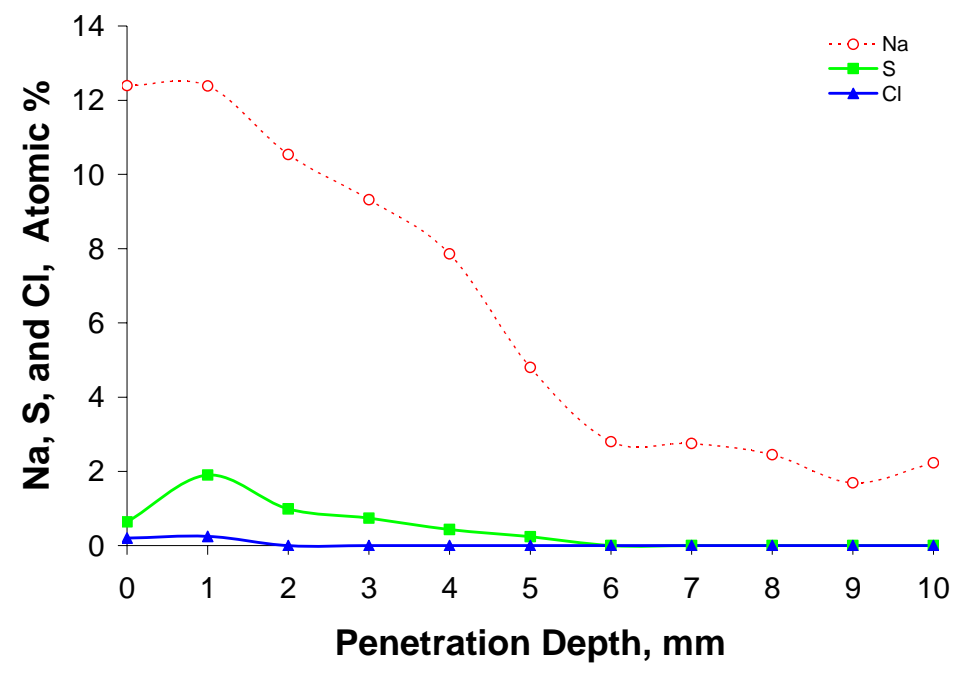

Figure 6.2. Concentration Profile by EDS of Compacted Vibrocast Mixed with 18 mass\% of Graphite

\subsection{Protecting CRB with AZS Tiles}

The AZS tiles were tested for their tendency to delaminate and allow low-viscosity MIS to penetrate behind the tile through the AZS/CRB interface. Dye penetration tests were performed to determine whether micro-fractures along the CRB-AZS interface form during a temperature increase to $800^{\circ} \mathrm{C}$. A molten-salt dye penetration test with $\mathrm{Na}_{2} \mathrm{SO}_{4}$ containing $1 \% \mathrm{Na}_{2} \mathrm{CrO}_{4}$ did not show any penetration through an AZS-CRB interface during a 2-hour hold at $900^{\circ} \mathrm{C}$ (Appendix B). However, AZS porosity was sufficiently open to allow penetration of molten salt into the tile. A room temperature test with ink showed that ink penetrated into both the CRB and the AZS tile, but no excessive ink penetration occurred along the interface. Thus, while the interface was well bonded, both molten salt and ink penetrated into AZS tiles as easily as in the CRB, which casts doubt on the protective ability of the tiles.

\subsection{Converting Tc/Re into Insoluble Form in the Upper CRB}

At sufficiently high temperatures, a salt condensate will react with the CRB cement to produce a glass phase that could encapsulate Tc salts. If condensate in the upper portion of the CRB was a significant contributor to the soluble Tc in the waste package, heating the upper portion of the CRB to higher temperatures by extending the melt duration and using an insulated lid might have reduced soluble Tc levels in the box. However, analyses of the CRB from a partially completed FS-38B test showed that condensation on the upper portions did not lead to significant Re migration into the CRB. As a result, efforts in this area were reduced to a series of scoping tests.

To produce simulated CRB samples that had known quantities of condensate with well characterized compositions, the salt condensate simulate was prepared and directly introduced into the CRB in the form of an aqueous solution. The composition of the condensate simulant was formulated based on the analysis of vapors condensed on a cold rod placed above a crucible of melted feed and SEM-EDS data from the upper walls of the FS-38B test (see Appendix C). The condensate simulant contained $\mathrm{NaNO}_{3}$ and $\mathrm{B}_{2} \mathrm{O}_{3}$. 
It was mixed with vibrocast $60 \mathrm{PC}$ to obtain the sodium levels seen in the upper portions of the CRB $\left(0.6\right.$ at\%). Three levels of $\mathrm{B}_{2} \mathrm{O}_{3}$ were tested, corresponding to the atomic fractions $\mathrm{Na} /(\mathrm{Na}+\mathrm{B})=0.50$, 0.75 , and 1.00 . The mixtures were heat-treated for 2 hours at $800^{\circ} \mathrm{C}, 1050^{\circ} \mathrm{C}$, and $1300^{\circ} \mathrm{C}$. Samples were then ground and leached in DI water at $60^{\circ} \mathrm{C}$ for 20 hours. The $\mathrm{pH}$ and electrical conductivity of the leachate was used as a measure for determining the chemical durability of the glass phase formed as a result of heat-treatment.

Both $\mathrm{pH}$ and electrical conductivity of leachates decreased as the temperature of the heat-treatment increased (Figure 6.3), showing a progressive incorporation of the condensate into a durable glass phase. As discussed in Appendix $\mathrm{C}$, the cement portion of the CRB turns into a glass phase on heating and absorbs oxides from the decomposing MIS. At the two higher test temperatures, the $\mathrm{B}_{2} \mathrm{O}_{3}$ content in the condensate had a positive impact on the durability of the glass. 

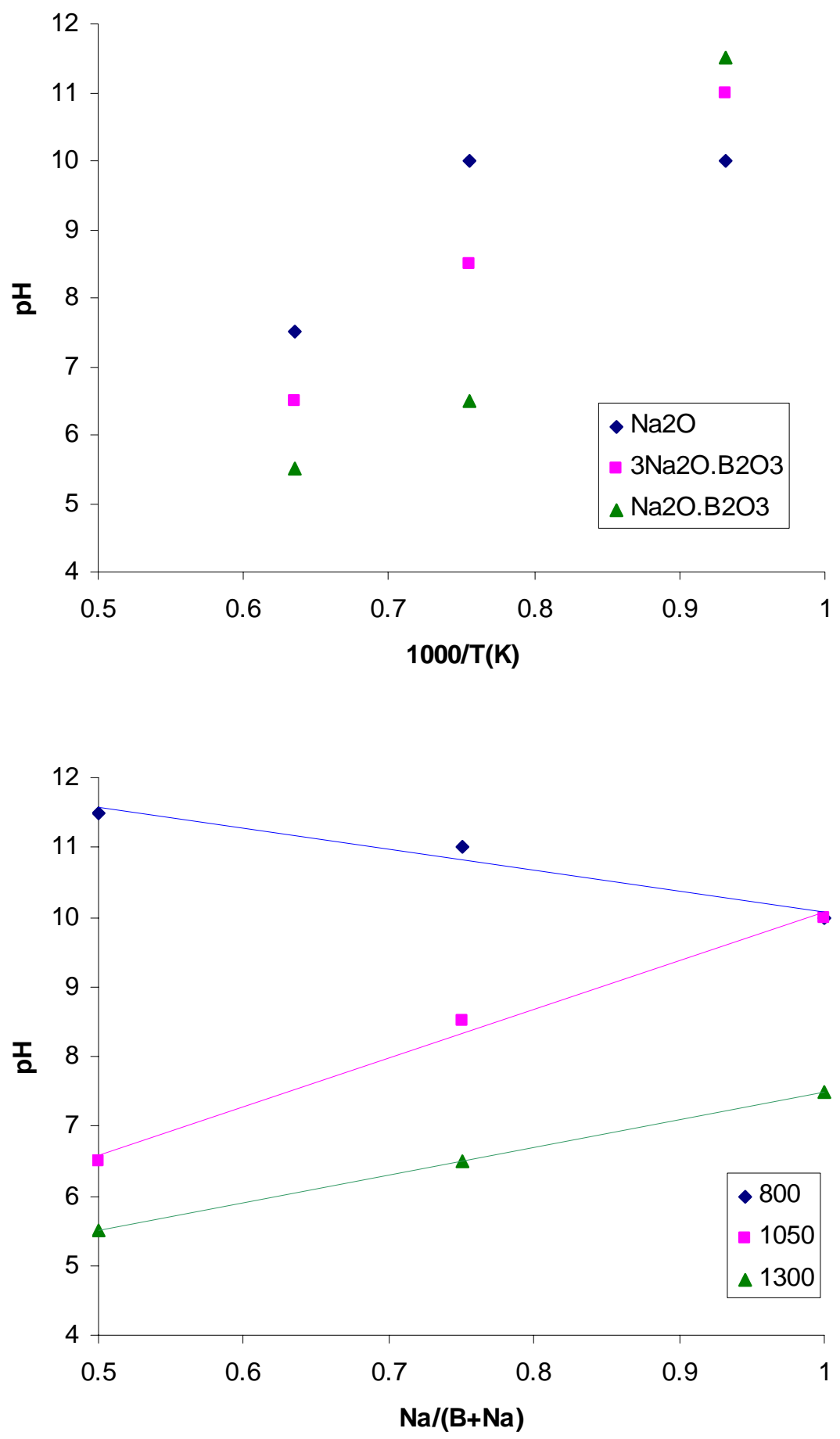

Figure 6.3. Effects of Temperature (top) and Condensate Composition (bottom) on the Leachate $\mathrm{pH}$ 


\subsection{MIS Immobilization by Increasing Specific Surface Area of Feed Solids}

At a constant temperature, two major parameters affect the amount of MIS that can be released from soilbased feed to the CRB. These parameters are the time of exposure and the specific surface area of the feed. The time of exposure is an important parameter if MIS availability is virtually unlimited. The time of contact of the feed with the refractory wall is controlled in the BV process by the method of feed loading. In feeds that cannot effectively retain the MIS, a thicker layer of feed results in a longer contact time and deeper MIS penetration. As the FS-38C experiment had shown, MIS can penetrate all the way through the refractory wall and continue flowing through it as long as it is supplied from the feed. However, a thick feed layer at the surface of the glass melt has important benefits, such as preventing the volatilization of radioactive components that condense on the upper CRB, the lid, the pipes, and in the offgas system. Preventing the MIS from migrating outside the feed would allow charging the BV melter in way that is optimal for the process.

MIS migration outside of the feed can be reduced by increasing the specific surface area of the feed solids or by destroying nitrates and nitrites in the feed before they can migrate. Increasing the specific surface area of solids in the feed helps adsorb and immobilize the excess MIS by allowing it to spread on solid particles and form bridges between them. As has been shown by Hrma et al. (1991), only the excess fraction of salt can flow freely in the open spaces between the grains. This free fraction readily migrates into the open pores of the CRB. Hence, the transfer of MIS from feed to CRB can be substantially reduced by increasing the surface area available for wetting by using feed solids with smaller particle sizes.

Tests were performed with feeds containing several different types of high specific surface area materials. Tests were also conducted with soil that was ground to generate the high specific surface area. When fine-grain materials were used, they partly replaced the soil so that they would not negatively impact the final glass properties. A partial replacement of soil with fine-grain materials did not significantly reduce MIS migration. However, excellent results were obtained from high surface area feeds generated through soil grinding.

An attempt was also made to decrease MIS mobility by dissolving $\mathrm{B}_{2} \mathrm{O}_{3}$ in aqueous LAW before blending it with other feed materials. It was expected that $\mathrm{B}_{2} \mathrm{O}_{3}$ would decrease MIS mobility by increasing its viscosity. Preliminary tests showed that $\mathrm{B}_{2} \mathrm{O}_{3}$ substantially increased the liquidus temperature of MIS. However, MIS penetration tests with feeds produced with LAW that had dissolved $\mathrm{B}_{2} \mathrm{O}_{3}$ did not produce promising results even when the $\mathrm{B}_{2} \mathrm{O}_{3}$ concentration was added at twice the concentration used in the current baseline glass formulation.

\subsection{Materials Tested}

The fine-grain materials tested included fine silica powder, diatomaceous earth, kaolin, bentonite, and fly ash. Table 7.1 lists the generic compositions of these materials. ${ }^{(a)}$ The particle size of silica powder was

(a) The diatomaceous earth used was taken from existing supplies. Table 7.1 lists an average of three typical compositions. 
$<75 \mu \mathrm{m}$. A typical particle size of clay minerals (kaolin and bentonite) is $<4 \mu \mathrm{m}$. Diatomaceous earth has 10 - to $200-\mu \mathrm{m}$ particles of $\sim 100 \mathrm{~m}^{2} / \mathrm{g}$ surface area. Ground soil and feeds from fine-grain minerals (replacing soil) were also tested as an alternate method to generate high surface areas of feed solids.

Table 7.1. Compositions of Fine Additives

\begin{tabular}{||l|c|c|c|c|c||}
\hline \hline & Silica $^{\text {(a) }}$ & $\begin{array}{c}\text { Diatomaceous } \\
\text { Earth }^{(\mathbf{b})}\end{array}$ & Kaolin $^{\text {(a) }}$ & Bentonite $^{(\mathbf{c})}$ & $\begin{array}{c}\text { Labadie } \\
\text { Fly Ash }^{(\mathbf{d})}\end{array}$ \\
\hline $\mathrm{Al}_{2} \mathrm{O}_{3}$ & & 0.0763 & 0.4590 & 0.2880 & 0.2025 \\
\hline $\mathrm{CaO}$ & & 0.0090 & & 0.0158 & 0.2442 \\
\hline $\mathrm{Fe}_{2} \mathrm{O}_{3}$ & & 0.0366 & & & 0.0674 \\
\hline $\mathrm{K}_{2} \mathrm{O}$ & & & & & 0.0067 \\
\hline $\mathrm{MgO}$ & & 0.0055 & & & 0.0506 \\
\hline $\mathrm{Na}_{2} \mathrm{O}$ & & 0.0135 & & 0.0175 & 0.0154 \\
\hline $\mathrm{P}_{2} \mathrm{O}_{5}$ & & & & & 0.0128 \\
\hline $\mathrm{SiO}_{2}$ & 1.0000 & 0.8591 & 0.5410 & 0.6787 & 0.3657 \\
\hline $\mathrm{SO}_{3}$ & & & & & 0.0201 \\
\hline $\mathrm{TiO}_{2}$ & & & & \\
\hline $\begin{array}{l}\text { (a) Stoichiometric composition. } \\
\text { (b) Average of three typical compositions. } \\
\text { (c) As montmorillonite from Mineralogy database (http://webmineral.com). } \\
\text { (d) Ameren Energy Fuels and Services. }\end{array}$ \\
\hline
\end{tabular}

Silica crucibles were used as model refractories. They had several advantages over the vibrocast CRB for testing purposes. First, silica crucibles were more uniform in composition, structure, and porosity; second, MIS penetration in silica crucibles was easier to detect because the penetrated areas had a distinct color and sharp boundary. ${ }^{\text {(a) }}$ Room-temperature modeling, using aqueous solutions that have a similar surface tension and viscosity as MIS was considered (see Appendix G) but the silica crucible tests proved to be the most reliable for predicting actual results with CRB materials.

Two types of silica crucibles were used for initial testing: small and medium. Scoping tests were performed in small crucibles, $\sim 50 \mathrm{~mm}$ tall, $\sim 20 \mathrm{~mm}$ inner diameter at the bottom, and $\sim 40 \mathrm{~mm}$ inner diameter at the top; the wall thickness was 3 to $5 \mathrm{~mm}$. The main testing was performed in medium-sized crucibles, $\sim 100 \mathrm{~mm}$ tall, $\sim 40 \mathrm{~mm}$ inner diameter at the bottom, and $\sim 80 \mathrm{~mm}$ inner diameter at the top; the wall thickness was 6 to $12 \mathrm{~mm}$. For tests with feeds containing six-tank composite LAW simulant, a CRB rod, $120 \mathrm{~mm}$ long with a square cross-section of 15- to 19-mm sides, was immersed in the feed in medium crucibles (see the schematic in Figure 7.1). CRB rod tests and silica crucible tests were conducted separately in the final series of tests with S-109 LAW simulant.

(a) The depth of MIS penetration in the CRB bodies is difficult to detect, both optically and with SEM-EDS. Optical detection by changing color can be misleading because the CRB color responds to various factors, such as heat-treatment temperature. Electronic detection is difficult because the beam is of the same size or smaller than large refractory grains that are not penetrated by MIS. 


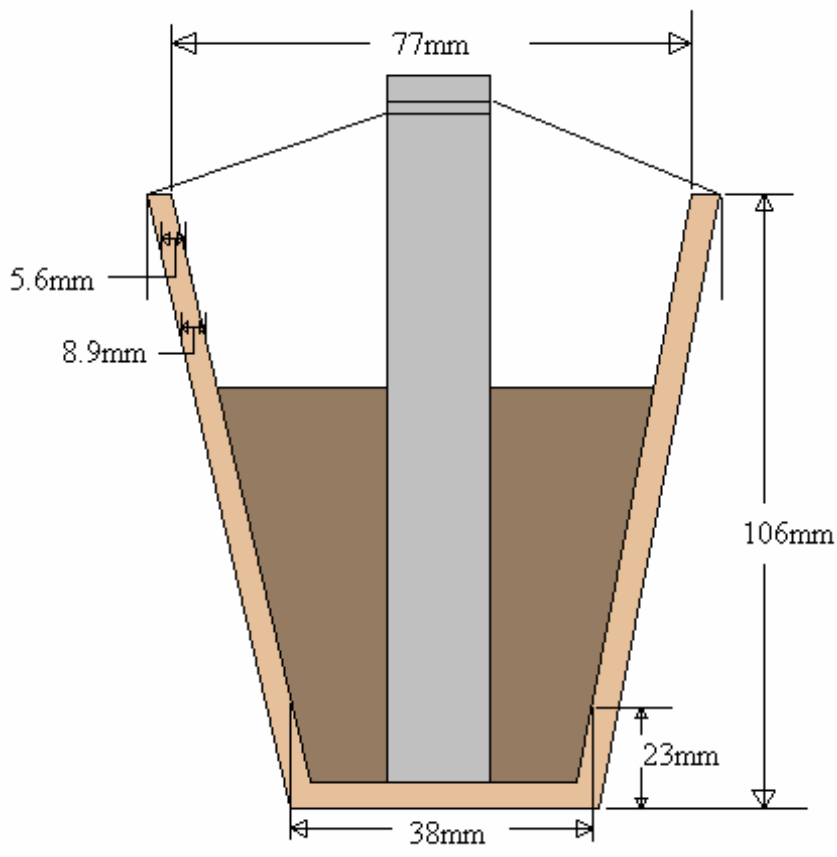

Figure 7.1. Dimensions of a Randomly Selected Medium-Sized Silica Crucible; a CRB Rod Is Adjusted by a Pt Wire in the Middle of the Feed

\subsection{Preliminary Studies}

Various scoping tests and preliminary tests were performed to explore the interaction between the finegrain additions and the MIS based on the six-tank simulant. Appendix D describes these tests.

- Experiments were designed to test whether MIS would wet fine-grain materials when the mixing was incomplete. The fines were placed in a silica crucible, overlaid with dry LAW, and heated to $1050^{\circ} \mathrm{C}$. The results showed that fine-grain silica and diatomaceous earth readily absorb the MIS, but silica flour failed to protect the crucible. Clays and fly ash formed a skin that blocked wetting of the internal portions of these materials.

- To assess the reactivity between fine-grain materials and MIS, when these components were well blended, fine-grain materials were loaded to silica crucibles and mixed with aqueous LAW to form plastic pastes, each of a similar consistency. Diatomaceous earth held more than twice as much liquid as other materials. The crucibles were heated to $1050^{\circ} \mathrm{C}$, held for 30 minutes, heated to $1300^{\circ} \mathrm{C}$, and then examined. Crucibles containing fine silica were severely damaged. The least damage was inflicted by the mixture containing kaolin.

- A test checking the interaction of a silica crucible with pore MIS in the absence of any feed solids during heating at $5^{\circ} \mathrm{C} / \mathrm{min}$ showed that MIS began to penetrate to the outer surface of the crucible at a temperature as low as $300^{\circ} \mathrm{C}$.

- Finally, heat-treating the Hanford soil overlaid with solid six-tank simulant at various temperatures showed that MIS did not readily permeate the soil but instead appeared to preferentially migrate to and react with the crucible. 
Scoping tests showed that thorough mixing of the liquid LAW simulant with the soil and fine-grained materials was necessary to promote spreading the MIS on the grain surfaces. Tests with fine-grained materials and tests with Hanford soil mixed with LAW liquid simulant to approximately the same consistency indicated that the wetting capacity by water was different from that by molten salt.

Three preliminary tests were performed. In the first, the baseline feed of composition defined in Table 4.2 was spiked with $\mathrm{Na}_{2} \mathrm{CrO}_{4}$ to enhance the color of MIS and heated in both small and medium silica crucibles at $5^{\circ} \mathrm{C} / \mathrm{min}$ to $1200^{\circ} \mathrm{C}$ with a 1 -hour hold. In the second test, the same experiment was performed with the feed used for the FS-38B test (this feed had identical composition, but was prepared by mixing dry LAW simulant chemicals with the glass-forming additives). Figure 7.2 shows the results.

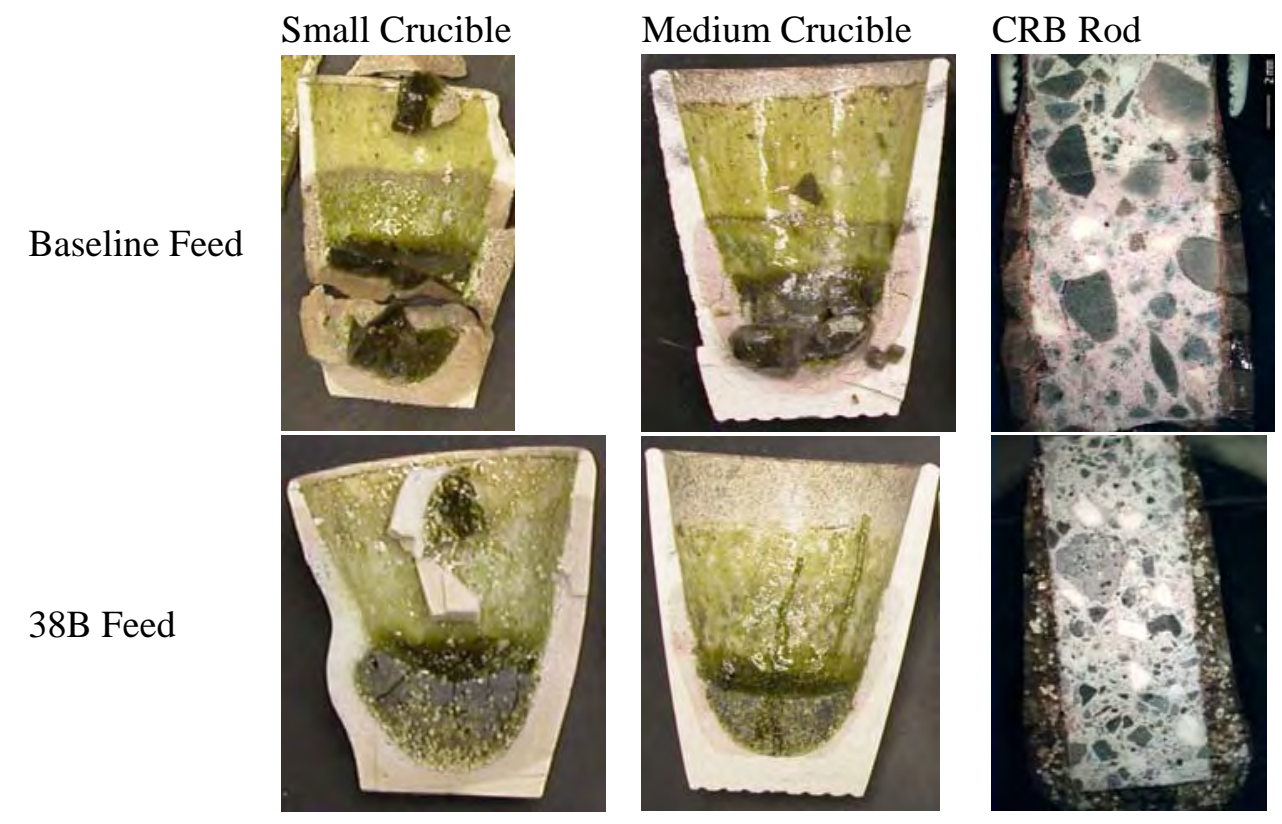

Figure 7.2. Comparison of MIS Penetration to Silica Crucibles and CRB Rod from Baseline Feed and 38B Feed

In the tests conducted with the baseline feed, the MIS penetrated through the entire side wall and bottom of the small crucible, but did not reach the outer surface of the side wall of the medium crucible (the bottom was partially protected by the CRB rod). In the tests conducted with the 38B feed, the wall of the small crucible was severely deformed; the MIS permeated nearly all the volume of the crucible except the rim. MIS penetration into the medium crucible was a similar to that from the baseline feed. The CRB rod was fully penetrated with MIS from both feeds and was visibly corroded below the final melt line. The pores and swelling developed in the rod as a result of the reaction between the MIS and the fine-grain portion of the CRB. Note that white particles of $\mathrm{ZrO}_{2}$ that was in the form of hollow $\sim 100-\mu \mathrm{m}$ baddeleyite spheres in 38B feed sank to the crucible bottom. Appendix D, Sections 2.2 and 2.3 provide the details of the tests.

The purpose of the third preliminary test was to investigate the effect of premixing $\mathrm{B}_{2} \mathrm{O}_{3}$ with LAW on MIS penetration into porous refractory. The test, described in Appendix D, Section 2.3, did not show any significant decrease in MIS penetration into the crucibles. 
After the completion of preliminary experiments, a more systematic study was undertaken. The study proceeded in three stages. In the first stage, the study focused on the response of MIS penetration to temperature. The second stage focused on the impact of fine-grain materials in the feed and the soil particle size (the specific surface area of soil). The third stage applied the techniques developed to feeds containing S-109 LAW simulant. Also in the third stage, successful attempts were made to decrease MIS migration by adding carbohydrates to the feed.

\subsection{Effect of Temperature and Time on MIS Penetration}

In the fist stage, feed was prepared separately for each experiment. The liquid LAW simulant was added to a metallic beaker on a hotplate. The fine-grain ingredient was added to the simulant and stirred into a homogenous suspension. The suspension was heated and stirred until all free water was evaporated. The dry suspension was mixed with the premixed additives (Hanford soil, $\mathrm{ZrO}_{2}, \mathrm{~B}_{2} \mathrm{O}_{3}$, and $\mathrm{Na}_{2} \mathrm{CrO}_{4}$ ) in the tungsten carbide mill for 1 minute. The feed was then heated in small and medium silica crucibles (with a CRB rod).

Four series of experiments were performed in the first stage:

- $\quad$ CRB samples ( 15 -mm cubes) were submerged in baseline feed and heated to temperatures of 550 to $1300^{\circ} \mathrm{C}$. The cubes were then fractured and examined. Appendix E, Section 1.1 describes these experiments. Specimen examination by SEM-EDS showed that all specimens were fully penetrated with MIS.

- $\quad$ A CRB chip was heated with feed under the hot-stage microscope (see Appendix E, Section 1.2). The test showed that the feed melts more slowly in the vicinity of the CRB sample, probably because the CRB sample removes fluxes from the feed.

- Small silica crucibles were filled with baseline feed and heated to $550^{\circ} \mathrm{C}$ and $800^{\circ} \mathrm{C}$ and held for 0 and 1 hour to compare the extent of MIS penetration (Appendix E, Section 1.3). The MIS decomposition was virtually complete at $800^{\circ} \mathrm{C}$; extended heating at this temperature did not increase the mass loss. About one half of the MIS was decomposed when the temperature reached $550^{\circ} \mathrm{C}$, and the decomposition continued when this temperature was held for 1 hour.

- Based on these results, a more systematic study of the response on MIS penetration into silica crucibles to temperature was performed (Appendix E, Section 1.4). Small silica crucibles with baseline feed were heated at $5^{\circ} \mathrm{C} / \mathrm{min}$ to $350,400,450$, and $500^{\circ} \mathrm{C}$. The feed was removed from the crucibles, and the amount of MIS in the crucible walls was determined. Empty crucibles were heated at $5^{\circ} \mathrm{C} / \mathrm{min}$ to $1200^{\circ} \mathrm{C}$ to allow the MIS to react with the crucible material. The MIS was fully decomposed, allowing an accurate determination of the migrated mass. Also, the crucibles could be wet-cut without dissolving the MIS.

The results showed the following MIS behavior:

o The MIS mobility within the feed is large, allowing a continuous supply of MIS from the bulk feed to the crucible wall.

o The first MIS that penetrates the refractory is composed of low-temperature eutectics.

0 As feed-melting reactions progress, MIS decomposition continues both in the feed and in the refractory, but possibly at different rates. 
o Significant MIS penetration occurs at temperatures as low as $350^{\circ} \mathrm{C}$.

Figure 7.3 shows the mass of MIS that entered the crucible wall per unit interface area as a function of temperature. Penetration depth did not increase as rapidly as penetrated mass, indicating that the MIS density in the refractory increased with temperature (from $0.14 \mathrm{~g} / \mathrm{mL}$ at $350^{\circ} \mathrm{C}$ to $0.24 \mathrm{~g} / \mathrm{mL}$ at $500^{\circ} \mathrm{C}$ ). It is likely that MIS reaction with the bonding medium (refractory cement) opens more space inside the refractory, allowing additional MIS to enter the space.

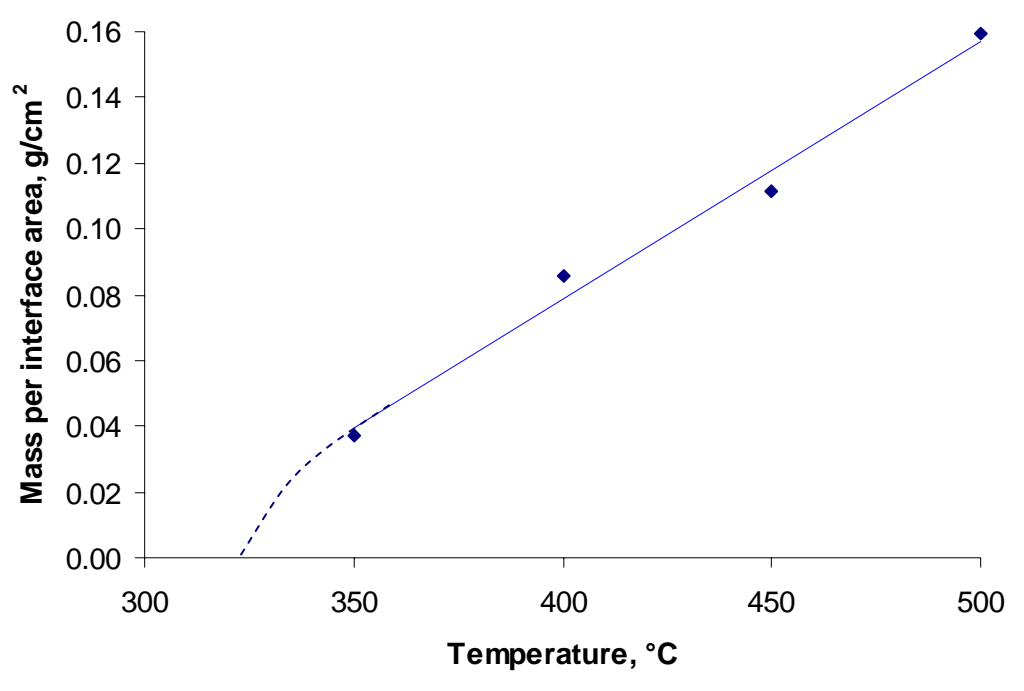

Figure 7.3. Mass of MIS Soaked into Crucible Wall Versus Temperature

\subsection{Effect of Fine-Grain Materials on MIS Penetration}

Appendix F lists the compositions of the feeds tested, the corresponding glass compositions, and the batch sheets. Figure 7.4 shows the results; see Appendix F for more images.

Appendix F describes the observations regarding crucible corrosion by the melts, melt foaming, sulfate segregation, and the appearance of CRB rods. For comparison, Figure 7.2 shows the impact of baseline feed on silica crucibles. As Figure 7.4 shows, diatomaceous earth substantially decreased MIS penetration into the crucible. Kaolin and bentonite slightly decreased MIS penetration. Fly ash brought little improvement. However, the low penetration depth from the feed with 10 mass\% of diatomaceous earth could be caused by feed shrinking away from the crucible sidewalls during the early stages of heating. 


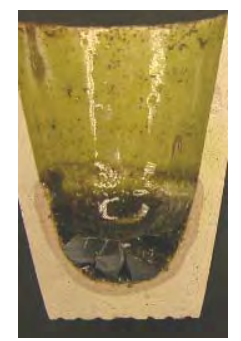

2 to $5 \mathrm{~mm}$

Kaolin

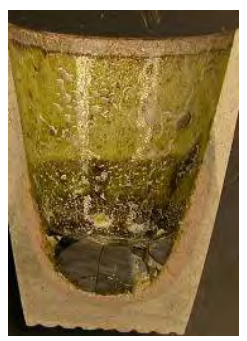

3 to $6 \mathrm{~mm}$

Fly Ash

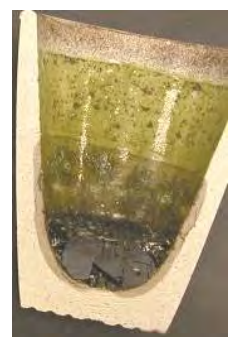

1 to $4 \mathrm{~mm}$

Diatomaceous

Earth

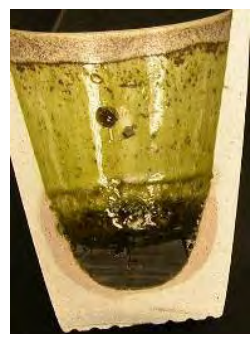

2 to $5 \mathrm{~mm}$

Bentonite

Figure 7.4. Sections Through Medium Crucibles with Feeds Containing Fine-Grain Ingredients Heated at $5^{\circ} \mathrm{C} / \mathrm{min}$ to $1200^{\circ} \mathrm{C}$ for 1 -Hour Dwell

The effect of diatomaceous earth additions on MIS penetration into porous refractory was investigated further by adding 15 to 25 mass\% of diatomaceous earth to the feed. Figure 7.5 shows the appearance of the crucibles after heating. The bottoms of small crucibles were severely damaged by MIS, turning a part of the bottom material into viscous foam. Heating probably destroyed the fine structure of the diatomaceous earth particles, releasing the MIS trapped inside. As the feed shrank away from the sidewalls, the MIS drained to the feed bottom. Medium crucibles were less damaged because the MIS soaked into the CRB rods.

\subsection{Effect of Ground Soil Fraction on MIS Penetration from Six-Tank Composite Feed into Porous Refractories}

This option was not considered in the original test plan because grinding the soil on a large scale is potentially an expensive process, and fine soil may be difficult for pneumatic transfer equipment. The initial test described below was performed to find out how much MIS penetration would be affected if all the soil were ground. The result was encouraging and led to extensive testing of the impact of soil grinding on the MIS. This testing has shown that grinding the soil appeared successful in limiting MIS migration into the CRB. All the tests in this section were performed using feeds prepared with the sixtank composite waste simulant.

In a preliminary test, CRB specimens were covered with a feed containing soil that was ground to a fine powder for 5 minutes in a tungsten carbide mill. The SEM-EDS profiles of Na (Figure 7.6) revealed that only the sample surface was contaminated by $\mathrm{Na}$, and possibly little penetration occurred at the bottom surface. This contrasted with the full penetration of samples treated in feeds containing the original soil.

The potential solution to the MS penetration problem via ground soil led to detailed investigation of the effects of soil grinding on MIS migration. These studies looked at how the fraction of ground soil mixed with the original soil impacted MIS penetration. The studies also looked at how varying the extent of grinding the soil impacted MIS penetration. Appendix $\mathrm{H}$ describes the details of testing. 
DE \%

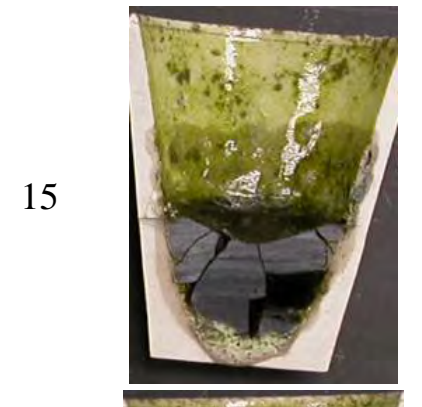

20

25
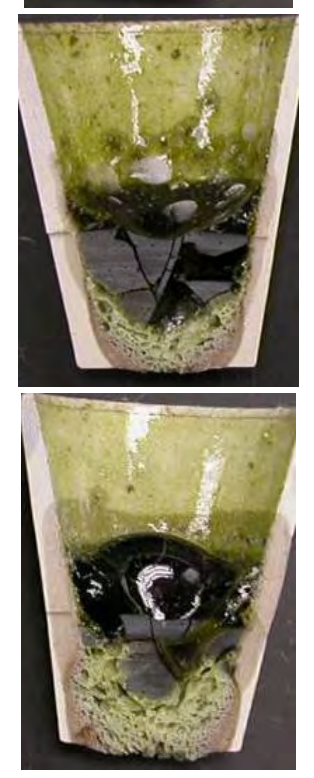

Small Crucible
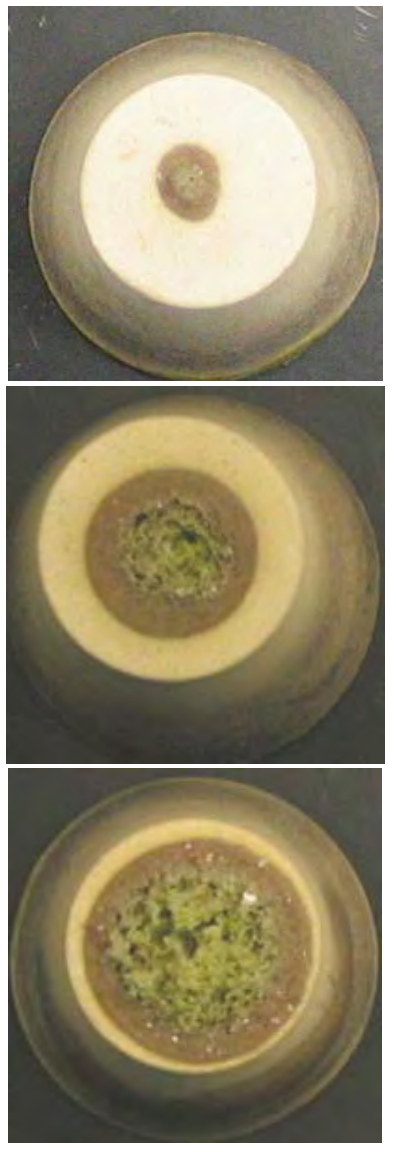

Medium

Crucible
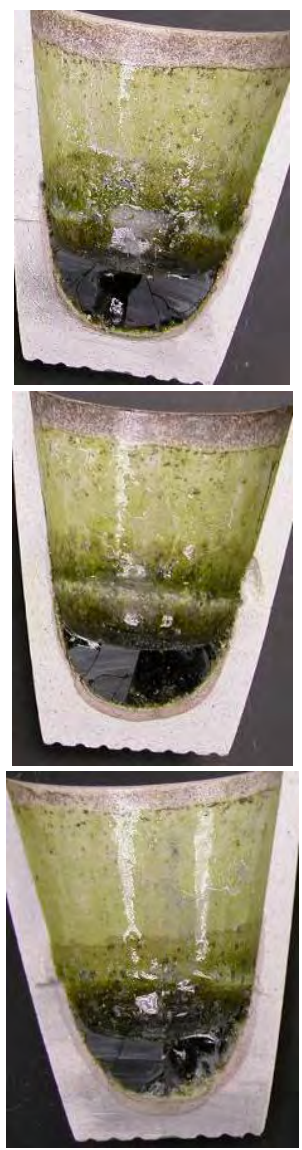

Figure 7.5. Sections Through Crucibles and Vibrocast Rods Exposed to Feed with 0 to 25 Mass\% of Diatomaceous Earth
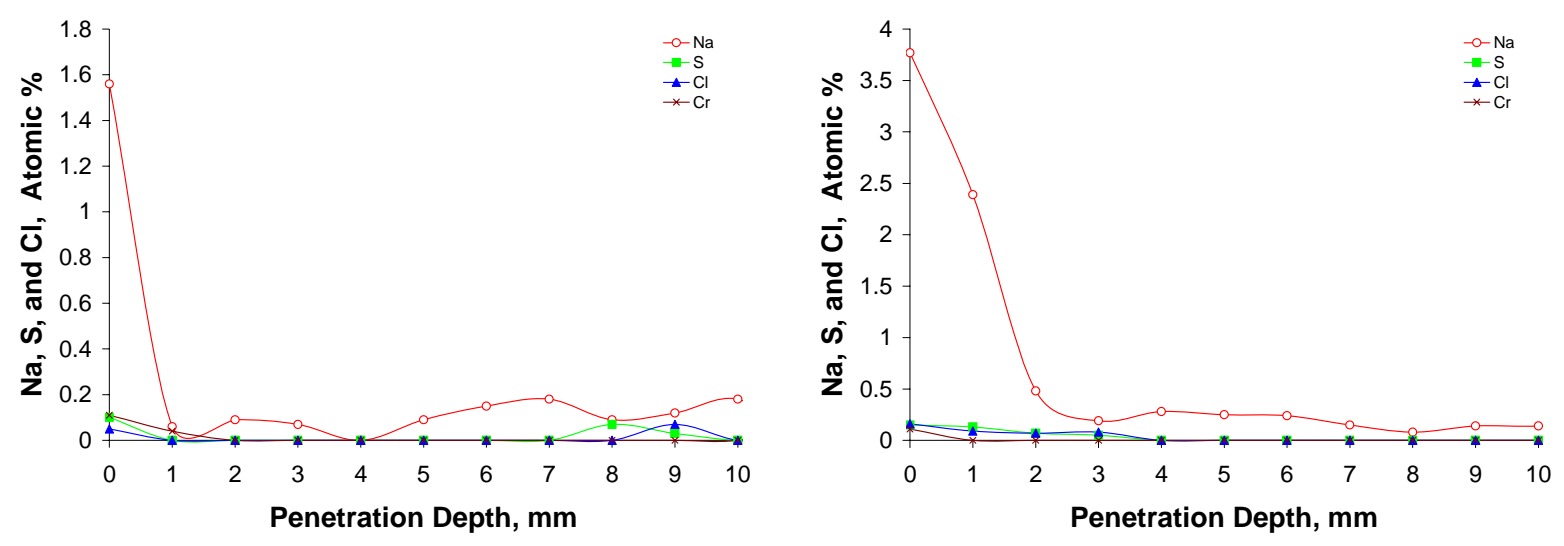

Figure 7.6. CRB Specimens Exposed to Feed with Ground Soil Heated to $800^{\circ} \mathrm{C}$; Sample Scan Went Through the Middle of Sample from Left to Right (left) and from Bottom to Top (right) 
Figure 7.7 shows the depth of MIS penetration into silica crucibles as a function of the fraction of soil that was ground in the tungsten mill for 2 minutes. From the exponential fit of the data, one can estimate that the depth of migration decreased by $25 \%$ if $15 \%$ of soil was ground, by $50 \%$ if $37 \%$ of soil was ground, by $75 \%$ if $73 \%$ of soil was ground, and by $85 \%$ if $100 \%$ of soil was ground for 2 minutes.

This experiment with silica crucibles confirmed the assumption that the main parameter influencing the extent of MIS migration from feed to CRB is the specific surface area of the feed. By extrapolation, one can assume that all MIS may become fully absorbed on soil particles if the specific surface area increases beyond a value at which the free-moving liquid phase is no longer present in the feed.

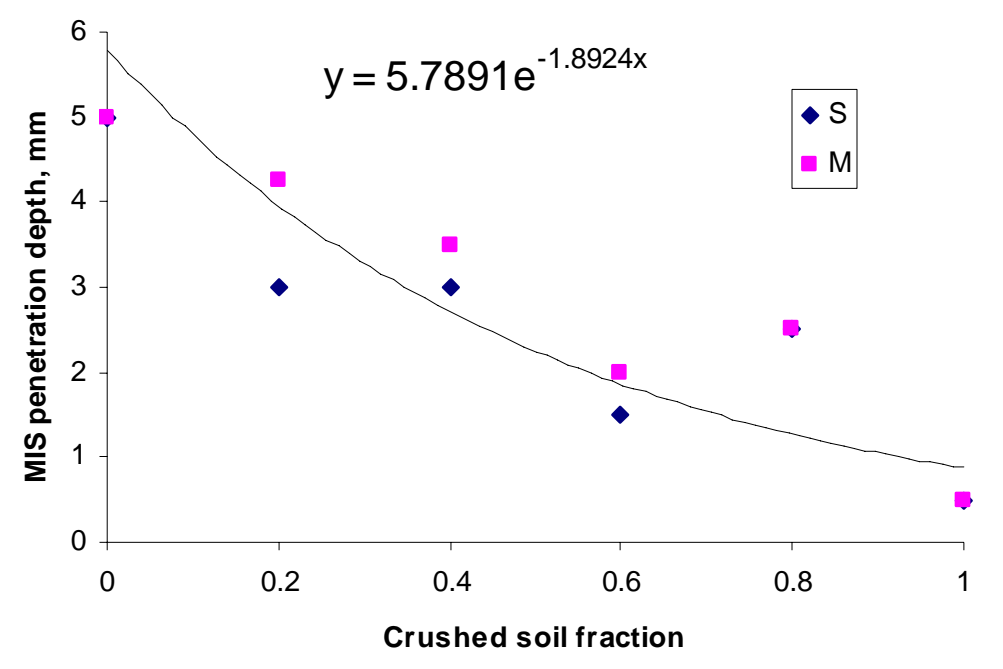

Figure 7.7. MIS Migration Depth Versus Fraction of Soil Ground (S-small crucible, $\mathrm{M}$-medium crucible)

Assessing the MIS migration depth is subjected to a high uncertainty because the depth is rather nonuniform over the crucible surface. A better way of quantifying MIS penetration is measuring the mass change. The problem of strong bonding between solidified glass and the refractory was overcome by heating the feed to a temperature at which the MIS was fully developed while the feed was not yet bonded to the refractory surface. The temperature range at which these two requirements were met lies between $350^{\circ} \mathrm{C}$ and $700^{\circ} \mathrm{C}$. The temperature range of 500 to $650^{\circ} \mathrm{C}$ was most frequently used in this study. When CRB samples were used, the feed was placed in Pt crucibles to avoid the competing effect of porous walls of silica crucibles.

For easier handling, tests with CRB samples were performed using the ESF that was available in large supply. An assembly consisting of a vertical CRB rod surrounded with $97 \mathrm{~g}$ of feed in a Pt crucible, seen in Figure 7.8, was placed in a furnace and heated at $5^{\circ} \mathrm{C} / \mathrm{min}$ to $550^{\circ} \mathrm{C}$. The $550^{\circ} \mathrm{C}$ temperature was chosen because all MIS is in a liquid state at this temperature while most of the nitrate in the MIS has not yet thermally decomposed. When comparing the results obtained with ground feed with feed prepared by mixing LAW simulant with ground soil, we need to keep in mind that grinding the feed decreases the efficiency of soil disintegration, but enhances the contact between the soil particles and the salt crystals. 
To determine the degree to which MIS was decomposed at $550^{\circ} \mathrm{C}, \mathrm{CRB}$ rods (removed from the feed and cleaned) underwent a second heat-treatment, this time in air, at $1200^{\circ} \mathrm{C}$ for 1 hour. At these conditions, the MIS was decomposed completely.

Generally, the rods were pinkish in color after the first heat-treatment and became white after the second. This color change was probably associated with the changes in the chromium valence, from $\mathrm{Cr}(\mathrm{VI})$ at $550^{\circ} \mathrm{C}$ to $\mathrm{Cr}(\mathrm{III})$ at $1200^{\circ} \mathrm{C}$. The first heat-treatment had little effect on the rod dimensions and texture. Figure 7.9 shows that even the 4-hour heat-treatment at $750^{\circ} \mathrm{C}$ of $\mathrm{CRB}$ rods in the original feed did not result in any detectable change in the sample appearance. Only subsequent heat-treatment of the rods at $1200^{\circ} \mathrm{C}$ caused the MIS-soaked portion of the rod to swell, crack (Figure 7.10), and develop macroscopic pores (Figure 7.11 and Figure 7.12).

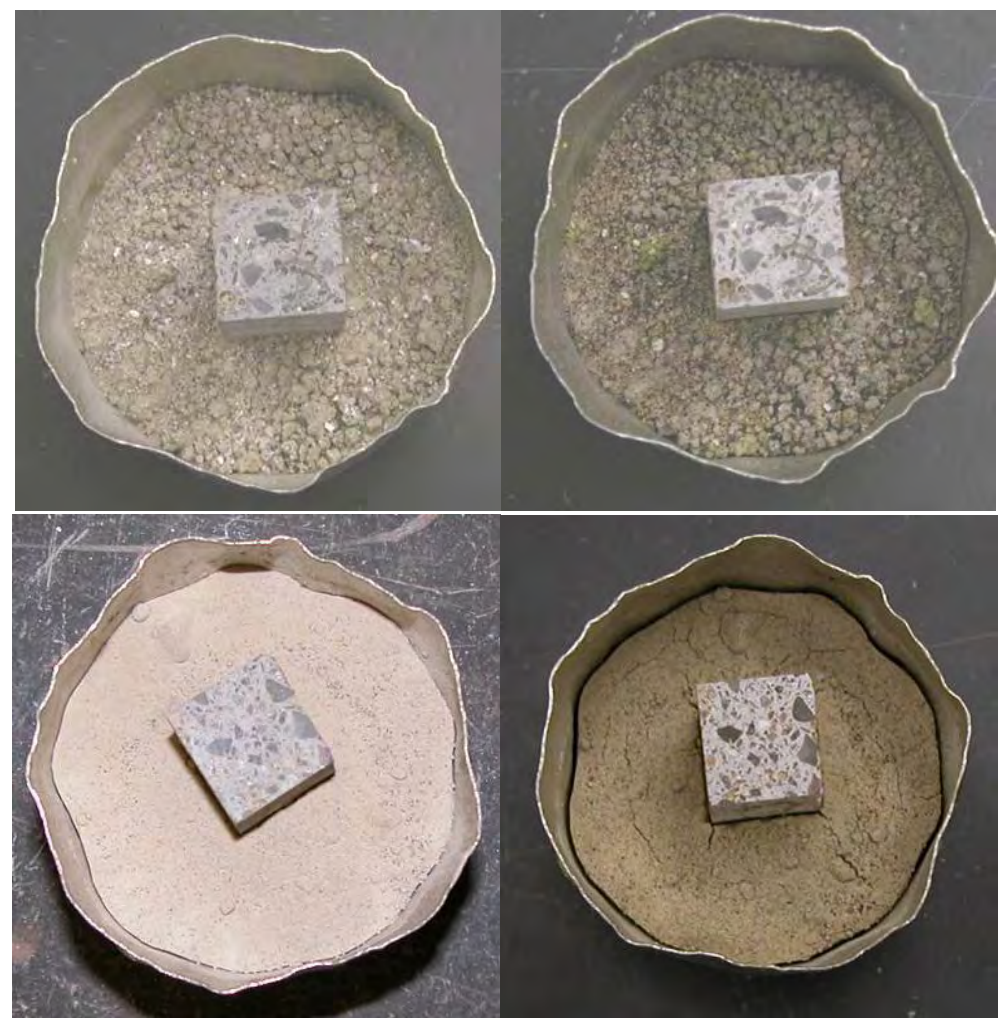

Figure 7.8. A Typical Assembly of CRB Rod and ESF (original feed top and ground feed bottom) in a Pt Crucible Before (left) and After (right) Heat-Treatment at $550^{\circ} \mathrm{C}$ for 1 Hour 


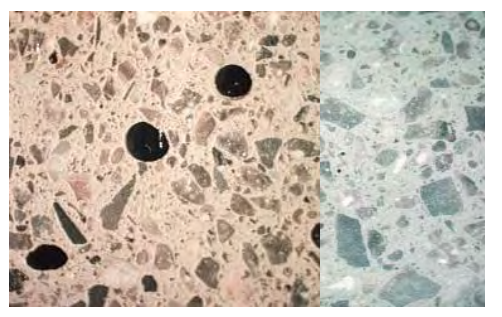

$550^{\circ} \mathrm{C}, 1$ hour

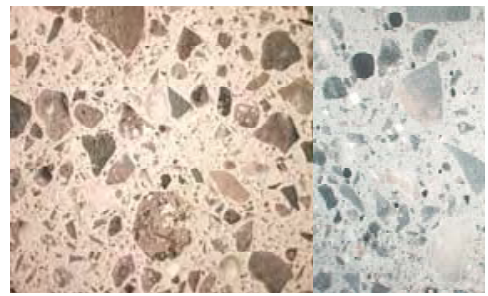

$650^{\circ} \mathrm{C}, 1$ hour

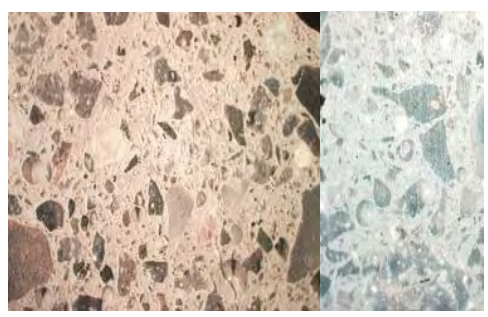

$550^{\circ} \mathrm{C}, 2$ hours

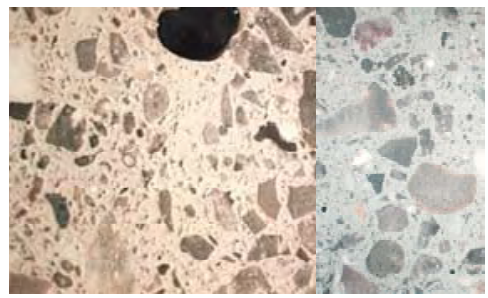

$650^{\circ} \mathrm{C}, 2$ hours

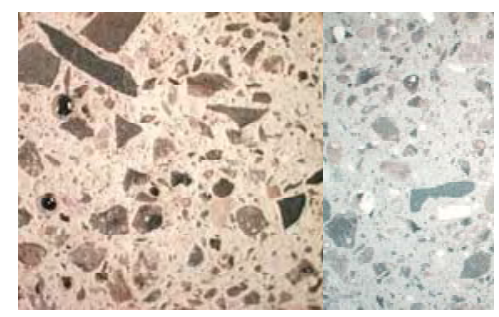

$550^{\circ} \mathrm{C}, 4$ hours

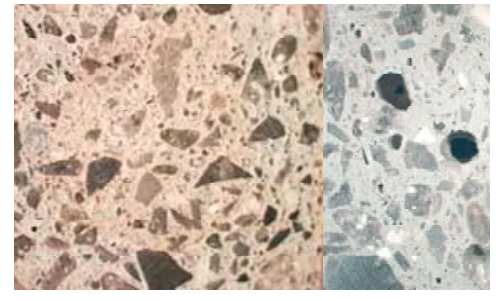

$750^{\circ} \mathrm{C}, 4$ hours

Figure 7.9. Micrographs of Thin Sections of CRB Samples Heat-Treated at Various Times and Temperatures Before (right image) and After (left image) Heat-Treatment in Baseline Feed

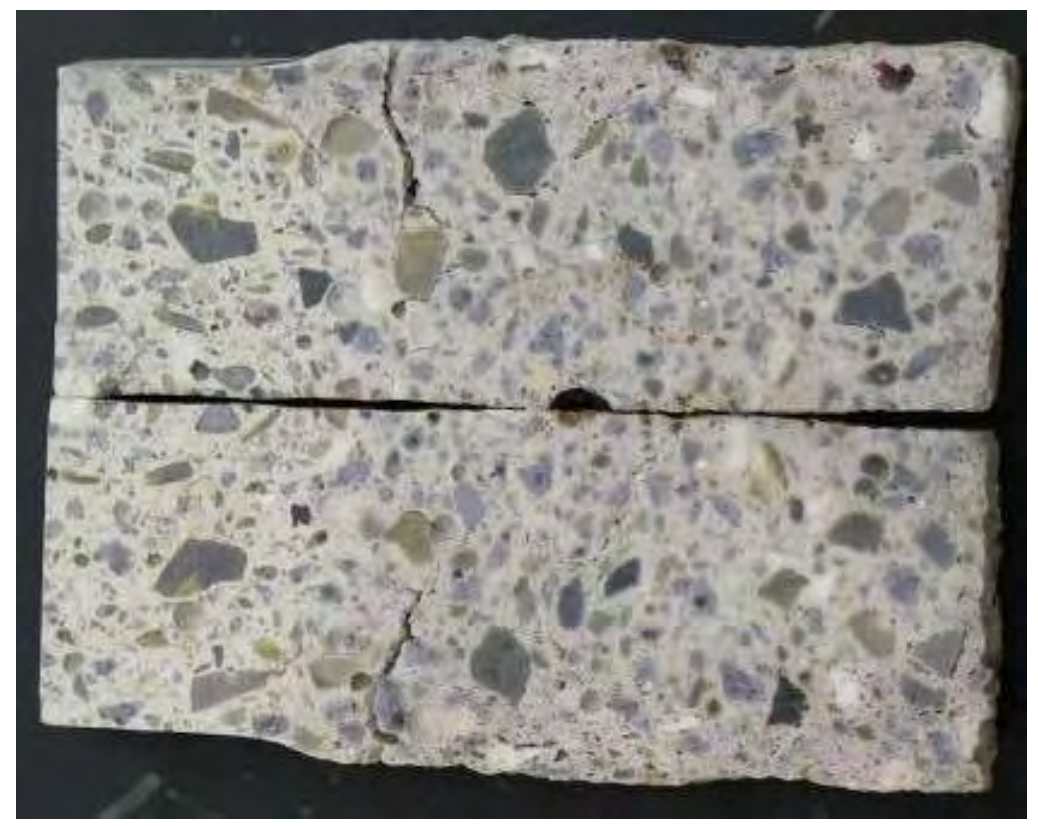

Figure 7.10. Cross-Section Through a CRB Rod Heat-Treated in Original (baseline) Feed at $550^{\circ} \mathrm{C}$ for $1 \mathrm{Hour}$ and then at $1200^{\circ} \mathrm{C}$ for $1 \mathrm{Hour}$ 


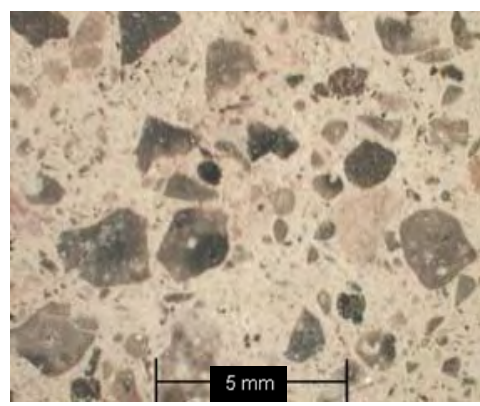

$550^{\circ} \mathrm{C}$ in feed

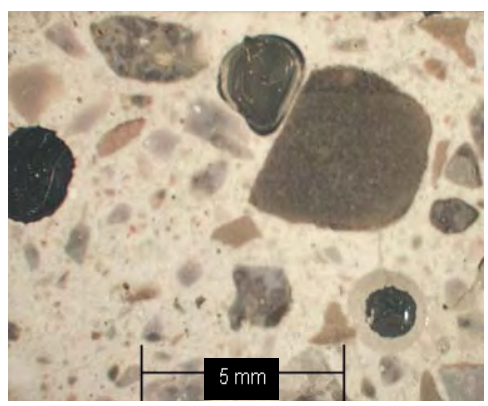

$1200^{\circ} \mathrm{C}$

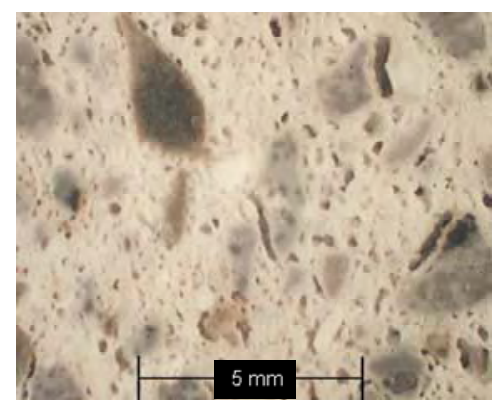

$1200^{\circ} \mathrm{C}$ in feed

Figure 7.11. Comparison of Microstructure of CRB Samples Heat-Treated With (left and right) and Without (center) the Presence of Feed

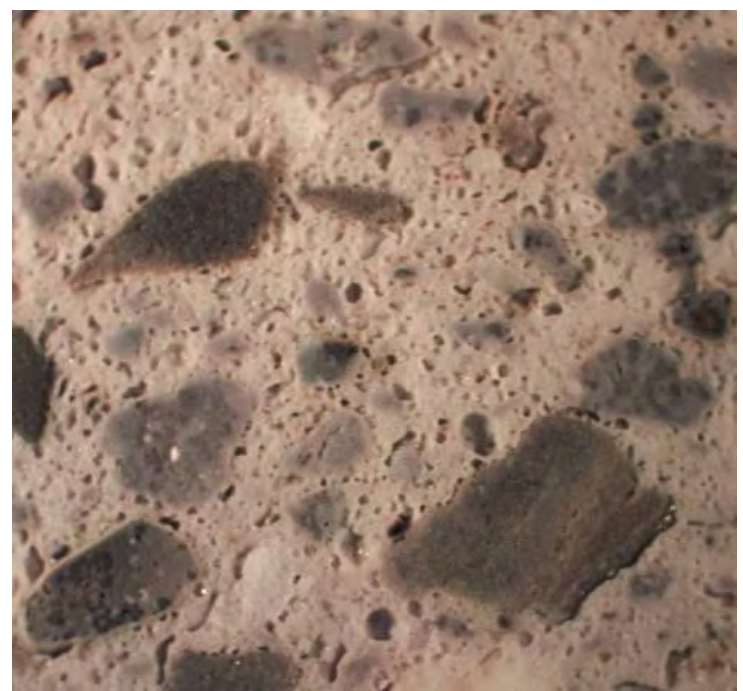

Figure 7.12. A Different Image of the Sample Shown in Figure 7.11 (heat-treated in feed to $550^{\circ} \mathrm{C}$ for 1 hour and then at $1200^{\circ} \mathrm{C}$ for 1 hour)

The rods were cut longitudinally in half and examined. Figure 7.10 shows the effect of heating in the original feed to $1200^{\circ} \mathrm{C}$. Note the crack at the level where the swollen part of the rod began. Because of wicking, a part of the rod above the feed level was also soaked with MIS. Also note that increased porosity was confined to the area close to the rod surface whereas the central portion was free of large pores.

For experiments with ground feed, ESF was ground in a tungsten carbide mill in 20-g batches for 2 minutes. To assess repeatability, three CRB rods were subjected to heating in the same arrangement and following the same procedure as for the experiment with the original (as-received) feed. After the second heat-treatment (for 1 hour at $1200^{\circ} \mathrm{C}$ ), the rods exposed to ground feed showed signs of MIS penetration only to a shallow depth from the surface $(<1 \mathrm{~mm})$. As seen in Figure 7.13, MIS penetrated to some depths at the bottom surface in the two rods and caused some swelling in the meniscus area in the third rod. 


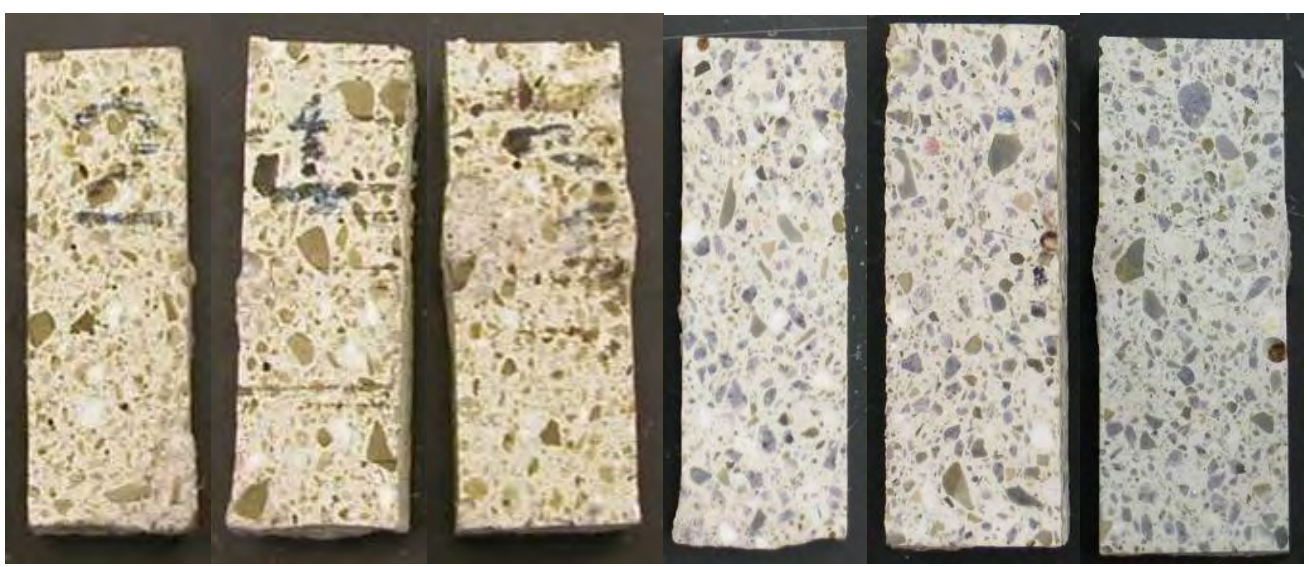

Figure 7.13. Three CRB Rods After Exposure to Ground Feed at $550^{\circ} \mathrm{C}$ and Subsequently Heated at $1200^{\circ} \mathrm{C}$; the Sections Are on the Right

The mass increase of the rod caused by MIS penetration was obtained for rods heat-treated at $1200^{\circ} \mathrm{C}$ and corrected for the LOI. This corrected value is called the net mass gain at $1200^{\circ} \mathrm{C}$. Appendix $\mathrm{H}$ shows the data tables and details of calculations.

The $1200^{\circ} \mathrm{C}$ net gain accounts for the solids from the MIS that penetrated into the CRB. It is plotted in Figure 7.14 as a function of the time exposure of CRB rods to the original and ground feeds at $550^{\circ} \mathrm{C}$.

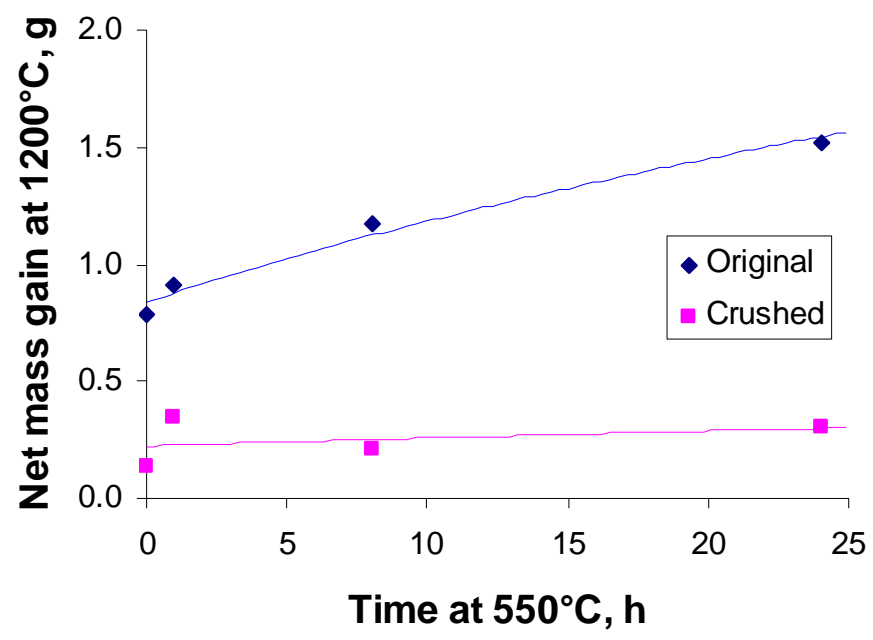

Figure 7.14. Effect of $550^{\circ} \mathrm{C}$ Heat-Treatment Time on MIS Penetration to CRB from Original and Ground Feed; the Lines Represent a Square-Root Fit

As Figure 7.14 shows, the total amount of MIS in the CRB decreased by an order of magnitude when the feed was ground. Its value no longer significantly increased with time, suggesting that only a small fraction of free liquid was present in the ground feed. Appendix $\mathrm{H}$ discusses the question of a mathematical description of MIS penetration. The mass losses from the feed were also measured and 
plotted as a function of time, showing that MIS is continuously decomposing as time progresses at $550^{\circ} \mathrm{C}$ - see Appendix $\mathrm{H}$ for the results.

Ground feed practically stopped MIS migration by preventing MIS from leaving the feed. As demonstrated above, experiments with ground feed showed that MIS migration was rendered negligible and did not progress with the time of exposure. Although grinding the soil can potentially solve the MIS penetration problem, it has disadvantages in the grinding cost and fine-powder manipulation. Therefore, experiments were undertaken to determine the minimum fraction of ground material and the minimum degree of grinding necessary to achieve the desired effect. The results are displayed in Figure 7.15 and Figure 7.16 for 1-hour heat-treatment time $\left(t_{H}\right)$ at $550^{\circ} \mathrm{C}$, while the details are described in Appendix $\mathrm{H}$.

Figure 7.15 indicates that 60 mass\% of ground feed was enough to substantially decrease MIS penetration into the CRB rod. The line in Figure 7.16 represents an exponential function. It appears that 3 minutes of grinding would virtually stop MIS penetration provided that extrapolation is permissible.

To select a large-scale grinding method, it is necessary to characterize the specific surface of solids in feed as well as other properties, such as the density, as functions of grinding parameters.

Figure 7.17 shows the impact of feed grinding on the feed bulk density. The increase in density was caused by widening the particle size distribution (PSD) as shown below. The consequence of higher feed density was that the void fraction decreased, and MIS had less room for flow.

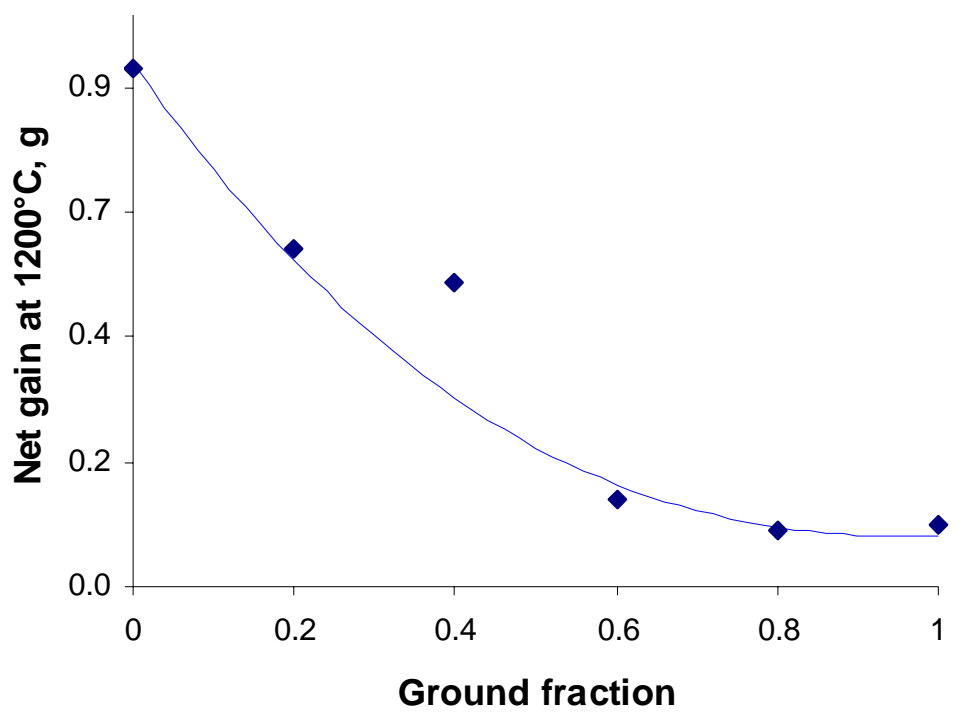

Figure 7.15. Effect of Ground Fraction on MIS Penetration to CRB ( $t_{\mathrm{H}}=1$ hour) 


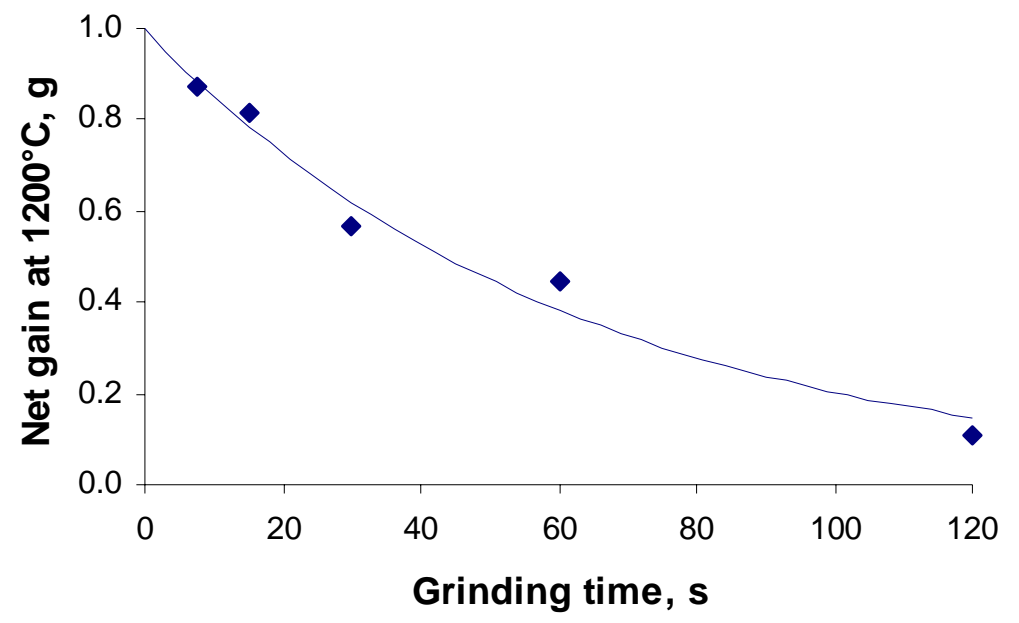

Figure 7.16. Effect of Grinding Time on MIS Penetration to CRB ( $t_{\mathrm{H}}=1$ hour)

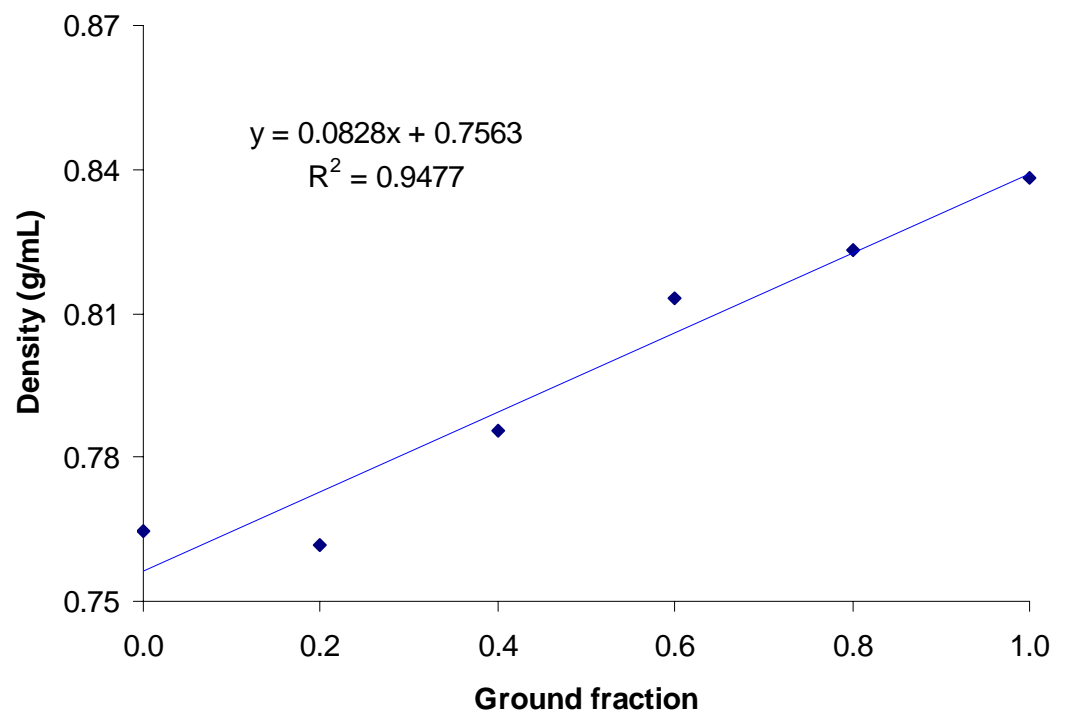

Figure 7.17. Feed Density as a Function of Ground Fraction

Whereas mixtures of the original and ground feed have bimodal PSDs, feeds ground to different degrees are more uniform. Tests were performed to determine the minimum necessary degree of grinding that would prevent MIS penetration into the CRB.

Figure 7.18 shows PSDs in original feed and feed ground for 2 minutes in the tungsten carbide mill. The median grain size was barely changed by grinding. Grinding removed large particles and created large fractions of small particles. Deagglomeration significantly shifted particle distribution towards small particle sizes, and grinding decreased the median grain size of deagglomerated powder. 


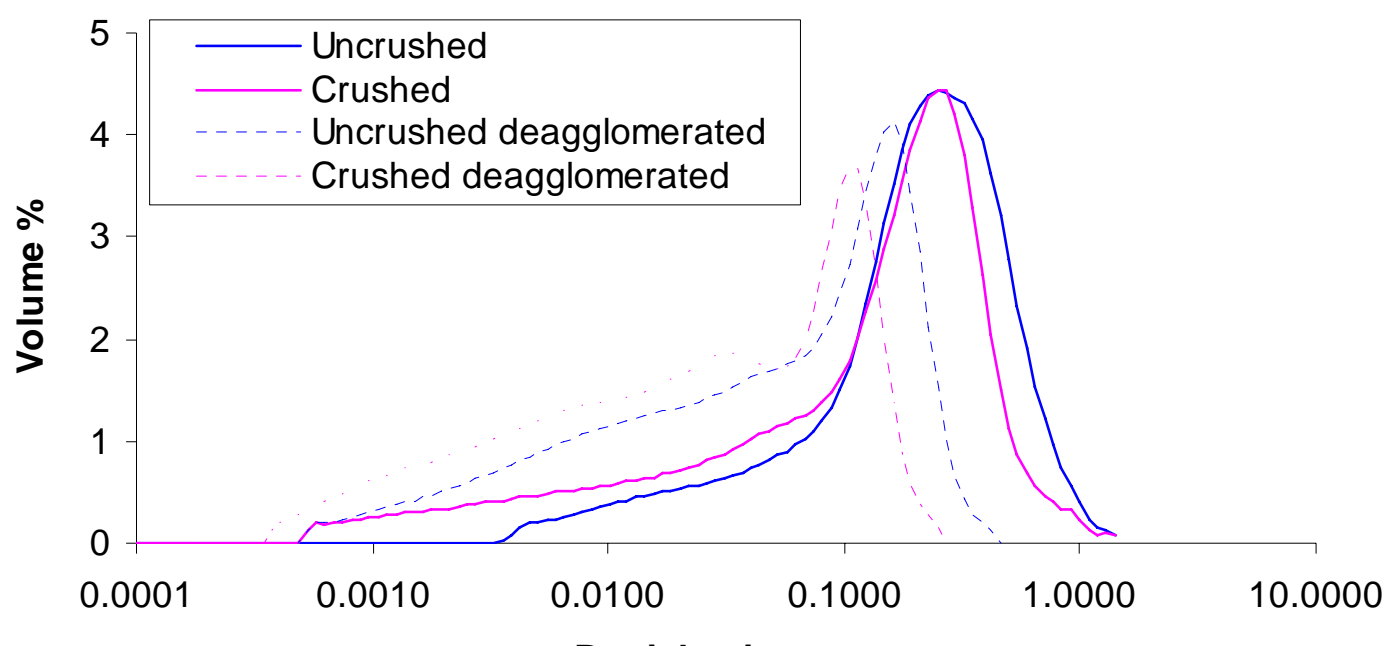

Particle size, $\mathrm{mm}$

Figure 7.18. Particle Size Distribution of Original and Ground (for 120 s) Feed, Untreated, and Deagglomerated

Figure 7.19 shows the relationship between the duration of grinding and the specific surface area estimated from the PSD. It is close to linear.

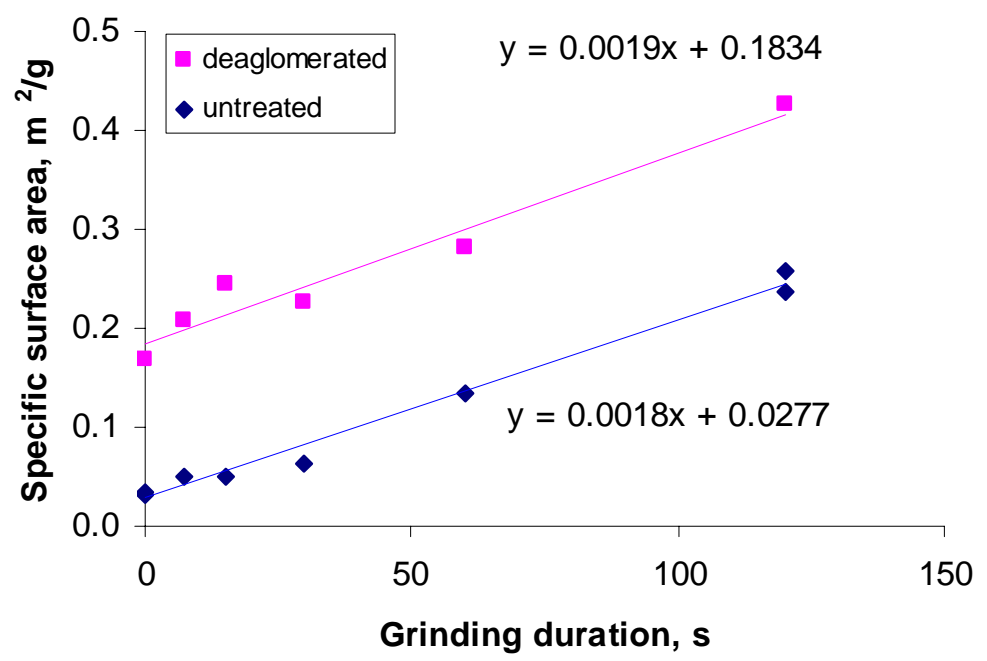

Figure 7.19. Particle Size Distribution of Original (as-received) and Ground (for 120 s) Feed, Untreated and Deagglomerated

Figure 7.20 shows the effect of the specific surface area on the amount of MIS in the vibrocast sample. The relationship appears to be linear, suggesting that MIS penetration will stop as the specific surface area reaches a critical size $\left(0.27 \mathrm{~m}^{2} / \mathrm{g}\right.$ or $0.45 \mathrm{~m}^{2} / \mathrm{g}$, whether the area is measured for untreated or deagglomerated feed). 


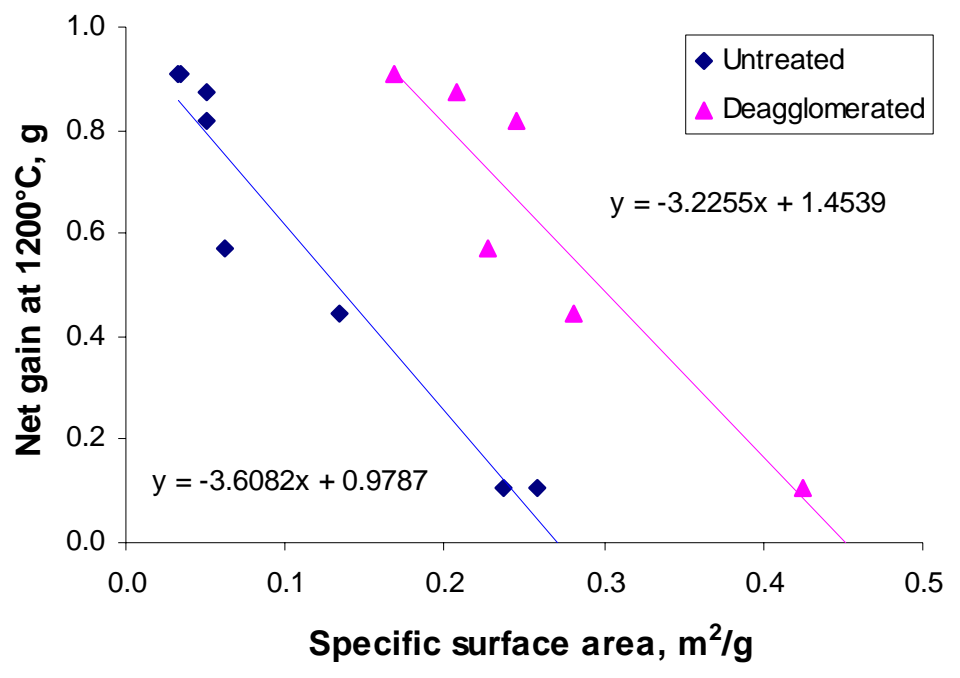

Figure 7.20. Effect of Specific Surface Area on MIS Penetration to CRB ( $t_{\mathrm{H}}=1$ hour)

\subsection{Conclusion}

Laboratory tests with six-tank composite LAW simulant have shown that grinding the feed to a finer particle size can decrease the movement of MIS within the feed, thus decreasing or preventing the transfer of MIS into the pores of the CRB. The solid particles in the feed physically retain the MIS by adsorbing the liquid onto the grain surfaces and reducing the total interfacial energy for all the phase boundaries present. The particles hold a portion of the MIS in place until it is converted, by batch reactions, to highviscosity glass-forming melt. As the specific surface area of feed solids increases, more MIS is retained in the feed until no MIS can drain or soak into the CRB pores by capillary forces. Hence, free MIS is no longer available for the competing capillary forces associated with the porous refractory material. 


\subsection{Study of MIS Penetration from Feeds Containing S-109 LAW Simulant}

The migration of MIS into porous refractories can be reduced by altering the refractory layer at the feedcontacting surface and by increasing the internal surface area of the feed. Research reported in Sections 6 and 7 showed that the following methods are potentially effective:

- making the inner layer of the refractory lining partially lyophobic by adding graphite powder into the vibrocast

- $\quad$ weakening the inner layer of the refractory lining by adding sodium silicate

- $\quad$ increasing the specific surface area of the feed solids by grinding.

The third of these methods appeared the most feasible for future MIS mitigation efforts. Potential waste tank candidates for the developmental bulk vitrification system (DBVS) were evaluated taking into consideration the Cs content and planned retrieval schedule. The best candidate for DBVS operations was Tank 241-S-109 (S-109). The waste in this tank was significantly different from the six-tank composite, so a new S-109 simulant was generated. A glass formulation that used the same soil and glass additives was developed and tested. S-109 simulant was tested in an earlier engineering-scale (ES) test (ES-31B), and there was no obvious difference in the processing of this simulant when compared to the six-tank composite feeds. The early FS work was conducted with feeds prepared with the six-tank composite simulant to tie back to the earlier Tc-spiked ES tests that also used six-tank composite feeds. However, because DBVS would be treating waste from S-109, a decision was made to shift to the S-109 simulant for the FS-38C test. Since six-tank simulant was used in all work described in previous sections of this report, additional tests were designed to obtain data on the impact of soil grinding on S-109 feed.

As Table 8.1 shows, the compositions of S-109 and six-tank simulant are different. The main difference between six-tank and S-109 compositions is that S-109 has a higher content of nitrates and nitrites and a lower content of organic components. The mass fraction of $\mathrm{KNO}_{3}+\mathrm{NaNO}_{2}+\mathrm{NaNO}_{3}$ is 0.850 in S-109 simulant and only 0.597 in six-tank simulant. The mass fraction of $\mathrm{NaCH}_{3} \mathrm{COO}+\mathrm{Na}_{2} \mathrm{C}_{2} \mathrm{O}_{4}$ is only 0.004 in S-109 simulant and 0.033 in six-tank simulant. Note that the six-tank simulant contains 13 mass\% of

$\mathrm{Na}_{2} \mathrm{CO}_{3}$, whereas S-109 simulant has $5 \%$. Sodium carbonate can substantially increase the liquidus temperature of MIS, thus decreasing MIS mobility at lower temperatures where little MIS decomposes. The $\mathrm{NaOH}$ content is also higher in six-tank simulant (7.8 mass\% compared to 1.9 mass\% in S-109). The reactivity of $\mathrm{NaOH}$ is high, so it tends to bond to the feed solids before it migrates into the refractory.

Table 8.2 shows the mass fractions of components entering the S-109-based glass from the feed constituents. Based on this information and the LOI data, a batch sheet was developed as shown in Table 8.3.

The LOI values were obtained by heating at $1050^{\circ} \mathrm{C}$ for 1 hour; $\mathrm{ZrO}_{2}$ and $\mathrm{B}_{2} \mathrm{O}_{3}$ were heated without drying, whereas the soil was dried before heating. Thus, the LOI for $\mathrm{ZrO}_{2}$ and $\mathrm{B}_{2} \mathrm{O}_{3}$ includes the loss on drying and was batched as-received, whereas the soil was batched after drying. Fine zirconia was used to keep the conditions the same as those used in the laboratory tests with six-tank composite simulant. 
Table 8.1. Comparison of S-109 and Six-Tank LAW Simulants (in mass fractions of components)

\begin{tabular}{||l|c|c||}
\hline & S-109 & Six-Tank \\
\hline $\mathrm{Al}\left(\mathrm{NO}_{3}\right)_{3} \cdot 9 \mathrm{H}_{2} \mathrm{O}$ & & 0.0629 \\
\hline $\mathrm{Al}(\mathrm{OH})_{3}$ & 0.0082 & - \\
\hline $\mathrm{Ca}(\mathrm{OH})_{2}$ & 0.0002 & - \\
\hline $\mathrm{Cr}_{2} \mathrm{O}_{3}$ & 0.0015 & - \\
\hline $\mathrm{Fe}_{2} \mathrm{O}_{3}$ & 0.0009 & - \\
\hline $\mathrm{KNO}_{3}$ & 0.0010 & 0.0033 \\
\hline $\mathrm{Na}_{2} \mathrm{C}_{2} \mathrm{O}_{4}$ & 0.0035 & 0.0042 \\
\hline $\mathrm{Na}_{2} \mathrm{CO}_{3}$ & 0.0502 & 0.1325 \\
\hline $\mathrm{Na}_{2} \mathrm{CrO}_{4}$ & 0.0022 & 0.0044 \\
\hline $\mathrm{Na}_{2} \mathrm{SO}_{4}$ & 0.0125 & 0.0336 \\
\hline $\mathrm{Na}_{3} \mathrm{PO}_{4} \cdot 12 \mathrm{H}_{2} \mathrm{O}$ & 0.0499 & 0.0492 \\
\hline $\mathrm{NaCH}_{3} \mathrm{COO}$ & 0.0003 & 0.0284 \\
\hline $\mathrm{NaCl}$ & 0.0010 & 0.0067 \\
\hline $\mathrm{NaF}$ & 0.0005 & 0.0035 \\
\hline $\mathrm{NaNO}$ & 0.0102 & 0.0770 \\
\hline $\mathrm{NaNO}$ & 0.8388 & 0.5163 \\
\hline $\mathrm{NaOH}$ & 0.0191 & 0.0779 \\
\hline \hline
\end{tabular}

Table 8.2. Mass Fractions of Glass Components from Feed Ingredients

\begin{tabular}{||r|r||}
\hline S-109 Simulant & 0.1928 \\
\hline HRTS05 Soil & 0.6872 \\
\hline $\mathrm{ZrO}_{2}$ & 0.0700 \\
\hline $\mathrm{B}_{2} \mathrm{O}_{3}$ & 0.0500 \\
\hline
\end{tabular}

Table 8.3. Batch Sheets for $800 \mathrm{~g}$ of Glass

\begin{tabular}{|r|c|c|l||}
\hline Materials & LOI & Recipe & \\
\hline Liquid S-109 Simulant & 0.6657 & 921.8 & $\mathrm{~mL}$ \\
\hline HRTS05 Soil & 0.0211 & 561.6 & $\mathrm{~g}$ \\
\hline $\mathrm{ZrO}_{2}$ & 0.0000 & 56.00 & $\mathrm{~g}$ \\
\hline $\mathrm{B}_{2} \mathrm{O}_{3}$ & 0.0464 & 41.95 & $\mathrm{~g}$ \\
\hline
\end{tabular}

The mass of $921.8 \mathrm{~mL}$ of the simulant solution is $1161.5 \mathrm{~g}$ (the simulant density is $1.260 \mathrm{~g} / \mathrm{mL}$ ), and its dry mass is $388.3 \mathrm{~g}$. Hence, the total mass of wet feed to make $800 \mathrm{~g}$ of glass is $1821.1 \mathrm{~g}$; the mass after drying is $1047.9 \mathrm{~g}$.

Three batches of feed were prepared, varying only the particle size of the HRTS soil. As-received soil, soil ground for 2 minutes, and soil ground for 5 minutes were used (see Section 7.3 for feed preparation description). Figure 8.1 displays the PSDs. Grinding the soil for 2 minutes decreased the sizes of nearly all particles. Further grinding to 5 minutes had a relatively small impact. Table 8.4 lists the specific surface areas measured by various methods. The Brunauer, Emmett, and Teller (BET) and Langmuir 
methods provide the same relative comparisons of samples. A reasonable relative comparison is achieved also with areas calculated from the PSD. The large intercept seen in Figure 8.2 can be attributed to the nonsphericity of the soil particles and their porosity. Appendix I presents more details.

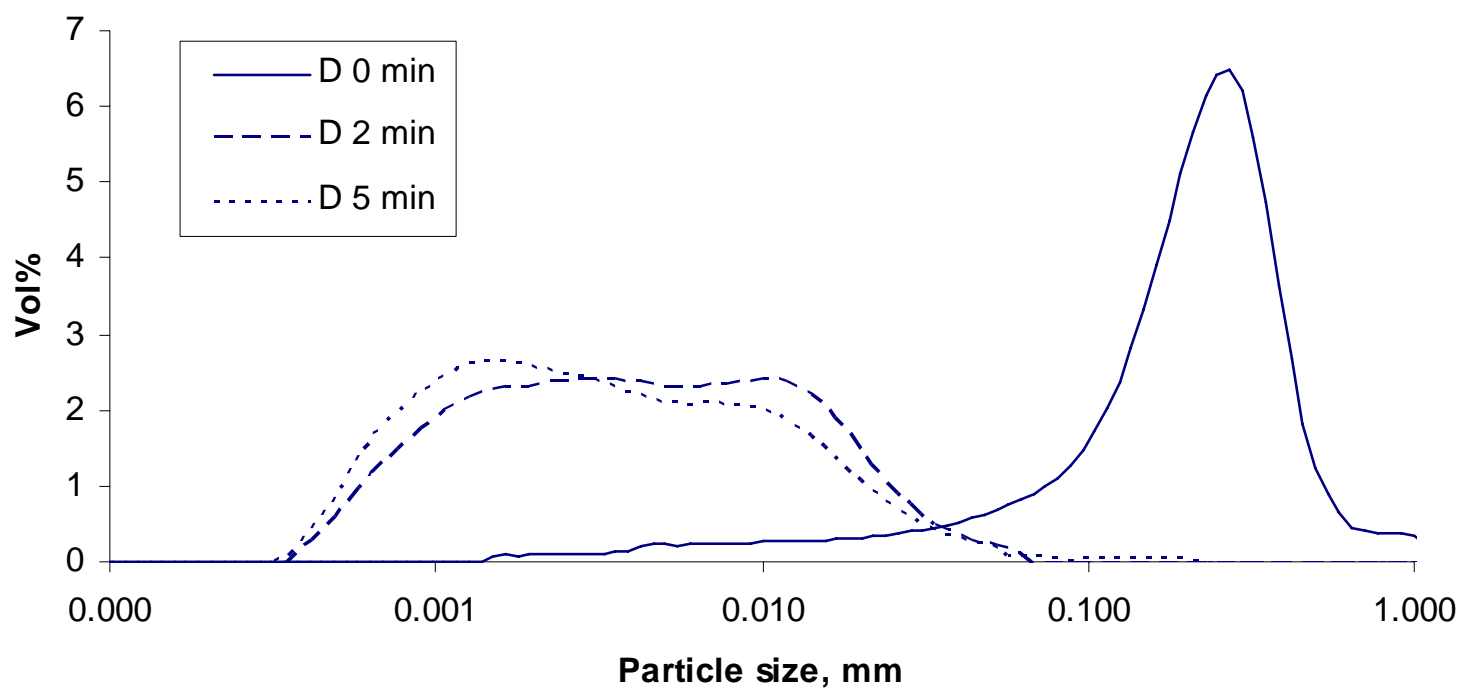

Figure 8.1. Comparison of PSD of Hanford Soil As-Received and Ground for 2 and 5 Minutes; Samples were Deagglomerated

Table 8.4. Specific Surface Area Measured with BET and Langmuir Methods and Calculated from Deagglomerated PSD for As-Received and Ground Soils

\begin{tabular}{||c|r|c|c||}
\hline \hline \multirow{2}{*}{$t$, min } & \multicolumn{3}{|c|}{$a, \mathbf{~ m}^{2} / \mathbf{g}$} \\
\cline { 2 - 4 } & BET & Langmuir & PSD \\
\hline 0 & 5.74 & 9.67 & 0.040 \\
\hline 2 & 9.75 & 16.59 & 1.047 \\
\hline 5 & 10.78 & 18.42 & 1.248 \\
\hline
\end{tabular}

Note the difference of the impact of grinding the feed (Figure 7.18) and soil (Figure 8.1). When feed was crushed, the salt particles in the feed may have cushioned the soil particles. The instrument used a watersuspended sample in which the salt was dissolved and did not influence the soil PSD. The PSDcalculated surface area of soil particles was only $0.4 \mathrm{~m}^{2} / \mathrm{g}$ in 2-min ground feed (Figure 7.19), whereas 2-min crushing of soil alone resulted in the PSD-calculated surface area of $1.0 \mathrm{~m}^{2} / \mathrm{g}$ (Table 8.4 ). However, grinding the soil and LAW salts together (in the form of the dried feed) improves the contact between soil particles and the MIS. This better contact compensates of the lower interface are as compared with soil-only grinding. 


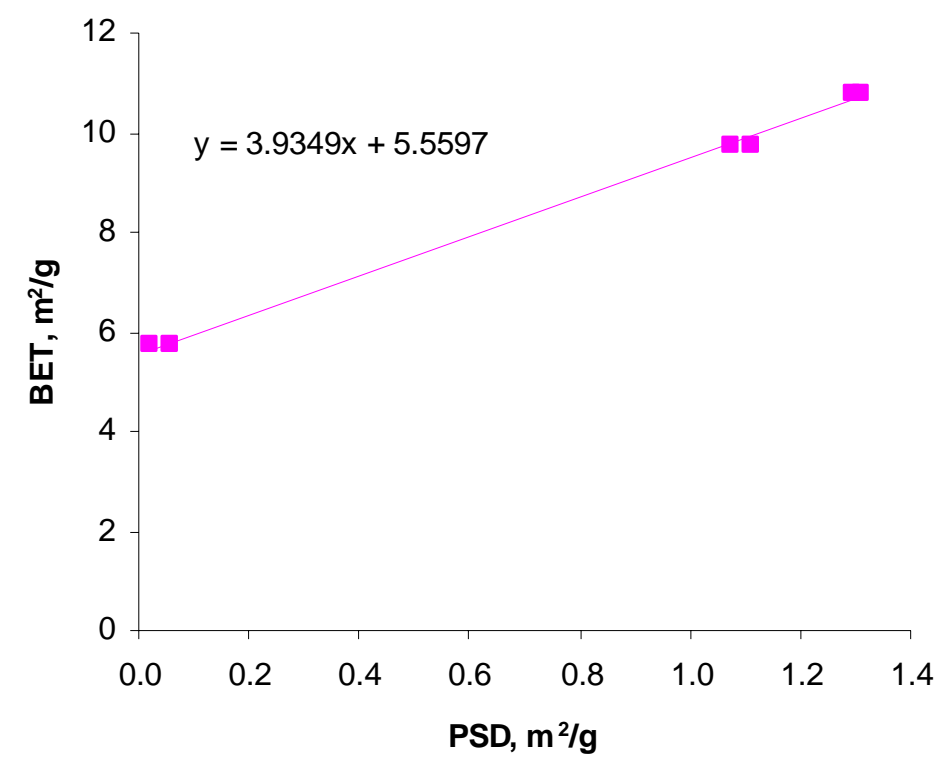

Figure 8.2. Comparison of Specific Surface Area of Hanford Soil Measured with BET Method with the Area Calculated from the Deagglomerated PSD

Medium silica crucible tests (see Section 7.1) were used to measure the extent of MIS penetration from S109 feeds as a function of the extent of grinding. The LOI values for silica crucibles were measured by heating at $5^{\circ} \mathrm{C} / \mathrm{min}$ to the set temperature of 500 and $1000^{\circ} \mathrm{C}$ and held for 1 hour. Table 8.5 lists the LOI results. Note that the LOI of silica crucibles is small, but needs to be considered because the mass loss of crucibles is comparable to the mass of MIS soaked into the crucible in some experiments.

All assemblies, crucibles with feed and CRB rods inserted in Pt crucibles with feed were heated at $5^{\circ} \mathrm{C} / \mathrm{min}$ to set temperatures and held for 30 minutes. Both as-received and ground soil were used. The soil was ground in 20-g batches in the tungsten carbide mill. The mass of the silica crucibles was measured before the test and after the first heating. The feed was removed from silica crucibles after the first heating, and the empty crucibles were subjected to a second heating at $1000^{\circ} \mathrm{C}$ for 1 hour. The crucibles were also weighed after the second heating. Crucibles were sectioned for examination. Appendix J presents detailed photographic documentation of the crucible appearance.

Table 8.5. LOI of Silica Crucibles (in mass fractions)

\begin{tabular}{||c|c|}
\hline \hline Temperature, ${ }^{\circ} \mathbf{C}$ & Silica Crucible \\
\hline 500 & 0.0018 \\
\hline 1000 & 0.0021 \\
\hline
\end{tabular}

The feed with as-received soil penetrated to the outer wall of the crucible heated to $500^{\circ} \mathrm{C}$. As Figure 8.3 shows, the net mass gain (the mass of fully decomposed MIS corrected for crucible LOI) was very low at $300^{\circ} \mathrm{C}$ and increased with increasing temperature with a decreasing rate (the line drawn through the data points are second-order polynomials). The amount of penetrated MIS from feed with 2-min ground soil was about $73 \%$ less than the MIS penetration for feeds prepared with as-received soil at both $400^{\circ} \mathrm{C}$ and $500^{\circ} \mathrm{C}$. 
Table 8.6 lists the fractional mass loss from feeds as a function of temperature. Figure 8.4 shows that the ground soil reacts faster at lower temperatures as seen by the higher LOI at $300^{\circ} \mathrm{C}$. However, the amount of MIS mass loss (decomposition) at $400^{\circ} \mathrm{C}$ and $500^{\circ} \mathrm{C}$ was smaller in the feed with 2-min ground soil than in the feed with as-received soil. This result is similar to that obtained for feed with the six-tank composite. The reason for the smaller amount of MIS decomposition at higher temperature in the ground soil case is not fully understood but could be a result of the MIS being held in the soil at the center of the crucible where it is not exposed to the higher temperatures that the MIS sees when it penetrates the crucible wall in the as-received soil case.

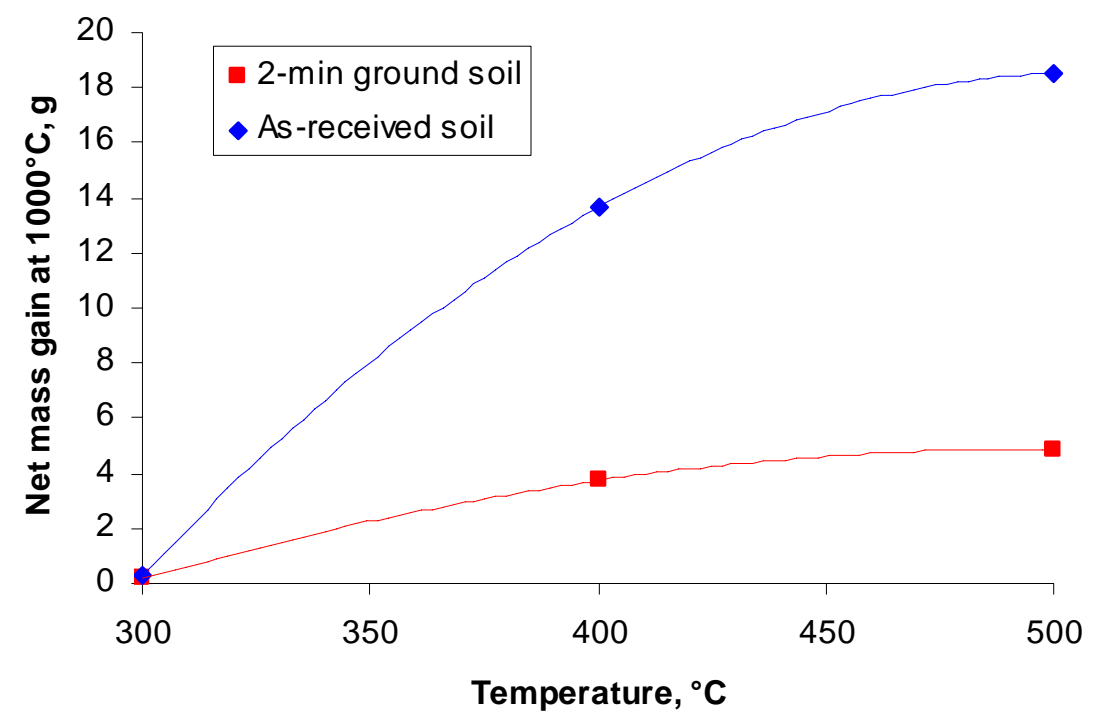

Figure 8.3. Effect of Temperature on MIS Penetration to Silica Crucible from Feed with S-109 Simulant

Table 8.6. Feed Mass Loss (in mass fractions) as a Function of Temperature

\begin{tabular}{||c|c|c||}
\hline Temperature & As-Received & 2-min Ground \\
\hline $300^{\circ} \mathrm{C}$ & 0.048 & 0.079 \\
\hline $400^{\circ} \mathrm{C}$ & 0.159 & 0.144 \\
\hline $500^{\circ} \mathrm{C}$ & 0.239 & 0.170 \\
\hline
\end{tabular}




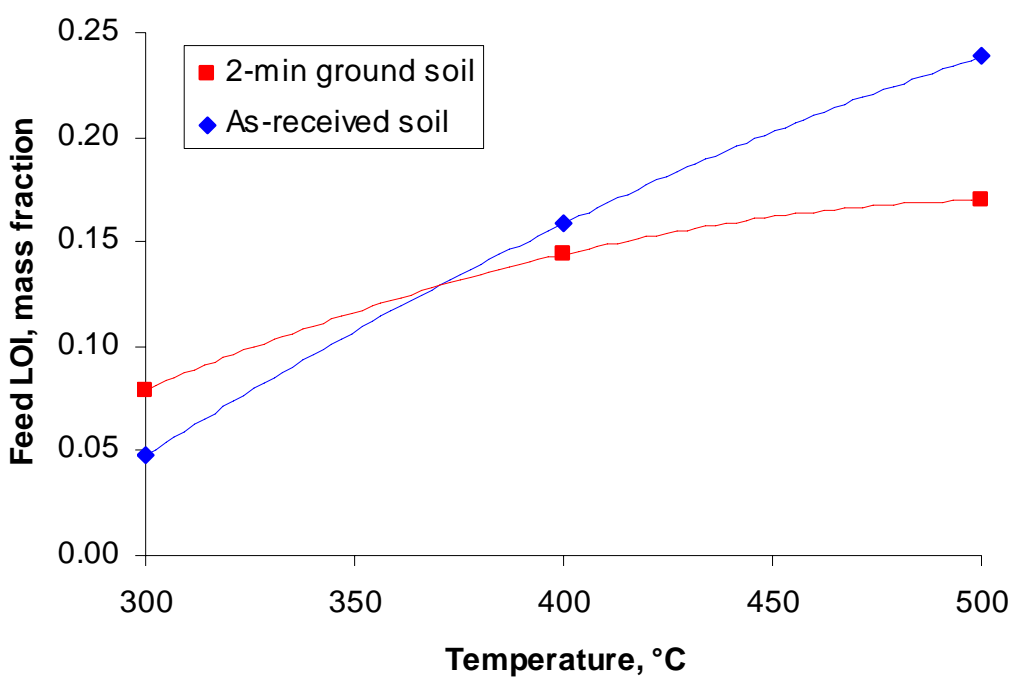

Figure 8.4. Mass Loss of Feed (with S-109 simulant and as-received or ground soil) Versus Temperature

Appendix $\mathrm{K}$ describes and analyzes in detail the experiments performed to determine the impact of grinding, additions of fine-grain feed components, and additions of carbohydrates on MIS migration to silica crucibles. It shows selected batch sheets, measured data (initial masses of feeds and crucibles, masses of feeds and crucibles after heat-treatment at $500^{\circ} \mathrm{C}$, and masses of crucibles after heat-treatment at $1000^{\circ} \mathrm{C}$ ), and photographic images of vertical sections through crucibles.

To understand the effect of feed alterations on MIS migrations, several processes need to be considered:

- LAW simulant forms MIS gradually, starting from low-temperature eutectics. Therefore, MIS composition changes until all LAW simulant becomes liquid.

- The organic and inorganic components in LAW simulant undergo oxidation-reduction reactions even in a solid state and continue as MIS is being formed.

- MIS reacts with feed components as it migrates through feed. It also reacts with crucible materials as it penetrates into crucible pores.

To obtain a better insight into the MIS migration mechanism, mass-balance calculations were performed, as described in Appendix K, for the simplified case where the MIS composition is assumed to remain constant as it migrates.

The mass balance has three items: 1) the amount of initial MIS that migrated to the crucible, 2) the mass of MIS lost as gas $\left(\mathrm{H}_{2} \mathrm{O}, \mathrm{NO}_{\mathrm{x}}, \mathrm{CO}_{\mathrm{x}}\right)$ because of feed reactions at temperatures below $500^{\circ} \mathrm{C}$, and 3$)$ the mass of initial MIS that remained in the feed, either as inorganic salt or in the form of glass-components (metal oxides, $\mathrm{SO}_{3}, \mathrm{Cl}, \mathrm{F}$ ). Results of calculations are displayed in Figure 8.5 through Figure 8.9 for several experiments.

Figure 8.5 shows that adding diatomaceous earth slightly decreased MIS migration into the silica crucible and slowed feed-conversion reactions. Diatomaceous earth probably held MIS inside the fractal-like silica particles that reacted with MIS at a slower rate than the soil. Grinding diatomaceous earth with the 
soil had no beneficial effect on MIS retention. A bentonite addition had no effect on MIS migration. Bentonite was probably present in the feed in the form of agglomerates that were not wetted by MIS, thus increasing the MIS fraction in soil and promoting its mobility.
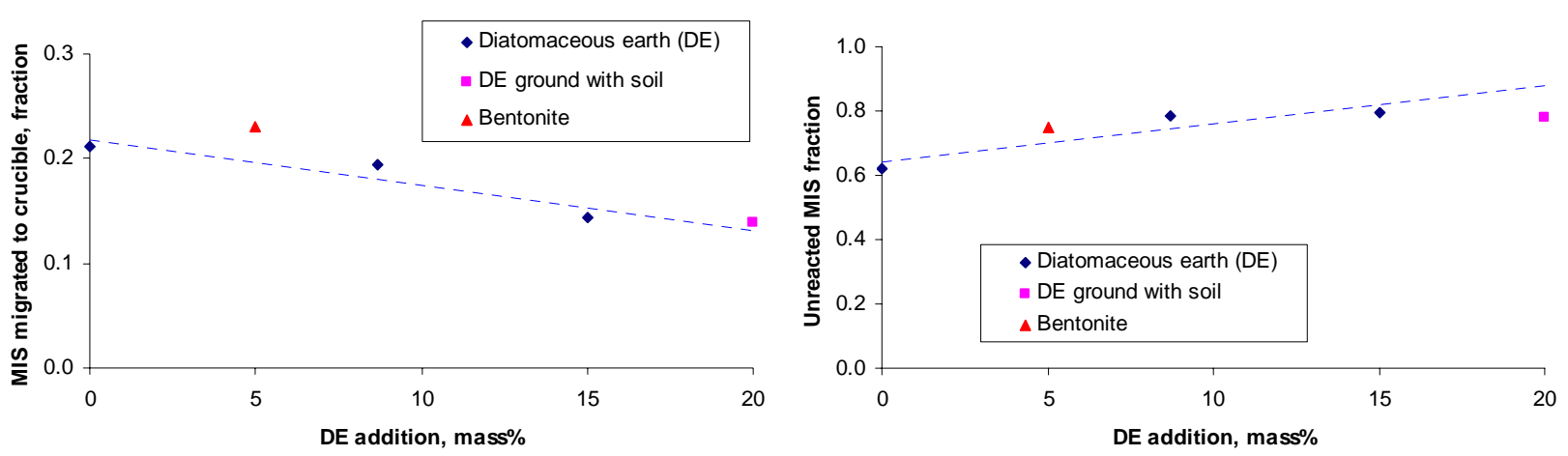

Figure 8.5. $\quad$ Effect of Additions of Diatomaceous Earth (DE) and Bentonite Addition on MIS Migration into Silica Crucible from Feed with 2-Min Ground Soil (left) and on Unreacted MIS Fraction in Feed at $500^{\circ} \mathrm{C}$ (right)

Figure 8.6 shows that adding sucrose decreased MIS migration and slightly decreased the extent of feed reactions in feeds with ground soil. In the feeds with as-received soil, the loss of MIS to the crucible from feed without sucrose addition was very high. Sucrose addition reduced this loss by nearly $80 \%$.
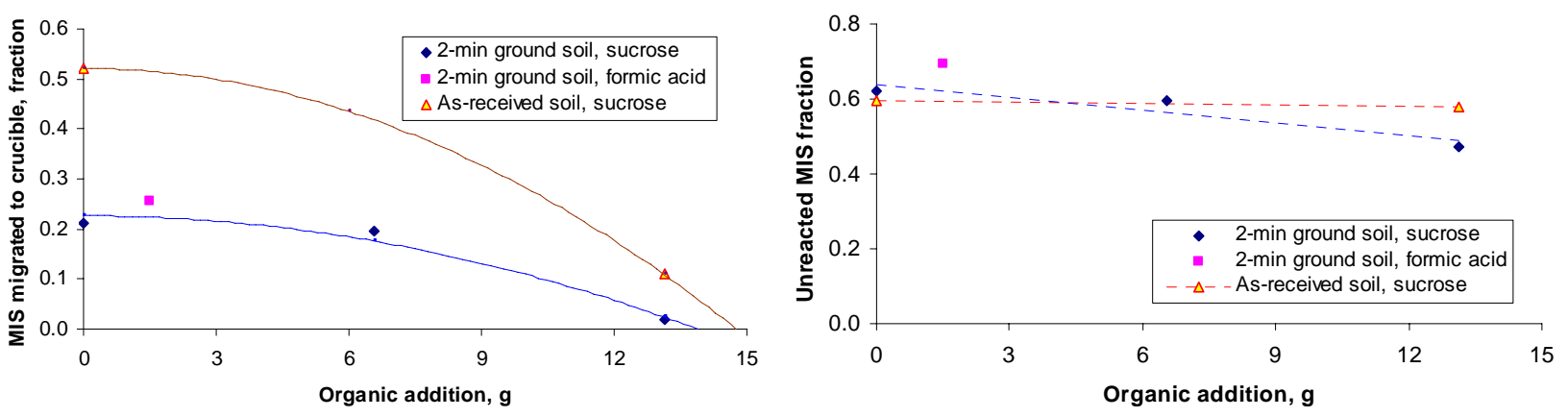

Figure 8.6. Effect of Organic Addition on MIS Migration into Silica Crucible from Feed with 2-Min Ground Soil (left) and on Unreacted MIS Fraction in Feed at $500^{\circ} \mathrm{C}$ (right)

In Figure 8.7, the percentage of sucrose (stoichiometric\%) is defined as the atomic ratio of carbon from sucrose to nitrogen in MIS multiplied by 100. Figure 8.7 shows the three items mentioned above (the fraction of MIS that migrated to the crucible, the fraction of MIS lost as gas, and the fraction of MIS that remained in the feed). Figure 8.8 shows the degree of decomposition of the MIS in feed (i.e., the MIS that did not enter crucible walls). Figure 8.9 shows the extent of decomposition of the MIS that penetrated into the crucible at $500^{\circ} \mathrm{C}$. The "complete reaction" bar represents the theoretical fractions of gas and glass components in MIS resulting from complete feed-to-glass conversion (40 mass\% is the expected fraction of MIS material that remains after all the $\mathrm{NO}_{\mathrm{x}}, \mathrm{CO}_{\mathrm{x}}$, and $\mathrm{H}_{2} \mathrm{O}$ gases escape). 


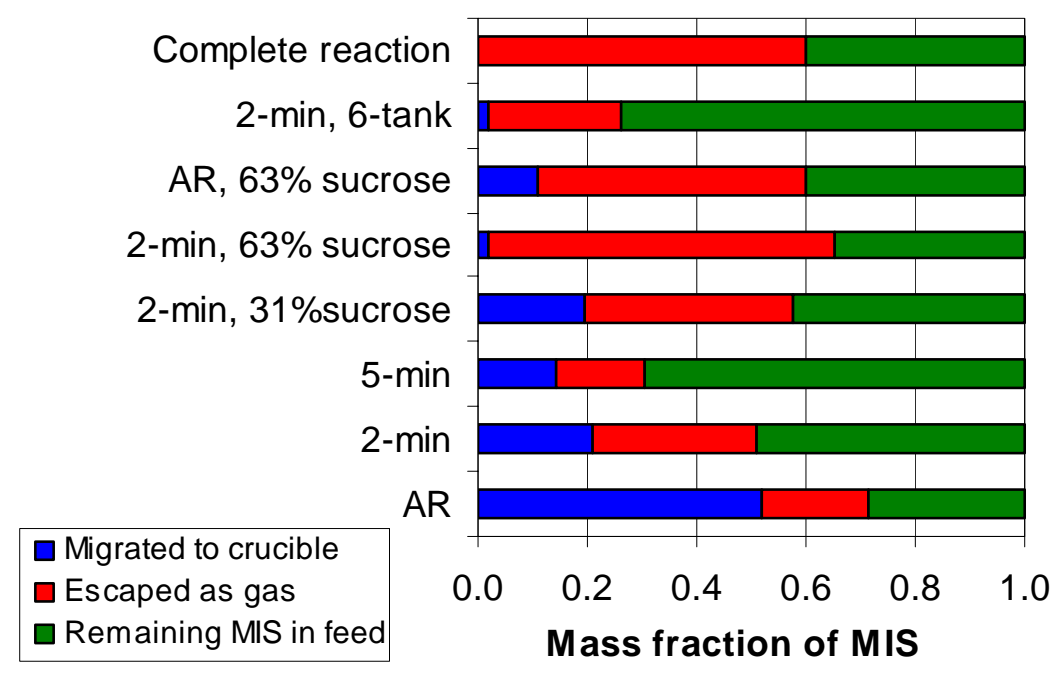

\section{Figure 8.7. Mass Balances for MIS at $500^{\circ} \mathrm{C}$ in Various Feeds Containing As-Received Soil (AR) and Ground Soil (for 2 and 5 min)}

Figure 8.7 shows that grinding decreased MIS penetration into crucible walls. Note that 31 stoichiometric\% addition of sucrose to the 2-min-ground feed had little effect on MIS penetration, whereas 63 stoichiometric\% addition had a dramatic effect. Adding sucrose decreased the fraction of unreacted MIS in the feed (Figure 8.8). The fraction of MIS that remained in the feed had not fully decomposed at $500^{\circ} \mathrm{C}$ in any feed except in the 2-min ground feed containing 63 stoichiometric\% of sucrose. The higher-than-theoretical loss may be attributed to experimental error or to partial segregation of MIS components during migration.

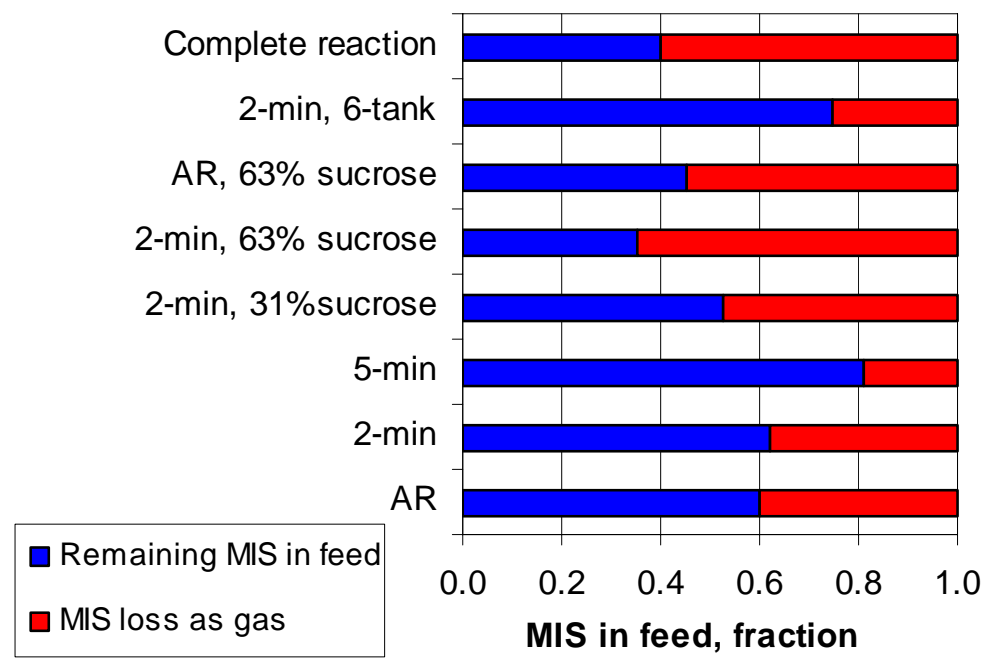

Figure 8.8. Extent of MIS Decomposition in Feed Heated to $500^{\circ} \mathrm{C}$ 


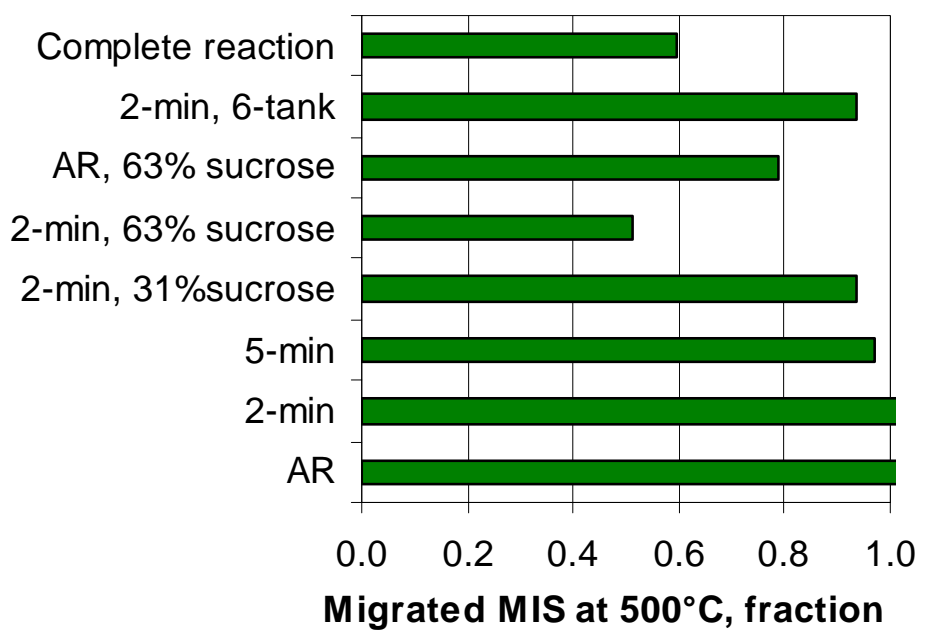

\section{Figure 8.9. MIS Present in Crucible Walls at $500^{\circ} \mathrm{C}$, Fraction of Total Estimated from $1000^{\circ} \mathrm{C}$ Data}

If the MIS does not decompose at temperatures of $500^{\circ} \mathrm{C}$ and penetrates into the crucible wall, the mass remaining in the wall after heating to $1000^{\circ} \mathrm{C}$ can be used to estimate the MIS quantity at $500^{\circ} \mathrm{C}$ by assuming that the mass present after heating to $1000^{\circ} \mathrm{C}$ is $40 \%$ of that present at $500^{\circ} \mathrm{C}$. If the measured mass of MIS at $500^{\circ} \mathrm{C}$ is less than the calculated mass using the $1000^{\circ} \mathrm{C}$ data, the MIS that migrated into the crucible must have partially decomposed before migrating. The fraction of total MIS in the crucible at $500^{\circ} \mathrm{C}$ can be expressed with the formula

$$
f_{500}=\frac{m_{500}}{m_{1000}} f_{1000}
$$

where $m_{500}$ and $m_{1000}$ are MIS mass inside crucible walls at $500^{\circ} \mathrm{C}$ and $1000^{\circ} \mathrm{C}$, respectively, and $f_{1000}$ is the fraction of glass solids in MIS that penetrated into the crucible walls. The plot in Figure 8.9 is based on measured values of $m_{500}$ and $m_{1000}$ (LOI corrected) and the assumption that $f_{1000}=f_{\text {theor }}$, where $f_{\text {theor }}=0.4023$. As seen in Figure 8.9, MIS had partially decompose before migrating to the crucible walls when feed was prepared from as-received or 2-min ground soil without carbohydrate additions (the calculated value of $f_{500}$ is 2 to $3 \%$ higher than the theoretical value).

In the case of sucrose addition, the MIS that migrated to the crucible walls contained smaller fractions of volatile gases than the LAW simulant and thus left a higher-than-theoretical solid residue $\left(f_{1000}>f_{\text {theor }}\right)$. Hence, the actual "fraction of total" (at $500^{\circ} \mathrm{C}$ ) is higher for feeds with sucrose addition than that displayed in Figure 8.9. We could assume that $f_{500}=1$ (this is a reasonable assumption, considering that $f_{500}=1$ for feeds with no sucrose addition) and calculate $f_{1000}=m_{1000} / m_{500}$. For the feed with 63 stoichiometric\% of sucrose and as-received soil, this ratio yields $f_{1000}=0.51$; for the feed with 63 stoichiometric\% of sucrose and 2-min ground soil, $f_{1000}=0.78$. Note that the amounts of MIS that migrated to the crucible walls are small for these two feeds (see Figure 8.7). 
Additional experiments were done with a special focus on feeds containing sucrose and ground soil, including soil ground by the Praxair Company, Seattle (see Table 8.7). Appendix L provides measured data for various feeds and the results of data analyses.

Table 8.7. Specific Surface Area of Soil Measured by the BET Method

\begin{tabular}{||l|r||}
\hline & $\mathbf{m}^{2} / \mathbf{g}$ \\
\hline As-received & 5.74 \\
\hline Plate ground and milled by Praxair & 8.00 \\
\hline Milled by Praxair & 8.80 \\
\hline Tungsten-carbide milled for 2 min & 9.75 \\
\hline Tungsten-carbide milled for 5 min & 10.78 \\
\hline
\end{tabular}

A linear relationship is likely to exist also between MIS migration and sucrose addition as seen in Figure 8.10. The relationship between MIS migration and the soil surface area is also linear (see Figure 8.11). This implies that the MIS retention is directly proportional to the internal surface of the solids in feed.

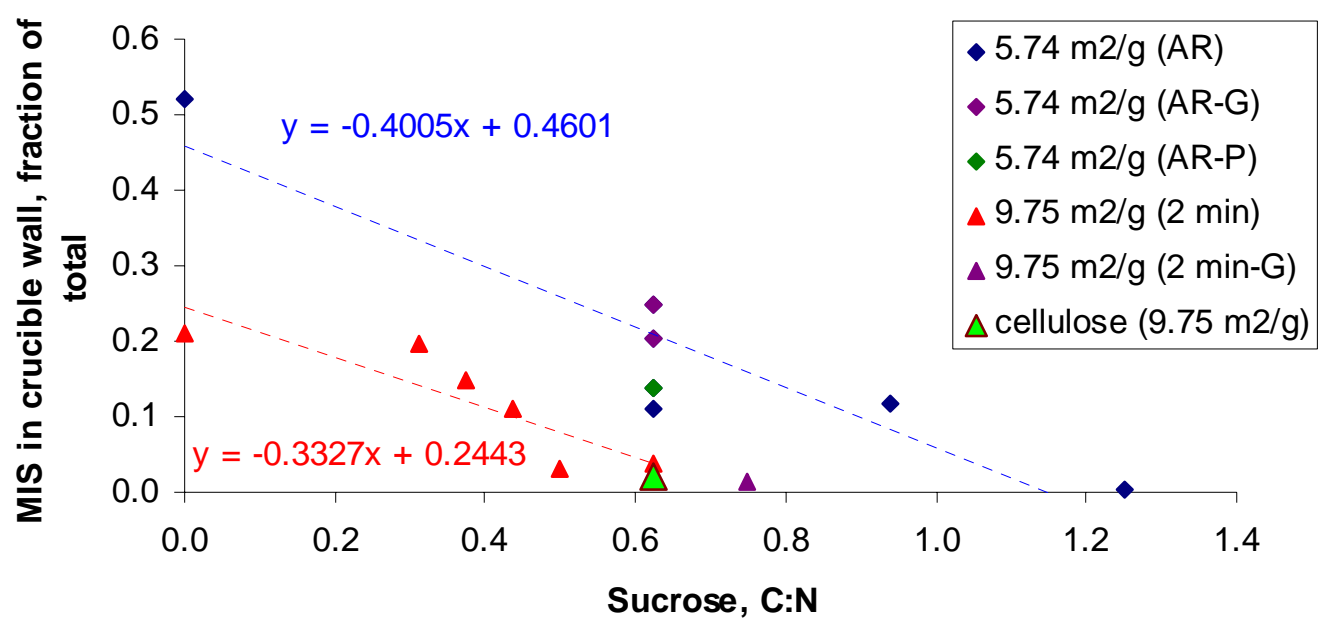

Figure 8.10. Effect of Sucrose Addition (in stoichiometric \%) on the Fraction of MIS Lost from Feed with Soil As-Received (5.74 $\mathrm{m}^{2} / \mathrm{g}$ specific surface area) and Ground $\left(9.75 \mathrm{~m}^{2} / \mathrm{g}\right.$ specific surface area) to Crucible Walls (G granular sucrose added to dry feed, $P$ powdered sucrose, $A R$ as-received soil)

The fraction of MIS that migrated outside the feed is a linear function of the soil specific surface area for a constant sucrose addition and appears to be close to linear as a function of the sucrose addition for a constant soil surface area. This suggests that linear regression can be applied to all available data.

Linear regression applied to data allows a region of the feed-modifying variables where MIS does not migrate outside the feed. Figure 8.12 indicates that MIS in unlikely to migrate to the crucible if $\mathrm{C}: \mathrm{N}>2.17-0.153 a$, where $a$ is the specific surface area of soil. 


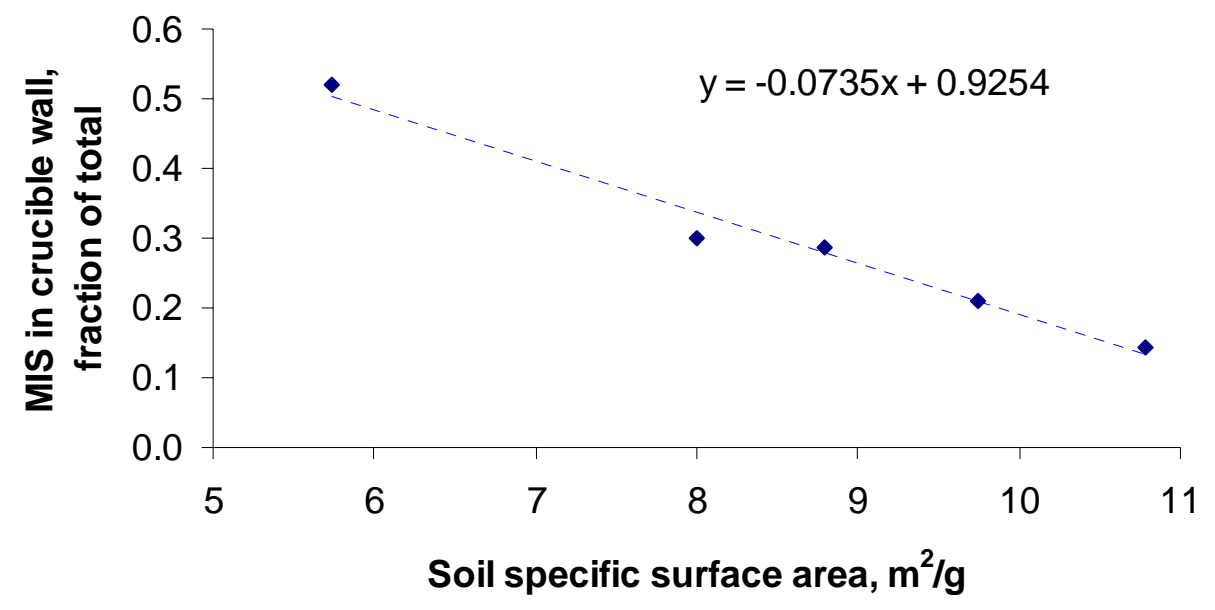

Figure 8.11. Effect of Soil Specific Surface Area (by BET) on the Fraction of MIS Lost from Feed to Crucible Walls (no sucrose has been added to feed)

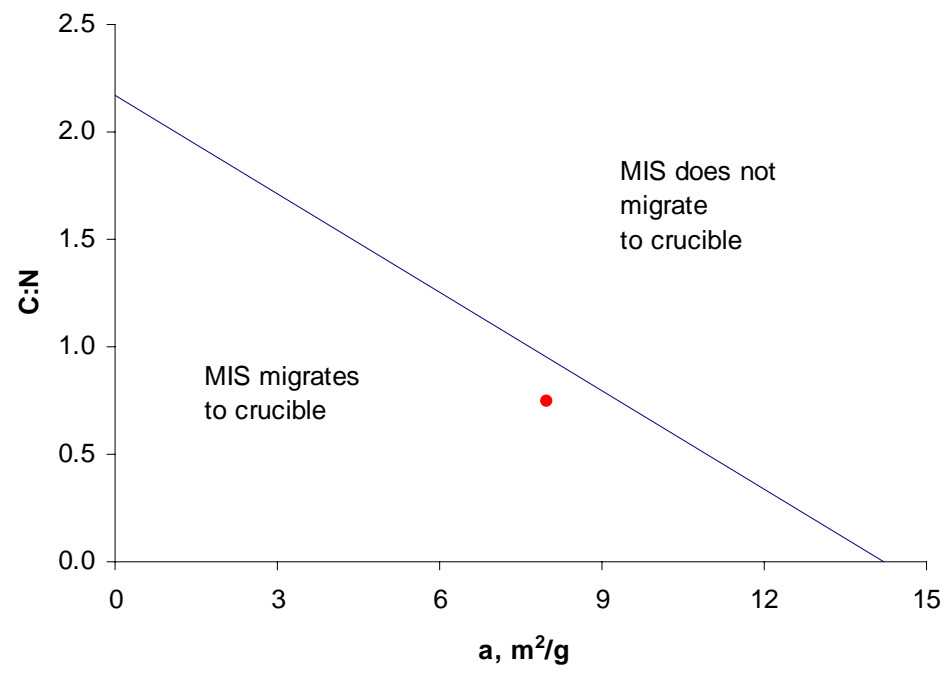

Figure 8.12. Preventable MIS Migration Diagram

The plot in Figure 8.12 is based on a rather small number of data points and only demonstrates the methodology. Also, it is based on the assumption that soil can effectively be ground to the required level and that dried feeds can be prepared with sucrose at any level without risking potential reactions in feed before it is charged into the BV melter. For example, if the maximum acceptable C:N is 0.75 and soil grinding to a specific surface area of only $8 \mathrm{~m}^{2} / \mathrm{g}$ is practicable (see the red point in the figure), MIS would not be $100 \%$ immobilized in the feed. 


\subsection{Feed Development for Large-Scale Applications}

This section is concerned with the immobilization of MIS within BV feeds. It reviews results from experiments performed in the exploratory phase of this task and adds new data obtained during the application phase in which the aim was to develop a BV melter feed that would effectively minimize MIS migration and could also be readily processed in the dryer. The drying operation for feed preparation was developed in parallel with this research and is reported separately. (Shimskey et. al. 2007)

As has been documented in the previous section, grinding the glass-forming portion of the feed (soil) to a finer particle size decreases the mobility of MIS within the feed, thus decreasing or preventing the transfer of MIS into the pores of the CRB. The solid particles in the feed physically retain the MIS by adsorbing the liquid onto the grain surfaces via surface forces and reducing the total interfacial energy for all the phase boundaries present. The particles hold the liquid in place until the MIS is immobilized through batch reactions, ultimately leading to the formation of high-viscosity glass melt.

After grinding in a small laboratory mill led to a successful immobilization of MIS, potential production soil-grinding methods were investigated by conducting tests at Praxair Specialty Ceramics. ${ }^{(a)}$ ) For largescale application, Praxair recommended and then performed bench-scale tests for two grinding methods, plate grinding and dry-ball milling. The plate-grinding method readily scales to a higher throughput while the dry-ball milling served as a bench-scale test for larger scale vibratory milling operations. Praxair used the specific surface area by the BET method and the particle-size distribution as the criteria to evaluate how well their product matched the PNNL baseline ground-soil samples. Praxair ground samples were shipped to PNNL for verification.

Feeds were prepared from as-received soil and soil ground at PNNL and Praxair. These solids were mixed with liquid S-109 tank waste simulate and pan dried to simulant dryer operations. The extent of MIS migration was measured using silica and vibrocast crucibles. As has been demonstrated in previous sections, silica crucibles allow visual evaluation and photographic documentation of the MIS penetration depth and also quantitative determination of the fraction of MIS that migrated to the crucible from the feed.

Large vibrocast crucibles (Figure 9.1) were obtained from E. J. Bartells Company. These were designed to evaluate the rate of MIS migration into, and possibly through, thicker refractory walls at a larger scale. They were made from the same material as that for the vibrocast-lined ES and full-scale melters. The crucibles were of a cylindrical shape, $275 \mathrm{~mm}$ high and $238 \mathrm{~mm}$ wide (outer diameter). The bottom thickness was $25 \mathrm{~mm}$, and the wall thickness was tapered from $25 \mathrm{~mm}$ at the bottom to $19 \mathrm{~mm}$ at the top (hence, the inner diameter was $188 \mathrm{~mm}$ at the bottom and $200 \mathrm{~mm}$ at the top). Their internal volume was nearly $7 \mathrm{~L}$.

To produce a homogenous mix on a small scale, feeds were prepared by mixing all solids with a liquid waste simulant while simultaneously drying the mixture; various other mixing methods were tried to evaluate the applicability of the results to dryer operation. Tests were performed by heating the crucibles at $5^{\circ} \mathrm{C} / \mathrm{min}$ to the set temperature $\left(500^{\circ} \mathrm{C}\right)$ and then holding for 30 minutes in silica crucibles and 20 hours in vibrocast crucibles. Feeds were then cooled down and removed from the crucibles. Empty crucibles

(a) Praxair Specialty Ceramics, Woodinville, WA. 
were ramp heated at $7^{\circ} \mathrm{C} / \mathrm{min}$ from 650 to $1000^{\circ} \mathrm{C}$ and held at $1000^{\circ} \mathrm{C}$ for 1 hour for silica crucibles and 2 hours for the vibrocast crucibles to determine the amount of MIS that migrated to crucible walls.

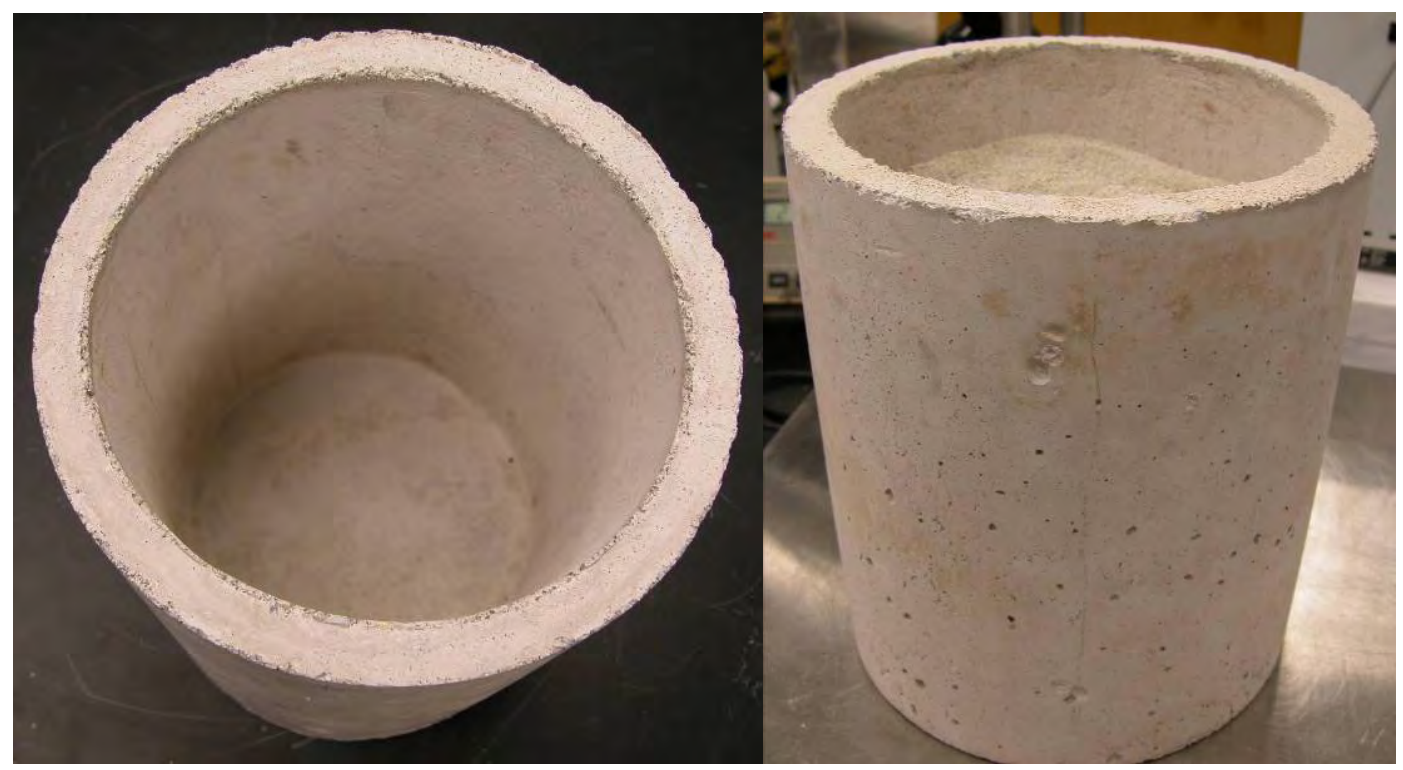

Figure 9.1. Vibrocast Crucible Before Heat-Treatment, Empty, and Filled with Feed

Most of the exploratory research was done with six-tank-composite LAW simulant. The S-109 LAW simulant planned for use in DBVS operations showed a higher mobility than six-tank-composite simulant. Testing with S-109 LAW simulant revealed that the grinding methods suggested by Praxair as potentially practical methods for grinding the large quantities of soil needed for DBVS operations did not provide a sufficiently fine material to limit MIS migration for S-109 feeds to desired levels. As an alternate or enhancement to fine solids, sucrose was added to the feed in an attempt to destroy the MIS before it had a chance to migrate. Sucrose reacts with nitrates at a temperature lower than $300^{\circ} \mathrm{C}$, above which substantial migration of MIS occurs. The reductant was changed to cellulose after dryer problems discouraged the use of sucrose as a reductant.

Tests performed with feeds containing the original Hanford soil showed that adding sufficient carbohydrates (sucrose or cellulose) to destroy enough nitrates and nitrites for MIS immobilization was large enough to raise safety concerns, i.e., a possibility of vigorous exothermic reaction in the dryer (the potential of such a reaction is under investigation). Hence, the presence of finer solids in feeds was considered necessary. However, tests at Praxair showed that grinding soil to the necessary particle size was a difficult operation and that it might be cost effective to purchase GFMs with the desired particle size. As an alternative to ground soil, feeds where readily available, high surface area GFMs replaced the Hanford soil component were made. The following feed variations were tested:

1) “No-iron glass feed” supplies all the soil components from GFMs except for $\mathrm{Fe}_{2} \mathrm{O}_{3}$ and increases waste loading by replacing $\mathrm{Na}_{2} \mathrm{O}$ from the soil with $\mathrm{Na}_{2} \mathrm{O}$ in the waste simulant while maintaining 20 mass $\%$ of $\mathrm{Na}_{2} \mathrm{O}$ in glass.

2) "GFM feed with low waste loading” has the same waste loading (18.43 mass\% of $\mathrm{Na}_{2} \mathrm{O}$ from the waste simulant) as the feed with soil. 
3) “GFM feed with high waste loading” replaces $\mathrm{Na}_{2} \mathrm{O}$ and $\mathrm{K}_{2} \mathrm{O}$ from the soil with $\mathrm{Na}_{2} \mathrm{O}$ (on molar basis) in the waste simulant to obtain glass with 21.24 mass\% of $\mathrm{Na}_{2} \mathrm{O}$.

The "no-iron glass feed" used a no-iron glass formulation developed by Kim et al. (2006). This feed may be of interest if process changes made in FS tests do not continue to control the precipitation of metallic $\mathrm{Fe}$ in the BV box. The GFM feeds (with high or low waste loading) contain $\mathrm{Fe}_{2} \mathrm{O}_{3}$ (added as hematite) to keep the glass composition the same as that based on Hanford soil in order to maintain the traceability to all the BV glass-composition work that has been conducted to date. This conservative approach was necessary to prevent unforeseen processing and/or regulatory problems associated with a major change in the glass composition. Testing various promising options offered by shifting to GFMs may be evaluated in future research. Upcoming engineering-scale and full-scale tests plan to use the "GFM feed with low waste loading”.

\subsection{LAW Simulants}

Two versions of S-109 simulants were prepared and used for testing. The new S-109 simulant was formulated to reduce the amount of solids that were generated with the old simulant recipe. The new S-109 simulant retains 11 components out of the original 16 of the (old) simulant recipe. The recipes, the content of glass components, the content of nitrates and nitrites, and selected properties of the simulants are listed in Table 9.1 through Table 9.4. Six-tank composite simulant data are also included for completeness. 
Table 9.1. Recipes of LAW Simulants

\begin{tabular}{|c|c|c|c|}
\hline \multirow[b]{2}{*}{ Reagent } & \multicolumn{3}{|c|}{ Mass Fraction, g/kg } \\
\hline & Six-Tank & $\begin{array}{c}\text { S-109 } \\
\text { (old) }\end{array}$ & $\begin{array}{l}\text { S-109 } \\
\text { (new) }\end{array}$ \\
\hline $\mathrm{Al}\left(\mathrm{NO}_{3}\right)_{3} \cdot 9 \mathrm{H}_{2} \mathrm{O}$ & 19.27 & & \\
\hline $\mathrm{Al}(\mathrm{OH})_{3}$ & & 2.76 & 0.69 \\
\hline $\mathrm{Ca}(\mathrm{OH})_{2}$ & & 0.07 & \\
\hline $\mathrm{NaCl}$ & 2.06 & 0.33 & 0.59 \\
\hline $\mathrm{Na}_{2} \mathrm{CrO}_{4}$ & 1.35 & 0.75 & \\
\hline $\mathrm{Na}_{2} \mathrm{CrO}_{4} \cdot 4 \mathrm{H}_{2} \mathrm{O}$ & & & 2.63 \\
\hline $\mathrm{Cr}_{2} \mathrm{O}_{3}$ & & 0.50 & \\
\hline $\mathrm{NaF}$ & 1.07 & 0.18 & \\
\hline $\mathrm{Fe}_{2} \mathrm{O}_{3}$ & & 0.29 & \\
\hline $\mathrm{KNO}_{3}$ & 1.01 & 0.34 & 0.34 \\
\hline $\mathrm{NaNO}_{2}$ & 23.60 & 3.42 & 3.43 \\
\hline $\mathrm{NaNO}_{3}$ & 158.15 & 281.06 & 281.74 \\
\hline $\mathrm{NaOH}$ & 23.85 & 6.39 & 6.23 \\
\hline $\mathrm{Na}_{3} \mathrm{PO}_{4} \cdot 12 \mathrm{H}_{2} \mathrm{O}$ & 15.08 & 16.71 & 16.75 \\
\hline $\mathrm{Na}_{2} \mathrm{SO}_{4}$ & 10.31 & 4.20 & 4.21 \\
\hline $\mathrm{Na}_{2} \mathrm{CO}_{3} \cdot \mathrm{H}_{2} \mathrm{O}$ & & 16.83 & 16.87 \\
\hline $\mathrm{Na}_{2} \mathrm{CO}_{3}$ & 40.60 & & \\
\hline $\mathrm{CH}_{3} \mathrm{COONa}$ & 8.70 & 0.10 & 0.82 \\
\hline $\mathrm{Na}_{2} \mathrm{C}_{2} \mathrm{O}_{4}$ & 1.27 & 1.17 & \\
\hline $\mathrm{CsNO}_{3}$ & 0.00 & & \\
\hline $\mathrm{H}_{2} \mathrm{O}$ & 693.55 & 664.91 & 665.71 \\
\hline
\end{tabular}

Table 9.2. Content of Glass Components in LAW Simulants

\begin{tabular}{||l|c|c|c|c|c|c||}
\hline \multirow{4}{*}{} & \multicolumn{3}{|c|}{ g/L } & \multicolumn{3}{c||}{ Mass Fraction } \\
\cline { 2 - 7 } & $\begin{array}{c}\text { Six- } \\
\text { Tank }\end{array}$ & $\begin{array}{c}\text { S-109 } \\
\text { (old) }\end{array}$ & $\begin{array}{c}\text { S-109 } \\
\text { (new) }\end{array}$ & $\begin{array}{c}\text { Six- } \\
\text { Tank }\end{array}$ & $\begin{array}{c}\text { S-109 } \\
\text { (old) }\end{array}$ & $\begin{array}{c}\text { S-109 } \\
\text { (new) }\end{array}$ \\
\hline $\mathrm{Al}_{2} \mathrm{O}_{3}$ & 3.25 & 2.24 & 0.568 & 0.0188 & 0.0135 & 0.0034 \\
\hline $\mathrm{CaO}$ & & 0.064 & & & 0.0004 & \\
\hline $\mathrm{Cl}$ & 1.55 & 0.251 & 0.447 & 0.0090 & 0.0015 & 0.0027 \\
\hline $\mathrm{Cr}_{2} \mathrm{O}_{3}$ & 0.79 & 1.06 & 1.55 & 0.0046 & 0.0064 & 0.0093 \\
\hline $\mathrm{F}$ & 0.60 & 0.101 & & 0.0035 & 0.0006 & \\
\hline $\mathrm{Fe}_{2} \mathrm{O}_{3}$ & & 0.361 & & & 0.0022 & \\
\hline $\mathrm{K}_{2} \mathrm{O}$ & 0.58 & 0.194 & 0.197 & 0.0034 & 0.0012 & 0.0012 \\
\hline $\mathrm{Na}_{2} \mathrm{O}$ & 154.95 & 154.97 & 157.60 & 0.8987 & 0.9331 & 0.9421 \\
\hline $\mathrm{P}_{2} \mathrm{O}_{5}$ & 3.49 & 3.88 & 3.94 & 0.0203 & 0.0234 & 0.0236 \\
\hline $\mathrm{SO}_{3}$ & 7.20 & 2.94 & 2.98 & 0.0418 & 0.0177 & 0.0179 \\
\hline $\mathrm{SUM}$ & 172.41 & 166.07 & 167.29 & 1.0000 & 1.0000 & 1.0000 \\
\hline
\end{tabular}


Table 9.3. Content of nitrates and nitrites in LAW simulants

\begin{tabular}{||l|c|c|c||}
\hline \hline & \multicolumn{3}{|c|}{ g/L } \\
\cline { 2 - 4 } & Six-Tank & $\begin{array}{c}\text { S-109 } \\
\text { (old) }\end{array}$ & $\begin{array}{c}\text { S-109 } \\
\text { (new) }\end{array}$ \\
\hline $\mathrm{NO}_{3}^{-}$ & 155.7 & 255.5 & 259.2 \\
\hline $\mathrm{NO}_{2}^{-}$ & 19.5 & 2.8 & 2.9 \\
\hline
\end{tabular}

Table 9.4. Properties of LAW Simulants

\begin{tabular}{|c|c|c|c|c|}
\hline & & Six-Tank & S-109 (old) & S-109 (new) \\
\hline Solid mass content in liquid simulant & $\mathrm{g} / \mathrm{kg}$ & 306.38 & 335.09 & 334.28 \\
\hline Solid mass density in liquid simulant & $\mathrm{g} / \mathrm{L}$ & 379.87 & 417.19 & 421.20 \\
\hline Density of $5 \mathrm{M}$ Na simulant & $\mathrm{kg} / \mathrm{L}$ & 1.240 & 1.245 & 1.260 \\
\hline Density of glass components in liquid simulant & $\mathrm{g} / \mathrm{L}$ & 172.41 & 166.07 & 167.29 \\
\hline Mass fraction of glass components in dry simulant & $\mathrm{g} / \mathrm{g}$ & 0.4539 & 0.3981 & 0.3972 \\
\hline Mass fraction of nitrates + nitrites in dry simulant ${ }^{(a)}$ & $\mathrm{g} / \mathrm{g}$ & 0.6595 & 0.8500 & 0.8541 \\
\hline
\end{tabular}

\subsection{Feed Formulation}

Previous research established the following two conclusions:

1. As the specific surface area of batch solids increases, more MIS is retained in the feed until no MIS can drain or get soaked into CRB pores by capillary forces.

2. As the carbohydrate addition to feed increases, more nitrates and nitrites are destroyed in the feed until no free MIS can be transferred into the CRB.

As mentioned above, neither of these phenomena alone can effectively operate in a large-scale situation for all LAWs stored at Hanford. Hence, fine solids and carbohydrates need to be used in combination. As an alternative to soil grinding, feeds were prepared with fine-grained GFMs available in large quantities from sources that provide these minerals for HLW vitrification or for commercial glass production. Feeds were tested with sands of various grain size, mostly with fine silica $(<5 \mu \mathrm{m})$, Min-USil 5. Table 9.5 lists compositions of the minerals used in crucible tests in this study.

The advantage of using several minerals instead of soil is a better control of glass composition. As mentioned above, one potential option is virtually eliminating $\mathrm{Fe}_{2} \mathrm{O}_{3}$, an oxide that produces a large volume of gas in molten glass at temperatures above $1000^{\circ} \mathrm{C}$ and can precipitate metallic Fe at glassprocessing temperatures to which Tc tends to partition and from which Tc can be easier released than from glass. Moreover, formulating feeds from minerals allows for a higher waste loading and, hence, a smaller volume of waste glass is generated, thus resulting in substantial savings that offset the higher cost of raw materials. These attractive options required further evaluation before they could be used in the BV process and were not considered for near-term engineering and FS testing. However, limited testing of no-Fe feeds and feeds with higher waste loadings were conducted in silica-crucible experiments. 
Table 9.5. Compositions of Minerals (in mass fractions)

\begin{tabular}{||l|c|c|c|l|c|c|c|}
\hline \hline Oxide & Hematite & Rutile & $\mathbf{Z r S i O}_{4}$ & Sand & Kyanite & Wollastonite & Olivine \\
\hline $\mathrm{Al}_{2} \mathrm{O}_{3}$ & 0.0149 & 0.0051 & 0.0025 & 0.0014 & 0.5718 & 0.0020 & 0.0019 \\
\hline $\mathrm{CaO}$ & 0.0004 & & & 0.0001 & 0.0003 & 0.4783 & 0.0002 \\
\hline $\mathrm{Cr}_{2} \mathrm{O}_{3}$ & & 0.0016 & & & & & 0.0013 \\
\hline $\mathrm{Fe}_{2} \mathrm{O}_{3}$ & 0.9657 & 0.0071 & 0.0008 & 0.0002 & 0.0078 & 0.0040 & 0.0776 \\
\hline $\mathrm{K}_{2} \mathrm{O}$ & & & & 0.0002 & 0.0042 & & 0.0003 \\
\hline $\mathrm{MgO}$ & 0.0010 & & & 0.0001 & 0.0001 & 0.0010 & 0.4852 \\
\hline $\mathrm{MnO}$ & 0.0012 & & & & & 0.0010 & 0.0037 \\
\hline $\mathrm{Na}_{2} \mathrm{O}$ & & & & & & & 0.0000 \\
\hline $\mathrm{P}_{2} \mathrm{O}_{5}$ & 0.0027 & & & & & & \\
\hline $\mathrm{SiO}_{2}$ & 0.0134 & 0.0223 & 0.3268 & 0.9979 & 0.4078 & 0.5135 & 0.4297 \\
\hline $\mathrm{SO}_{3}$ & 0.0007 & & & 0.0000 & 0.0000 & & \\
\hline $\mathrm{TiO}_{2}$ & & 0.9447 & 0.0010 & 0.0001 & 0.0079 & 0.0002 & \\
\hline $\mathrm{ZrO}_{2}$ & & 0.0193 & 0.6688 & & & & \\
\hline \hline
\end{tabular}

The glasses made from GFMs were not optimized for the best-possible properties. The goal of this study was to eliminate MIS migration to the highest possible extent without making additional changes to the glass formulation. Substantial changes in glass composition would require extensive testing of glass properties and feed melting behavior. As argued above, this was beyond the scope of the present study. Therefore, glasses from minerals were formulated to obtain compositions as close as possible to the baseline glass with soil. Only minor ( $<1$ mass\%), less-desirable components, such as $\mathrm{Cl}, \mathrm{SrO}$, and $\mathrm{BaO}$, were avoided when reformulating with the GFMs.

Table 9.6 shows recipes for three GFM feeds, and Table 9.7 lists the corresponding glass compositions. Feed A is "no-iron glass feed," Feed B is "GFM feed with low waste loading," and Feed C is "GFM feed with high waste loading." Feed B was formulated to produce glass of the same waste sodium loading as the baseline glass made from feed containing Hanford soil, and Feed $\mathrm{C}$ was formulated to have a higher waste sodium loading than the baseline glass. Because $\mathrm{Na}_{2} \mathrm{O}$ and $\mathrm{K}_{2} \mathrm{O}$ from soil were replaced by $\mathrm{Na}_{2} \mathrm{O}$ from waste in Glass C, the waste loading increased by 15\%, from 18.43 mass $\%$ to 21.24 mass\%. The noiron Glass A contains 0.45 mass $\% \mathrm{Fe}_{2} \mathrm{O}_{3}$ from impurities in various minerals. 
Table 9.6. Recipes for Feeds from Minerals and S-109 LAW Simulant (new) To Make $100 \mathrm{~g}$ of Glass

\begin{tabular}{|c|c|c|c|c|c|}
\hline Material & Unit & $\begin{array}{c}\text { Grain size } \\
\mu \mathrm{m}\end{array}$ & A & B & C \\
\hline Kyanite $\left(\mathrm{Al}_{2} \mathrm{SiO}_{5}\right)$ & $\mathrm{g}$ & $<44$ & 10.24 & 13.60 & 13.59 \\
\hline $\mathrm{B}_{2} \mathrm{O}_{3}$ (AMEC) & $\mathrm{g}$ & & 5.50 & 5.00 & 5.00 \\
\hline Wollastonite $\left(\mathrm{CaSiO}_{3}\right)$ & $\mathrm{g}$ & $<44$ & 10.51 & 5.31 & 5.33 \\
\hline Olivine $\left(\mathrm{MgSiO}_{4}\right)$ & $\mathrm{g}$ & $<74$ & 4.13 & 2.55 & 2.55 \\
\hline Sand $\left(\mathrm{SiO}_{2}\right)$ & $\mathrm{g}$ & $<5$ & 41.57 & 39.19 & 36.20 \\
\hline Zircon $\left(\mathrm{ZrSiO}_{4}\right)$ & $\mathrm{g}$ & $<44$ & & 10.45 & 10.45 \\
\hline Baddeleyite $\left(\mathrm{ZrO}_{2}\right)$ & $\mathrm{g}$ & $<590$ & 7.00 & & \\
\hline Hematite $\left(\mathrm{Fe}_{2} \mathrm{O}_{3}\right)$ & $\mathrm{g}$ & $<44$ & & 3.78 & 3.78 \\
\hline Rutile $\left(\mathrm{TiO}_{2}\right)$ & $\mathrm{g}$ & air floated & & 0.56 & 0.55 \\
\hline LAW S-109 simulant (new) & $\mathrm{mL}$ & & 126.90 & 116.94 & 134.77 \\
\hline Liquid simulant mass & g & & 159.90 & 147.35 & 169.81 \\
\hline Dry simulant mass & $\mathrm{g}$ & & 53.45 & 49.25 & 56.77 \\
\hline Wet feed mass & g & & 238.85 & 227.78 & 247.27 \\
\hline Dry feed mass & g & & 132.40 & 129.69 & 134.22 \\
\hline
\end{tabular}

Table 9.7. Compositions (in mass fractions) of Glasses Made from Minerals and S-109 LAW Simulant

\begin{tabular}{||l|c|c|c||}
\hline & \multicolumn{1}{|c|}{ A } & B & C \\
\hline $\mathrm{Al}_{2} \mathrm{O}_{3}$ & 0.0600 & 0.0800 & 0.0800 \\
\hline $\mathrm{B}_{2} \mathrm{O}_{3}$ & 0.0550 & 0.0500 & 0.0500 \\
\hline $\mathrm{BaO}$ & 0.0000 & 0.0000 & 0.0000 \\
\hline $\mathrm{CaO}$ & 0.0500 & 0.0255 & 0.0256 \\
\hline $\mathrm{Cl}$ & 0.0006 & 0.0005 & 0.0006 \\
\hline $\mathrm{Cr}_{2} \mathrm{O}_{3}$ & 0.0020 & 0.0019 & 0.0021 \\
\hline $\mathrm{Fe}_{2} \mathrm{O}_{3}$ & 0.0045 & 0.0400 & 0.0400 \\
\hline $\mathrm{K}_{2} \mathrm{O}$ & 0.0008 & 0.0009 & 0.0009 \\
\hline $\mathrm{MgO}^{2}$ & 0.0200 & 0.0125 & 0.0125 \\
\hline $\mathrm{MnO} \mathrm{O}$ & 0.0003 & 0.0002 & 0.0002 \\
\hline $\mathrm{Na}_{2} \mathrm{O}$ & 0.2000 & 0.1843 & 0.2124 \\
\hline $\mathrm{P}_{2} \mathrm{O}_{5}$ & 0.0050 & 0.0047 & 0.0054 \\
\hline $\mathrm{SiO}_{2}$ & 0.5272 & 0.5195 & 0.4898 \\
\hline $\mathrm{SO}_{3}$ & 0.0038 & 0.0035 & 0.0041 \\
\hline $\mathrm{SrO}_{|l|} 0.0000$ & 0.0000 & 0.0000 \\
\hline $\mathrm{TiO}_{2}$ & 0.0009 & 0.0065 & 0.0064 \\
\hline $\mathrm{ZrO}_{2}$ & 0.0700 & 0.0700 & 0.0700 \\
\hline $\begin{array}{l}\mathrm{A}-\mathrm{No} \text {-iron glass feed, B-GFM feed with } \\
\text { low waste loading, C-GFM feed with high } \\
\text { waste loading. }\end{array}$ \\
\hline
\end{tabular}


Table 9.8 summarizes the additional cost for minerals used in Feeds B and C compared to feeds with soil. Table 9.8 also includes estimated cost savings resulting from high waste loading in Feed C. The additional cost for GFMs strongly depends on the choice of sand: the feed with fine sand $(<5 \mu \mathrm{m})$ costs more than twice the feed with coarse sand $(<75 \mu \mathrm{m})$. Table 9.8 suggests that for Feed $\mathrm{C}$ with high waste loading, the additional cost for GFMs is roughly compensated for by the cost savings from increased waste loading. Considering that the current estimation for the cost of fine sand (Min-U-Sil 5) is conservative, it is likely that there would be a net cost savings for Feed $\mathrm{C}$ if an economical source of fine sand is available.

Table 9.8. Cost Comparison Between GFM Feeds with Low (Feed B) and High (Feed C) Waste Loading

\begin{tabular}{||l|c|c||}
\hline \multicolumn{1}{|c|}{ Material } & Feed B & Feed C \\
\hline Waste oxide loading, mass\% & 19.56 & 22.55 \\
\hline Additional cost for GFMs with coarse sand $(<75 \mu \mathrm{m})^{(\mathrm{a})}$ & $\$ 9,517$ & $\$ 9,218$ \\
\hline Additional cost for GFMs with fine sand $(<5 \mu \mathrm{m})^{(\mathrm{a})}$ & $\$ 20,241$ & $\$ 19,125$ \\
\hline Estimated cost savings from waste loading increase ${ }^{(\mathrm{b})}$ & - & $\$ 19,847$ \\
\hline (a) Assumes that the cost of soil is negligible; the cost of GFMs is at WTP design basis except for \\
fine sand, present at the market cost; the shipping cost is based on $\$ 3700$ per full truck load of \\
18 MT for all GFMs. \\
(b) Assumes that the total cost of BV box production (currently estimated at $\$ 150,000$ per box) is \\
proportional to the total glass mass.
\end{tabular}

Feeds for silica crucible experiments were prepared with a target glass mass of $150 \mathrm{~g}$. Dry chemicals were mixed in a plastic bag for 2 to 3 minutes and then added to a steel boat that contained the liquid waste simulant and stirred on a hotplate until the feed was dry. Stirring the feed continued until it was cool enough to form small, 1 to $6 \mathrm{~mm}$, granules. Granulated feed was dried in the oven at $90^{\circ} \mathrm{C}$ overnight. Sucrose and cellulose were added to the dried granulated feed in a few experiments, but, generally, the sucrose or cellulose was blended with the dry ingredients before they were added to the steel boat that contained liquid-waste simulant.

To prepare enough as-received soil-containing feed to produce $10 \mathrm{~kg}$ of glass for vibrocast crucible experiments, the liquid simulant $(14.9 \mathrm{~kg})$ was heated to evaporate some water. Then $6.19 \mathrm{~kg}$ of dried HRTS soil, $0.1068 \mathrm{~kg}$ of $\mathrm{Al}(\mathrm{OH})_{3}$, and $0.281 \mathrm{~kg}$ of $\mathrm{SiO}_{2}$ were added. The mixture was dried in a water bath heater and then heated on drying pans in a 6-cm layer at $90^{\circ} \mathrm{C}$ overnight in the oven. The dry hard mixture was crushed and sieved through a 9-mm sieve. The resulting feed was divided into three equal portions to which $0.363 \mathrm{~kg}$ of $\mathrm{ZrSiO}_{4}$ and $0.173 \mathrm{~kg}$ of $\mathrm{B}_{2} \mathrm{O}_{3}$ were added and blended for 30 minutes in a $\mathrm{V}$ - blender with the intensifier bar running for 1 to 2 minutes at the beginning and end of each blending period. One 10-kg batch was also prepared in a similar manner with the addition of sucrose to the wet mixing process.

To prepare enough simulated AMEC 38-C feed (i.e., dry blended feed) to produce $10 \mathrm{~kg}$ of glass, $4.926 \mathrm{~kg}$ of dry AMEC simulant, $0.0958 \mathrm{~kg}$ of $\mathrm{NaOH}, 6.126 \mathrm{~kg}$ of dried as-received HRTS soil, $0.0880 \mathrm{~kg}$ of $\mathrm{Al}(\mathrm{OH})_{3}, 0.337 \mathrm{~kg}$ of silica sand, $1.090 \mathrm{~kg}$ of $\mathrm{ZrSiO}_{4}$, and $0.520 \mathrm{~kg}$ of $\mathrm{B}_{2} \mathrm{O}_{3}$ were batched in two portions and blended for 1 hour in the V-blender.

For GFM feeds, WTP minerals, silica (Min-U-Sil 5), and cellulose were premixed and added to a 22-L dryer. S-109 liquid simulant (5 molar $\mathrm{Na}$ ) was gradually added to the 22-L dryer while simultaneously 
drying and stirring the blend. Adding sucrose as the carbon source in the dryer was also attempted, but adding sugar at the end of operations or pre-dissolved in the S-109 liquid simulant caused the feed in the dryer to become extremely sticky and freeze the stirring paddles. Dryer runs with Praxair ground soils with and without cellulose were also successfully completed.

\subsection{MIS Migration: Experiments with Silica Crucibles}

Table 9.9 summarizes data obtained with soil, as received or ground in a tungsten-carbide mill at PNNL, and the new S-109 LAW simulant (see Table 9.1 through Table 9.4). Fractions of MIS that migrated to the crucible from the feed during heating at $5^{\circ} \mathrm{C} / \mathrm{min}$ to the set temperature of $500^{\circ} \mathrm{C}$ held for 30 minutes are listed as functions of the grinding time, or the specific surface area $(a)$, and the addition of two types of carbohydrate: sucrose and cellulose.

The carbohydrate addition is quantified in terms of the carbohydrate carbon to feed nitrogen (C:N) ratio. As introduced in the previous section, the carbohydrate $\mathrm{C}: \mathrm{N}$ ratio is defined as the ratio of moles of $\mathrm{C}$ from carbohydrates (sucrose and cellulose) to moles of $\mathrm{N}$ from nitrates plus nitrites.

Figure 9.2 displays MIS migration data as a function of the $\mathrm{C}: \mathrm{N}$ ratio (c) for as-received soil ( $a=5.74 \mathrm{~m}^{2} / \mathrm{g}$ ). At $c=0.63$, data scatter is large. At $c \geq 0.63$ (excluding an outlying data point), the fraction of migrated MIS $(y)$ decreases as $c$ increases by the linear relationship $y=0.343-0.260 c$, suggesting that MIS migration stops at $c \cong 1$.3. Indeed, as Table 9.9 shows, the addition of sucrose corresponding to $c=1.25$ stopped MIS migration (the negative number of migrated MIS is indistinguishable from zero, considering uncertainties associated with small crucible mass differences comparable to LOI).

As Table 9.9 indicates, MIS migration is not significantly affected by the form of sucrose (ground or powder) or the mode of mixing (added to wet or dried feed). More significantly, there is no difference between sucrose and cellulose with respect to their effects on MIS migration.

For laboratory-ground soil to $a=9.75 \mathrm{~m}^{2} / \mathrm{g}$, carbohydrate addition became effective at $c>0.3$ (see Figure 9.3). At $c \geq 0.3$, the fraction of migrated MIS decreases with the linear relationship $y=0.280-0.400 c$, indicating that MIS migration stops at $c=0.70$. As Table 9.9 shows, the value of $y=0.01$ was obtained at $c=0.75$. Most of the data were obtained with sucrose addition. A single experiment performed with cellulose confirms the observation that MIS migration is not significantly affected by the form of carbohydrate.

The single experiment with soil ground to $a=10.78 \mathrm{~m}^{2} / \mathrm{g}$ (Figure 9.3) with no carbohydrate addition indicates the extent to which grinding impacts MIS mobility (see also Figure 8.11). 
Table 9.9. Fractions of Crucible-Migrated MIS: Feeds with Soil (as received or ground at PNNL) and S-109 LAW Simulant (new)

\begin{tabular}{|c|c|c|c|c|}
\hline $\bar{a}$ & \multicolumn{2}{|c|}{ C:N Ratio } & \multirow{2}{*}{$\begin{array}{c}\text { Migrated MIS } \\
\text { Fraction }\end{array}$} & \multirow[b]{2}{*}{ Note } \\
\hline $\mathrm{m}^{2} / \mathrm{g}$ & Sucrose & Cellulose & & \\
\hline \multicolumn{5}{|c|}{ 5-min grinding } \\
\hline 10.78 & 0.00 & 0.00 & 0.13 & \\
\hline \multicolumn{5}{|c|}{ 2-min grinding } \\
\hline 9.75 & 0.75 & 0.00 & 0.01 & \\
\hline 9.75 & 0.50 & 0.00 & 0.03 & \\
\hline 9.75 & 0.63 & 0.00 & 0.03 & \\
\hline 9.75 & 0.44 & 0.00 & 0.10 & \\
\hline 9.75 & 0.38 & 0.00 & 0.14 & \\
\hline 9.75 & 0.31 & 0.00 & 0.18 & \\
\hline 9.75 & 0.00 & 0.00 & 0.19 & \\
\hline 9.75 & 0.63 & 0.00 & 0.02 & \\
\hline 9.75 & 0.00 & 0.63 & 0.02 & \\
\hline \multicolumn{5}{|c|}{ As-received } \\
\hline 5.74 & 1.25 & 0.00 & -0.01 & \\
\hline 5.74 & 0.94 & 0.00 & 0.11 & \\
\hline 5.74 & 0.63 & 0.00 & 0.10 & \\
\hline 5.74 & 0.63 & 0.00 & 0.13 & (a) \\
\hline 5.74 & 0.63 & 0.00 & 0.19 & (b) \\
\hline 5.74 & 0.63 & 0.00 & 0.23 & (c) \\
\hline 5.74 & 0.00 & 0.00 & 0.47 & \\
\hline 5.74 & 0.13 & 0.63 & 0.13 & \\
\hline 5.74 & 0.13 & 0.75 & 0.14 & \\
\hline 5.74 & 0.13 & 0.88 & 0.03 & \\
\hline 5.74 & 0.13 & 1.00 & 0.04 & \\
\hline 5.74 & 0.13 & 1.13 & 0.02 & \\
\hline 5.74 & 0.00 & 0.63 & 0.21 & \\
\hline 5.74 & 0.00 & 0.63 & 0.40 & (d) \\
\hline 5.74 & 0.00 & 0.75 & 0.20 & \\
\hline 5.74 & 0.00 & 0.88 & 0.08 & \\
\hline 5.74 & 0.00 & 0.88 & 0.09 & \\
\hline 5.74 & 0.00 & 1.00 & 0.12 & \\
\hline 5.74 & 0.00 & 1.13 & 0.09 & \\
\hline 5.74 & 0.00 & 1.25 & 0.02 & \\
\hline 5.74 & 0.00 & 0.00 & 0.47 & \\
\hline \multicolumn{5}{|c|}{$\begin{array}{l}\text { Notes: } \\
\text { (a) Ground sucrose added to dried feed, } \\
\text { (b) Sucrose added to dried feed, } \\
\text { (c) Powder sucrose added to dried feed, } \\
\text { (d) Dryer dried feed. }\end{array}$} \\
\hline
\end{tabular}




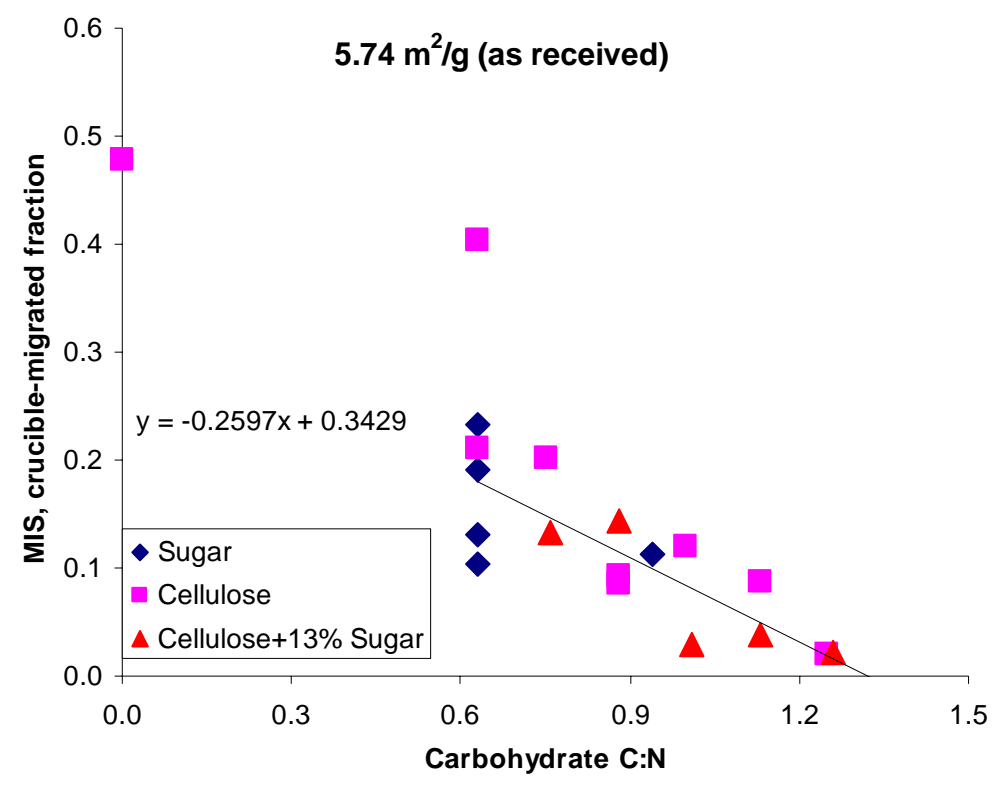

Figure 9.2. Mass Fraction of Silica-Crucible-Migrated MIS from Feed with As-Received Soil Versus Carbohydrate Addition

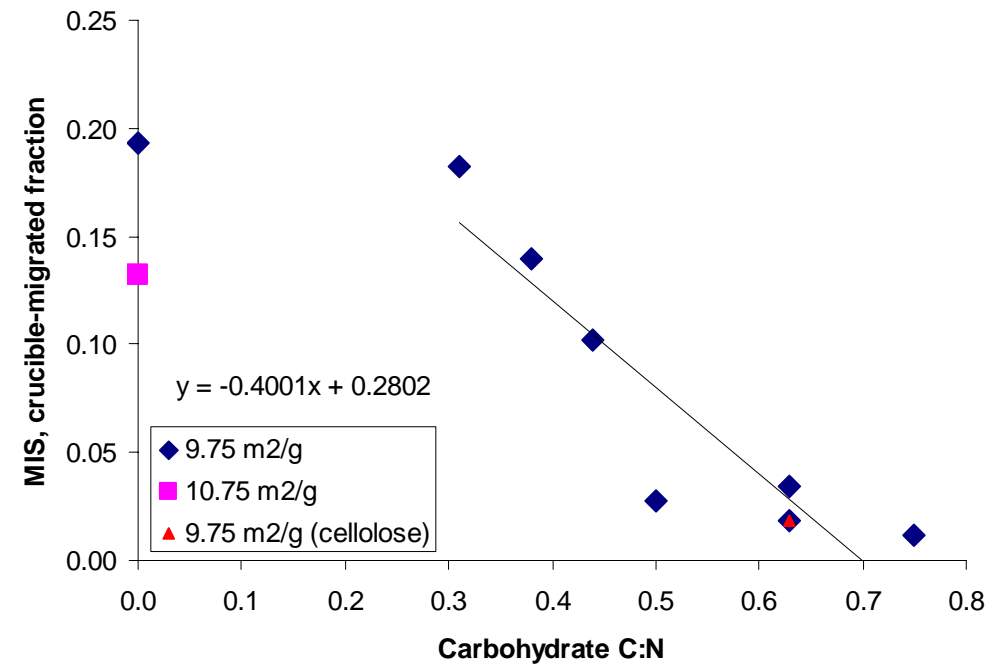

Figure 9.3. Mass Fraction of Silica-Crucible-Migrated MIS from Feed with Ground Soil Versus Carbohydrate Addition

Table 9.10 and Figure 9.4 summarize data obtained with soil ground at Praxair listing fractions of MIS migrated to silica crucibles from the feed during heating at $5^{\circ} \mathrm{C} / \mathrm{min}$ to the set temperature held for 30 minutes. Figure 9.4 demonstrates that even with the finest grinding achievable at Praxair, carbohydrate addition would have to exceed the C:N value of approximately 0.7 to prevent MIS migration. 
Table 9.10. Fractions of Crucible-Migrated MIS: Feeds with Soil (ground at Praxair) and S-109 LAW Simulant (new)

\begin{tabular}{|c|c|c|c|c|}
\hline$a$ & \multicolumn{2}{|c|}{ C:N Ratio } & \multirow{2}{*}{$\begin{array}{c}\text { Migrated MIS } \\
\text { Fraction }\end{array}$} & \multirow{2}{*}{ Note } \\
\hline $\mathrm{m}^{2} / \mathrm{g}$ & Sucrose & Cellulose & & \\
\hline $7.7-10.2$ & 0.00 & 0.63 & 0.10 & \\
\hline $7.7-10.2$ & 0.00 & 0.63 & 0.14 & \\
\hline 8.8 & 0.63 & 0.00 & 0.02 & \\
\hline 8.8 & 0.00 & 0.00 & 0.26 & \\
\hline $7.6-8.2$ & 0.00 & 0.00 & 0.42 & \\
\hline $7.6-8.2$ & 0.00 & 0.00 & 0.50 & \\
\hline 8 & 0.50 & 0.00 & 0.02 & \\
\hline 8 & 0.00 & 0.00 & 0.27 & \\
\hline 6.7 & 0.00 & 0.63 & 0.07 & \\
\hline 5.6 & 0.00 & 0.63 & 0.08 & \\
\hline 5.1 & 0.00 & 0.63 & 0.14 & \\
\hline \multirow[t]{3}{*}{5.1} & 0.00 & 0.00 & 0.27 & \\
\hline & 0.00 & 0.00 & 0.52 & (a) \\
\hline & 0.00 & 0.88 & 0.19 & (b) \\
\hline $\begin{array}{l}\text { Notes: } \\
\text { (a) Sieved } \\
\text { (b) Dryer } \\
\text { specifi }\end{array}$ & $\begin{array}{l}-35 \text { mesh, s } \\
\text { ed with } \alpha \text { c } \\
\text { surface are }\end{array}$ & $\begin{array}{l}\text { cific surface } \\
\text { ulose ( } 4 \times \text { vil } \\
\text { fformation ne }\end{array}$ & $\begin{array}{l}\text { anformation not a } \\
\text { ation-milled, drye } \\
\text { available). }\end{array}$ & $\begin{array}{l}\text { ilable), } \\
\text { Aried; }\end{array}$ \\
\hline
\end{tabular}

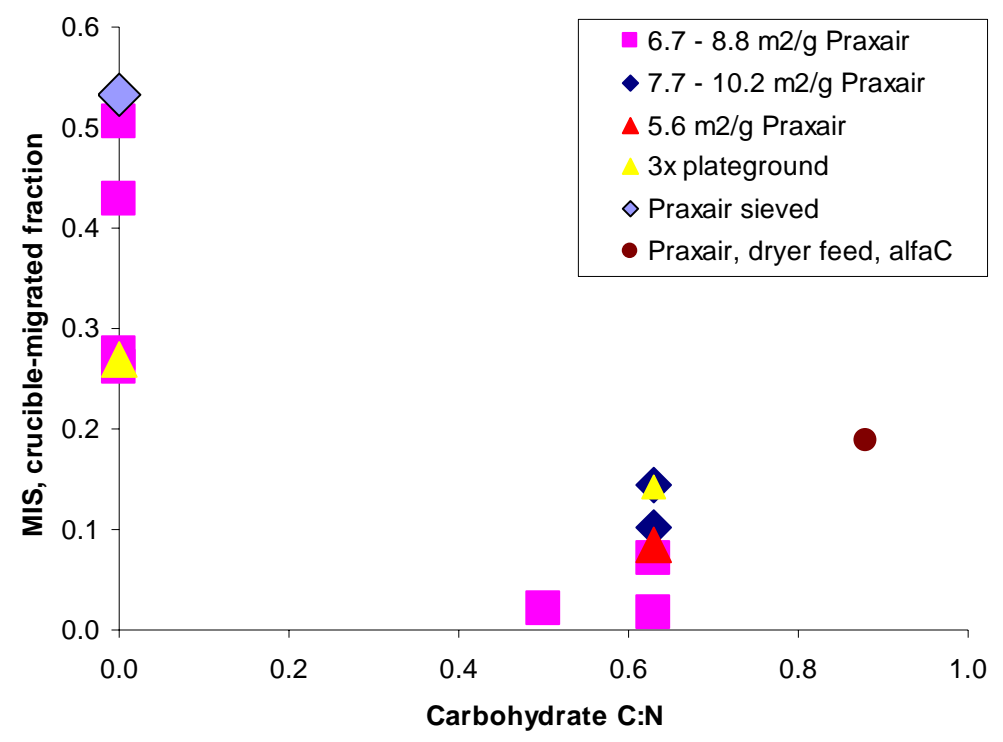

Figure 9.4. Mass Fraction of Silica-Crucible-Migrated MIS from Feed with Praxair Ground Soil Versus Carbohydrate Addition 
Table 9.11 and Figure 9.5 summarize data obtained with GFMs. Figure 9.5 shows that silica with grains of $\leq 75 \mu \mathrm{m}$ (-200 mesh) is not effective in decreasing MIS migration. Neither the form of cellulose (pure cellulose, wheat bran, dog wheat, shredded newspaper) nor the mode of drying had an impact on MIS retention. One data point (Dryer feed I) is an outlier (the red triangle in Figure 9.5), probably related to the problems experienced during dryer operation for this feed. The feed designated as "Dryer feed II" was prepared under identical conditions after correcting the dryer-operation issue and produced a comparable result to other feeds with the same cellulose addition).

Table 9.11. Fractions of Crucible-Migrated MIS: Feeds with Minerals, S-109 LAW Simulant (new), and Cellulose Addition

\begin{tabular}{|c|c|c|c|c|c|}
\hline $\begin{array}{c}\text { Waste } \\
\text { Loading }\end{array}$ & $\begin{array}{c}\mathbf{a} \\
\mathrm{m}^{2} / \mathrm{g}\end{array}$ & C:N Ratio & $\begin{array}{l}\text { Migrated MIS } \\
\text { Fraction }\end{array}$ & $\begin{array}{c}\text { Feed } \\
\text { Formulation }^{(a)}\end{array}$ & Note \\
\hline 0.2123 & 3.83 & 0.00 & 0.223 & $\mathrm{~A}$ & NFS \\
\hline 0.2255 & 4.12 & 0.54 & 0.252 & $\mathrm{C}$ & S75 \\
\hline 0.2255 & 4.12 & 0.75 & 0.086 & $\mathrm{C}$ & DI \\
\hline 0.2255 & 4.12 & 0.75 & 0.021 & $\mathrm{C}$ & B \\
\hline 0.2255 & 4.12 & 0.54 & 0.039 & $\mathrm{C}$ & B \\
\hline 0.2255 & 4.12 & 0.75 & 0.038 & $\bar{C}$ & DII \\
\hline 0.2255 & 4.12 & 0.54 & 0.049 & $\mathrm{C}$ & $\mathrm{ADF}$ \\
\hline 0.1956 & 4.12 & 0.62 & 0.041 & B & LW \\
\hline 0.2255 & 4.16 & 0.75 & 0.015 & $\mathrm{C}$ & BW \\
\hline 0.2255 & 4.16 & 0.75 & 0.017 & $\bar{C}$ & CB \\
\hline 0.2255 & 4.12 & 0.75 & 0.035 & $\mathrm{C}$ & DW \\
\hline 0.1956 & 4.12 & 0.75 & 0.003 & $\mathrm{~B}$ & ELW \\
\hline 0.1956 & 4.12 & 0.75 & 0.024 & B & ELW-LD \\
\hline 0.2255 & 4.16 & 0.75 & 0.021 & $\mathrm{C}$ & $\mathrm{N}$ \\
\hline 0.2255 & 3.83 & 0.75 & 0.021 & $\mathrm{C}$ & SWB0.4 \\
\hline 0.2255 & 4.12 & 0.75 & 0.034 & $\bar{C}$ & SWB2 \\
\hline 0.1956 & 4.12 & 0.00 & 0.023 & $\bar{B}$ & LW \\
\hline $\begin{array}{l}\text { (a) See Ta } \\
\text { Notes: Unl } \\
\text { high waste } \\
\text { ADF } \\
\text { B bas } \\
\text { BW b } \\
\text { CB 4 } \\
\text { DI dr } \\
\text { DW c } \\
\text { ELW } \\
\text { ELW } \\
\text { LW l } \\
\text { N shr } \\
\text { NFS } \\
\text { S75 I } \\
\text { SWB } \\
\text { SWB }\end{array}$ & $\begin{array}{l}\text { le } 9.6 \mathrm{f} \\
\text { ss indic } \\
\text { oading. } \\
\text { ellulose } \\
\text { line fee } \\
\text { an whe } \\
\text { \% pure } \\
\text { er dried } \\
\text { g whea } \\
\text { S-31F } \\
\text { D ES- } \\
\text { w waste } \\
\text { dded ne } \\
\text { Fe fee } \\
\text { in-U-Si } \\
4 \text { soft }\end{array}$ & $\begin{array}{l}\mathrm{r} \text { feed recipes. } \\
\text { ted otherwise, } \\
\text { source added } \\
\mathrm{s} \text { (high waste } \\
\mathrm{t} \text { as cellulose s } \\
\text { ellulose and } 3 \\
\mathrm{I} \text {, DII dryer dr } \\
\text { as cellulose s } \\
\text { eed dryer drie } \\
1 \mathrm{~F} \text { feed lab dr } \\
\text { loading with o } \\
\text { vspaper as cell } \\
\text {, sand with } 2 . \\
75 \text { as the silic } \\
\text { hite bran as ce } \\
\text { te bran as cell }\end{array}$ & $\begin{array}{l}\text { feeds contained Mi } \\
\text { dry feed } \\
\text { oading with } \alpha \text {-cellu } \\
\text { urce } \\
\% \text { bran wheat as ce } \\
\text { d II } \\
\text { urce } \\
\text { (low waste loading } \\
\text { d (low waste loadi } \\
\text { cellulose } \\
\text { lose source } \\
\mathrm{m}^{2} / g \text { specific surfa } \\
\text { source } \\
\text { lulose source, } 0.4-\text {-r } \\
\text { lose source, } 2 \text {-min }\end{array}$ & $\begin{array}{l}\text {-U-Sil } 5 \text { as the silic } \\
\text { ose) } \\
\text { lulose source }\end{array}$ & source and \\
\hline
\end{tabular}




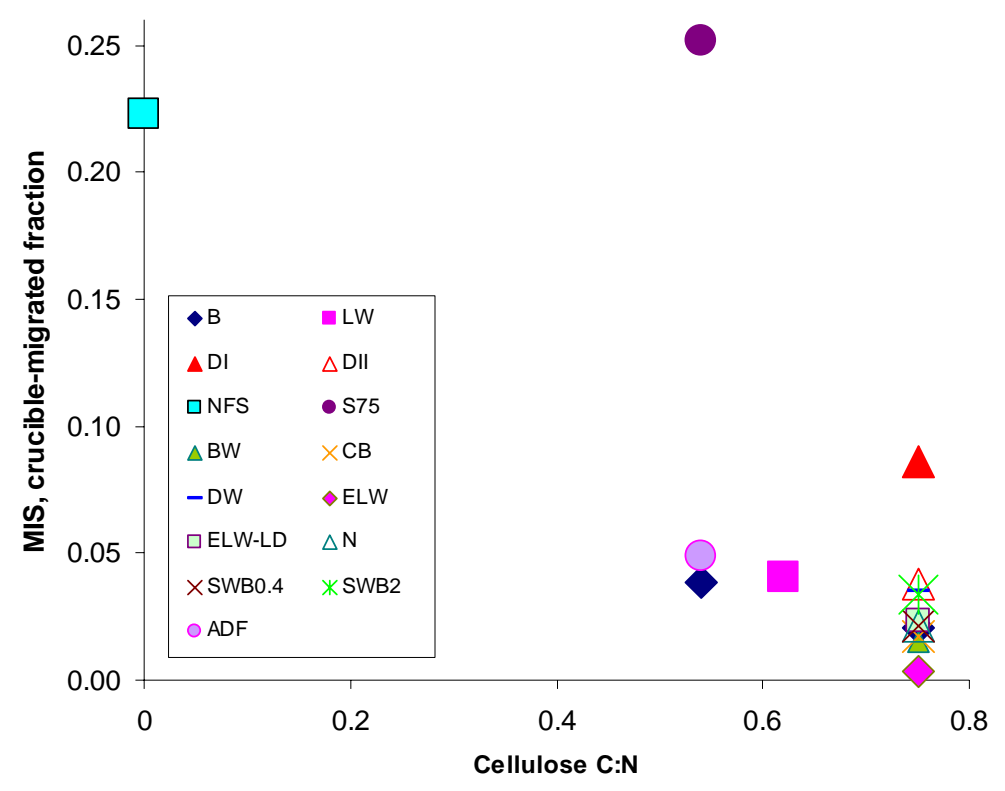

\section{Figure 9.5. Mass Fraction of Silica-Crucible-Migrated MIS from Feed with GFMs Versus Cellulose Addition (see Table 9.11 notes for legend acronyms)}

Figure 9.6 shows a high-cellulose-addition area of the Figure 9.5 diagram (the DI outlier is not included). Most of the data were obtained with cellulose addition corresponding to $\mathrm{C}: \mathrm{N}=0.75$. For this ratio, the MIS-migration-fraction (from feed to crucible) falls within the interval of 1.5 to 2.5 mass\%. Three data points are above 2.5 mass $\%$ and one below 1.5 mass $\%$. These differences are not readily attributable to experimental conditions.

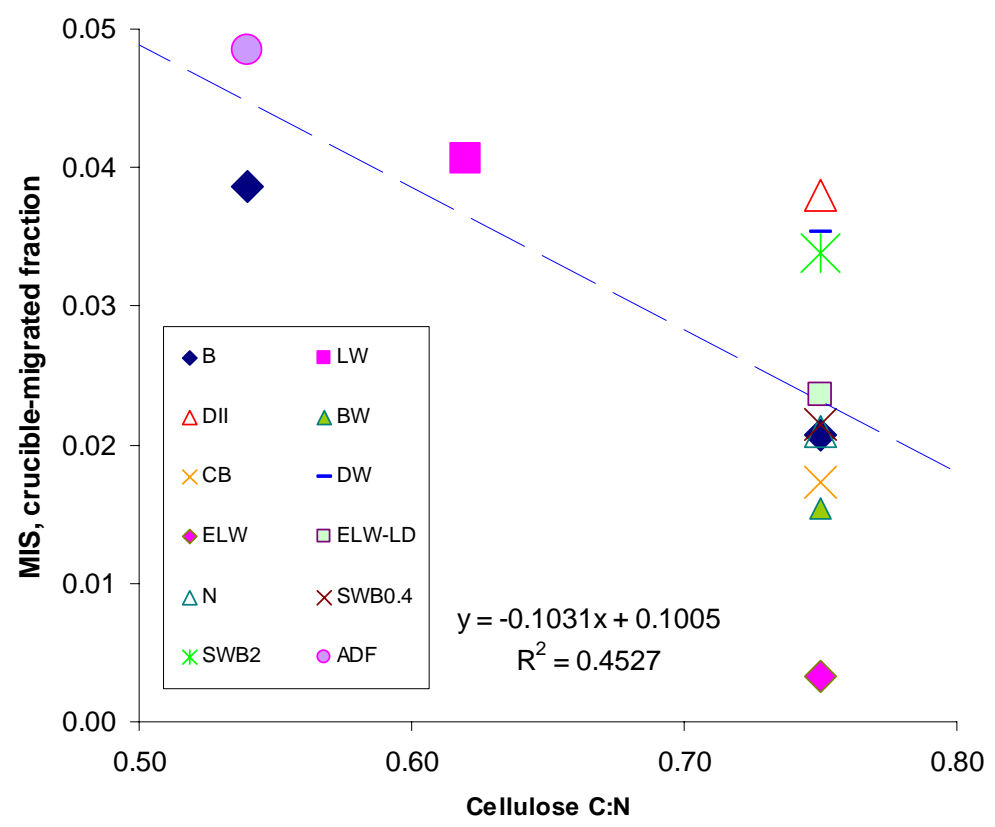

Figure 9.6. Mass Fraction of Silica-Crucible-Migrated MIS from Feed with GFMs Versus Cellulose Addition of $\mathrm{C}: \mathrm{N}>0.5$ (see Table 9.11 notes for legend acronyms) 
Figure 9.7 displays the order of feeds with zero carbohydrate addition. No attempt was made to understand the poor performance of several Praxair-ground samples with MIS migration comparable to, or exceeding, feeds with as-received soil.

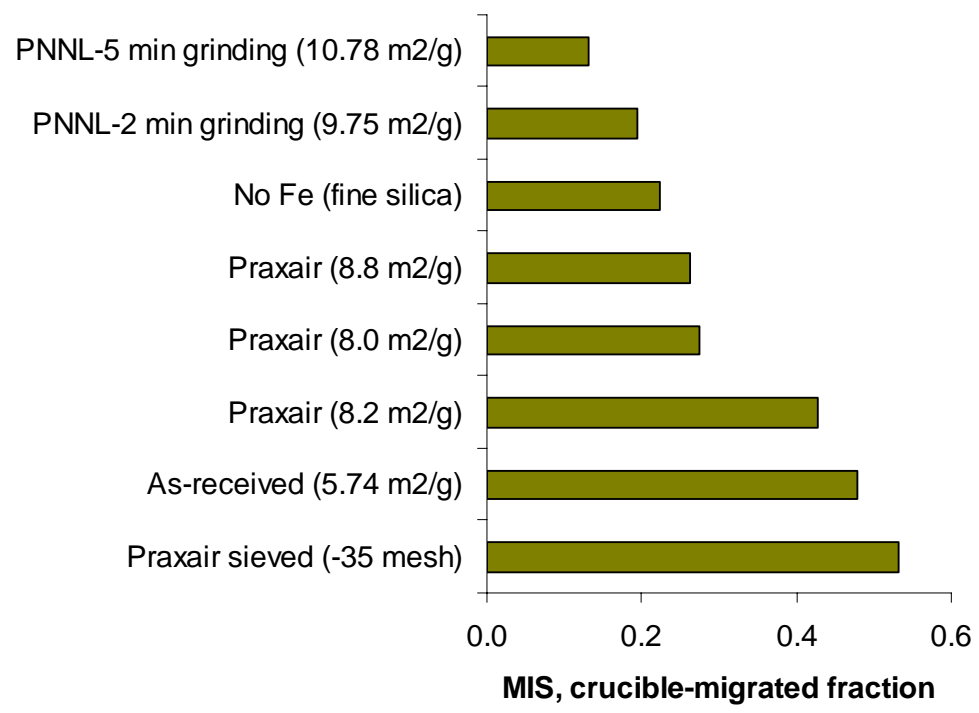

Figure 9.7. Mass Fraction of Silica-Crucible-Migrated MIS from Feed with Zero Carbohydrate Addition

With zero addition of carbohydrates, the fraction of MIS that migrated to the crucible from no-Fe feed with GFMs is comparable to soil ground for $2 \mathrm{~min}$. If we assume that the specific surface area of minerals has the same impact on MIS migration as the specific surface area of ground soil, then, by Figure 8.11, we would estimate that $a=9.2 \mathrm{~m}^{2} / \mathrm{g}$ for the mineral mix in no-Fe feed with fine silica (of unknown specific surface area). As Table 9.12 shows, the specific surface of the mineral portion in feeds with Min-USil-5 silica is likely less than half of this value $\left(3.8 \mathrm{~m}^{2} / \mathrm{g}\right.$ for Feed A, $4.2 \mathrm{~m}^{2} / \mathrm{g}$ for Feed B-see Table 9.6, and $4.1 \mathrm{~m}^{2} / \mathrm{g}$ for Feed C; the $a$ values are also listed in Table 9.11). No attempt was made to examine the cause of the difference in MIS migration in feed with soil and feeds with GFMs.

Table 9.12. Specific Surface Area of Minerals by BET and Particle Size Distribution (PSD) ${ }^{(a)}$

\begin{tabular}{|c|c|c|c|c|}
\hline & \multirow{2}{*}{$\begin{array}{c}\text { Feed B } \\
\text { Mass Fraction }\end{array}$} & \multicolumn{3}{|c|}{$\bar{a}, \mathrm{~m}^{2} / \mathrm{g}$} \\
\hline & & BET & PSD & Feed B \\
\hline Min-U-Sil 5 & 0.519 & 5.15 & 2.652 & 2.68 \\
\hline Air-floated rutile & 0.007 & 1.29 & 1.557 & 0.01 \\
\hline Zircon & 0.139 & 1.47 & 1.492 & 0.20 \\
\hline Olivine & 0.034 & 3.87 & 0.426 & 0.13 \\
\hline Kyanite & 0.180 & 2.26 & 1.462 & 0.41 \\
\hline Wollastonite & 0.070 & 1.70 & 1.090 & 0.12 \\
\hline Hematite & 0.050 & 12.27 & 2.454 & 0.61 \\
\hline Total Batch B area $^{(b)}$ & & & & 4.16 \\
\hline $\begin{array}{l}\text { (a) See Section 7.5. } \\
\text { (b) } a_{\text {total }}=\Sigma a_{\mathrm{i}} x_{\mathrm{i}} \text {, where } \\
\text { and } x_{\mathrm{i}} \text { is tha i-th m }\end{array}$ & ral mass fraction & & & BET), \\
\hline
\end{tabular}


Linear regression is applied to data listed in Table 9.9 and Table 9.10 for feeds with soil and in Table 9.11 for feeds with GFMs. The MIF migrated fraction (y) was expressed as a function of the specific surface area (a) and the $\mathrm{C}: \mathrm{N}$ ratio (c) as follows:

$$
y=y_{0}+y_{\mathrm{a}} a+y_{\mathrm{c}} c
$$

where the values of $y_{0}, y_{\mathrm{a}}$, and $y_{\mathrm{c}}$ coefficients are listed in Table 9.13 (for $a$ in $\mathrm{m}^{2} / \mathrm{g}$ ). In spite of a large number of removed outliers (3 out of 30 data for feeds with as-received or PNNL-ground soil and 9 out of 14 data with Praxair-ground soil), the $R^{2}$ was only 0.85 for feeds with soil. Figure 9.8 shows the plot of estimated versus measured data (hollow points represent outliers).

Table 9.13. MIS Migration Linear Regression Coefficients

\begin{tabular}{||l|c|c||}
\hline Coefficient & Soil & GMF \\
\hline$y_{0}$ & 0.6066 & 0.311 \\
\hline$y_{\mathrm{a}}$ & -0.0408 & -0.023 \\
\hline$y_{\mathrm{c}}$ & -0.2869 & -0.262 \\
\hline$R^{2}$ & 0.85 & 0.96 \\
\hline
\end{tabular}

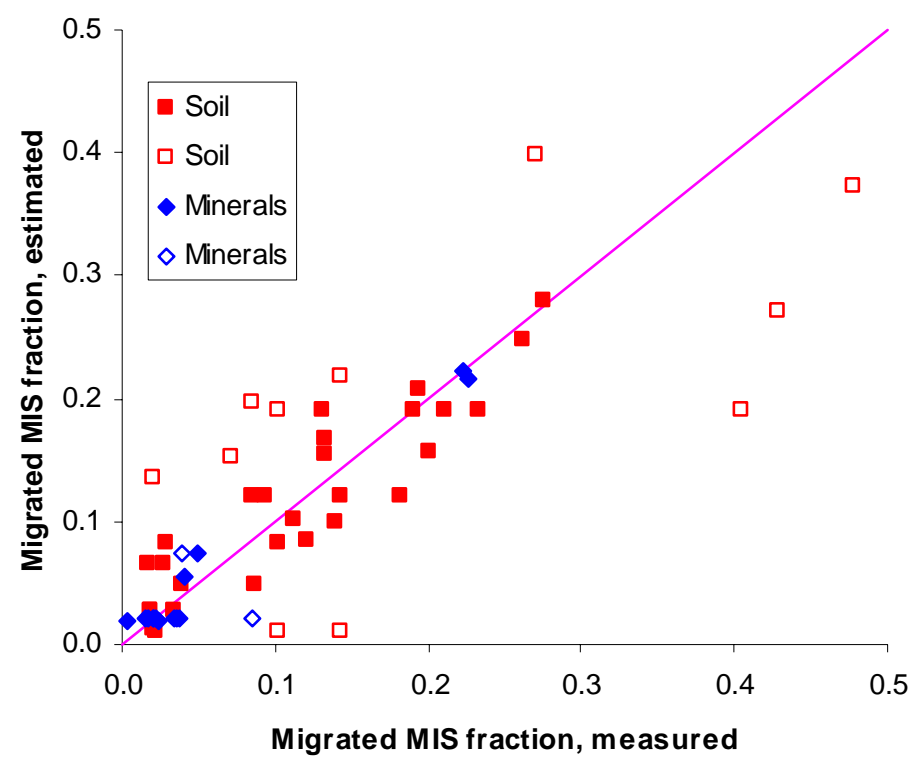

Figure 9.8. Migrated MIS Fraction, Estimated Versus Measured (full points: data used for calculation; outliers: hollow points)

Figure 9.9 shows the line representing $y=0$ on the $c-a$ plane as $c=-y_{0} / y_{c}-\left(y_{\mathrm{a}} / y_{\mathrm{c}}\right) a$. The line for the soilcontaining feeds is identical as the line shown in Figure 8.12 that was based on a smaller number of data. All but one of the measured samples (represented by the points) are inside the area where $y>0$, i.e., MIS migrated to the crucible. Note that data for GFM-containing feeds are contained in a smaller area. To stop MIS migration, feeds with no cellulose require a smaller specific surface area when minerals are used: $13.5 \mathrm{~m}^{2} / \mathrm{g}$ for minerals as compared with $14.9 \mathrm{~m}^{2} / \mathrm{g}$ for soil. Also, there is a major difference in the 
slope of the lines: -0.09 for minerals versus -0.14 for soil. According to Wilburn and Thomasson (1958), fine silica reacts with soda at temperatures below $300^{\circ} \mathrm{C}$. Since the main component in GFMs is fine silica, a low-temperature reaction can occur also between silica and sodium nitrate, whereas aluminosilicates in soil are less reactive. The observation that GFMs are more effective in reducing MIS migration than soil may be attributed to this early reaction. It should be noted that the database for feeds with minerals is limited, and more crucible data are needed.

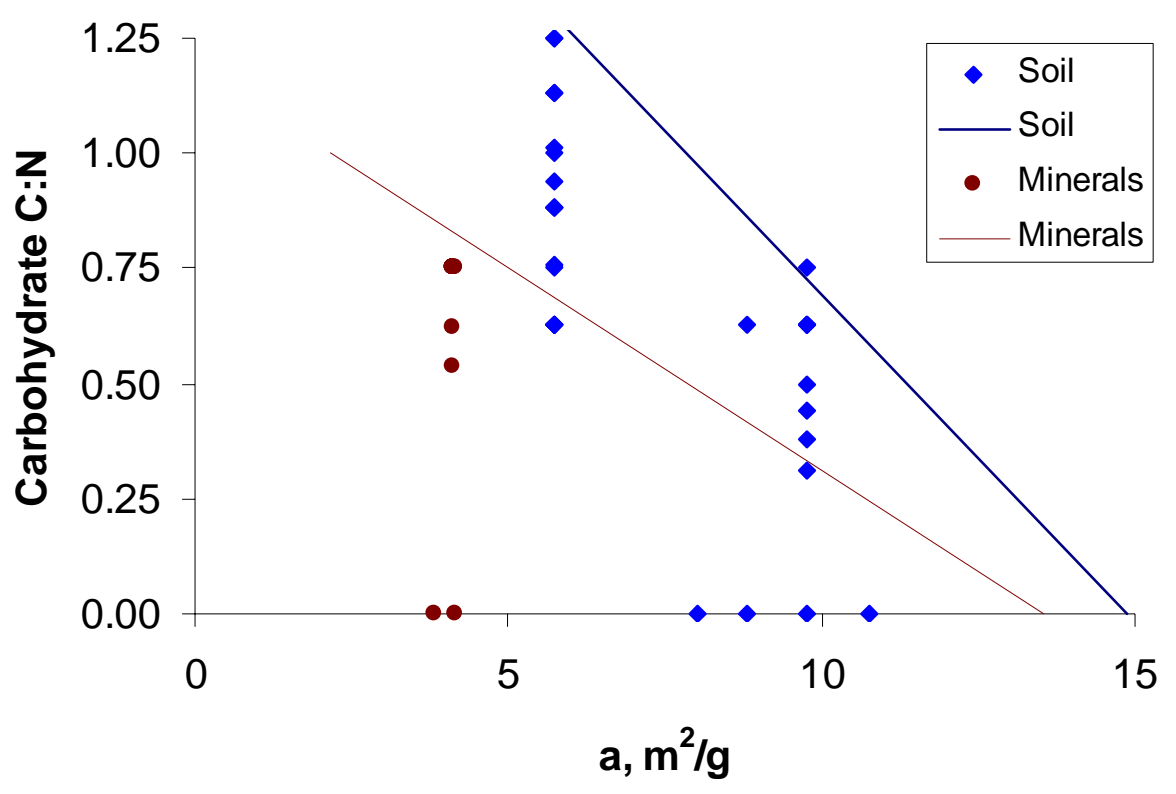

Figure 9.9. Preventable MIS Migration Diagram

The feed appearance was affected by both the feed makeup and the drying method. Figure 9.10 through Figure 9.19 show several examples. Figure 9.10 shows the appearance of dry feeds, illustrating that the feed granules varied in size and size distribution. When removed from the crucible, feeds with no carbohydrate additions had a relatively smooth texture. When sucrose or cellulose was added, heattreatment produced sponge-like feeds (Figure 9.11). Feeds with carbohydrates imprinted themselves on the internal crucible surface differently: after $500^{\circ} \mathrm{C}$ heat-treatment, feeds without carbohydrate additions resulted in a homogeneous coloration of the crucible internal surface, whereas carbohydrate-containing feeds left a spotted crucible surface (Figure 9.12). The spotting effect was possibly associated with nonuniform distribution of carbohydrates in granulated feeds. 


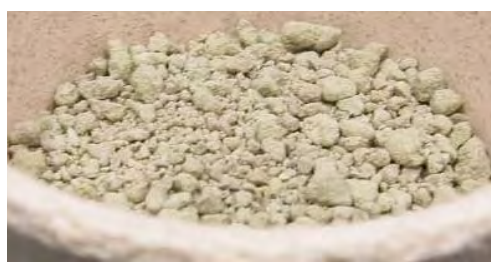

No-Fe

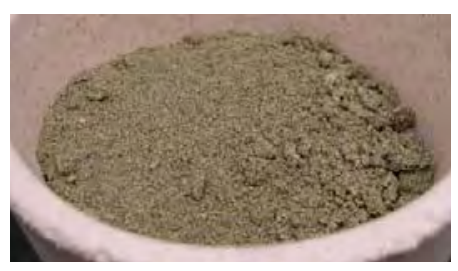

PSC sieved (-35 mesh)

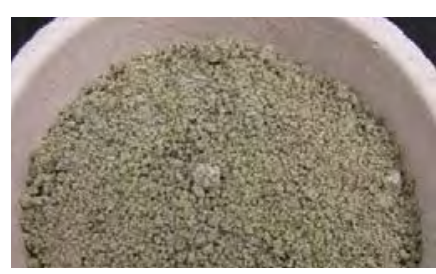

AR $(C: N=0.63)$

Figure 9.10. Appearance of Dry Feeds

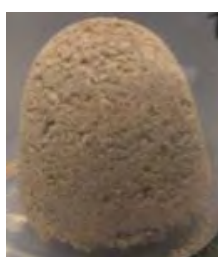

PSC

$\mathrm{C}: \mathrm{N}=0$

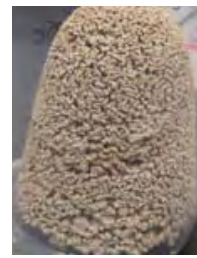

PSC

0.63

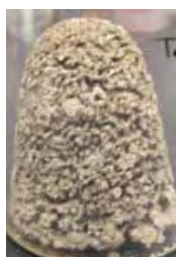

AR

0.76

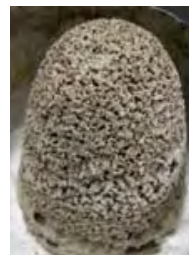

AR

1.00

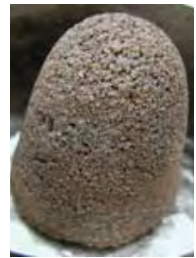

GFM

0.54

Figure 9.11. Appearance of Feeds as Removed from Crucibles (PSC Praxair Seattle Company, AR as received, GFMs)

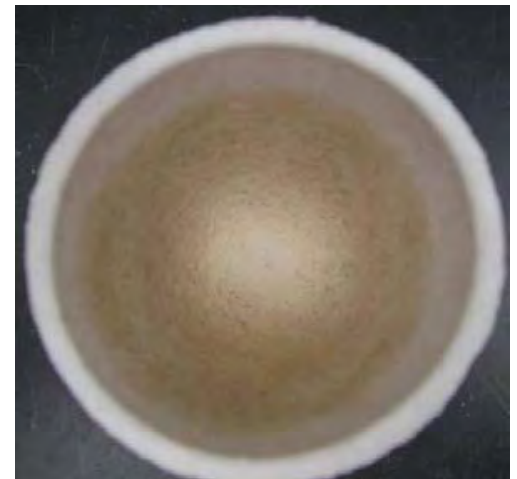

PSC

$\mathrm{C}: \mathrm{N}=0$

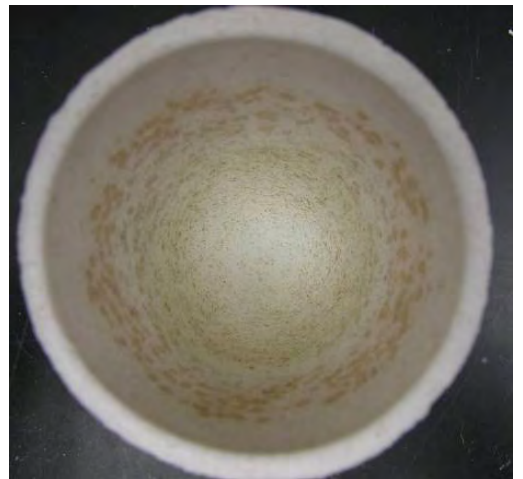

PSC

0.63

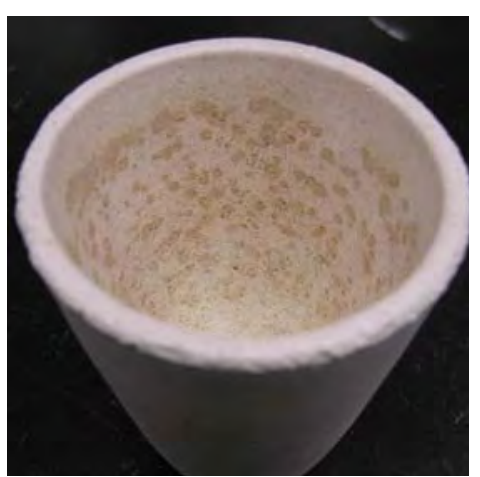

AR

0.63

Figure 9.12. Appearance of Crucibles After Feeds Were Removed

Figure 9.13 illustrates the impact of the preparation method and the solid (glass-forming) portion of feed on the feed appearance after $500^{\circ} \mathrm{C}$ heat-treatment. Figure 9.14 sows the impact of cellulose and sucrose addition. 


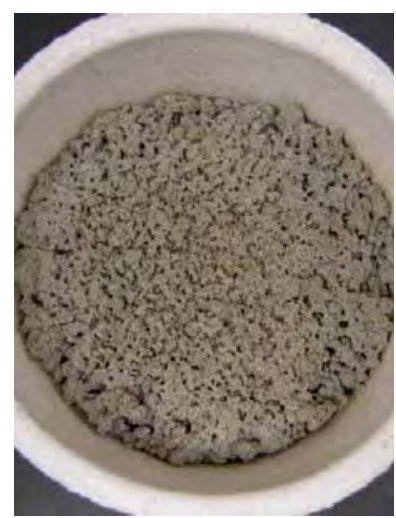

PSC

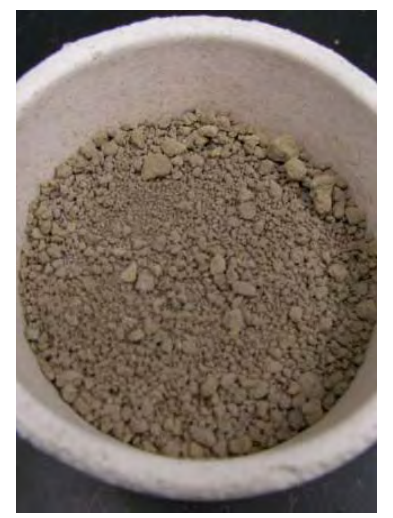

2-min ground

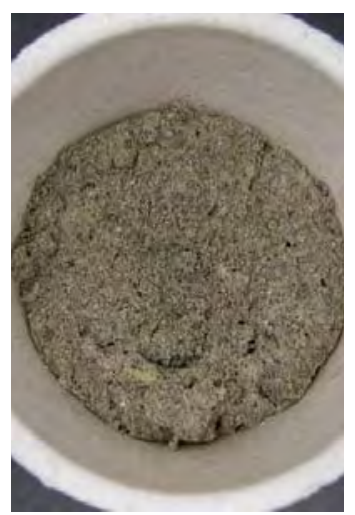

AR, dryer dried

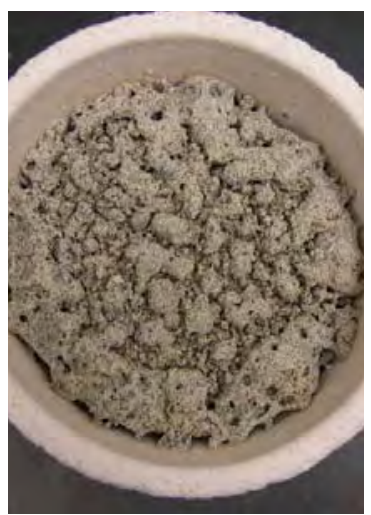

AR

Figure 9.13. Appearance of Various Feeds Processed at $500^{\circ} \mathrm{C}(\mathrm{C}: \mathrm{N}=0.63)$

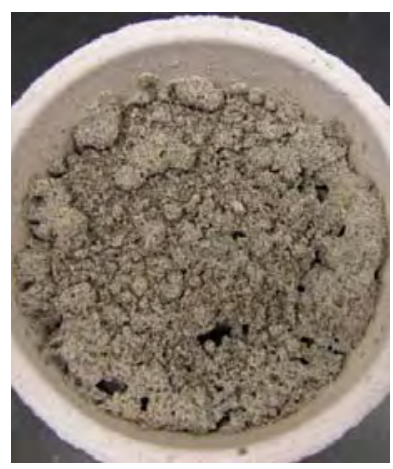

$\mathrm{C}: \mathrm{N}=0.75$

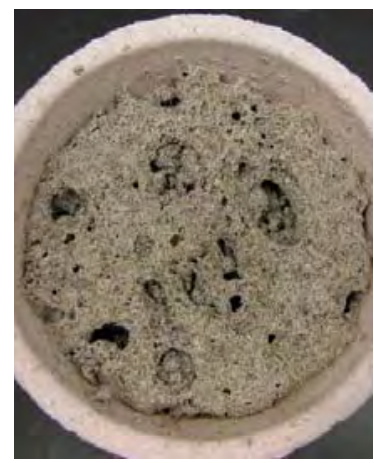

$\mathrm{C}: \mathrm{N}=0.88$

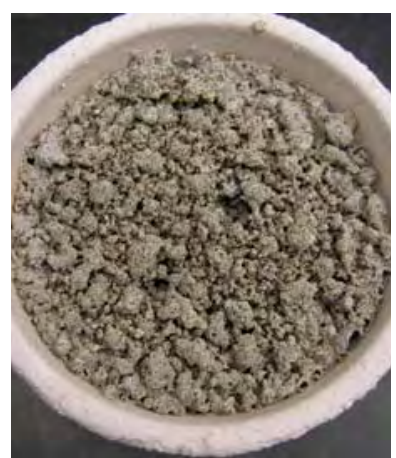

0.88

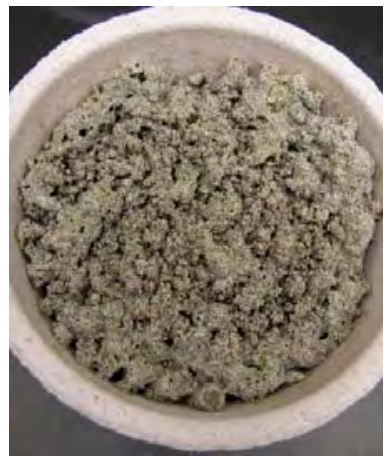

1.01

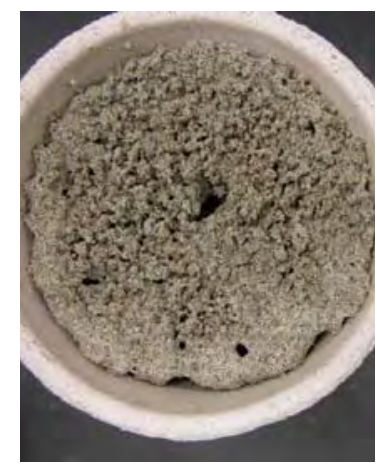

1.00

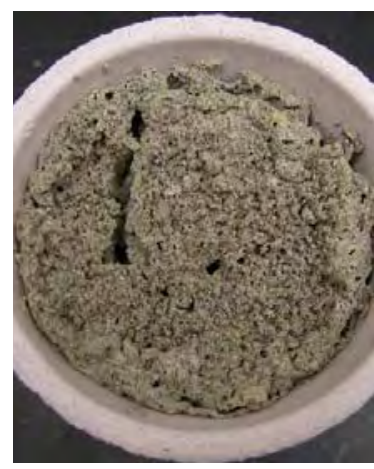

1.13

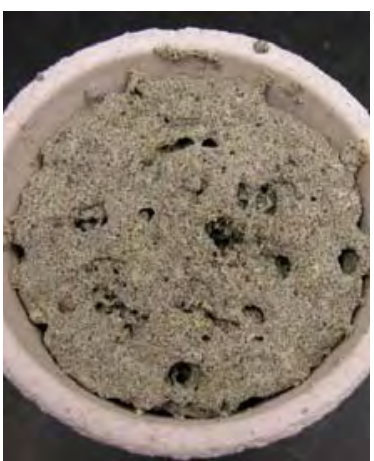

1.13

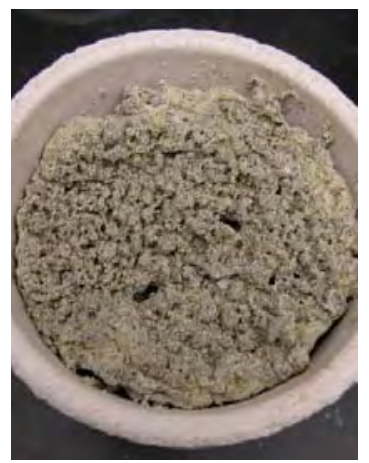

1.26

Figure 9.14. Feeds from As-Received Soil with Various Additions of Carbohydrates Processed at $500^{\circ} \mathrm{C}$ (top: all carbon from cellulose; bottom: 0.13 carbon from sucrose, the rest from cellulose)

Interface patterns became more pronounced after $1000^{\circ} \mathrm{C}$ heat-treatment. Figure 9.15 and Figure 9.16 display top views of crucible appearances after $1000^{\circ} \mathrm{C}$ heat-treatment (feeds were removed after $500^{\circ} \mathrm{C}$ heat-treatment). The sponge-like texture of feeds with carbohydrates heated to $500^{\circ} \mathrm{C}$, seen in Figure 9.11 and Figure 9.14, is caused by vigorous evolution of reaction gases and is most likely responsible for the spotted pattern of the internal crucible surfaces that were in contact with feed (see Figure 9.12, Figure 9.15, Figure 9.16, Figure 9.18, and Figure 9.19). When heated to $1000^{\circ} \mathrm{C}$, the MIS in 
crucible pores reacted with the bonding cement, forming glassy melt that in some cases were forced out of the crucible wall by expanding gases.

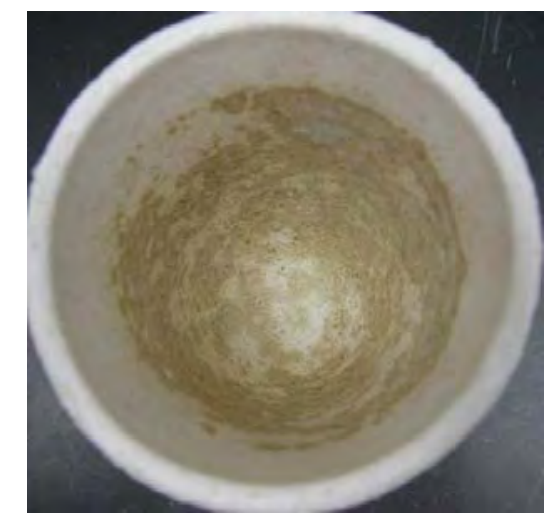

2-min ground, $\mathrm{C}: \mathrm{N}=0.63$

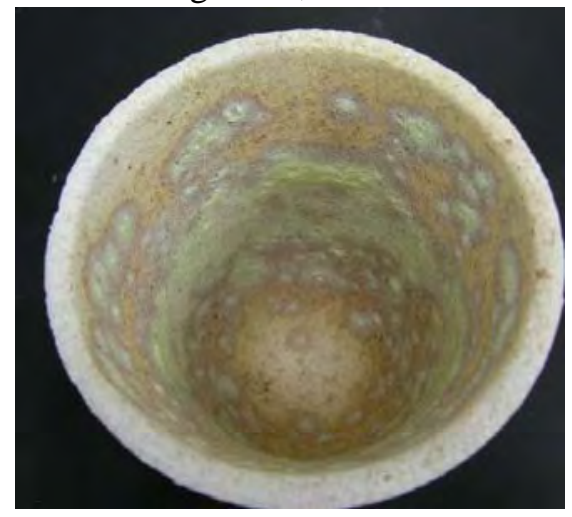

AR, $\mathrm{C}: \mathrm{N}=1.00$

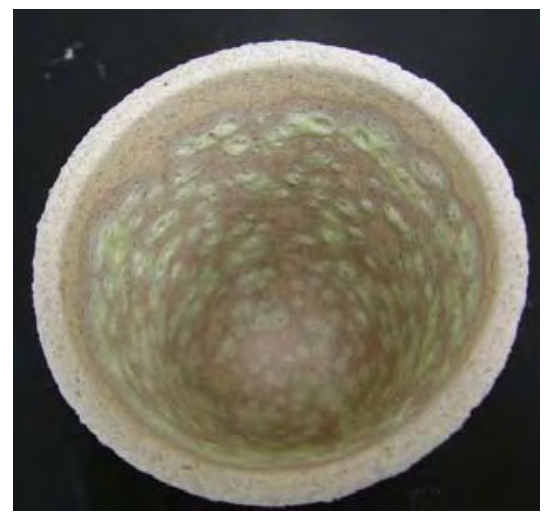

AR, $\mathrm{C}: \mathrm{N}=0.63$

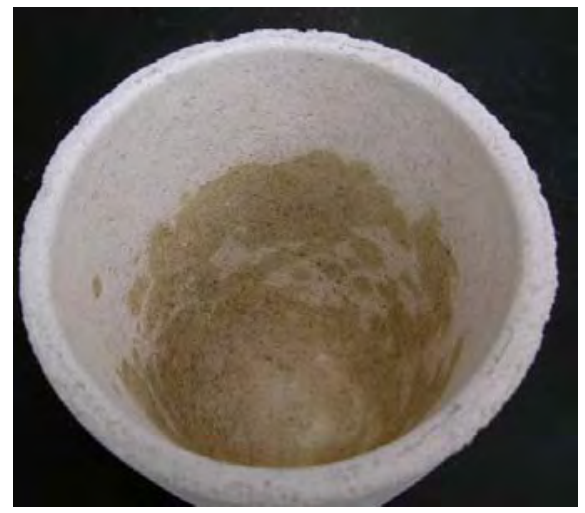

GFM, C:N = 0.62

Figure 9.15. Appearance of Crucibles Heat-Treated at $1000^{\circ} \mathrm{C}$

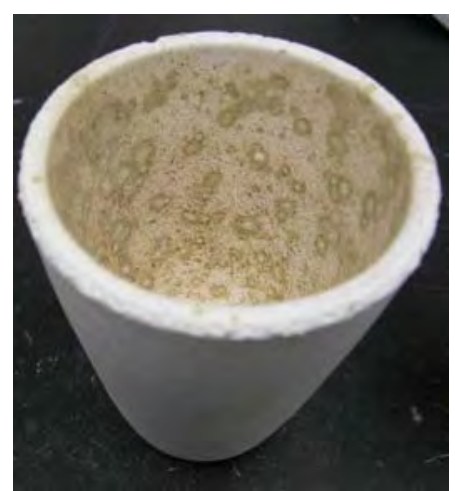

$\mathrm{AR}, \mathrm{C}: \mathrm{N}=1.25$

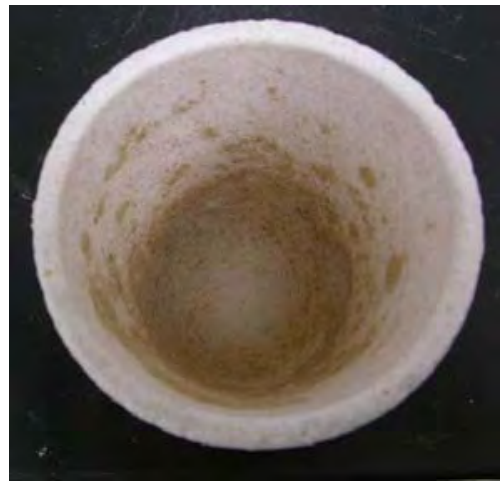

GFM, C:N = 0.54

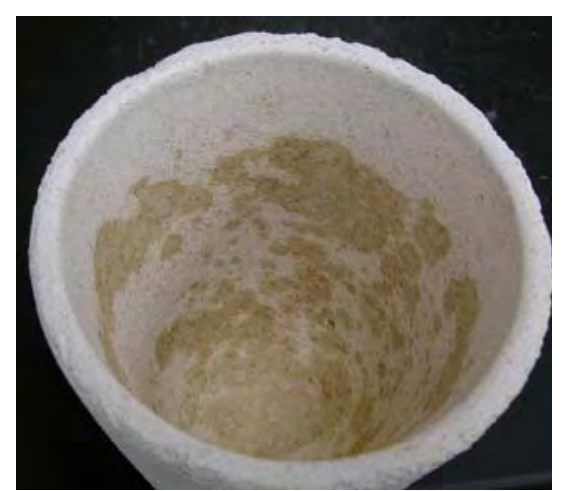

GFM, C:N = 0.75

Figure 9.16. Appearance of Crucibles Heat-Treated at $1000^{\circ} \mathrm{C}$ (carbohydrate was added to the feeds removed at $500^{\circ} \mathrm{C}$ ) 
Deep penetration of MIS, often to the external surface of the crucible, frequently occurred in feeds prepared without carbohydrate addition (Figure 9.17). Penetration decreased, but did not disappear when carbohydrates were added to as-received soils (Figure 9.18). With large silica particles $(\leq 75 \mu \mathrm{m})$ or insufficient carbohydrate addition (C:N = 0.54), GFMs did not stop MIS migration (Figure 9.19). Using small silica particles $(<5 \mu \mathrm{m})$ and a carbohydrate addition of C: $\mathrm{N}=0.75$ performed well and prevented most MIS penetration.

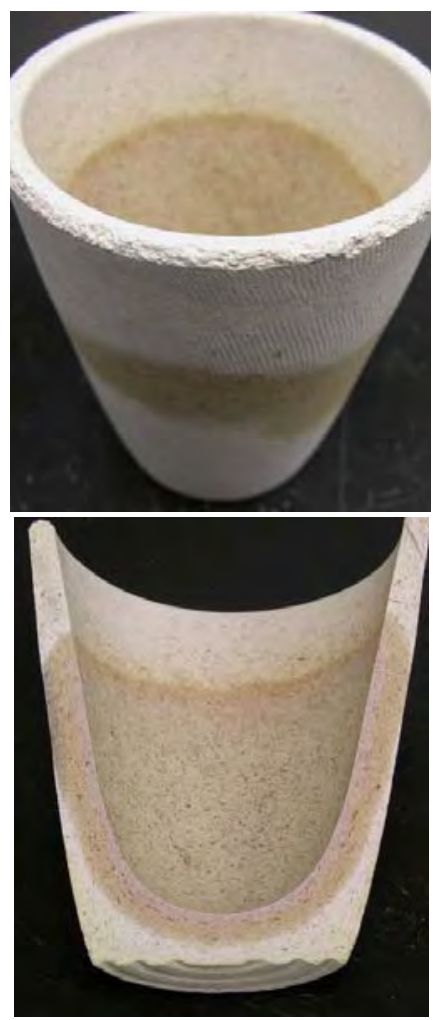

No-Fe GFM
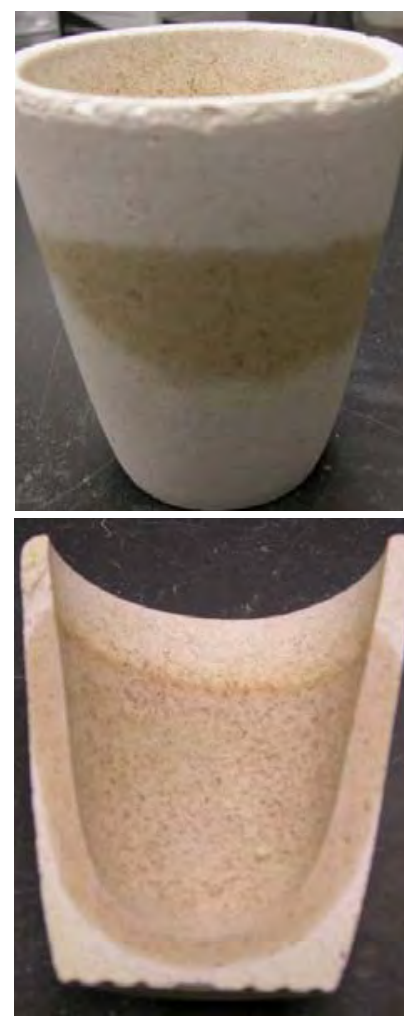

PSC $3 \times$ plate ground

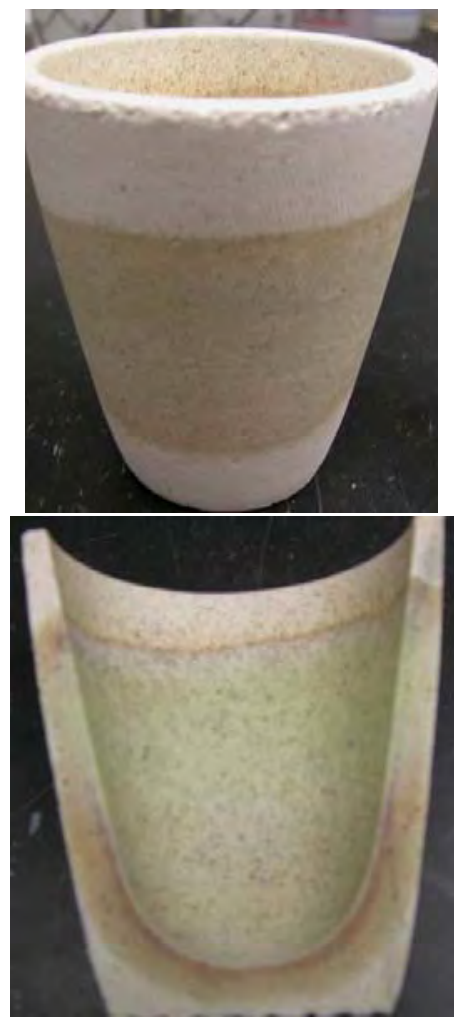

PSC $8.2 \mathrm{~m}^{2} / \mathrm{g}$

Figure 9.17. Appearance of Crucibles Heat-Treated at $1000^{\circ} \mathrm{C}$ (feeds were removed at $500^{\circ} \mathrm{C}$; no carbohydrate was added) 


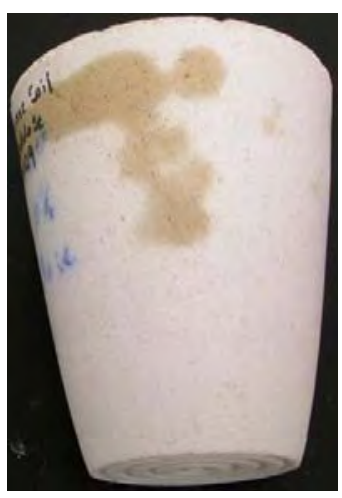

$\mathrm{C}: \mathrm{N}=0.63$

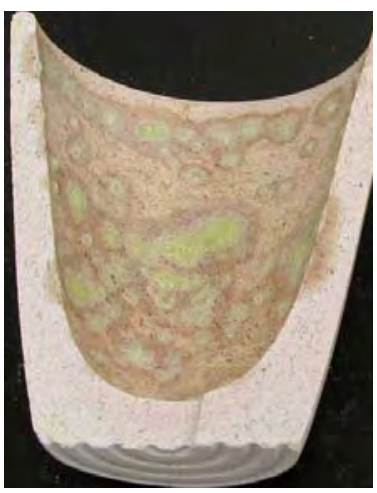

0.88

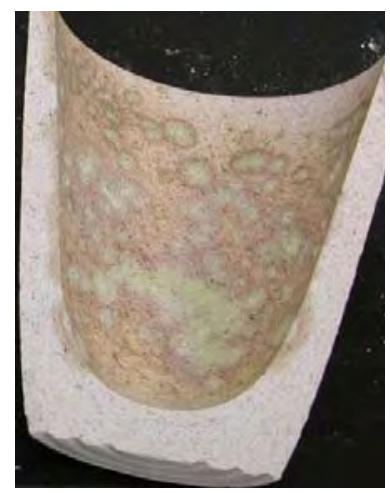

1.13

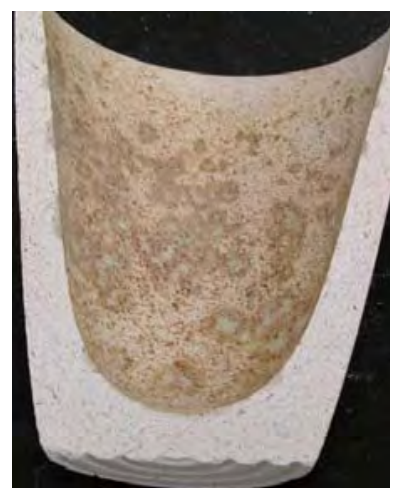

1.26

Figure 9.18. Appearance of Crucibles Heat-Treated at $1000^{\circ} \mathrm{C}$ (feeds with as-received soil were removed at $500^{\circ} \mathrm{C}$ )

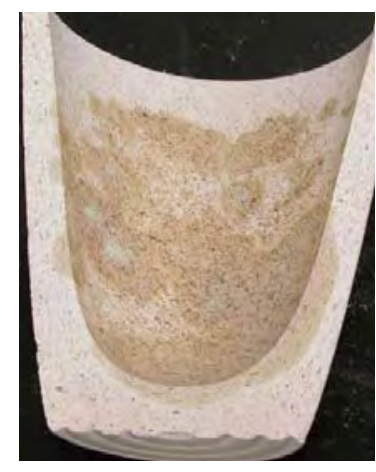

$\mathrm{C}: \mathrm{N}=0.54(\leq 5 \mu \mathrm{m}$ silica $)$

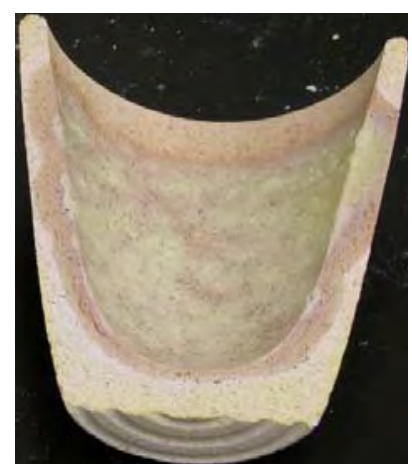

0.54 ( $\leq 75 \mu \mathrm{m}$ silica $)$

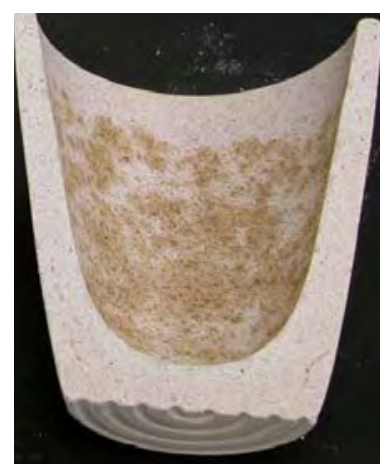

$0.75(\leq 5 \mu \mathrm{m}$ silica $)$

Figure 9.19. Appearance of Crucibles Heat-Treated at $1000^{\circ} \mathrm{C}$ (carbohydrate was added to GFM-containing feeds removed at $500^{\circ} \mathrm{C}$ )

The temperature evolution in feeds was measured with two thermocouples (TCs): one placed at the crucible axis about half way between the bottom of the crucible and the surface of the feed (M), and a second one on the feed top surface (T) - see Figure 9.20. These temperatures were recorded as functions of the furnace temperature read by the furnace-controlling TC and are listed in Appendix M. The furnace temperature ramp rate was set at $5^{\circ} \mathrm{C} / \mathrm{min}$. However, when the cellulose addition was high (C:N $\left.>1\right)$, the exothermic chemical reaction within the feed was sufficient to increase the furnace temperature at a rate faster than the programmed ramp rate. 


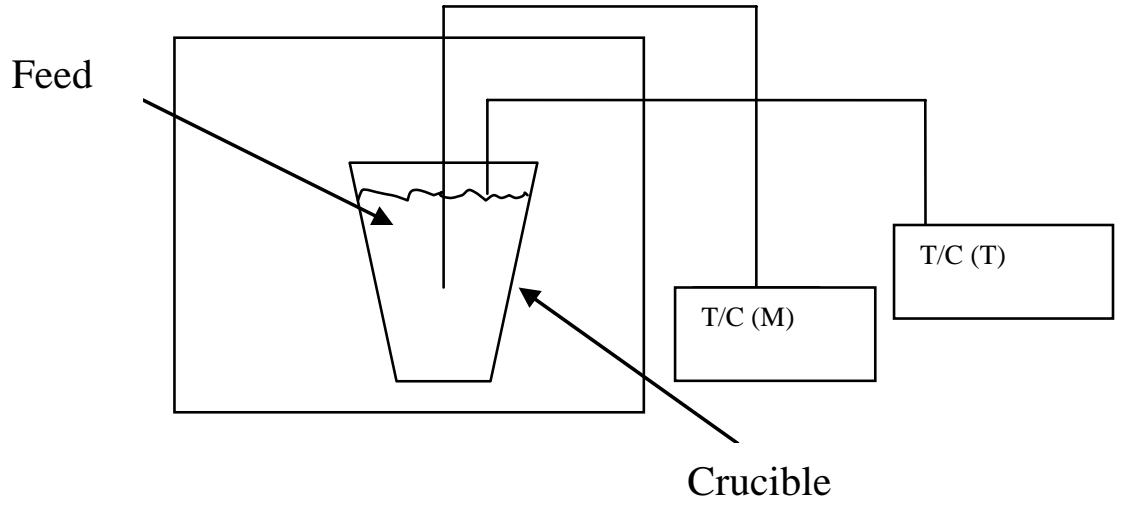

Figure 9.20. Thermocouple Placement in Silica Crucible

Figure 9.21 shows in graphical form the results listed in Appendix M. The temperature gradually increased to the point at which the reaction front reached the TC. A black smoke began to come out from the furnace around that time. The temperature jumped rapidly to a maximum and then gradually decreased to a minimum; then it increased gradually again, approaching the furnace temperature. Black smoke stopped evolving shortly after the temperature spiked. Occasionally, two temperature maxima occurred, separated by a shallow minimum. This could be caused by a sudden motion of feed, bringing colder material to the TC position. Note that two GFM feeds with different kinds of bran responded differently to heating (Figure 9.21f).

Figure 9.21d-f compare the temperature development in the feed at the surface (T) and in the center (M). Generally, the feed at the surface was at a higher temperature than the center when the rapid temperature rise started. The reaction starting temperatures $\left(T_{0}\right)$, the maximum temperature seen from the reaction ( $T_{\max }$ ), the minimum temperature $\left(T_{\min }\right)$ after $T_{\max }$ was reached, the extent of temperature increase $\left(\Delta T_{\max }=T_{\max }-T_{0}\right)$, and subsequent decrease $\left(\Delta T_{\min }=T_{\max }-T_{\min }\right)$ are summarized in Table 9.14 and Table 9.15 (note that three additional measurements were performed with only one TC placed in the feed center).

As Figure 9.22 shows, the reaction started in the feed center earlier when the carbohydrate addition was higher. A curve fit of the data indicates that a $1 \%$ increase in C: $\mathrm{N}$ resulted in a $1.5^{\circ} \mathrm{C}$ decrease in $T_{0}$. All other temperatures and temperature differences $\left(T_{\max }, T_{\min }, \Delta T_{\max }\right.$, and $\left.\Delta T_{\min }\right)$ varied around an average value without showing a clear dependence on the $\mathrm{C}: \mathrm{N}$ value.

The TC data indicate that the reaction was initiated at a spot where the local temperature was the highest. The reaction front then spread through the colder areas of feed until it reached the TC. The highest starting temperature measured by a TC was $T_{0}=276^{\circ} \mathrm{C}$, and its average value was $T_{0}=246 \pm 23^{\circ} \mathrm{C}$. It is likely that the recorded $T_{0}$ value was lower than the reaction initiation temperature (because the TC at the top surface was not right at the point where the reaction initiation occurred and the furnace temperature was generally higher than the TC measured temperature). 


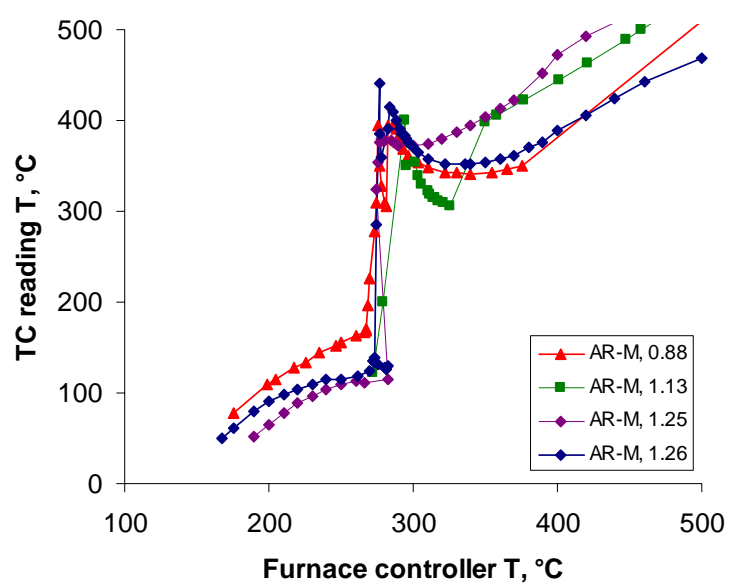

a

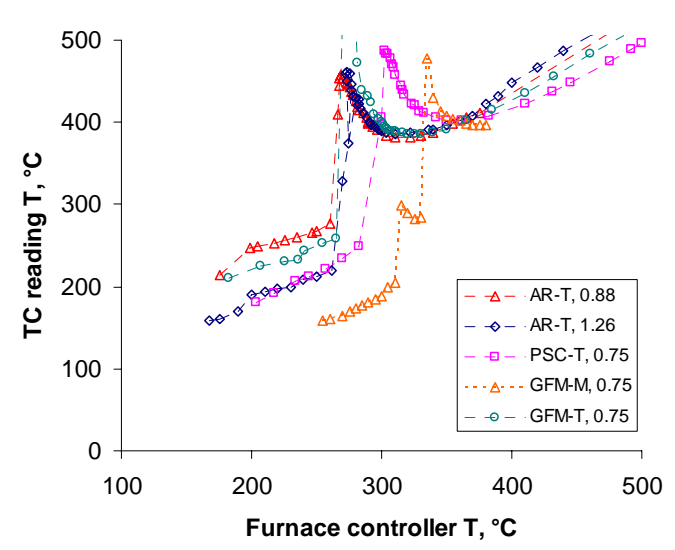

b

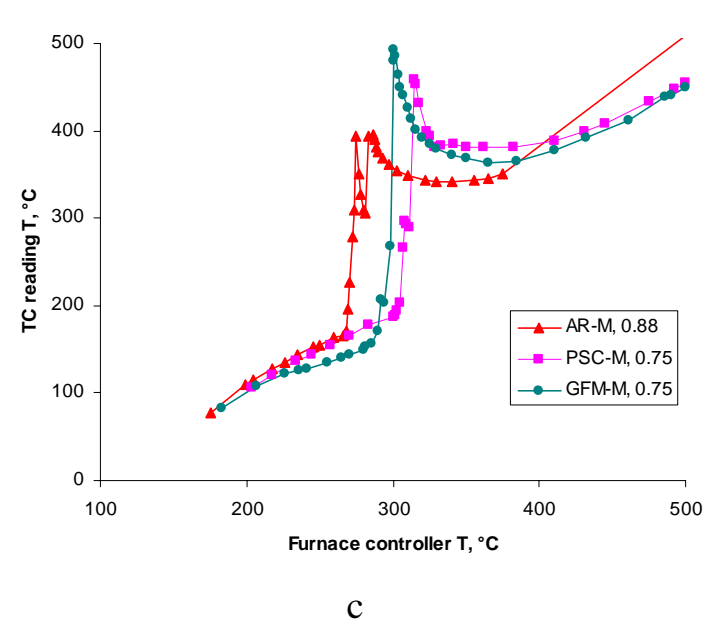

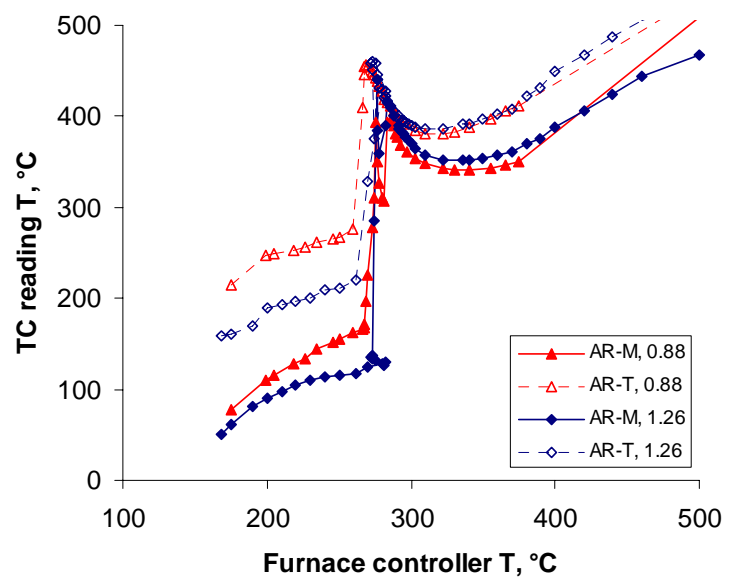

d

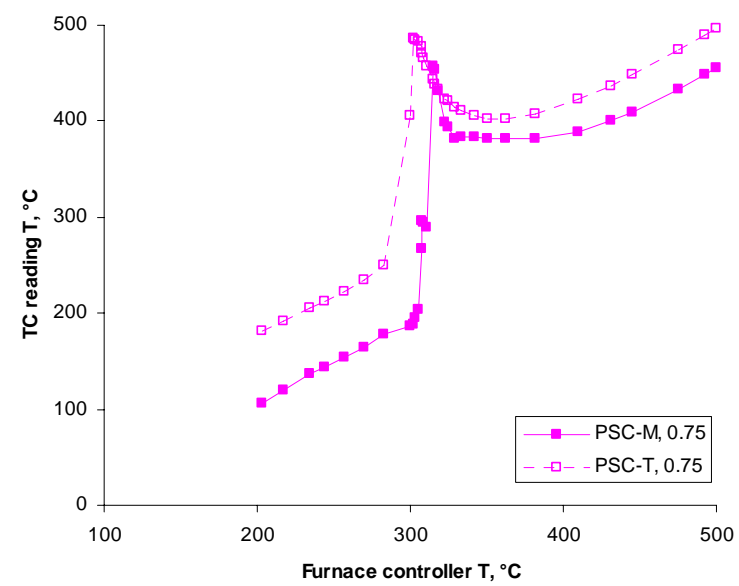

e

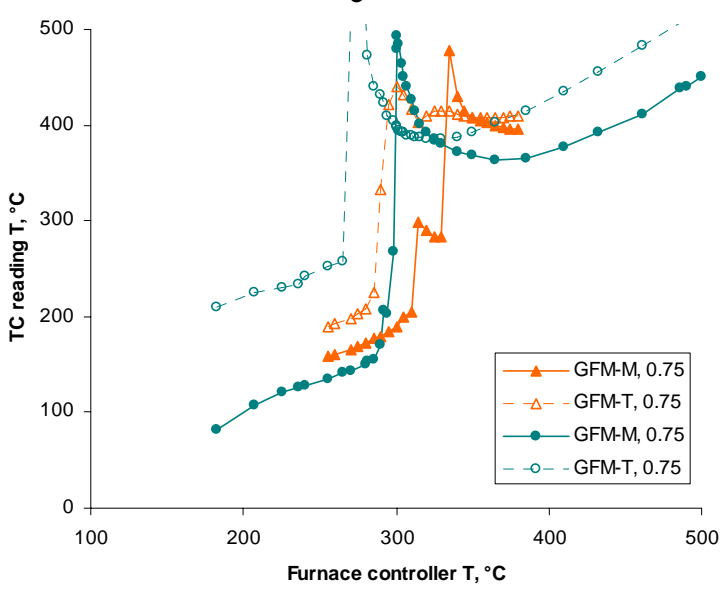

$\mathrm{f}$

Figure 9.21. Feed Temperature Response to Furnace Temperature Increasing at $5^{\circ} \mathrm{C} / \mathrm{min}$ (see Appendix M; AR as received soil, PSC soil ground at Praxair, GFMs, M feed body center, $\mathrm{T}$ feed top surface; numbers after the feed acronym represent the $\mathrm{C}: \mathrm{N}$ ratio) 
If the assumption that the reaction initiates at the feed surface is correct, the value of $T_{0}$ as measured in the crucible center indicates how fast the reaction front progressed through the feed. Lower $T_{0}$ values suggest that the reaction front moved faster. Based on Figure 9.22, we may reason that the reaction front velocity increased as the $\mathrm{C}: \mathrm{N}$ ratio increased. However, this reasoning calls for independent verification, the importance of which should not be underestimated. For example, a propensity of feeds to reaction propagation at a low temperature from a point at which the reaction was initiated should be thoroughly checked to assess a possibility of such an occurrence in feed outside the melting box.

The TC data also point out another side benefit of adding carbohydrates to the melter feeds. The presence of carbohydrates in feed accelerates conversion reactions and allows the reactions to proceed more uniformly within the feed volume. Without the presence of carbohydrates, the temperature distribution within the feed is controlled by conductive heat transfer, which is rather slow because the thermal conductivity of feed is low. Heat transfer is assisted by reaction gases percolating through the feed, but there is little generation of reaction gases at lower temperatures. Exothermic reactions between carbohydrates and nitrates provide an internal heat source that brings the feed pile from temperatures that range from 150 to $200^{\circ} \mathrm{C}$ to about 400 to $500^{\circ} \mathrm{C}$. Once ignited, the reaction spreads through the entire volume of feed (similar to igniting a pile of wood).

Table 9.14. Temperature Development (in ${ }^{\circ} \mathrm{C}$ ) in the Center Point of Feeds

\begin{tabular}{||l|r|r|r|r|r|r||}
\hline Feed & C:N & $\boldsymbol{T}_{\mathbf{0}}$ & $\boldsymbol{T}_{\max }$ & $\boldsymbol{T}_{\min }$ & $\boldsymbol{\Delta} \boldsymbol{T}_{\max }$ & $\boldsymbol{\Delta} \boldsymbol{T}_{\min }$ \\
\hline AR & 1.00 & 122 & 400 & 306 & 278 & 94 \\
\hline AR & 1.25 & 115 & 379 & 372 & 265 & 8 \\
\hline AR & 1.13 & 138 & 440 & 351 & 302 & 89 \\
\hline AR & 0.88 & 171 & 394 & 341 & 223 & 53 \\
\hline GFM & 0.75 & 204 & 477 & 396 & 273 & 81 \\
\hline GFM & 0.75 & 156 & 493 & 364 & 337 & 129 \\
\hline PSC & 0.75 & 203 & 458 & 381 & 255 & 77 \\
\hline Average & & 158 & 411 & 346 & 276 & 76 \\
\hline STD & & 36 & 66 & 37 & 36 & 38 \\
\hline
\end{tabular}

Table 9.15. Temperature Development (in ${ }^{\circ} \mathrm{C}$ ) on a Surface Point of Feeds

\begin{tabular}{|l|l|l|r|r|r|r||}
\hline \hline Feed & C:N & $\boldsymbol{T}_{\mathbf{0}}$ & $\boldsymbol{T}_{\max }$ & $\boldsymbol{T}_{\min }$ & $\Delta \boldsymbol{T}_{\max }$ & $\Delta \boldsymbol{T}_{\min }$ \\
\hline AR & 1.13 & 220 & 429 & 386 & 209 & 43 \\
\hline AR & 0.88 & 276 & 457 & 381 & 181 & 76 \\
\hline GFM & 0.75 & 225 & 440 & 408 & 215 & 32 \\
\hline GFM & 0.75 & 258 & 523 & 386 & 265 & 137 \\
\hline PSC & 0.75 & 250 & 484 & 402 & 234 & 82 \\
\hline Average & & 246 & 467 & 393 & 221 & 74 \\
\hline STD & & 23 & 38 & 12 & 31 & 41 \\
\hline \hline
\end{tabular}




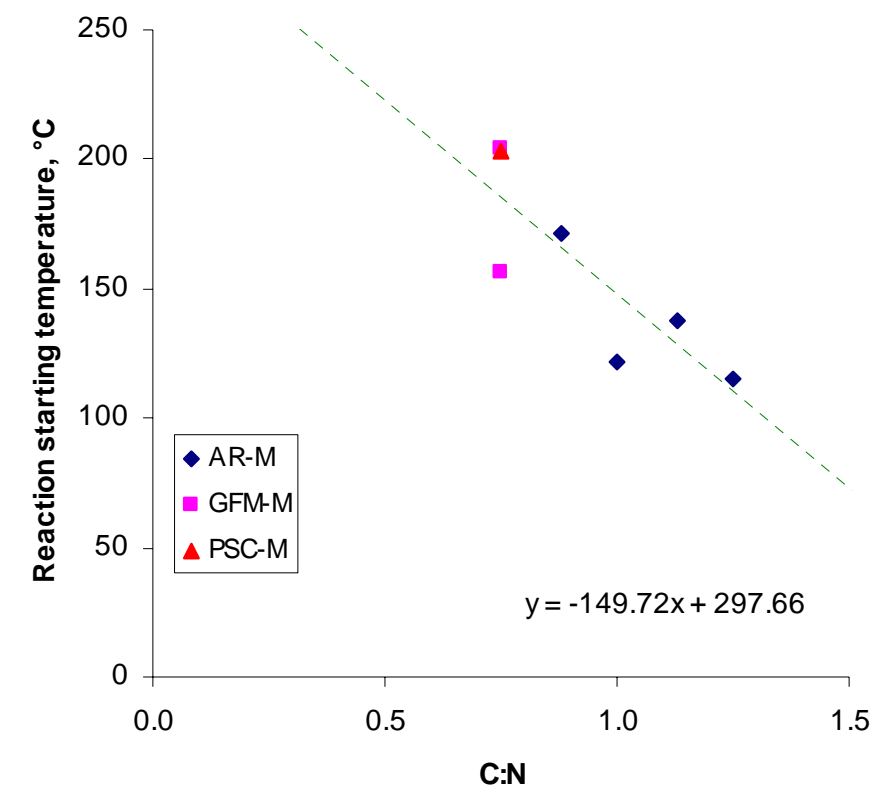

Figure 9.22. Reaction Starting Temperature in Feed Center as a Function of Carbohydrate Addition

\subsection{MIS Migration: Experiments with Large Vibrocast Crucibles}

Vibrocast crucibles containing 7.3 to $9.1 \mathrm{~kg}$ of feed were placed in the furnace and heated at $5^{\circ} \mathrm{C} / \mathrm{min}$ from room temperature to $500^{\circ} \mathrm{C}$ and held for 20 hours. One crucible was cut and rebonded with the refractory cement used for joints in engineering and FS melters (Figure 9.23). Crucibles were removed from the furnace 2 days after the furnace was turned off. Feed was removed from crucibles (see Figure 9.24), and empty crucibles were placed in the furnace at room temperature and heated at $5^{\circ} \mathrm{C} / \mathrm{min}$ to $1000^{\circ} \mathrm{C}$ and held for 2 hours. The crucibles were removed from the furnace after cooling to room temperature.

Feeds with as-received soil leaked MIS through the crucible bottom. The crucible was stuck to the course alumina used to protect the heart plate and had to be dislodged. It was necessary to clean off some $\mathrm{Al}_{2} \mathrm{O}_{3}$ grains from the crucible bottom. The crucible with a joint turned to a light brown after heating to $500^{\circ} \mathrm{C}$. A nitrate odor was perceived when removing either of the crucibles that used as-received soils. An estimated $100 \mathrm{~g}$ of $\mathrm{Al}_{2} \mathrm{O}_{3}$ grains was stuck to the bottom. The joint remained intact (Figure 9.23). Figure 9.25 shows the appearance of feeds after the $500^{\circ} \mathrm{C}$ heat-treatment, and Figure 9.26 shows the appearance of crucibles after the $1000^{\circ} \mathrm{C}$ heat-treatment. Raw data are summarized in Table 9.16. 


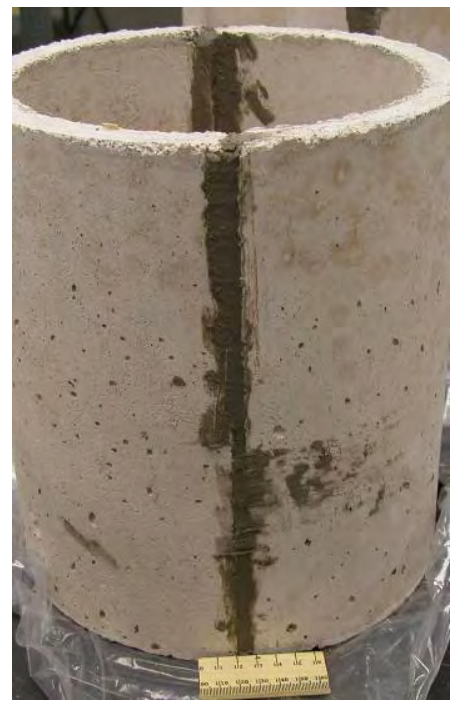

RT

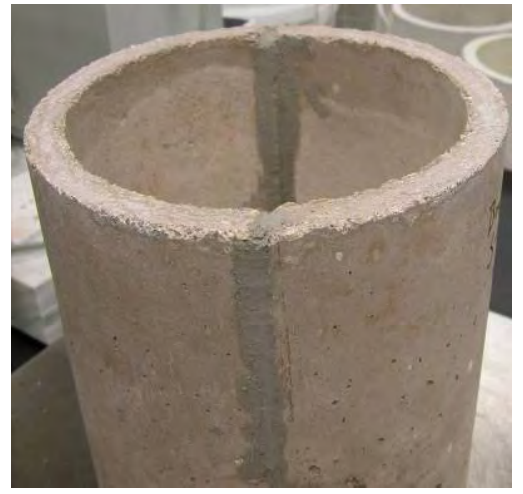

$500^{\circ} \mathrm{C}$

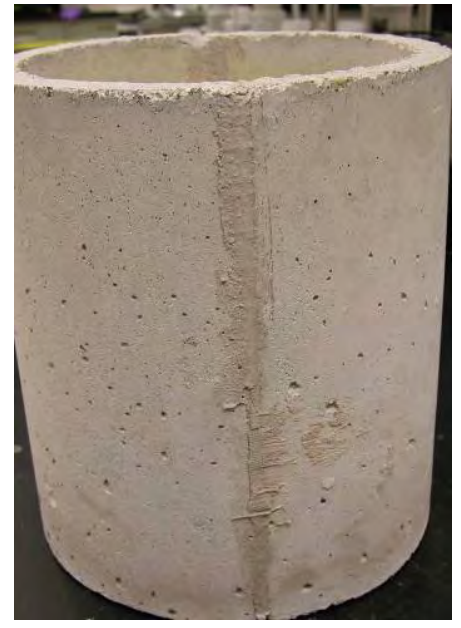

$1000^{\circ} \mathrm{C}$

Figure 9.23. Crucible with a Joint Before and After the Heat-Treatments

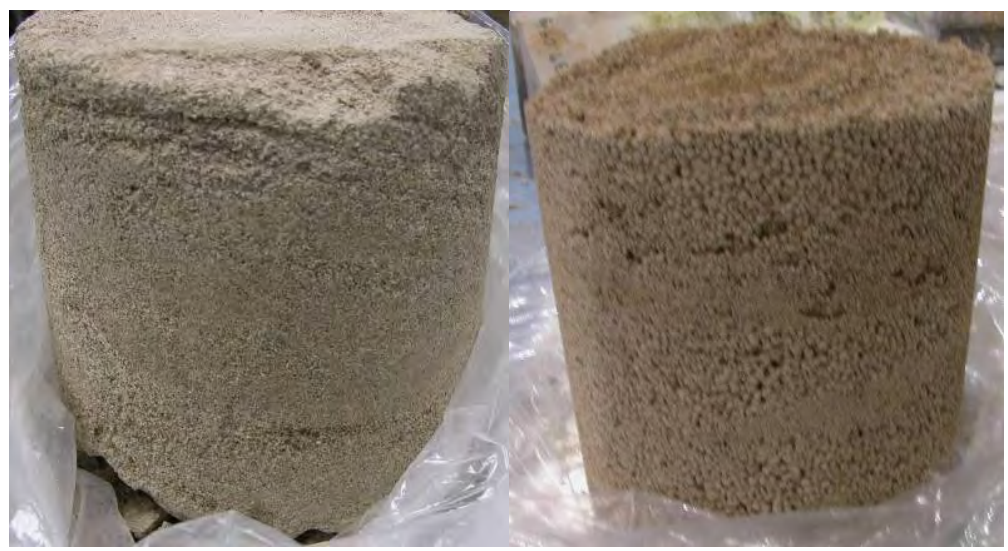

Figure 9.24. Feeds Removed from Crucible after 20 Hours at $500^{\circ} \mathrm{C}$ (38-C feed left and Praxair feed right)
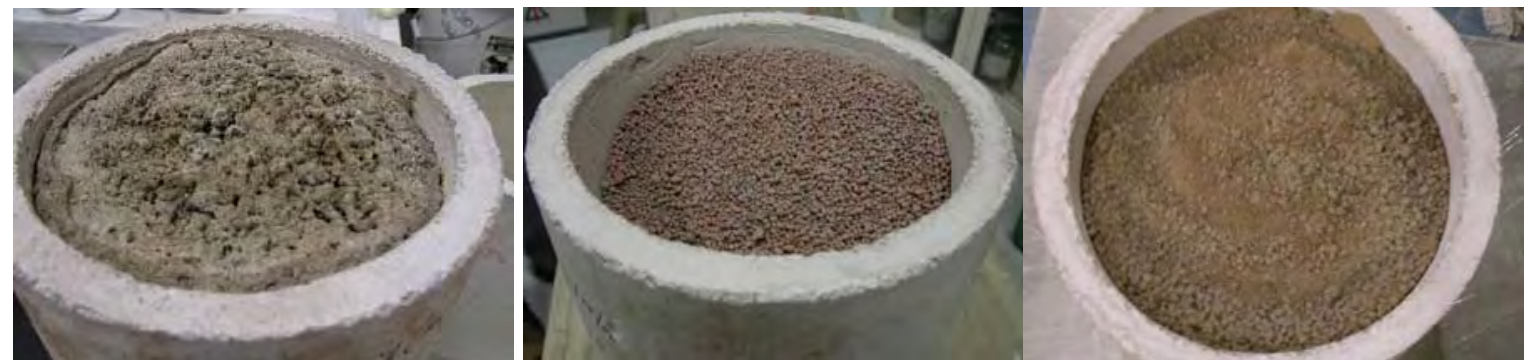

Figure 9.25. Crucibles with Feed after $500^{\circ} \mathrm{C}$ Heat-Treatment; from Left to Right: Feed with Sucrose, Feed with GFMs (dryer I), and Feed with Praxair-Ground Soil 


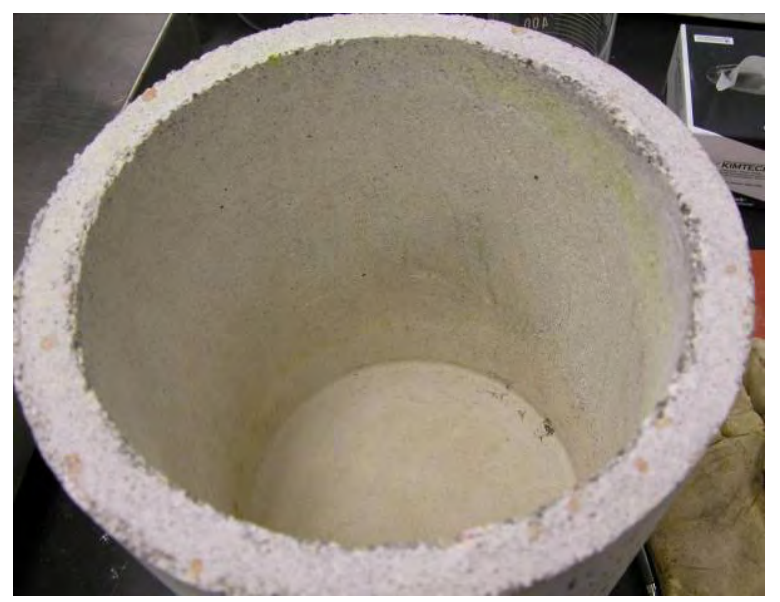

As-received soil (lab dried)

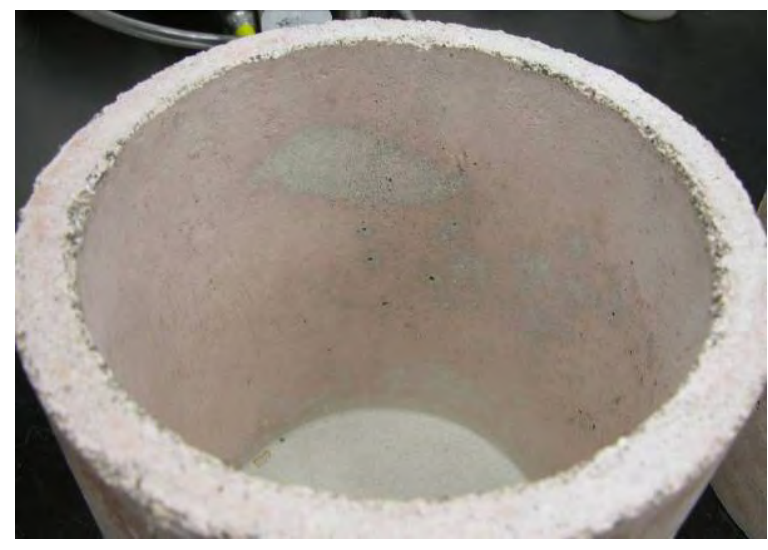

GFMs, dryer dried I with cellulose, C:N = 0.63

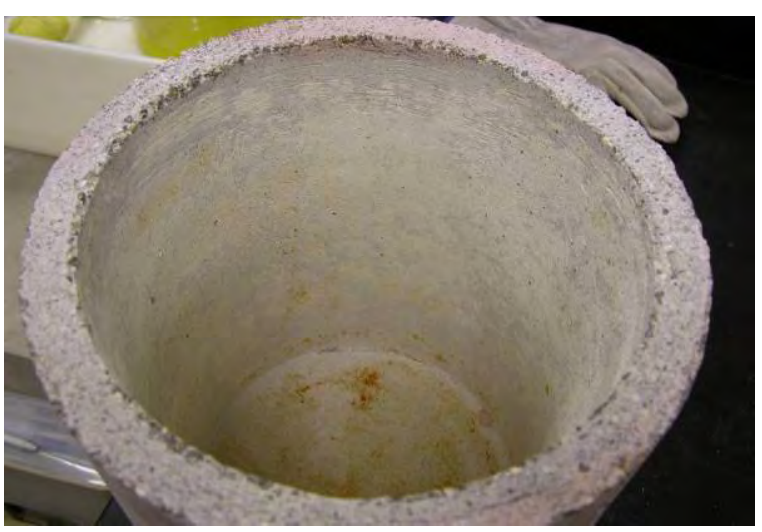

As-received soil, with sucrose, C:N = 0.50 (lab dried)

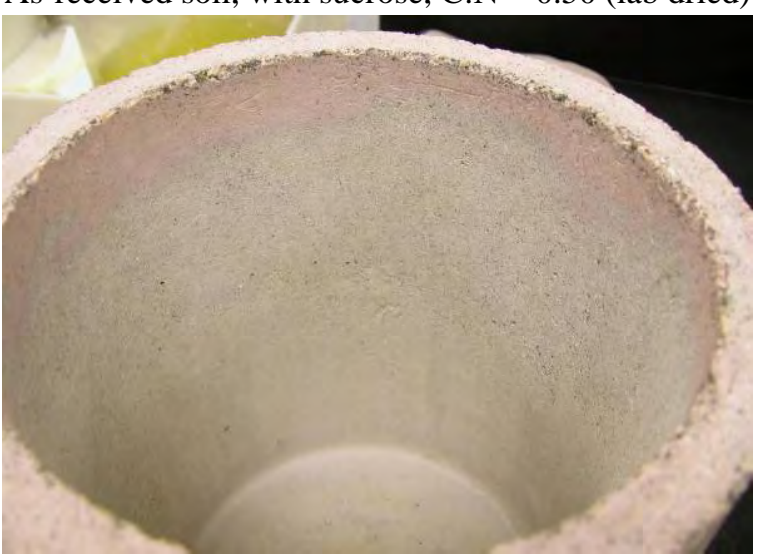

Praxair (4× vibratory-milled), dryer dried with cellulose, $\mathrm{C}: \mathrm{N}=0.50$

Figure 9.26. Vibrocast Crucibles after $1000^{\circ} \mathrm{C}$ Heat-Treatment

Table 9.16. Masses of Vibrocast Crucibles and Feeds in kg

\begin{tabular}{|c|c|c|c|c|c|c|}
\hline \multirow[b]{2}{*}{ Feed made with: } & \multirow[b]{2}{*}{$\mathrm{C}: \mathrm{N}$ ratio } & \multicolumn{3}{|c|}{ Crucible } & \multicolumn{2}{|c|}{ Feed } \\
\hline & & RT & $500^{\circ} \mathrm{C}$ & ${ }_{1000}{ }^{\circ} \mathrm{C}$ & RT & $500^{\circ} \mathrm{C}$ \\
\hline As-received soil (lab dried) & 0 & 12.190 & 13.605 & 12.635 & 8.925 & 7.145 \\
\hline As-received soil (lab dried, crucible with joint) & 0 & 12.390 & 13.860 & 12.865 & 9.07 & 7.132 \\
\hline 38-C Feed (old S-109 dry chemicals) & 0 & 13.110 & 14.385 & 13.490 & 8.265 & 6.595 \\
\hline As-received soil & 0.63 & 12.255 & 12.805 & 12.470 & 7.555 & 5.455 \\
\hline Minerals (dryer dried I) & 0.75 & 12.385 & 12.450 & 12.340 & 7.335 & 5.490 \\
\hline Minerals (dryer dried II) & 0.75 & 12.615 & 12.670 & 12.585 & 7.465 & 5.570 \\
\hline Praxair (4× vibratory-milled, dryer dried) & 0.75 & 12.265 & 12.685 & 12.415 & 8.735 & 6.520 \\
\hline CRB large crucible LOI test & 0 & 12.425 & 12.390 & 11.950 & & \\
\hline
\end{tabular}


Table 9.17 lists MIS migration fractions obtained with vibrocast crucibles filled with feeds without carbohydrates (sucrose or cellulose). The average of two tests (with feed prepared from new S-109 LAW liquid simulant) was 43.2 mass\% of total MIS migrated from the feed to the crucible wall and bottom. The corresponding number from a much smaller silica crucible was similar: 47.8 mass\%. When dry chemicals were used in lieu of liquid simulant in a simulated 38C feed, 45.8 mass\% of MIS was lost to the crucible. A comparison of the feed prepared with the liquid simulant and the simulated 38C feed showed that the MIS penetration was not simply a result of using a non-prototypic dry blended feed in 38C and that dryer-prepared feed using as-received Hanford soil would also show significant MIS migration. These three results clearly demonstrate the high mobility of MIS within the feed. The free MIS tends to escape from the feed even when the migration distance in the large crucible was increased to several inches.

Table 9.17. Fractions of Crucible-Migrated MIS: Baseline Feeds

\begin{tabular}{||l|c|c|c||}
\hline \multicolumn{1}{|c|}{ Crucible } & \multicolumn{2}{c||}{ Migrated MIS Fraction } \\
\hline & Silica & Vibrocast & $\begin{array}{c}\text { Vibrocast/Silica } \\
\text { Ratio }\end{array}$ \\
\hline As-received soil (lab dried) & 0.478 & 0.441 & 0.92 \\
\hline As-received soil (lab dried, crucible with joint) & & 0.423 & 0.89 \\
\hline 38C Feed (old S-109 dry-blended chemicals) & & 0.458 & 0.96 \\
\hline
\end{tabular}

Table 9.18 lists MIS migration fractions obtained with silica and vibrocast crucibles filled with feeds containing carbohydrates (sucrose and cellulose). The table shows that the fraction of total MIS that migrated to the crucible dropped to 10.3 mass\% in the small silica crucible when sucrose was added at a C:N ratio of 0.63 to a feed with as-received soil and new S-109 LAW liquid simulant. However, the sucrose addition alone was not as effective in the large CRB crucible with 31.8 mass\% of the MIS penetrating into the crucible wall. In the small crucible, the heating of the feed may be more uniform and allow most of the nitrate and sucrose to react at about the same time. In the large crucible, there are significant gradients such that some portions of the feed are at significantly higher temperatures than other portions of the feed. When the sucrose/nitrate reaction starts in the warmer portions of the feed, the heat generated may propagate to the cooler areas and rapidly melt the MIS that drains away from the more viscous sucrose before it can react. Alternately, the longer duration of the CRB crucible test may have allowed enough additional time for the MIS that was not destroyed in the nitrate/sucrose reaction to migrate to the CRB. Further experiments would be required to better understand the cause of these scale effects.

The data in Table 9.18 show that the addition of fine-grained materials improves the effectiveness of carbohydrate additions. An addition of cellulose at a C:N of 0.75 and the use of fine-grain GFMs in place of soil resulted in a decrease of migrated MIS in the silica crucibles to an average of only 6.2 mass\%, and this excellent performance was maintained in the larger scale vibrocast crucibles with an average migrated MIS fraction of only 6.8\%. Apparently, the fine-grained material is able to slow the migration of the MIS enough to allow the carbon/nitrate reaction to occur to a large enough extent to prevent most MIS migration even at the larger scale. Tests were also conducted with the finest ground soil that Praxair could provide without further development efforts. This material was passed through a vibratory ball mill four times. The ground soil performed better than the as-received soil in the large vibrocast crucibles but not as well the GFMs. 
The data in Table 9.17 and Table 9.18 along with Figure 9.27 through Figure 9.29 show that the simpler silica crucible test was an effective screening method and generally yielded MIS penetration results that were consistent with the large vibrocast crucible results. However, the as-received soil/sucrose test showed that some MIS reduction methods may not be as effective when applied at larger scale and that large-scale verification of any proposed method is necessary.

Table 9.18. Fractions of Crucible-Migrated MIS: Feeds with Carbohydrates

\begin{tabular}{|c|c|c|c|c|c|}
\hline & \multirow{2}{*}{$C: N$} & \multirow{2}{*}{ CH } & \multicolumn{3}{|c|}{ Migrated MIS Fraction } \\
\hline & & & Silica & Vibrocast & Vibrocast/Silica \\
\hline As-received soil & 0.63 & $\mathrm{~S}$ & 0.103 & 0.318 & 3.09 \\
\hline Praxair (4× vibration-milled, dryer dried $)^{(\mathrm{a})}$ & 0.75 & $\mathrm{C}$ & 0.188 & 0.225 & 1.20 \\
\hline GFMs (dryer dried II) & 0.75 & $\mathrm{C}$ & 0.038 & 0.074 & 1.94 \\
\hline GFMs (dryer dried I) $^{(a)}$ & 0.75 & $\mathrm{C}$ & 0.086 & 0.062 & 0.72 \\
\hline \multicolumn{6}{|c|}{$\begin{array}{l}\mathrm{CH} \text { - carbohydrate, } \mathrm{S} \text { - sucrose, } \mathrm{C} \text { - cellulose } \\
\text { (a) Note that the migrated MIS faction for Praxair feed and dyer-dried feed I in silica crucibles are outliers—see } \\
\text { Figure 9.4 (Praxair, dryer-dried feed with } \alpha \text { cellulose, see also Table 9.10) and Figure 9.5 (DI, see also Table 9.11). }\end{array}$} \\
\hline
\end{tabular}
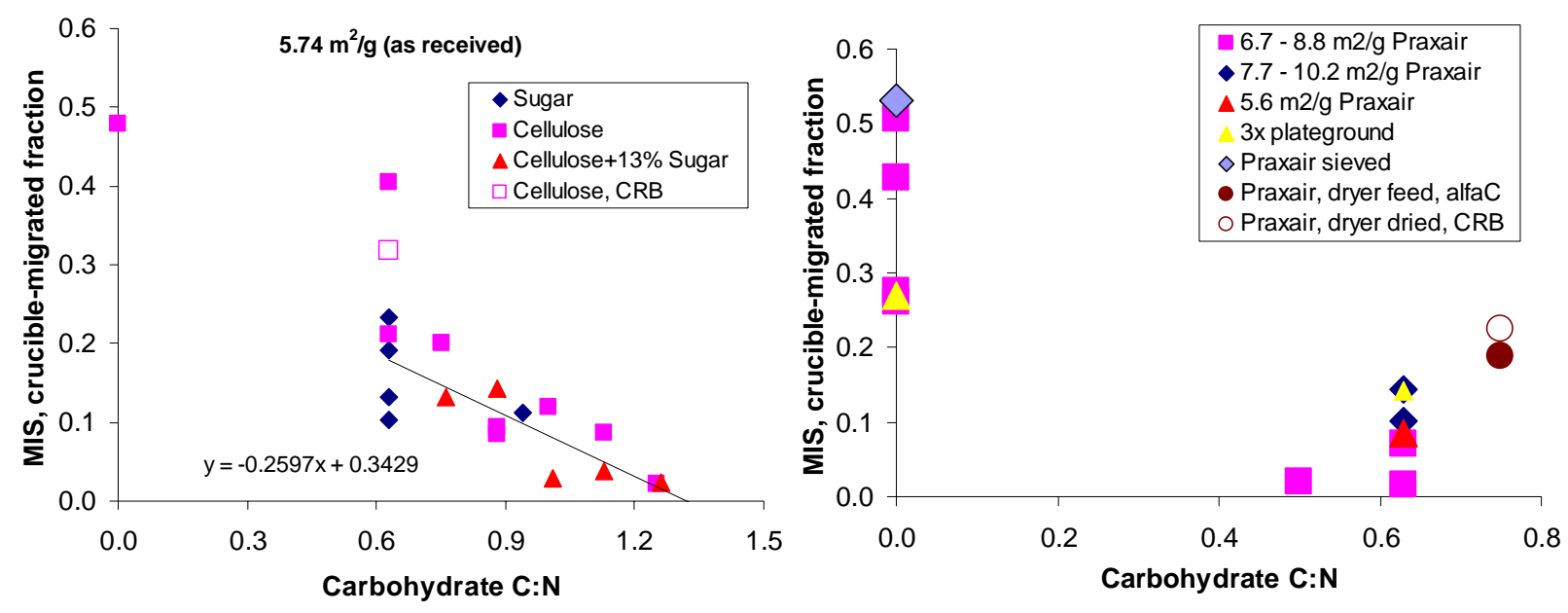

Figure 9.27. Mass Fraction of Silica-Crucible-Migrated MIS from Feed with Soil Versus Carbohydrate Addition, Including Vibrocast (CRB) Crucible Data 


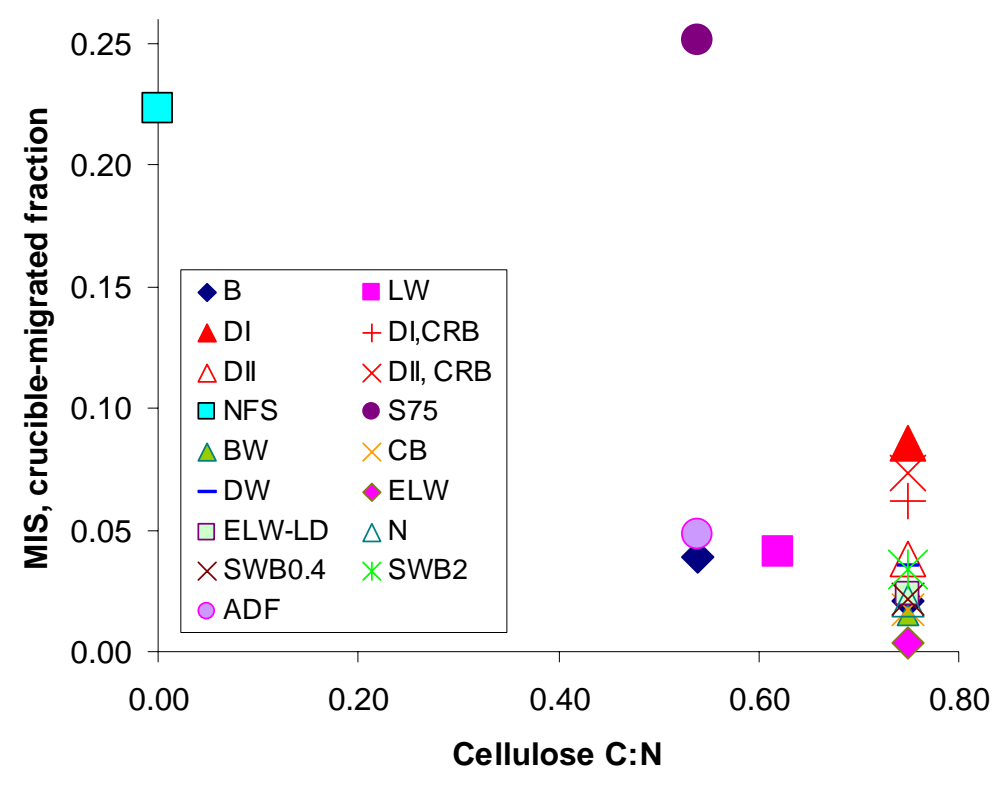

Figure 9.28. Mass Fraction of Silica-Crucible-Migrated MIS from Feed with GFMs Versus Carbohydrate Addition, Including Vibrocast (CRB) Crucible Data

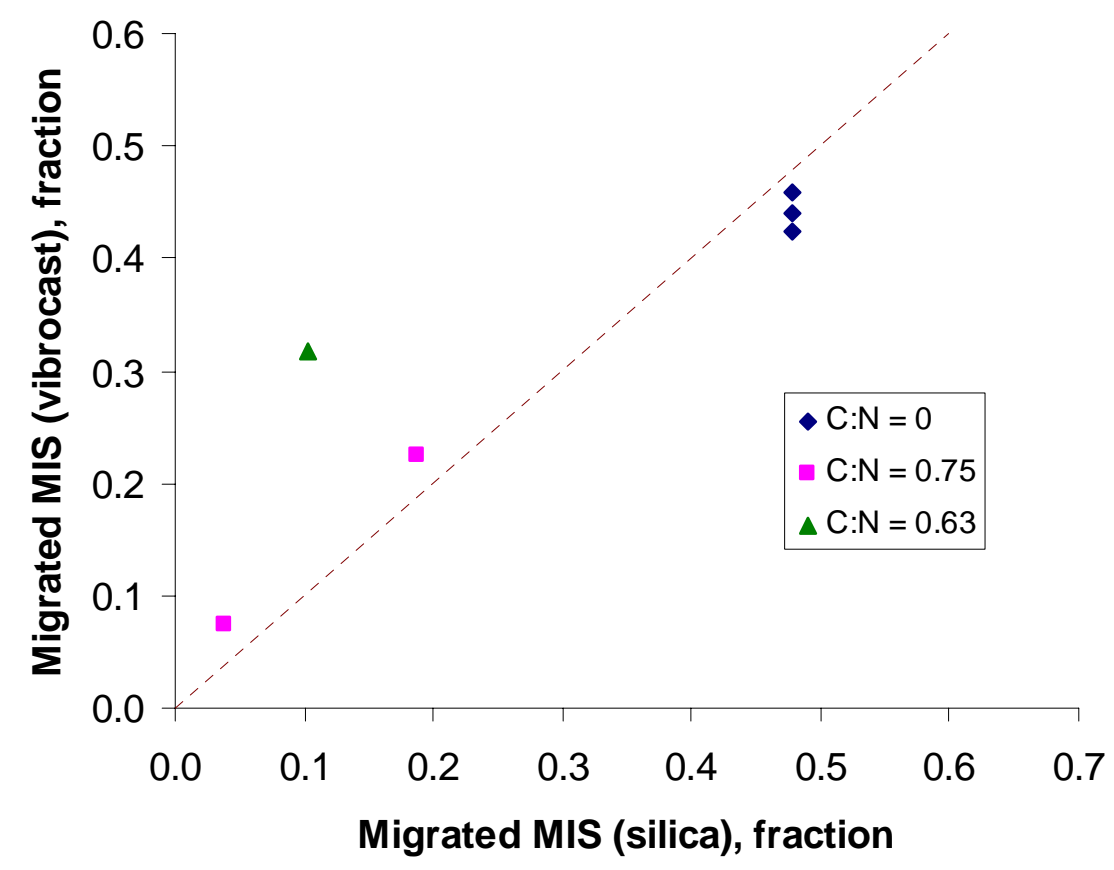

Figure 9.29. Migrated MIS Fraction, Vibrocast Versus Silica Crucibles 
Feed temperatures in a large vibrocast crucible were measured for a GFM feed having a C:N ratio of 0.75. The feed was prepared in a 22-L dryer. Figure 9.30 describe the TC placement. One TC (T) was placed at the top surface of the feed one-third the radius from the wall (the top); TC B was close to the bottom of the crucible and one-third the radius from the wall (the bottom); TC $\mathrm{S}$ was about $3 \mathrm{~cm}$ below the surface of the feed and one-quarter radius from the wall (the side); and TC M was at the crucible axis at about half the depth of the feed (the middle). Table 9.19 shows the coordinates of TC placement in terms of the radial coordinate ( $r$, the distance from crucible axis) and the axial coordinate ( $z$, the distance from the top of the feed).

Table 9.19. Thermocouple Placement ( $r$ is the distance from crucible axis, and $z$ is the distance from the top of the feed $)^{(a)}$

\begin{tabular}{||l|c|c||}
\hline \hline TC Location & $\boldsymbol{r}, \mathbf{c m}$ & $\boldsymbol{z}, \mathbf{c m}$ \\
\hline Top (T) & 6 & 0 \\
\hline Middle (M) & 0 & 10.5 \\
\hline Bottom (B) & 6 & 18 \\
\hline Side (S) & 7.5 & 3 \\
\hline $\begin{array}{l}\text { (a) The crucible inner radius was } 10 \mathrm{~cm}, \\
\text { and the feed depth } 20.5 \pm 0.5 \mathrm{~cm} .\end{array}$ \\
\hline
\end{tabular}

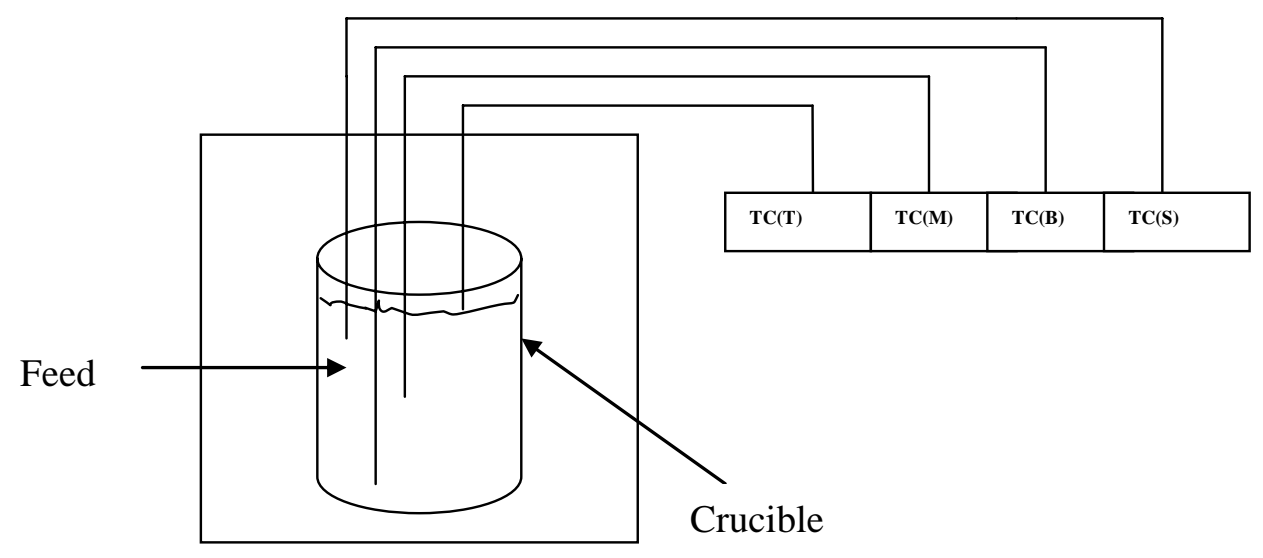

Figure 9.30. Thermocouple Placement in Large CRB Crucible

Table 9.20 lists the temperatures registered on these four TCs and the furnace control temperature. As Figure 9.31 shows, the temperature history, as recorded at fixed spatial points within the CRB crucible feed, is rather complex. In the first hour of heating, as the furnace temperature increased from ambient to $410^{\circ} \mathrm{C}$, the temperature increased gradually with time at all four locations (Figure 9.32). The temperature profiles at different locations in the crucible changed dramatically after 1 hour of heat-treatment while the furnace temperature increased from $410^{\circ} \mathrm{C}$ to $500^{\circ} \mathrm{C}$. Rapid changes in temperature continued for $40 \mathrm{~min}$ when the furnace temperature was maintained at $500^{\circ} \mathrm{C}$. After $40 \mathrm{~min}$ at $500^{\circ} \mathrm{C}$, the feed temperature was fairly uniform. 
Table 9.20. Temperature Distribution Evolution in a CRB Crucible Feed

\begin{tabular}{|c|c|c|c|c|c|c|c|c|c|c|c|}
\hline \multirow{3}{*}{$\begin{array}{l}\text { Time } \\
\text { min }\end{array}$} & \multicolumn{5}{|c|}{ "Temperature, ${ }^{\circ} \mathrm{C}$} & \multirow{3}{*}{$\begin{array}{c}\text { Time } \\
\text { min }\end{array}$} & \multicolumn{5}{|c|}{ 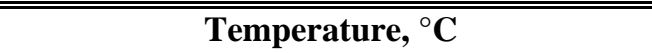 } \\
\hline & \multirow[b]{2}{*}{ Furnace } & \multicolumn{4}{|c|}{ Thermocouple } & & \multirow[b]{2}{*}{ Furnace } & \multicolumn{4}{|c|}{ Thermocouple } \\
\hline & & $\mathbf{T}$ & $\mathbf{B}$ & $\mathrm{S}$ & $\mathbf{M}$ & & & $\mathbf{T}$ & $\mathbf{B}$ & $\mathrm{S}$ & $\mathbf{M}$ \\
\hline 0 & 95 & 41 & 21.0 & 23.5 & 20.6 & 69.8 & 444 & 383 & 59.5 & 443.9 & 47.7 \\
\hline 4.2 & 116 & 54 & 21.3 & 25.5 & 20.7 & 70.6 & 448 & 365 & 59.8 & 438.1 & 86.6 \\
\hline 7.0 & 130 & 57 & 21.4 & 27.2 & 20.7 & 71.6 & 453 & 328 & 60.6 & 428.1 & 98.8 \\
\hline 9.0 & 140 & 62 & 21.6 & 28.9 & 20.7 & 72.6 & 458 & 306 & 62.7 & 422.5 & 103.5 \\
\hline 11.0 & 150 & 69 & 21.7 & 30.4 & 20.6 & 73.8 & 464 & 285 & 71.9 & 412.1 & 108.2 \\
\hline 15.8 & 174 & 77 & 22.3 & 36.2 & 20.7 & 75.4 & 472 & 273 & 89.8 & 402.7 & 110.3 \\
\hline 19.6 & 193 & 86 & 22.8 & 40.8 & 20.6 & 78.8 & 489 & 256 & 100.5 & 394.9 & 110.7 \\
\hline 22.0 & 205 & 97 & 23.4 & 44.7 & 20.6 & 80.4 & 497 & 252 & 108.2 & 400.2 & 103.4 \\
\hline 24.8 & 219 & 102 & 24.1 & 49.3 & 20.6 & 81.0 & 500 & 251 & 104.8 & 403.7 & 109.5 \\
\hline 28.2 & 236 & 110 & 25.1 & 56.1 & 20.6 & 81.4 & 500 & 252 & 155.0 & 407.3 & 110.0 \\
\hline 31.0 & 250 & 116 & 26.1 & 61.7 & 20.7 & 82.2 & 500 & 255 & 338.0 & 409.3 & 112.2 \\
\hline 33.4 & 262 & 123 & 27.4 & 67.5 & 20.8 & 83.3 & 500 & 256 & 365.0 & 409.7 & 111.6 \\
\hline 35.4 & 272 & 129 & 28.3 & 72.1 & 20.8 & 84.8 & 500 & 258 & 376.5 & 410.1 & 109.3 \\
\hline 37.8 & 284 & 135 & 29.6 & 78.0 & 21.0 & 86.8 & 500 & 260 & 360.7 & 411.0 & 108.5 \\
\hline 40.0 & 295 & 141 & 31.0 & 82.6 & 21.1 & 89.1 & 500 & 260 & 349.2 & 412.3 & 106.2 \\
\hline 42.0 & 305 & 145 & 32.3 & 86.5 & 21.2 & 91.8 & 500 & 260 & 442.0 & 414.0 & 114.6 \\
\hline 45.0 & 320 & 153 & 35.0 & 93.0 & 21.5 & 94.8 & 500 & 276 & 440.5 & 415.9 & 159.0 \\
\hline 49.0 & 340 & 163 & 38.3 & 99.9 & 21.8 & 98.3 & 500 & 328 & 441.0 & 416.9 & 237.0 \\
\hline 53.0 & 360 & 173 & 42.6 & 107.2 & 22.3 & 102.2 & 500 & 336 & 440.5 & 418.0 & 330.0 \\
\hline 55.0 & 370 & 177 & 44.5 & 110.8 & 22.7 & 106.4 & 500 & 380 & 438.1 & 419.0 & 284.0 \\
\hline 59.0 & 390 & 188 & 49.3 & 118.0 & 23.4 & 111.0 & 500 & 400 & 434.6 & 420.6 & 260.0 \\
\hline 61.0 & 400 & 193 & 51.8 & 121.8 & 23.9 & 116.0 & 500 & 406 & 432.8 & 421.6 & 273.0 \\
\hline 63.0 & 410 & 200 & 54.2 & 122.0 & 24.5 & 122.0 & 500 & 404 & 428.6 & 423.9 & 426.0 \\
\hline 65.0 & 420 & 216 & 56.6 & 307.0 & 25.6 & 129.0 & 500 & 403 & 424.5 & 425.4 & 419.0 \\
\hline 65.8 & 424 & 221 & 57.3 & 336.0 & 26.0 & 137.0 & 500 & 403 & 419.9 & 427.6 & 418.7 \\
\hline 67.4 & 432 & 355 & 58.7 & 439.5 & 27.3 & 146.0 & 500 & 404 & 418.1 & 429.2 & 411.3 \\
\hline 68.2 & 436 & 354 & 59.2 & 441.4 & 29.5 & & & & & & \\
\hline
\end{tabular}




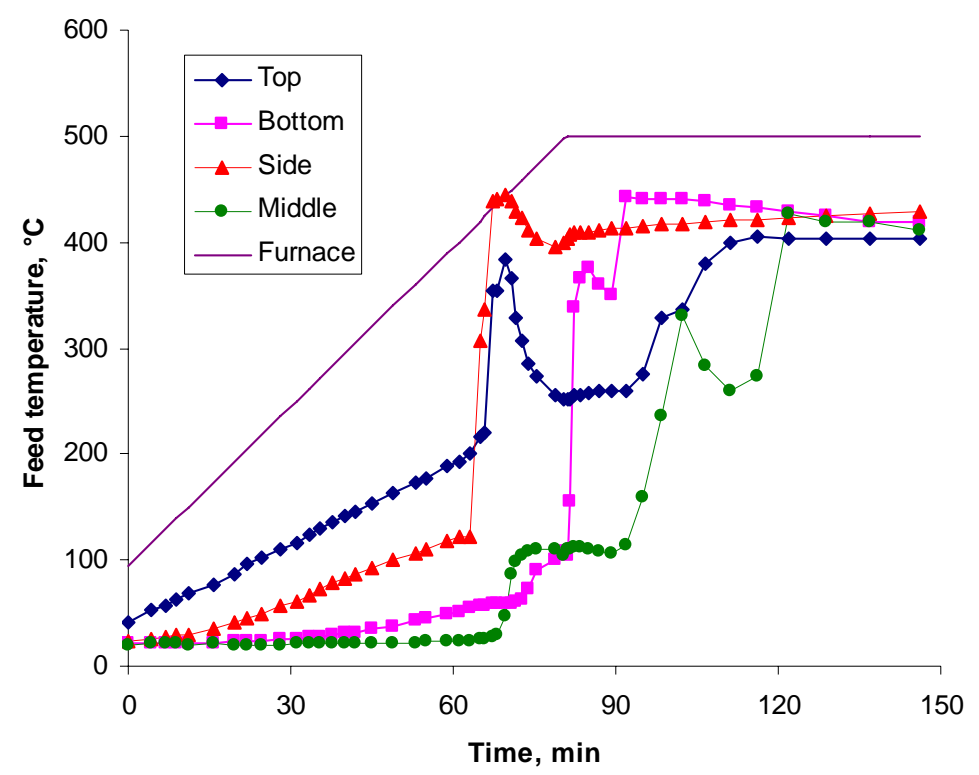

Figure 9.31. Temperature Field Evolution of CRB Crucible Feed

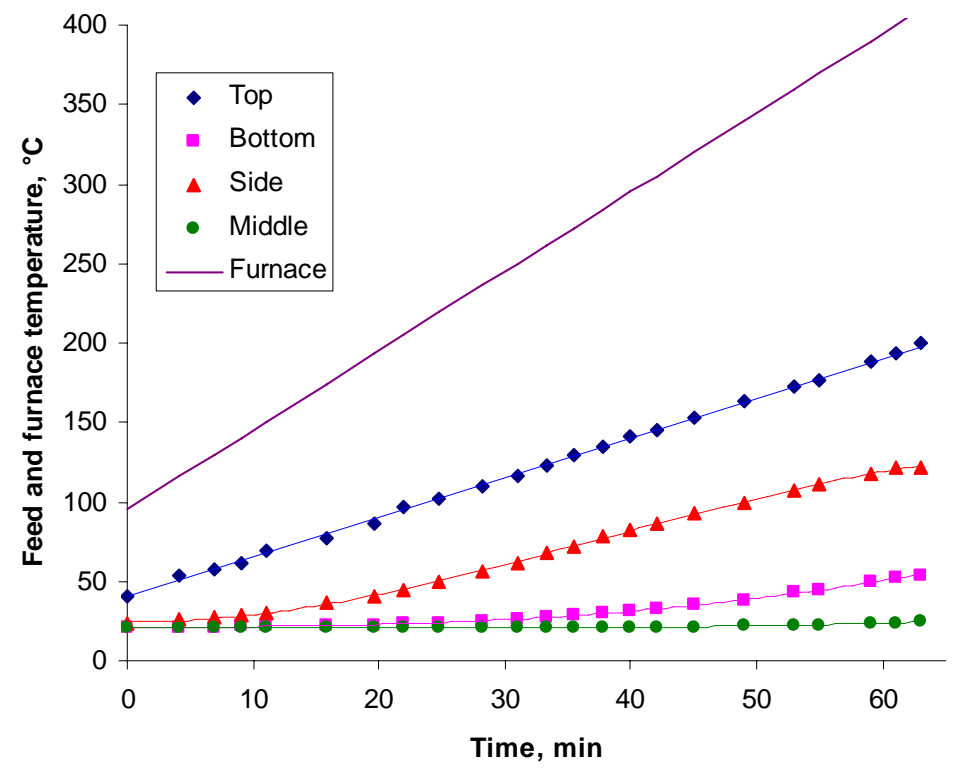

Figure 9.32. The Initial Stage of Temperature Field Evolution of CRB Crucible Feed

During the initial stage (Figure 9.32), the fastest feed temperature increase was $2.5^{\circ} \mathrm{C} / \mathrm{min}$ measured on the feed top surface (TC T). This rate was only half the rate of the furnace temperature increase. After about a 15-min delay, nearly the same rate was recorded by TC S located about $2.5 \mathrm{~cm}$ from the wall and $\sim 3 \mathrm{~cm}$ from the top. TC B, placed about $5 \mathrm{~cm}$ from the feed bottom, experienced a much longer delay, about $30 \mathrm{~min}$, and a much slower temperature increase rate afterwards. The point in the very center of the 
feed (TC M) experienced the largest delay in temperature increase- the temperature changed by a mere $4^{\circ} \mathrm{C}$ within the first hour in the furnace.

After 1 hour of heating, the rate of temperature increase in the feed increased. Figure 9.33 shows the temperature evolution at four fixed points as a function of the furnace temperature that increased at the rate of $5 \% \mathrm{~min}$. The first temperature jump was recorded by the S TC placed to the side below the feed surface-at 63 min into the heat-treatment, when the feed temperature was $122^{\circ} \mathrm{C}$. At that moment, black smoke was observed coming out from the furnace. The S TC temperature increased by $320^{\circ} \mathrm{C}$ within $5 \mathrm{~min}$ and then decreased again by $\sim 45^{\circ} \mathrm{C}$ within the following $7 \mathrm{~min}$. Shortly after the $\mathrm{S}$ TC event, a temperature jump at the feed top surface (T TC) occurred. The T TC event occurred at 66 min into the heat-treatment, when the feed temperature was $221^{\circ} \mathrm{C}$, a temperature $99^{\circ} \mathrm{C}$ above that of the temperature jump recorded by S TC. The temperature increased by $180^{\circ} \mathrm{C}$ within $4 \mathrm{~min}$ and then decreased again by $\sim 130^{\circ} \mathrm{C}$ within the following $11 \mathrm{~min}$.

A possible explanation for this behavior is that the fast chemical reaction (burning) of nitrate with cellulose was triggered in the hottest spot, probably in the area where feed top surface meets the crucible wall, and spread throughout the feed in areas that were near the surface where the feed had enough time to completely dry. Based on the S TC data, the reaction started at a temperature above $220^{\circ} \mathrm{C}$ and then propagated into increasingly colder feed. When reached by the reaction front, the dry-feed temperature increased rapidly and then decreased again because of the internal heat sink caused by the heat capacity of the inert granular material (the GFMs) and/or the heat lost to the escaping gasses. The effect was more pronounced at the internal point (S TC) than on the surface point (T TC).

The feed close to the surface was gradually heated to the temperature above the boiling point of water until the cellulose-nitrate reaction was triggered (see TCs T and S). During this initial period, the heat was supplied from the external source through conduction through the crucible wall and by direct radiation to the feed top surface. The internal areas of the feed remained cold until the influx of hot, highhumidity gas phase generated at the reaction front rapidly increased the feed temperature via direct heat exchange and steam condensation. When the feed temperature reached the boiling point of water, recorded at $70 \mathrm{~min}$ by TC $\mathrm{M}$ and at $74 \mathrm{~min}$ by TC B, the excess water from the feed was evaporated and the nitrate-cellulose reaction could start. 


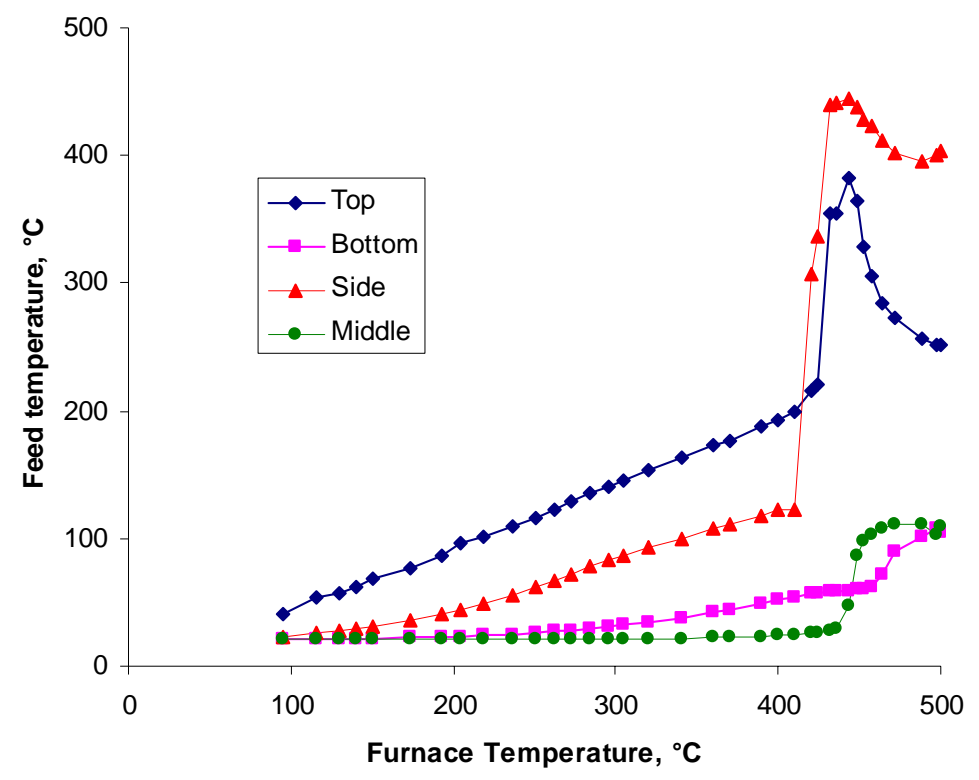

Figure 9.33. Temperature Field Evolution of CRB Crucible Feed During the Heat-Up Period

As Figure 9.34 shows, the rate of temperature change continued to fluctuate after 80 min of heattreatment when the furnace temperature became constant at $500^{\circ} \mathrm{C}$. After the center and bottom portions of the feed were completely dry, they started to heat up because of the thermal gradient set up by the $500^{\circ} \mathrm{C}$ furnace. Eventually, a portion of the feed at the bottom of the crucible got warm enough to start the nitrate/cellulose reaction in that area, causing an increase in the rate of temperature rise in TC B. Eventually, the materials in the middle of the feed (TC M) dried out and allowed the nitrate/cellulose reaction to proceed and result in an accelerated heating rate. After $120 \mathrm{~min}$ from the beginning of heattreatment ( $40 \mathrm{~min}$ at the furnace temperature of $500^{\circ} \mathrm{C}$ ), the feed temperature reached a nearly uniform value of $\sim 420^{\circ} \mathrm{C}$ in the bulk and $\sim 400^{\circ} \mathrm{C}$ on the top surface, indicating that the cellulose nitrate reactions were essentially complete. 


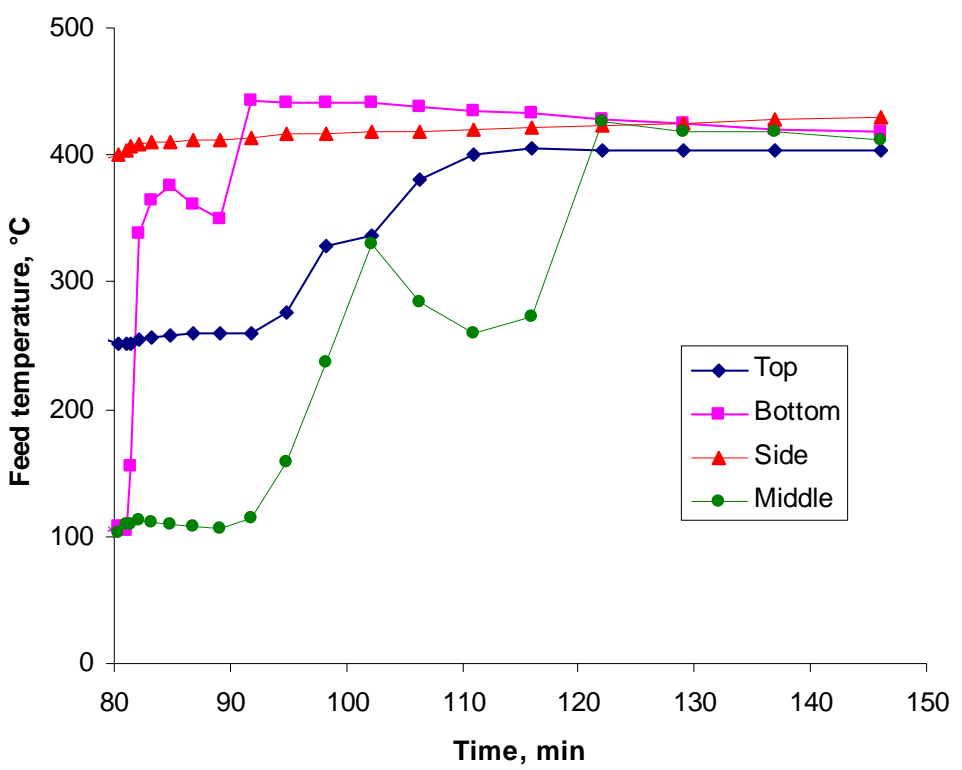

Figure 9.34. Temperature Field Evolution of CRB Crucible Feed During the Constant Furnace Temperature Period 


\subsection{Conclusions and Recommendations}

Measurements and calculations of the MIS, CRB, and feed properties were performed to improve the understanding of MIS penetration. The results of these measurements are summarized below:

- The volume fraction of MIS in six-tank composite feeds before decomposing was $41.6 \%$.

Considering that $\mathrm{B}_{2} \mathrm{O}_{3}$ can combine with MIS to form a common liquid phase, the volume fraction of liquid phase in the feed at temperatures above $450^{\circ} \mathrm{C}$ could be as high as $46 \%$.

- Although the CRB material (Viborcast 60PC) is dense by refractory standards, it still contains 11 to $15 \%$ open porosity.

- The results of evolved gas analyses, thermal gravimetric analysis, differential thermal analysis, and hot-stage microscopy show that the waste ingredients undergo minor reactions with organic carbon in the feed temperatures as low as $150^{\circ} \mathrm{C}$ but remain essentially unchanged to temperatures of about $300^{\circ} \mathrm{C}$. At temperatures above $300^{\circ} \mathrm{C}$, the waste materials begin to melt and form a single molten MIS phase. This MIS remains stable until roughly $550^{\circ} \mathrm{C}$ where it begins to thermally decompose with the evolution of gas. By $800^{\circ} \mathrm{C}$, the thermal decomposition of the nitrate salts is complete.

Modification of the CRB was not deemed practical as a method to prevent the large quantities of MIS penetration seen in FS-38C. However, these methods may be useful to reduce the impacts of the lower levels of MIS penetration. The conclusions from the methods attempted in this study are given below:

\section{$\underline{\text { Sacrificial Layer at CRB-Feed Contact Surface }}$}

- Tests showed that a sacrificial layer could be made by adding 20 to 50 mass $\% \mathrm{Na}_{2} \mathrm{SiO}_{3}$ (NS) to the 60PC material. Adding 25 mass\% NS appeared to give the best results in that the material stayed intact to high enough temperatures to be in place during the MIS attack but completely dissolved in the glass melt when heated to $1200^{\circ} \mathrm{C}$.

- Manufacturability tests indicated that a large composite sample of 60PC with a sacrificial layer made from 75\% 60PC and 25 mass\% NS could be fabricated. This composite had good structural integrity as-prepared and after cooling from a heat-treatment to $1200^{\circ} \mathrm{C}$. This qualitative test indicated that this type of composite could be prepared at the larger scales required for BV.

Treating the CRB with a Lyophobic Material (Carbon)

- Of the various carbon sources added to 60PC, the best results were obtained by adding 18 mass\% of graphite. This 18\% addition of ground-graphite decreased the depth of MIS penetration into a CRB sample from $10 \mathrm{~mm}$ to less than $6 \mathrm{~mm}$.

- Manufacturability tests indicated that a large composite sample of 60PC with an inner layer of 60PC containing 17.4 mass\% of graphite could be fabricated. The altered layer formed a porous solid with fine cracks running through the material. It appeared to bond well to the layer, but it 
crumbled under pressure, showing that this type of composite might not be durable enough for BV applications.

Protecting the CRB Surface with alumina-zirconia-silica (AZS) Tiles

- Room-temperature dye-penetration tests and molten-salt dye-penetration tests showed that AZS tiles could be effectively bonded to CRB materials and that there was no preferential penetration of the dye or molten salt through the AZS-CRB interface.

- The AZS porosity was sufficiently open to allow penetration of molten salt into the tile. Thus, while the interface was well bonded, both molten salt and ink penetrated into AZS tiles as easily as in to the CRB, indicating that the tiles would supply minimal protection against MIS penetration.

Immobilizing Contaminants in the Upper Portion of the CRB with Higher Temperatures

- Analyses of the CRB from a partially completed full-scale test (FS-38B) showed that condensation on the upper portions did not lead to significant Re migration into the CRB. As a result, efforts in this area were reduced to a series of scoping tests.

- Both pH and electrical conductivity of leachates decreased as the temperature of the heattreatment increased, showing a progressive incorporation of the condensate into a durable glass phase.

Immobilizing the MIS in the BV feeds by increasing the surface area of the feed solids proved to be a more effective way to reduce MIS penetration. The conclusions from the early portions of the MIS penetration studies are discussed below. All the initial studies were performed using six-tank composite waste-feed simulant.

- Dissolving $\mathrm{B}_{2} \mathrm{O}_{3}$ in aqueous LAW before blending it with other feed materials did not decrease MIS mobility. Preliminary tests showed that $\mathrm{B}_{2} \mathrm{O}_{3}$ substantially increased the liquidus temperature of MIS. However, MIS penetration tests showed extensive penetration even when $\mathrm{B}_{2} \mathrm{O}_{3}$ was added at twice the concentration used in the current baseline glass formulation.

- Silica crucibles were effective model refractories and had several advantages over CRB tests. The silica crucibles were more uniform in composition, structure, and porosity; and MIS penetration areas had a distinct color and a sharp boundary, allowing unambiguous assessment of MIS penetration levels.

- Tests of MIS penetration from feed into silica crucibles showed that significant penetration starts as low as $350^{\circ} \mathrm{C}$ and increases as the temperature increases to $500^{\circ} \mathrm{C}$. At higher temperatures, MIS decomposition becomes more extensive. At $800^{\circ} \mathrm{C}$, the MIS is fully decomposed and little additional penetration occurs.

- MIS penetration has no visual effect on the $60 \mathrm{PC}$ material at temperatures up to $750^{\circ} \mathrm{C}$. At temperatures of $1200^{\circ} \mathrm{C}$ or higher, the penetrated MIS causes significant swelling of the CRB and 
can lead to crack formation at an interface between an MIS-saturated region and a dry portion of the CRB.

- Crucible experiments indicated that the atypical nature of the dry-blended feed used for the FS-38C test did not contribute to the extensive MIS penetration. Dryer-prepared feed would have produced similar results.

- A partial replacement of soil with fine-grain materials, including silica powder ( $<75 \mu \mathrm{m})$, kaolin clay, bentonite clay, diatomaceous earth, and fly ash, did not significantly reduce MIS migration. The best initial candidate was a $10-w t \%$ addition of diatomaceous earth, but increasing the quantity of this material from 10 to 15,20 , and $25 \mathrm{wt} \%$ led to a progressively worse crucible degradation.

- $\quad$ CRB and silica crucible tests showed that grinding the Hanford soil reduced MIS penetration depths. The degree of reduction was a function of the extent of grinding and the fraction of ground material added. These results indicated that the reduction in MIS migration was a function of surface area and that sufficiently high surface areas in the feed solids could eliminate MIS penetration.

- Tests with CRB rods showed that the extent of MIS penetration at $550^{\circ} \mathrm{C}$ continued to increase for periods up to 24 hours for feeds prepared with as-received Hanford soil. Ground soils showed no increased MIS penetration for time periods as long as 24 hours, indicating that high-surfacearea materials would prevent MIS penetration in FS melts.

The early FS work was conducted with feeds prepared with the six-tank composite simulant to tie back to the earlier Tc-spiked ES tests that also used six-tank composite feeds. However, because the Demonstration Bulk Vitrification System would be treating waste from S-109, a decision was made to shift to the S-109 simulant for the FS-38C test. Since six-tank simulant was used in all work described in previous sections of this report, additional tests were designed to obtain data on the impact of soil grinding on S-109 feed. The MIS migration behavior was different for the S-109 simulant. The conclusions from the S-109 MIS migration studies are discussed below.

- The S-109 simulant showed significantly higher MIS penetration levels than the six-tank composite. The main difference in the compositions is that S-109 has a higher content of nitrates and nitrites and a lower content of organic components. The S-109 simulant also contains a significantly lower concentration of $\mathrm{Na}_{2} \mathrm{CO}_{3}$ and $\mathrm{NaOH}$. All these differences are expected to play a role in the different MIS penetration behavior, but the degree of impact for the various factors were not investigated in this study.

- Feed grinding and soil grinding showed significantly different results. The soil grinding operations showed significant diminishing returns when grinding times were increased from 2 to 5 minutes. This indicated that soil grinding as the sole MIS migration mitigation might have practical limits. 
- The MIS penetration behavior for the S-109 simulant was similar to that seen for the six-tank composite. Little penetration occurred at a temperature of $300^{\circ} \mathrm{C}$, but significant penetration occurred at temperatures of 400 and $500^{\circ} \mathrm{C}$.

- The ground soil reduced MIS penetration for the S-109 simulant by $75 \%$ but did not reduce penetration to the low levels seen for the six-tank composite feed. This indicated that soil grinding alone would not be enough to reduce MIS penetration to the desired levels.

- Adding diatomaceous earth and high-surface-area bentonite clay to ground soil did not significantly reduce the MIS penetration levels.

- Adding organic carbon in the form of sucrose significantly reduced the level of MIS penetration. Mass-loss calculations confirmed that this was the result of nitrate destruction at low enough temperatures to prevent MIS migration. Sugar additions were effective in both as-received soil and in ground soil.

- Combining the effects of increased surface area in the feed solids and the effects of sucrose additions indicated that a family of solutions where MIS penetration could be essentially eliminated existed. However, practical limits for soil grinding and sugar-addition levels narrowed the range of possible solutions.

The early laboratory work established that MIS migration could be controlled through different combinations of high-surface-area solid materials and the use of carbon supplied through the addition of sucrose. The next phase of the study used this laboratory knowledge to explore practical implementation of this knowledge for FS operations. The conclusions from the application phase of this study are discussed below.

- Problems associated with drying the bulk vitrification feed with sucrose additions required investigation of alternate forms of carbon. Alpha cellulose, which performed better in dryer tests, was shown to be as effective as sucrose in the reduction of MIS migration. Sucrose, cellulose, or combinations of the two produced essentially the same results. The reduction in MIS penetration was a linear function of the amount of carbon added to the feed.

- Small silica crucible tests showed that MIS penetration in feeds with as-received Hanford soil could be reduced to low levels with carbon additions in the range of a 1.2 carbon:nitrogen $(\mathrm{C}: \mathrm{N})$ ratio. Feeds with ground soils only required a carbon addition of $0.75 \mathrm{C}: \mathrm{N}$ ratio to achieve low MIS penetration levels.

- Studies conducted by Praxair under the direction of Pacific Northwest National Laboratory indicated that grinding Hanford soil to levels equivalent to that obtained in the laboratory grinding operations would be an expensive operation that would reduce the benefits of using Hanford soil as an inexpensive feedstock for bulk vitrification melting operations.

- GFMs similar to those used by the Waste Treatment Plant did not significantly reduce MIS migration until the -200 mesh silica material was replaced with silica that had a particle size of $<5 \mu$. 
- The use of GFMs allowed for higher waste loading by replacing the sodium and potassium flux materials in the soil with waste sodium. A rough-cost estimate showed that the increased waste loading would essentially balance the cost of purchasing and shipping the GFMs.

- The measured surface area of the GFMs with the fine silica $(<5 \mu)$ was only half the value of the as-received Hanford soil. If confirmed, this result indicates that reducing the surface area in a specific type of feed solid will tend to reduce MIS penetration but that it is not possible to predict MIS penetration reduction only from measurements of the surface areas of feed solids. The reasons for the difference in the performance of the GFMs versus the Hanford soil were not investigated further in this study.

- Feeds without carbohydrate additions resulted in a homogeneous coloration of the crucible internal surface. Carbohydrate-containing feeds left a spotted crucible surface. The spotting effect tended to be more pronounced for coarser feed solids. The spotting effect was probably associated with non-uniform distribution of carbohydrates in granulated feeds.

- Feeds with higher C:N carbohydrate additions tended to produce foamy feeds and smoke at high enough rates to be noticeable outside the treatment furnace. Although higher C:N carbohydrate additions further reduced MIS penetration, levels greater than 0.75 are not recommended due to the rapid feed reactions.

- Small silica crucible tests indicated that carbohydrate additions resulted in temperature spikes in the feed. These temperature spikes were initiated at the outer portion of the crucible at an average temperature of $246^{\circ} \mathrm{C}$ and resulted in an average maximum temperature of $467^{\circ} \mathrm{C}$. The initiation temperature and maximum temperature were independent of carbohydrate levels. However, propagation of the reaction to the center of the crucible appeared to happen faster with higher carbohydrate additions. This thermal behavior indicated that, as desired, the carbohydrate reactions started and destroyed a portion of the nitrates before a significant quantity of MIS formed. The average maximum temperature of the feed indicates that the energy given off in the nitrate-destruction reaction and retained in the feed was not excessive and was only sufficient to raise the temperature of the feed to the furnace temperature.

- A side benefit to the presence of carbohydrates in feed is accelerated conversion reactions that proceed more uniformly within the feed volume.

The last set of confirmatory tests was conducted in large CRB crucibles. The conclusions from those tests are discussed below:

- The large CRB crucible tests proved to be an effective way to test possible solutions at a scale that better represented the cold-cap thicknesses that might be expected in the FS BV melter. The large CRB tests generally confirmed the results obtained in the silica crucible tests.

- Three baseline tests were conducted. These tests showed that more than $40 \%$ of the MIS migrated to the wall of the CRB crucible, and a significant portion passed through the wall. A CRB crucible with a cemented seam performed no worse than an as-received crucible, indicating 
that the seams in the FS CRB are not the weak points for MIS penetration. A comparison of the feed prepared with the liquid simulant and the simulated FS-38C feed showed that the MIS penetration was not simply a result of using a non-prototypic dry blended feed in FS-38C and that dryer prepared feed using as-received Hanford soil would also show significant MIS migration. The baseline results demonstrated the high mobility of MIS and demonstrated that the MIS would travel at least several inches if not further.

- Limited large CRB crucible tests indicated that using only carbohydrate additions with asreceived soil to reduce MIS migration was not as effective at the larger CRB crucible scale. Total migrated MIS dropped to 10.3 mass\% in the small silica crucible when sucrose was added at a $\mathrm{C}: \mathrm{N}$ ratio of 0.63 to a feed with as-received soil, but the same sucrose addition allowed 31.8 mass\% of the MIS to penetrate into the large CRB crucible wall.

- Large CRB crucible tests confirmed that adding fine-grained materials improved the effectiveness of carbohydrate additions to reduce MIS penetration. An addition of cellulose at a C:N of 0.75 and the use of fine-grain GFMs in place of soil resulted in a decrease of migrated MIS to an average value of $6.8 \%$.

- Thermocouples placed in multiple locations in a large CRB crucible melt showed similar temperature spikes to those seen in the silica crucible tests. These spikes were roughly the same magnitude as those seen in the silica crucibles, but the center portion of the feed showed a significant delay of 15 to 30 minutes. After the outer portions reacted, the center portions of the feed heated to $100^{\circ} \mathrm{C}$ but plateaued at this temperature for 15 minutes before reacting further. This temperature behavior indicates that the outer portions of the feed dry out and react but that the propagation of the reaction to nearby areas is delayed by the energy requirements necessary to drive off the residual moisture in the feed and heat the solids in those locations. These results indicate that runaway reactions of large beds of cellulose-containing feed are unlikely.

The results of this extensive study leads to several recommendations related to future activities.

- Near term ES and FS tests should focus on formulations that include the use of fine-grained GFMs and alpha-cellulose. The GFMs should include silica sources that have a particle size of $<5 \mu \mathrm{m}$ and an addition of cellulose at a C: $\mathrm{N}$ of 0.75 .

- Investigations should be conducted to find less expensive sources of suitable fine silica and cellulose material. These two materials account for the majority of the formulation modification costs that were necessary to reduce the MIS migration.

- Investigations should be conducted to take advantage of the flexibility supplied by the shift from Hanford soil to GFMs and produce improved glass waste forms. Potential improvements that might be realized include higher waste loadings, reduced metal precipitation, better sulfate tolerance, and improved processing rates.

- Investigations should be conducted to better understand why the surface area of the GFMs and Hanford soil appear to have different MIS migration reduction abilities. The improved understanding might lead to better formulations with reduced costs. 
- Investigations should be conducted to better understand the difference in the MIS penetration behavior of the six-tank composite and the S-109 simulants. These investigations would help ensure that the S-109 simulant represents the most difficult wastes from a MIS mitigation standpoint and might suggest tank blending schemes or additions that could be made that would reduce MIS migration.

- Further investigations of the cellulose/nitrate/nitrite waste reactions should be conducted to ensure that the exothermic reactions necessary to prevent MIS migration are properly controlled in other portions of the BV process to address any safety concerns.

- If the small levels of MIS penetration seen with the GFM/cellulose glass formulations prove to be a concern, modifying the inner layer of the CRB with sodium silicate appeared to be an effective way to create a sacrificial layer on the inner surface that might help return any penetrated Tc back to the glass melt. ES tests would be the next step in a process to see if this method could further reduce the levels of Tc that remain in the CRB after melting. 


\subsection{References}

Darab JG, and PA Smith. 1996. "Chemistry of Technetium and Rhenium Species during Low-Level Radioactive Waste Vitrification.” Chem. Mater. 8:1004-1021.

Cooley SK, EM Pierce, LM Bagaasen, and MJ Schweiger. 2006. Analysis of Soluble Rhenium Concentrations in the Refractory from Bulk Vitrification Full-Scale Test 38B. PNNL-15868, Pacific Northwest National Laboratory, Richland, WA.

Gibson JK. 1993a. “High Temperature Oxide and Hydroxide Vapor Species of Technetium.” Radochim. Acta 60:121-126.

Gibson JK. 1993b. "Mass Spectrometric Identification of Potassium and Cesium Pertecnetates and Bimetallic Technetium-Rhenium Oxide Dimers.” Radiochim. Acta 62:127-132.

Hrma P, CE Goles, and DD Yasuda. 1991. “Drainage of Primary Melt in a Glass Batch.” Ceram. Trans. 23:361-367.

Hrma P, J Matyas, LM Bagaasen, KBC Mimister, AE Beck, MJ Schweiger, TM Brouns, DM Strachan, DD Caldwell, BP Tinsley, ML Elliott, and GW Hollenberg. 2005a. Bulk Vitrification Castable Refractory Block Protection Study. PNNL-15193, Pacific Northwest National Laboratory, Richland, WA.

Hrma P, J Matyas, AE Beck, MJ Schweiger, DD Caldwell, BP Tinsley, DM Strachan, ML Elliott, LM Bagaasen, TM Brouns, and GW Hollenberg. 2005b. "Bulk Vitrification Castable Refractory Block Protection Against Molten Ionic Salt Penetration.” In: Proceedings of International Symposium on Radiation Safety Management. Daejeon, S. Korea.

Kim D, CZ Soderquist, JP Icenhower, BP McGrail, RD Scheele, BK McNamara, LM Bagaasen, MJ Schweiger, JV Crum, JD Yeager, J Matyáš, LP Darnell, HT Schaef, AT Owen, AE Kozelisky, LA Snow, and MJ Steele. 2005. Tc Reductant Chemistry and Crucible Melting Studies with Simulated Hanford Low-Activity Waste. PNNL-15131, Pacific Northwest National Laboratory, Richland, WA.

Kim D, MJ Schweiger, J Matyas, AB Gallegos, A Fluegel, JV Crum, and ML Elliott. 2006. Letter Report: No-Iron Glass Formulation Crucible Tests Results. PNNL-15706, Pacific Northwest National Laboratory, Richland, WA.

R. W. Shimskey, W. C. Buchmiller, and M. R. Elmore. 2007. Testing Report: Littleford-Day Dryer Operation: Dryer Operation Impacts of Proposed MIS Mitigation Changes. PNNL-16659, Pacific Northwest National Laboratory, Richland, Washington.

Wilburn FW, and CV Thomasson. 1958. "The Application of Differential Thermal Analysis and Thermogravimetric Analysis to the Study of Reactions between Glass Materials: Part 1, The Sodium Carbonate System.” J. Soc. Glass Tech. 42:156T-175T. 


\subsection{Technical Procedures}

APEL-PAD-V, Rev. 2. 2002. Operation of Scintag Pad-VX-Ray Diffractometer. Safe Operating Procedure.

GDL-GBM, Rev. 3. 2002. Glass Batching and Melting. Pacific Northwest National Laboratory, Technical Procedure.

GDL-XRD. 2002. Quantitative and Semi-quantitative analysis using X-Ray Diffraction, Pacific Northwest National Laboratory, Technical Procedure. 



\section{Appendix A}

\section{CRB Inner-Surface Treatment}




\section{Appendix A: CRB Inner-Surface Treatment}

Previous research showed that molten ionic salt penetration appears to play a role in moving Tc and Re into the CRB (Hrma et al. 2005). Tests were performed where the inner layer of the CRB was altered to reduce the content of soluble Tc/Re in the final BV block. Two possible alterations were examined. First, the inner CRB layer was modified to form a durable glass that would immobilize the MIS near the surface of the refractory. Second, the inner layer of the CRB was filled with a carbonaceous material in an attempt to slow MIS penetration and accelerate nitrate decomposition. Laboratory tests were performed using crucible melts and hot-stage microscopy.

\section{A.1. Creating a Sacrificial Inner Layer on CRB}

The vibrocast porosity is unavoidable, but mixing fluxes with the refractory material may soften the innermost layer of the CRB that would eventually be vitrified and fused with the bulk glass without compromising the integrity of the refractory lining of the BV block. Adding fluxes would not prevent MIS penetration in the CRB because the sealing of the inner layer will proceed only at temperatures well above the nitrate melting point.

A fluxed sacrificial layer can immobilize Tc/Re only if it is thicker than the MIS penetration depth. In engineering scale test ES 31B, the estimated thickness of Tc/Re penetration was $15 \mathrm{~mm}$ (Hrma et al. 2005). A sacrificial layer thicker than $15 \mathrm{~mm}$ would seal and possibly incorporate Tc/Re in the glass phase when the temperature rises above the softening point of the sacrificial layer. Eventually, it may fuse with the BV glass, thus effectively returning the Tc/Re back to the main body of the BV melt. The 15-mm layer was established as an initial target, but subsequent FS tests have indicated that the penetration may be deeper than that seen in the ES tests. For example, in FS-38C, the CRB was in contact with feed for an extended period of time, and MIS penetrated through the entire wall of the CRB. A sacrificial layer would not protect the CRB in this case.

Because of its potency as a flux and its low cost, sodium metasilicate, $\mathrm{Na}_{2} \mathrm{SiO}_{3}$ (NS), was selected as a suitable modifier to be mixed into the surface layer of the CRB. Only layers with constant NS fractions were tested in these scoping studies. However, if layers with constant concentrations of NS were not mechanically sound up to the required temperatures, the NS content could be graded to reduce mechanical stresses.

Sodium metasilicate was prepared by melting sodium carbonate $\left(\mathrm{Na}_{2} \mathrm{CO}_{3}\right)$ with silica $\left(\mathrm{SiO}_{2}\right)$. The components for making $300 \mathrm{~g}$ of NS were mixed in the agate mill for $1 \mathrm{~min}$, placed into a platinum crucible, and heat-treated at $1250^{\circ} \mathrm{C}$ for 1 hour. The melt then was poured on a steel plate. The glass was ground in the tungsten carbide mill for $4 \mathrm{~min}$ and kept in a desiccator to prevent hydration from moisture in the air.

To determine the optimum content of NS in the sacrificial layer, powdered vibrocast was mixed with NS in several ratios from 20 to 50 mass\% NS in vibrocast. Vibrocast powder was mixed with NS in the agate mill for 1 min to prepare 100-g batches, of which 30-g samples were placed into silica crucibles and ramp heated at $5^{\circ} \mathrm{C} / \mathrm{min}$ to $1200^{\circ} \mathrm{C}$ with a 2-hour hold. Crucibles were then sectioned as shown in Figure A.1. 
NS, mass $\%$

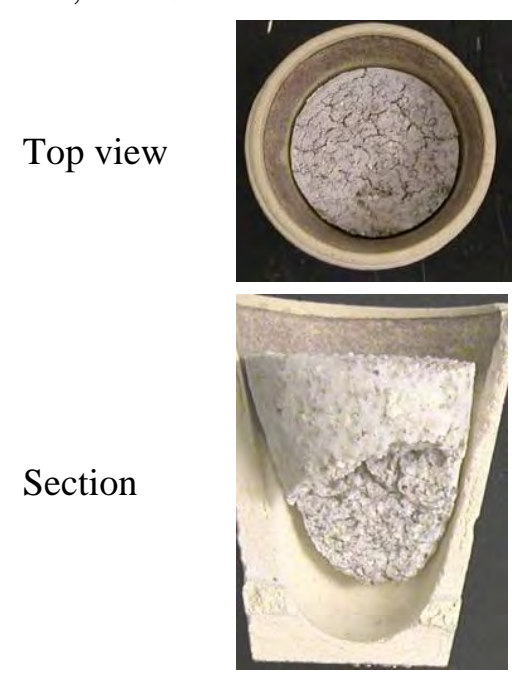

30
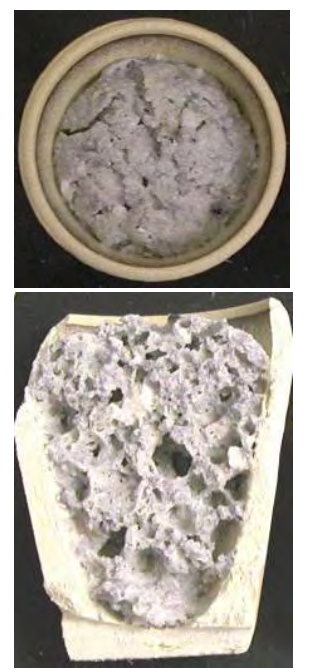

40
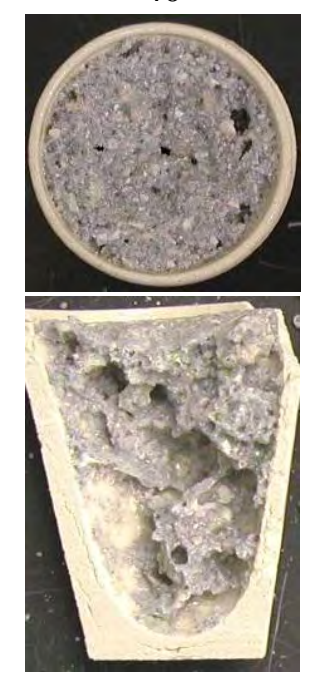
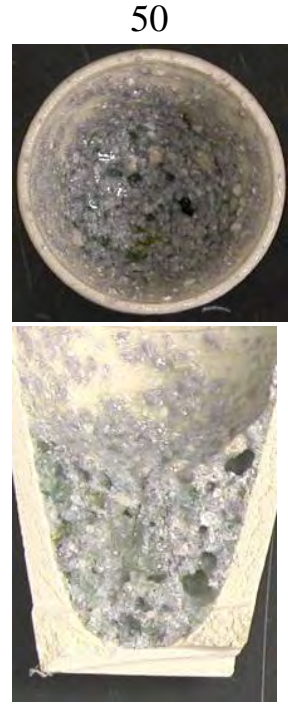

Figure A.1. Mixtures of Vibrocast with NS Heat-Treated at $1200^{\circ} \mathrm{C}$ for 2 Hours

The mixture with $20 \%$ NS formed a porous, white, concrete-like solid. Mixtures with 30 to 50 mass\% of NS expanded on heating, forming a foamy mass with large irregular cavities. After initial foaming, the mixture containing 50 mass\% of NS partially collapsed to glassy mass that encapsulated refractory grains (Figure A.2).

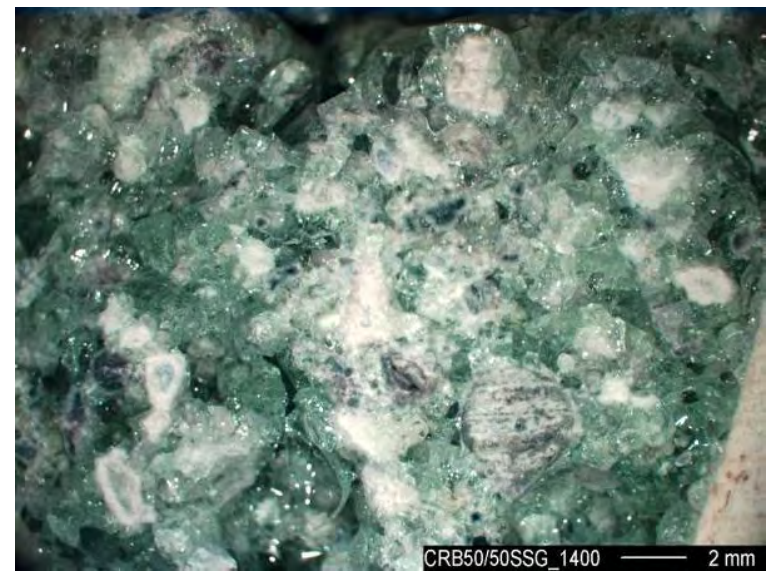

Figure A.2. Vibrocast with 50 Mass\% NS Heat-Treated at $1200^{\circ} \mathrm{C}$ for 2 Hours

In the following series of tests, 30-g samples of vibrocast with 20 and 50 mass\% of NS were heated in Pt crucibles at $5^{\circ} \mathrm{C} / \mathrm{min}$ to temperatures of $800^{\circ} \mathrm{C}$ to $1400^{\circ} \mathrm{C}$ with $100^{\circ} \mathrm{C}$ increments. Table A.1 shows the LOI of the mixtures as a function of temperature and the NS content. Extrapolation to zero NS content yields 0.0076 for the vibrocast LOI. Using this value for the vibrocast LOI, the NS LOI increased with temperature as shown in Figure A.3, stabilizing at 2.1 mass $\%$ at $1200^{\circ} \mathrm{C}$. The CRB LOI would release $17 \mathrm{~mL} / \mathrm{g}$ of gas phase at $1000^{\circ} \mathrm{C}$. Foaming is caused by the glass phase trapping some of the gas from the $\mathrm{CRB}$ and more gas from it own loss of chemical water. 
Table A.1. LOI of Vibrocast-NS Mixtures as a Function of Temperature and NS Content (in mass fractions)

\begin{tabular}{|r|c|c||}
\hline $\boldsymbol{T},{ }^{\circ} \mathbf{C}$ & $\mathbf{0 . 2}$ & $\mathbf{0 . 5}$ \\
\hline 800 & 0.0083 & 0.0103 \\
\hline 900 & 0.0093 & 0.0127 \\
\hline 1000 & 0.0097 & 0.0127 \\
\hline 1100 & 0.0100 & 0.0133 \\
\hline 1200 & 0.0103 & 0.0143 \\
\hline 1300 & 0.0103 & 0.0133 \\
\hline 1400 & 0.0103 & 0.0147 \\
\hline
\end{tabular}

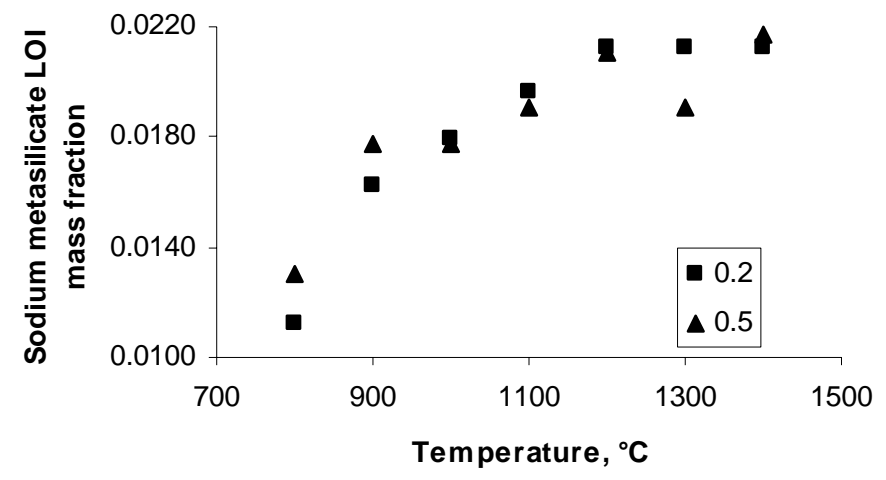

Figure A.3. LOI of NS as a Function of Temperature and NS Content (in mass fractions)

Figure A.4 shows the melt appearance after the heat-treatments. Generally, 20 mass\% of NS affected the vibrocast response to heating significantly less than 50-mass\% addition. The beginning of sintering was discernible already at $800^{\circ} \mathrm{C}$, even with 20 -mass $\%$ addition of NS, but the mixture would easily disintegrate. The mixture became solid at $900^{\circ} \mathrm{C}$, increasingly shrank and cracked at 1000 to $1200^{\circ} \mathrm{C}$, showed signs of closing porosity at $1300^{\circ} \mathrm{C}$, and began to expand at $1400^{\circ} \mathrm{C}$ (see Figure A.5). With 50 -mass $\%$ addition, the mixture became solid at $800^{\circ} \mathrm{C}$, substantially shrank at $900^{\circ} \mathrm{C}$, foamed and expanded at 1000 to $1100^{\circ} \mathrm{C}$, gradually collapsed at 1200 to $1300^{\circ} \mathrm{C}$, and was largely vitrified at $1400^{\circ} \mathrm{C}$ (see Figure A.6 and Figure A.7). 


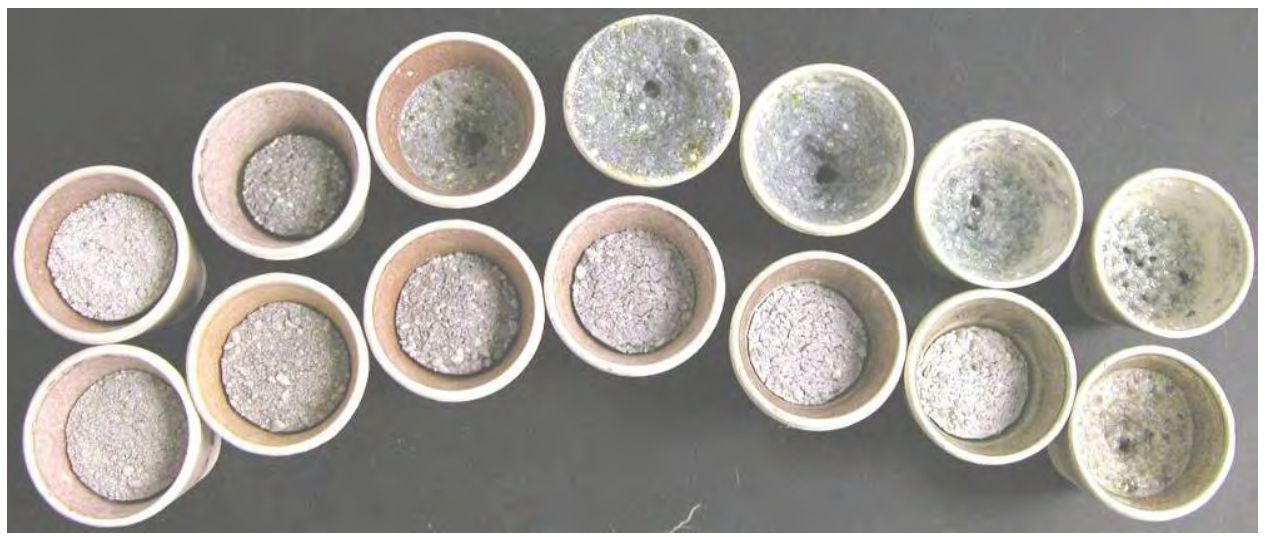

Figure A.4. Vibrocast with 50 Mass\% of NS (top) and with 20 Mass\% of NS (bottom) After Heating at $5^{\circ} \mathrm{C} / \mathrm{min}$ to the Following Temperatures (from left to right): 800, 900, 1000, $1100,1200,1300$, and $1400^{\circ} \mathrm{C}$
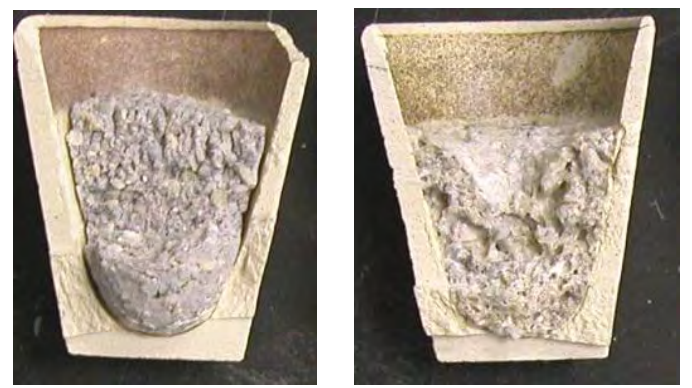

Figure A.5. Fractured Vibrocast with 20 -Mass \% of NS Heated to $1300^{\circ} \mathrm{C}$ (left) $1400^{\circ} \mathrm{C}$ (right)
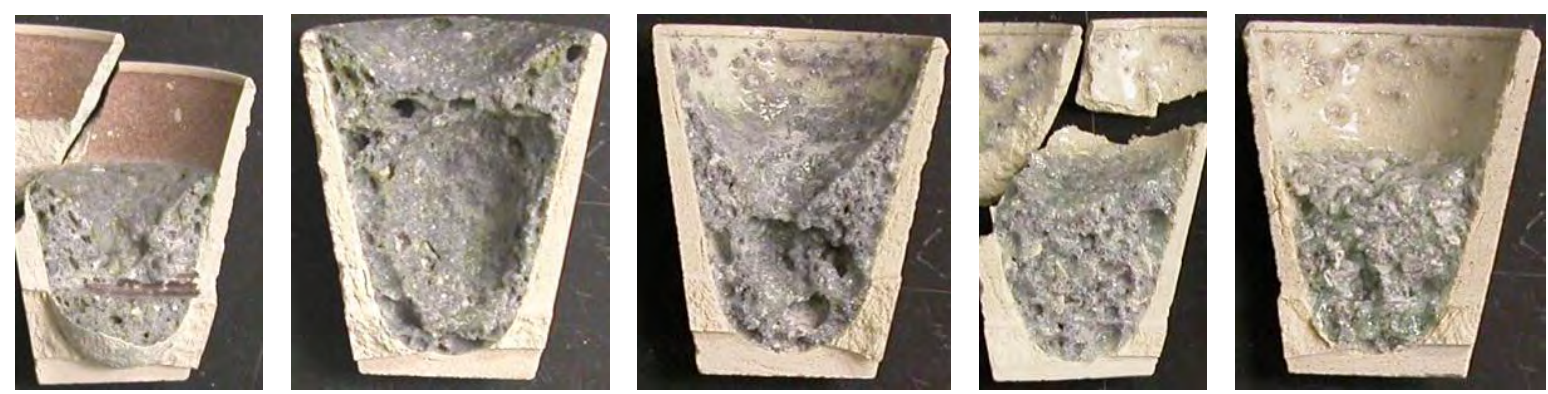

Figure A.6. Fractured Vibrocast with 50-Mass\% of NS Heated to (from left to right) $1000^{\circ} \mathrm{C}, 1100^{\circ} \mathrm{C}, 1200^{\circ} \mathrm{C}, 1300^{\circ} \mathrm{C}$, and $1400^{\circ} \mathrm{C}$ 

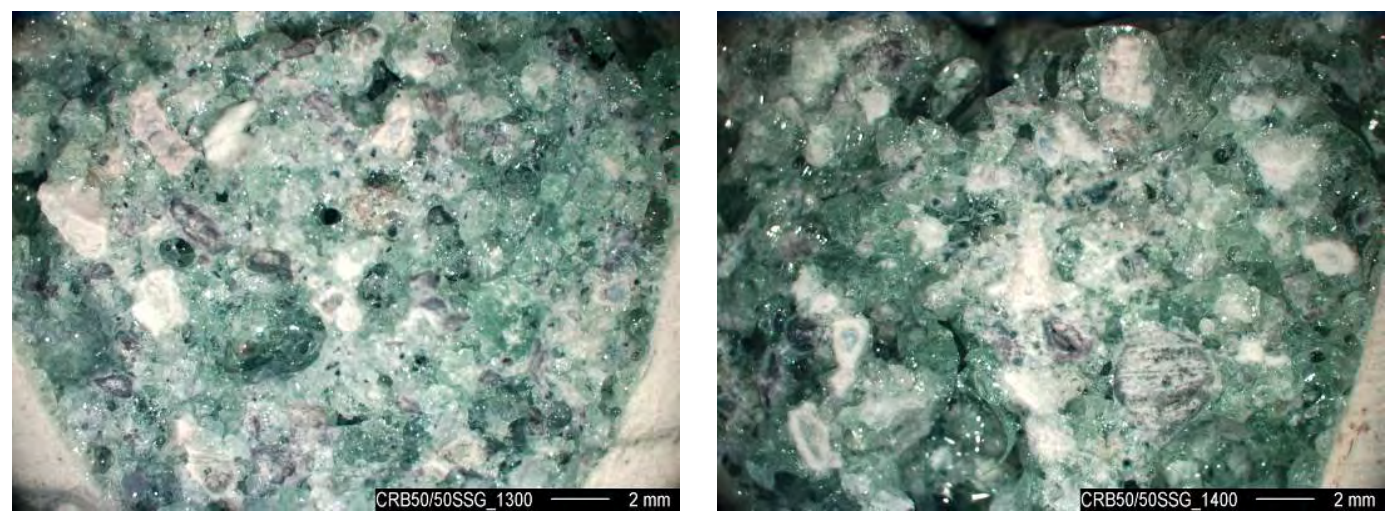

Figure A.7. Detail of Fractured Vibrocast with 50-mass\% of NS Heated to $1300^{\circ} \mathrm{C}$ (left) and $1400^{\circ} \mathrm{C}$ (right)

As the next step, samples of vibrocast and their mixtures with NS were exposed to engineering-scale-test feed (ESF) and heated at $5^{\circ} \mathrm{C} / \mathrm{min}$. The ESF has the same composition as that shown in Table 4.2. The heat-treatments were performed in high-density alumina crucibles. Three arrangements were used: unaltered compacted vibrocast, vibrocast with NS, and a CRB specimen cut from the producer-rammed vibrocast (Figure A.8, left), all covered with the BV feed.
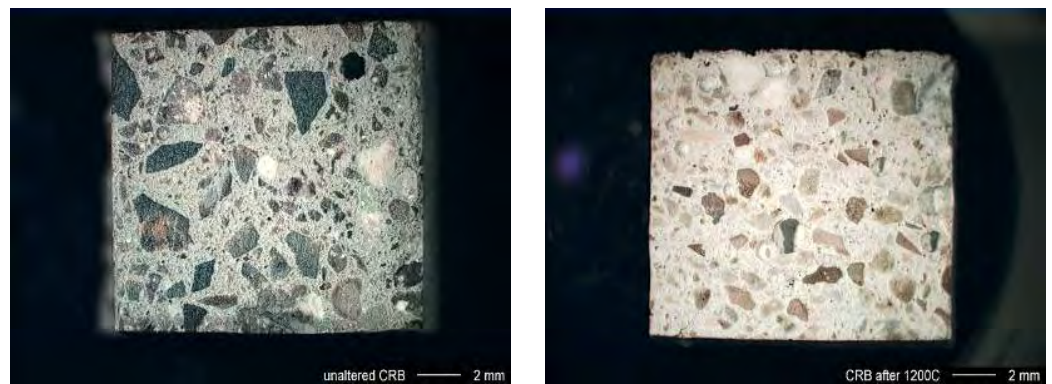

Figure A.8. CRB Specimen, as Cut (left) and After 1 Hour at $1200^{\circ} \mathrm{C}$ (right)

Three sets of heat-treatments were performed. In the first sets of heat-treatments, one crucible was filled with $5 \mathrm{~g}$ of vibrocast and the other with $5 \mathrm{~g}$ of vibrocast with 20 mass\% of NS. Both powders were firmly compacted into the crucibles, periodically adding a few drops of water until the volume of the compacted powders was minimized. The total volume of water added to each compact was $1 \mathrm{~mL}$. The compacts were dried at $110^{\circ} \mathrm{C}$ for 3 hours. A CRB specimen was placed in another crucible. Both compacts and the specimen were covered with $15 \mathrm{~g}$ of the ESF and ramp-heated at $5^{\circ} \mathrm{C} / \mathrm{min}$, starting at $200^{\circ} \mathrm{C}$ and advancing to $1300^{\circ} \mathrm{C}$ and then held for 1 hour. Then the crucibles were sectioned and examined.

As Figure A.9 shows, the ESF melt broke down the compact vibrocast, dissolving its fine-grain portion and leaving pieces of large grains scattered throughout the melt. The ESF melt corroded away approximately one half of the CRB specimen, turning the other half into a porous, glossy, sponge-like body. Finally, the ESF melt broke down all of the compact vibrocast with 20 mass\% of NS. Even the large grains were attacked, leaving only residues in the melt. 

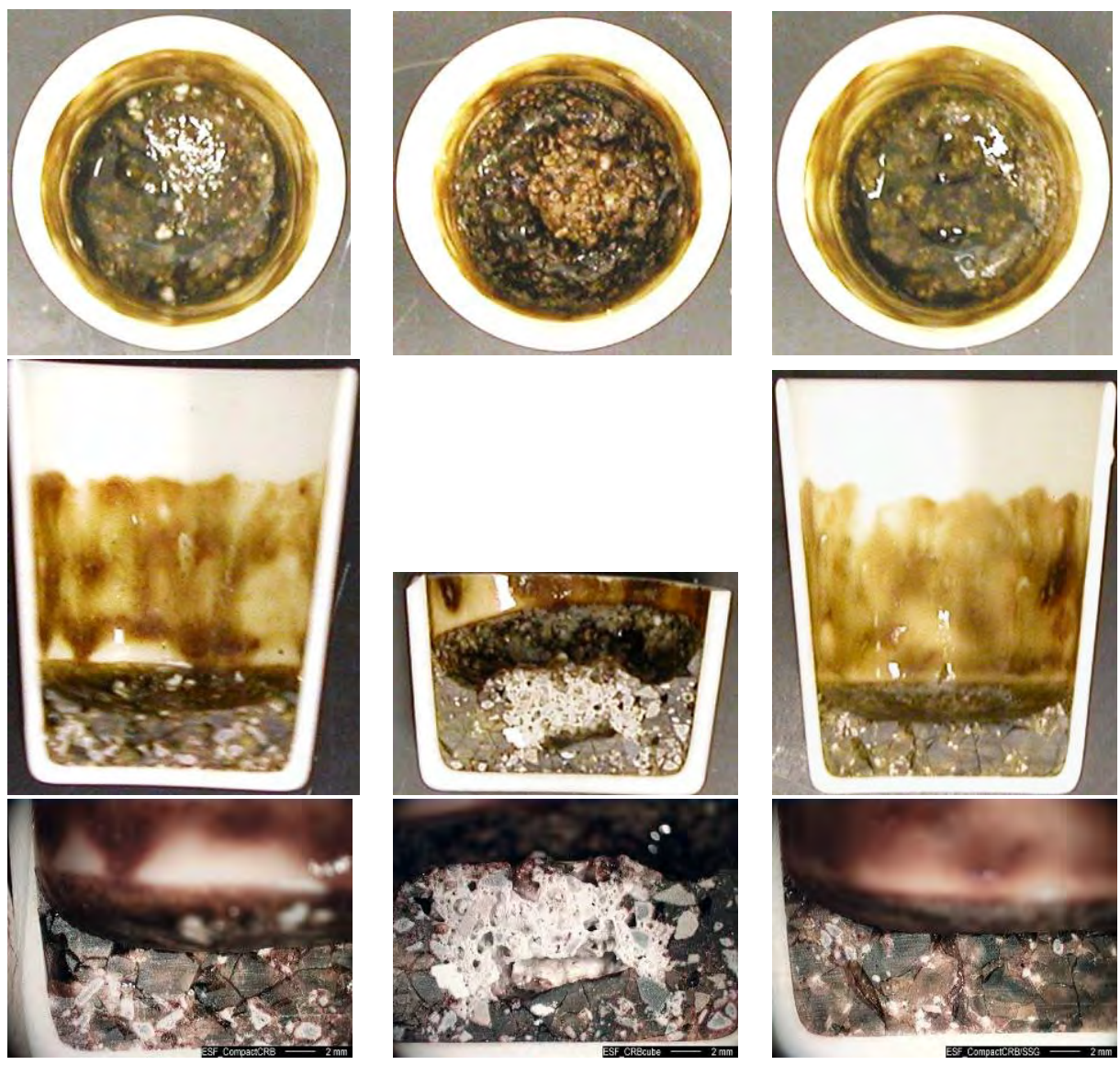

Figure A.9. ESF Melted over Compact Vibrocast (left), CRB Specimen (middle), and Compact Vibrocast with $20 \%$ of NS (right) $1300^{\circ} \mathrm{C}, 1 \mathrm{Hr}$

These experiments showed that the conditions at 1 hour at $1300^{\circ} \mathrm{C}$ were too harsh even for the unaltered CRB. These conditions are probably rarely experienced in the walls of FS BV melters. Therefore, the second set of heat-treatments was performed. Crucible assemblies were heated at $5^{\circ} \mathrm{C} / \mathrm{min}$ to $1200^{\circ} \mathrm{C}$ with a 1-hour hold with 15 grams of ESF. Testing was done in high-density alumina crucibles containing $15 \mathrm{~g}$ of vibrocast, $15 \mathrm{~g}$ of vibrocast with 20\% of NS, and a CRB specimen as seen in Figure A.10. 

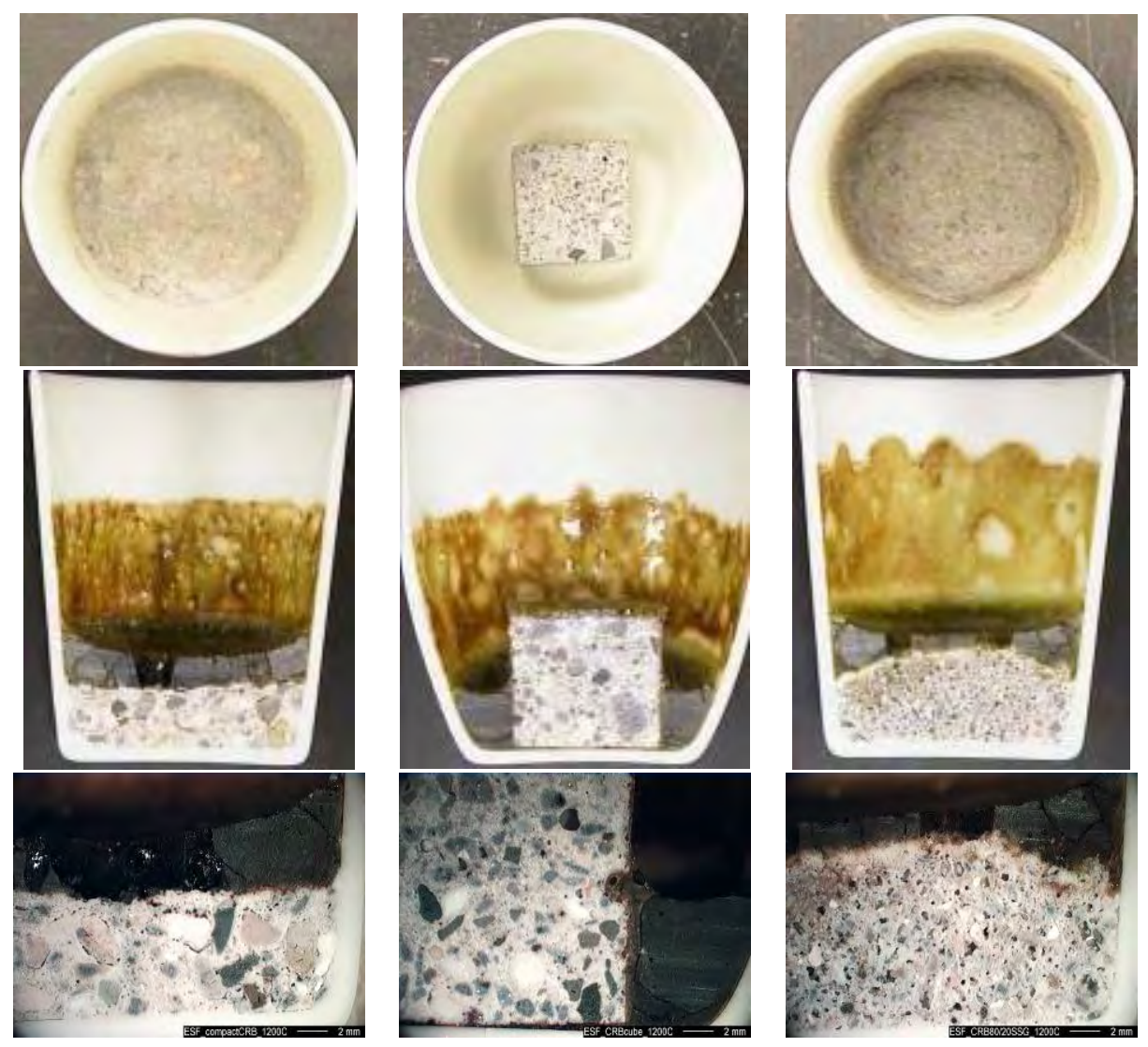

Figure A.10. Alumina Crucibles with Compact Vibrocast (left), CRB Specimen (middle), and Compact Vibrocast with $20 \%$ of NS (right); the Refractories Were Covered with ESF and Heat-Treated; Sections Through the Crucibles Are in the Bottom Row of Images $1200^{\circ} \mathrm{C}, 1 \mathrm{Hr}$

The interface between the ESF and the compact vibrocast showed minor corrosion, leaving the vibrocast integrity uncompromised. The melt also did not attack the CRB specimen. The light pink tint of both the compact and the CRB specimen indicated MIS penetration. The ESF began to fuse with the NScontaining vibrocast, forming a corrosion layer between the glass and the compact mixture. The compacted vibrocast is more porous than the CRB specimen, resembling the CRB in FS BV melters. Rounded, shiny-smooth pores are visible in the fine-grained portion of vibrocast, both in the compact and in the specimen, indicating that $\mathrm{Na}_{2} \mathrm{O}$ from the MIS reacted with the refractory cement, creating a viscous glass-forming melt. A CRB specimen subjected to the same heat-treatment without the presence of ESF (shown in Figure A.8, right) exhibits a different texture: the microcracks in the fine-grained portion have rough edges without a viscous smoothing tendency.

The third set of heat-treatments was performed with four large alumina crucibles containing $15 \mathrm{~g}$ of vibrocast, $15 \mathrm{~g}$ of vibrocast with $20 \%$ of NS, $30 \mathrm{~g}$ of vibrocast with $30 \%$ of NS, and a CRB specimen. Large alumina crucibles allowed an increase of the amount of feed to $45 \mathrm{~g}$. The heat-treatment was $5^{\circ} \mathrm{C} / \mathrm{min}$ to $1200^{\circ} \mathrm{C}$ held for 1 hour.

As in the second set, the compact vibrocast and the CRB specimen were permeated with MIS but were only slightly attacked by molten glass. Partial vitrification and development of closed pores can be seen 
in details shown in the bottom row of Figure A.11. The presence of $20 \%$ of NS in vibrocast caused a partial vitrification of the refractory and a significant, but not severe, corrosion by the melt. The refractory layer containing 30\% of NS in the vibrocast reacted with the glass, forming a nearly uniform heterogeneous mass of glass with suspended solid particles and gas bubbles. Based on these results, a $25 \%$ addition of NS to vibrocast was recommended as an optimum composition of a sacrificial layer.
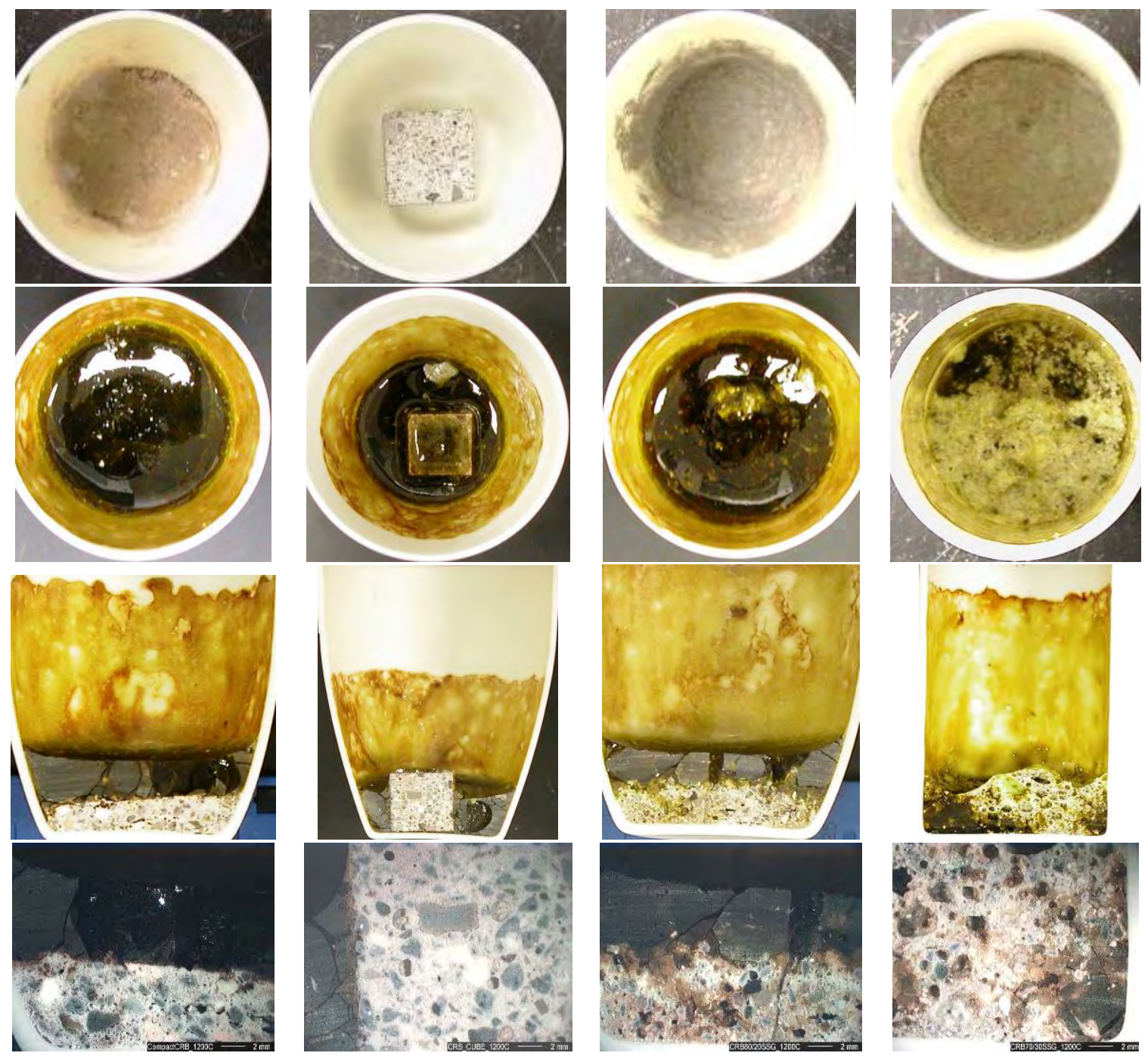

Figure A.11. Alumina Crucibles with Compact Vibrocast (left), CRB Specimen (second from left), Compact Vibrocast with $20 \%$ of NS (second from right), and Compact Vibrocast with $30 \%$ of NS (right); the Refractories Were Covered with ESF and Heat-Treated at $1200^{\circ} \mathrm{C}$ for 1 Hour; the Rows Show (from above down) Raw Refractory Materials, Melts, Sections Through Crucibles, and Details of Refractory Materials After HeatTreatment 
The final test was performed with vibrocast containing 25 mass\% of NS. A CRB specimen was placed into the center of an alumina crucible. The space around the specimen was filled with compact vibrocast to make an $\sim 20$-mm-thick layer (Figure A.12). On top of this layer, an 6-mm layer of vibrocast with $25 \%$ of NS was rammed. This assembly was dried at $110^{\circ} \mathrm{C}$ for 3 hours and ramped to $200^{\circ} \mathrm{C}$ at $30^{\circ} \mathrm{C} /$ hour to drive away moisture. During drying, the NS-containing vibrocast began to rise and was compacted back down into the crucible. The dry layer had a concrete-like hardness. Three times the mass of the dried refractory of ESF was added to the crucibles and heated at $5^{\circ} \mathrm{C} / \mathrm{min}$ to $1200^{\circ} \mathrm{C}$ with a 4 hour hold.
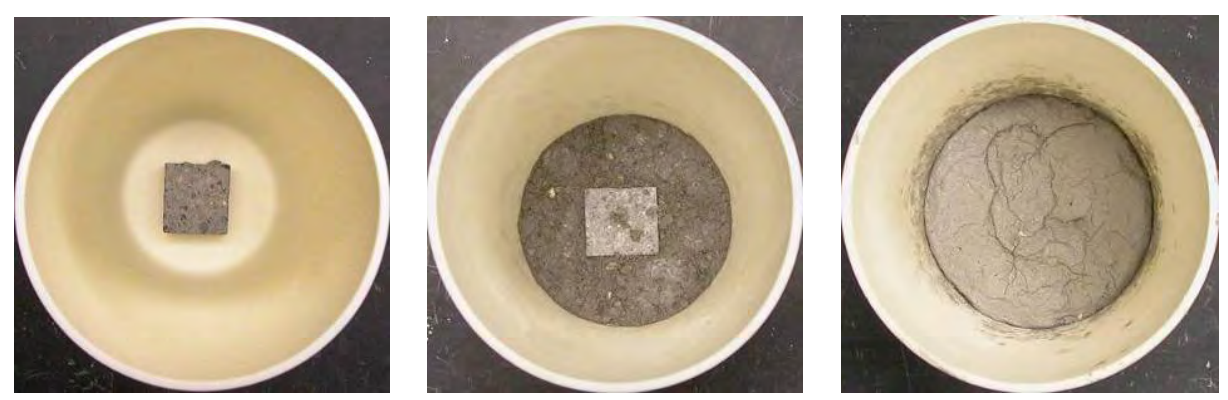

Figure A.12. Preparation of CRB Assembly: CRB Specimen, Specimen and Vibrocast Compact, and Vibrocast with 25 mass\% NS on Top

As the section through the crucible shows (Figure A.13), the fine-grain portion of the vibrocast fused with the NS component, forming a yellow transparent glass. A part of this glass, together with undissolved large grains, floated to the top of the waste glass melt where it remained with segregated yellow sulfate. The NS melt also attacked the vibrocast compact and specimen. As the compacted vibrocast is more porous than the CRB specimen, slightly more of it was corroded away (Figure A.14). The pink tint of the bottom layer (the compact and the CRB specimen) indicates that MIS penetrated the whole refractory assembly. The MIS-penetrated fine-grained portion of vibrocast shows signs of vitrification: the pores are rounded and shiny-smooth.
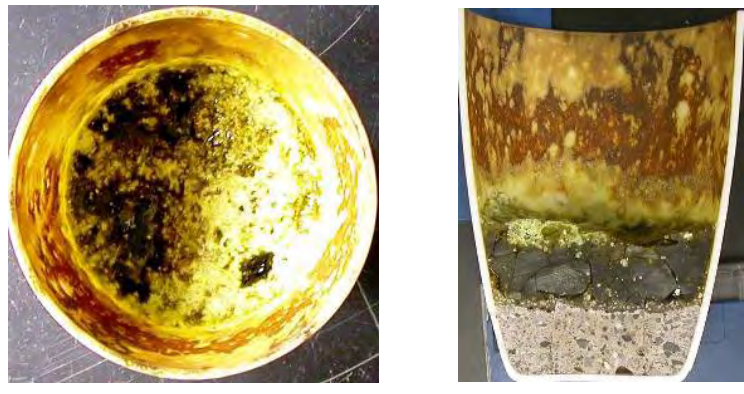

Figure A.13. Crucible Section Showing the Destruction of the NS-Containing Layer and Partly Corroded Vibrocast Compact and Specimen (the assembly was heat-treated for 4 hours at $1200^{\circ} \mathrm{C}$ ) 


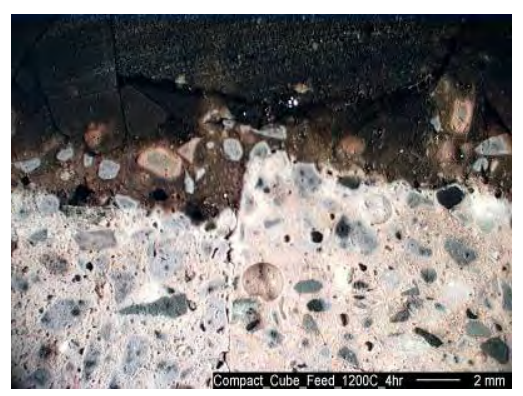

Figure A.14. Details of the Refractory-Melt Assembly Heat-Treated for 4 Hours at $1200^{\circ} \mathrm{C}$. In the area on top of CRB specimen (right) and vibrocast compact (left), only a thin layer of grain-containing glass remains from NS-containing vibrocast.

A thicker internal layer of vibrocast with 25 mass\% of NS would catch more of the advancing MIS and return it to the bulk melt. In the BV melter, most MIS penetration was seen in vertical walls. In the past tests, the horizontal base of the CRB was protected by melting glass frit at the base of the refractory. The penetration of MIS into the bottom-positioned refractory material in the crucible is promoted by gravity in addition to capillary suction. To observe MIS penetration through a vertical interface, vibrocast with 25 mass\% of NS was compacted vertically into a large cylindrical high-density alumina crucible and exposed to ESF at $1200^{\circ} \mathrm{C}$.

The crucible was vertically divided into halves with aluminum foil. In a plastic container, $20 \mathrm{~mL}$ of water was added to $240 \mathrm{~g}$ of vibrocast mixed with 25 mass\% of NS. The moist material was compacted in one half of the crucible and dried first at $110^{\circ} \mathrm{C}$ for 3 hours. The material expanded to three quarters of the crucible and was compacted back to a half of the crucible volume. The crucible was then heated at $5^{\circ} \mathrm{C} / \mathrm{min}$ to $200^{\circ} \mathrm{C}$ and held for 1 hour. A small expansion occurred during the treatment as seen in Figure A.15. The open half of the crucible was filled with ESF, and the crucible was ramp-heated to $500^{\circ} \mathrm{C}$. Then the feed was removed. Extending the feed-refractory contact to higher temperature was not considered necessary because experiments with silica crucibles (Sections 8 and 9) indicated that most of the MIS penetration occurs before the temperature reaches $500^{\circ} \mathrm{C}$, a temperature allowing an easy removal of the feed. The crucible with the refractory material was further heated to $1200^{\circ} \mathrm{C}$ and held for 1 hour. This operation was performed to decompose the MIS and thus make wet cutting possible. As Figure A.15 shows, the material swelled and became porous. Figure A.16 clearly indicates a limited penetration of MIS into the refractory. 

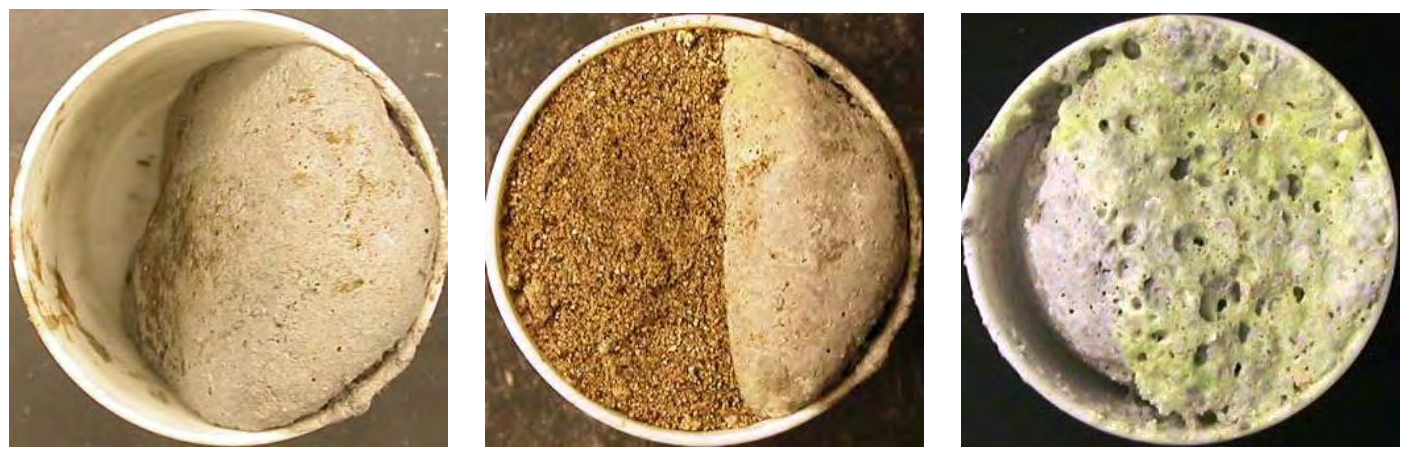

Figure A.15. From Left to Right: Crucible Containing Vibrocast with 25 Mass\% of NS Dried at $20{ }^{\circ} \mathrm{C}$; Same Crucible with ESF Heated to $500^{\circ} \mathrm{C}$; Same Crucible After Feed Removal and Heating to After $1200^{\circ} \mathrm{C}$
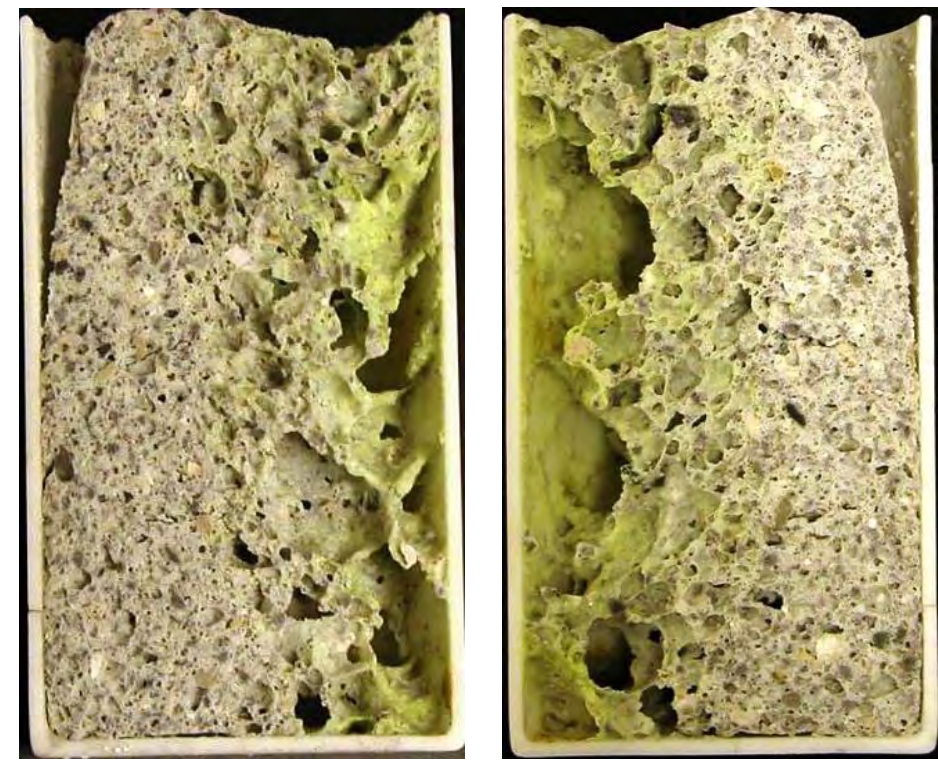

Figure A.16. Vertical Section Through the Crucible Shown in Figure A.15, Right

As an indicator of the manufacturability of the CRB with an inner layer of vibrocast containing 25 mass\% of NS, a composite of vibrocast and vibrocast with 25 mass\% of NS was prepared. The materials were compacted into a $100 \times 90 \times 5$-mm box made out of aluminum foil. To ease compacting, $1 \mathrm{~mL}$ of water was added for every $15 \mathrm{~g}$ of the vibrocast (the bottom layer), and $2 \mathrm{~mL}$ of water was added for every $15 \mathrm{~g}$ of the vibrocast with NS (the top layer). The thickness of the vibrocast layer was 15 to $20 \mathrm{~mm}$ and the thickness of the vibrocast with 25 mass\% NS was 10 to $15 \mathrm{~mm}$. The vibrocast-with-NS layer expanded to between 20 to $25 \mathrm{~mm}$ on heating to $200^{\circ} \mathrm{C}$. The composite was heated at $5^{\circ} \mathrm{C} / \mathrm{min}$ to $1200^{\circ} \mathrm{C}$ and removed from the furnace to cool down. The composite was cut perpendicular to the interface. Figure A.17 shows micrographs of the interface. The vibrocast with NS contains large pores, mostly $\leq 1 \mathrm{~mm}$. The interface is reddish in color and contains small $(\leq 0.1-\mathrm{mm})$ pores. A tiny crack can be discerned between the reddish interface and the vibrocast layer below. However, the composite appeared well 
connected without any tendency to fall apart when hit by a hammer, indicating that it may be possible to manufacture large sections of CRB with sacrificial inner layers.
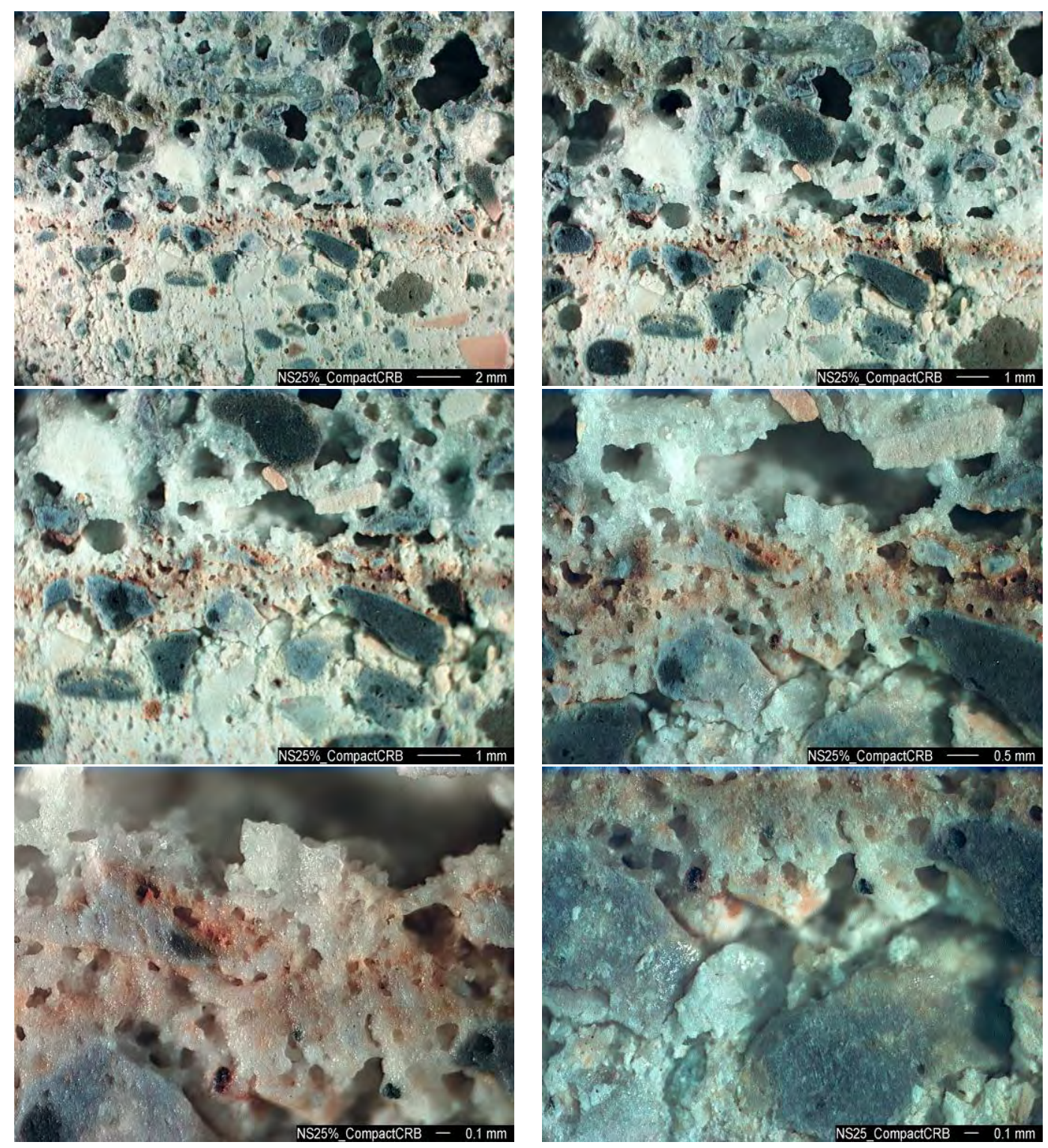

Figure A.17. Interface Between Vibrocast and Vibrocast-NS (25\%) Mixture

\section{A.2. Creating an Inner Layer on CRB that is less Susceptible to MIS Penetration}

The penetration of MIS into the CRB pores is mainly driven by capillary forces. Since the CRB is readily wetted by molten salts and MIS has a low viscosity, filling the pores proceeds virtually unhindered. On the other hand, carbon is not readily wetted by ionic salts because the contact angle between ionic

materials and materials with a metallic bond is generally large. Therefore, filling the inner layer of CRB 
with carbon or an organic material, such as sucrose, which produces carbon on heating by pyrolysis, is likely to interfere with MIS penetration by making the material more lyophobic with respect to molten salts. Moreover, fine carbon may also present a purely mechanical obstacle by clogging the pores. Also, carbon may react with the MIS, reducing its components, bringing about early decomposition of nitrates, possible reduction of sulfates, and even reducing the pertechnetate and perrhenates to insoluble oxides $\mathrm{TcO}_{2}$ and $\mathrm{ReO}_{2}$. Additionally, offgas from the carbon reaction with nitrates may generate a back pressure that would further reduce MIS penetration into the CRB pores. It is also possible that the $\mathrm{Na}_{2} \mathrm{O}_{2}$ generated by the nitrate reduction (Abe et al. 1983) would react with the CRB components at lower temperatures and create viscous melts that would immobilize Tc/Re and seal the refractory surface earlier than in the standard CRB.

For preliminary experiments, an attempt was made to soak CRB specimens with molten sucrose, molten wax, and molten polyvinyl alcohol. In another set of experiments, carbon black, carbocite, and graphite were mixed with the vibrocast powder and compacted to make a CRB sample.

The soaking tests were performed with CRB specimens, typically $\sim 20 \times 20 \times 18 \mathrm{~mm}$. The specimens were placed into alumina crucibles and heated under parowax (m.p. $60^{\circ} \mathrm{C}$ ), sucrose (m.p. $160^{\circ} \mathrm{C}$ ), and polyvinyl alcohol. The test with polyvinyl alcohol was aborted because polyvinyl alcohol sintered and foamed, making a viscous solid mass that had no tendency to penetrate into CRB pores. Sucrose was allowed to soak into the CRB specimen for 3 hours at $160^{\circ} \mathrm{C}$ and parowax for 4 hours at $90^{\circ} \mathrm{C}$ (as long as releasing of bubbles from the specimen continued). Figure A.18 shows the specimens. The sucrosesoaked specimen had a caramel coating on the surface. Sectioning showed that wax penetrated the specimen completely, while the sucrose only penetrated $\sim 0.5 \mathrm{~mm}$ from the specimen surface.
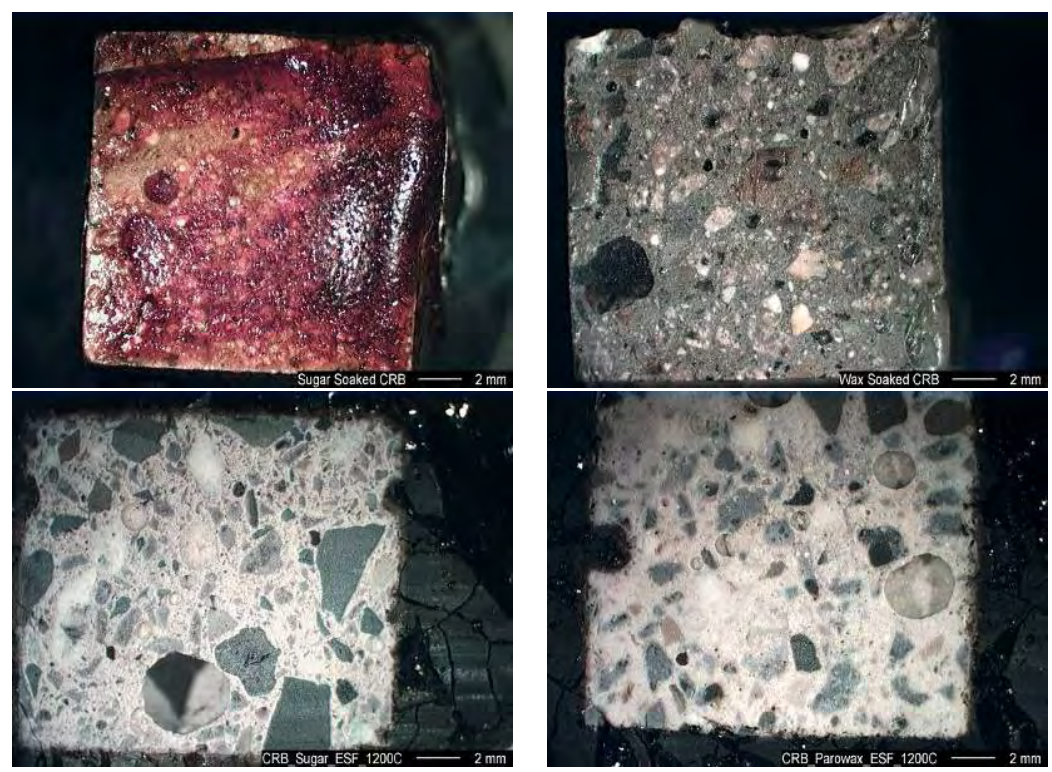

Figure A.18. CRB Soaked with Caramel (top left) and with Parowax (top right); Bottom Pictures Show Specimens Sectioned After Heat-Treatment in Baseline Feed 
Saturated specimens were covered with three times their mass of ESF. The crucibles were heated to $1200^{\circ} \mathrm{C}$ at $5^{\circ} \mathrm{C} / \mathrm{min}$ and held at $1200^{\circ} \mathrm{C}$ for 1 hour. Sectioned crucibles were examined for MIS penetration. The wax-soaked specimen became white with a pinkish tint throughout the specimen, indicating MIS penetration. The sucrose-soaked specimen did not show any pink tint inside the specimen, indicating some level of protection against MIS penetration. Vacuum impregnation was attempted to increase the level of sugar penetration but was not successful due to the viscosity of the sugar melt.

Five different forms of carbon were used to prepare mixtures of vibrocast with carbonaceous materials: anthracite coal, carbocite, lampblack, graphite, and carbon flakes. These materials, except lampblack, were powdered in a tungsten carbide mill for $5 \mathrm{~min}$ and then mixed with vibrocast. The mixtures, $20 \mathrm{~g}$ of each, were compacted into alumina crucibles while moisturizing them with 4-mL of water to achieve dense compacts. The compacts were dried at $105^{\circ} \mathrm{C}$ for 3 hours and ramped to $200^{\circ} \mathrm{C}$ at $5^{\circ} \mathrm{C} / \mathrm{min}$ with a 1-hour hold. Finally, $40 \mathrm{~g}$ of the ESF was put to each crucible on top of the compact. The crucibles were heated at $5^{\circ} \mathrm{C} / \mathrm{min}$ to $1200^{\circ} \mathrm{C}$ and held for 1 hour.

Table A.2 shows the measured masses of CRB mixes, the feed, and the glass. The glass mass was calculated using the glass/feed ratio of 0.765 . The measured mass of the crucible content (CRB + glass + carbonaceous additive [CA]), also shown in Table A.2, is smaller than the calculated mass. The difference was attributed to a loss of the carbonaceous material by oxidation, volatilization, an overspill of melt due to foaming, and the vibrocast LOI. As the glass line in Figure A.19 indicates, overflow could occur from all crucibles except from that containing carbon flakes. If attributed to the CA oxidation only, the CA loss was $\sim 1$ to 11 mass\%, except for anthracite, where overflow was a likely occurrence.

Table A.2. Masses in g of CRB with CAs, Feed, Corresponding Glass (79.5 mass\% of feed), Crucible Content after $1200^{\circ} \mathrm{C}$ Heat-Treatment, and Losses

\begin{tabular}{||l|c|c|c|c|c|c|c||}
\hline & CRB+CA & CA & Feed & Glass & CRB+CA+G & \multicolumn{2}{||}{ Loss } \\
\hline & Mass & Mass\% & Mass & Mass & Mass & Mass & CA fraction \\
\hline Anthracite & 19.93 & 17.2 & 39.86 & 31.68 & 48.40 & 3.21 & 0.589 \\
\hline Carbocite & 19.43 & 14.8 & 38.87 & 30.90 & 48.89 & 1.44 & 0.096 \\
\hline Lampblack & 19.69 & 22.4 & 39.39 & 31.31 & 49.32 & 1.68 & 0.114 \\
\hline Graphite & 19.84 & 22.4 & 39.68 & 31.54 & 49.98 & 1.40 & 0.048 \\
\hline Carbon flakes & 19.68 & 18.0 & 39.36 & 31.28 & 49.75 & 1.21 & 0.011 \\
\hline CA = carbonaceous additive, G glass \\
\hline \multicolumn{7}{|l|}{} \\
\hline
\end{tabular}

Figure A.19 shows sections through crucibles. The anthracite, carbocite, and carbon flake mixtures formed concrete-like compacts. The graphite compact was soft and crumbled away when a small pressure was applied to it. The carbon lampblack disintegrated during cutting. 


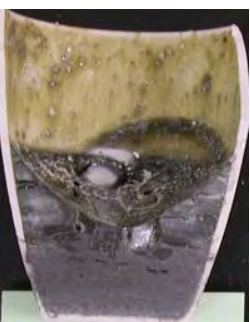

Anthracite

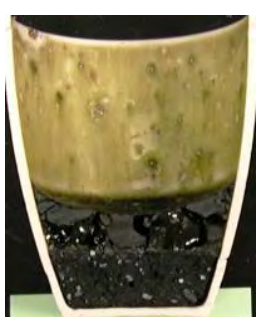

Carbocite

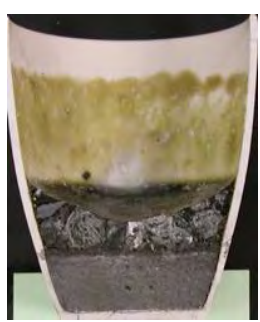

Carbon Flakes

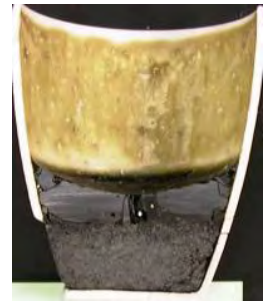

Graphite

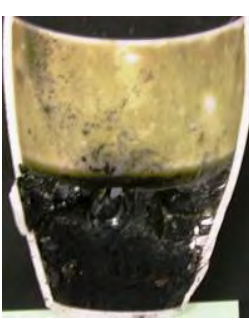

Lampblack

Figure A.19. Sections Through Crucibles with CRB Mixed with 20 Mass\% Carbonaceous Material at the Bottom and Glass on the Top

EDS analysis of the cut surface was performed for vibrocast mixed with carbon flakes and graphite exposed to ESF heated to $1200^{\circ} \mathrm{C}$ and held for 1 hour. The sample scan, shown in Figure A.20, went through the middle of the sample from the top to the bottom. As Figure A.20 shows, an $18 \%$ addition of ground-graphite decreased the amount of MIS that penetrated into the CRB.
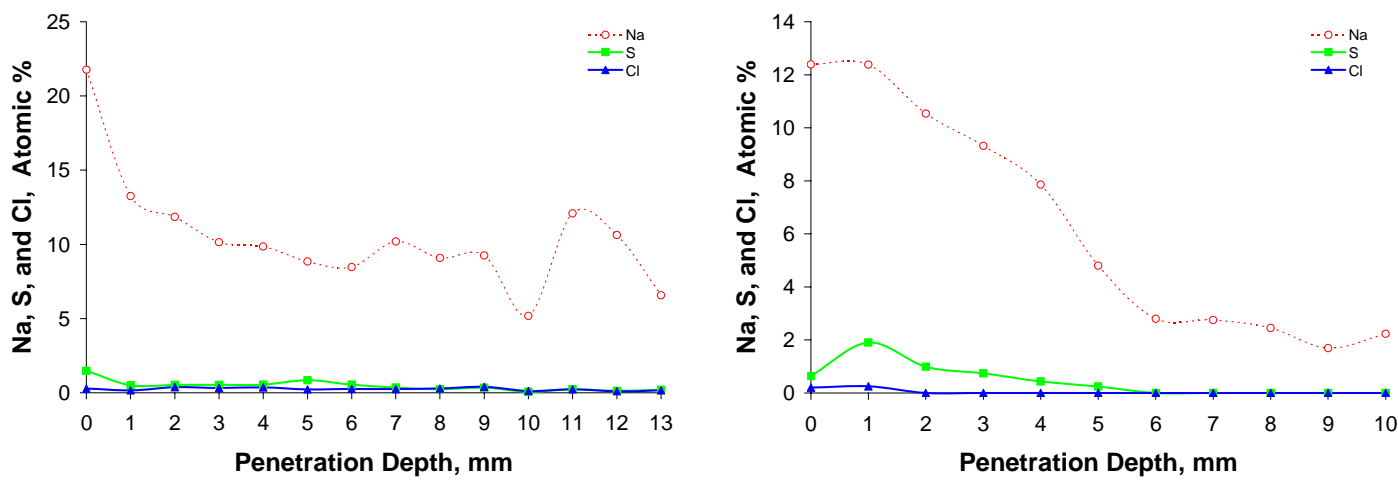

Figure A.20. Concentration Profiles by EDS of Compacted Vibrocast Mixed with 22.4 Mass\% of Carbon Flakes (left) and 18.0 Mass\% Graphite (right)

Additional experiments were performed with various additions of lampblack and graphite. Ground materials were combined with vibrocast at various proportions (6 to 22 mass $\%$ of CA in the mixture). Approximately $5 \mathrm{~mL}$ water and $1 \mathrm{~mL}$ of detergent were added to $20 \mathrm{~g}$ of each mixture that was compacted into an alumina crucible. The compacts were dried at $110^{\circ} \mathrm{C}$ for 1 hour, ramped to $200^{\circ} \mathrm{C}$ at $5^{\circ} \mathrm{C} / \mathrm{min}$, and held at $200^{\circ} \mathrm{C}$ for 1 hour. The compact was then covered with a double mass of ESF, ramp heated at $5^{\circ} \mathrm{C} / \mathrm{min}$ to $1200^{\circ} \mathrm{C}$, and held at $1200^{\circ} \mathrm{C}$ for 1 hour. The crucibles were sectioned and examined for MIS penetration.

Table A.3 lists the measured masses of CRB mixes, the feed, and the glass. The loss of mass during heattreatment can be attributed to oxidation of the carbonaceous material, melt overflow due to foaming, and the vibrocast LOI. 
Table A.3. Masses in g of CRB with CA, Feed, Corresponding Glass (76.5 mass \% of feed), Crucible Content after $1200^{\circ} \mathrm{C}$ Heat-Treatment, and Losses

\begin{tabular}{||l|c|c|c|c|c|c||}
\hline & & CRB+CA & Feed & Glass & CRB+CA+G & Loss \\
\hline CA & CA Fraction & Mass & Mass & Mass & Mass & Mass \\
\hline Lampblack & 0.224 & 21.85 & 43.70 & 33.43 & 55.28 & 2.44 \\
\hline & 0.170 & 21.09 & 42.18 & 32.27 & 53.36 & 1.84 \\
\hline & 0.114 & 20.36 & 40.72 & 31.15 & 51.51 & 0.94 \\
\hline & 0.057 & 20.45 & 40.90 & 31.29 & 51.74 & 1.32 \\
\hline Graphite & 0.224 & 20.7 & 41.4 & 31.67 & 52.37 & 0.67 \\
\hline & 0.170 & 21.17 & 42.34 & 32.39 & 53.56 & 1.37 \\
\hline & 0.114 & 20.43 & 40.86 & 31.26 & 51.69 & 0.77 \\
\hline & 0.057 & 20.51 & 41.02 & 31.38 & 51.89 & 1.98 \\
\hline CA = carbonaceous additive, G glass & & & \\
\hline
\end{tabular}

Figure A.21 shows top views of the crucibles and the sections. Compacts with 22.4 mass\% of carbonaceous material, both lampblack and graphite, were weak and easily broke apart under pressure. Compacts with 17.0 mass\% of CA, both lampblack and graphite, and with 11.4 mass\% of lampblack were somewhat stronger, but still rather weak. Compacts with 11.4 mass\% of graphite formed a strong solid that did not easily disintegrate. The compact with 5.7 mass\% of lampblack was attacked by molten glass and partly dispersed in the melt. The compact with 5.7 mass\% of graphite formed a concrete-like porous solid that resisted molten glass. The lampblack filling is not recommended because it weakens the CRB at fractions where it reduces MIS penetration. Graphite appears a better choice when added at 12 to 15 mass\%.

The manufacturability of the CRB with an inner layer of lyophobic vibrocast was tested by making a composite slab. A layer of $250 \mathrm{~g}$ of pure vibrocast was compacted into an aluminum box. Over this layer, a second layer of $250 \mathrm{~g}$ of vibrocast with 17.4 mass\% of graphite was compacted. The size of the slab, the layer thickness, and the procedure followed were similar to the test performed with the NSvibrocast mixture. To facilitate compacting, $1 \mathrm{~mL}$ of water was added for every $15 \mathrm{~g}$ of vibrocast, and 50 $\mathrm{mL}$ of water and $12.5 \mathrm{~mL}$ of detergent were used for the graphite-vibrocast mixture. A composite slab was also prepared from the lampblack-vibrocast mixture. The slabs were dried at $110^{\circ} \mathrm{C}$ for 3 hours, ramped at $5^{\circ} \mathrm{C} / \mathrm{min}$ to $200^{\circ} \mathrm{C}$, and held for 1 hour. The slabs were removed from the boxes, ramp-heated at $5^{\circ} \mathrm{C} / \mathrm{min}$ to $1200^{\circ} \mathrm{C}$, and held at $1200^{\circ} \mathrm{C}$ for 1 hour. The dimensions of the slabs after heating were $100 \times 90 \times 45 \mathrm{~mm}$ (graphite) and 115×110×45 mm (lampblack).

Figure A.22 shows sections through the composite slabs. After the $200^{\circ} \mathrm{C}$ heat-treatment, the graphitevibrocast layer broke when pressure was applied, and the lampblack-vibrocast layer tended to disintegrate into powder with minimal pressure. After the $1200^{\circ} \mathrm{C}$ heat-treatment, the carbon in both composite layers burned out. The graphite-vibrocast layer formed a porous solid with fine cracks running through the material. It appeared to bond to the vibrocast layer; however, it crumbled under pressure. The lampblack-vibrocast layer formed a solid with fine cracks and disintegrated easier than the graphitevibrocast layer when pressure was applied. The slab with graphite had better mechanical properties, but was relatively fragile. If carbon-based protection of the CRB were tried on a large scale, the recommended starting graphite addition is 10 to $15 \mathrm{vol} \%$. 


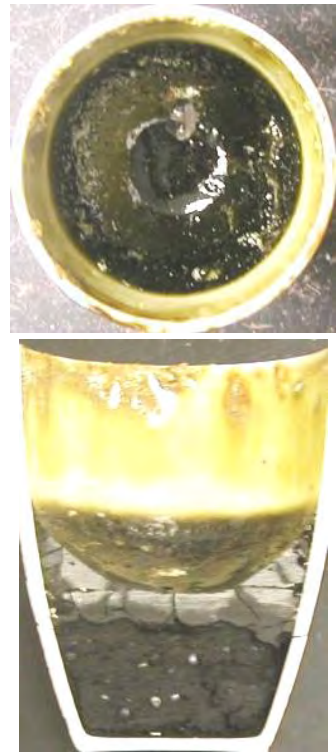

Lampblack $22.4 \%$

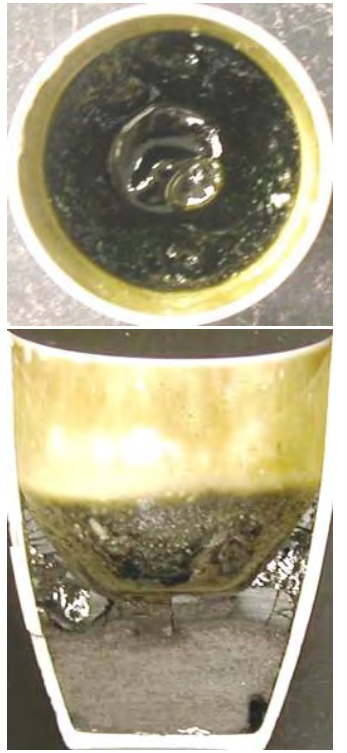

Graphite 22.4\%
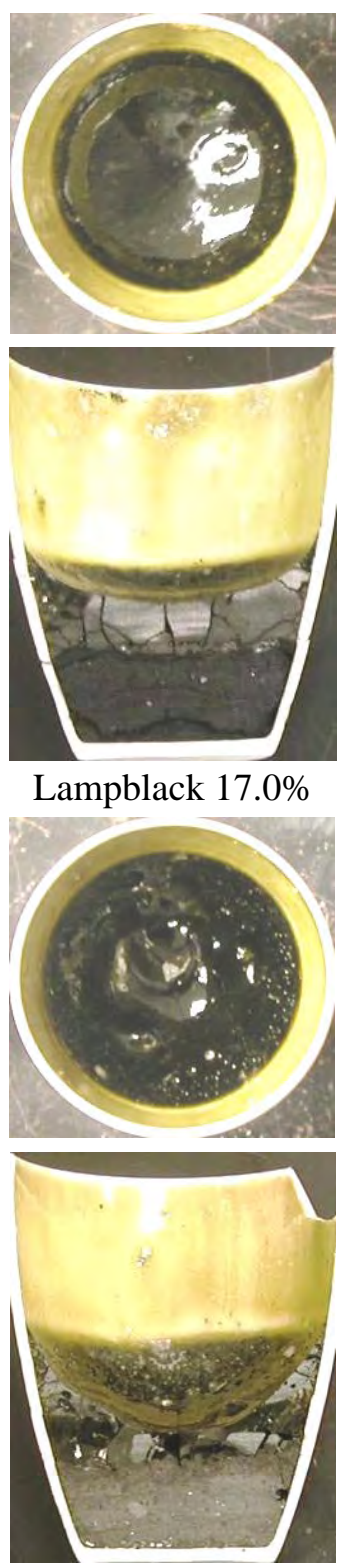

Graphite 17.0\%

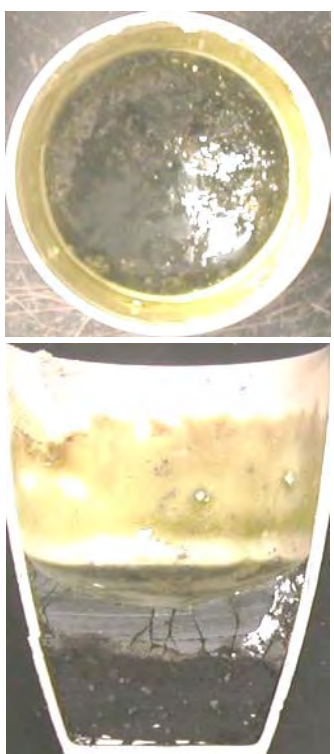

Lampblack $11.4 \%$
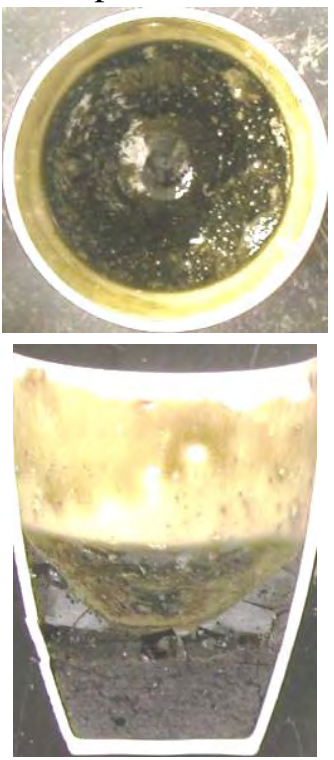

Graphite $11.4 \%$

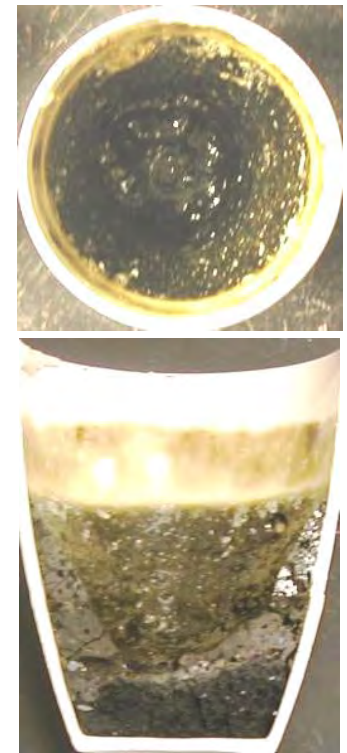

Lampblack 5.7\%
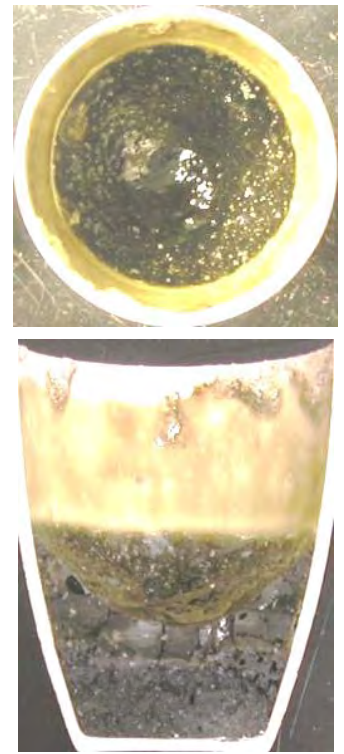

Graphite 5.7\%

Figure A.21. Alumina Crucibles with Compacted CRB-Lampblack and CRB-Graphite Mixtures and Glass Melted at $1200^{\circ} \mathrm{C}$ for 1 Hour 

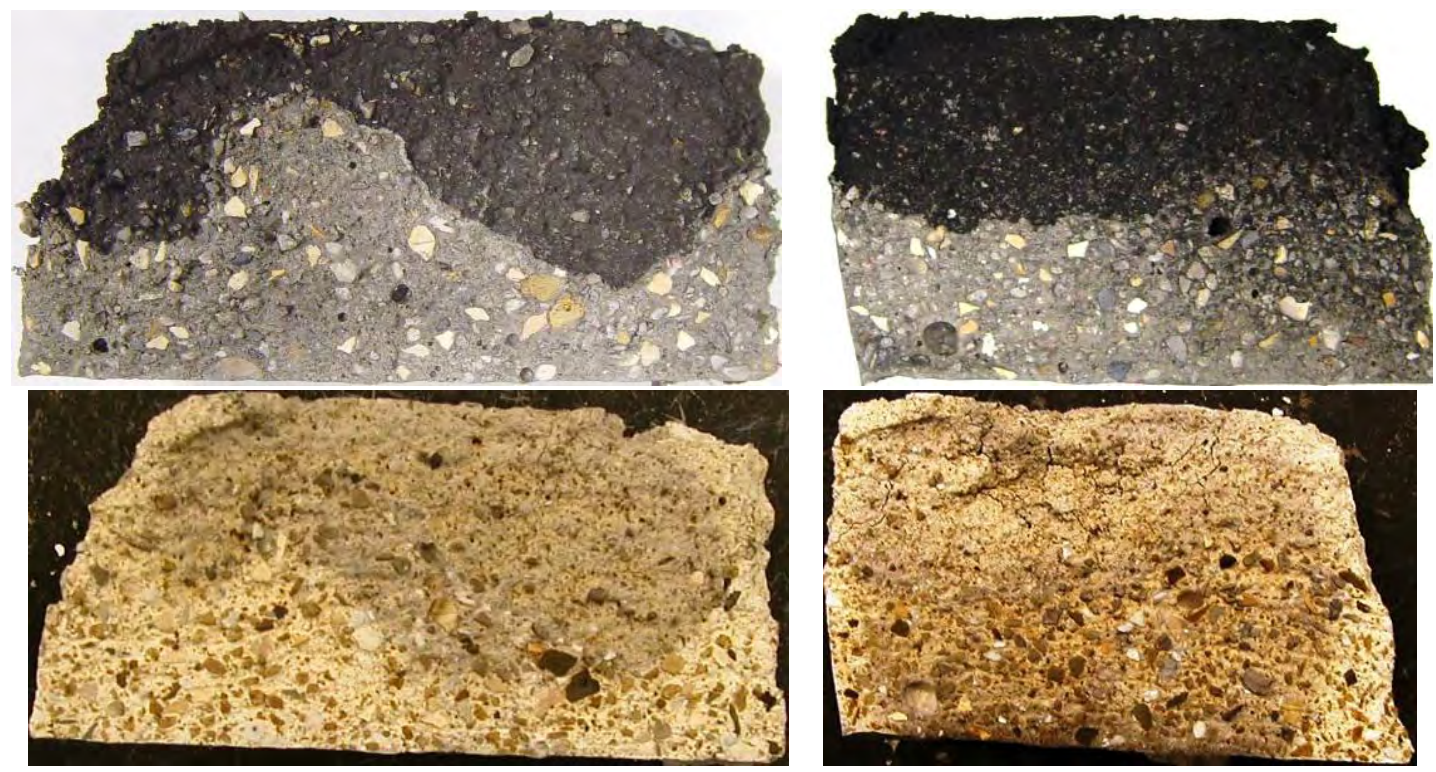

Figure A.22. Double Slab with Lampblack After $200^{\circ} \mathrm{C}$ (above left) and $1200^{\circ} \mathrm{C}$ (below left) Heat-Treatment; the Slabs with Graphite Are on the Right

\section{A.3 References}

Abe O, T Utsunomiya, and Y Hoshino. 1983. The Reaction of Sodium Nitrate with Silica.” Bulletin of the Chemical Society of Japan 56, 249-433.

Hrma P, J Matyas, LM Bagaasen, KBC Mimister, AE Beck, MJ Schweiger, TM Brouns, DM Strachan, DD Caldwell, BP Tinsley, ML Elliott, and GW Hollenberg. 2005. Bulk Vitrification Castable Refractory Block Protection Study. PNNL-15193, Pacific Northwest National Laboratory, Richland, WA. 
Appendix B

\section{Protecting CRB with AZS Tiles}




\section{Appendix B: Protecting CRB with AZS Tiles}

When grey stone was used as a CRB inner lining in the ES-31E test, significant concentrations of Re were found behind the tiles. The penetration of MIS behind the grey stone tiles was attributed to poor bonding of the tiles. Controlled laboratory experiments were conducted to demonstrate whether well-bonded AZS tiles prevent Re penetration between the tiles and the CRB. Positive results from these tests would lead to an incorporation of AZS tiles into future ES scale tests (e.g., Series 33) in conjunction with other actions that would help achieve the desired decrease in the Tc/Re penetration. The tests performed in this study showed that AZS tiles bond very well with the CRB, but are themselves permeable to MIS.

The AZS tiles have reportedly low open porosity and superior corrosion resistance, and they have been shown to bond well to the CRB material (Hrma et al. 2005). Their only disadvantage is a relatively high cost. Generally, tiles may delaminate from the CRB as a result of even a small difference in the thermal expansion between the tile and the block if the composite is exposed to a thermal shock. A partial delamination may not necessarily result in the loss of structural integrity. A partly delaminated tile may maintain a strong bond and yet allow low-viscosity MIS to penetrate behind through the AZS/CRB interface via refractory-cement joints between tiles. If the amount of MIS behind the tiles is as large as the amount that penetrates into the untiled CRB, the purpose of tiling would be defeated. Therefore, the quality of an AZS-CRB interface in a composite that has been subjected to heat-treatment must be thoroughly characterized.

Figure B.1 indicates that there is little difference in thermal expansion between vibrocast and AZS at temperatures below $800^{\circ} \mathrm{C}$, where MIS is present. If the AZS-CRB composite is not exposed to thermal shocks above $800^{\circ} \mathrm{C}$, then it is likely that the bond between AZS and CRB will remain strong when MIS contacts the wall, disallowing MIS penetration through joints and behind the tile. Any cracks appearing at temperatures above $800^{\circ} \mathrm{C}$, or during cooling, will not impact Tc/Re migration via MIS flow.

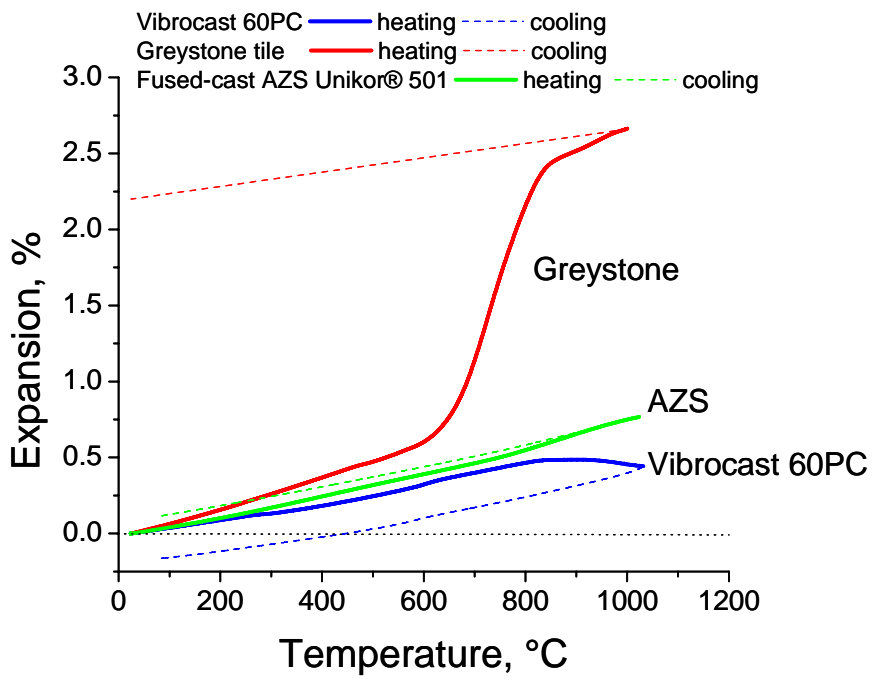

Figure B.1. Thermal Expansion of Greystone Tile, AZS, and Vibrocast 60PC Specimen (specimens heated and cooled down at $2^{\circ} \mathrm{C} / \mathrm{min}$ ) (Hrma et al. 2005a) 
Dye penetration tests were performed to determine whether micro-fractures along the CRB/AZS interface form during a temperature increase to $800^{\circ} \mathrm{C}$.

\section{B.1. Molten-Salt Dye Penetration Test}

To prepare the composite, a sample of AZS tile $(5 \times 75 \times 150 \mathrm{~mm})$ was placed into an aluminum form, and a layer of vibrocast $(\sim 25 \times 25 \times 100 \mathrm{~mm})$ was compacted on top of it. During the compaction, a square metallic block $(25 \times 25 \mathrm{~mm}$ ) was placed in the middle of the composite to create a cavity, perpendicular to the tile, for the dye to be introduced. A clean tile surface was left at the bottom of the cavity to allow the dye access to the tile.

The dye consisted of $99 \% \mathrm{Na}_{2} \mathrm{SO}_{4}$ and $1 \% \mathrm{Na}_{2} \mathrm{CrO}_{4}$. Sodium sulfate was chosen because it remains solid to a temperature above $800^{\circ} \mathrm{C}$ (its m.p. is $884^{\circ} \mathrm{C}$ ) and thus would not penetrate to cracks formed during cooling. Sodium chromate was added to act as a coloring agent.

Once formed, the AZS-vibrocast composite was removed from the form and allowed to dry overnight. The following morning, the sample was further dried in an oven at $150^{\circ} \mathrm{C}$ for 3 hours. Both the sample and the salt mixture (0.24-g $\mathrm{Na}_{2} \mathrm{CrO}_{4}$ in 23.76-g Na $\mathrm{SO}_{4}$, put into a Pt crucible) were placed into the furnace and ramp heated to $900^{\circ} \mathrm{C}$ at a rate of $5^{\circ} \mathrm{C} / \mathrm{min}$. When the furnace reached $900^{\circ} \mathrm{C}$, the dye was poured into the cavity and allowed to soak for $5 \mathrm{~min}$ in one experiment and for 2 hours in another. The furnace was then turned off, allowing the sample to cool slowly for $15 \mathrm{~min}$. The dye material was carefully chipped from the cavity, after which the sample was notched using a diamond saw and chiseled in half (Figure B.2).
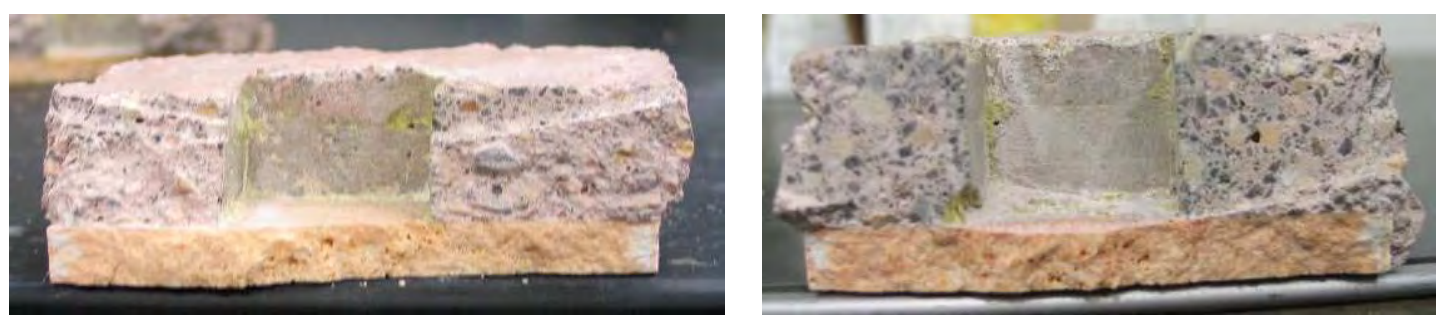

Figure B.2. Fractured CRB-AZS Composite After 5-min (left) and 2-hour (right) Sulfate-Chromate Dye Penetration Test

As Figure B.2 shows, no dye penetration into the AZS-CRB interface was discernible in both the 5-min and the 2-hour tests. In the 2-hour test, molten sulfate penetrated into the vibrocast as deep as $5 \mathrm{~mm}$ in the upper third of the layer (the color change is not visible on the photograph in Figure B.2) and also penetrated in the AZS tile inside the cavity. Even though the AZS open porosity is almost zero, the closed porosity in the particular tile used was connected enough (thus becoming virtually open within a limited portion of the tile) to allow penetration of molten salt. Regardless, the dye showed no propensity towards the interface, as observed from the chiseled portion. 


\section{B.2. Ink Penetration Test at Room Temperature}

The AZS-CRB composite was prepared and heat-treated in the same manner as described for the moltensalt-dye penetration tests, except the dye used was stamp ink that was poured into the cavity after the sample was heat-treated and cooled to room temperature. The composite with the dye was then held at room temperature for $45 \mathrm{~min}$, dried at $150^{\circ} \mathrm{C}$ for 1 hour, and fractured (Figure B.3).

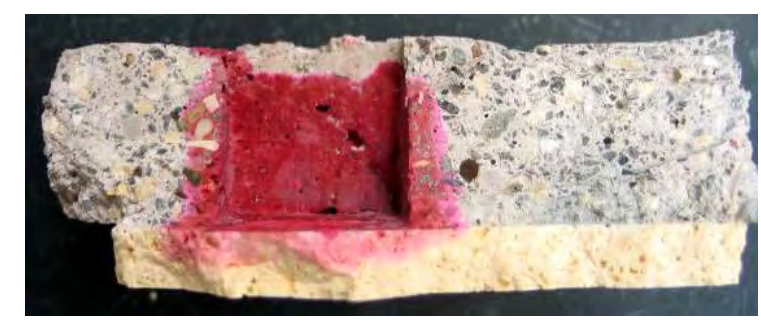

Figure B.3. Fractured CRB-AZS Composite After Room-Temperature Dye-Penetration Test

The ink penetrated into both the CRB and the AZS tile, but no excessive ink penetration occurred along the interface. The taper-like penetration (the increase of the penetration depth in the vertical direction from top down) was caused by the sinking level of the ink in the cavity, leaving the bottom portion to soak longer.

The lack of ink penetration into the interface shows that the interface was well bonded. A fracture parallel to the interface went through the vibrocast rather than through the interface, leaving residual vibrocast attached to the tile. However, hammer-and-chisel blows caused a portion of vibrocast to cleanly delaminate from the AZS. The ink did not penetrate along the interface any deeper than into the other portions of the block (Figure B.4), indicating that no debonding occurred before the ink was poured into the cavity.

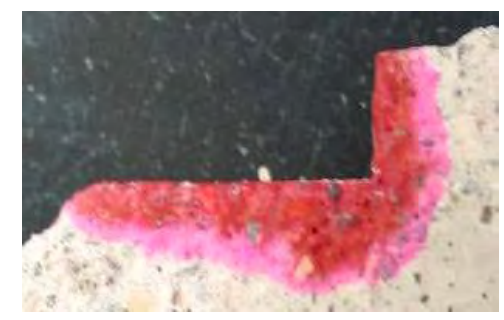

Figure B.4. Fractured CRB-AZS Composite After Room-Temperature Dye-Penetration Test, Bottom View from Delaminated Interface

To summarize, the integrity of AZS-CRB interface heated to $900^{\circ} \mathrm{C}$ was sufficient to keep a liquid from seeping into it. It is not likely that any micro-fractures ever form during heating or cooling from room temperature to $900^{\circ} \mathrm{C}$. Surprisingly, both molten salt and ink penetrated into AZS tiles as easily as in the $\mathrm{CRB}$, which casts doubt on the protective capability of the tiles. 


\section{B.3 References}

Hrma P, J Matyas, LM Bagaasen, KBC Mimister, AE Beck, MJ Schweiger, TM Brouns, DM Strachan, DD Caldwell, BP Tinsley, ML Elliott, and GW Hollenberg. 2005. Bulk Vitrification Castable Refractory Block Protection Study. PNNL-15193, Pacific Northwest National Laboratory, Richland, WA. 


\section{Appendix C}

Converting Tc/Re into Insoluble Form in the Upper CRB 


\section{Appendix C: Converting Tc/Re into Insoluble Form in the Upper CRB}

Vapors of Tc/Re condense on the upper section of the CRB. Though both Tc/Re and Na compounds volatilize from the $B V$ melt and condense on the CRB, the temperature of the upper portions of the CRB may not increase high enough to immobilize the deposit into a durable amorphous phase. In this study, the CRB was heated with simulated condensate to determine the temperature required to immobilize the deposits.

As Section 2 (Background) described, sulfate and chloride residues from the MIS that remain in the main body of glass after nitrites and nitrates are decomposed may find their way to the melt surface (in gas bubbles or salt droplets), where they vaporize. Because Tc/Re from the LAW tends to remain in the molten salt phases and Tc/Re salts are volatile, Tc/Re salts readily enter the gas phase. Common volatilization of sodium borates and Tc/Re dissolved in glass continues simultaneously with the evaporation of salts. Furthermore, bubbles produced by the decomposition of sulfate dissolved in the glass bring both $\mathrm{SO}_{\mathrm{x}}$ and $\mathrm{Tc} / \mathrm{Re}$ vapors to the glass surface. The space above the melt is thus saturated with salt vapors, including Tc/Re. Some of the vapors deposit on solid surfaces, including the CRB.

Although the deposits contain $\mathrm{Na}_{2} \mathrm{O}$ and $\mathrm{K}_{2} \mathrm{O}$ (see Hrma et al. 2005; Kim et al. 2005), the Tc/Re deposits are not immobilized into the glass phase as effectively as in the submerged portions of the CRB because the temperature is not high enough. The upper wall temperature might be increased by using an insulated lid on the BV box, which would immobilize a substantial portion of the condensed Tc/Re on the upper portion of the CRB into a glass phase. This glass phase would be produced by the reaction between alkali oxides and CRB components.

It was originally proposed that vapors from heated feed are deposited on a CRB surface using the technique described in the previous report (Hrma et al. 2005). The problem with this arrangement was that it would not reproduce the conditions in the BV processing and thus would not yield a similar deposition pattern. The actual extent of deposition and the concentration of the deposit as it occurred in the recent FS-38B test have been reported by Kim et al. 2006. Additional data are available from the cold-rod testing, where the condensate composition was carefully measured as a function of various parameters (Kim et al. 2005).

An alternative to direct vapor deposition is introducing the condensate directly into the CRB in the form of aqueous solution. This would achieve a uniform distribution of the deposit within the CRB at the specified concentration.

Soaking a solid monolithic piece of CRB with a solution and subsequent drying was considered, but was rejected because evaporating water would deposit the salts on the CRB sample surface rather than inside the pores. This would defeat the goal of achieving a uniform distribution of the condensate in the bulk of the CRB sample.

Instead, the condensate solution was mixed with powdered vibrocast, creating a slurry. The slurry was then dried and heat-treated to different temperatures. Once heat-treated, samples were ground and leached. Budget, time, and priority constraints did not allow analysis of the solutions and the solids for 
Re. Obtaining the $\mathrm{pH}$ and electrical conductivity of the solution was deemed a sufficient measure for determining the chemical durability of the glass phase that would form as a result of heat-treatment. These results are likely to correlate with Re release.

\section{C.1. Condensate Composition}

Two sources for estimating the condensate composition are available. One is the analysis of vapors condensed on a cold rod and the other consists of SEM-EDS tests on CRB samples taken from the upper walls of the FS-38B test.

Kim et al. (2005) trapped vapors from a BV feed on a cold rod and determined the condensate composition as a function of temperature. Table C.1 shows the averages of the high-temperature data $\left(1000^{\circ} \mathrm{C}\right.$ and $\left.1200^{\circ} \mathrm{C}\right)$ for major components. Table C.2 lists the estimated condensate composition, based on these data. The fraction of sodium was adjusted to meet the charge compensation requirement based on anion concentrations as determined by ion chromatography. Boron was treated as an oxide (not as sodium borate).

Table C.1. Cold-Rod Condensate Composition in mg

\begin{tabular}{||l|r|r|c||}
\hline \hline $\mathbf{T}\left({ }^{\circ} \mathbf{C}\right)$ & $\mathbf{1 0 0 0}$ & $\mathbf{1 2 0 0}$ & Average \\
\hline $\mathrm{Cl}$ & 6.12 & 24.83 & 15.48 \\
\hline $\mathrm{F}$ & 0.59 & 5.58 & 3.09 \\
\hline $\mathrm{NO}_{3}$ & 10.15 & 7.86 & 9.01 \\
\hline $\mathrm{SO}_{4}$ & 0.51 & 12.62 & 6.57 \\
\hline $\mathrm{K}$ & 2.36 & 7.30 & 4.83 \\
\hline $\mathrm{Na}$ & 5.31 & 18.92 & 12.11 \\
\hline $\mathrm{B}$ & 0.264 & 3.76 & 2.01 \\
\hline $\mathrm{Re}$ & 0.204 & 0.404 & 0.304 \\
\hline
\end{tabular}

Table C.2. A Possible Condensate Composition Based on Cold-Rod Data

\begin{tabular}{||l|r||}
\hline \hline $\mathrm{KCl}$ & $\begin{array}{c}\text { Mass } \\
\text { Fraction }\end{array}$ \\
\hline $\mathrm{NaCl}$ & 0.1950 \\
\hline $\mathrm{NaF}$ & 0.2458 \\
\hline $\mathrm{NaNO}_{3}$ & 0.1066 \\
\hline $\mathrm{Na}_{2} \mathrm{SO}_{4}$ & 0.1929 \\
\hline $\mathrm{B}_{2} \mathrm{O}_{3}$ & 0.1517 \\
\hline $\mathrm{Na}_{2} \mathrm{Re}_{2} \mathrm{O}_{7}$ & 0.1012 \\
\hline & 0.0068 \\
\hline & 1.0000 \\
\hline
\end{tabular}

The condensate composition shown in Table C.2 is typical for vaporization dominated by feed. However, this composition may not be applicable to the feeding mode in which a free melt surface dominates, as was the case with the 38B test. 
The main components that evaporate from the free surface of molten glass are $\mathrm{Na}_{2} \mathrm{O}$ and $\mathrm{B}_{2} \mathrm{O}_{3}$. Because of sulfate accumulation on the melt surface, it can be expected that $\mathrm{Na}_{2} \mathrm{SO}_{4}$ is also a major component of the vapor phase.

Figures C.1 through C.3 summarize data from SEM-EDS analysis [Kim et al. 2006] of the upper refractory wall in the FS-38B test; these include the results for $\mathrm{Cl}, \mathrm{S}$, and $\mathrm{Na}$, but not for $\mathrm{B}$, which has too low of an atomic number to be detected by EDS.

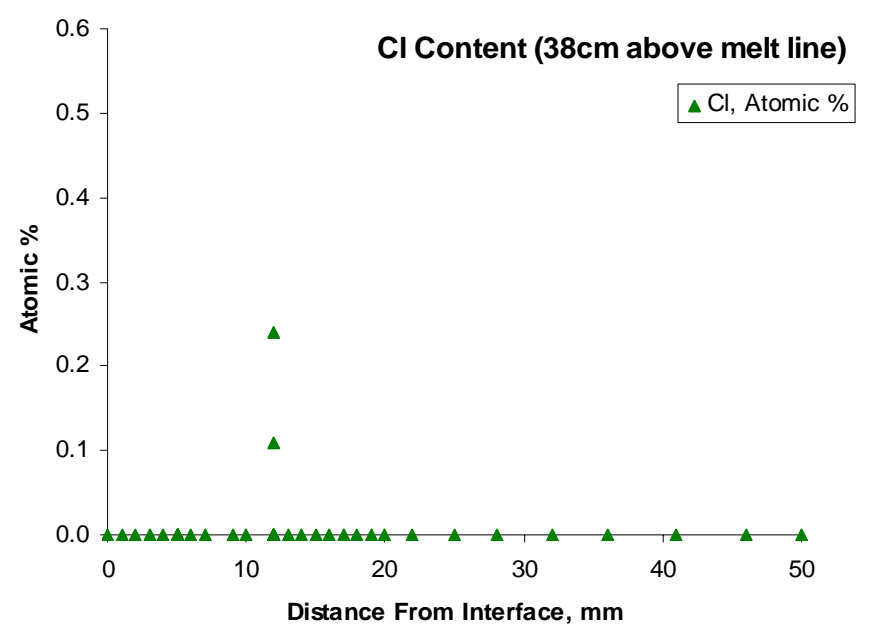

Figure C.1. Distribution of $\mathrm{Cl}$ in the Upper CRB (38B)

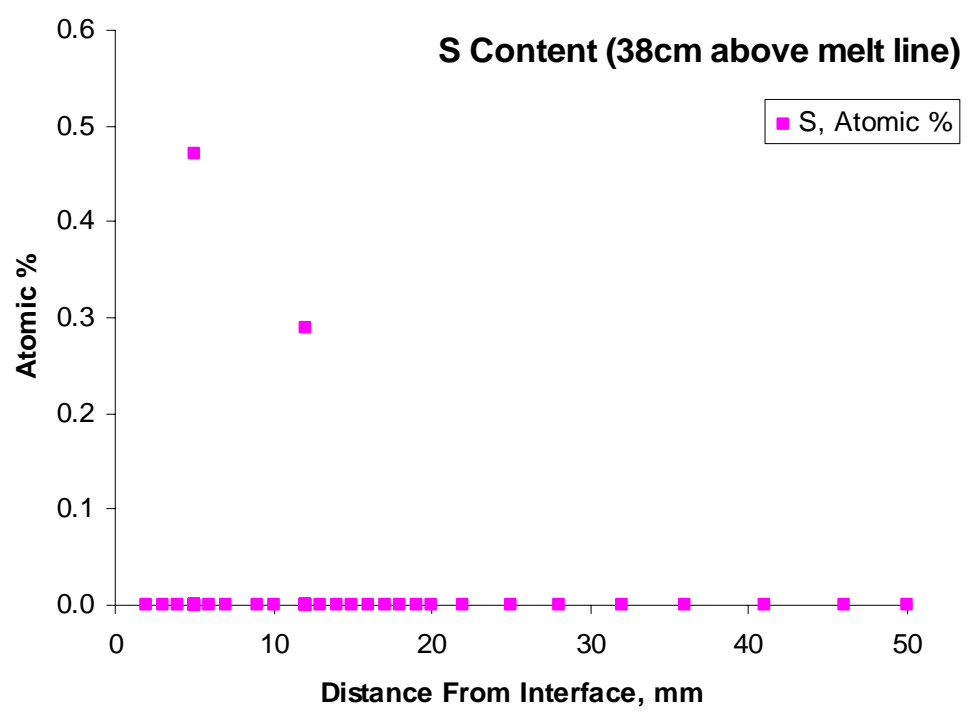

Figure C.2. Distribution of $S$ in the Upper CRB (38B) 


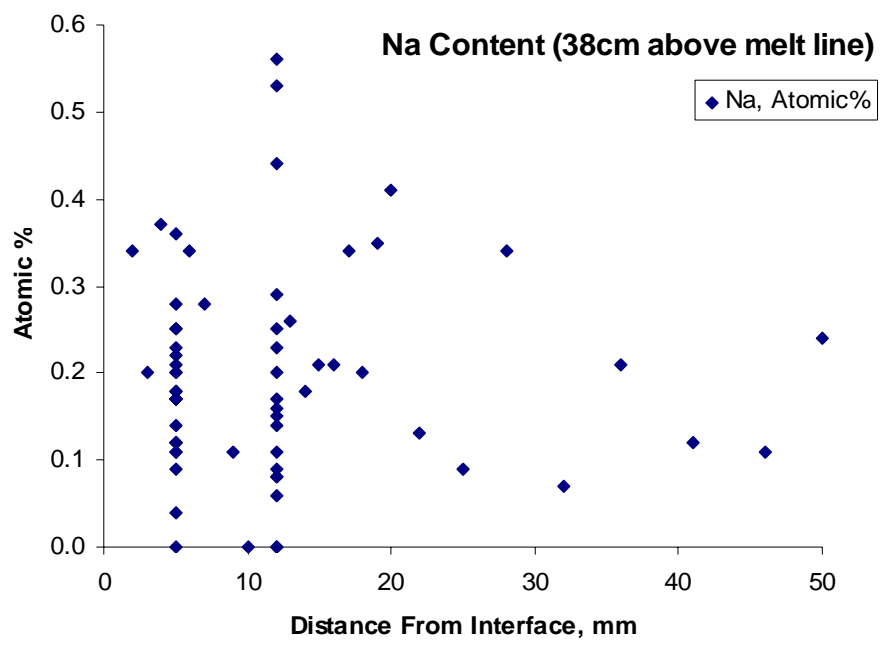

Figure C.3. Distribution of Na in the Upper CRB (38B)

As Figures C.1 through C.3 show, the content of $\mathrm{Cl}$ and $\mathrm{S}$ in the upper refractory wall is virtually zero except for a couple of spikes that can be disregarded. Consequently, the condensate contained mainly species that evaporated from molten glass $\left(\mathrm{Na}_{2} \mathrm{O}\right.$ and $\left.\mathrm{B}_{2} \mathrm{O}_{3}\right)$. It is not clear why $\mathrm{SO}_{3}$ was not present.

Sodium content at or below 0.2 at\% is considered to be background content. Based on the SEM-EDS data, the average Na content minus the background content was $\sim 0.2$ at $\%$. The corresponding mass fraction of $\mathrm{Na}_{2} \mathrm{O}$ deposited inside the $\mathrm{CRB}$ was estimated as 0.003 . This value was obtained by assuming, for convenience, that the CRB was $100 \%$ mullite. Table 4.1 shows the vibrocast composition provided by the manufacturer. However, considering the large scatter of data, as seen in Figure C.3, and the low accuracy of EDS analysis, a more accurate composition would not present any significant benefit.

The vapors depositing $\mathrm{Na}$ into the CRB could be $\mathrm{Na}_{2} \mathrm{O}$ (possibly in the form of $\mathrm{NaOH}$ ) or $\mathrm{Na}_{2} \mathrm{O} \cdot \mathrm{B}_{2} \mathrm{O}_{3}$. Because the fraction of $\mathrm{B}_{2} \mathrm{O}_{3}$ was unknown, three levels of $\mathrm{B}_{2} \mathrm{O}_{3}$ were considered, corresponding to the atomic fractions $\mathrm{Na} /(\mathrm{Na}+\mathrm{B})=0.5,0.75$, and 1 . To facilitate experimental detection of dissolved species in the leachates, the fraction of $\mathrm{Na}$ in the CRB was increased to 0.6 at\%. Table C.3 shows the compositions of the three condensates and the fractions of these condensates mixed with the vibrocast to maintain the atomic fraction of $\mathrm{Na}$ at 0.6 at\%.

Table C.3. Condensate Composition and Condensate Addition Based on SEM-EDS Data

\begin{tabular}{||l|c|c|c||}
\hline \hline $\mathrm{Na} /(\mathrm{Na}+\mathrm{B})$ & 1.0 & 0.75 & 0.5 \\
\hline Condensate composition, mass fractions \\
\hline $\mathrm{Na}_{2} \mathrm{O}$ & 1.0000 & 0.7276 & 0.4710 \\
\hline $\mathrm{B}_{2} \mathrm{O}_{3}$ & 0.0000 & 0.2724 & 0.5290 \\
\hline Condensate in CRB, mass fractions & \\
\hline Condensate & 0.0092 & 0.0126 & 0.0195 \\
\hline CRB & 0.9908 & 0.9874 & 0.9805 \\
\hline \hline
\end{tabular}


In the 38B test, from which the FS data were taken, a large fraction of the free surface of molten glass was uncovered by feed. In the following test (38C), the free surface of melt was minimal; feeding was done in the cold-cap mode, keeping the feed spread over the surface from wall to wall. Though the upper-wall deposit composition and concentration were not available at the time this study was performed, one can expect that the mode of feeding would greatly affect the conditions in the atmosphere as is obvious from the much lower extent of deposits on the lid and in the offgas system of the 38C test. The design of experiments described below was based on the FS-38B test data. It is possible that the cold-rod condensate would be more representative for 38C test conditions.

\section{2. Test Procedure and Results}

Sodium oxide was introduced in the form of $\mathrm{NaNO}_{3}$, which decomposes at temperatures below $800^{\circ} \mathrm{C}$. The mixtures of $\mathrm{NaNO}_{3}$ and $\mathrm{B}_{2} \mathrm{O}_{3}$ listed in Table C.3 were dissolved in water before adding vibrocast. The solution was mixed with vibrocast powder in proportions shown in Table C.3 (to reach 0.6 at\% Na in dry vibrocast). The resulting slurry was dried on a hot plate and then heat-treated for 2 hours at temperatures of $800^{\circ} \mathrm{C}, 1050^{\circ} \mathrm{C}$, and $1300^{\circ} \mathrm{C}$. The samples were then taken from the furnace and air cool. Once cooled, the samples were ground in a tungsten mill for 3 seconds. The ground samples ( $20 \mathrm{~g}$ of sample material) were then placed into Teflon containers and leached in $30 \mathrm{~mL}$ of DI water at $60^{\circ} \mathrm{C}$ for 20 hours. After leaching, the solution was removed using a syringe and filtered through a $0.45-\mu \mathrm{m}$ filter.

Figures C.4 and C.5 show the effects of temperature and condensate composition on the $\mathrm{pH}$ and electrical conductivity of the leachate. Figure C.5 also shows that $\mathrm{pH}$ and electrical conductivity have a strong correlation to each other. As expected, both $\mathrm{pH}$ and electrical conductivity of leachates decrease as the temperature of the heat-treatment increases. This behavior shows the progressive incorporation of the condensate into a durable glass phase produced by the reaction between the condensate and the cement that bonds the mullite and other refractory particles of vibrocast together.
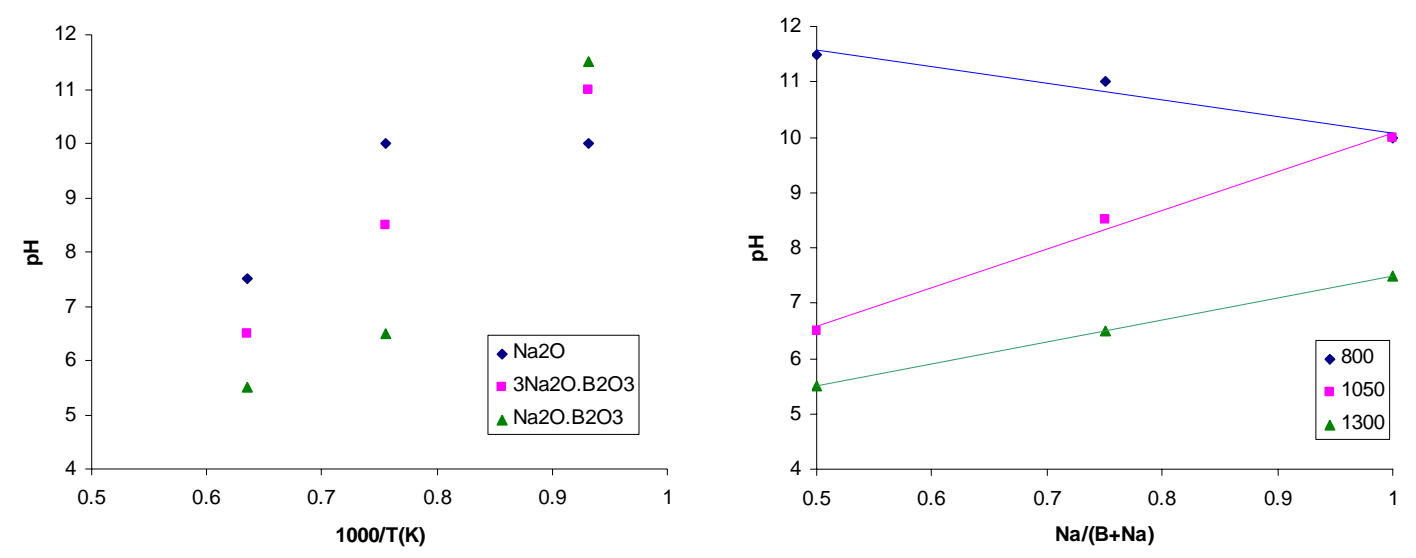

Figure C.4. Effects of Temperature (left) and Condensate Composition (right) on the Leachate pH 

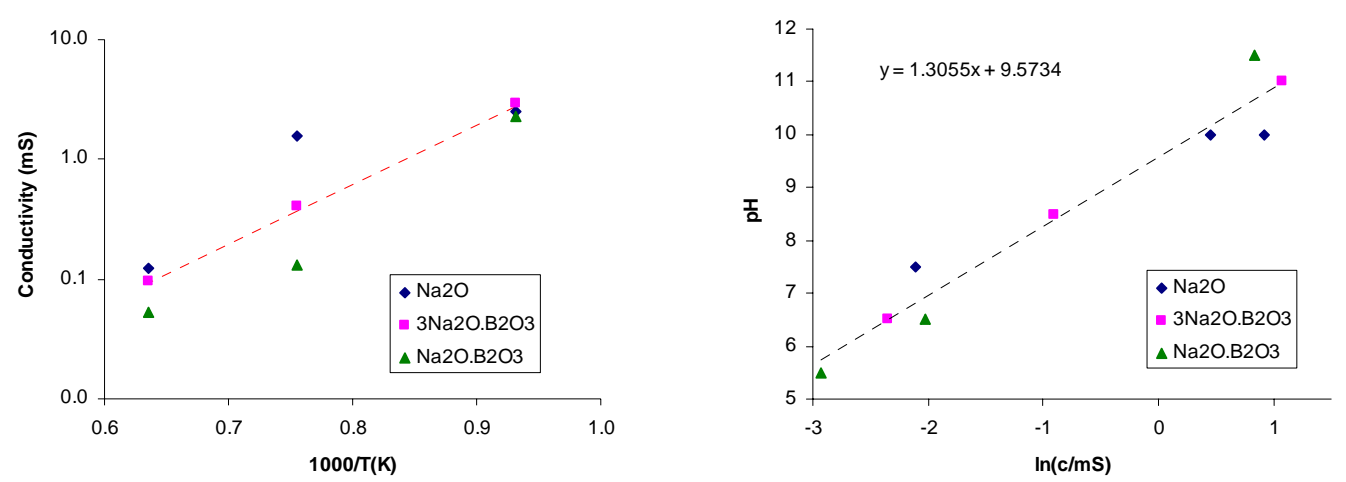

\section{Figure C.5. Effects of Temperature on the Leachate Electrical Conductivity (left); Correlation Between pH and Electrical Conductivity (right)}

The impact of $\mathrm{B}_{2} \mathrm{O}_{3}$ depends on the heat-treatment temperature. In samples heat-treated at $800^{\circ} \mathrm{C}$, the increasing fraction of $\mathrm{B}_{2} \mathrm{O}_{3}$ leads to an increased release of $\mathrm{Na}$ into the leachate. This indicates that $\mathrm{Na}_{2} \mathrm{O}$ preferably forms a nondurable borosilicate glass with $\mathrm{B}_{2} \mathrm{O}_{3}$ instead of forming a somewhat more durable aluminosilicate glass with the refractory cement. The opposite trend seen at the heat-treatment temperature of $1050^{\circ} \mathrm{C}$ and $1300^{\circ} \mathrm{C}$ indicates that the aluminosilicate and calcium components of the vitrified cement fuse with the borosilicate glass, creating a single durable glass phase. The $\mathrm{B}_{2} \mathrm{O}_{3}$ content in this glass phase had a positive impact on glass durability.

Figures C.6 and C.7 show optical images of the finest fraction of the vibrocast samples taken before and after the heat-treatment. A glass-like phase is present in the original vibrocast as supplied by the manufacturer, and its content increases when heated. Adding the condensate does not visibly increase the glass-phase content.
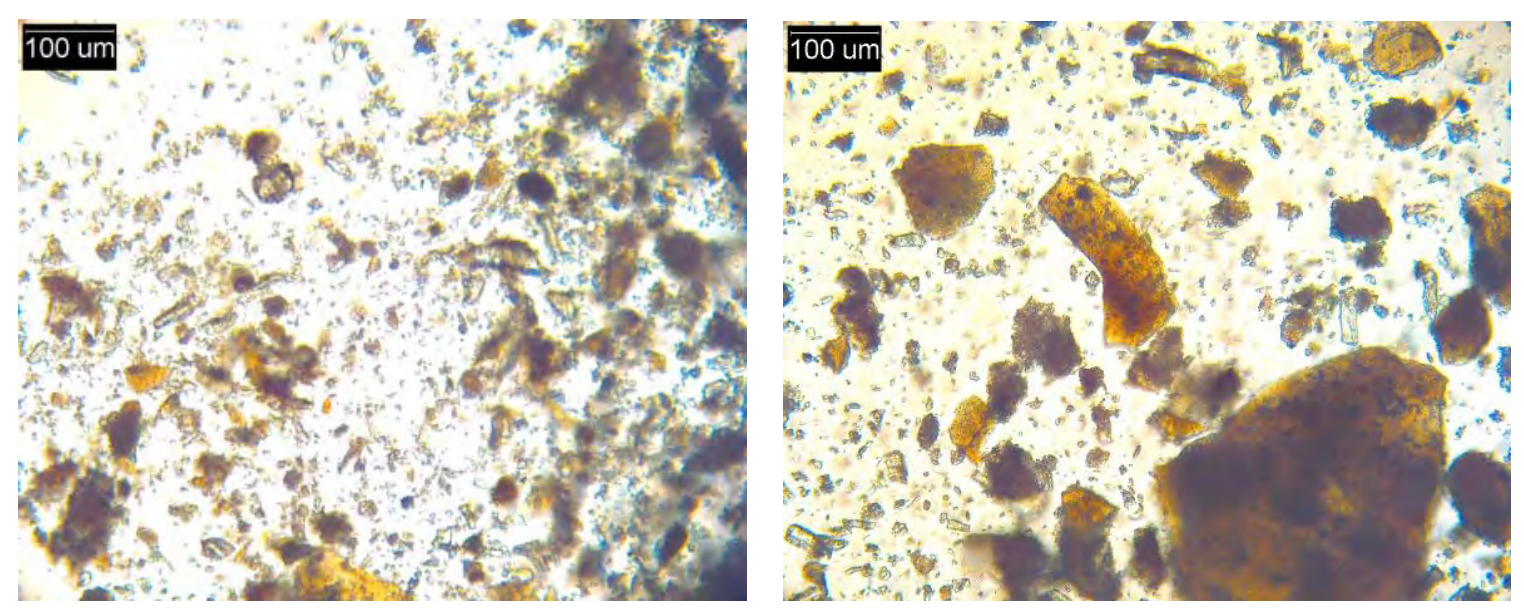

Figure C.6. Vibrocast Original (left) and Heat-Treated at $1300^{\circ} \mathrm{C}$ for 2 Hours (right) 

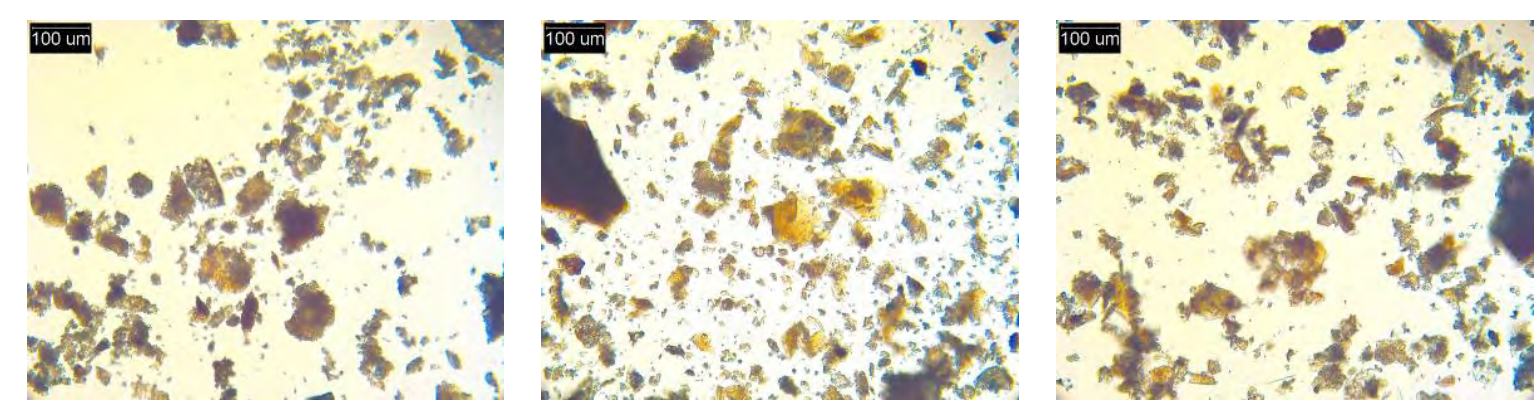

Figure C.7. Vibrocast Containing $\mathrm{Na}_{2} \mathrm{O}$ (left) $3 \mathrm{Na}_{2} \mathrm{O} \cdot \mathrm{B}_{2} \mathrm{O}_{3}$ (middle) and $\mathrm{Na}_{2} \mathrm{O} \cdot \mathrm{B}_{2} \mathrm{O}_{3}$ (right) and Heat-Treated at $1300^{\circ} \mathrm{C}$ for 2 Hours

Results shown in tables C.4-C.7 shows why the content of the glass phase might not be significantly affected by adding the condensate. Table C.4 lists SEM-EDS data obtained by Matyas and reported in Hrma et al. (2005) for cement portions of CRB samples treated with simulated condensate (the simulate composition was different from that used in this study). Atomic fractions of selected elements were renormalized (Table C.5) to compare them with vibrocast composition reported by the manufacturer (Table 4.1). The average composition of the cement portion of the CRB is compared with the overall vibrocast composition in Table C.6.

Table C.4. Compositions in at\% of Cement Areas of CRB Samples Exposed to Simulated Condensate and Reported in Hrma et al. (2005), Appendix B

\begin{tabular}{||l|c|c|c|c|c||}
\hline \hline$T,{ }^{\circ} \mathrm{C}$ & 800 & 800 & 900 & 1000 & 1100 \\
\hline Figure & 13 & 13 & 9 & 11 & 12 \\
\hline Area No & 2 & 3 & 2 & 1 & \multicolumn{1}{c||}{2} \\
\hline Table & \multicolumn{1}{c|}{7} & \multicolumn{1}{c|}{7} & \multicolumn{1}{c|}{4} & \multicolumn{1}{c|}{5} & \multicolumn{1}{c||}{} \\
\hline $\mathrm{O}$ & 38.94 & 35.23 & 32.44 & 34.25 & 35.47 \\
\hline $\mathrm{Na}$ & 16.38 & 2.61 & 10.74 & 0.28 & 2.47 \\
\hline $\mathrm{Mg}$ & & & & 0.59 & \\
\hline $\mathrm{Al}$ & 22.01 & 31.51 & 20.65 & 28.09 & 29.7 \\
\hline $\mathrm{Si}$ & 16.72 & 27.29 & 25.85 & 31.07 & 26.27 \\
\hline $\mathrm{P}$ & & & & 0.51 & \\
\hline $\mathrm{Zr}$ & 0.27 & 0.14 & & & \\
\hline $\mathrm{S}$ & 0.68 & & 1.92 & & \\
\hline $\mathrm{Cl}$ & 1.61 & 0.24 & 4.42 & & \\
\hline $\mathrm{K}$ & 1.56 & 0.58 & 0.73 & & 0.52 \\
\hline $\mathrm{Ca}$ & 0.85 & 1.96 & 3.25 & 4.31 & 4.44 \\
\hline $\mathrm{Ti}$ & 0.48 & 0.13 & & 0.24 & 0.47 \\
\hline $\mathrm{Fe}$ & 0.5 & 0.3 & & 0.66 & 0.66 \\
\hline
\end{tabular}


Table C.5. Compositions in at\% of Cement Areas of CRB Samples Exposed to Simulated Condensate and Reported in Hrma et al. (2005), Appendix B

\begin{tabular}{||l|c|c|c|c|c||}
\hline \hline$T,{ }^{\circ} \mathrm{C}$ & 800 & 800 & 900 & 1000 & 1100 \\
\hline Figure & 13 & 13 & 9 & 11 & 12 \\
\hline Area No & 2 & 3 & 2 & 1 & 2 \\
\hline Table & 7 & 7 & 4 & 5 & 6 \\
\hline $\mathrm{Al}$ & 0.5427 & 0.5150 & 0.4151 & 0.4364 & 0.4826 \\
\hline $\mathrm{Si}$ & 0.4122 & 0.4460 & 0.5196 & 0.4827 & 0.4269 \\
\hline $\mathrm{Fe}$ & 0.0123 & 0.0049 & 0.0000 & 0.0103 & 0.0107 \\
\hline $\mathrm{Ti}$ & 0.0118 & 0.0021 & 0.0000 & 0.0037 & 0.0076 \\
\hline $\mathrm{Ca}$ & 0.0210 & 0.0320 & 0.0653 & 0.0670 & 0.0721 \\
\hline
\end{tabular}

Table C.6. Composition in at\% of Vibrocast, Cement Portion of CRB (average from data listed in Table C.4) and its Mixtures with Condensate Simulants

\begin{tabular}{||l|l|l|l|l|l||}
\hline & Vibrocast & Cement & \multicolumn{3}{|c|}{ Cement + Condensate } \\
\hline $\mathrm{Na} /(\mathrm{Na}+\mathrm{B})$ & & & 1.00 & 0.75 & 0.50 \\
\hline Condensate fraction & & & 0.0092 & 0.0126 & 0.0195 \\
\hline $\mathrm{Al}_{2} \mathrm{O}_{3}$ & 0.6556 & 0.4372 & 0.4332 & 0.4317 & 0.4287 \\
\hline $\mathrm{SiO}_{2}$ & 0.3156 & 0.4928 & 0.4883 & 0.4866 & 0.4832 \\
\hline $\mathrm{Fe}_{2} \mathrm{O}_{3}$ & 0.0007 & 0.0109 & 0.0108 & 0.0108 & 0.0107 \\
\hline $\mathrm{TiO}_{2}$ & 0.0124 & 0.0073 & 0.0072 & 0.0072 & 0.0071 \\
\hline $\mathrm{CaO}$ & 0.0157 & 0.0518 & 0.0513 & 0.0511 & 0.0508 \\
\hline $\mathrm{Na}_{2} \mathrm{O}$ & & & 0.0092 & 0.0092 & 0.0092 \\
\hline $\mathrm{B}_{2} \mathrm{O}_{3}$ & & & 0.0000 & 0.0034 & 0.0103 \\
\hline
\end{tabular}

Assuming that all $\mathrm{CaO}$ present is only in the cement fraction (not in the large refractory grains), we can estimate that vibrocast contains 30.5 mass\% of the cement. Assuming further that the condensate predominantly reacts with the cement, and using data listed in Table C.3, we can estimate the composition of the mixtures of the cement when it is fused with the condensate. The results are listed in the last three columns of Table C.6.

The mixtures of the cement vibrocast portion with the condensate shown in Table C.6 contain 43 mass\% of $\mathrm{Al}_{2} \mathrm{O}_{3}$. The glass phase from these mixtures is not capable of incorporating this large fraction of alumina. Table C.7 shows compositions of the glass phase provided that only 5 mass $\%$ of $\mathrm{Al}_{2} \mathrm{O}_{3}$ dissolves in the glass. The fractions of the condensate components $\left(\mathrm{Na}_{2} \mathrm{O}\right.$ and $\left.\mathrm{B}_{2} \mathrm{O}_{3}\right)$ in these hypothetical glasses are still very low (less than 2 mass $\%$ of $\mathrm{Na}_{2} \mathrm{O}$ ), and they would remain low even if the glasses contained less $\mathrm{SiO}_{2}$. 


\section{Table C.7. Hypothetical Composition in at\% of Glass Phase from Cement Portion of CRB Fused with Condensate Simulants}

\begin{tabular}{||l|l|l|l||}
\hline \hline & \multicolumn{3}{|c|}{ Cement + Condensate } \\
\hline $\mathrm{Na} /(\mathrm{Na}+\mathrm{B})$ & 1.00 & 0.75 & 0.50 \\
\hline $\mathrm{Al}_{2} \mathrm{O}_{3}$ & 0.0500 & 0.0500 & 0.0500 \\
\hline $\mathrm{SiO}_{2}$ & 0.8184 & 0.8135 & 0.8035 \\
\hline $\mathrm{Fe}_{2} \mathrm{O}_{3}$ & 0.0182 & 0.0181 & 0.0178 \\
\hline $\mathrm{TiO}_{2}$ & 0.0120 & 0.0120 & 0.0118 \\
\hline $\mathrm{CaO}$ & 0.0860 & 0.0854 & 0.0844 \\
\hline $\mathrm{Na}_{2} \mathrm{O}$ & 0.0154 & 0.0153 & 0.0153 \\
\hline $\mathrm{B}_{2} \mathrm{O}_{3}$ & 0.0000 & 0.0057 & 0.0172 \\
\hline \hline
\end{tabular}

This small concentration of fluxes explains the observation that additions of the condensate did not increase the observable amount of the glass phase in the vibrocast as seen in Figures C.6 and C.7. The amount of the deposit observed in the upper wall of the CRB in the 38B test is small compared with the fraction of the refractory cement in the vibrocast refractory (estimated to be as high as 30 mass\%). As the temperature increases, more and more refractory components are incorporated into the glass phase, making it increasingly durable. This being the case, the Tc/Re will be incorporated into this glass phase, thus retarding its release.

\section{C.3 References}

Hrma P, J Matyas, LM Bagaasen, KBC Mimister, AE Beck, MJ Schweiger, TM Brouns, DM Strachan, DD Caldwell, BP Tinsley, ML Elliott, and GW Hollenberg. 2005. Bulk Vitrification Castable Refractory Block Protection Study. PNNL-15193, Pacific Northwest National Laboratory, Richland, WA.

Kim, D-S, CZ Soderquist, JP Icenhower, BP McGrail, RD Scheele, BK McNamara, LM Bagaasen, MJ Schweiger, JV Crum, JD Yeager, J Matyáš, LP Darnell, HT Schaef, AT Owen, AE Kozelisky, LA Snow, MJ Steele. 2005. Tc Reductant Chemistry and Crucible Melting Studies with Simulated Hanford LowActivity Waste. PNNL-15131, Pacific Northwest National Laboratory, Richland, WA.

Kim D, LM Bagaasen, JV Crum, A Fluegel, A Gallegos, B. Martinez, J Matyáš, PA Meyer, DR Paulsen, BJ Riley, MJ Schweiger, CW Stewart. RG Swoboda, JD Yeager. 2006. Investigation of Tc Migration Mechanism During Bulk Vitrification Process Using Re Surrogate. PNNL-16267, Pacific Northwest National Laboratory, Richland, WA. 

Appendix D

\section{MIS Immobilization by Increasing Specific Surface Area of Feed Solids: Preliminary Testing}




\section{Appendix D: MIS Immobilization by Increasing Specific Surface Area of Feed Solids: Preliminary Testing}

\section{D.1. Scoping Tests}

To obtain an initial insight into the complexity of the interaction between the soil, fine-grain materials, MIS, and silica crucibles that were used as a model porous refractory, four preliminary scoping tests were performed. Scoping Test I was designed to test whether MIS would wet fine-grain materials even when the mixing was incomplete, i.e., to find out whether MIS would spontaneously soak into the granular material, or would a saturated skin form that would prevent further penetration of MIS into the powder. In Scoping Test II, MIS was thoroughly mixed with fine-grain materials, and the focus was on the reaction of MIS with both the fine-grain material and the crucible as a response to heating. Scoping Test III checked the interaction of a silica crucible with pure MIS in the absence of any feed solids. Finally, Scoping Test IV was performed to assess the interaction of MIS with Hanford soil and the crucible. This was done to better assess the effectiveness of fine-grain materials in decreasing the MIS mobility. The scoping tests served as a rough guide to the design of more systematic studies that followed.

\section{D.1.1. Scoping Test I: MIS Penetration into Fine-Grain Materials}

The mixing sequence of feed components may impact the reaction path. As is well known from making ceramic pastes or preparing plaster of Paris, blending powders with liquids proceeds more effectively if the liquid is gradually added to the powder. If fine-grain materials are mixed with other solids before adding LAW, the fine powder could clump into aggregates that would not be uniformly wetted by the MIS.

To assess the soaking ability of fine-grain materials with respect to molten MIS, the fines were placed in a silica crucible, overlaid with dry LAW, and heated to a temperature at which the MIS became liquid. The dry LAW used in this test was a mixture of chemicals shown in Table 4.3 except $\mathrm{NaOH}$ and $\mathrm{Al}\left(\mathrm{NO}_{3}\right)_{3} \cdot 9 \mathrm{H}_{2} \mathrm{O}$. The fine-grain materials were added to the crucibles to approximately one half of its height (11 to $15 \mathrm{~g}$ of material except for diatomaceous earth and kaolin that weighed 4 and $5 \mathrm{~g}$, respectively) and overlaid with 12 to $15 \mathrm{~g}$ dry LAW as shown in Table D.1. The amount of waste was approximately equal to that of fines except for diatomaceous earth and kaolin, where the masses of waste were 3.2 and 2.7 times larger than those of fines, respectively.

Table D.1. Dry LAW Overlaid on Fines: Scoping Test Results

\begin{tabular}{|l|c|c|c|c|c||}
\hline & Silica & $\begin{array}{c}\text { Diatomaceous } \\
\text { Earth }\end{array}$ & Bentonite & Kaolin & Fly Ash \\
\hline Fine-grain material, g & 14.73 & 4.27 & 11.15 & 5.17 & 13.00 \\
\hline Dry LAW, g & 14.86 & 13.52 & 12.11 & 13.97 & 13.88 \\
\hline LOI $^{(\mathrm{a})}$ & 0.68 & 0.53 & 0.65 & 0.59 & 0.56 \\
\hline Waste/Fines & 1.01 & 3.17 & 1.09 & 2.70 & 1.07 \\
\hline (a) Mass loss of dry LAW during the heat-treatment. \\
\hline
\end{tabular}


The crucibles were heated at the rate of $5^{\circ} \mathrm{C} / \mathrm{min}$ to $550^{\circ} \mathrm{C}$ and held for $\sim 30 \mathrm{~min}$. The crucible content was checked every $25^{\circ} \mathrm{C}$. Crucibles were then removed from the furnace and examined. The heattreatment was repeated, except that the crucibles were heated to $1100^{\circ} \mathrm{C}$. The crucible content was checked every $100^{\circ} \mathrm{C}$. Because some crucibles became deformed, the temperature was decreased from $1100^{\circ} \mathrm{C}$ to $1050^{\circ} \mathrm{C}$ and then held for $\sim 30 \mathrm{~min}$. Cold crucibles were dry-cut and examined.

Generally, the volume of the material in the crucibles slightly shrank as the temperature increased to $450^{\circ} \mathrm{C}$ and then began to expand at $\sim 500^{\circ} \mathrm{C}$, forming a dome-like shape of the waste on top of the fines. Except for silica and diatomaceous earth, melt was visible on the slopes of the dome. The color of the waste became bright yellow when the temperature increased above $300^{\circ} \mathrm{C}$ and turned to orange above $450^{\circ} \mathrm{C}$.

As Figure D.1 shows, the top surface of fine silica $(<75 \mu \mathrm{m})$ was flat at $550^{\circ} \mathrm{C}$ except for a small dome in the center. The flat surface was covered with pits, probably from burst bubbles. Similar shallow pits were visible also on other materials outside the dome or on its edges. The dome was preserved on diatomaceous earth and bentonite. These materials also shrank horizontally, leaving a gap around crucible walls. The top of the dome was typically white after the $550^{\circ} \mathrm{C}$ heat-treatment, while yellow patches - probably segregated sulfate melt with dissolved chromates-remained at the dome edges and on the top of the fines.

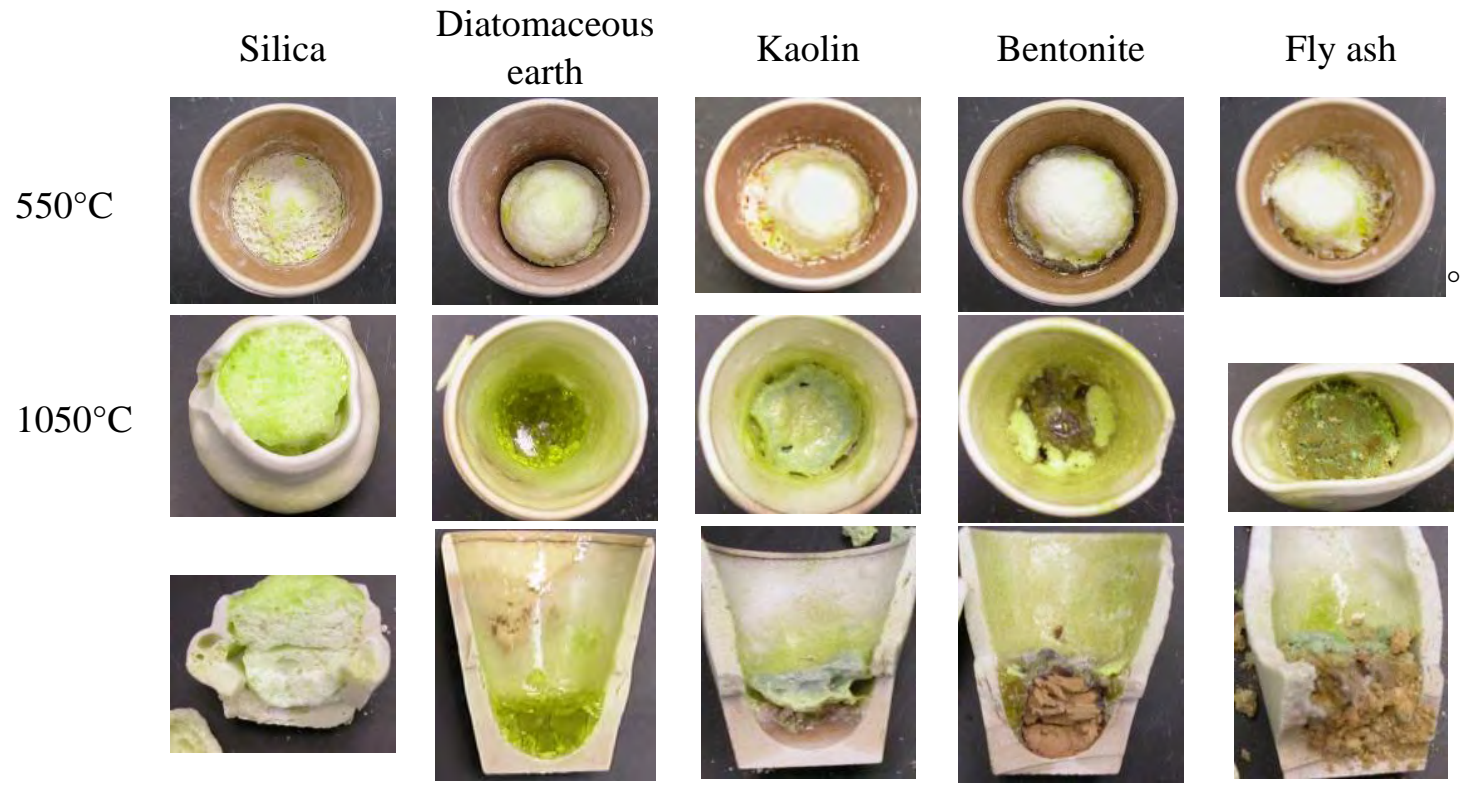

Figure D.1. Fine-Grain Materials Overlaid with Dry LAW and Heated to $550^{\circ} \mathrm{C}$ and $1050^{\circ} \mathrm{C}$

During the second heating, the crucible with fine silica bulged and sagged. The crucible with fly ash deformed. Diatomaceous earth formed melt that released gas and foamed, but the foam quickly collapsed upon lowering the crucible from the furnace. The sample with bentonite foamed to a lesser extent. Bright orange spots were typical at temperatures below $1000^{\circ} \mathrm{C}$.

In crucibles after cooling (see Figure D.1), the material became greenish in color except for yellow patches (probably sulfate to which chromate partitioned providing the yellow color) near the crucible 
walls; no such patches were seen on fine silica. Except for diatomaceous earth that formed transparent melt, loose fines remained on the bottoms of crucibles. Even kaolin, which was covered with a larger amount of waste than silica, bentonite, and fly ash, formed a layer of bluish viscous glass with large bubbles while the bottom portion was loose and fell out after the crucible was cut. An interesting pattern appeared in the bentonite sample: glass formed between the crucible and the core of bentonite into which the molten salt did not penetrate. Unlike other materials, fine silica appeared sintered, but was otherwise unchanged. The crucible containing fine silica was severely damaged; it was bloated with large bubbles, and it sagged. Fly ash reacted in a similar manner as kaolin.

To summarize, fine-grain silica and diatomaceous earth readily absorb the MIS, but silica flour failed to protect the crucible. Clays and fly ash tend to form a skin that blocks wetting of the internal portions of these materials. In subsequent tests, fine-grain materials were blended with the waste before mixing them with other feed components.

\section{D.1.2. Scoping Test II: Reaction of MIS with Fine-Grain Materials}

To assess the reactivity between fine-grain materials and MIS when these components were well blended, fine-grain materials were loaded into silica crucibles and mixed with liquid LAW to form plastic pastes, each of a similar consistency. As Table D.2 shows, diatomaceous earth absorbed the largest quantity of liquid waste, more than three times as much as other fine-grain materials. Compared to the experiment with dry LAW (Table D.1), the ratio of dry waste to fines was about three times lower when the fines were soaked with the aqueous LAW. The liquid LAW and the corresponding dry LAW contained all chemicals listed in Table 4.3, including $\mathrm{NaOH}$ and $\mathrm{Al}\left(\mathrm{NO}_{3}\right)_{3} \cdot 9 \mathrm{H}_{2} \mathrm{O}$ that were missing in the soaking tests shown in Figure D.1.

Table D.2. Liquid LAW Blended with Fines: Scoping Test Results

\begin{tabular}{||l|c|c|c|c|c||}
\hline \hline & Silica & $\begin{array}{c}\text { Diatomaceous } \\
\text { Earth }\end{array}$ & Bentonite & Kaolin & Fly Ash \\
\hline Fines, g & 14.75 & 4.27 & 11.16 & 5.18 & 13.01 \\
\hline Waste, mL & 10 & 12 & 10 & 4 & 10 \\
\hline Waste/Fines, mL/g & 0.68 & 2.81 & 0.90 & 0.77 & 0.77 \\
\hline Dry waste/Fines, g/g & 0.26 & 1.07 & 0.34 & 0.29 & 0.29 \\
\hline Dry waste mass fraction & 0.206 & 0.517 & 0.254 & 0.225 & 0.225 \\
\hline
\end{tabular}

The pastes were dried at $103^{\circ} \mathrm{C}$ for 15 hours, heated at $5^{\circ} \mathrm{C} / \mathrm{min}$ to $550^{\circ} \mathrm{C}$, held for 1 hour, and then examined. A second heating followed at $5^{\circ} \mathrm{C} / \mathrm{min}$ to $1050^{\circ} \mathrm{C}$ and held for $30 \mathrm{~min}$. Finally, the crucibles were heated to $1300^{\circ} \mathrm{C}$ and then broken and examined.

As Figure D. 2 shows, mixtures containing diatomaceous earth and bentonite turned to glass at $1050^{\circ} \mathrm{C}$. The mixture containing fly ash began to vitrify at $1300^{\circ} \mathrm{C}$. Bentonite and fly ash formed a foamy melt with large bubbles. Kaolin sintered into a glass ceramic that broke to pieces while cutting the crucible. Silica sintered into a grainy mass that easily crumbled. 


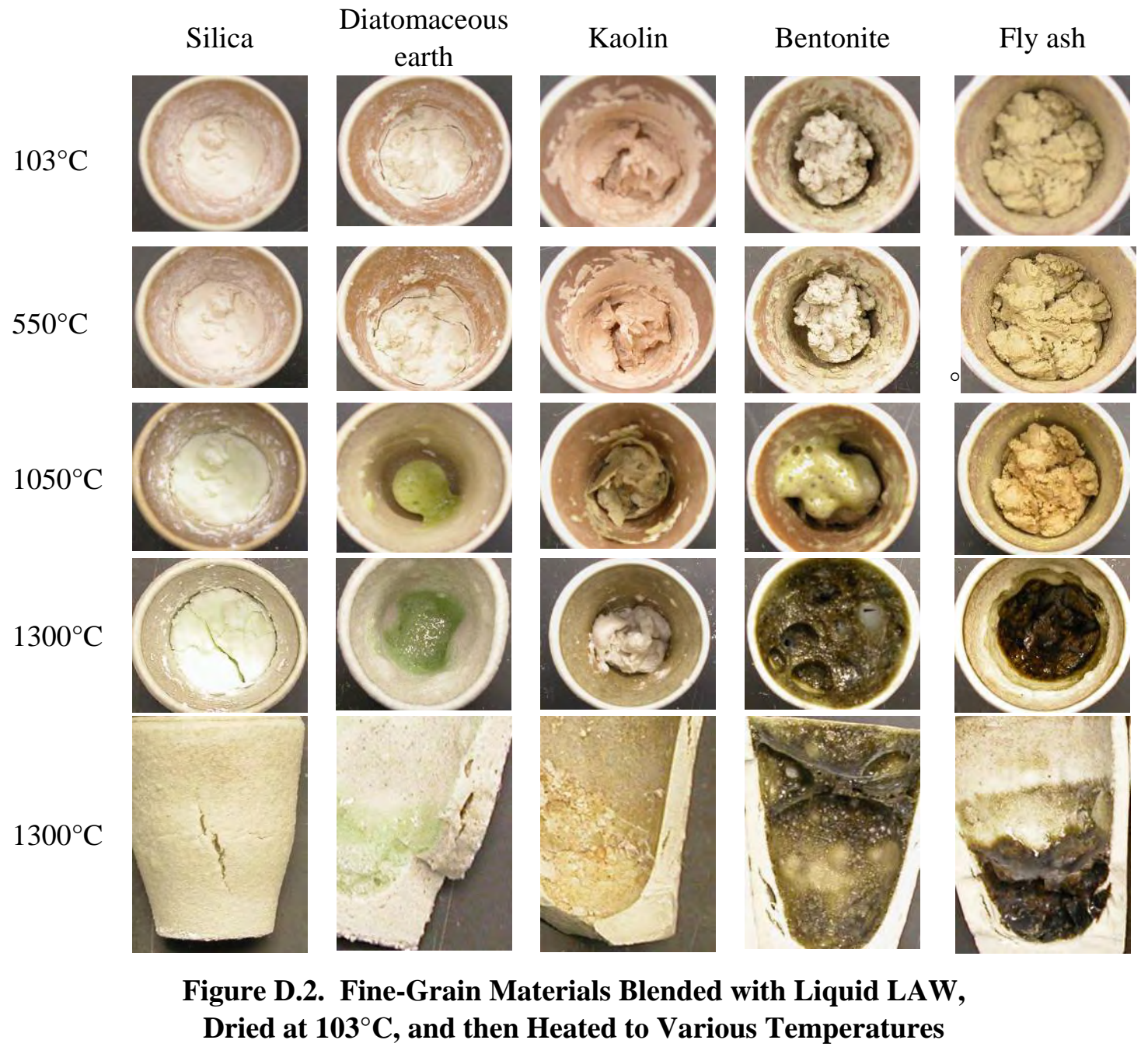

All crucibles were damaged. The least damage was done by the mixture containing kaolin, where only a minor internal crack occurred. The most severe damage was caused by the mixture containing fine silica: a crack appeared on the outer wall that was swollen and deformed. Other mixtures caused internal cracking and swelling.

Note that the mass fraction of dry waste was more than twice as high in diatomaceous earth as in other materials (the last raw in Table D.1). This explains why the crucible with diatomaceous earth was damaged. This damage would probably not occur at waste to fines ratios that were similar to the other materials tested.

Materials other than diatomaceous earth absorbed approximately $0.3 \mathrm{~g}$ of aqueous LAW per $\mathrm{g}$ of fines, which was not enough form a bubble-free glass when heated to $1300^{\circ} \mathrm{C}$. Additional tests with diatomaceous earth are reported on pages F.6 to F.10. 


\section{D.1.3. Scoping Test III: MIS in a Silica Crucible}

To further investigate the interaction between MIS and the porous walls of silica crucibles, 13.6-g dry LAW was added to the crucible and heated at $10^{\circ} \mathrm{C} / \mathrm{min}$ to $300^{\circ} \mathrm{C}$ and then at $5^{\circ} \mathrm{C} / \mathrm{min}$ to $450^{\circ} \mathrm{C}$ (while checking the content every $50^{\circ} \mathrm{C}$ ). The following observations were made.

The outside wall of the crucible appeared wet at $300^{\circ} \mathrm{C}$, indicating the penetration of MIS through the crucible wall ( $\sim 5 \mathrm{~mm}$ thick) while the waste in the center of the crucible appeared dry. The chromate grains turned bright yellow at $300^{\circ} \mathrm{C}$, the waste surface became bright yellow with orange spots, and the liquid phase became noticeable near the inside wall of the crucible at $360^{\circ} \mathrm{C}$. The waste turned dark yellow with orange areas and foamed at $400^{\circ} \mathrm{C}$. The dark orange sample became fully molten at $450^{\circ} \mathrm{C}$ when the test was terminated. As Figure D.3 shows, the outer surface of the crucible was saturated with yellowish molten salt that also appeared on the bottom surface. The dry LAW lost 8.55 mass\% on heating to $450^{\circ} \mathrm{C}$.

This test indicates that the porous crucible walls soak up low-temperature eutectic melt from LAW at temperatures as low as $300^{\circ} \mathrm{C}$.
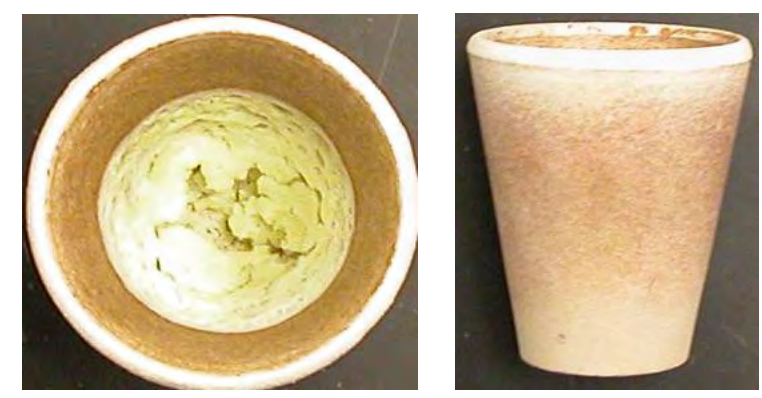

Figure D.3. Silica Crucible with LAW Simulant Heated to $450^{\circ} \mathrm{C}$ :

Top View (left) and Side View (right)

\section{D.1.4. Scoping Test IV: Comparison with Hanford Soil}

To compare the effect of fine-grain materials with Hanford soil on MIS penetration, two additional experiments were performed. In the first experiment, $17.26 \mathrm{~g}$ of soil was overlaid in a silica crucible with $13.68 \mathrm{~g}$ of solid LAW (waste/soil ratio of 0.793 ). The crucible was heated at $5^{\circ} \mathrm{C} / \mathrm{min}$ up to $550^{\circ} \mathrm{C}$ and held at this temperature for $30 \mathrm{~min}$.

As in previous experiments and in the experiment with pure LAW, the outer surface of the crucible appeared wet with MIS at the level of the LAW as soon as the temperature reached $300^{\circ} \mathrm{C}$. By $500^{\circ} \mathrm{C}$, most of the waste melted and soaked into the soil except far a bright yellow residue with orange spots.

Heating was interrupted at $550^{\circ} \mathrm{C}$ when the crucible was removed from the furnace and photographed (Figure D.4, right). The MIS penetrated through the crucible bottom to the alumina grains on which the crucible rested during heating (Figure D.5). 
The crucible was returned to the furnace and heated at the same rate as before. A waste residue, probably sulfate, remained on the top of the soil above $800^{\circ} \mathrm{C}$. The crucible content began to expand at $900^{\circ} \mathrm{C}$. Expansion continued at $1000^{\circ} \mathrm{C}$. The experiment was terminated at $1050^{\circ} \mathrm{C}$ after a 1-hour hold because the crucible was severely deformed and began to sag (see Figure D.4, right). The LOI of the sample (soil + waste) was 26.1 mass\%.

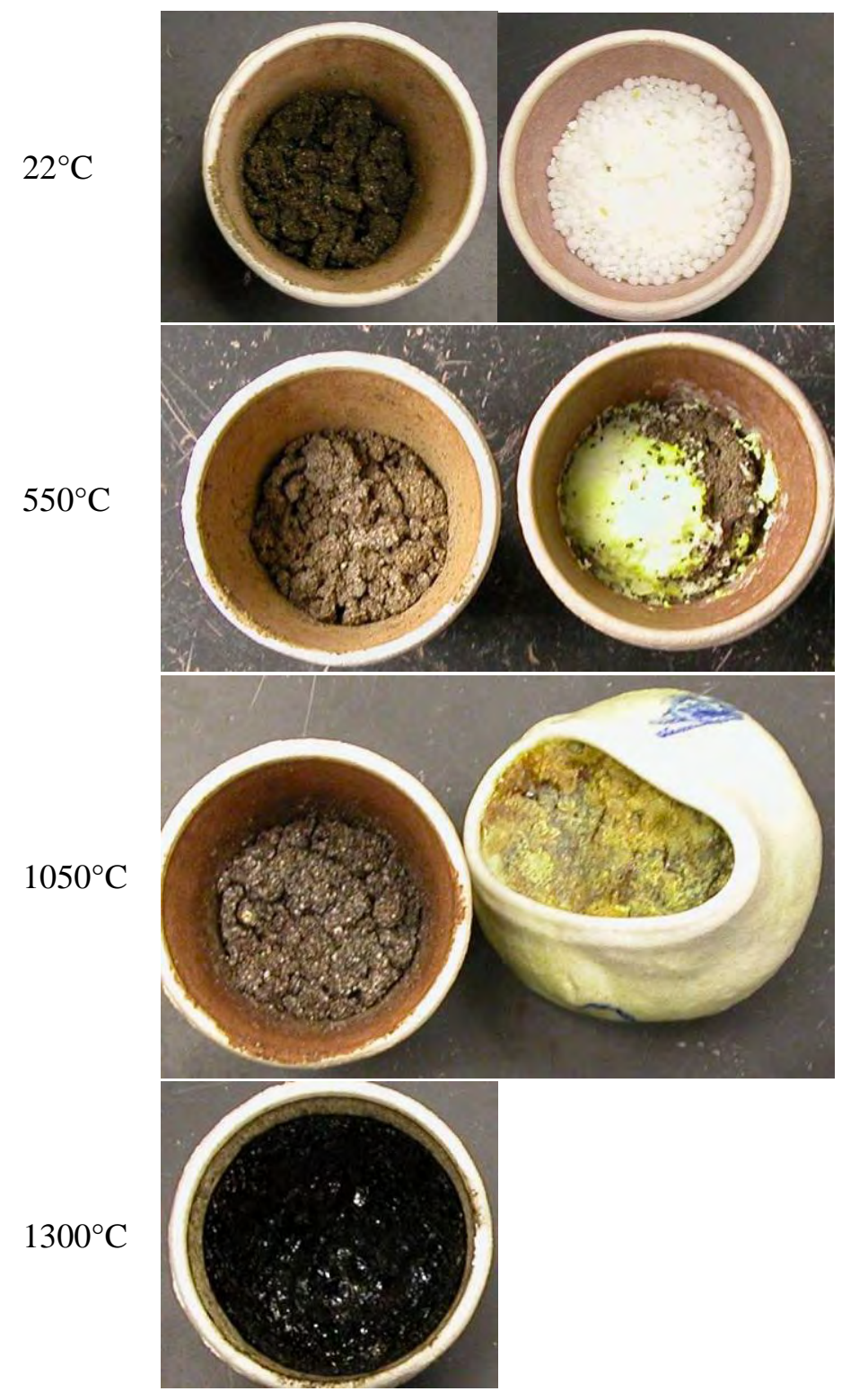

Figure D.4. Sample Appearance after Heat-Treatments at Different Temperatures: Soil Mixed with Wet LAW (left) and Soil Overlaid with Dry LAW (right) 


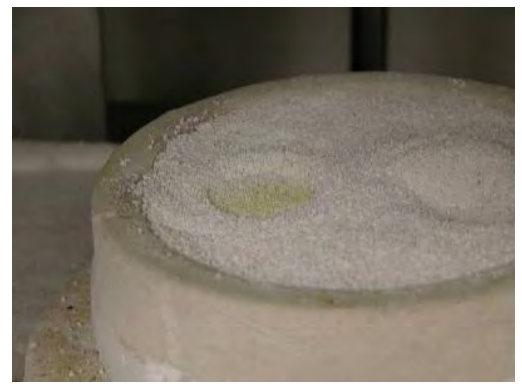

Figure D.5. Traces of MIS Under Crucible Bottom left at $500^{\circ} \mathrm{C}$

The crucible was cut on a diamond-tipped saw-water was used for lubrication. As Figure D.6 shows, most of the soil was sintered except near the bottom, where the soil grains were only weakly sintered. The very top of the sample turned into a viscous bubbly melt with separated patches of bright yellow sulfate melt. The crucible wall was soaked with MIS that began to react with silica at temperatures above $1000^{\circ} \mathrm{C}$ to form viscous glassy melt. Gas trapped in the viscous glass expanded, forming large cavities within the wall. The fragile portions of the wall fractured.

A comparison with tests shown in Figure D.1 shows that fine-grain materials protected the crucible better than the soil except silica flour, which appeared to be worse.

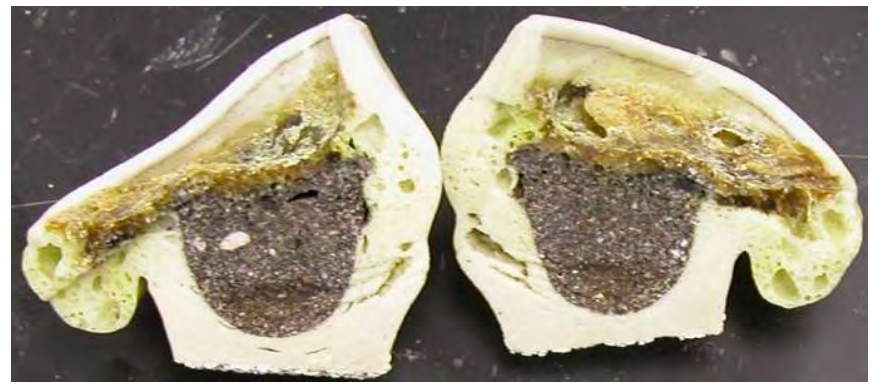

Figure D.6. Cross-Section of Silica Crucible with Soil Overlaid with Dry LAY and Heated to $1050^{\circ} \mathrm{C}$

In the second experiment, liquid LAW was added to make a paste; $5 \mathrm{~mL}$ of LAW was needed for $17.3 \mathrm{~g}$ of soil $(0.290 \mathrm{~mL} / \mathrm{g})$. The sample was dried at $100^{\circ} \mathrm{C}$ for $\sim 48$ hours and then heated at $5^{\circ} \mathrm{C} / \mathrm{min}$ to $550^{\circ} \mathrm{C}$, when the crucible was removed from the furnace and photographed. No change in the texture of the sample was observed (Figure D.5, left).

When heating resumed, the level of the sample somewhat dropped from the previous level by $700^{\circ} \mathrm{C}$ and continued to decrease without a change in texture until $1050^{\circ} \mathrm{C}$. The sample was held at $1050^{\circ} \mathrm{C}$ for 1 hour, removed from the furnace, and examined (Figure D.5, left).

When heating continued, melting began at $1120^{\circ} \mathrm{C}$. At $1300^{\circ} \mathrm{C}$, the level of melt rose because of vigorous foaming. After a 1-hour hold at $1300^{\circ} \mathrm{C}$, the crucible was removed from the furnace. The wall of the crucible was slightly deformed by the penetrating MIS. 
The crucible was cut on a diamond-tipped saw. As Figure D.7 shows, the sample was only partially vitrified. The dark glass was porous with irregular cavities. The MIS penetrated through the crucible sidewall and bottom to the outer surface, where its presence was clearly visible.

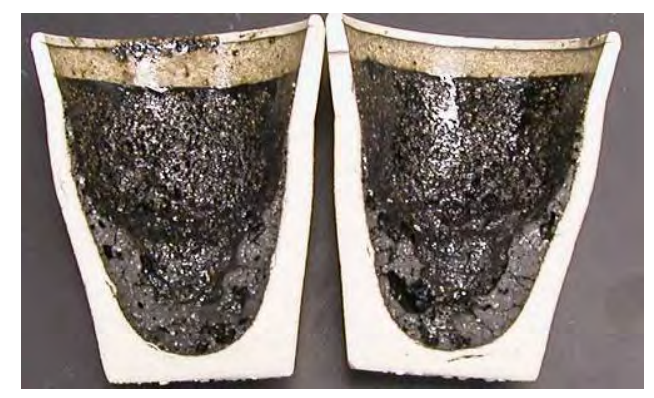

Figure D.7. Cross-Section of Silica Crucible with Soil Mixed with Liquid LAW and Heated to $1300^{\circ} \mathrm{C}$

The crucible was less damaged with soil mixed with LAW than when LAW was mixed with fine-grain materials (compare Figure D.7 and Figure D.2) because the liquid LAW addition that created the same plasticity was only $0.290 \mathrm{~mL} / \mathrm{g}$ of soil, whereas 0.68 to $2.81 \mathrm{~mL} / \mathrm{g}$ was needed for materials with a large specific surface area (see Table D.2). The LOI of the sample (soil + waste) was 25.4 mass\% (the sample mass did not change on heating from $1050^{\circ} \mathrm{C}$ to $1300^{\circ} \mathrm{C}$ ).

\section{D.2. Preliminary Studies}

Scoping tests showed that fine-grain silica failed to protect the crucible. Therefore, it was dropped from further testing. Diatomaceous earth appeared the most promising additive. Tests with various finegrained materials (kaolin, bentonite, fly ash, and diatomaceous earth) showed that good mixing with the liquid LAW simulant was necessary for MIS immobilization.

The purpose of the following series of preliminary tests was to develop a suitable experimental setup for assessing MIS penetration into porous refractory from BV feeds and to perform tests with the baseline feed to gauge the performance of modified feeds. Two tests were performed to compare the behavior of the baseline feed with the feed used in the 38B test and to assess the effect of premixing $\mathrm{B}_{2} \mathrm{O}_{3}$ with LAW MIS penetration into the porous refractory.

\section{D.2.1. MIS Penetration into Porous Refractory: Experimental Setup}

In the initial testing, the feed was heated to $1200^{\circ} \mathrm{C}$ in two silica crucibles: a small crucible of the same size as that used for scoping studies ( $\sim 50 \mathrm{~mm}$ tall, $\sim 20 \mathrm{~mm}$ inner diameter at the bottom, and $\sim 40 \mathrm{~mm}$ inner diameter at the top, wall thickness 3 to $5 \mathrm{~mm}$ ) and a medium crucible ( $\sim 100 \mathrm{~mm}$ tall, $\sim 40 \mathrm{~mm}$ inner diameter at the bottom, and $\sim 80 \mathrm{~mm}$ inner diameter at the top, wall thickness 6 to $12 \mathrm{~mm}$ ), in which the CRB rod was immersed in the feed (see the schematic in Figure D.8). The rods used for testing were $\sim 120 \mathrm{~mm}$ long and had a square cross-section (15 to $19 \mathrm{~mm}$ in size). 

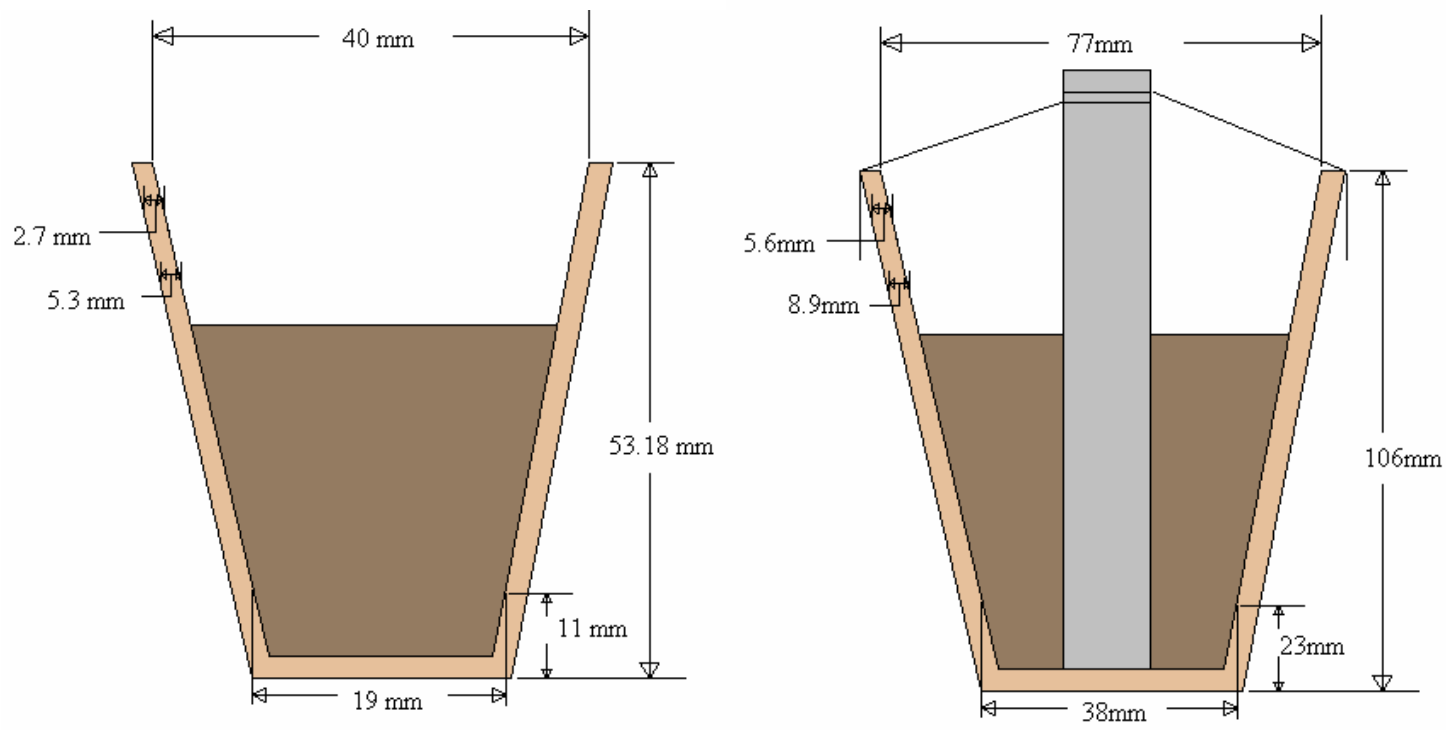

Figure D.8. Dimensions of Randomly Selected Silica Crucibles: a Small Crucible (left) and a Medium Crucible with a CRB Rod Adjusted by a Pt Wire (right)

\section{D.2.2. MIS Penetration into Porous Refractory from Baseline Feed}

To evaluate the effects of adding fine-grain materials, thorough testing was performed first with the baseline feed. The feed of composition defined in Table 4.2 was spiked with $\mathrm{Na}_{2} \mathrm{CrO}_{4}$ to enhance the color of MIS, thus making it more apparent in the refractory walls of silica crucibles and in rectangular CRB rods that were partly immersed into the feed.

For the baseline feed testing, dry solids (soil, $\mathrm{ZrO}_{2}, \mathrm{~B}_{2} \mathrm{O}_{3}$, and $\mathrm{Na}_{2} \mathrm{CrO}_{4}$ ) to make $200 \mathrm{~g}$ of glass were mixed in the proportion given in Table 4.2, except that the quantity of $\mathrm{Na}_{2} \mathrm{CrO}_{4}$ was increased by $200 \%$. The solids were mixed in a tungsten carbide mill in two separate batches. The liquid LAW was concentrated in a stainless steel beaker on a hot plate until its volume dropped to half of the original level. The solids were then added to the beaker, and the mixture was dried while continuously stirring with a spatula.

For the initial test, a small silica crucible was loaded with 24.4 grams of dry feed. In a second medium silica crucible, a square CRB rod was installed (Figure D.8) and $97.5 \mathrm{~g}$ of feed was added. The rod was secured by a Pt wire as seen in Figure D.9. The crucibles were put in the furnace and heated at $5^{\circ} \mathrm{C} / \mathrm{min}$ to a temperature of $1200^{\circ} \mathrm{C}$ and held for 1 hour. 


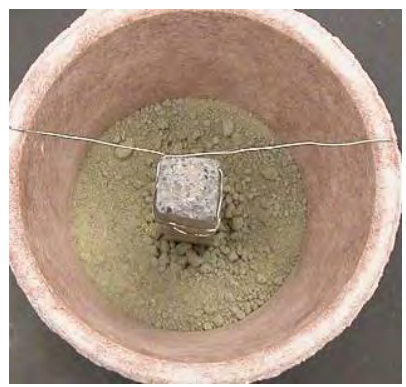

Figure D.9. Silica Crucible with a CRB Rod and Feed

As Figure D.10 shows, two melt lines are visible on the inner surface of the small crucible wall. The lower melt line indicates the level of the feed when the first melt appeared as the temperature was increasing. The second melt line indicates the foam height as the melt volume expanded with trapped gas after the glass-forming melt became connected. In the case of the small crucible shown in Figure D.10, the foam reached the rim of the crucible, but did not overflow.
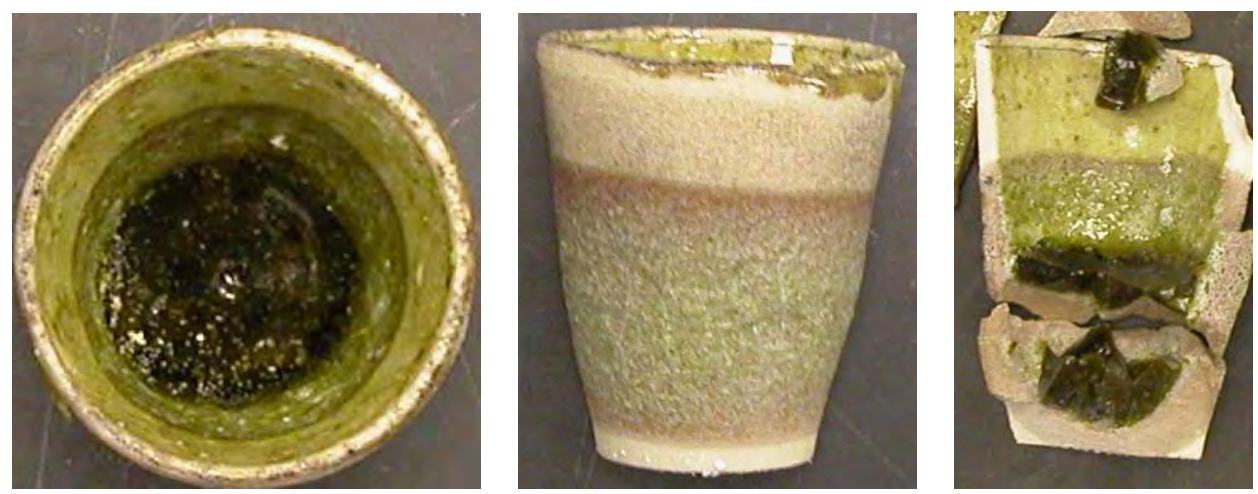

Figure D.10. Baseline Feed Molten at $1200^{\circ} \mathrm{C}$ in Small Crucible

One hour at $1200^{\circ} \mathrm{C}$ did not allow complete the removal of all gas bubbles and small bubbles remained at the top layer of the glass. The "funnel-like crater" in the glass is caused by thermal shrinkage of gas bubbles and glass during cooling (the glass around the crucible axis sunk because the perimeter solidified faster than the center). The mass of glass in the crucible was $19.3 \mathrm{~g}$, corresponding to a mass loss of $20.9 \%$ from the feed.

As in previous tests, the MIS penetrated through the crucible wall at the feed level. It also penetrated through the crucible bottom except for a small area around the edge at which the conical wall meets with the bottom. The extent of MIS penetration was clearly visible in the crucible cross-section as a dark brown area with a green-hue coloration, probably caused by green crystals of eskolaite, $\mathrm{Cr}_{2} \mathrm{O}_{3}$, to which chromate would reduce at temperatures above $850^{\circ} \mathrm{C}$. The crucible wall was swollen, mostly around the melt meniscus area (the final melt line), where MIS made a glass phase with silica, trapping gas that expanded, creating cavities inside the wall.

Figure D.11 shows a cross-section through the medium crucible. The MIS did not reach the outer surface of the crucible wall. The inside of the crucible wall exhibits the two levels as described above, one of the 
foam (just below the rime) and the other where the feed began to melt. This level coincides with the top level at which MIS penetrated into the wall. Because the CRB rod was removed at the end of the experiment, the final level of the melt was lower than that during the experiment.

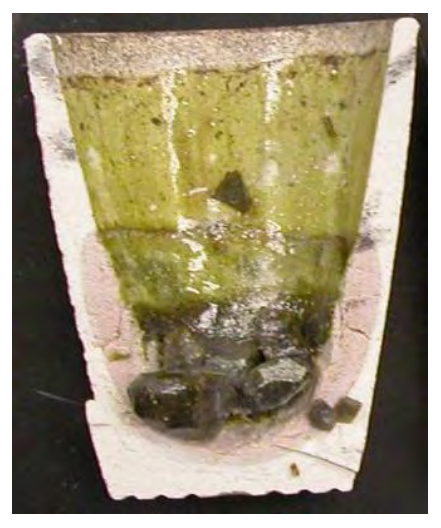

Figure D.11. Cross-Section Through the Medium Crucible with Baseline Feed Heated to $1200^{\circ} \mathrm{C}$

Within the area of penetration, clearly marked by pink coloring, the wall cracked because of expansion caused by vitrification followed by bubble expansion. In the bottom part of the inner surface, the glass penetrated into the MIS-soaked area and began dissolving the crucible material. Silica grains released from the wall dispersed in the melt, giving the green glass a murky appearance.

The CRB rod was visibly corroded below the final melt line. Figure D.12 shows an irregular interface of the CRB with the melt, the altered (pinkish) color of the bottom part of the rod, round pores to a distance of 2 to $3 \mathrm{~mm}$ from the interface, and the corrosion products in the adjacent layer of the glass. The pores that developed as a result of the reaction between the MIS and the fine-grain portion of the CRB cause CRB swelling.

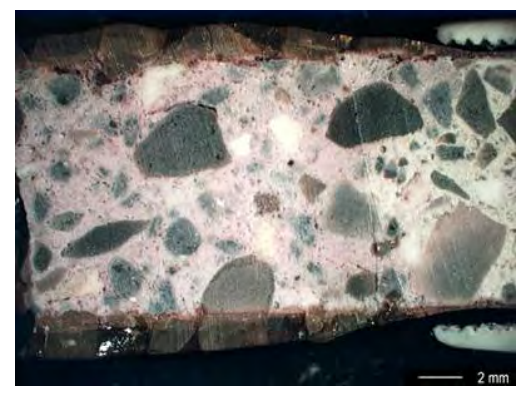

Figure D.12. The Bottom Part of the Sectioned CRB Rod as Removed from Baseline Melt

\section{D.2.3. MIS Penetration into Porous Refractory from 38B Feed}

The MIS penetration test was repeated with the AMEC feed that was used for FS-38B melter testing. Unlike in the feed used in this study, in which fine $\mathrm{ZrO}_{2}$ powder was used, zirconium oxide in 38B feed was in the form of hollow $\sim 100-\mu \mathrm{m}$ baddeleyite spheres.

The test outcome is shown in Figures D.13 through D.16. The wall of the small crucible was severely deformed (Figure D.13); the MIS permeated nearly all the volume of the crucible except the rim, while 
$\mathrm{ZrO}_{2}$ particles remained virtually undissolved and mostly sunk to the melt bottom (see details shown in Figure D.14).
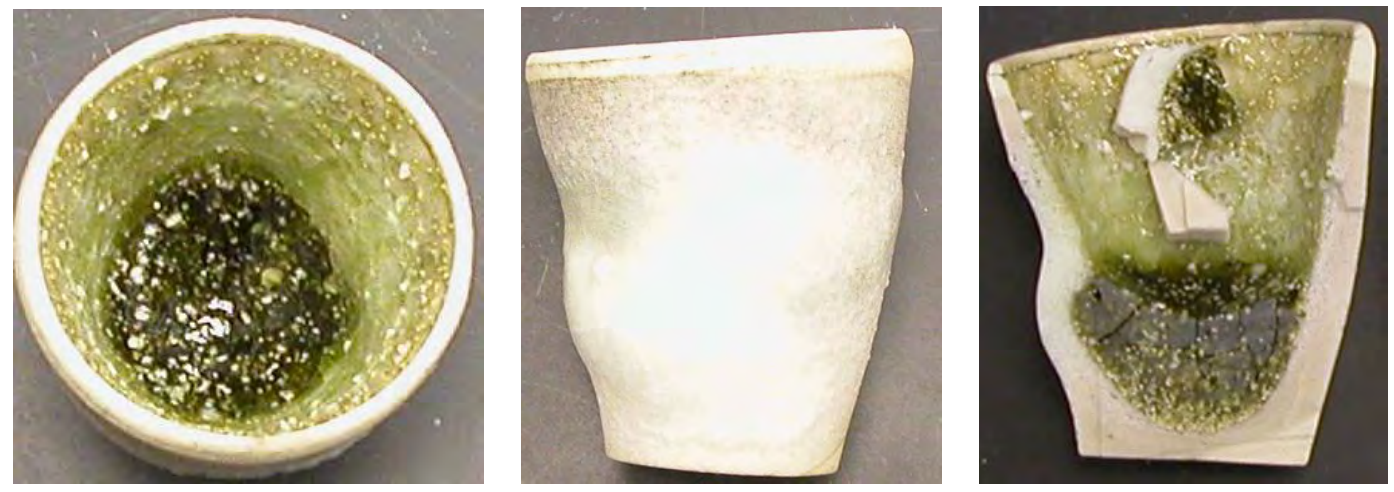

Figure D.13. AMEC 38B Feed Molten at $1200^{\circ} \mathrm{C}$ in Small Crucible
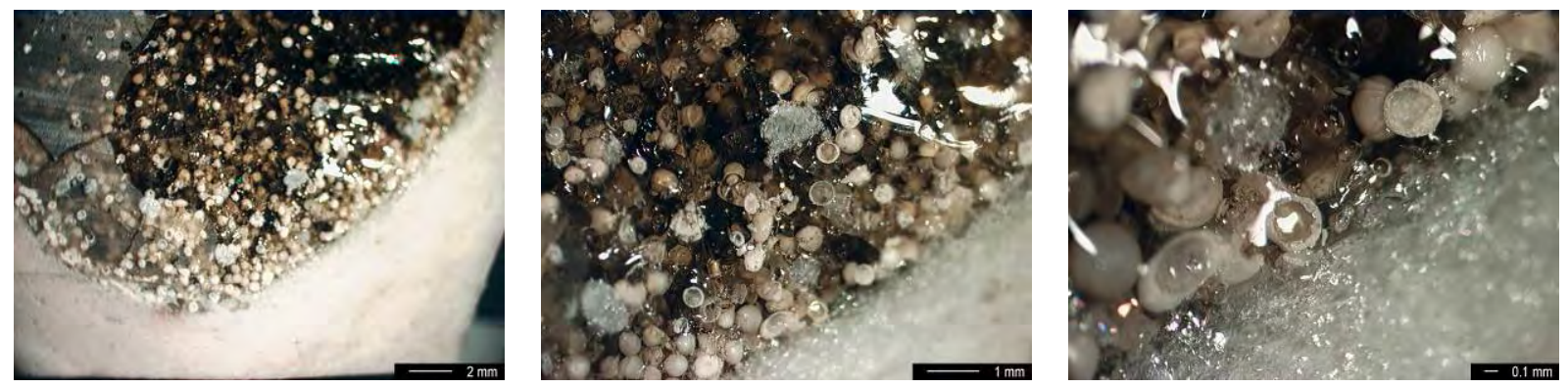

Figure D.14. Detail of AMEC 38B Melt

Figure D.15 shows a cross-section through the medium crucible. The penetration of the MIS is visible as a zone of a darker fleshy color. Undissolved particles of $\mathrm{ZrO}_{2}$ are visible as wide spots in the glass.

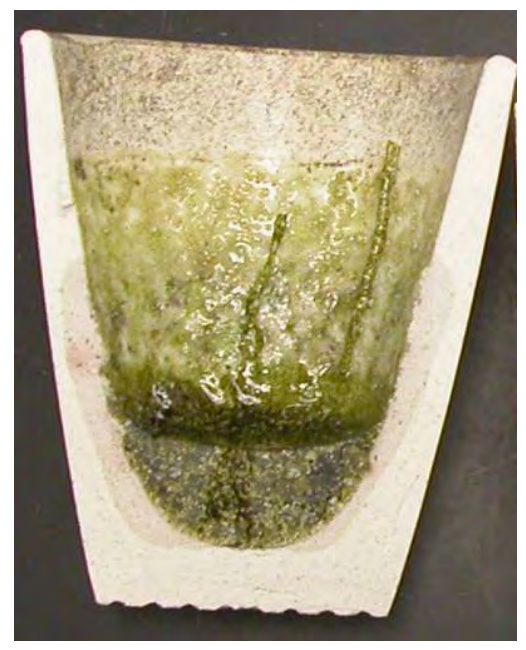

Figure D.15. Cross-Section Through the Medium Crucible with AMEC 38B Feed Molten at $1200^{\circ} \mathrm{C}$

D.12 
Figure D.16 shows the CRB rod. In the areas where the CRB rod was contacted by melt, pits appeared in the rod surface, indicating that the bonding cement of the CRB dissolve faster. In the cross-section, the color of the rod became somewhat darker below the melt line, not as pink as in samples exposed to melt with an increased concentration of Cr. Spherical cavities provided additional evidence of the penetration of MIS into the rod.
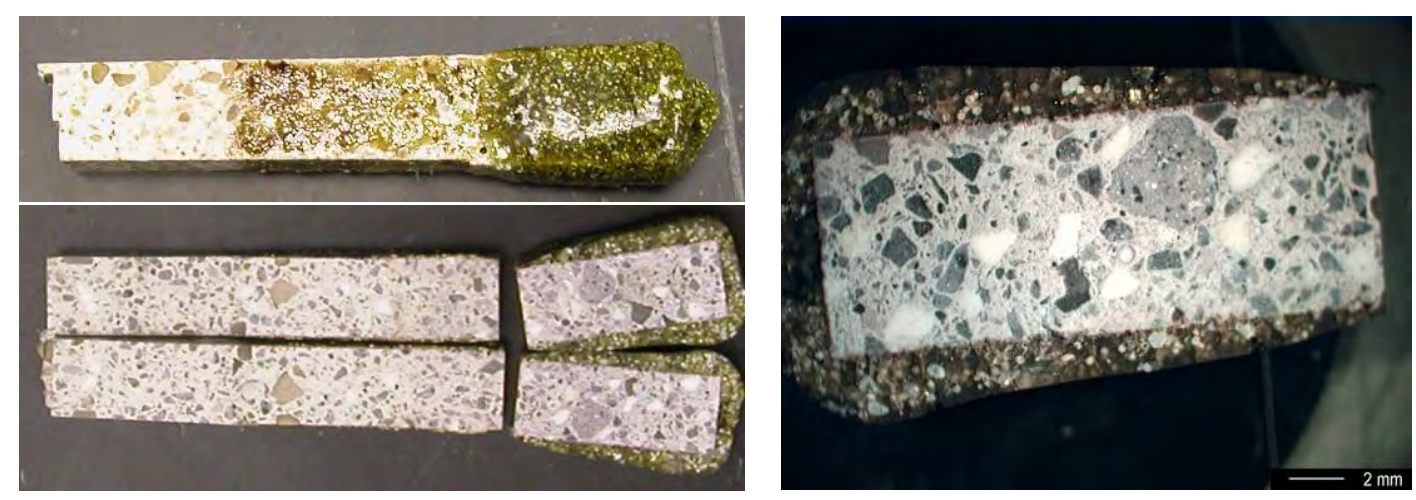

Figure D.16. CRB Rod, as Removed from AMEC 38B Melt and Sectioned

A comparison of small crucible results (compare Figure D.10 with Figures D.13 and D.14) indicates a more severe attack by 38B feed. However, a comparison of medium crucible results (Figure D.11 versus Figure D.15) indicates a similar or even an opposite tendency. Hence, the results of crucible experiments do not allow any definite conclusion regarding which of the two feeds is associated with a more severe MIS penetration. Neither does any difference show up when comparing Figure D.12 with Figure D.16 (CRB rods). This is somewhat surprising because the LAW simulant was thoroughly blended with the additive components (soil, $\mathrm{ZrO}_{2}$, and $\mathrm{B}_{2} \mathrm{O}_{3}$ ) in the baseline feed, whereas a dry simulant with relatively large salt crystals (not co-precipitated) was used in the 38B feed. These results clearly showed that MIS penetration was not simply the result of using non-prototypic feed in the early full-scale tests.

\section{D.2.4. Effect of Premixing $\mathrm{B}_{2} \mathrm{O}_{3}$ with LAW on MIS Penetration into Porous Refractory:}

It was expected that $\mathrm{B}_{2} \mathrm{O}_{3}$ would increase the viscosity of MIS, thus hindering MIS mobility and decreasing its penetration into the porous refractory. To check the effect of $\mathrm{B}_{2} \mathrm{O}_{3}$ on the MIS, $\mathrm{B}_{2} \mathrm{O}_{3}$ was dissolved in liquid six-tank LAW simulant in several $\mathrm{B}_{2} \mathrm{O}_{3}$-MIS ratios. The simulant solution was thoroughly stirred to disperse the undissolved fraction, poured into a glass beaker, and stirred with $\mathrm{B}_{2} \mathrm{O}_{3}$ until all the $\mathrm{B}_{2} \mathrm{O}_{3}$ dissolved while the temperature was gradually increased. $\mathrm{B}_{2} \mathrm{O}_{3}$ fully dissolved as the temperature reached $55^{\circ} \mathrm{C}$.

In the preliminary experiment, a mixture with the proportion of LAW and $\mathrm{B}_{2} \mathrm{O}_{3}$ as shown in Table 4.2 was dried in a Pt crucible and then gradually heated at the rate of $0.78^{\circ} \mathrm{C} / \mathrm{min}$ to $525^{\circ} \mathrm{C}$ and $1.72^{\circ} \mathrm{C}$ to $800^{\circ} \mathrm{C}$. At this slow rate, the reactions between organic and nitrite/nitrate components were allowed to reach near equilibrium. The mixture appeared solid, keeping its original shape, until $725^{\circ} \mathrm{C}$, when it appeared wet. It became a "boiling" liquid with a fraction of solid material at $775^{\circ} \mathrm{C}$ and turned into clear liquid at $800^{\circ} \mathrm{C}$. The liquid had a low, water-like viscosity. 
This experiment showed that $\mathrm{B}_{2} \mathrm{O}_{3}$ does not significantly increase the viscosity of MIS, but keeps it solid to a high temperature; with $\mathrm{B}_{2} \mathrm{O}_{3}$, MIS became a melt at $775^{\circ} \mathrm{C}$ instead of at $450^{\circ} \mathrm{C}$ without $\mathrm{B}_{2} \mathrm{O}_{3}$ (see D.3.1)

The experiment was repeated with a faster rate of heating, $5^{\circ} \mathrm{C} / \mathrm{min}$. The mixture began to form melt at $700^{\circ} \mathrm{C}$ and became fully liquid (the last trace of solid phase disappeared) at $800^{\circ} \mathrm{C}$. When the amount of $\mathrm{B}_{2} \mathrm{O}_{3}$ was decreased to one half, the mixture appeared "wet" at $400^{\circ} \mathrm{C}$ and became fully molten at $775^{\circ} \mathrm{C}$. Table D.3 shows data obtained from these experiments. The "full load" corresponds to the proportion of LAW and $\mathrm{B}_{2} \mathrm{O}_{3}$ shown in Table 4.2, and the "half load" mixture contains $50 \%$ of the $\mathrm{B}_{2} \mathrm{O}_{3}$ required by the feed recipe.

Table D.3. Blending $\mathrm{B}_{2} \mathrm{O}_{3}$ with Liquid LAW Simulant

\begin{tabular}{||r|c|c||}
\hline & Full Load & Half Load \\
\hline $\mathrm{B}_{2} \mathrm{O}_{3}(\mathrm{~g})$ & 9.91 & 5.50 \\
\hline Liquid LAW simulant (g) & 286.03 & 317.48 \\
\hline Dry mixture (g) & 98.24 & 103.55 \\
\hline Mixture at $800^{\circ} \mathrm{C}(\mathrm{g})$ & 75.82 & 82.49 \\
\hline LOI (fraction) & 0.254 & 0.215 \\
\hline
\end{tabular}

Both melts had a low viscosity, and when poured on a steel plate at $800^{\circ} \mathrm{C}$, they solidified into a compact yellow solid cake that appeared homogeneous with no signs of segregation of sulfates and halides. The mass loss was $21.5 \%$ for the half-load mixture and $25.4 \%$ for the full-load mixture (Table D.3). Considering that losses occurred also while pouring the melt from the crucible on the steel plate and transferring the cake from the plate to a beaker, it appears that the true loss due to MIS decomposition was below 20\%. Compared to the LOI, 54.6 mass\%, it appears that only the organic constituents of the MIS decomposed at temperatures below $800^{\circ} \mathrm{C}$.

This impact of $\mathrm{B}_{2} \mathrm{O}_{3}$ on MIS is surprising. Remaining solid up to $700^{\circ} \mathrm{C}$ and delayed decomposition were not anticipated. This change was caused by simply modifying the mixing sequence, i.e., dissolving $\mathrm{B}_{2} \mathrm{O}_{3}$ in the liquid waste instead of premixing it with other solid additives. The question is whether the mixing sequence impacts the MIS mobility and its migration into the CRB.

To check previously obtained results of the melting behavior of dry LAW $13.7 \mathrm{~g}$ of dry LAW was placed into a silica crucible and heated at $5^{\circ} \mathrm{C} / \mathrm{min}$. Although the melt was not visible in the sample itself, its first occurrence was indicated by the wet appearance on the outer walls of the porous crucible. Inside the crucible, melt was first observed close to the crucible walls at $360^{\circ} \mathrm{C}$. The sample began to foam at $400^{\circ} \mathrm{C}$ and was completely molten at $450^{\circ} \mathrm{C}$.

The difference between the TGA shown in Figure 5.2 and the crucible test could be attributed to two circumstances: 1) the 42-mg TGA sample decomposed under a smaller partial pressure of $\mathrm{NO}_{\mathrm{x}}$ than the 76-g crucible sample and 2) frequently lowering the sample from the hot zone of the furnace to check its appearance could cause its temperature to be lower than the furnace TC indicated.

Based on these results, we may conclude that $\mathrm{B}_{2} \mathrm{O}_{3}$ delays complete melting of LAW. Once molten, the mixture has a low viscosity, not visibly affected by the presence of $\mathrm{B}_{2} \mathrm{O}_{3}$. Because of lack of phase 
diagrams of $\mathrm{B}_{2} \mathrm{O}_{3}$-ionic salt mixtures, the mechanism at which $\mathrm{B}_{2} \mathrm{O}_{3}$ delays LAW melting into MIS is difficult to understand.

To check whether the mixing sequence impacts the MIS mobility and its migration into the CRB, the MIS penetration test was repeated with the baseline feed composition, except that the soil and $\mathrm{ZrO}_{2}$ were premixed in the agate mill, and the excess $\mathrm{Na}_{2} \mathrm{CrO}_{4}$ and $\mathrm{B}_{2} \mathrm{O}_{3}$ were added to the liquid simulant that was subsequently heated and stirred until the solids were dissolved.

Heating and stirring continued until most of water evaporated. The mixture was then dried in the oven at $100^{\circ} \mathrm{C}$ for over 48 hours. To make the feed, both parts, the soil with $\mathrm{ZrO}_{2}$ and the dried LAW with $\mathrm{Na}_{2} \mathrm{CrO}_{4}$ and $\mathrm{B}_{2} \mathrm{O}_{3}$, were mixed in a tungsten carbide mill for 1 min. The feed was heated in a small and a medium silica crucible with a CRB rod as in the previous tests.

As seen in Figure D.17, the level of the feed is marked by the lower melt line that coincides with the top boundary of the dark areas caused by MIS soaked into the wall and bottom of the crucibles. Crucible walls are somewhat corroded, releasing silica grains into the melt. Bubbles are also visible inside the walls, where MIS reacted with silica. The wall penetration is somewhat less severe than in the case of $\mathrm{B}_{2} \mathrm{O}_{3}$ premixed with the solids (compare Figure D.17 with Figures D.10 and D.11) though this conclusion is somewhat subjective due to the irregularities of MIS penetration.
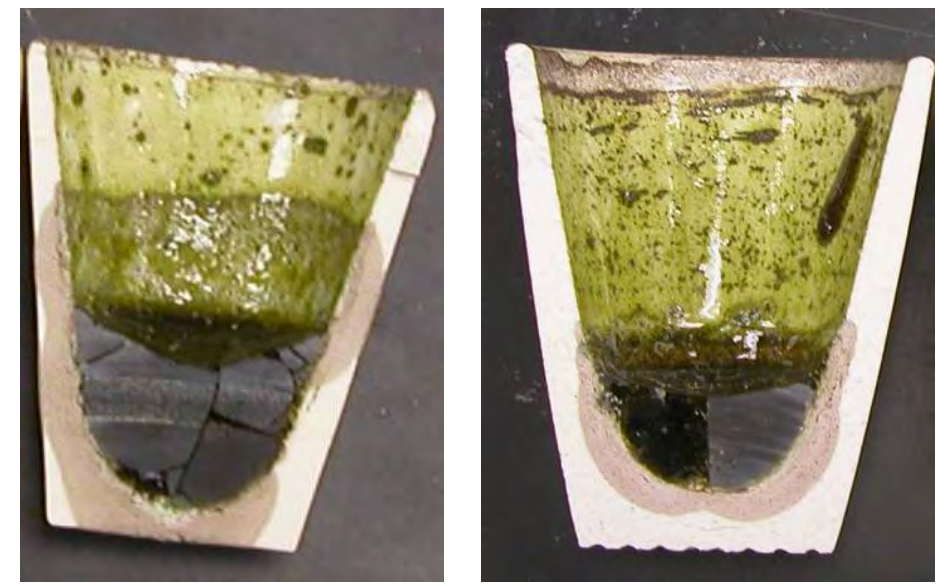

Figure D.17. Melt with $\mathrm{B}_{2} \mathrm{O}_{3}$ Premixed with LAW: Small Crucible (left), Medium Crucible (right)

As seen in Figure D.18, the outer surface of the CRB rod became rough with corrosion pits in the area contacted by the foaming feed. The edges of the rod were rounded. At the meniscus area and below, the CRB expanded and become more porous, and its color turned pink in the cross-section. 


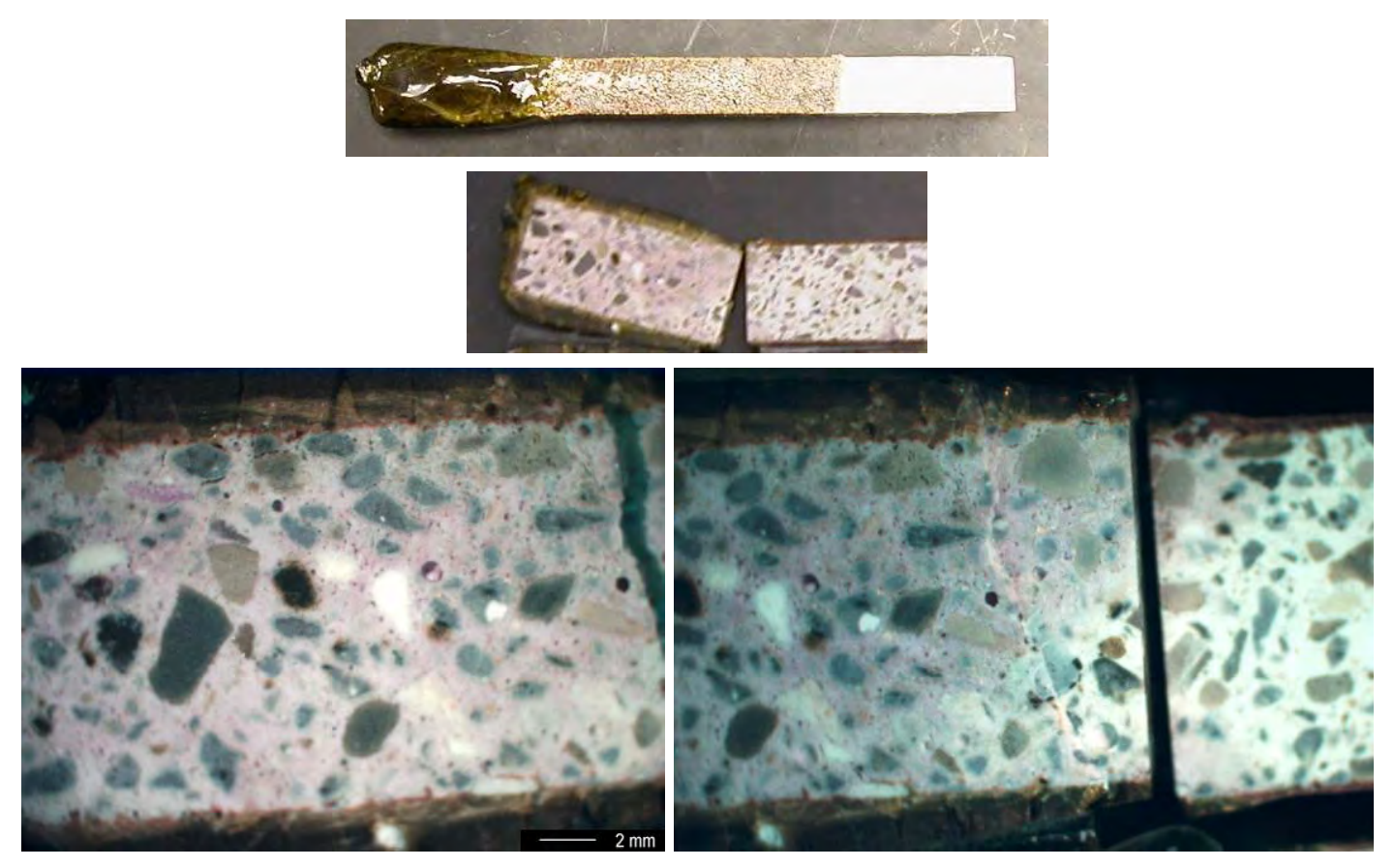

Figure D.18. CRB Rod as Removed from Feed with $\mathrm{B}_{2} \mathrm{O}_{3}$ Dissolved in LAW and Sectioned

Because the irregularities of MIS penetration, as seen in Figure D.17, the experiment was repeated. This time, the chromate addition was doubled to enhance the color. The MIS penetrated through the small crucible wall and bottom as is clearly seen in Figure D.19. The extent of penetration appears more severe than in Figure D.17 and is comparable to the case of baseline feed in which $\mathrm{B}_{2} \mathrm{O}_{3}$ was mixed with other additives (Figure D.10). The extent of MIS penetration in the medium crucible (Figure D.20) is nearly the same as in the baseline experiment (Figure D.11).
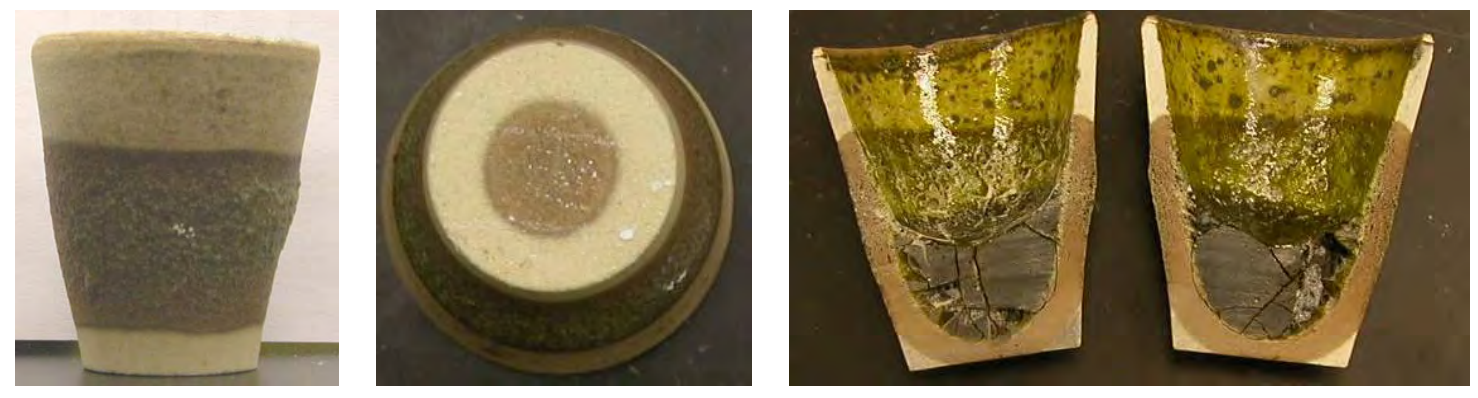

Figure D.19. Melt wit $\mathrm{B}_{2} \mathrm{O}_{3}$ Premixed with LAW: Small Crucible 


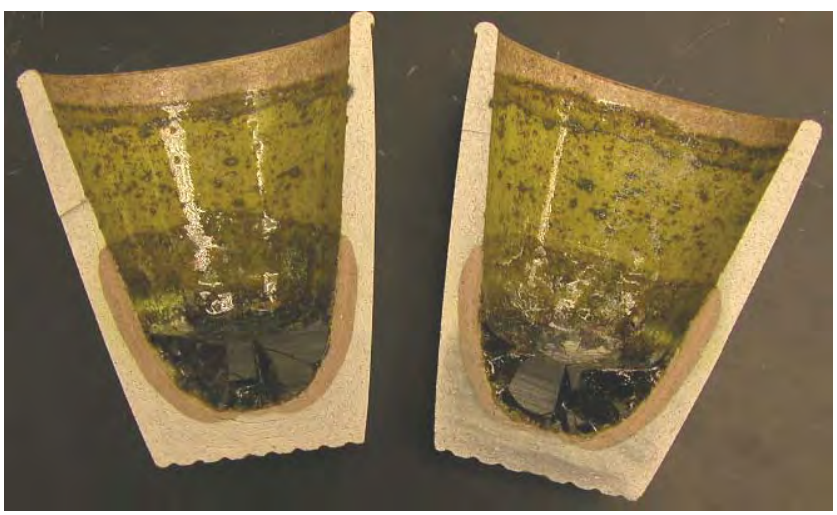

Figure D.20. Melt wit $\mathrm{B}_{2} \mathrm{O}_{3}$ Premixed with LAW: Medium Crucible

The color of the CRB rod turned to a light pink below the feed level except for the central area (Figure D.21). This result is different from that of previous experiments (Figures D.12 and D.18), where the whole bottom portion of the rod turned pink.

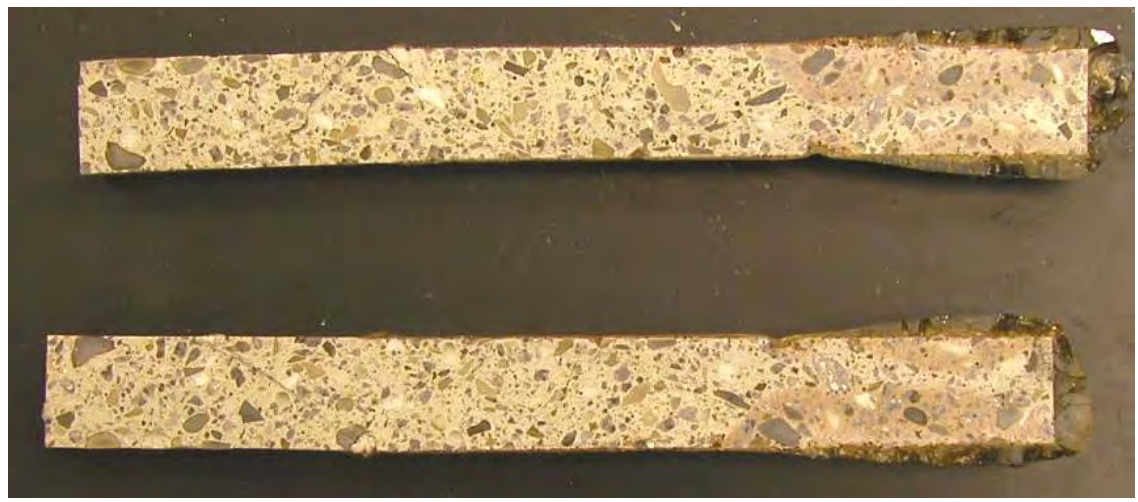

Figure D.21. Section Through CRB Rod Removed from Feed with $\mathrm{B}_{2} \mathrm{O}_{3}$ Dissolved in LAW

The results of crucible experiments show that the persistence of solid phases in the LAW to higher temperatures caused by premixing LAW with $\mathrm{B}_{2} \mathrm{O}_{3}$ has little impact on MIS penetration into porous refractory. The MIS containing $\mathrm{B}_{2} \mathrm{O}_{3}$ probably penetrated into the refractory wall at a higher temperature to the same depth as the MIS from LAW that has not been premixed with $\mathrm{B}_{2} \mathrm{O}_{3}$. It is even possible that $\mathrm{B}_{2} \mathrm{O}_{3}$ may increase the MIS mobility by increasing its volume without substantially increasing its viscosity.

To further clarify the effect of $\mathrm{B}_{2} \mathrm{O}_{3}$, two additional experiments were performed with a $2 \mathrm{X}$ addition of $\mathrm{B}_{2} \mathrm{O}_{3}$ predissolved into the liquid LAW. The doubled amount of $\mathrm{B}_{2} \mathrm{O}_{3}$ was added without changing the proportions of other feed constituents. As before, the soil and $\mathrm{ZrO}_{2}$ were premixed in the tungsten mill for 1 min; $\mathrm{Na}_{2} \mathrm{CrO}_{4}+\mathrm{B}_{2} \mathrm{O}_{3}$ were dissolved in the liquid simulant that was dried, mixed with soil $+\mathrm{ZrO}_{2}$, and homogenized in the tungsten mill for $1 \mathrm{~min}$. In the first experiment, the feed was heated in the small and medium crucibles at $5^{\circ} \mathrm{C} / \mathrm{min}$ to $1200^{\circ} \mathrm{C}$ and held for 1 hour. In the second experiment, a small crucible was heated at $5^{\circ} \mathrm{C} / \mathrm{min}$ to $500^{\circ} \mathrm{C}$ and then removed from the furnace. The feed was removed, and the empty crucible was heated from $500^{\circ} \mathrm{C}$ at $5^{\circ} \mathrm{C} / \mathrm{min}$ to $1200^{\circ} \mathrm{C}$. 
As Figure D.22 shows, MIS penetrated the walls of the small crucible in areas that were in contact with the feed and reached the outside surface of the crucible on the side walls and the bottom. The MISsaturated areas of the refractory have increased porosity; cavities were formed by expanding gases, resulting in bulging and cracking of the refractory material. The weakened refractory material was attached by molten glass.

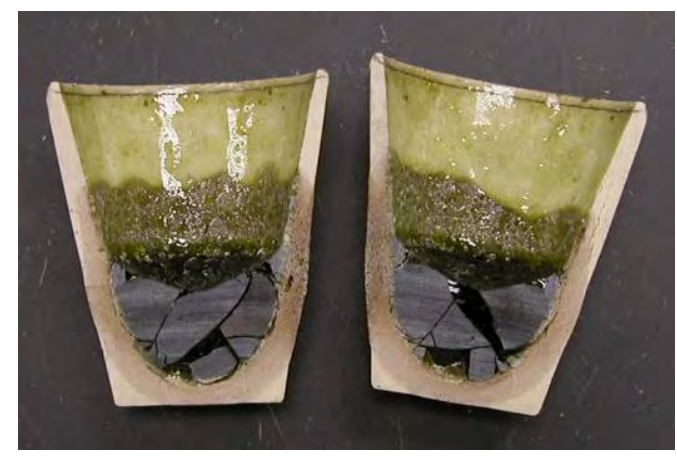

Figure D.22. Small Crucible with Feed Containing a Double Addition of $\mathrm{B}_{2} \mathrm{O}_{3}$

Figure D.23 shows that the impact of MIS was less severe on the medium crucible. The bottom was protected by the refractory rod. The penetration into the walls was limited, possibly by the competing impact of the CRB rod. As in the small crucible, MIS penetrated into the walls where the walls were in contact with the feed, creating large pores.

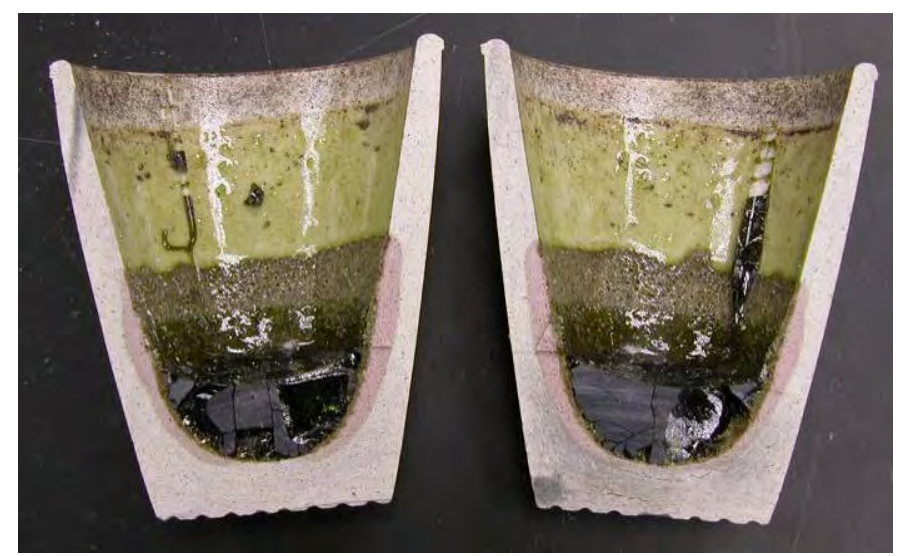

Figure D.23. Medium Crucible with Feed Containing a Double Addition of $\mathbf{B}_{2} \mathbf{O}_{3}$

Judging by the light pink color of the immersed portion of the vibrocast rod, MIS penetrated in all the way through (Figure D.24). In the pink area, the rod was more porous than the gray area, and the porosity was increased near the rod surface. 


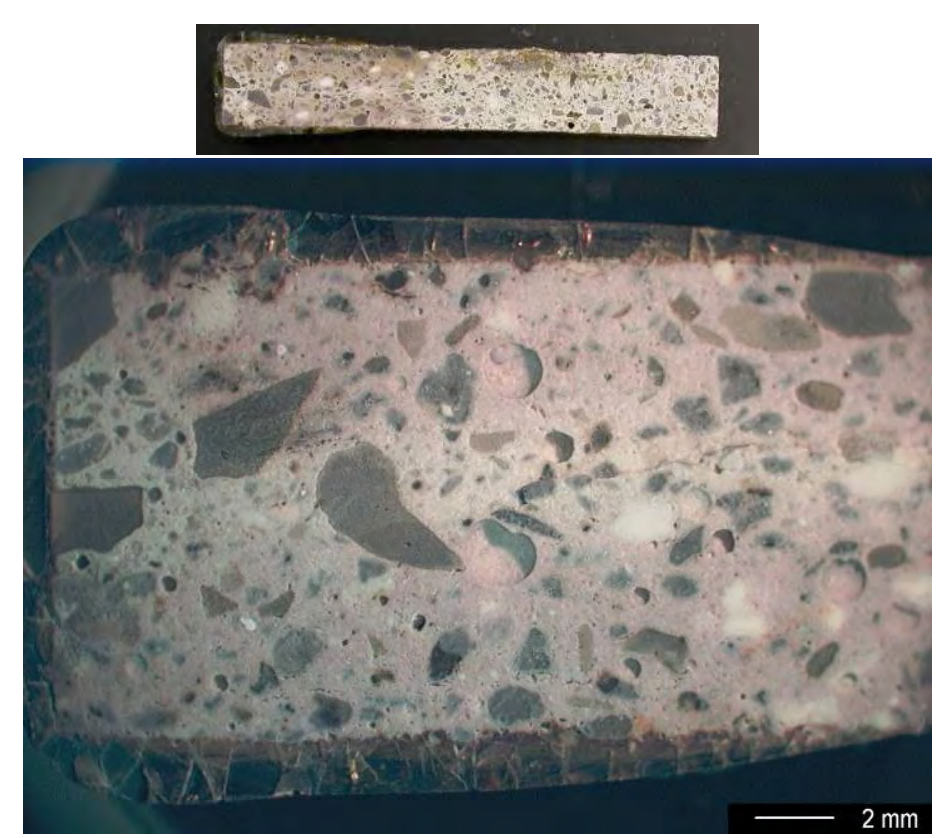

Figure D.24. CRB Rod Immersed in Feed Containing a Double Addition of $\mathrm{B}_{2} \mathrm{O}_{3}$

Figure D.25 shows that MIS penetrated the small crucible wall by $500^{\circ} \mathrm{C}$ in spite the double amount of $\mathrm{B}_{2} \mathrm{O}_{3}$ being premixed with the LAW. Based on the weight difference before and after the heat-treatment, the feed lost $9.8 \%$ of its mass. Based on the weight difference between the full and empty crucible after the heat-treatment, $2.08 \mathrm{~g}$ of MIS penetrated into the crucible out of $24.37 \mathrm{~g}$ of the original amount of the feed. Based on the weight difference before and after the heat-treatment of the empty crucible, the MIS lost $54.3 \%$ of its mass during heating from $500^{\circ} \mathrm{C}$ to $1200^{\circ} \mathrm{C}$. Figure D.26 shows the appearance of the crucible after heating to $1200^{\circ} \mathrm{C}$. The dark brown area where the MIS penetrated through the wall is swollen both outside and inside (see Figure D.27). Bulged regions have large pores.
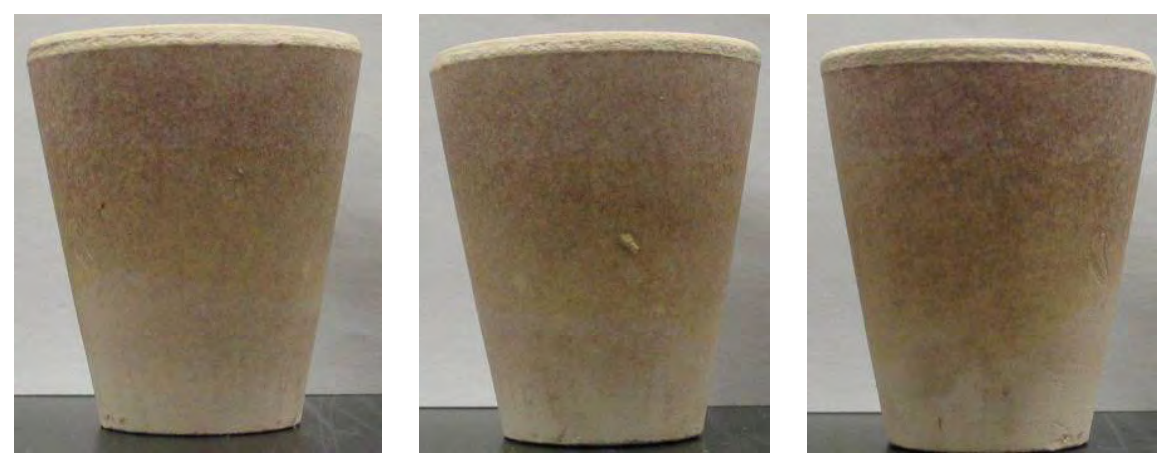

Figure D.25. Small Crucible with Feed Containing a Double Addition of $\mathrm{B}_{2} \mathrm{O}_{3}$ Heated to $500^{\circ} \mathrm{C}$ 

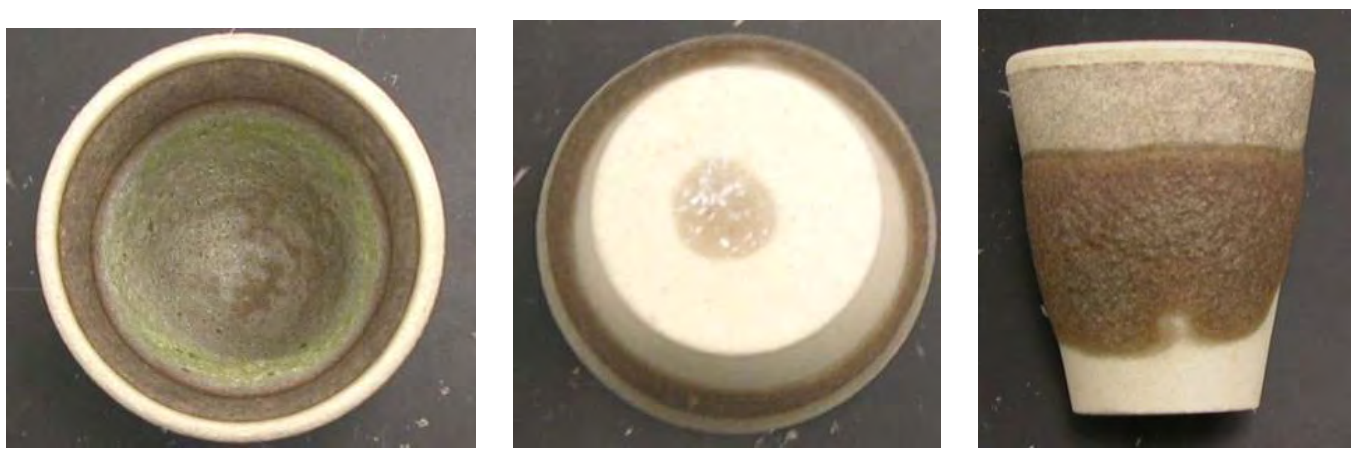

Figure D.26. Small Crucible Heated to $1200^{\circ} \mathrm{C}$ with Feed Removed (see Figure D.25)

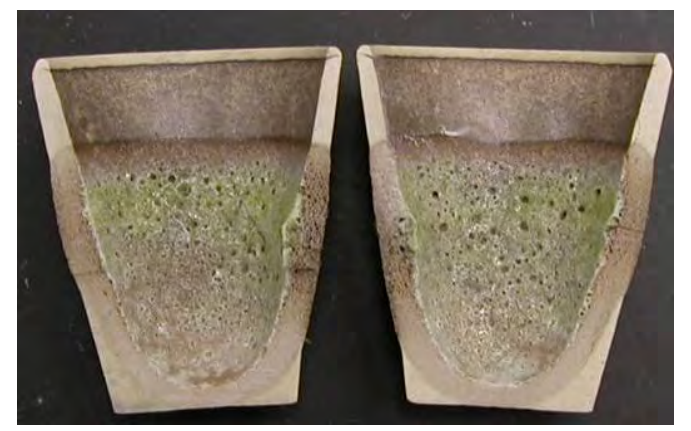

Figure D.27. Small Crucible Heated to $1200^{\circ} \mathrm{C}$ with Feed Removed (see Figure D.26)

These results indicate that the presence of $\mathrm{B}_{2} \mathrm{O}_{3}$ in the MIS does not slow down the penetration of molten salt into the crucible even at a temperature as low as $500^{\circ} \mathrm{C}$. The lack of melt observed when MIS was heated with $\mathrm{B}_{2} \mathrm{O}_{3}$ in the absence of a solid feed component is probably not duplicated when the solid feed components are present. No attempt was made to study this complicated behavior in detail because, in spite of initial optimism, the experiments performed showed that decreasing MIS migration into the CRB via premixing $\mathrm{B}_{2} \mathrm{O}_{3}$ with LAW is unlikely to happen. 


\section{Appendix E}

Effect of Time, Temperature, and Particle Size on MIS Penetration into Porous Refractory 


\section{Appendix E: Effect of Time, Temperature, and Particle Size on MIS Penetration into Porous Refractory}

\section{E.1. Kinetics of MIS Penetration into Porous Refractory}

\section{E.1.1. Interaction Between Feed and CRB Samples During Melting}

Feeds for the CRB tests were prepared by mixing dry solids (soil, $\mathrm{ZrO}_{2}, \mathrm{~B}_{2} \mathrm{O}_{3}$, and extra $\mathrm{Na}_{2} \mathrm{CO}_{4}$ ) in an agate mill. The liquid simulant was evaporated under constant stirring to approximately one half of the original volume. The blended solids were added to the simulant, mixed, and dried while mixing the mass. The feed samples with a CRB specimen $(\sim 15 \times 15 \times 15 \mathrm{~mm})$ were heated in Pt crucibles in a furnace at $5^{\circ} \mathrm{C} / \mathrm{min}$ and removed when temperatures reached $550,800,900,100,1100,1200$, and $1300^{\circ} \mathrm{C}$. The mass loss was $10.6 \%$ for the sample heated to $550^{\circ} \mathrm{C}$ and $\sim 21 \%$ (20.4 to $21.7 \%$ ) for samples heated to 800 to $1300^{\circ} \mathrm{C}$. Figure E. 1 shows the appearance of the melts.

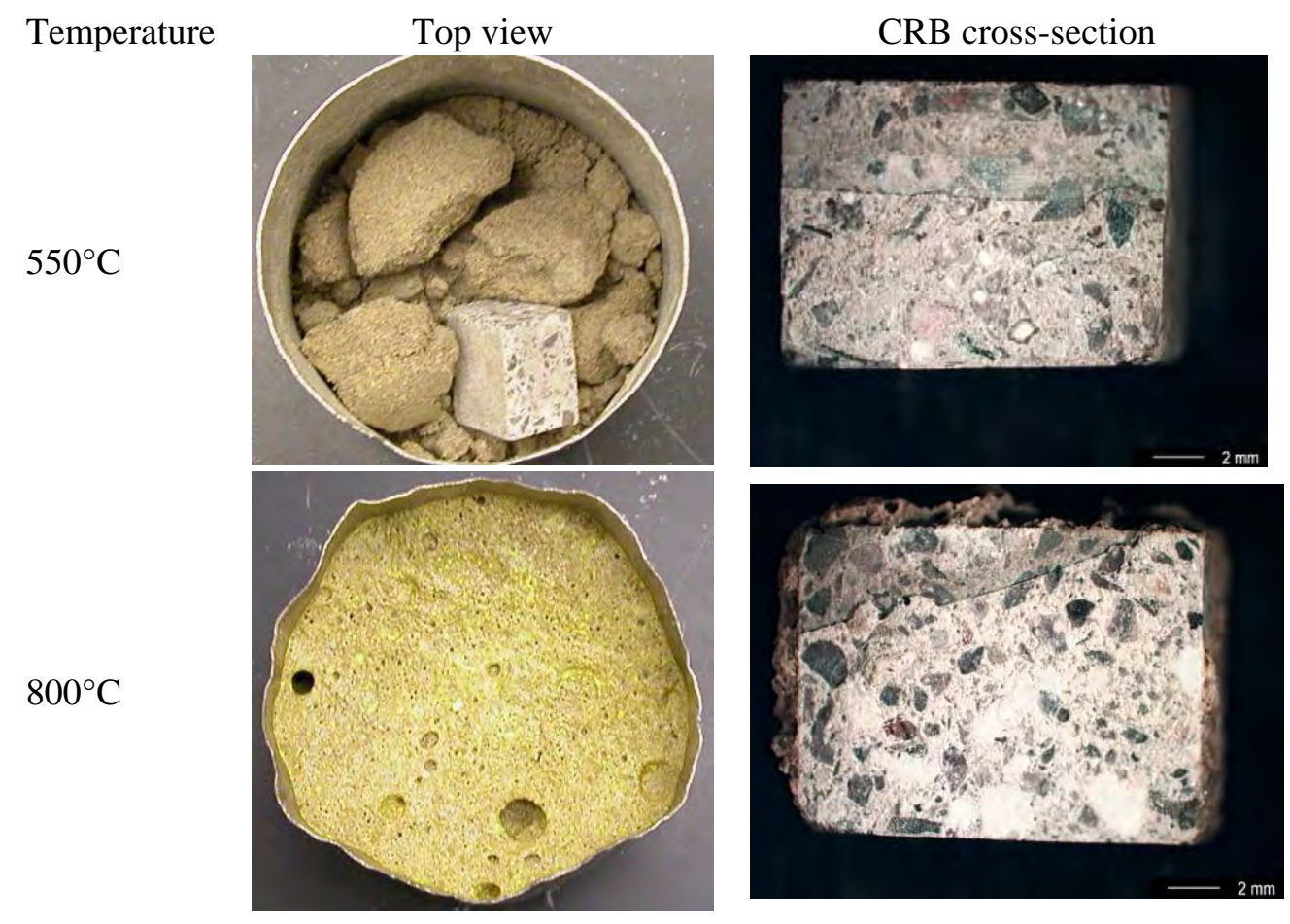

Figure E.1. Feed/Melt Appearance After Heat-Treatment at Different Temperatures; Fracture Surfaces Through CRB Specimens (after a 4 to $5 \mathrm{~mm}$ cut with a dry saw) 
Temperature

$900^{\circ} \mathrm{C}$

$1000^{\circ} \mathrm{C}$

$1100^{\circ} \mathrm{C}$

$1200^{\circ} \mathrm{C}$
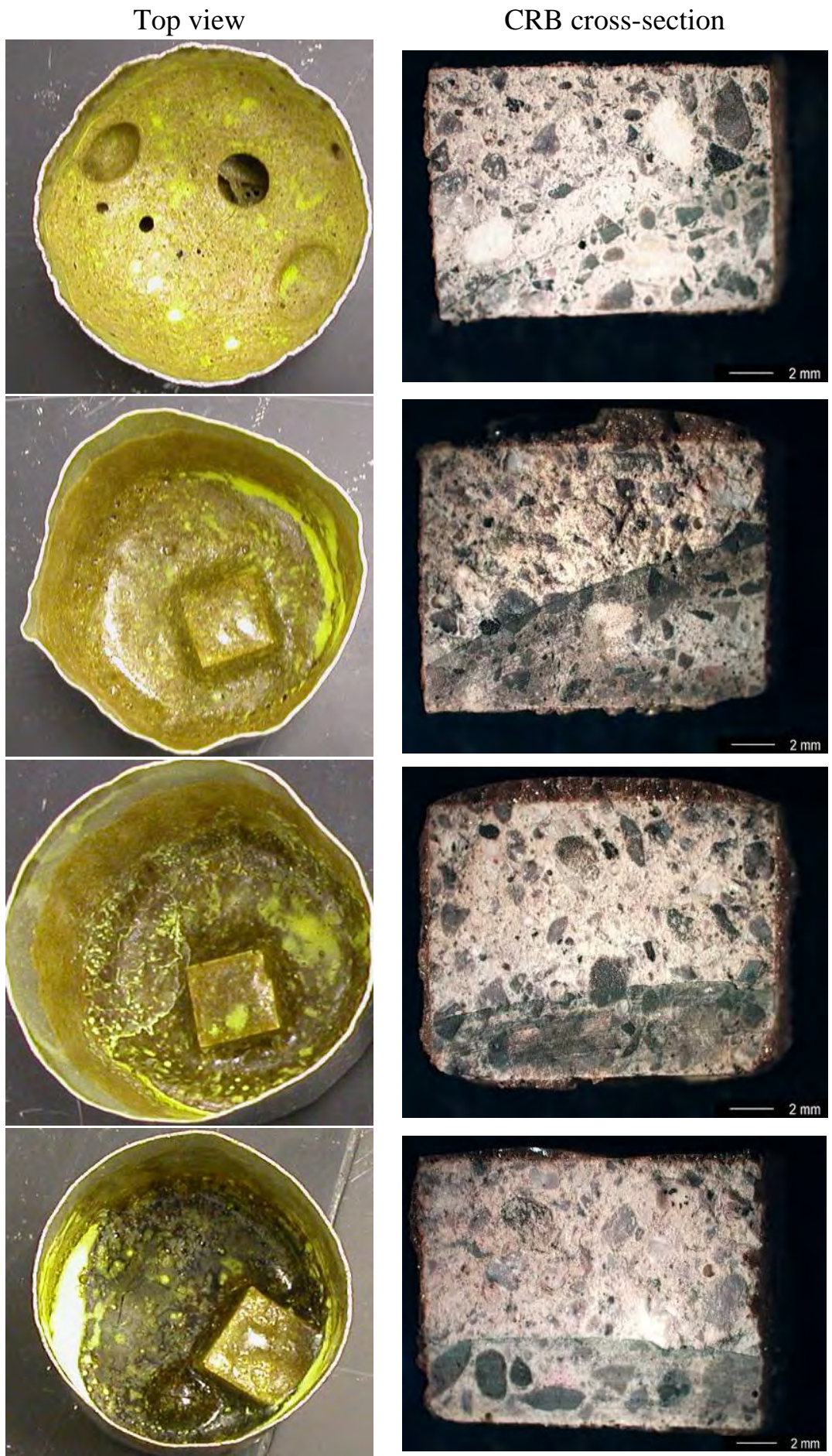

Figure E.1 (Contd.) 

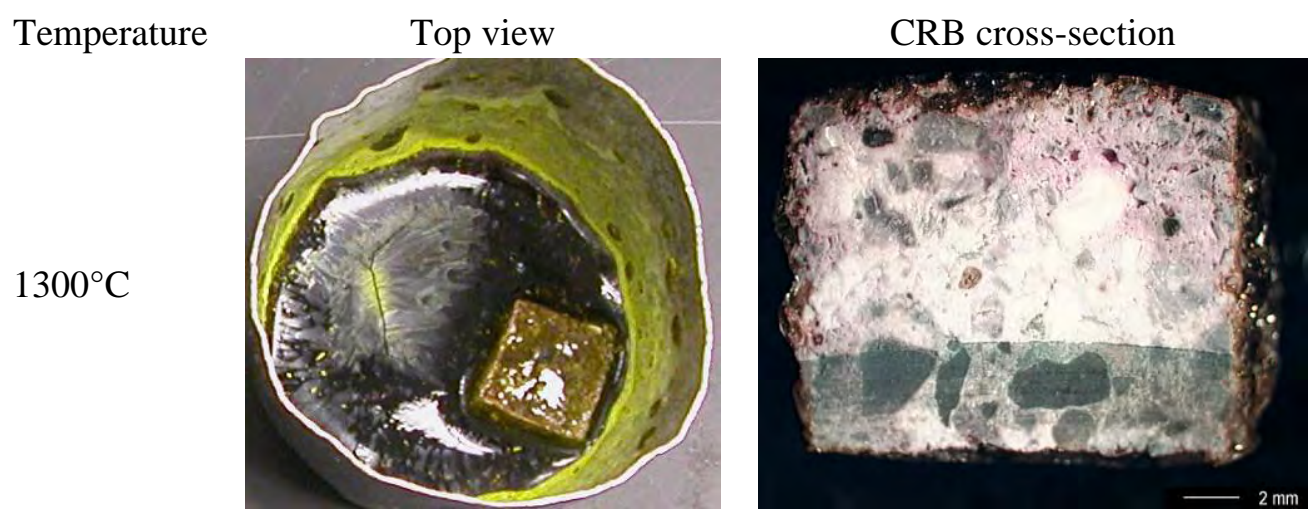

Figure E.1 (Contd.)

The feed hardened at $550^{\circ} \mathrm{C}$. Figure E.1 shows pieces of the hardened feed after the feed was removed from the crucible and put back. The feed become foamy by $800^{\circ} \mathrm{C}$. The foam extended to near the top of the crucible. Small isolated yellow spots of sulfate are visible on the top surface of the melt. The level of foam was lower at $900^{\circ} \mathrm{C}$. The amount of segregated sulfate increased and was seen also in bubbles throughout the feed. The feed was nearly vitrified at $1000^{\circ} \mathrm{C}$. The level of glass was lower after the foam collapsed, but the glass retained some bubbles. Yellow sulfate was on the glass surface, mostly in the meniscus area, but also inside bubbles.

At $1100^{\circ} \mathrm{C}$, the glass was too viscous to be poured out of the crucible. Figure E.1 shows the glass surface after an unsuccessful attempt to pour the glass. The CRB specimen broke when solid glass was removed from the crucible. No attempts to pour glass were made when the $1200^{\circ} \mathrm{C}$ and $1300^{\circ} \mathrm{C}$ melts were completed. In each of these melts, the CRB specimen drifted from the center to the wall. In the $1200^{\circ} \mathrm{C}$ melt, a few remaining bubbles contained sulfate. Sulfate was also present on the glass surface, mostly in the meniscus area on the opposite side to the CRB. The glass surface was smooth with a few small bubbles near the crucible wall in the $1300^{\circ} \mathrm{C}$ melt. Bright yellow sulfate was spread on the inside wall.

The CRB specimens were dry-cut vertically (with respect to their position in the crucible) to some distance from the surface (as is clearly visible in photographs shown in Figure E.1) and then cracked open. Figure E. 1 shows the fracture surfaces. At $550^{\circ} \mathrm{C}$, MIS penetrated about $3 \mathrm{~mm}$ into the specimen from the top (opposite to the cut, as in all other specimens except the one heated to $800^{\circ} \mathrm{C}$ ) and side walls. The penetrated area was yellow in color from the sodium chromate. At $800^{\circ} \mathrm{C}$, the CRB specimen was penetrated by the MIS from the top (where the specimen was cut) and sidewalls, except for a small area near the center. Here too, the penetrated area was yellow-colored by sodium chromate. At $1000^{\circ} \mathrm{C}$, large pores indicate that MIS reacted with the fine-grain portion of the CRB. The pore size increased at $1100^{\circ} \mathrm{C}$ and grew further in $1200^{\circ} \mathrm{C}$. At $1300^{\circ} \mathrm{C}$, the specimen was corroded by glass at all except the bottom surfaces.

The concentration distribution of $\mathrm{Na}, \mathrm{S}$, and $\mathrm{Cl}$ in selected CRB samples was measured with SEM-EDS. Figure E. 2 displays the results, which indicate that the samples were fully permeated with MIS. The large local concentration fluctuations result from the inhomogeneous structure of vibrocast, consisting of large solid zero-open porosity grains and a connected body of fine open porosity cement. The plots give the impression that the Na content in samples decreased as the temperature increased, but this is not likely because all samples see the same temperature history up to their hold temperatures. This effect may be do 
to a more complete reaction of the MIS with the CRB cement at higher temperatures that reduces the concentration of sodium at the cement particle surfaces. The SEM-EDS analysis has a limited penetration depth and may not detect the $\mathrm{Na}$ at greater depths.
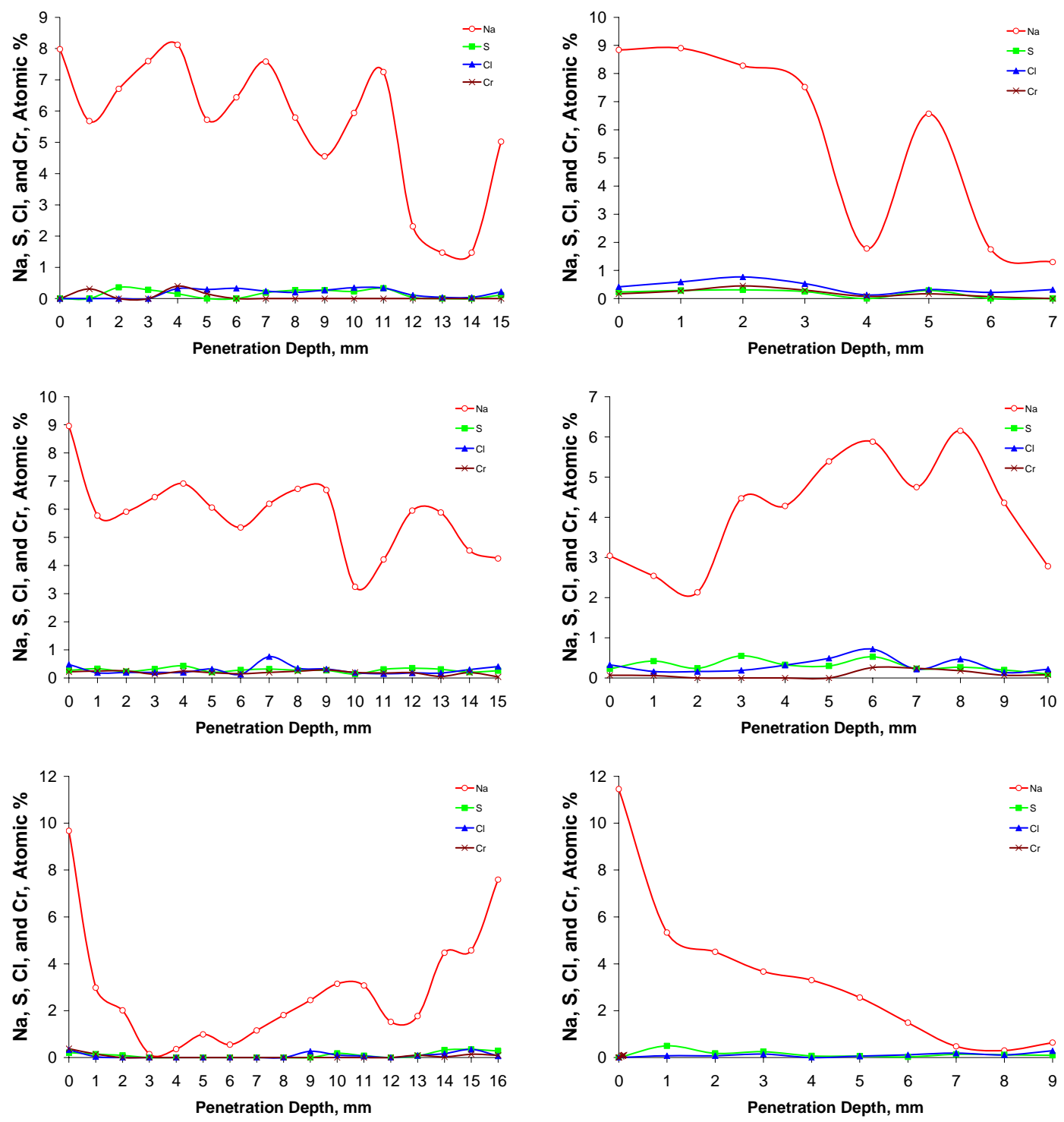

Figure E.2. CRB Specimens Exposed to Baseline Feed Heated to $550^{\circ} \mathrm{C}$ (top), $800^{\circ} \mathrm{C}$ (middle), and $1300^{\circ} \mathrm{C}$ (bottom): EDS Analysis of the Sample Surface; the Sample Scan Went Through the Middle of the Sample from the Left Side to the Right Side (left) and from the Bottom to the Top (right)

\section{E.1.2. Hot-Stage Microscopy}

Hot-stage microscopy was used in an attempt to gain a better insight into the kinetics of feed melting reactions and MIS penetration into the CRB. Figure E.3 shows pictures of feed with the CRB sample. Because of the featurelessness of low-temperature samples, pictures of feed with the CRB sample were 
not taken at temperatures below $900^{\circ} \mathrm{C}$. In both feeds, the baseline six tank composite feed and the 38B feed, the CRB samples appear fully saturated with MIS and undergo little change as the temperature rises to $1300^{\circ} \mathrm{C}$. In the presence of the CRB sample, the baseline feed melts more slowly compared to the feed alone (see Figure 5.3), possibly because the CRB sample removed some fluxes from it in the from of MIS. The 38B feed appears to melt faster but it is likely that composition fluctuations associated with taking a small sample of the dry mixed 38B feed resulted in higher quantities of salt that caused a faster meting rate. This was not the case in the baseline feed that was carefully prepared and well mixed starting with the liquid LAW simulant.

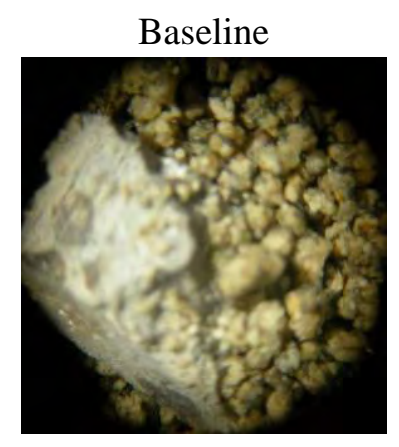

100

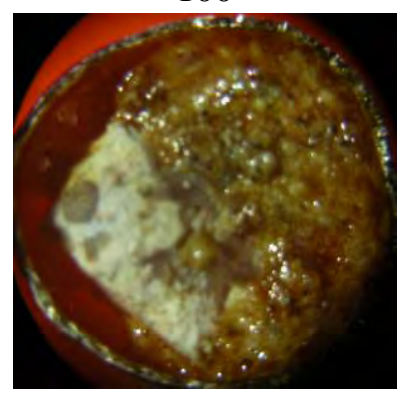

950

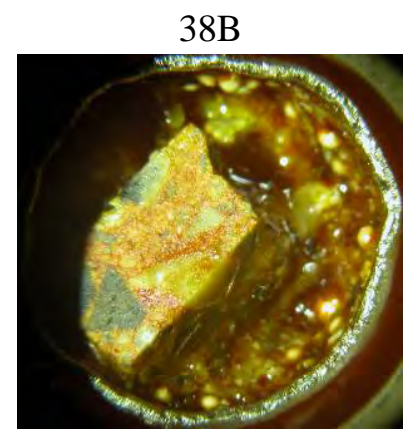

900

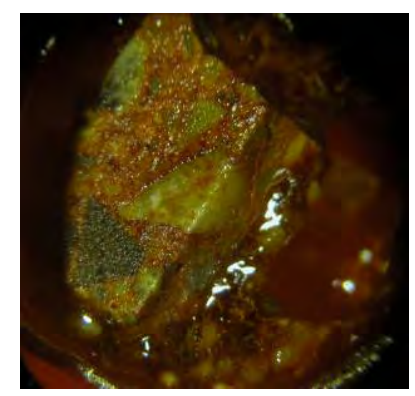

950

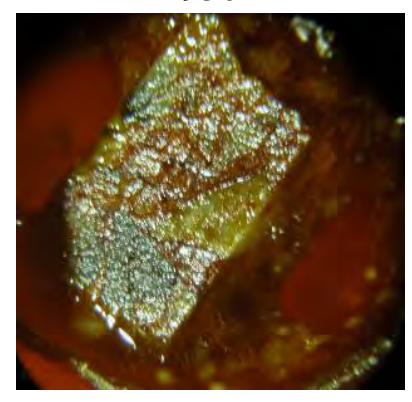

1000

Figure E.3. Hot Stage Microscopy Images of the Baseline Feed (left) and the 38-B Feed (right) with a CRB Sample; Numbers Under the Images Indicate Temperature in ${ }^{\circ} \mathrm{C}$ (the rate of heating was $5^{\circ} \mathrm{C} / \mathrm{min}$, and the crucible diameter was $6 \mathrm{~mm}$ ) 


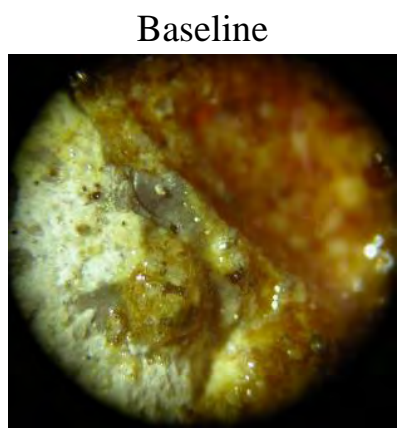

1100

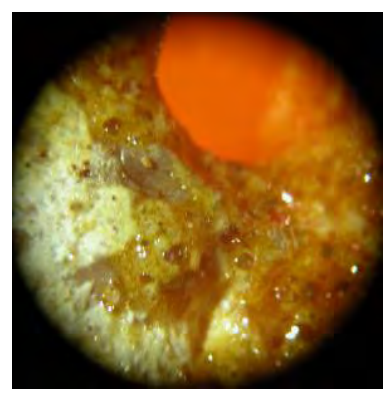

1200

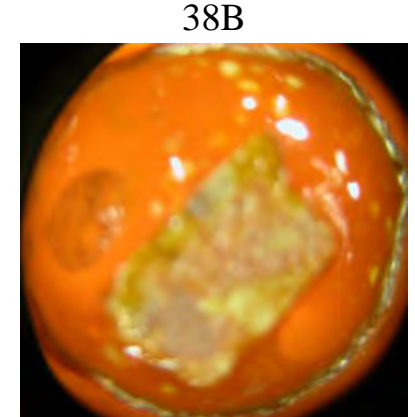

1100

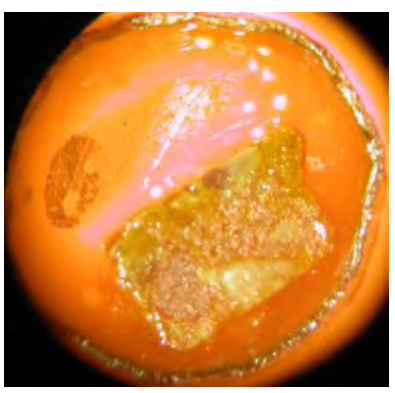

1200

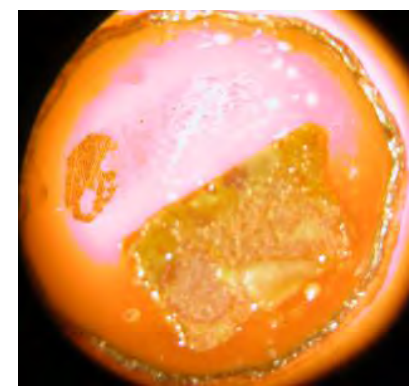

1300

38B

Baseline

Figure E.3 (Contd.)

\section{E.1.3. Effect of Time and Temperature on MIS Penetration into Small Silica Crucibles}

The preliminary experiment indicated that CRB samples are fully saturated with MIS at temperatures as low as $500^{\circ} \mathrm{C}$. To better assess the impact of time and temperature on MIS penetration, small silica crucibles were filled with the baseline feed and heated to $550^{\circ} \mathrm{C}$ and $800^{\circ} \mathrm{C}$ with no hold and 1-hour hold at each of these two temperatures.

As Figure E.4 shows, the stain of MIS penetrating to the outer wall is irregular, most likely resulting from the irregular thickness and porosity of the crucible wall. 


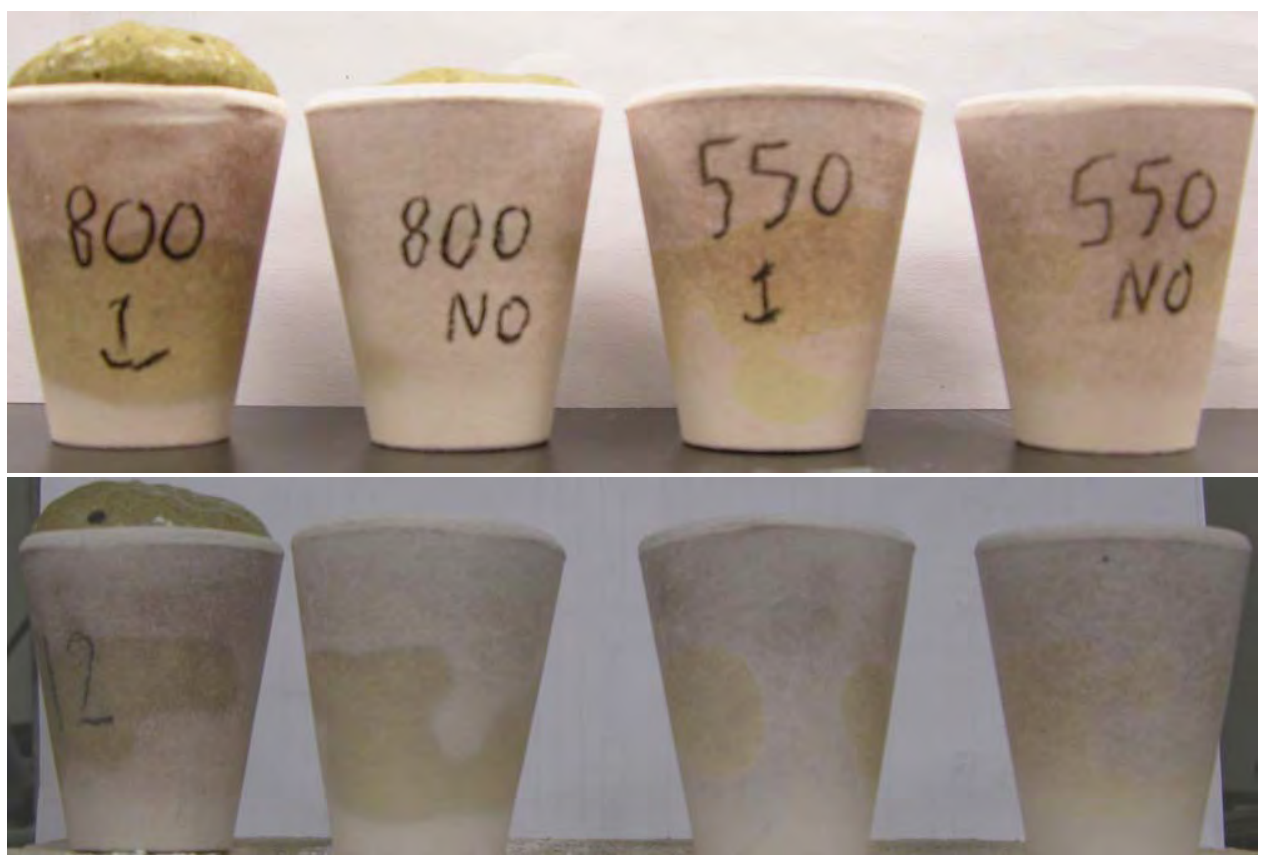

Figure E.4. Small Silica Crucible with Baseline Feed Heated at $5^{\circ} \mathrm{C}$ to the Temperatures Indicated (in ${ }^{\circ} \mathrm{C}$ ) with No Hold and 1-Hour Hold: Views from Two Opposite Sides

The MIS decomposition was virtually complete at $800^{\circ} \mathrm{C}$; extended heating at this temperature did not increase the mass loss (Table E.1). About one half of the MIS was decomposed when the temperature reached $550^{\circ} \mathrm{C}$, and the decomposition continued when this temperature was held for 1 hour.

Table E.1. LOI of Baseline Feed Heated at $5^{\circ} \mathrm{C} / \mathrm{Min}$ to 550 and $800^{\circ} \mathrm{C}$ and Held for 0 and 1 Hour

\begin{tabular}{|c|c|c|}
\hline T, $^{\circ} \mathbf{C}$ & Time, $\mathbf{h}$ & LOI \\
\hline 550 & 0 & 0.100 \\
\hline 550 & 1 & 0.136 \\
\hline 800 & 0 & 0.207 \\
\hline 800 & 1 & 0.206 \\
\hline
\end{tabular}

Based on Table E.1 data, one would expect that the extent of MIS soaking would increase during the 1-hour hold at $550^{\circ} \mathrm{C}$ and during heating from $550^{\circ} \mathrm{C}$ to $800^{\circ} \mathrm{C}$, but not during the 1-hour hold at $800^{\circ} \mathrm{C}$. However, as Figure E. 4 shows, little difference exists between the stained areas on the four crucibles displayed. This observation suggests that the soaking occurred early as the salts melted and did not substantially advance with subsequent heating. Instead, more MIS kept accumulating in the areas to which MIS penetrated at early stages.

\section{E.1.4. A Systematic Study of the Response on MIS Penetration into Silica Crucibles to Temperature}

Based on previous results, experiments were performed to determine MIS penetration into silica crucibles at temperatures as low as $350^{\circ} \mathrm{C}$. Small crucibles with $F_{0}=24.37 \mathrm{~g}$ of baseline feed were heated at $5^{\circ} \mathrm{C} / \mathrm{min}$ to $350,400,450$, and $500^{\circ} \mathrm{C}$ and then removed from the furnace and set to cool. The feed was 
removed from the crucibles. This was easy because the feed was still in a loose granular form (Figure E.5).
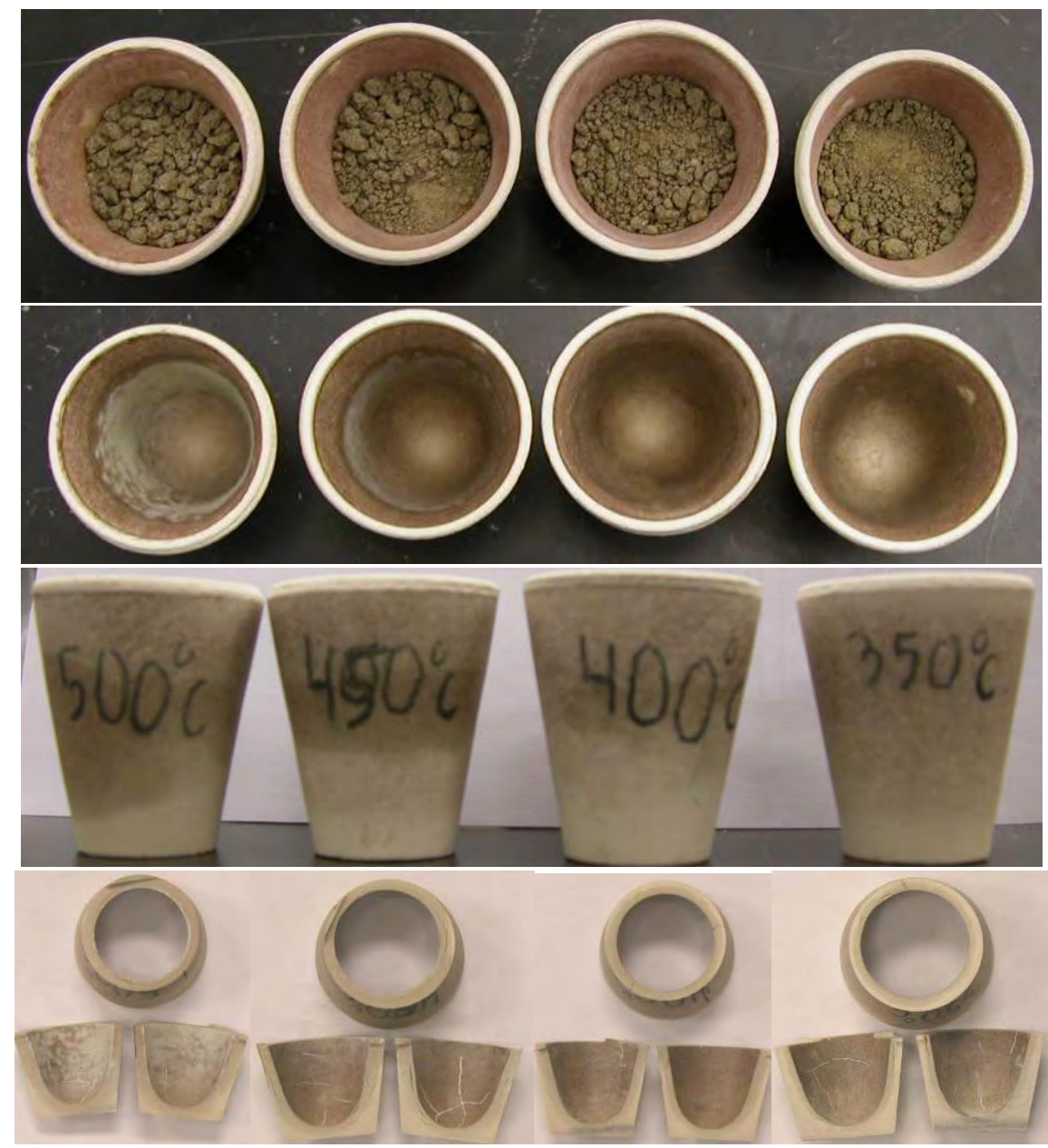

Figure E.5. Small Silica Crucibles with Baseline Feed; Heat-Treatment Temperature Decreases from Left $\left(500^{\circ} \mathrm{C}\right)$ to Right $\left(350^{\circ} \mathrm{C}\right)$; from Top to Bottom: Feed After Heat-Treatments, Empty Crucibles After Feed Removal and Heating to $1200^{\circ} \mathrm{C}$ (top view, side view, and sections)

Empty crucibles were heated at $5^{\circ} \mathrm{C} / \mathrm{min}$ to $1200^{\circ} \mathrm{C}$ to allow the MIS to react with the crucible material, so the crucibles could be wet-cut without any loss of MIS. Empty crucibles after the $1200^{\circ} \mathrm{C}$ heattreatment are shown in Figure E.5. The crucibles were sectioned to view the extent of MIS penetration into their walls. The sections are also shown in Figure E.5.

Crucibles were weighed at each stage. Table E.2 summarizes the measured values. The nomenclature used is in Table E.3. 
Table E.2. Small Crucible Experiment: Measured Data

\begin{tabular}{|r|c|c|c|c|c||}
\hline \hline $\boldsymbol{T},{ }^{\circ} \mathbf{C}$ & $\boldsymbol{C}, \mathbf{g}$ & $\boldsymbol{C}^{+\boldsymbol{F}_{\mathbf{0}}, \mathbf{g}}$ & $\boldsymbol{C +}_{\boldsymbol{1}}, \mathbf{g}$ & $\boldsymbol{C}^{+\boldsymbol{S}_{\mathbf{1}}, \mathbf{g}}$ & $\boldsymbol{C}^{+\boldsymbol{S}_{\mathbf{2}}, \mathbf{g}}$ \\
\hline 350 & 39.79 & 64.16 & 63.46 & 40.17 & 39.86 \\
\hline 400 & 40.26 & 64.62 & 63.46 & 41.14 & 40.57 \\
\hline 450 & 39.68 & 64.05 & 62.74 & 40.83 & 40.12 \\
\hline 500 & 40.31 & 64.68 & 63.01 & 41.95 & 41.00 \\
\hline \hline
\end{tabular}

Table E.3. Small Crucible Experiment: Definitions, Symbols, Values, and Units

\begin{tabular}{|r|c|c|c|c||}
\hline Quantity & Symbol & Definition & Value & Unit \\
\hline Temperature & $T$ & & & ${ }^{\circ} \mathrm{C}$ \\
\hline Crucible mass & $C$ & & & $\mathrm{~g}$ \\
\hline Feed mass & $F_{0}$ & & 24.37 & $\mathrm{~g}$ \\
\hline Feed mass after $1^{\text {st }}$ heating & $F_{1}$ & & & $\mathrm{~g}$ \\
\hline Feed loss after $1^{\text {st }}$ heating & $D_{1}$ & $F_{0}-F_{1}$ & & $\mathrm{~g}$ \\
\hline Crucible mass change after $1^{\text {st }}$ heating & $S_{1}$ & & & $\mathrm{~g}$ \\
\hline MIS in crucible wall after 2 ${ }^{\text {nd }}$ heating & $S_{2}$ & & & $\mathrm{~g}$ \\
\hline Migrated MIS loss during 2 ${ }^{\text {nd }}$ heating & $L_{2}$ & $S_{1}-S_{2}$ & & $\mathrm{~g}$ \\
\hline LAW mass fraction in feed & $m_{0}$ & & 0.354 & \\
\hline Theoretical loss, fraction of LAW & $f$ & & 0.546 & \\
\hline Dry LAW in crucible & $M_{0}$ & $F_{0} m_{0}$ & 8.64 & $\mathrm{~g}$ \\
\hline Theoretical loss & $L$ & $f M_{0}$ & 4.72 & $\mathrm{~g}$ \\
\hline Migrated layer thickness & $Z$ & & & $\mathrm{~mm}$ \\
\hline MIS fraction migrated to crucible & $S_{1}$ & $S_{1} / M_{0}$ & & \\
\hline Migrated MIS loss fraction after 2d heating & $f_{2}$ & $L_{2} / S_{1}$ & & \\
\hline \hline
\end{tabular}

The feed in each crucible contained $M_{0}=F_{0} m_{0}=24.37 \times 0.3544=8.64 \mathrm{~g}$ of dry LAW (the mass fraction of dry LAW in the feed, $m_{0}$, is listed in Table E.3). The difference in the mass of the crucible with feed before and after the first heat-treatment corresponds to the feed lost by gas-evolving reactions $\left(D_{1}=F_{0}-\right.$ $F_{1}$ ). For example, the heat-treatment to $500^{\circ} \mathrm{C}$ resulted in the mass loss of $D_{1}=1.67 \mathrm{~g}$. Table E. 4 summarizes this and other calculated values.

Table E.4. Small Crucible Experiment: Calculated Data

\begin{tabular}{||r|c|c|c|c|c|c|c||}
\hline $\mathbf{T}^{\circ}{ }^{\circ} \mathbf{C}$ & $\boldsymbol{F}_{\mathbf{1}}, \mathbf{g}$ & $\boldsymbol{D}_{\mathbf{1}}, \mathbf{g}$ & $\boldsymbol{S}_{\mathbf{1}}, \mathbf{g}$ & $\boldsymbol{S}_{\mathbf{2}}, \mathbf{g}$ & $\boldsymbol{L}_{\mathbf{2}}, \mathbf{g}$ & $\boldsymbol{s}_{\mathbf{1}}$ & $\boldsymbol{f}_{\mathbf{2}}$ \\
\hline 350 & 23.67 & 0.70 & 0.38 & 0.07 & 0.31 & 0.044 & 0.816 \\
\hline 400 & 23.20 & 1.16 & 0.88 & 0.31 & 0.57 & 0.102 & 0.648 \\
\hline 450 & 23.06 & 1.31 & 1.15 & 0.44 & 0.71 & 0.133 & 0.617 \\
\hline 500 & 22.70 & 1.67 & 1.64 & 0.69 & 0.95 & 0.190 & 0.579 \\
\hline
\end{tabular}

Assuming that the mass loss during the first heat-treatment is entirely due to LAW components (no gases were evolved from the soil), we can estimate the fraction of lost MIS (i.e., melted LAW) during heating to $500^{\circ} \mathrm{C}$ as $D_{1} / M_{0}=1.67 / 8.64=0.193$. Note that $D_{1}$ consists of two contributions: the MIS that left the feed into the crucible wall and the gas that escaped from the feed. 
Table E.4 lists the amounts of MIS migrated into crucible after heating, $S_{1}$, calculated as the difference between the mass of the crucible with the feed removed and the original crucible. After heating to $500^{\circ} \mathrm{C}$, $S_{1}=1.64 \mathrm{~g}$.

The first molten salt that enters the crucible pores is a low-temperature eutectic melt while the rest of the MIS is still in a solid form. Table E.5 lists gas-releasing components of MIS. Also, the gas-evolving reaction is influenced by the nature of solid present (soil in the feed and silica in the crucible wall) and the partial pressure of the gas evolved. The partial pressure can be different, possibly higher, in the porous material. Finally, a chromatographic absorption-desorption effect can operate that segregates MIS components as they penetrate into the porous material. If the MIS that was migrated during the first heating at lower temperatures $\left(350^{\circ} \mathrm{C}\right)$ was mainly composed of components with a high content of gas, then most of the MIS found after the second heating would be lost as the gas phase.

Table E.5. Gas Release from MIS Components (normalized to $800^{\circ} \mathrm{C}$ )

\begin{tabular}{||l|c|c|c|c||}
\hline & Mass Fraction & $\begin{array}{c}\text { Gas release at 800 } \\
\text { L/kg } \mathbf{C I S}\end{array}$ & $\begin{array}{c}\text { Gas/Solid } \\
\text { L/kg Solid }\end{array}$ & $\begin{array}{c}\text { M.P. } \\
{ }^{\circ} \mathbf{C}\end{array}$ \\
\hline $\mathrm{NaNO}_{3}$ & 0.5163 & 614.5 & 3.26 & 307 \\
\hline $\mathrm{Al}_{\left(\mathrm{NO}_{3}\right)_{3} \cdot 9 \mathrm{H}_{2} \mathrm{O}}$ & 0.0623 & 194.9 & 23.02 & 73 \\
\hline $\mathrm{Na}_{3} \mathrm{PO}_{4} \cdot 12 \mathrm{H}_{2} \mathrm{O}$ & 0.0492 & 125.7 & 5.92 & 75 \\
\hline $\mathrm{NaC}_{2} \mathrm{H}_{3} \mathrm{O}_{2}$ & 0.0284 & 98.1 & 9.14 & 382 \\
\hline $\mathrm{NaOH}$ & 0.0779 & 78.8 & 1.31 & 323 \\
\hline $\mathrm{Na}_{2} \mathrm{CO}_{3}$ & 0.1326 & 50.6 & 0.65 & 858 \\
\hline $\mathrm{NaNO}_{2}$ & 0.0770 & 45.2 & 1.31 & 271 \\
\hline $\mathrm{Na}_{2} \mathrm{C}_{2} \mathrm{O}_{4}$ & 0.0042 & 5.1 & 2.61 & 250 \\
\hline $\mathrm{KNO}_{3}$ & 0.0033 & 3.3 & 2.15 & 337 \\
\hline $\mathrm{Total}$ & & 1216.1 & & \\
\hline
\end{tabular}

The amount of MIS found in crucible after second heating, $S_{2}$, was calculated as the difference between the mass of the crucible after second heating and the original crucible. For crucible first heated to $500^{\circ} \mathrm{C}$, $S_{2}=0.69 \mathrm{~g}$. The amount of MIS lost to gas from MIS migrated to crucible, $L_{2}$, was calculated as the difference between the mass of the crucible after second and first heating. For crucible first heated to $500^{\circ} \mathrm{C}, L_{2}=0.95 \mathrm{~g}$. Assuming that no migrated MIS was lost to gas during the first heating, the total loss occurred during the second heating. The fraction of the original MIS that migrated to crucible then was $s_{1}$ $=S_{1} / M_{0}$ and the lost fraction of migrated MIS to gas was $f_{2}=L_{2} / S_{1}$. Hence, $s_{1}=1.64 / 8.64=0.190$ and $f_{2}=$ $0.95 / 1.64=0.579$ for the $500^{\circ} \mathrm{C}$ experiment (see $f_{2}$ in Table E.4). Since $f_{2}$ is close to the theoretical values, $f=0.546$ (Table E.3), we may conclude that the loss of migrated MIS to gas during the first heating was negligible.

Based on these calculations, up to 19 mass\% of the original MIS migrated to the crucible walls. Provided that no gas was evolved from the MIS in the crucible walls during first heating, the gas content in the MIS that migrated at $350^{\circ} \mathrm{C}$ was as high as 82 mass\%. However, these calculations are subjected to error because the crucible LOI was not taken into account.

According to Figure E.6, both estimates of total losses are higher than the theoretical value (represented by the horizontal straight line, the LAW LOI, $f=0.546)$ and decrease as the temperature decreases. This 
Figure E.6 demonstrates another interesting aspect. The left diagram shows the mass of MIS that entered the crucible wall per unit interface area $\left(S_{1} / A\right)$. Based on crucible dimensions (Table E.6 and Figure D.8) and $2 / 3$ of filling height, $A=10.3 \mathrm{~cm}^{2}$. Table E.4 lists the values of $S_{1}$. The amount of MIS in the porous wall increased as the temperature increased with the rate of $8 \mathrm{~g} /\left(\mathrm{m}^{2} \cdot \mathrm{K}\right)$. Since the temperature increased at $5^{\circ} \mathrm{C} / \mathrm{min}$, the mass per unit interface area increased at $40 \mathrm{~g} /\left(\mathrm{m}^{2} \cdot \mathrm{min}\right)$.
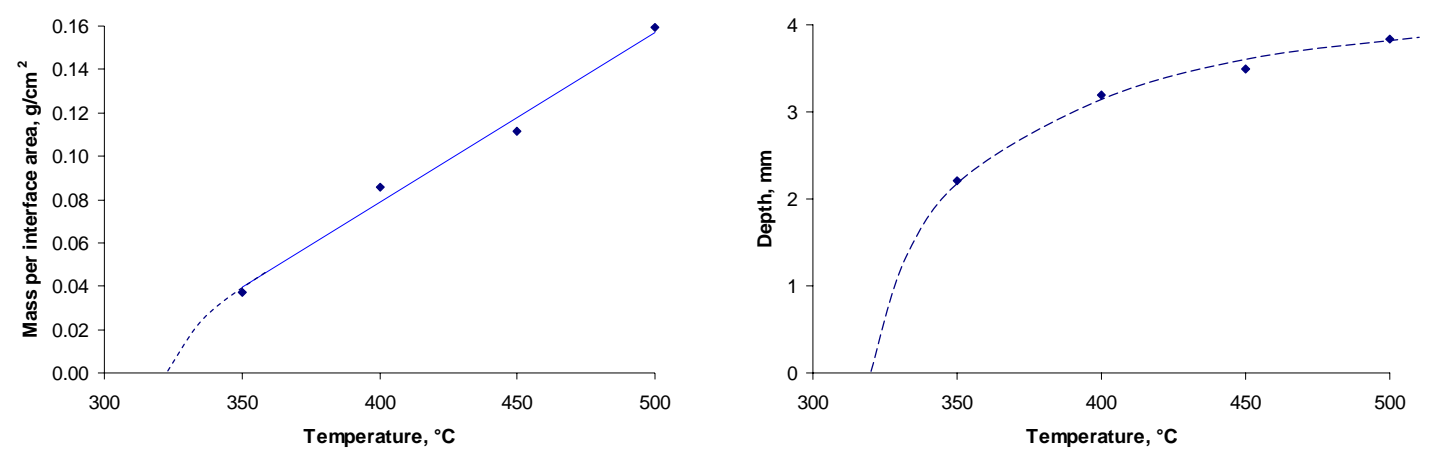

Figure E.6. Mass (left) and Penetration Depth (right) of MIS Migrated into Crucible Wall Versus Temperature

\section{Table E.6. Small Crucible Dimensions}

\begin{tabular}{||l|r|l||}
\hline Bottom inner diameter & 16 & $\mathrm{~mm}$ \\
\hline Inner height & 48 & $\mathrm{~mm}$ \\
\hline Bottom inner surface area & 201 & $\mathrm{~mm}^{2}$ \\
\hline Side wall feed contact surface area & 829 & $\mathrm{~mm}^{2}$ \\
\hline Feed/crucible interface area & 1030 & $\mathrm{~mm}^{2}$ \\
\hline \hline
\end{tabular}

Table E.7 summarizes the measured values of penetration thickness $(Z)$. The right diagram in Figure E.6 shows that the average $Z$ increased with increasing temperature, but with a decreasing rate. Hence, the amount of migrated MIS per the volume of the refractory wall, $\rho=S_{1} /(A Z)$, was not constant, but increased with temperature, as shown in Figure E.7. The amount of MIS pushed out of the wall by evolving gases during the second heat-treatment also increased as the temperature increased—see the top views of crucibles shown in Figure E.5.

Table E.7. MIS Penetration Depth ( $Z$, in $\mathrm{mm})$, Total Volume ( $V$, $\mathrm{mL})$, and Density $(\rho, \mathrm{g} / \mathrm{mL})$

\begin{tabular}{||c|l|l|l|c|c|c|c||}
\hline \hline & \multicolumn{2}{|c|}{ Wall } & \multicolumn{2}{c|}{ Bottom } & \multirow{2}{*}{${ }^{(a)}$} & $\boldsymbol{V}$ & $\boldsymbol{\rho}$ \\
\cline { 1 - 5 } $\boldsymbol{T},{ }^{\circ} \mathbf{C}$ & $\boldsymbol{Z}_{\min }$ & $\boldsymbol{Z}_{\max }$ & $\boldsymbol{Z}_{\min }$ & $\boldsymbol{Z}_{\max }$ & $\boldsymbol{Z}_{\text {ave }}$ & & $\boldsymbol{\rho}$ \\
\hline 350 & 2 & 3 & 0 & 2 & 2.2 & 2.27 & 0.136 \\
\hline 400 & 2.5 & 4 & 3 & 3 & 3.2 & 3.30 & 0.173 \\
\hline 450 & 3 & 4 & 3 & 4 & 3.5 & 3.61 & 0.197 \\
\hline 500 & 3 & 4.5 & 3.4 & 5 & 3.8 & 3.95 & 0.240 \\
\hline (a) Weighted average based on wall and bottom surface areas. \\
\hline
\end{tabular}




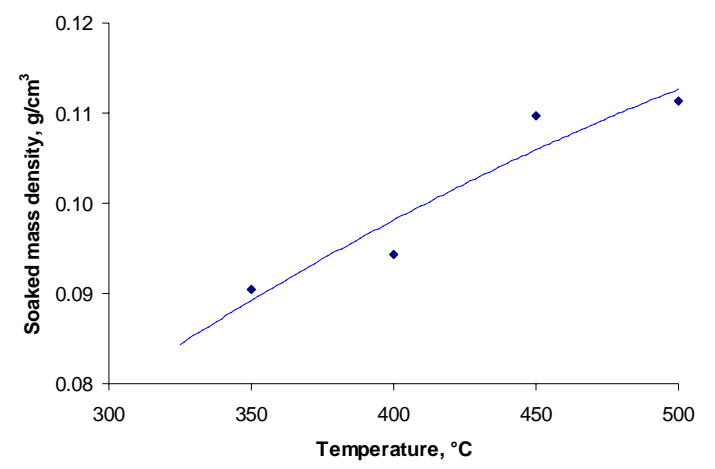

Figure E.7. Mass Density of MIS Migrated into Crucible Wall Versus Temperature

As the wicking experiment (Kim et. al. 2006) showed, MIS climbs vertically upward (against the force of gravity) in the CRB material according to the formula $t=a h^{2}$, where $t$ is the time, $h$ the distance, and $a=$ $26.5 \mathrm{~s} / \mathrm{mm}^{2}$ for $450^{\circ} \mathrm{C}$ data. Thus, $450^{\circ} \mathrm{C}$ MIS reaches $12 \mathrm{~mm}$ vertically up in 1 hour. During the 1 -hour hold time plus additional exposure during the $5^{\circ} \mathrm{C} / \mathrm{min}$ heating to the set temperature, a vibrocast wall would be completely saturated with MIS.

As Figure E.6 indicates, MIS penetrated only $4 \mathrm{~mm}$ deep into the silica crucible at $500^{\circ} \mathrm{C}$. This is less than expected based on the wicking experiment. One reason is that MIS may penetrate more slowly to sintered silica than to vibrocast material. The other difference is that penetration from feed is likely to be slower than from the $100 \%$ molten MIS. The penetration of MIS naturally slows down as a layer of feed adjacent to the wall is depleted of the free-flowing MIS. However, observed massive penetration of MIS through the joints and cracks in FS melter experiments casts doubt on this hypothesis. It appears that an MIS migration through the feed may be as easy as that of groundwater through saturated sand.

The penetration of MIS during the period of increasing temperature also affects the process. As Table E.5 indicates, components with low melting temperature are most likely to become major components of the early MIS and release large volumes of gas. The extensive release of gas may hinder further penetration of MIS into the wall. Sodium oxide that remains when gas is liberated probably reacts with fine fractions of MIS (refractory cement) creating sodium aluminosilicates. This opens more space into which additional MIS can flow instead of penetrating deeper into the CRB.

\section{E.2. References}

Kim D-S, LM Bagaasen, JV Crum, A Fluegel, A Gallegos, B. Martinez, J Matyáš, PA Meyer, DR Paulsen, BJ Riley, MJ Schweiger, CW Stewart. RG Swoboda, JD Yeager. 2006. Investigation of Tc Migration Mechanism During Bulk Vitrification Process Using Re Surrogate. PNNL-16267, Pacific Northwest National Laboratory, Richland, WA. 


\section{Appendix F}

\section{MIS Penetration into Porous Refractory from Feeds Containing Fine-Grain Materials}




\section{Appendix F: MIS Penetration into Porous Refractory from Feeds Containing Fine-Grain Materials}

Table F.1 shows examples of feed compositions, i.e., the baseline feed and feeds with fine-grain materials. Table F.2 lists the corresponding glass compositions. The batches were formulated to keep the $\mathrm{Na}_{2} \mathrm{O}$ content in glass at 20 mass\%.

Table F.1. Fractions of Glass Originating from Feed Ingredients

\begin{tabular}{||l|c|c|c|c|c||}
\hline & Baseline & $\begin{array}{c}\text { Diatom. } \\
\text { Earth }\end{array}$ & Kaolin & Bentonite & Fly ash \\
\hline six-tank simulant & 0.2023 & 0.2039 & 0.2036 & 0.2028 & 0.2029 \\
\hline $\mathrm{HRTS05}$ & 0.6777 & 0.5761 & 0.6364 & 0.6372 & 0.6371 \\
\hline $\mathrm{ZrO}_{2}$ & 0.0700 & 0.0700 & 0.0700 & 0.0700 & 0.0700 \\
\hline $\mathrm{B}_{2} \mathrm{O}_{3}$ & 0.0500 & 0.0500 & 0.0500 & 0.0500 & 0.0500 \\
\hline $\mathrm{SiO}_{2}$ & & & & & \\
\hline Diatomaceous earth & & 0.1000 & & & \\
\hline Kaolin & & & 0.0400 & & \\
\hline Montmorillonite & & & & 0.0400 & \\
\hline Fly ash & & & & & 0.0400 \\
\hline
\end{tabular}

Table F.2. Compositions (in mass fractions) of Glasses with Batch Ingredients Listed in Table F.1

\begin{tabular}{||l|c|c|c|c|c||}
\hline & Baseline & $\begin{array}{c}\text { Diatom. } \\
\text { Earth }\end{array}$ & Kaolin & Bentonite & Fly ash \\
\hline $\mathrm{Al}_{2} \mathrm{O}_{3}$ & 0.0878 & 0.0829 & 0.1010 & 0.0943 & 0.0909 \\
\hline $\mathrm{B}_{2} \mathrm{O}_{3}$ & 0.0500 & 0.0500 & 0.0500 & 0.0500 & 0.0500 \\
\hline $\mathrm{BaO}$ & 0.0005 & 0.0005 & 0.0005 & 0.0005 & 0.0005 \\
\hline $\mathrm{CaO}$ & 0.0295 & 0.0260 & 0.0277 & 0.0284 & 0.0375 \\
\hline $\mathrm{Cl}$ & 0.0018 & 0.0018 & 0.0018 & 0.0018 & 0.0018 \\
\hline $\mathrm{Cr}_{2} \mathrm{O}_{3}$ & 0.0010 & 0.0010 & 0.0010 & 0.0010 & 0.0010 \\
\hline $\mathrm{F}$ & 0.0007 & 0.0007 & 0.0007 & 0.0007 & 0.0007 \\
\hline $\mathrm{Fe}_{2} \mathrm{O}_{3}$ & 0.0462 & 0.0430 & 0.0434 & 0.0435 & 0.0461 \\
\hline $\mathrm{K}_{2} \mathrm{O}$ & 0.0222 & 0.0190 & 0.0209 & 0.0209 & 0.0212 \\
\hline $\mathrm{MgO}$ & 0.0144 & 0.0128 & 0.0136 & 0.0136 & 0.0156 \\
\hline $\mathrm{MnO} 0.0007$ & 0.0006 & 0.0007 & 0.0007 & 0.0007 \\
\hline $\mathrm{Na}_{2} \mathrm{O}$ & 0.2000 & 0.2000 & 0.2000 & 0.2000 & 0.2000 \\
\hline $\mathrm{P}_{2} \mathrm{O}_{5}$ & 0.0057 & 0.0055 & 0.0056 & 0.0056 & 0.0061 \\
\hline $\mathrm{SiO}_{2}$ & 0.4532 & 0.4712 & 0.4473 & 0.4533 & 0.4407 \\
\hline $\mathrm{SO}_{3}$ & 0.0085 & 0.0085 & 0.0085 & 0.0085 & 0.0093 \\
\hline $\mathrm{SrO}^{2}$ & 0.0003 & 0.0002 & 0.0003 & 0.0003 & 0.0003 \\
\hline $\mathrm{TiO}_{2}$ & 0.0074 & 0.0063 & 0.0069 & 0.0069 & 0.0075 \\
\hline $\mathrm{ZrO}_{2}$ & 0.0700 & 0.0700 & 0.0700 & 0.0700 & 0.0700 \\
\hline \hline
\end{tabular}


The LOI of fine-grain materials and soil was determined by heating 10-g samples in Pt crucibles at $1200^{\circ} \mathrm{C}$ for $30 \mathrm{~min}$. Table F. 3 lists the results. Figure F.1 shows these materials as-received and after the heat-treatment. After the heat-treatment, the soil was sintered into a black porous mass, diatomaceous earth was shrank away from crucible walls into a hard white cake, bentonite was sintered and cracked, kaolin was shrank and cracked, but did not sinter, and fly ash became a porous brown glass.

\section{Table F.3. LOI of Hanford Soil and Fine-Grain Materials}

\begin{tabular}{|l|c|}
\hline Material & LOI \\
\hline Soil & 0.020 \\
\hline Diatomaceous Earth & 0.003 \\
\hline Bentonite & 0.099 \\
\hline Kaolin & 0.141 \\
\hline Fly Ash & 0.095 \\
\hline
\end{tabular}

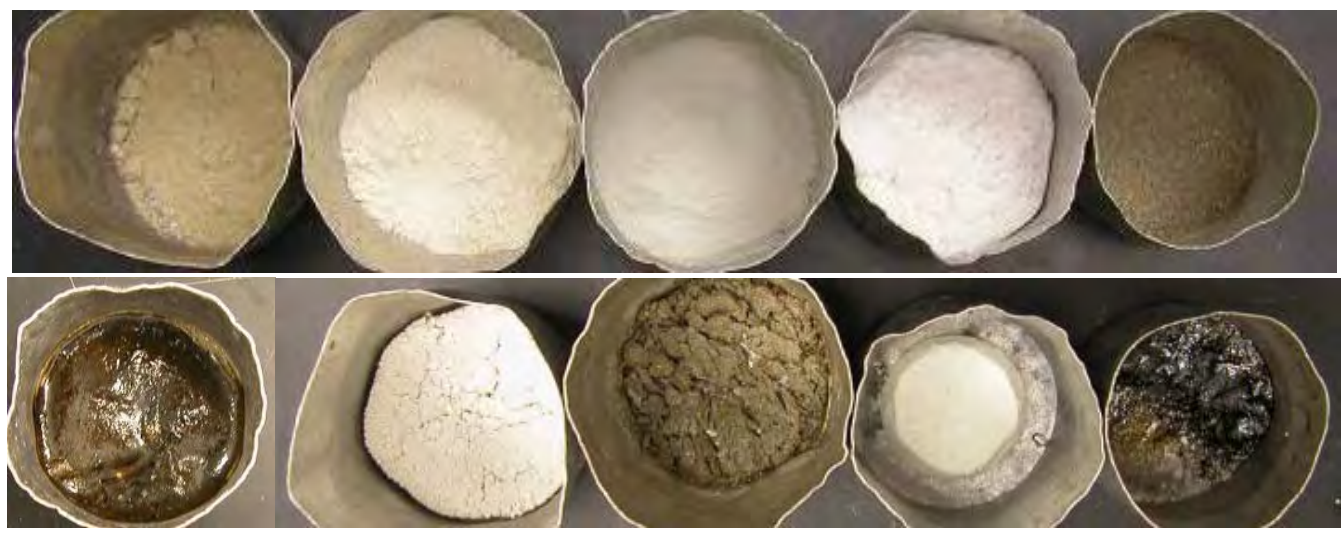

Figure F.1. From the Left to Right: Fly Ash, Kaolin, Bentonite, Diatomaceous Earth, and Hanford Soil As-Received (above) and After $30 \mathrm{~min}$ at $1200^{\circ} \mathrm{C}$ (below)

Table F.4 lists the batch sheets for glass with fine-grain materials. The batch sheets were calculated using data listed in Tables F.1 through F.3.

To prepare feeds with fine-grain materials, the Hanford soil, $\mathrm{ZrO}_{2}, \mathrm{~B}_{2} \mathrm{O}_{3}$, and $\mathrm{Na}_{2} \mathrm{CrO}_{4}$ were premixed in the agate mill for $1 \mathrm{~min}$. The liquid LAW simulant was added to a metallic beaker on a hotplate. The fine-grain ingredient was added to the simulant and stirred into a homogenous suspension. The suspension was heated and stirred until all free water was evaporated. The dry suspension was mixed with the premixed additives in the tungsten carbide mill for 1 min.

Preliminary experiments with feed containing diatomaceous earth and CRB specimens were performed in Pt crucibles as shown in Figure F.2. Note extensive yellow phase segregation at $1000^{\circ} \mathrm{C}$. All CRB specimens were fully penetrated with MIS that reacted with refractory cement to form into a smooth glass-ceramic phase. As a result, pores in the CRB swelled. As Figure F.3 shows, SEM detected Na within the samples at concentrations comparable to and perhaps slightly lower than in the case of the baseline feed (see Figure E.2). 
Table F.4. Batch Sheets, in g, for 20-g Glass

\begin{tabular}{|r|r|r|r|r|r||}
\hline & Baseline & D. Earth & Kaolin & Bentonite & Fly Ash \\
\hline six-tank dry simulant & 8.915 & 8.985 & 8.972 & 8.937 & 8.941 \\
\hline six-tank liquid simulant & 28.865 & 29.093 & 29.050 & 28.936 & 28.950 \\
\hline $\mathrm{HRTS05}$ & 13.831 & 11.757 & 12.988 & 13.004 & 13.002 \\
\hline $\mathrm{ZrO}_{2}$ & 1.400 & 1.400 & 1.400 & 1.400 & 1.400 \\
\hline $\mathrm{B}_{2} \mathrm{O}_{3}$ & 1.000 & 1.000 & 1.000 & 1.000 & 1.000 \\
\hline Silica & & & & & \\
\hline Kaolin & & & 0.931 & & \\
\hline Diatomaceous earth & & 2.006 & & & \\
\hline Montmorillonite & & & & 0.888 & \\
\hline Fly ash & & & & & 0.884 \\
\hline Sum (wet feed) & 45.095 & 45.256 & 45.369 & 45.228 & 45.236 \\
\hline
\end{tabular}
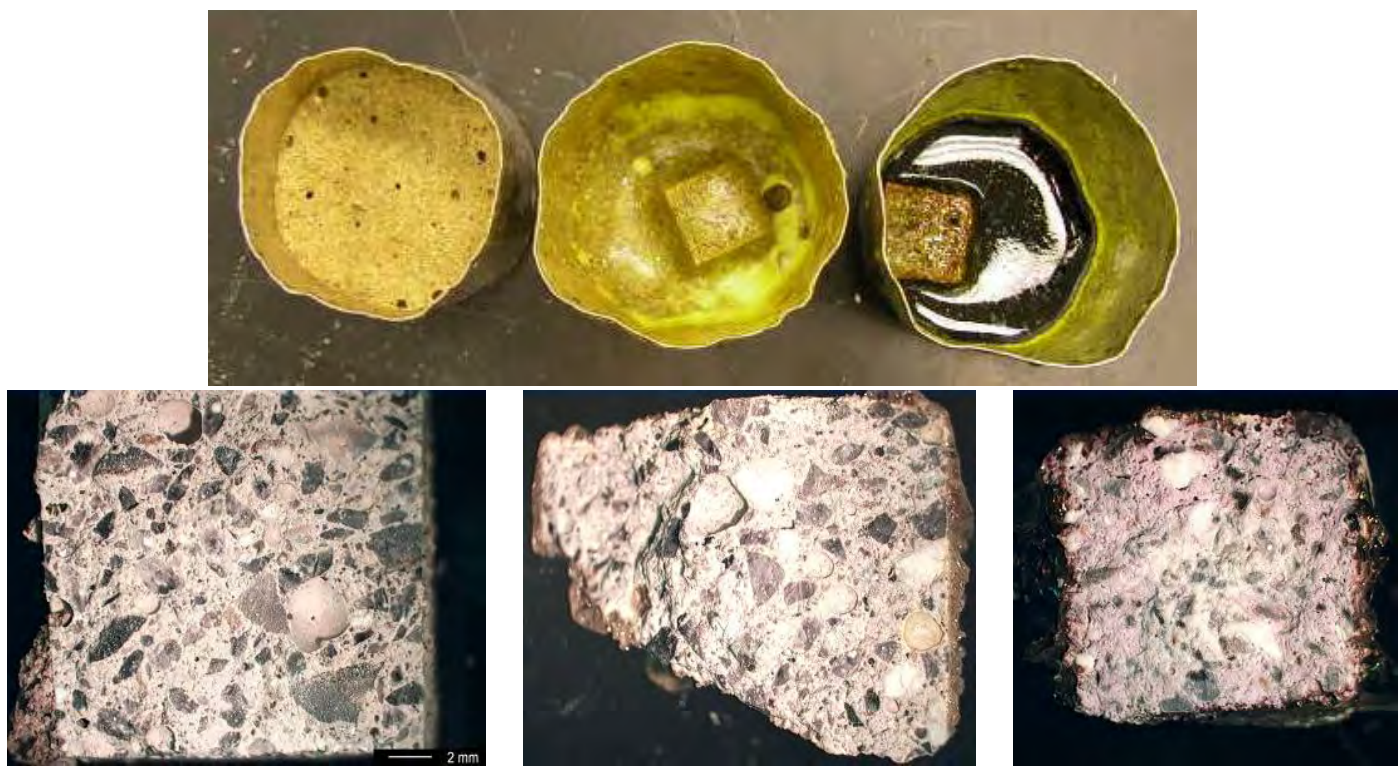

Figure F.2. Melts from Feed with Diatomaceous Earth and the Appearance of Sectioned Specimens; Left to Right: $800^{\circ} \mathrm{C}, 1000^{\circ} \mathrm{C}$, and $1300^{\circ} \mathrm{C}$ 

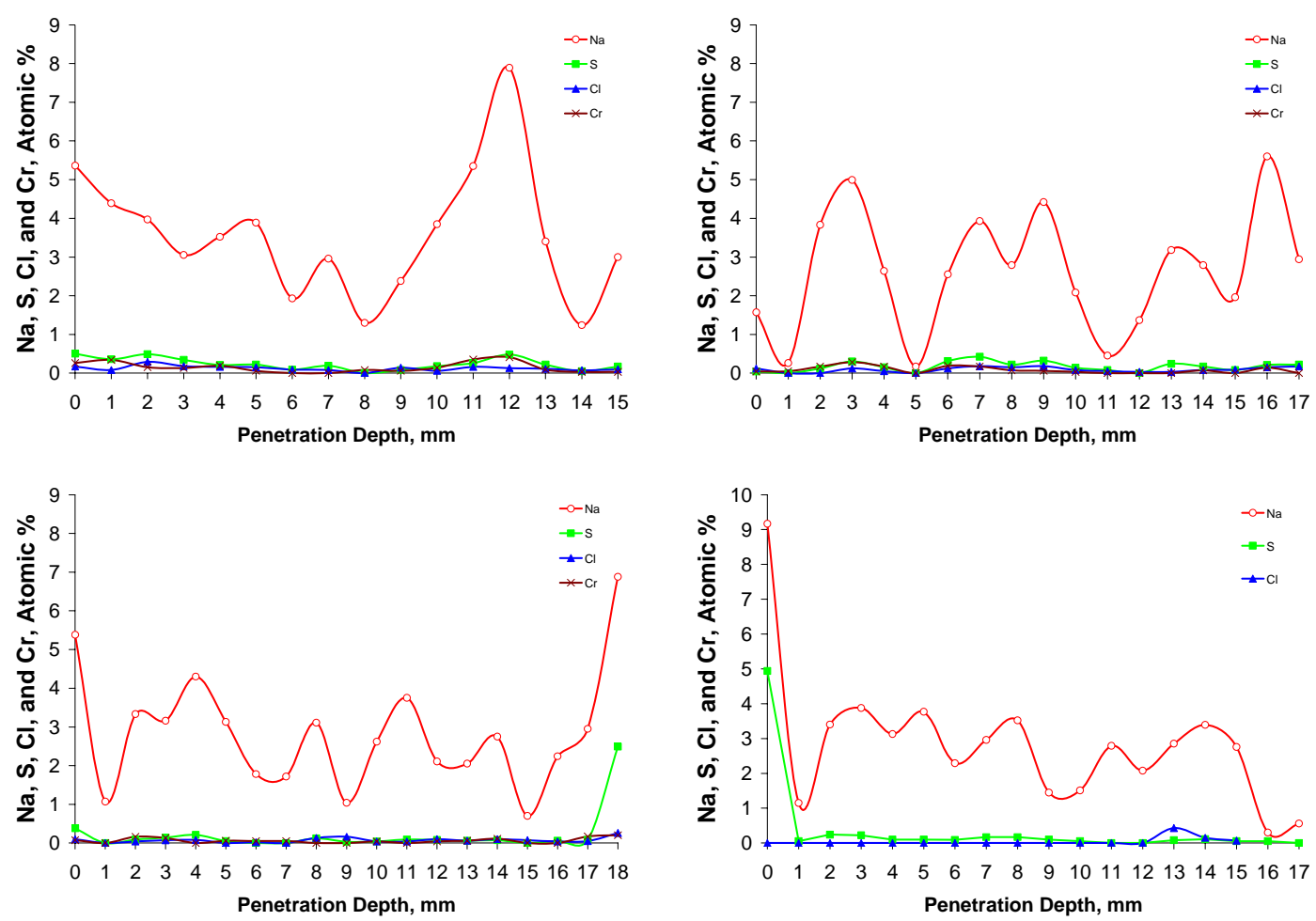

Figure F.3. CRB Specimens Exposed to Feed with Diatomaceous Earth Heated to $800^{\circ} \mathrm{C}$ (top) and $1300^{\circ} \mathrm{C}$ (bottom): EDS Analysis of the Sample Surface. The Sample Scan Went Through the Middle of the Sample from the Left Side to the Right Side (left) and from the Bottom to the Top (right).

MIS penetration tests for all fine-grain additives were performed in silica crucibles. The feed was heated in small and medium silica crucibles (with a CRB rod) as described above. In the case of the ground additives (soil with $\mathrm{ZrO}_{2}$ and $\mathrm{B}_{2} \mathrm{O}_{3}$ ), the feed preparation was the same as that used for the baseline feed experiment. Figure F.4 displays the results. Melts with ground additives are also shown for comparison (these tests are reported in Appendix H). 


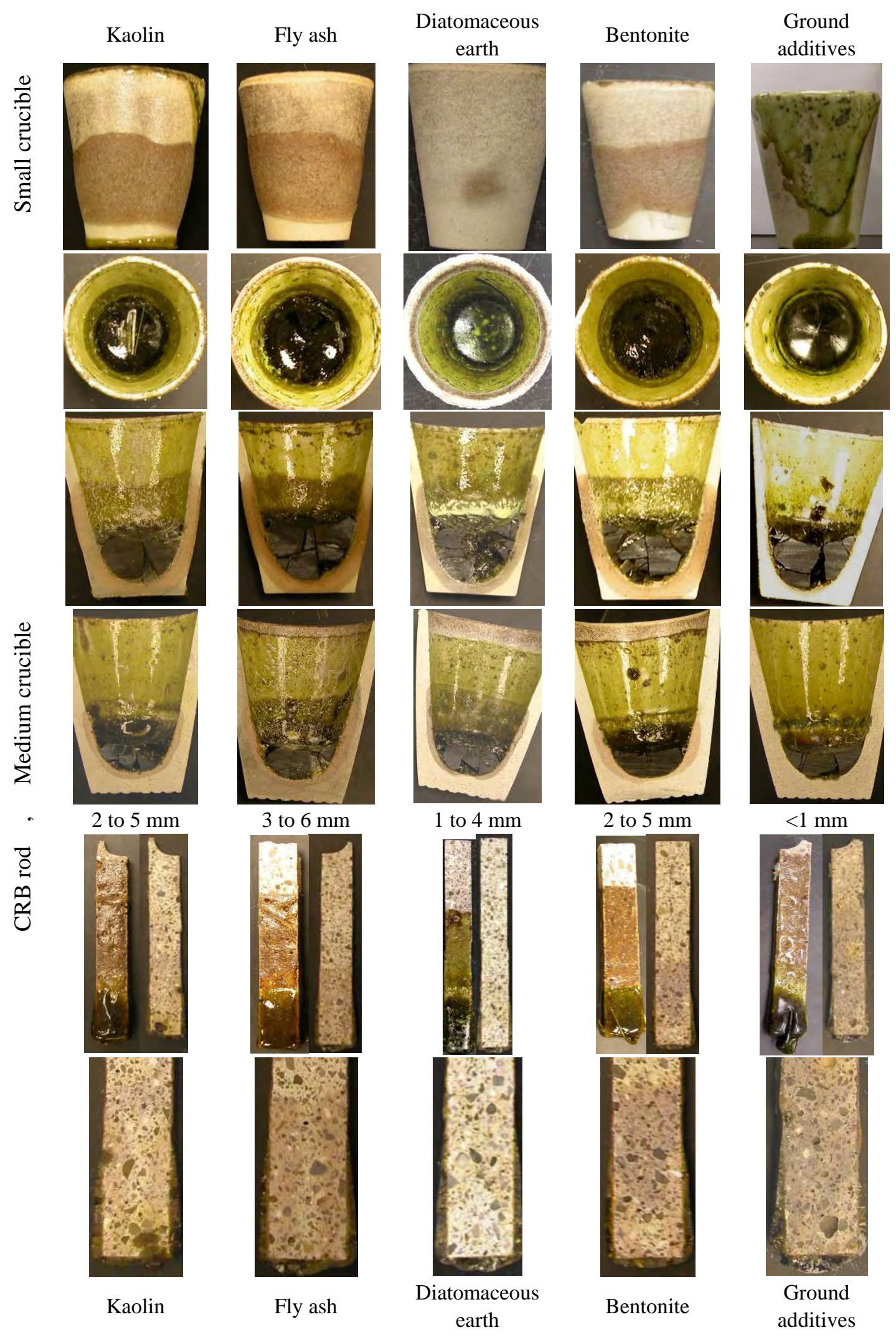

Figure F.4. Summary of MIS Penetration Tests 
The observations made can be summarized as follows.

Corrosion: A white transition layer, possibly a sodium silicate, formed on the glass-contact area.

Crucible walls were slightly corroded by the melt. Melt from ground soil made a distinct corrosion groove in the meniscus area.

Foaming: Feed with kaolin and especially feed with ground soil have foamed. Melt from ground soil overflowed the small crucible.

Sulfate segregation: Sulfate segregated from all melts. It formed a ring in the meniscus area in all melts except kaolin. In the melt with diatomaceous earth, patches of segregated sulfate floated on the melt surface and also outside the meniscus area.

MIS penetration into crucibles: Nearly zero penetration occurred in feed containing ground soil.

Diatomaceous earth substantially decreased MIS penetration. Kaolin and bentonite slightly decreased MIS penetration, and fly ash brought little improvement. Areas of sidewalls of small crucibles that were penetrated with MIS from feeds containing fly ash, kaolin, and bentonite deformed and slightly swelled or bulged.

MIS penetration into CRB rods: Generally, CRB rods turned pink and developed large pores in the submerged area. In the case of feed with kaolin, the CRB rod did not turn pink in the area around the axis. The rod slightly swelled in the feed with bentonite. Less MIS penetrated into the CRB rod from the feed with diatomaceous earth than from other fine-containing feeds. Although the lower part of the CRF rod appeared slightly pink in the ground-soil feed, no increase of pore size was observed.

The effect of diatomaceous earth additions on MIS penetration into porous refractory was investigated in greater detail. Feeds with diatomaceous earth were prepared as described earlier. Briefly, the soil, $\mathrm{ZrO}_{2}$, $\mathrm{B}_{2} \mathrm{O}_{3}$, and $\mathrm{Na}_{2} \mathrm{CrO}_{4}$ were premixed in the agate mill for $1 \mathrm{~min}$. Diatomaceous earth was added to liquid simulant. Table F.5 lists the masses of ingredients used for the feeds. The mixture was stirred and dried in a steel pot on a hotplate and then mixed with the premixed solids in the agate mill for $1 \mathrm{~min}$. The feed was loaded in the crucibles (one small and one medium with a CRB rod, see Table F.5). Crucibles were heated at $5^{\circ} \mathrm{C} / \mathrm{min}$ to $1200^{\circ} \mathrm{C}$ and held for 1 hour. Crucibles were removed from the furnace during heating to break foam when necessary.

Table F.5 shows the mass losses of feeds on heating. The losses from small crucibles appear small because the MIS penetrated through their bottoms, and some of the alumina powder from the underlying plate remained attached to the crucibles.

Figures F.5 and F.6 show the appearance of the crucibles after heating. Images for the baseline feed and the feed with 10 mass\% of diatomaceous earth are included for comparison. 
Table F.5. Masses of Batched Materials, Feeds, and Losses on Heating (in g, except for mass fractions)

\begin{tabular}{||l|r|r|r||}
\hline DE mass fraction & $\mathbf{0 . 1 5}$ & $\mathbf{0 . 2 0}$ & $\mathbf{0 . 2 5}$ \\
\hline Hanford soil & 128.66 & 116.23 & 103.80 \\
\hline $\mathrm{ZrO}_{2}$ & 16.80 & 16.80 & 16.80 \\
\hline $\mathrm{B}_{2} \mathrm{O}_{3}$ & 12.00 & 12.00 & 12.00 \\
\hline Diatomaceous Earth & 36.11 & 48.14 & 60.18 \\
\hline $\mathrm{Na}_{2} \mathrm{CrO}_{4}$ & 2.16 & 2.16 & 2.16 \\
\hline Liquid simulant & 350.37 & 351.68 & 352.99 \\
\hline Feed made & 546.10 & 547.01 & 547.93 \\
\hline Feed in small crucible & 24.37 & 24.37 & 24.37 \\
\hline Feed in medium crucible & 97.48 & 97.48 & 97.48 \\
\hline \multicolumn{4}{|c||}{ Mass loss on heating } \\
\hline Small crucible & 5.71 & 5.27 & 6.33 \\
\hline Medium crucible & 25.97 & 25.24 & 27.15 \\
\hline \multicolumn{4}{|c|}{ LOI, mass fraction } \\
\hline Small crucible & 0.234 & 0.216 & 0.260 \\
\hline Medium crucible & 0.266 & 0.259 & 0.279 \\
\hline \hline
\end{tabular}




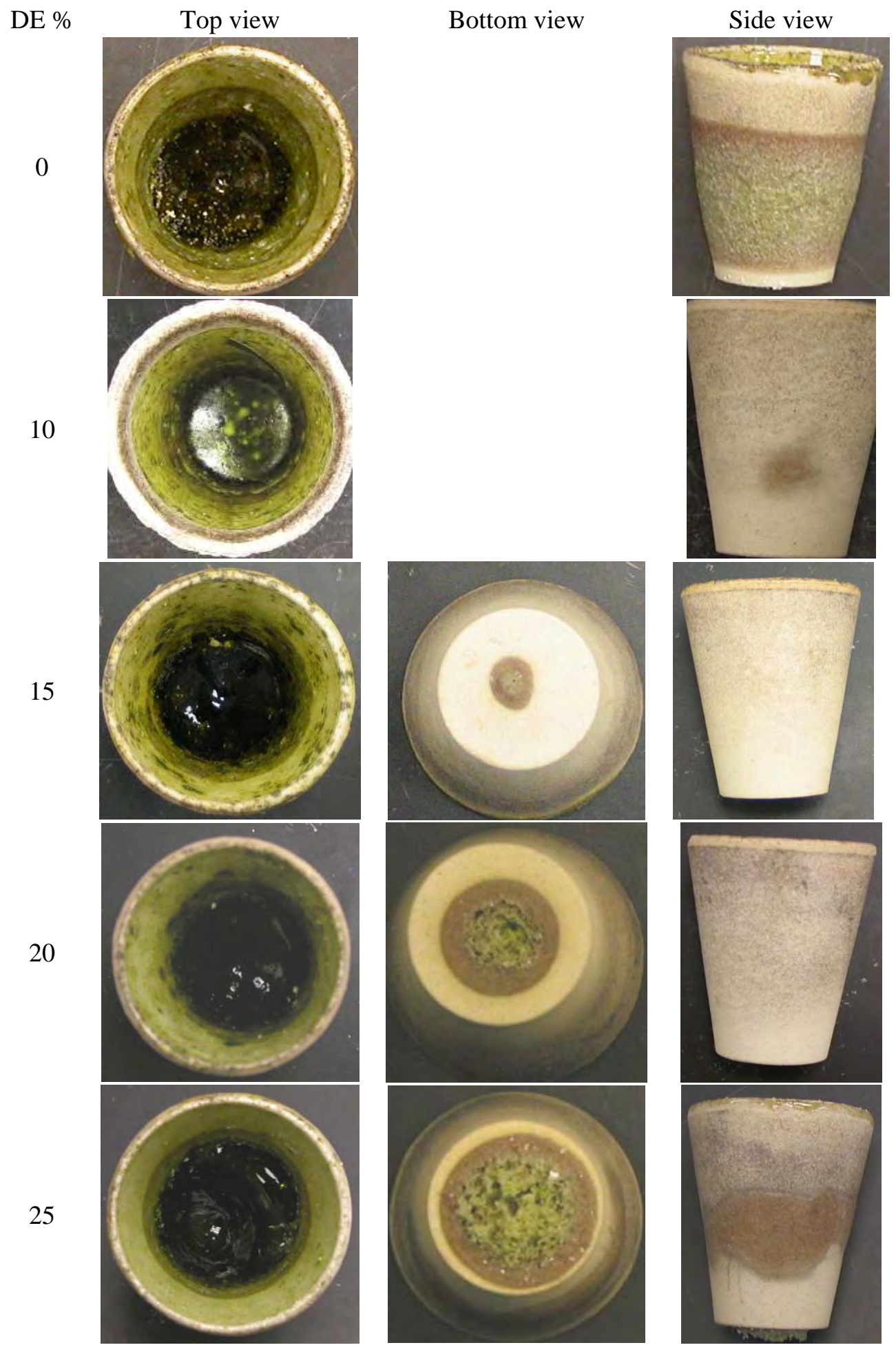

Figure F.5. Small Crucibles with Feed Containing 0 to 25 Mass\% of Diatomaceous Earth 


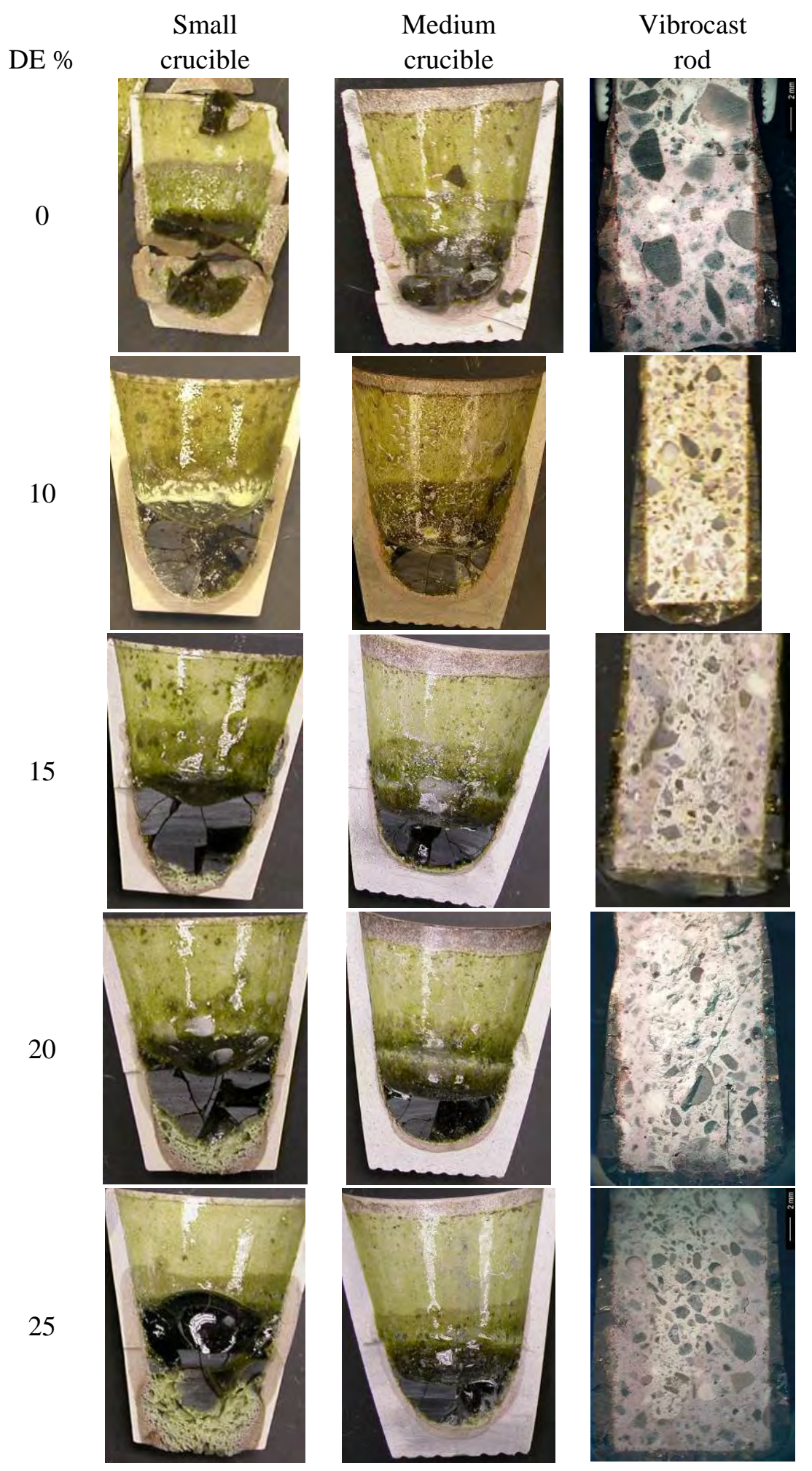

Figure F.6. Sections Through Crucibles and Vibrocast Rods Exposed to Feed with 0 to 25 Mass\% of Diatomaceous Earth 
The striking feature of small crucibles in which feeds with 15 to 25 mass\% of diatomaceous earth were melted is the penetration of their bottoms by MIS. Except 25 mass\% of diatomaceous earth, MIS did not penetrate to the outside surfaces of the sidewalls. Part of the bottom material turned into a viscous melt that turned into foam and swelled. This indicates that some MIS sunk under the feed. It is unclear how this happened and how the diatomaceous earth facilitated this to happen. A likely scenario can be suggested as follows. Heating destroyed the fine structure of the diatomaceous earth particles. As a result, the feed shrank away from the sidewalls. Simultaneously, the MIS trapped inside the diatomaceous earth particles was released and drained to the feed bottom, which corroded the crucible.

Compared to the baseline feed, the MIS penetrated medium crucibles slightly less when diatomaceous earth was a feed component. However, the depth of penetration does not change when the content of diatomaceous earth increases above 15 mass\%. It is possible that the presence of the vibrocast rods limited the penetration depth of MIS into the sidewalls. The increased porosity and vitrification of the wall material is noticeable on the bottoms, resembling the deterioration, on a small scale, that occurred in the small crucibles.

The color of the vibrocast material turned pink where the rod was in touch with the feed. The pink area did not reach to the central part of the rod. Its depth was $\sim 5 \mathrm{~mm}$ in the rod immersed in the feed containing 15 mass\% of diatomaceous earth. In the rod immersed in the feed containing 25 mass\% of diatomaceous earth, the rod turned pink up to $10 \mathrm{~mm}$ from the bottom. The pink areas have increased porosity, especially close to the rod surface. 
Appendix G

\section{Room-Temperature Physical Modeling}




\section{Appendix G: Room-Temperature Physical Modeling}

Room-temperature experiments with liquids of similar or nearly identical properties (viscosity, surface tension, and wetting angle) can accelerate and simplify studies of the MIS flow as it penetrates into the CRB. Table G.1 compares viscosity and surface tension of molten $\mathrm{NaNO}_{3}$, a major component of MIS, at $500^{\circ} \mathrm{C}$ with water at $20^{\circ} \mathrm{C}$, indicating that water is a suitable surrogate of the MIS (the wetting angle of both on the CRB is assumed to be close to zero).

Table G.1. Viscosity and Surface Tension of Liquid $\mathrm{NaNO}_{3}$ and $\mathrm{H}_{2} \mathrm{O}$

\begin{tabular}{||l|c|c||}
\hline & Viscosity (mPa·s) & Surface Tension (N/m) \\
\hline Water $\left(\right.$ at $\left.20^{\circ}\right)$ & 1.305 & 0.0728 \\
\hline $\mathrm{NaNO}_{3}\left(\right.$ at $\left.500^{\circ} \mathrm{C}\right)$ & 1.002 & 0.1088 \\
\hline
\end{tabular}

To make water penetration visible, a dye is needed. Preliminary tests were done using red ink. No attempt was made determine the impact of ink on water properties. It was assumed that the viscosity was slightly increased, and the surface tension decreased.

The soaking experiments were conducted with CRB rods sealed on three sides with silicon grease and immersed into a wet material, allowing liquid penetration from one side only. The rods were removed after 3 and 6 hours, dried, dry-cut with a diamond saw, and photographed.

The first experiment was done with soil mixed with 48 vol\% (27 mass\%) of liquid (undiluted red ink). Contrary to expectation, liquid penetration was rather shallow even after the 4-hour contact (Figure G.1). Since we suspected that water may interact with soil differently than MIS, we decided to continue testing with simple, well defined, granular materials, namely, silica sand (60 $\mu \mathrm{m}$ average particle size), ground silica ( $2 \mu \mathrm{m}$ average particle size), and diatomaceous earth. These materials were saturated with a waterink mixture containing 25\% red ink.
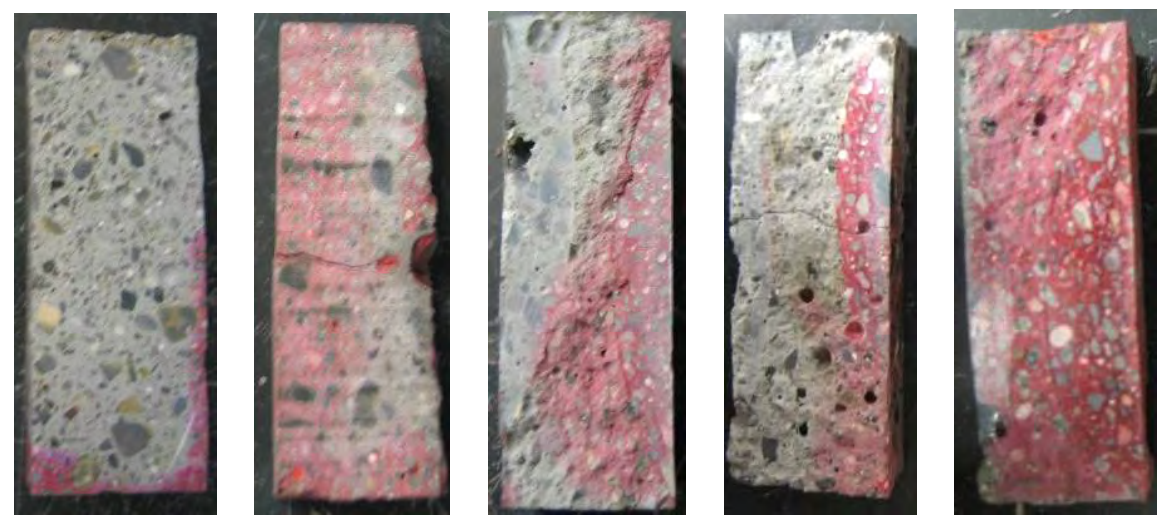

Figure G.1. Ink-Colored Water Penetration into Vibrocast Samples from Various Wet Solids; from Left to Right: Hanford Soil (4 hours), Silica Sand (3 and 6 hours), and Ground Silica (3 and 6 hours) 
Though no systematic study of water-based liquid penetration was performed because this was beyond the intended purpose of the test, Figure G.1 shows that room-temperature modeling is a possible option. The results indicate that the rate of liquid penetration from a saturated granular material is similar to that of wicking of pure liquid into a vibrocast cylinder. It was possible to observe a change of saturated granular material into an unsaturated one in the vicinity of the sample. Similar results were obtained with a saturated diatomaceous earth. 
Appendix $\mathbf{H}$

\section{Effect of Ground Soil Fraction on MIS \\ Penetration into Porous Refractory}




\section{Appendix H: Effect of Ground Soil Fraction on MIS Penetration into Porous Refractory}

\section{H.1. Initial Experiment}

(NOTE: All experiments described in Appendix H were conducted with six-tank composite LAW simulant. Experiments described in Sections H.1 and H.2 were performed with feeds prepared by mixing ground soil with LAW simulant. Experiments described in Sections H.3 were performed with ground feed that was available from a previous project.)

The first experiment with soil ground to a fine powder by milling it for $5 \mathrm{~min}$ in a tungsten carbide mill was performed with the baseline composition. The feed with a CRB specimen was heated in Pt crucibles at $5^{\circ} \mathrm{C} / \mathrm{min}$ to temperatures of $800^{\circ} \mathrm{C}, 1000^{\circ} \mathrm{C}$, and $1300^{\circ} \mathrm{C}$. Figure H.1 shows the melts and sectioned specimens. Figure H.2 shows that the MIS penetration was small with the only sodium concentration higher than the background level was on the sample surface. The appearance of the specimens (pink coloration) wrongly indicated that the fine soil did not protect the specimens from MIS penetration. better than the original soil. This result indicated that relying on the qualitative color assessment to determine MIS penetration could be misleading and mass change was generally adopted as the preferred method in subsequent analyses.
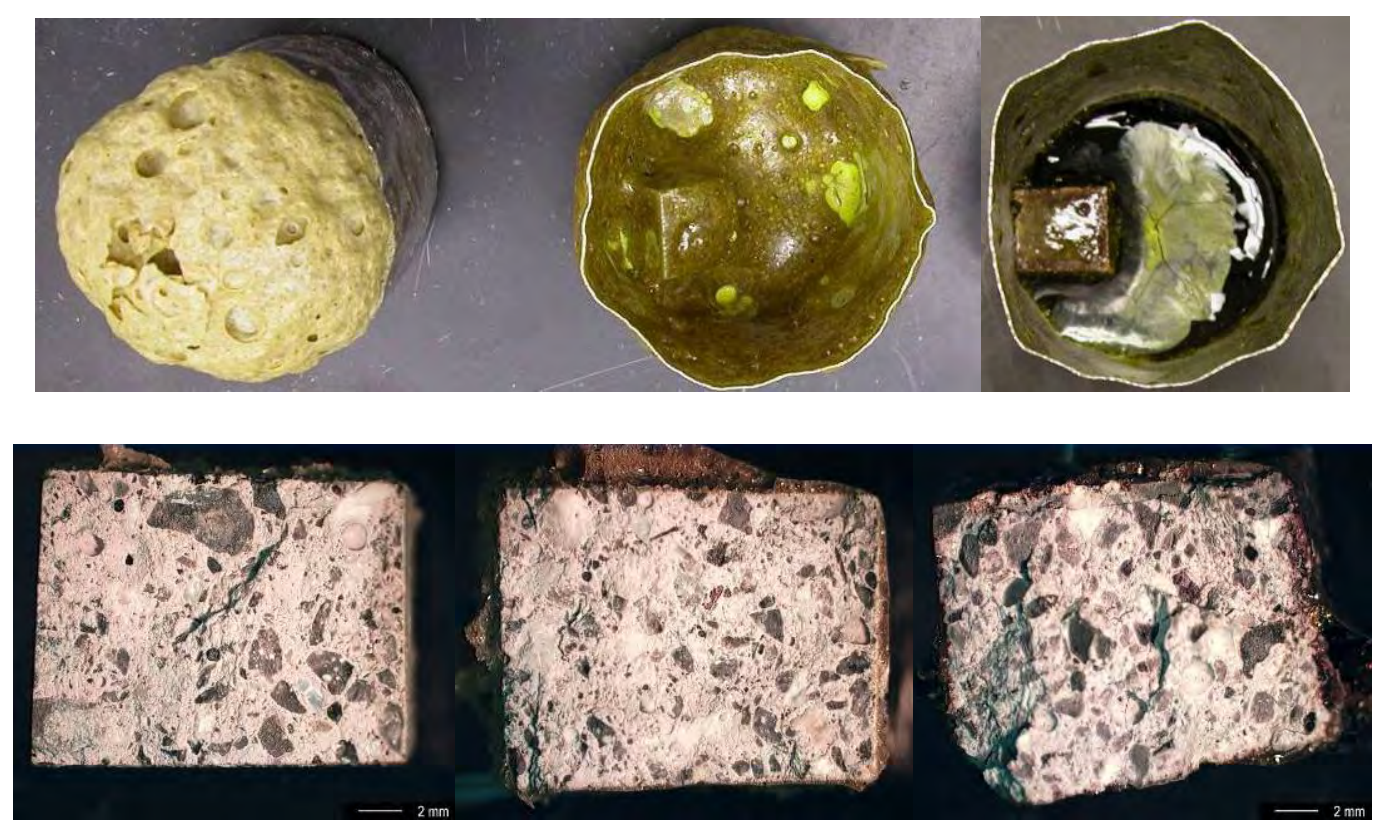

Figure H.1. Melts from Feed with Ground Soil and the Appearance of Sectioned Specimens; Left to Right: $800^{\circ} \mathrm{C}, 1000^{\circ} \mathrm{C}$, and $1300^{\circ} \mathrm{C}$ 

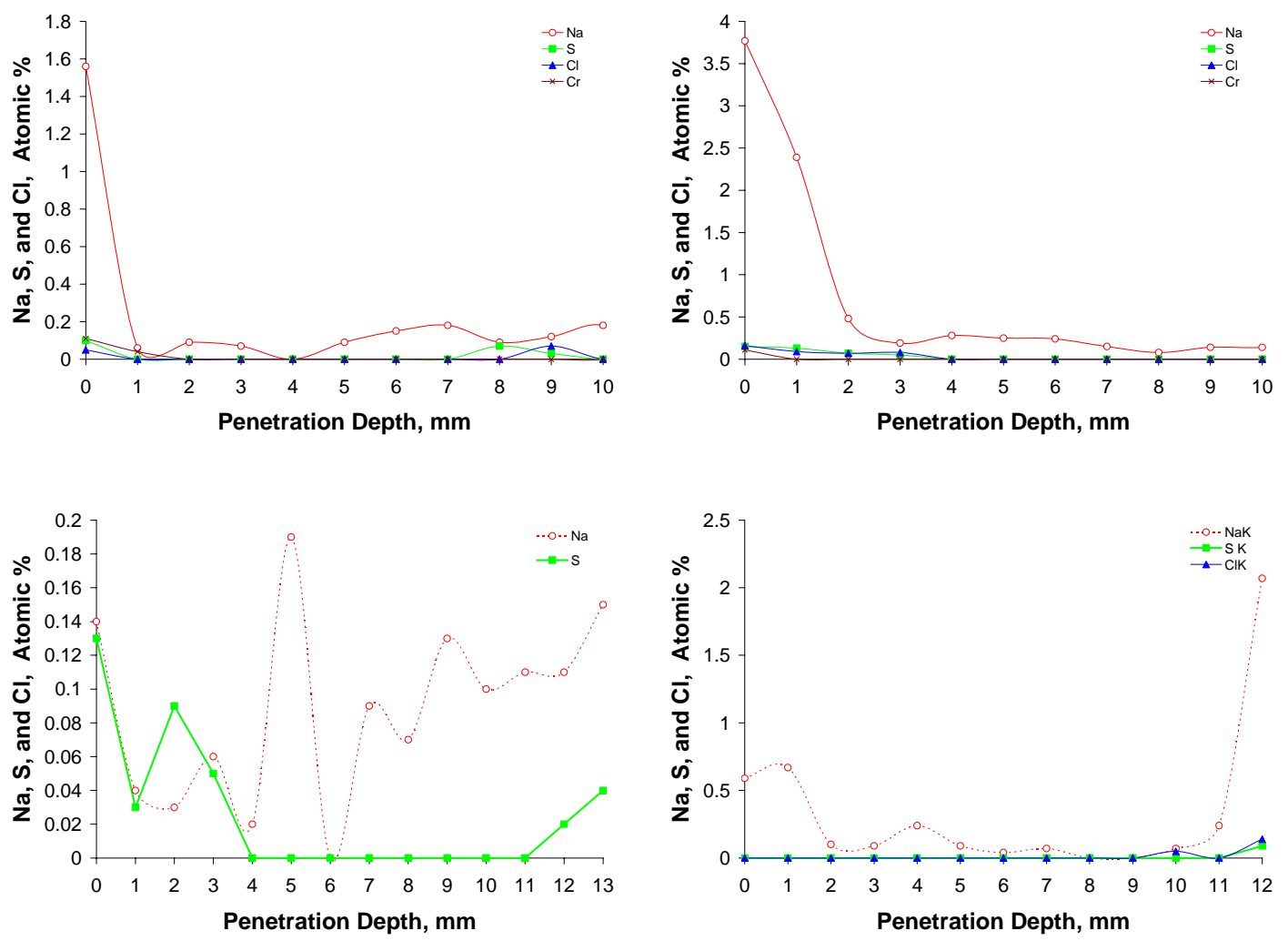

Figure H.2. CRB Specimens Exposed to Feed with Ground Soil Heated to $800^{\circ} \mathrm{C}$ (top) and $1300^{\circ} \mathrm{C}$ (bottom). The sample scan went through the middle of the sample from left to right (left) and from bottom to top (right).

\section{H.2. Experiments with Silica Crucibles}

To find out the fraction of ground soil that keeps MIS in the feed without allowing it to soak into a porous refractory wall, the soil was ground in the tungsten mill for $2 \mathrm{~min}$. The additives containing soil of which $20,40,60$, and $80 \%$ was ground were mixed in the agate mill for $1 \mathrm{~min}$ and then used to prepare the feed in the same way as the baseline feed. Briefly, liquid simulant was condensed by boiling in a steel beaker. The additives were added and stirred with the simulant, and the mixture was dried in the beaker. The dry feed was further homogenized in the agate mil for $1 \mathrm{~min}$. The MIS penetration test was performed in silica crucibles, medium and small. The feeds were heated at $5^{\circ} \mathrm{C} / \mathrm{min}$ to $1200^{\circ} \mathrm{C}$ with a 1 -hour hold.

Feed with ground soil foamed extensively. Small crucibles were removed from the furnace at $800^{\circ} \mathrm{C}$, and the foam was broken by mechanically pushing the feed back down into the crucible with a steel rod. This was performed once with the feed containing $20 \%$ ground soil and at least twice with feeds containing $40 \%$ and $60 \%$ of ground soil. The foam had to be destroyed in the $40 \%$ and $60 \%$ ground soil medium crucibles tests as well. No excessive foaming occurred in these melts after the temperature reached $900^{\circ} \mathrm{C}$. With the feed containing $100 \%$ ground soil, both crucibles had to be removed every 2 to 3 min to suppress foaming that stopped only at $995^{\circ} \mathrm{C}$. 
Figure H.3 shows the top, bottom, and side views of crucibles after the heat-treatment. Note the diminishing extent at which MIS penetrated to the outside surface of the crucibles as the fraction of ground soil increased.

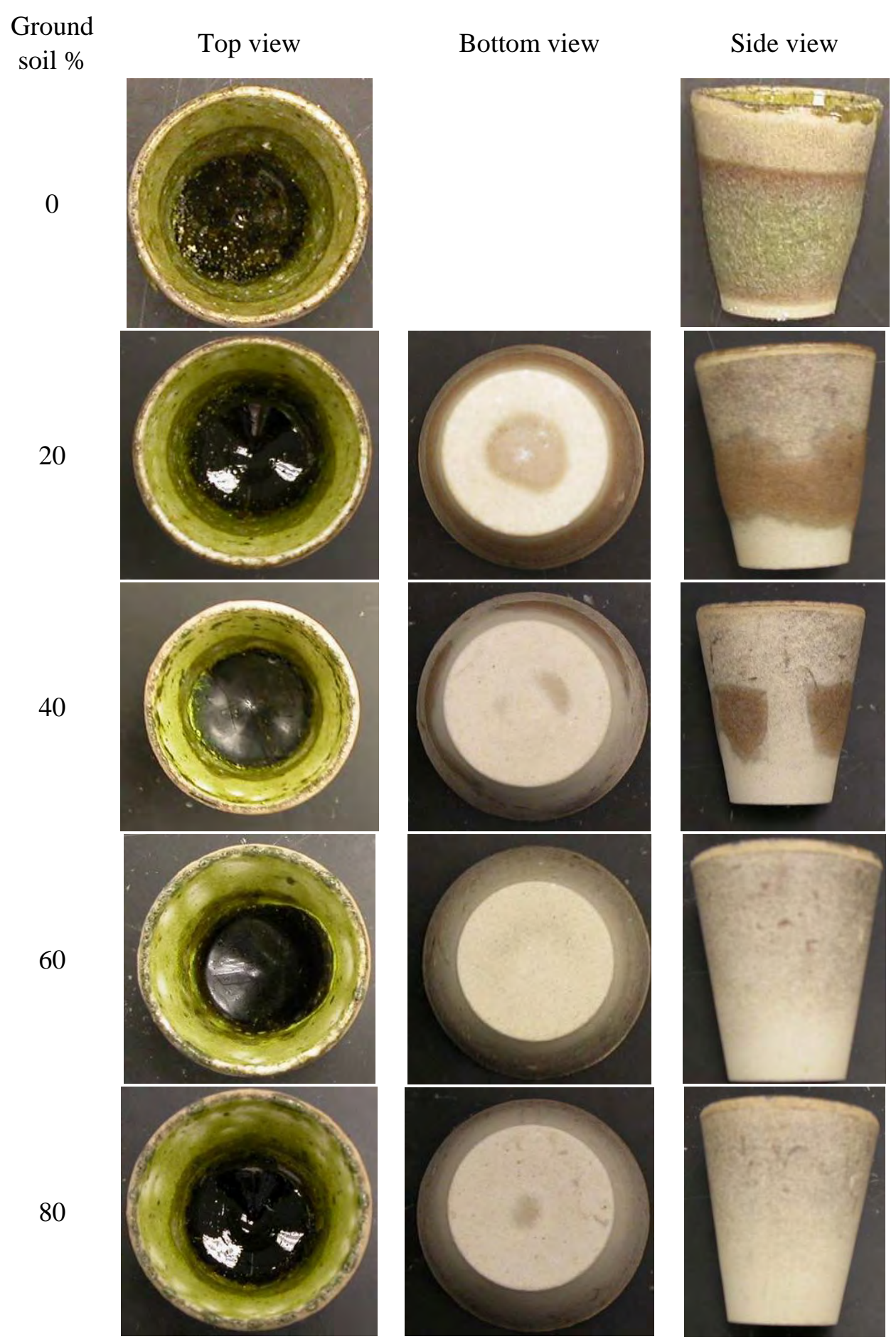

Figure H.3. Small Crucibles with Baseline Feed Composition and 0 to $100 \%$ Soil Ground 
Sections through small and medium crucibles, shown in Figure H.4, demonstrate that MIS penetration decreased as the fraction of ground soil increased until it virtually stopped at $100 \%$ ground soil. Pictures obtained previously with the original and fully ground soil are added for comparison.

Weighing the melts before and after heat-treatments allowed the determination of the mass losses. Based on small crucible data, the LOI of the feed was $0.219 \pm 0.007$.

The miss penetration depth was measured for both small and medium crucibles. It was nearly equal for sidewalls and bottoms of small crucibles and sidewalls of medium crucibles. Figure H.5 displays the averages of maximum and minimum penetration depth versus the percentage of ground soil.
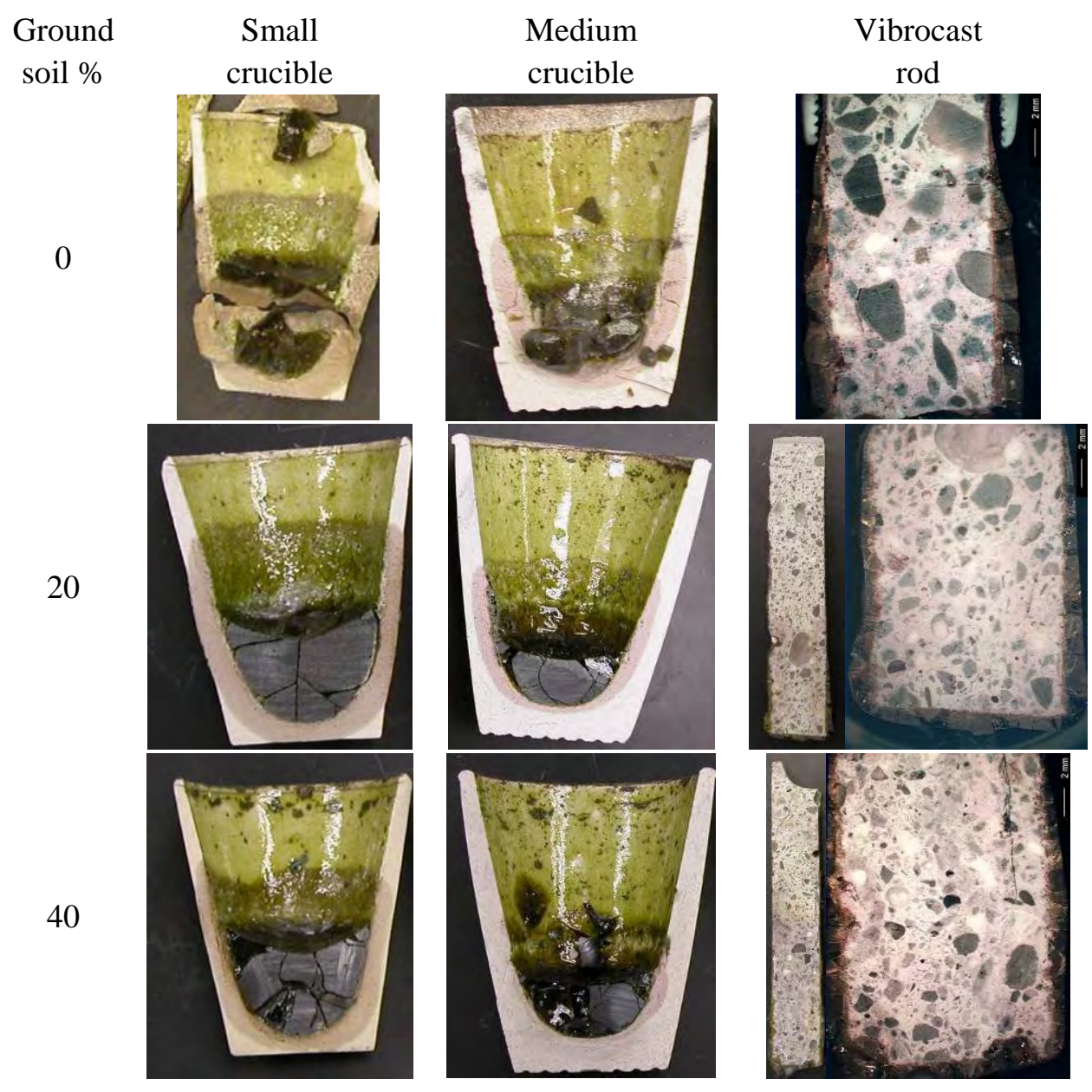

Figure H.4. Sections Through Crucibles and Vibrocast Rods Exposed to Feed of Baseline Composition and 0 to $100 \%$ Soil Ground 
Ground
soil \%
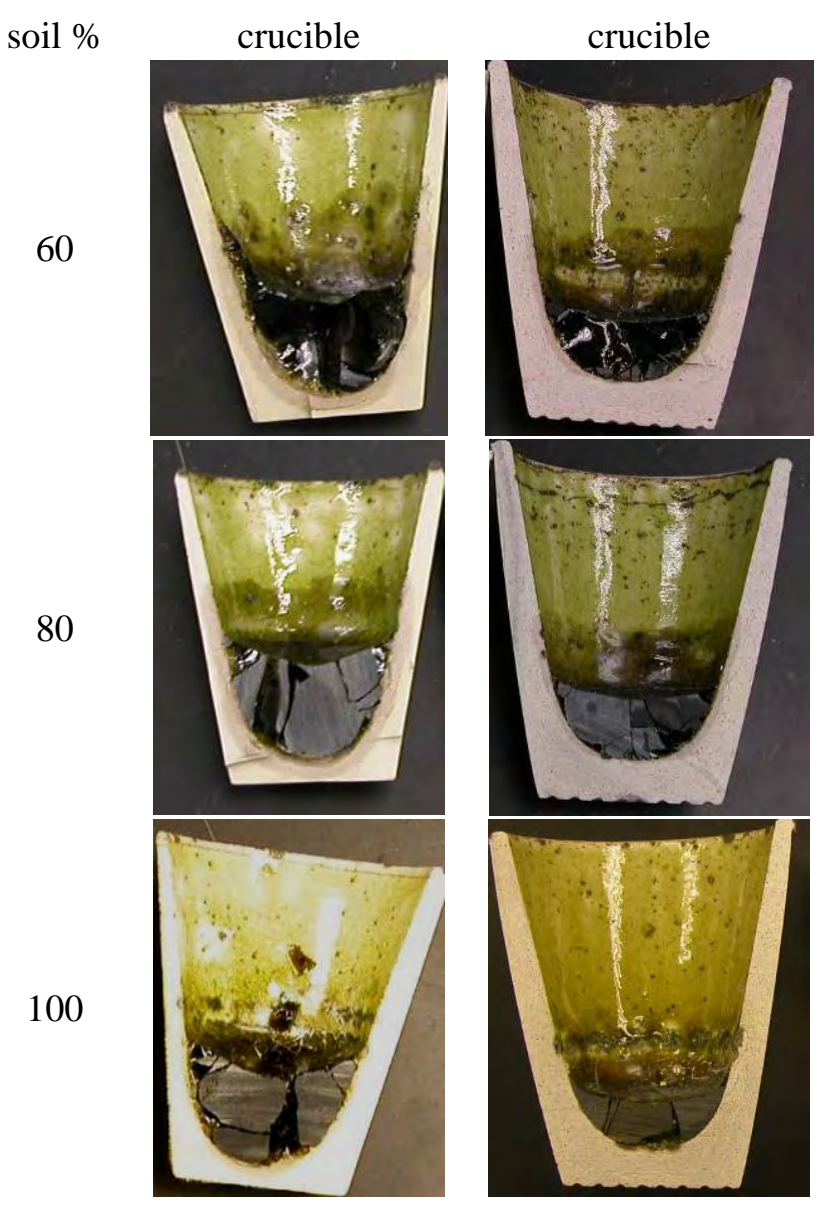

Figure H.4 (Contd.)

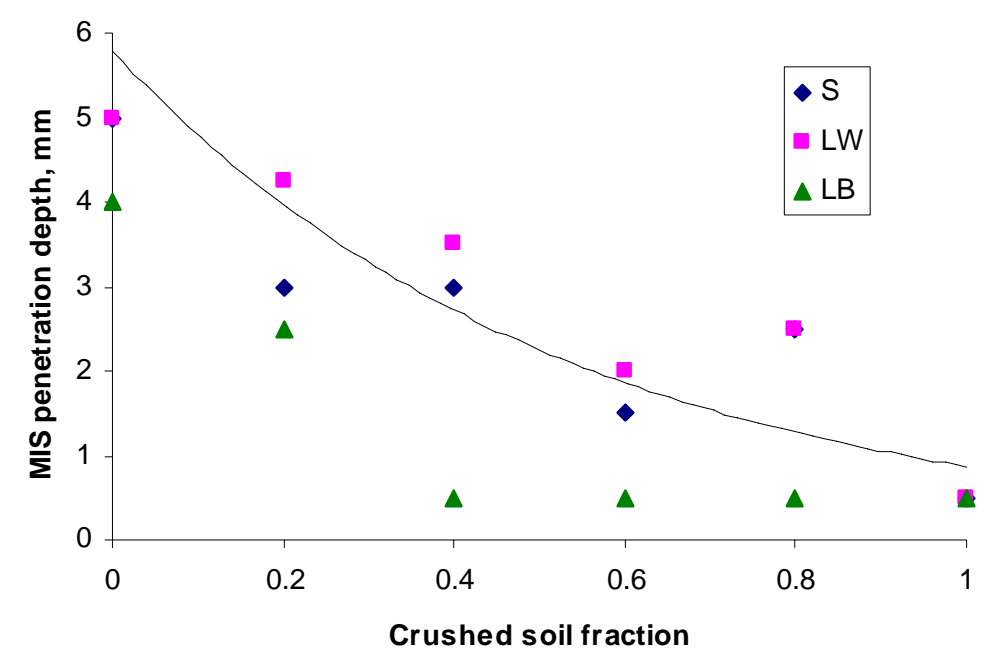

Figure H.5. MIS Penetration Depth Versus Fraction of Soil Ground (S - small crucible, $\mathrm{L}$ - medium crucible, $\mathrm{W}$ - wall, $\mathrm{B}$ - bottom) 
The penetration depth into the bottoms of medium crucibles is relatively small because the vibrocast rods rested on the bottoms. The solid line is an exponential fit to small crucible and medium crucible sidewalls data. Accordingly, the depth of penetration is decreased by $25 \%$ if $15 \%$ of the soil is ground, by $50 \%$ if $37 \%$ of the soil is ground, by $75 \%$ if $73 \%$ of the soil is ground, and by $85 \%$ if $100 \%$ of the soil is ground.

\section{H.3. Experiments with CRB Rods}

For CRB rod testing, the assembly consisted of a vertical rod, $\sim 18 \times 18 \times 50 \mathrm{~mm}$, surrounded with $97 \mathrm{~g}$ of feed added in a Pt crucible. The assembly was placed in a furnace and heated at $5^{\circ} \mathrm{C} / \mathrm{min}$ to $550^{\circ} \mathrm{C}$. Typically, the feed shrank during the heat-treatment, and the MIS wicked a few millimeters up to the protruding rod. The rods underwent a second heat-treatment, this time in air, in which they were heated at $1200^{\circ} \mathrm{C}$ for 1 hour. The purpose of this operation was to determine the mass change resulting from the complete decomposition of the absorbed MIS.

Rods were weighed before and after the heat-treatment to obtain the change in the rod mass. The net gain was obtained by adding the LOI of the rod heat-treated in the absence of feed to the measured difference. Table H.1 compares data obtained for the original and ground feed. Table H.2 summarizes the averages of the mass gains and losses. The net gains/losses are corrected for the LOIs using the values listed in Table 5.1. Note that the net mass gains at $1200^{\circ} \mathrm{C}$ that measure the mass of MIS that penetrated into the rod from the feed are non-zero, but are substantially (by 88\%) lower for the ground feed than those from the original feed.

Table H.1. Data Obtained from CRB Rods Exposed to Original and Ground Feeds ${ }^{(a)}$

\begin{tabular}{||l|c|c|c|c|c|c||}
\hline Feed & \multicolumn{3}{|c|}{ Original } & \multicolumn{3}{c||}{ Ground } \\
\hline Sample & 1 & 2 & 3 & 1 & 2 & 3 \\
\hline Initial mass, g & 51.09 & 45.98 & 46.76 & 45.77 & 46.14 & 51.23 \\
\hline Mass after $550^{\circ} \mathrm{C}, \mathrm{g}$ & 52.62 & 47.70 & 48.91 & 45.08 & 45.42 & 50.60 \\
\hline Mass after $1200^{\circ} \mathrm{C}, \mathrm{g}$ & 51.02 & 46.17 & 47.19 & 44.89 & 45.25 & 50.36 \\
\hline Mass gain at $550^{\circ} \mathrm{C}^{\mathrm{a}}, \mathrm{g}$ & 1.53 & 1.72 & 2.15 & -0.69 & -0.72 & -0.63 \\
\hline Mass loss at $1200^{\circ} \mathrm{C}, \mathrm{g}$ & 1.60 & 1.53 & 1.72 & 0.19 & 0.17 & 0.24 \\
\hline Mass gain at $1200^{\circ} \mathrm{C}^{\mathrm{a}}, \mathrm{g}$ & -0.07 & 0.19 & 0.43 & -0.88 & -0.89 & -0.87 \\
\hline Rod volume, $\mathrm{mL}$ & 20.23 & 17.99 & 17.79 & 18.40 & 17.46 & 19.23 \\
\hline Initial density, $\mathrm{g} / \mathrm{mL}$ & 2.525 & \multicolumn{7}{|c|}{2.556} & 2.628 & 2.488 & 2.642 & 2.664 \\
\hline (a) The vibrocast $\mathrm{LOI}$ has not been applied. This causes some gains to have negative values. \\
\hline
\end{tabular}


Table H.2. Summary of Averaged Gains/Losses Resulting from Heat-Treatments of CRB Rods Exposed to Original and Ground Feeds

\begin{tabular}{||l|c|c||}
\hline \hline Feed & Original & Ground \\
\hline Mass gain at $550^{\circ} \mathrm{C}, \mathrm{g}$ & 1.80 & -0.68 \\
\hline Mass loss at $1200^{\circ} \mathrm{C}, \mathrm{g}$ & 1.62 & 0.20 \\
\hline Mass gain at $550^{\circ} \mathrm{C}, \mathrm{g}$ & 0.18 & -0.88 \\
\hline Net mass gain at $550^{\circ} \mathrm{C}, \mathrm{g}$ & 2.70 & 0.22 \\
\hline Net mass loss at $1200^{\circ} \mathrm{C}, \mathrm{g}$ & 1.49 & 0.07 \\
\hline Net mass gain at $1200^{\circ} \mathrm{C}, \mathrm{g}$ & 1.22 & 0.15 \\
\hline
\end{tabular}

The values in Tables H.1 and H.2 were calculated as follows.

- The mass gain at $550^{\circ} \mathrm{C}\left(G_{1}\right)$ is the difference between the initial CRB mass $\left(m_{0}\right)$ and the mass after exposure to feed at $550^{\circ} \mathrm{C}\left(m_{1}\right)$.

- The mass loss at $1200^{\circ} \mathrm{C}(L)$ is the difference between $m_{1}$ and the mass after exposure to feed at $1200^{\circ} \mathrm{C}\left(m_{2}\right)$.

- The mass gain at $1200^{\circ} \mathrm{C}\left(G_{2}\right)$ is the difference between the initial CRB mass $\left(m_{0}\right)$ and the mass after exposure to feed at $550^{\circ} \mathrm{C}\left(m_{2}\right)$.

Thus,

$$
\begin{gathered}
G_{1}=m_{1}-m_{0} \\
L=m_{1}-m_{2} \\
G_{2}=m_{2}-m_{0}
\end{gathered}
$$

- The net mass gains and losses are defined as

$$
\begin{gathered}
G_{\mathrm{N} 1}=G_{1}+m_{0} l_{550} \\
L_{\mathrm{N}}=L-m_{0}\left(l_{1200}-l_{550}\right) \\
G_{\mathrm{N} 2}=G_{2}+m_{0} l_{1200}
\end{gathered}
$$

where $l$ is the average LOI, and subscripts 550 and 1200 denote the heat-treatment temperatures in ${ }^{\circ} \mathrm{C}$.

Note that

$$
G_{\mathrm{N} 1}-G_{\mathrm{N} 2}=L_{\mathrm{N}}
$$

The following subsections report the results from the test duration at $550^{\circ} \mathrm{C}$ as a function of time (from 0 to 24 hours) and temperature $\left(350^{\circ} \mathrm{C}\right.$ to $\left.850^{\circ} \mathrm{C}\right)$. The main focus, however, is on the impact of feed grinding. The CRB rod was exposed to feeds in which ground and original fractions were mixed in the 
agate mill for $1 \mathrm{~min}$ and to feeds that were ground for various durations. The specific surfaces of feeds were estimated from the grain-size distribution.

\section{H.3.1. Effect CRB of Exposure Time to Feed on MIS Penetration}

The exposure time of CRB rods to the original and ground feeds varied from 0 to 24 hours. The heattreatments were repeated in the same manner as described above. Tables H.3 and H.4 show the results. Figure H.6 displays the net gains/losses.

Table H.3. Effect of $550^{\circ} \mathrm{C}$ Heat-Treatment Time on MIS Penetration to CRB from Original Feed

\begin{tabular}{||l|r|r|r|r||}
\hline Time at $\mathbf{5 5 0} \mathbf{}^{\circ} \mathbf{C}, \mathbf{~ h}$ & \multicolumn{1}{|c|}{$\mathbf{0}$} & \multicolumn{1}{c|}{$\mathbf{1}$} & \multicolumn{1}{c|}{$\mathbf{8}$} & \multicolumn{1}{c|}{$\mathbf{2 4}$} \\
\hline Initial mass, g & 42.01 & 48.15 & 44.28 & 44.11 \\
\hline Mass after $550^{\circ} \mathrm{C}, \mathrm{g}$ & 42.98 & 48.89 & 45.09 & 45.00 \\
\hline Mass after $1200^{\circ} \mathrm{C}, \mathrm{g}$ & 41.89 & 48.02 & 44.50 & 44.68 \\
\hline Mass gain at $550^{\circ} \mathrm{C}, \mathrm{g}$ & 0.97 & 0.74 & 0.81 & 0.89 \\
\hline Mass loss at $1200^{\circ} \mathrm{C}, \mathrm{g}$ & 1.09 & 0.87 & 0.59 & 0.32 \\
\hline Mass gain at $1200^{\circ} \mathrm{C}, \mathrm{g}$ & -0.12 & -0.13 & 0.22 & 0.57 \\
\hline Net mass gain at $550^{\circ} \mathrm{C}, \mathrm{g}$ & 1.73 & 1.61 & 1.61 & 1.69 \\
\hline Net mass loss at $1200^{\circ} \mathrm{C}, \mathrm{g}$ & 0.98 & 0.74 & 0.47 & 0.20 \\
\hline Net mass gain at $1200^{\circ} \mathrm{C}, \mathrm{g}$ & 0.76 & 0.88 & 1.15 & 1.50 \\
\hline \hline
\end{tabular}

Table H.4. Effect of $550^{\circ} \mathrm{C}$ Heat-Treatment Time on MIS Penetration to CRB from Ground Feed

\begin{tabular}{||l|r|r|r|r||}
\hline Time at $\mathbf{5 5 0} \mathbf{}^{\circ} \mathbf{C}, \mathbf{h}$ & \multicolumn{1}{c|}{$\mathbf{0}$} & \multicolumn{1}{c|}{$\mathbf{1}$} & \multicolumn{1}{c|}{$\mathbf{8}$} & \multicolumn{1}{c|}{$\mathbf{2 4}$} \\
\hline Initial mass, g & 46.54 & 49.66 & 50.37 & 52.83 \\
\hline Mass after $550^{\circ} \mathrm{C}, \mathrm{g}$ & 45.87 & 49.23 & 49.65 & 52.16 \\
\hline Mass after $1200^{\circ} \mathrm{C}, \mathrm{g}$ & 45.67 & 48.94 & 49.50 & 52.00 \\
\hline Mass gain at $550^{\circ} \mathrm{C}, \mathrm{g}$ & -0.67 & -0.43 & -0.72 & -0.67 \\
\hline Mass loss at $1200^{\circ} \mathrm{C}, \mathrm{g}$ & 0.20 & 0.29 & 0.15 & 0.16 \\
\hline Mass gain at $1200^{\circ} \mathrm{C}, \mathrm{g}$ & -0.87 & -0.72 & -0.87 & -0.83 \\
\hline Net mass gain at $550^{\circ} \mathrm{C}, \mathrm{g}$ & 0.17 & 0.47 & 0.19 & 0.29 \\
\hline Net mass loss at $1200^{\circ} \mathrm{C}, \mathrm{g}$ & 0.07 & 0.16 & 0.01 & 0.02 \\
\hline Net mass gain at $1200^{\circ} \mathrm{C}, \mathrm{g}$ & 0.11 & 0.32 & 0.19 & 0.28 \\
\hline \hline
\end{tabular}



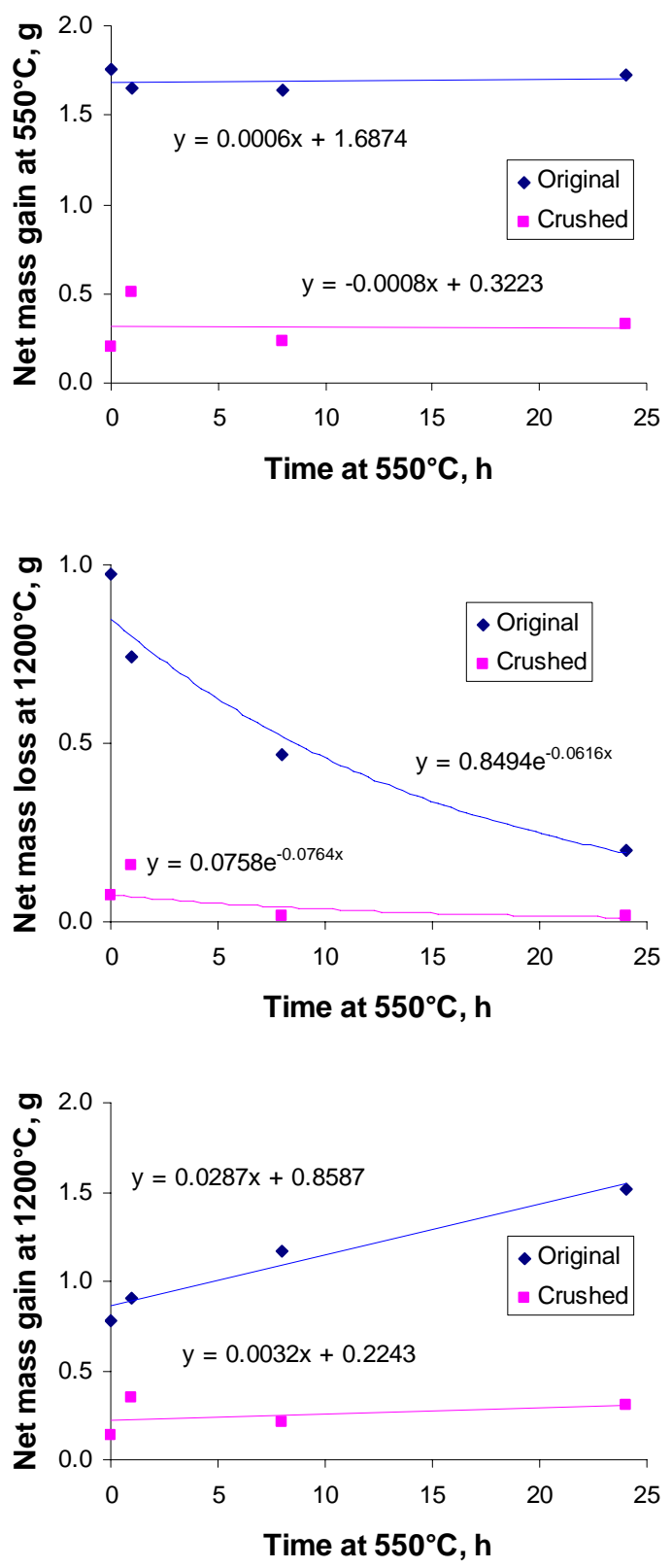

Figure H.6. Effect of $550^{\circ} \mathrm{C}$ Heat-Treatment Time on MIS Penetration to CRB from Original and Ground Feed

The data show that the exposure time does not affect the net mass gain at $550^{\circ} \mathrm{C}$ (see Figure H.6, top). However, the net mass loss during heating from $550^{\circ} \mathrm{C}$ to $1200^{\circ} \mathrm{C}$ decreases with the exposure time at $550^{\circ} \mathrm{C}$ (Figure H.6, middle). This indicates that MIS present in the CRB was decomposing while more MIS continued to penetrate. Hence, at $550^{\circ} \mathrm{C}$, the increase of the mass of MIS by penetration was compensated by the loss of MIS by decomposition. The net mass gain at $1200^{\circ} \mathrm{C}$, which accounts for the glass-forming solids in the total amount of MIS that penetrated into the CRB, continuously increased with the exposure time at $550^{\circ} \mathrm{C}$ (Figure H.6, bottom). 
The total amount of MIS in the CRB decreased by an order of magnitude when the feed was ground, and its value no longer significantly increased with time, suggesting that free liquid was virtually absent in the ground feed.

The straight lines fitted in Figure H.6 to $G_{\mathrm{N} 2}$ versus the $550^{\circ} \mathrm{C}$ heat-treatment time $\left(t_{\mathrm{H}}\right)$ cannot be extrapolated and do not fit data very well. The left plot in Figure H.7 shows an exponential fit using the function

$$
G_{N 2}=a+b\left(1-e^{-t_{H} / t_{0}}\right)
$$

where $a, b$, and $t_{0}$ are coefficients whose values are listed in Table H.5. The extremely high value of $t_{0}$ for the ground feed indicates that MIS penetration will virtually never progress beyond the short-time level.
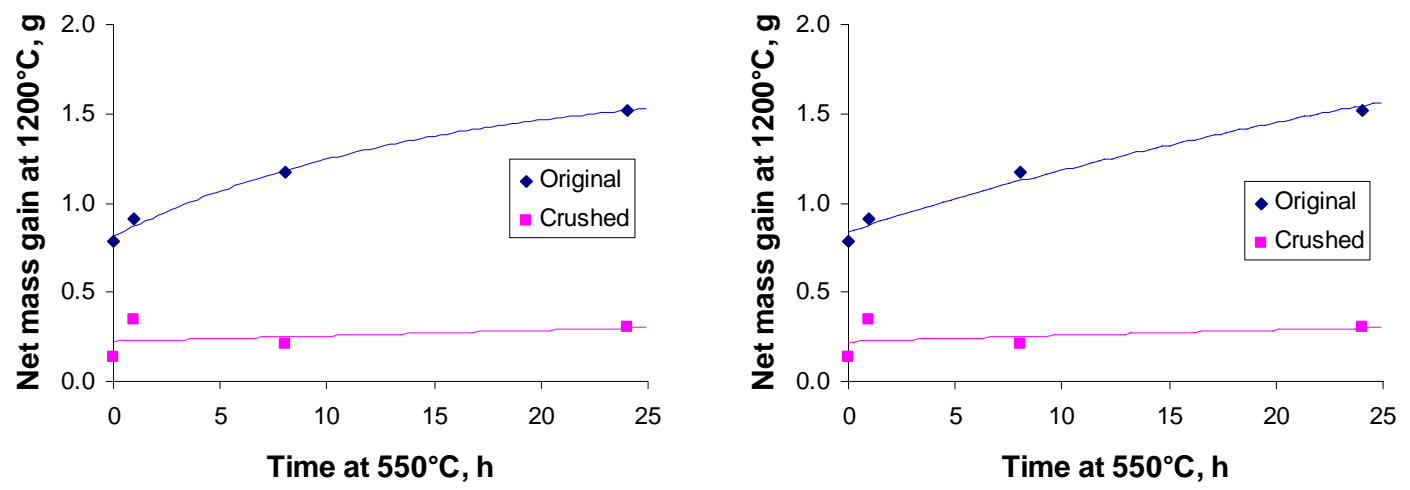

Figure H.7. Effect of $550^{\circ} \mathrm{C}$ Heat-Treatment Time on MIS Penetration to CRB from Original and Ground Feed: Exponential Fit (left) and Square-Root Fit (right)

Table H.5. Equation (8a) and (8b) Coefficients

\begin{tabular}{||c|c|c|c||}
\hline & & Original & Ground \\
\hline$a$ & $\mathrm{~g}$ & 0.813 & 0.225 \\
\hline$b$ & $\mathrm{~g}$ & 0.872 & 51.75 \\
\hline$t_{0}$ & $\mathrm{~h}$ & 14.52 & 16764 \\
\hline$A$ & $\mathrm{~g}$ & 0.265 & 0.0404 \\
\hline$t_{1}$ & $\mathrm{~h}$ & 9.94 & 30.7 \\
\hline
\end{tabular}

The right plot in Figure H.7 shows a square-root using the function

$$
G_{N 2}=A\left(t+t_{1}\right)^{1 / 2}
$$

where $A$ and $t_{1}$ are coefficients whose values are listed in Table H.5. The coefficient $t_{1}$ compensates from the amount of MIS that penetrated during initial heating of the sample to $550^{\circ} \mathrm{C}$. 
It is important to note that neither the linear, polynomial, exponential, nor power-law function can express the complex process of simultaneous MIS penetration and decomposition. Since the process normally occurs at an increasing temperature, a realistic mathematical description would be even more difficult.

Feed data shown in Figure H.8 verify that MIS is indeed progressively decomposing as time progresses at $550^{\circ} \mathrm{C}$. By the time the feed temperature reached $550^{\circ} \mathrm{C}$ (at $\left.5^{\circ} \mathrm{C} / \mathrm{min}\right)$, the feed lost $8 \%$ of its mass. After 24 hours at $550^{\circ} \mathrm{C}$, the mass loss increased to $23 \%$. The right plot in Figure H.8 shows that the mass lost during heating $\mathrm{CRB}$ samples with MIS from $550^{\circ} \mathrm{C}$ to $1200^{\circ} \mathrm{C}$ linearly decreased with the increasing mass loss from feed at temperature $550^{\circ} \mathrm{C}$, which is as expected. Note that slightly less (by $10 \%$ on average) MIS decomposed when the feed was ground.
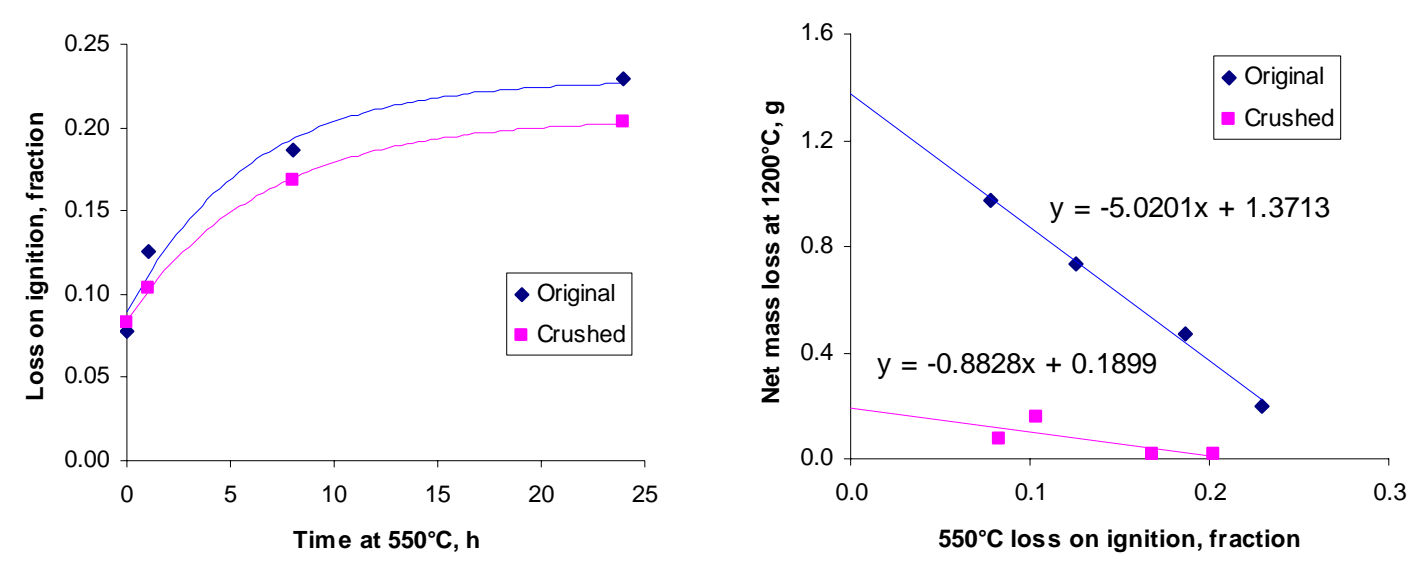

\section{Figure H.8. Mass Loss of Feed (original and ground) Versus Time at $550^{\circ} \mathrm{C}$ (left) and $1200^{\circ} \mathrm{C}$-net Mass Loss of MIS in CRB Versus Mass Loss of Feed (right)}

The lines in the left plot represent exponential functions

$$
L_{F}=a+b\left(1-e^{-t_{H} / t_{0}}\right)
$$

where $L_{\mathrm{F}}$ is the feed LOI, $t_{\mathrm{H}}$ is the heat-treatment time at $550^{\circ} \mathrm{C}$, and $a, b$, and $t_{0}$ are coefficients listed in Table H.6. As $t_{\mathrm{H}} \rightarrow \infty, L_{\mathrm{F}} \rightarrow a+b$, a value that should not depend on whether the feed is ground. This value is $0.229 \mathrm{~g}$ for original feed and $0.206 \mathrm{~g}$ for ground feed; the difference is attributable to experimental error. The large value of $t_{0}$ for ground feed (6.6 hours) as compared to original feed (5.9 hours) indicates that the ground feed reacts more slowly. The reason for the smaller amount of MIS decomposition at higher temperature in the ground soil case is not fully understood but could be a result of the MIS being held in the soil at the center of the crucible where it is not exposed to the higher temperatures that the MIS sees when it penetrates the crucible wall in the as-received soil case. 
Table H.6. Equation (9) Coefficients

\begin{tabular}{||c|c|c|c||}
\hline \hline & & Original & Ground \\
\hline$a$ & $\mathrm{~g}$ & 0.089 & 0.084 \\
\hline$b$ & $\mathrm{~g}$ & 0.140 & 0.121 \\
\hline$t_{0}$ & $\mathrm{~h}$ & 5.865 & 6.593 \\
\hline
\end{tabular}

\section{H.3.2. Effect of Temperature on MIS Penetration to CRB}

A CRB rod was surrounded by baseline feed and placed in a Pt crucible. This assembly was placed in a furnace preheated to a set temperature and held for 1 hour. The rod was then pulled out of the feed, cleaned to remove any feed attached to its surface, and weighed. Table H.7 shows the measured data. The net mass gain is plotted in Figure H.9 against temperature, except the $600^{\circ} \mathrm{C}$-data point, an obvious outlier. The line drawn through the data points suggests that the amount of MIS does not increase when the temperature rises above $750^{\circ} \mathrm{C}$ and in fact, the amount of MIS penetration decreased because MIS decomposes before it can penetrate into the CRB (see the last row in Table H.7).

Table H.7. Effect of Temperature on 1-Hour MIS Penetration into CRB Sample

\begin{tabular}{||c|c|c|c|c||}
\hline $\mathbf{T ,}{ }^{\circ} \mathbf{C}$ & $\begin{array}{c}\text { Initial } \\
\text { Mass, } \mathbf{g}\end{array}$ & $\begin{array}{c}\mathbf{5 0 0}^{\circ} \mathbf{C} \\
\mathbf{M a s s}^{\mathbf{g}}\end{array}$ & $\begin{array}{c}\mathbf{1 2 0 0}^{\circ} \mathbf{C} \\
\mathbf{M a s s}^{\mathbf{g}}\end{array}$ & $\begin{array}{c}\text { Net Mass } \\
\text { Gain, } \mathbf{g}\end{array}$ \\
\hline 350 & 46.32 & 46.73 & 45.87 & 0.569 \\
\hline 450 & 44.89 & 45.96 & 44.76 & 0.858 \\
\hline 450 & 82.85 & 83.44 & 82.02 & 0.993 \\
\hline 550 & 79.32 & 80.22 & 79.25 & 1.675 \\
\hline 650 & 78.71 & 79.36 & 79.01 & 2.032 \\
\hline 500 & 76.90 & 78.66 & 77.36 & 2.152 \\
\hline 600 & 80.45 & 82.64 & 81.58 & 2.900 \\
\hline 850 & 81.29 & 81.53 & 80.94 & 1.438 \\
\hline
\end{tabular}

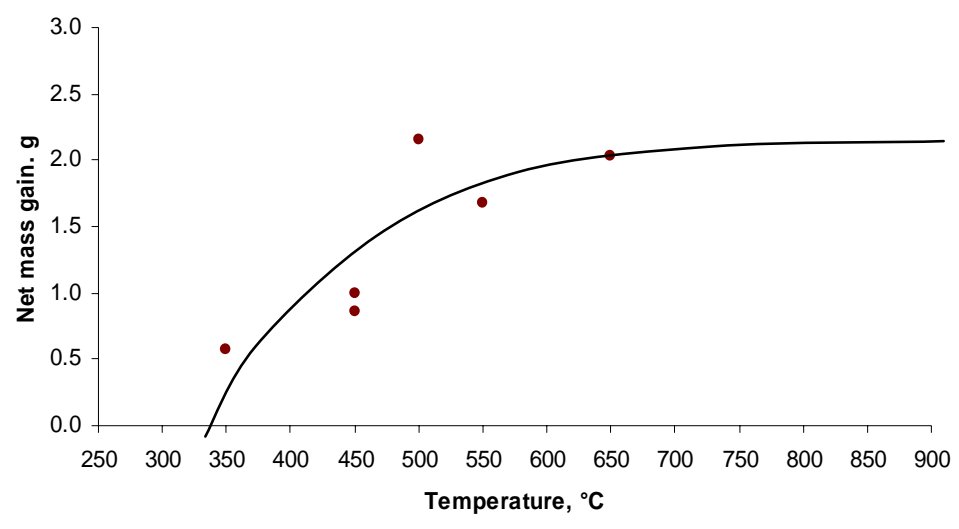

Figure H.9. Net Mass Gain Versus Temperature

The results shown in Figure H.9 are probably subjected to a large experimental error because (unlike in all other experiments) the heat-treatment was not done at a controlled rate of $5{ }^{\circ} \mathrm{C} / \mathrm{min}$, but was performed 
at a fast rate by placing the crucible in the furnace preheated to the set temperature. Steep temperature gradients and rapidly evolving gases caused a large uncertainty in the data. However, the data is reported to show that rapid heating alone is not likely to prevent MIS penetration.

\section{H.3.3. Effect of Ground-Feed Fraction}

Table H.8 lists data on the extent of MIS penetration into CRB rods from mixtures of the original feed and ground feed.

Table H.8. Effect of Ground-Feed Fraction on MIS Penetration to CRB at $550^{\circ} \mathrm{C}$

\begin{tabular}{||r|r|r|r|r||}
\hline Ground feed, fraction & \multicolumn{1}{c|}{$\mathbf{0 . 2}$} & \multicolumn{1}{c|}{$\mathbf{0 . 4}$} & \multicolumn{1}{c|}{$\mathbf{0 . 6}$} & \multicolumn{1}{c|}{$\mathbf{0 . 8}$} \\
\hline Initial mass, g & 49.85 & 47.89 & 45.70 & 45.90 \\
\hline Mass after $550^{\circ} \mathrm{C}, \mathrm{g}$ & 50.08 & 47.95 & 45.05 & 45.22 \\
\hline Mass after $1200^{\circ} \mathrm{C}, \mathrm{g}$ & 49.37 & 47.39 & 44.87 & 45.01 \\
\hline Mass gain at $550^{\circ} \mathrm{C}, \mathrm{g}$ & 0.23 & 0.06 & -0.65 & -0.68 \\
\hline Mass loss at $1200^{\circ} \mathrm{C}, \mathrm{g}$ & 0.71 & 0.56 & 0.18 & 0.21 \\
\hline Mass gain at $1200^{\circ} \mathrm{C}, \mathrm{g}$ & -0.48 & -0.50 & -0.83 & -0.89 \\
\hline Net mass gain at $550^{\circ} \mathrm{C}, \mathrm{g}$ & 1.17 & 0.96 & 0.21 & 0.18 \\
\hline Net mass loss at $1200^{\circ} \mathrm{C}, \mathrm{g}$ & 0.57 & 0.43 & 0.06 & 0.09 \\
\hline Net mass gain at $1200^{\circ} \mathrm{C}, \mathrm{g}$ & 0.59 & 0.53 & 0.15 & 0.10 \\
\hline \hline
\end{tabular}

The $550^{\circ} \mathrm{C}$-net mass gain $\left(G_{\mathrm{N} 1}\right)$ of samples (i.e., the gain compensated for the LOI but not for MIS decomposition) decreased with increasing mass fraction of the ground feed. The extent of MIS decomposition, measured as $1200^{\circ} \mathrm{C}$-net loss, also decreased as the mass fraction of the ground feed increased. The $1200^{\circ} \mathrm{C}$-net mass gain $\left(G_{\mathrm{N} 2}\right)$ of samples (i.e., the gain compensated for the LOI but was not biased by the extent of MIS decomposition) sharply decreased with the increasing mass fraction of the ground feed.

Figure H.10 confirms the observation that the extent of MIS decomposition in feed slightly decreases with the ground fraction of feed (see Figure H.8).
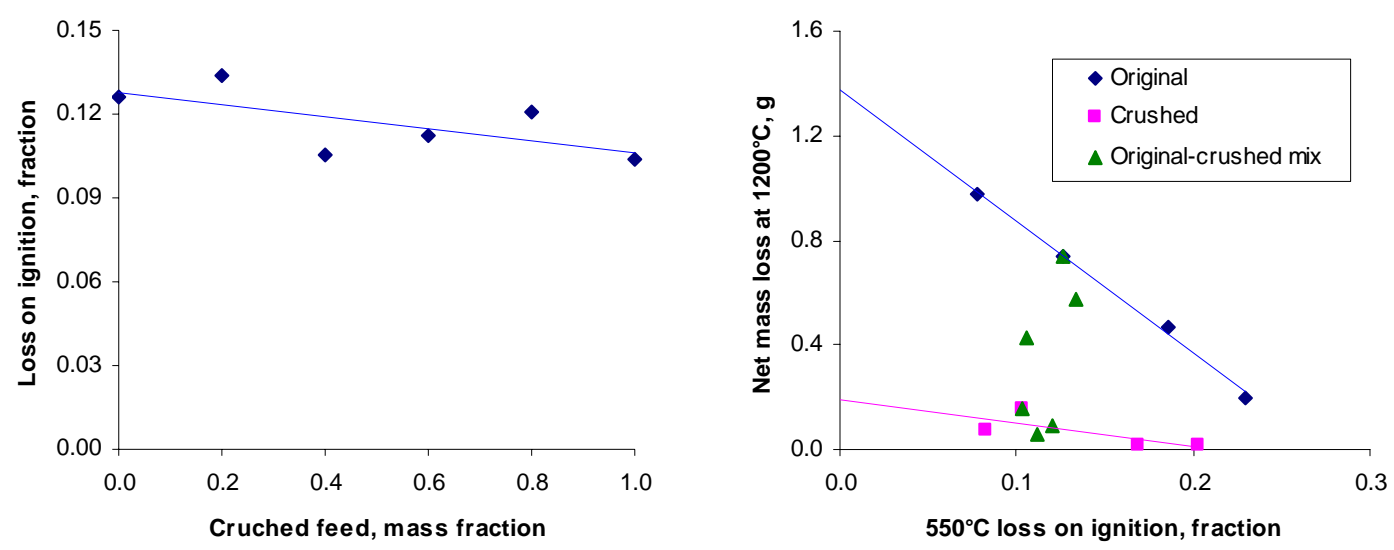

Figure H.10. Mass Loss of Feed at $550^{\circ} \mathrm{C}$ Versus Ground Fraction 
Figure H.11 displays $G_{\mathrm{N} 2}$ as a function of the fraction of ground feed. The line represents the function

$$
G_{N 2}=a+b(1-f)^{n}
$$

where $f$ is the ground fraction, and $a, b$, and $n$ are coefficients listed in Table H.9. The coefficients were fitted to data except the $G_{\mathrm{N} 2}$ value at $f=0.4$, which seems to be an outlier. It appears that the fraction above $60 \%$ of ground feed no longer affects MIS penetration.

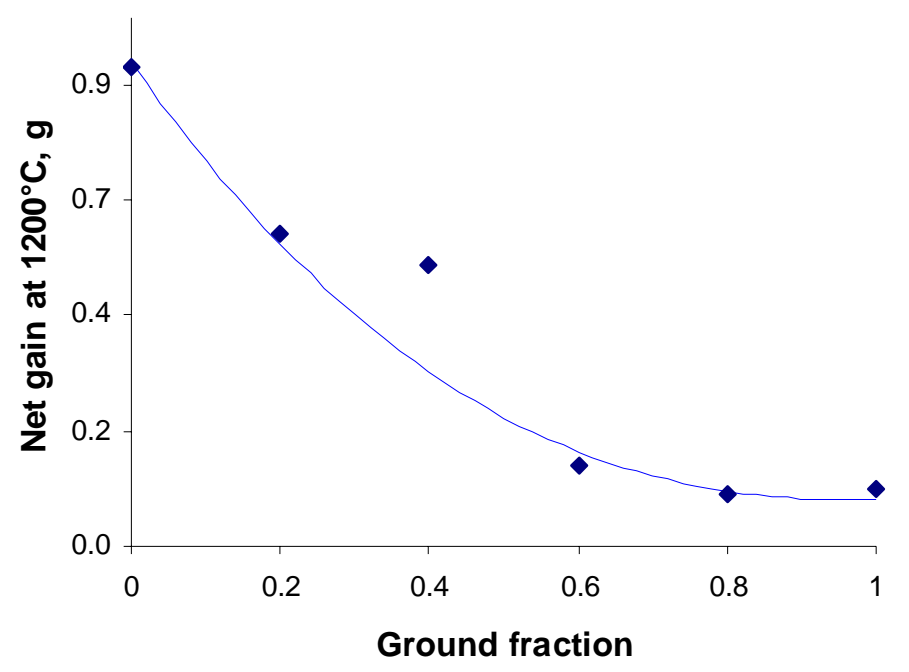

Figure H.11. Effect of Ground Fraction on MIS Penetration to CRB ( $t_{\mathrm{H}}=1$ hour)

Table H.9. Equation (10) Coefficients ( $t_{\mathrm{H}}=1$ hour)

\begin{tabular}{||l|l|l||}
\hline$a$ & $\mathrm{~g}$ & 0.087 \\
\hline$b$ & $\mathrm{~g}$ & 0.829 \\
\hline$n$ & - & 2.40 \\
\hline
\end{tabular}

\section{H.3.4. Effect of Feed Grinding Time}

Whereas mixtures of the original and ground feed have bimodal PSDs, feeds ground to different degrees are more uniform. The purpose of experiments described in this section was to find the minimum necessary degree of grinding that would prevent MIS penetration into the CRB.

Table H.10 lists data on the extent of MIS penetration into CRB rods from feed as a function of the time of grinding the feed in the tungsten carbide mill on 20 -g batches. Figure H.12 displays $G_{\mathrm{N} 2}$ versus the time of feed grinding. The line represents the function

$$
G_{N 2}=a e^{-t_{C} / t_{0}}
$$


where $t_{\mathrm{C}}$ is the grinding time, and $a$ and $t_{0}$ are coefficients listed in Table H.11. The coefficients were fitted to data. It appears that 3 min of grinding (approximately $3 t_{0}$ ) would virtually stop MIS penetration provided that extrapolation of the curve is accurate .

Table H.10. Effect of Grinding Time on MIS Penetration to CRB at $550^{\circ} \mathrm{C}$

\begin{tabular}{||l|c|c|c|c||}
\hline Grinding time, $\mathbf{s}$ & $\mathbf{6 0}$ & $\mathbf{3 0}$ & $\mathbf{1 5}$ & $\mathbf{7 . 5}$ \\
\hline Initial mass, $\mathrm{g}$ & 42.88 & 47.33 & 51.96 & 52.13 \\
\hline Mass after $550^{\circ} \mathrm{C}, \mathrm{g}$ & 42.67 & 47.53 & 52.51 & 52.8 \\
\hline Mass after $1200^{\circ} \mathrm{C}, \mathrm{g}$ & 42.40 & 46.88 & 51.66 & 51.88 \\
\hline Mass gain at $550^{\circ} \mathrm{C}, \mathrm{g}$ & -0.210 & 0.200 & 0.550 & 0.670 \\
\hline Mass loss at $1200^{\circ} \mathrm{C}, \mathrm{g}$ & 0.270 & 0.650 & 0.850 & 0.920 \\
\hline Mass gain at $1200^{\circ} \mathrm{C}, \mathrm{g}$ & -0.48 & -0.45 & -0.30 & -0.25 \\
\hline Net mass gain at $550^{\circ} \mathrm{C}, \mathrm{g}$ & 0.60 & 1.09 & 1.53 & 1.65 \\
\hline Net mass loss at $1200^{\circ} \mathrm{C}, \mathrm{g}$ & 0.15 & 0.52 & 0.71 & 0.78 \\
\hline Net mass gain at $1200^{\circ} \mathrm{C}, \mathrm{g}$ & 0.44 & 0.57 & 0.82 & 0.87 \\
\hline
\end{tabular}

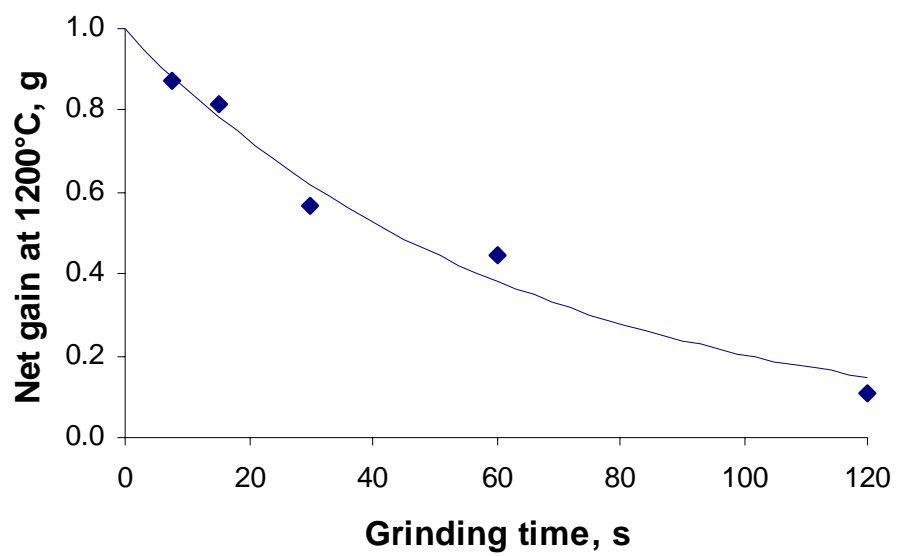

Figure H.12. Effect of Grinding Time on MIS Penetration to CRB $\left(t_{\mathrm{H}}=1\right.$ hour)

Table H.11. Equation (11) Coefficients for $t_{\mathrm{H}}=1$ Hour

\begin{tabular}{||c|c|c||}
\hline \hline$a$ & $\mathrm{~g}$ & 0.997 \\
\hline$t_{0}$ & $\mathrm{~s}$ & 62.82 \\
\hline \hline
\end{tabular}

\section{H.3.5. Ground Feed Density}

To determine the impact of feed grinding on the feed bulk density, ground feed was mixed with original feed in the agate mill for $1 \mathrm{~min}$. The density was determined by filling a 10 -mL plastic container with the feed and measuring the mass. The container was "tapped" five times to settle the feed. For consistency, both ground and original feed were mixed in the agate mill for 1 min before density measurement.

Table H.12 lists the results. Grinding the feed increased its bulk density by $0.083 \mathrm{~mL} / \mathrm{g}$, and the density increased linearly with the ground fraction (Figure H.13). 
Table H.12. Feed Density as a Function of Ground Fraction

\begin{tabular}{||c|c||}
\hline \hline Ground fraction & Density (g/mL) \\
\hline 1.0 & 0.838 \\
\hline 0.8 & 0.823 \\
\hline 0.6 & 0.813 \\
\hline 0.4 & 0.786 \\
\hline 0.2 & 0.762 \\
\hline 0.0 & 0.765 \\
\hline
\end{tabular}

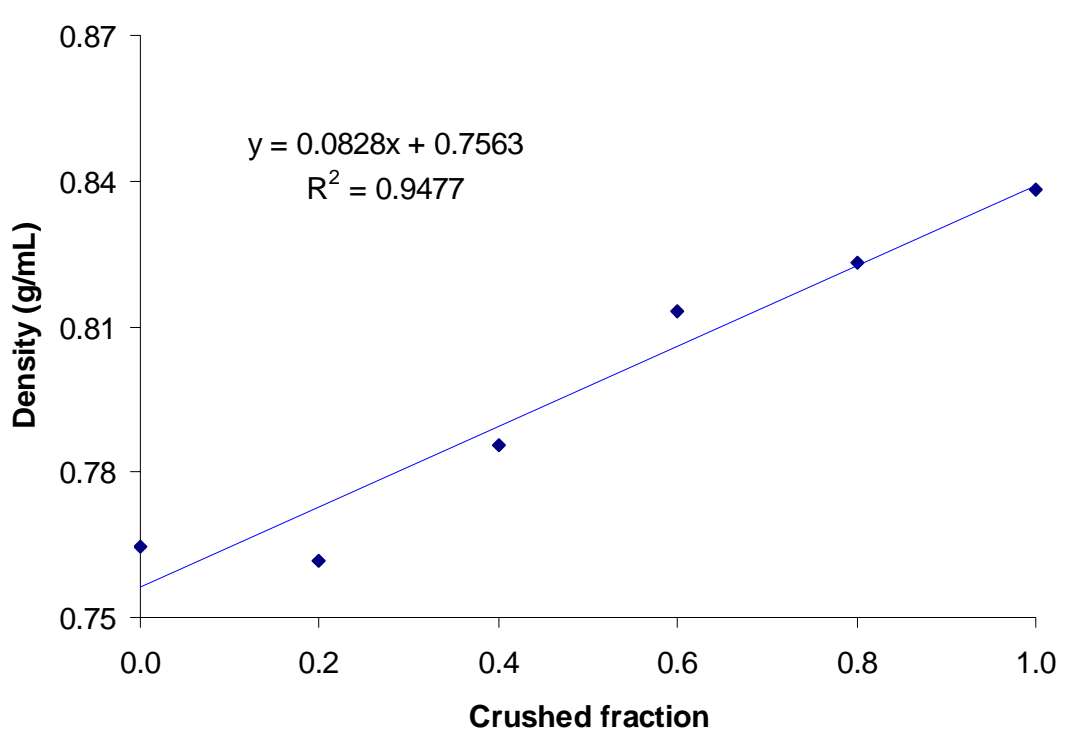

Figure H.13. Feed Density as a Function of Ground Fraction

\section{H.3.6. Ground Feed PSD}

Figure H.14 shows PSDs in original feed and feed ground for 2 min in the tungsten carbide mill. The median grain size was not significantly changed by grinding. Grinding removed large particles and created large fractions of small particles. Deagglomeration significantly shifted particle distribution towards a small particle size, and grinding decreased the median grain size of deagglomerated powder. A question arises whether MIS wets individual deagglomerated particles or particle agglomerates. 


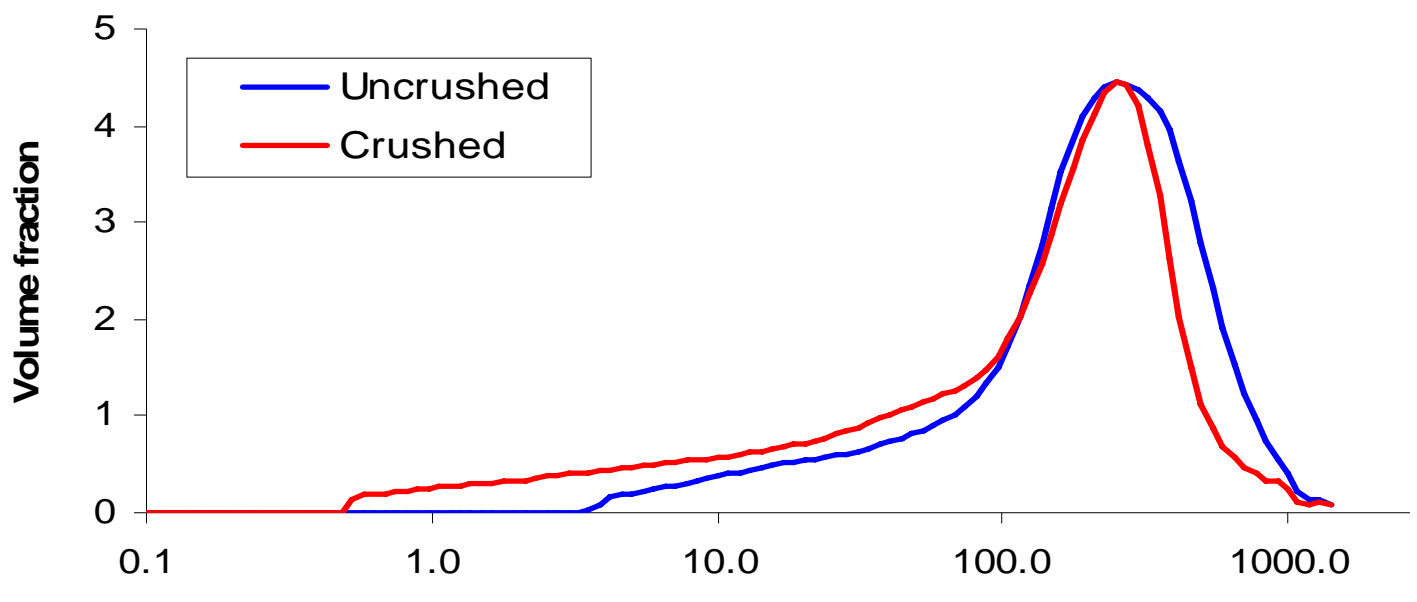

Particle size, $\mu \mathbf{m}$

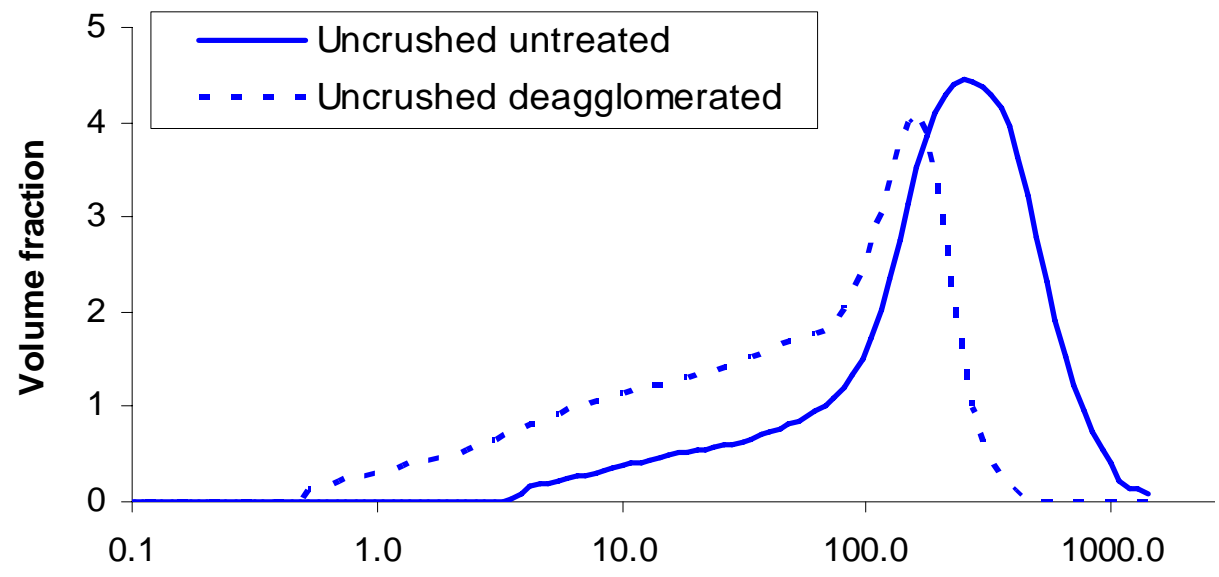

Particle size, $\mu \mathrm{m}$

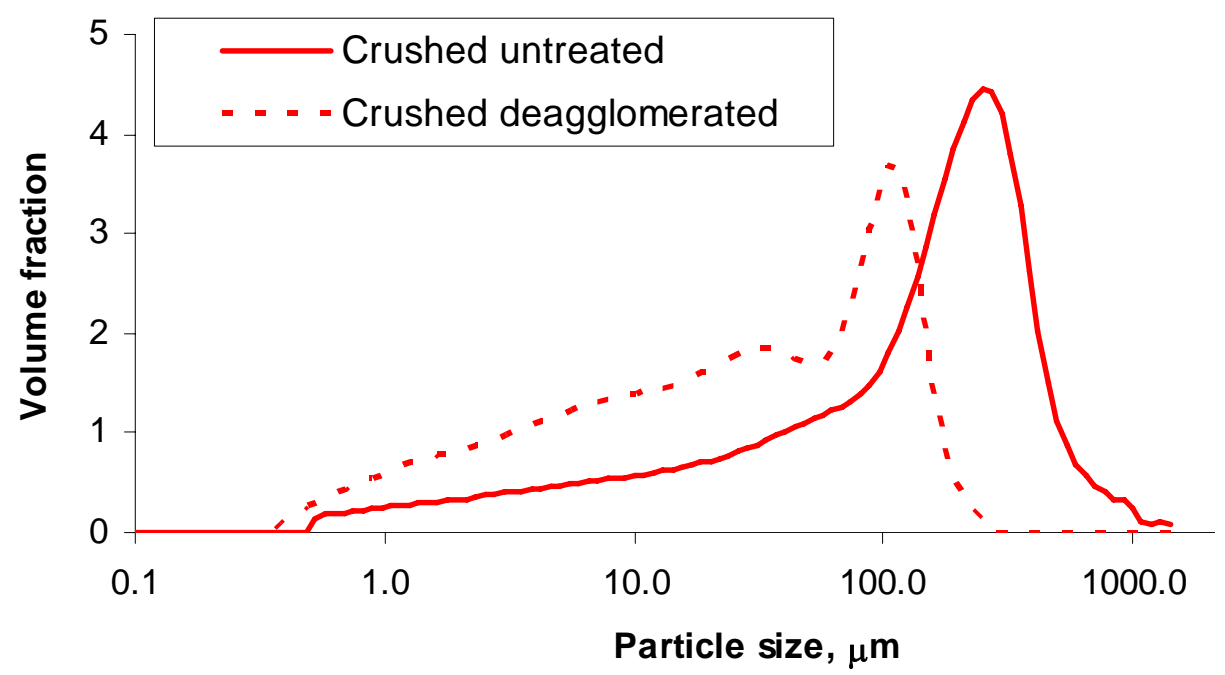

Figure H.14. PSD of Original and Ground (for 120 seconds) Feed, Untreated and Deagglomerated 
To obtain a measure for the specific surface area, we assume that the particles are spherical. The particle volume is $\pi d_{\mathrm{i}}{ }^{3} / 6$, and the particle area is $\pi d_{\mathrm{i}}{ }^{2}$, where $d_{\mathrm{i}}$ is the $i$-th particle effective diameter. The specific surface area of a particle is then $6 / d_{\mathrm{i}}$, and the surface area per unit volume is $6 \Sigma C_{\mathrm{i}} d_{\mathrm{i}}$, where $C_{\mathrm{i}}$ is the $i$-th volume fraction. Hence, the specific surface area $(a)$ is

$$
a=\frac{6}{\rho} \sum_{i} \frac{C_{i}}{d_{i}}
$$

where $\rho$ is the density.

Table H.13 lists the resulting values for feeds ground in the tungsten carbide mill (using an estimated value $\rho=2500 \mathrm{~kg} / \mathrm{m}^{3}$ ). Figure H.15 shows that the relationship is close to linear.

Table H.13. Specific Surface Area as a Function of Feed Grinding Time

\begin{tabular}{|r|c|c|c|c||}
\hline & \multicolumn{2}{|c|}{$\mathbf{s , ~ m m}^{-\mathbf{1}}$} & \multicolumn{2}{c||}{$\mathbf{a} \mathbf{~ m}^{\mathbf{2}} \mathbf{g}$} \\
\hline $\boldsymbol{t}_{\mathrm{C}}, \mathbf{s}$ & Untreated & Deagglomerated & Untreated & Deagglomerated \\
\hline 0 & 82 & 419 & 0.033 & 0.168 \\
\hline 0 & 85 & & 0.034 & \\
\hline 7.5 & 126 & 517 & 0.050 & 0.207 \\
\hline 15 & 125 & 611 & 0.050 & 0.244 \\
\hline 30 & 156 & 568 & 0.063 & 0.227 \\
\hline 60 & 335 & 702 & 0.134 & 0.281 \\
\hline 120 & 592 & 1063 & 0.237 & 0.425 \\
\hline 120 & 644 & & 0.258 & \\
\hline \hline
\end{tabular}

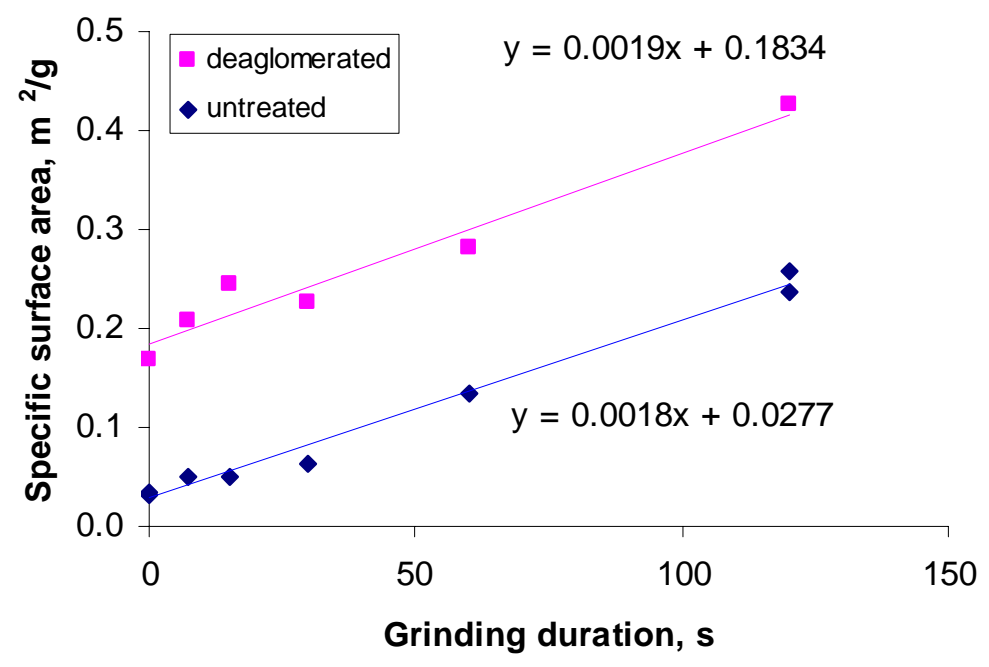

Figure H.15. PSD of Original (as-received) and Ground (for 120 seconds) Feed, Untreated and Deagglomerated 
At a constant temperature, two major parameters affect the amount of MIS that can be released from feed to the CRB. These parameters are the time of exposure and the specific surface area of the feed. Provided that the amount of free MIS is unlimited, the MIS will penetrate into the CRB, driven by capillary and hydrostatic forces. As the FS-38C experiment had shown, MIS can penetrate through the refractory wall and continue flowing through it as long as it is supplied from the feed.

The availability of free MIS from the feed is limited if the volume of feed is small (the feed layer-the cold cap-in the BV melter is thin), or the specific surface area of feed is large. If the relationship between the specific surface area and the grinding time is linear, as Figure H.15 suggests, then the exponential law, Equation (11), will be valid for the specific surface area in the same form, i.e.,

$$
V=V_{0} e^{-a / a_{0}}
$$

where $V$ is the volume of liquid (MIS) that entered the refractory wall, $V_{0}$ is the volume of liquid available in the absence of solid (molten MIS without other feed components), $a$ is the specific surface area of the solid portion of the feed, and $a_{0}$ is a constant. However, plotting the amount of MIS in vibrocast samples against the specific surface area, as shown in Figure H.16, leads to a conclusion that the actual relationship may be linear.

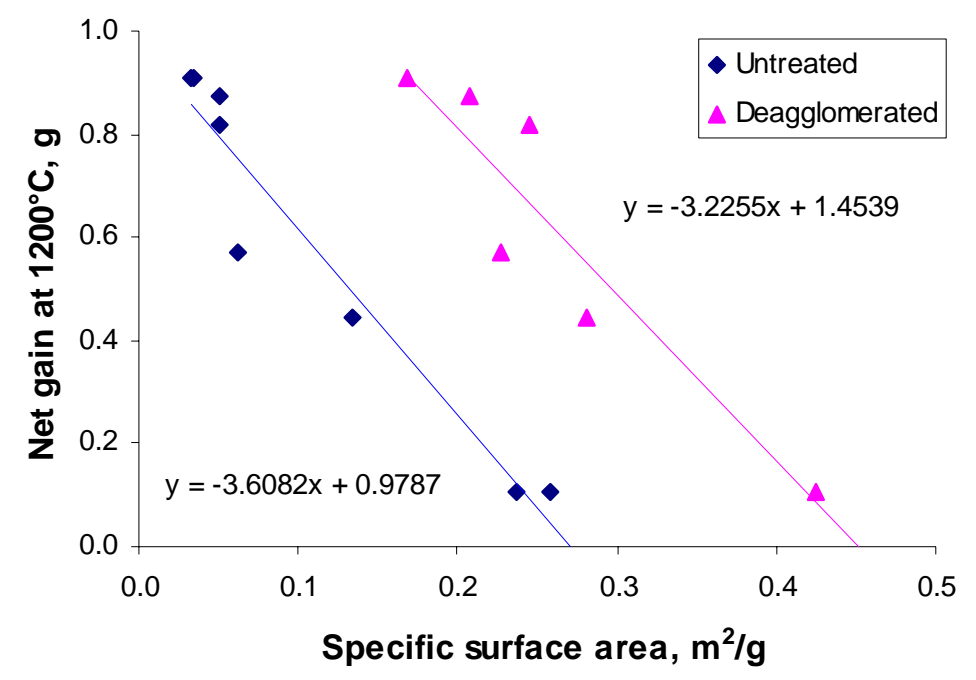

Figure H.16. Effect of Specific Surface Area on MIS Penetration to CRB ( $t_{\mathrm{H}}=1$ hour) 

Appendix I

Impact of Soil Grinding on the Particle Size Distribution and the Specific Surface Area 


\section{Appendix I: Impact of Soil Grinding on the Particle Size Distribution and the Specific Surface Area}

Samples of ground Hanford soil were analyzed for PSD. Figure I.1 shows all data. Note one probable outlier in the 5-min series. Figure I.2 displays averages for each type of sample. Note that deagglomeration had little impact on as-received soil, but substantially shifted the size distribution from large particles to small particles for both of the ground samples. The comparison between original and deagglomerated data shows that a large fraction of large particles are in fact agglomerates. Finally, Figure I.3 compares all averaged data. Grinding for 2 min significantly decreased the soil particle size. It decreased the size of nearly all particles and shifted the particle size from the range between 40 and $500 \mu$ to the range between 0.4 and $40 \mu \mathrm{m}$. The original bell-shaped curve (in semilogarithmic scale) turned into a plateau (uniform distribution) of particle sizes within 1 and $10 \mu \mathrm{m}$ intervals. Further grinding to 5 min had a relatively small impact. 

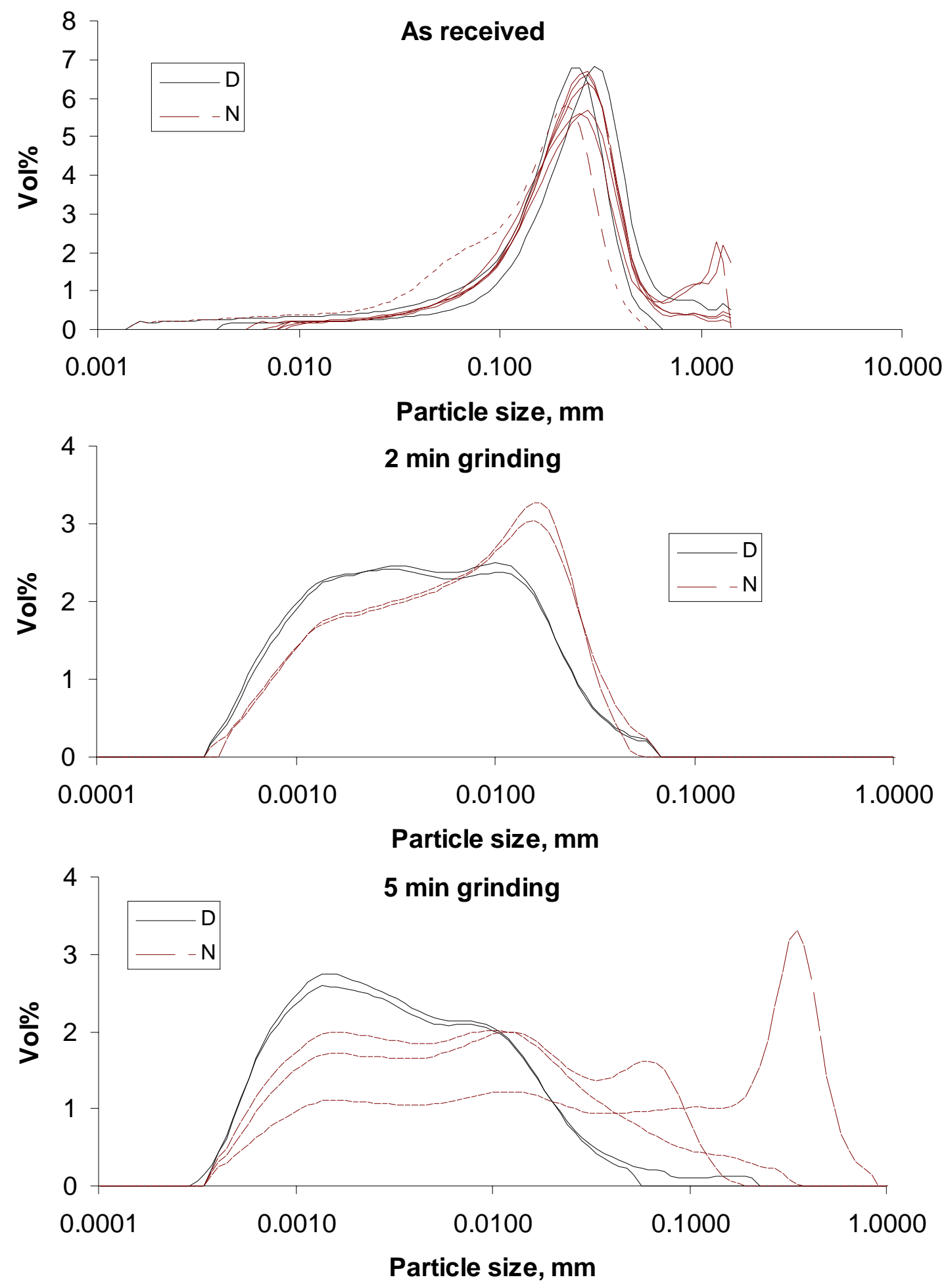

Figure I.1. Repeated Measurements of PSD of Hanford Soil As-Received (top), Ground for 2 Minutes (middle), and Ground for 5 Minutes (bottom); D Deagglomerated Samples, N Original Samples (not deagglomerated) 

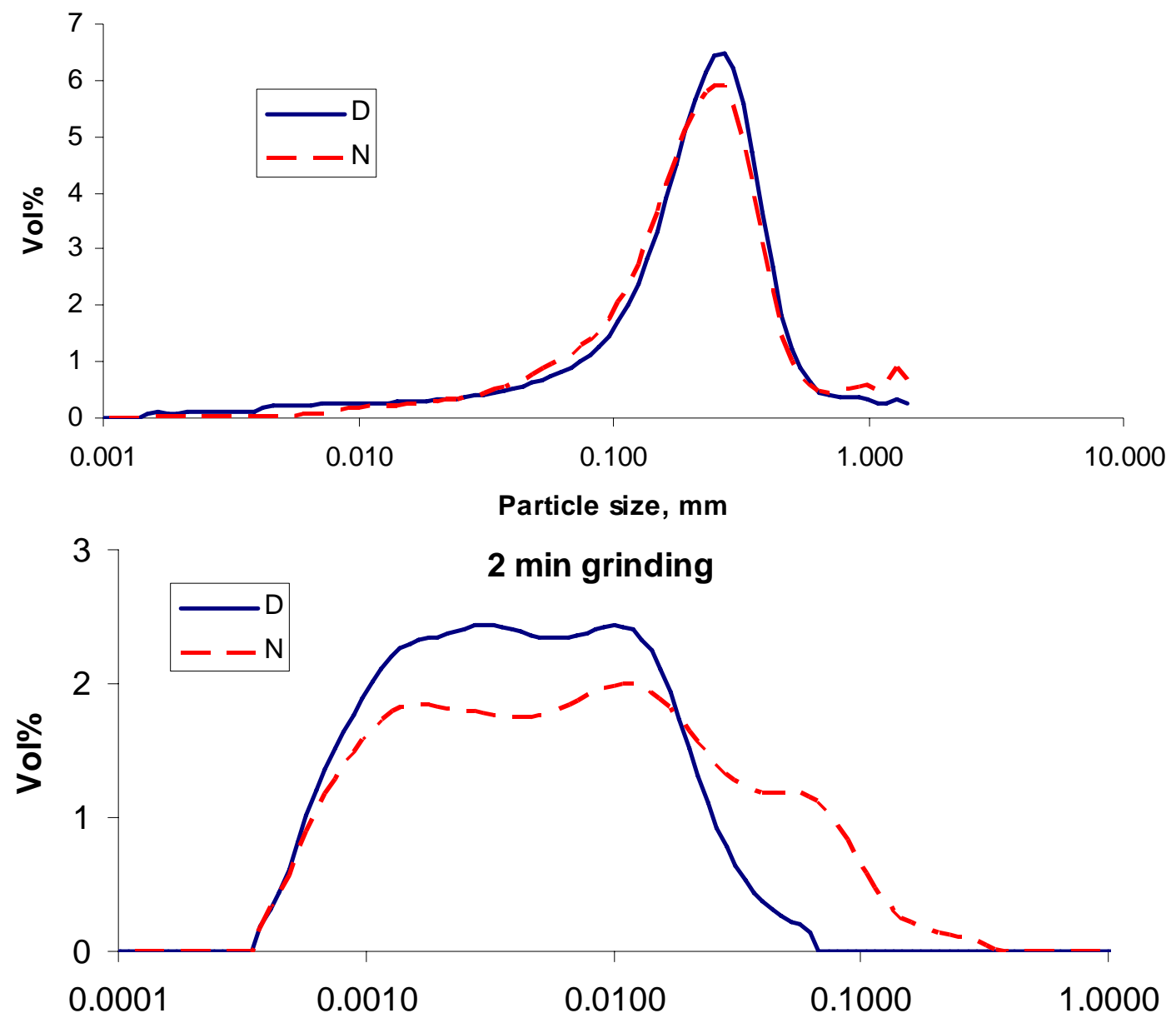

Particle size, $\mathrm{mm}$

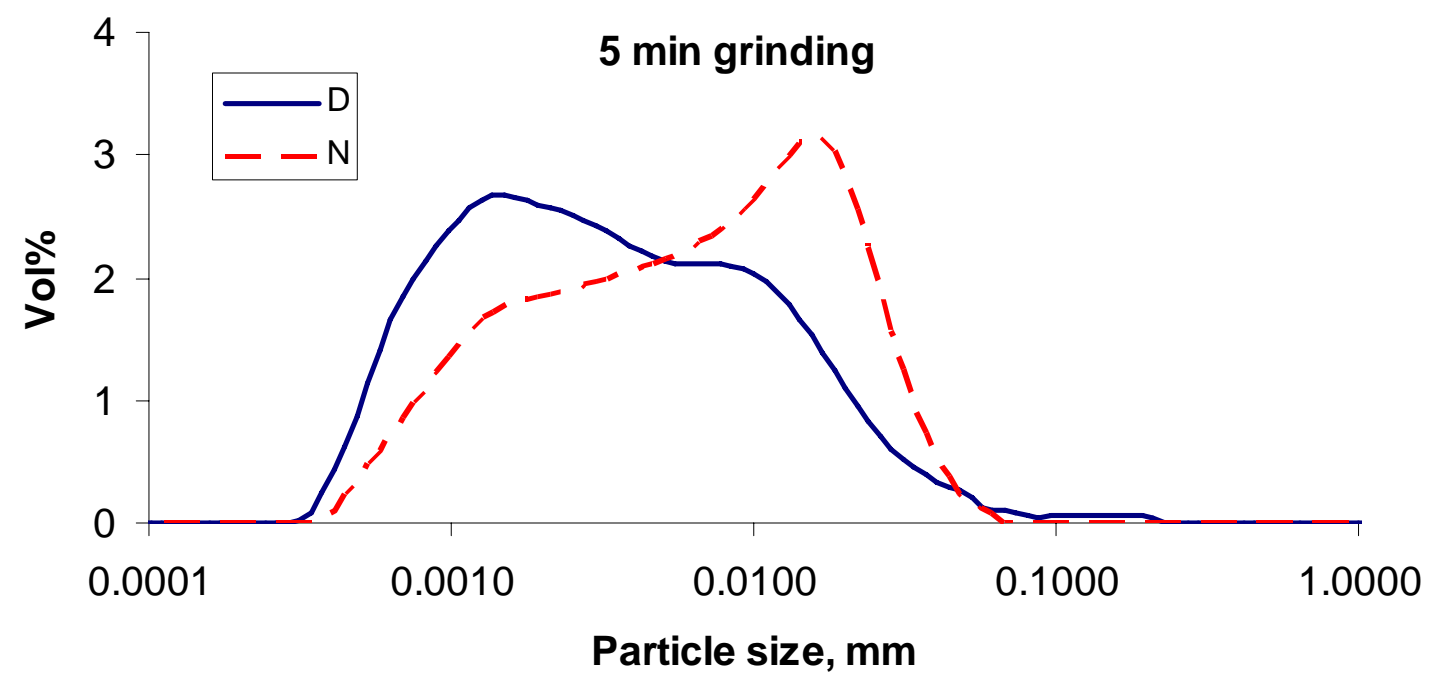

Figure I.2. Averaged Measurements of PSD of Hanford Soil As-Received (top), Ground for 2 Minutes (middle), and Ground for 5 Minutes (bottom); D Deagglomerated Samples, N Original Samples (not deagglomerated) 


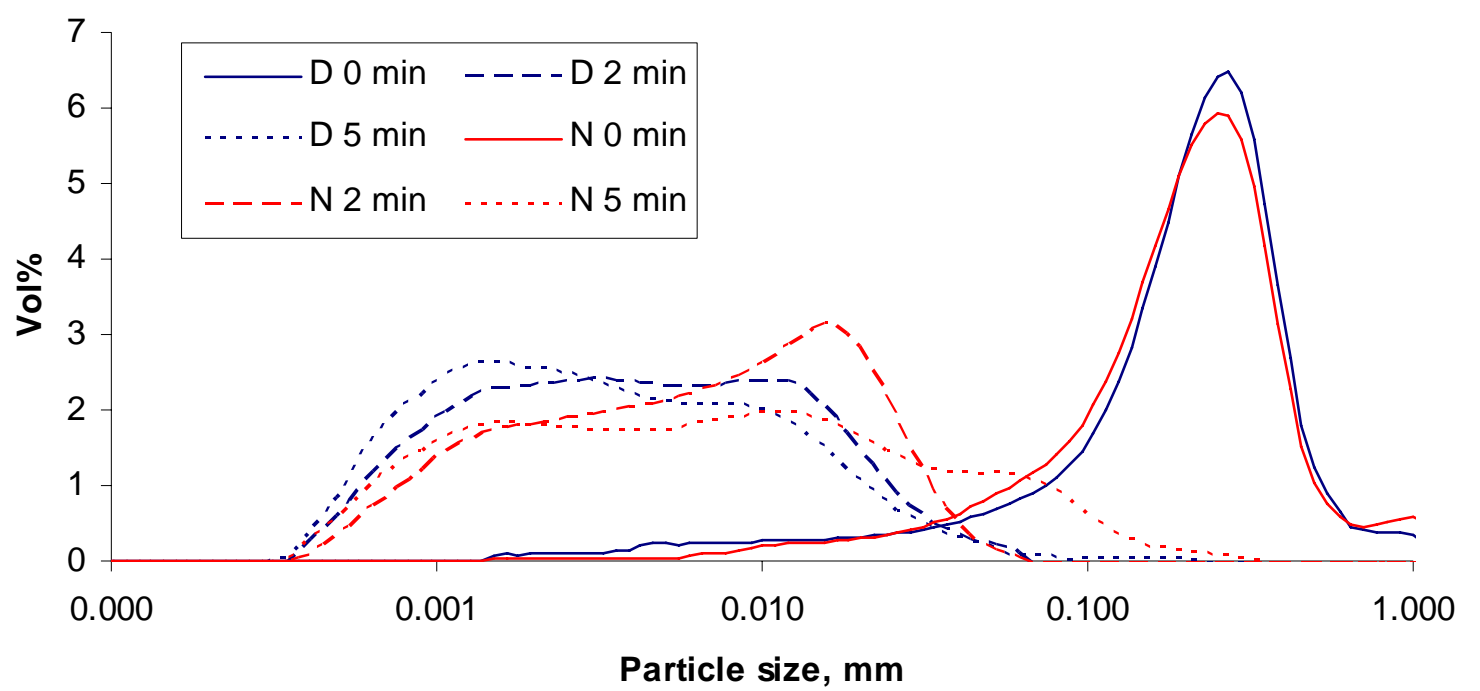

Figure I.3. Comparison of PSD of Hanford Soil As-Received and Ground for 2 and 5 Minutes; D Deagglomerated Samples, N Original Samples (not deagglomerated)

Table I.1 summarizes the main results. Deagglomeration increased the specific surface area of samples by 30 to 50\%. In Figure I.4, the specific surface area (a) of Hanford soil calculated from the PSD is plotted against the grinding time $(t)$ for both original $(\mathrm{N})$ and deagglomerated samples $(\mathrm{D})$. The trend lines were calculated as exponential functions with fitted coefficients shown in the figure. The characteristic time is $\sim 1$ min. Hence, no significant increase of the surface area can be expected after 3 min of grinding. Deagglomerated data show smaller scatter and seem to better represent the surface area.

Table I.1. Specific Surface Area (a) of Hanford Soil Calculated from the PSD as a Function of Grinding Time ( $t$ ) Based on Original (N) and Deagglomerated Samples (D)

\begin{tabular}{||c|c|c||}
\hline \hline $\boldsymbol{t}, \mathbf{m i n}$ & \multicolumn{2}{|c|}{$\boldsymbol{a}, \mathbf{~ m}^{2} / \mathbf{g}$} \\
\hline & $\mathrm{D}$ & $\mathrm{N}$ \\
\hline 0 & 0.040 & 0.027 \\
\hline 2 & 1.047 & 0.790 \\
\hline 5 & 1.248 & 0.884 \\
\hline
\end{tabular}




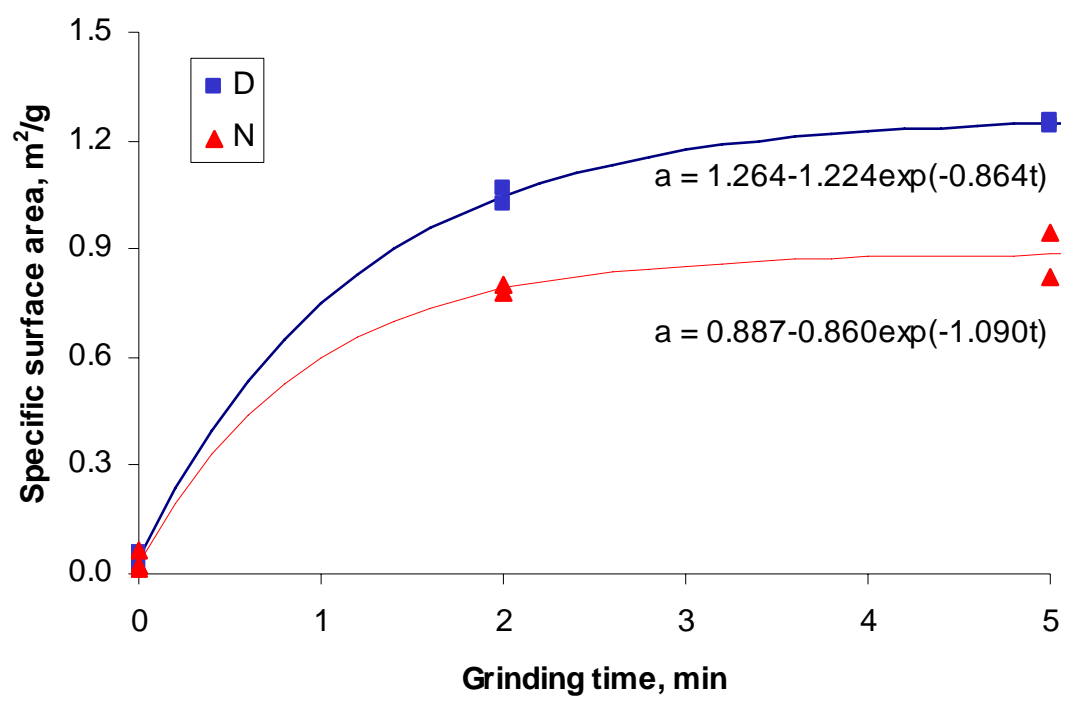

Figure I.4. Specific Surface Area of Hanford Soil Calculated from the PSD as a Function of Grinding Time Based on Original (N) and Deagglomerated Samples (D)

The specific surface area of Hanford soil samples was measured by BET and Langmuir methods at Praxair $^{1}$. Table I.2 lists the results as a function of the time of grinding. As Figure I.5 demonstrates, the BET and Langmuir methods provide the same relative comparisons of samples. A reasonable relative comparison is achieved also with areas calculated from the PSD, even though the absolute values, though linearly related, are not directly proportional—see Figure I.6. Values for deagglomerated samples appear better correlated with BET values. The large intercept can be attributed to the nonsphericity of the soil particles and their porosity.

Table I.2. Specific Surface Area of Hanford Soil Measured with BET and Langmuir Methods

\begin{tabular}{||c|r|c||}
\hline \multirow{2}{*}{ t, min } & \multicolumn{2}{|c|}{$\boldsymbol{a}, \mathbf{~ m}^{2} / \mathbf{g}$} \\
\cline { 2 - 3 } & BET & Langmuir \\
\hline 0 & 5.74 & 9.67 \\
\hline 2 & 9.75 & 16.59 \\
\hline 5 & 10.78 & 18.42 \\
\hline
\end{tabular}

\footnotetext{
${ }^{1}$ Praxair Specialty Ceramics, Woodinville, WA
} 


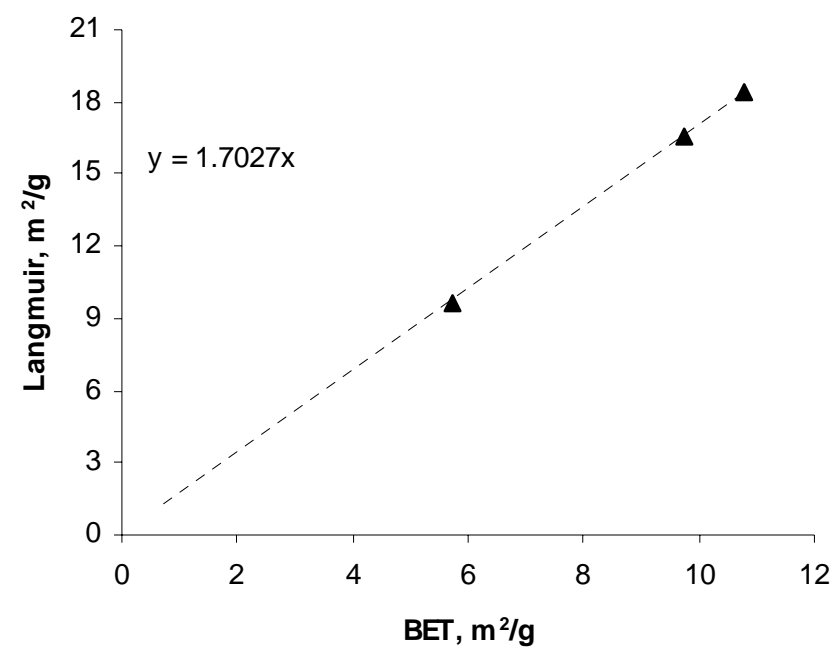

Figure I.5. Comparison of Specific Surface Area of Hanford Soil Measured with BET and Langmuir Methods

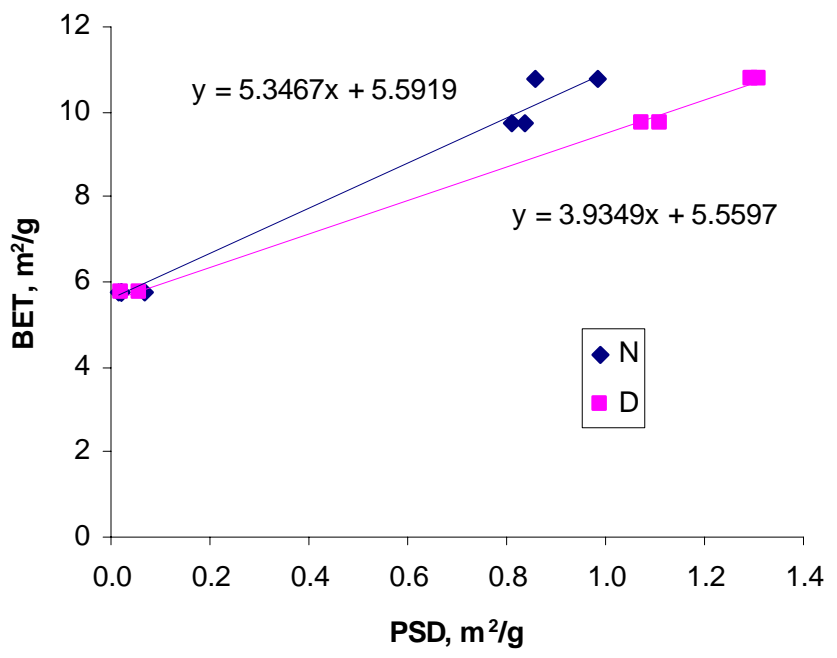

Figure I.6. Comparison of Specific Surface Area of Hanford Soil Measured with BET Method with the Area Calculated from the PSD; D Deagglomerated Samples, N Original Samples (not deagglomerated) 
Appendix $\mathbf{J}$

\section{Effect of Temperature on MIS Migration for Feeds with S-109 Simulant}




\section{Appendix J: Effect of Temperature on MIS Migration for Feeds with S-109 Simulant}

Tables J.1 and J.2 list the results of MIS penetration measurements for the original (as-received) and 2-min ground soil in the feeds with S-109 simulant. The net mass gain (the mass of fully decomposed MIS corrected for crucible LOI) was very low at $300^{\circ} \mathrm{C}$ and increased with increasing temperature with a decreasing rate (Figure J.1). The penetrated MIS from feed with 2-min ground soil amounted to 27 mass $\%$ of that from feed with as-received soil at both $400^{\circ} \mathrm{C}$ and $500^{\circ} \mathrm{C}$.

Table J.1. Effect of Temperature on MIS Penetration to Silica Crucible from Feed with S-109 Simulant and As-Received Soil

\begin{tabular}{||l|r|r|r||}
\hline \hline Temperature $\mathbf{( T ) , ~}{ }^{\circ} \mathbf{C}$ & \multicolumn{1}{c|}{$\mathbf{3 0 0}$} & \multicolumn{1}{c|}{$\mathbf{4 0 0}$} & \multicolumn{1}{c|}{$\mathbf{5 0 0}$} \\
\hline Initial mass, g & 357.05 & 358.91 & 356.26 \\
\hline Mass after $T$, g & 357.32 & 375.16 & 385.36 \\
\hline Mass after $1000^{\circ} \mathrm{C}, \mathrm{g}$ & 356.59 & 364.83 & 367.12 \\
\hline Mass gain at $T, \mathrm{~g}$ & 0.27 & 16.25 & 29.10 \\
\hline Mass loss at $1000^{\circ} \mathrm{C}, \mathrm{g}$ & 0.73 & 10.33 & 18.24 \\
\hline Mass gain at $1000^{\circ} \mathrm{C}, \mathrm{g}$ & -0.46 & 5.92 & 10.86 \\
\hline Net mass gain at $T, \mathrm{~g}$ & 0.91 & 23.00 & 35.80 \\
\hline Net mass loss at $1000^{\circ} \mathrm{C}, \mathrm{g}$ & 0.62 & 10.22 & 18.13 \\
\hline Net mass gain at $1000^{\circ} \mathrm{C}, \mathrm{g}$ & 0.29 & 13.64 & 18.53 \\
\hline \hline
\end{tabular}

Table J.2. Effect of Temperature on MIS Penetration to Silica Crucible from Feed with S-109 Simulant and 2-Minute Ground Soil

\begin{tabular}{||l|r|r|r||}
\hline Temperature $\mathbf{( T )},{ }^{\circ} \mathbf{C}$ & \multicolumn{1}{c|}{$\mathbf{3 0 0}$} & \multicolumn{1}{c|}{$\mathbf{4 0 0}$} & \multicolumn{1}{c|}{$\mathbf{5 0 0}$} \\
\hline Initial mass, g & 355.75 & 354.54 & 355.33 \\
\hline Mass after $T$, g & 355.57 & 363.74 & 367.11 \\
\hline Mass after $1000^{\circ} \mathrm{C}, \mathrm{g}$ & 355.19 & 357.53 & 359.47 \\
\hline Mass gain at $T, \mathrm{~g}$ & -0.18 & 9.2 & 11.78 \\
\hline Mass loss at $1000^{\circ} \mathrm{C}, \mathrm{g}$ & 0.38 & 6.21 & 7.64 \\
\hline Mass gain at $1000^{\circ} \mathrm{C}, \mathrm{g}$ & -0.56 & 2.99 & 4.14 \\
\hline Net mass gain at $T, \mathrm{~g}$ & 0.46 & 9.84 & 12.42 \\
\hline Net mass loss at $1000^{\circ} \mathrm{C}, \mathrm{g}$ & 0.27 & 6.10 & 7.53 \\
\hline Net mass gain at $1000^{\circ} \mathrm{C}, \mathrm{g}$ & 0.19 & 3.73 & 4.89 \\
\hline \hline
\end{tabular}




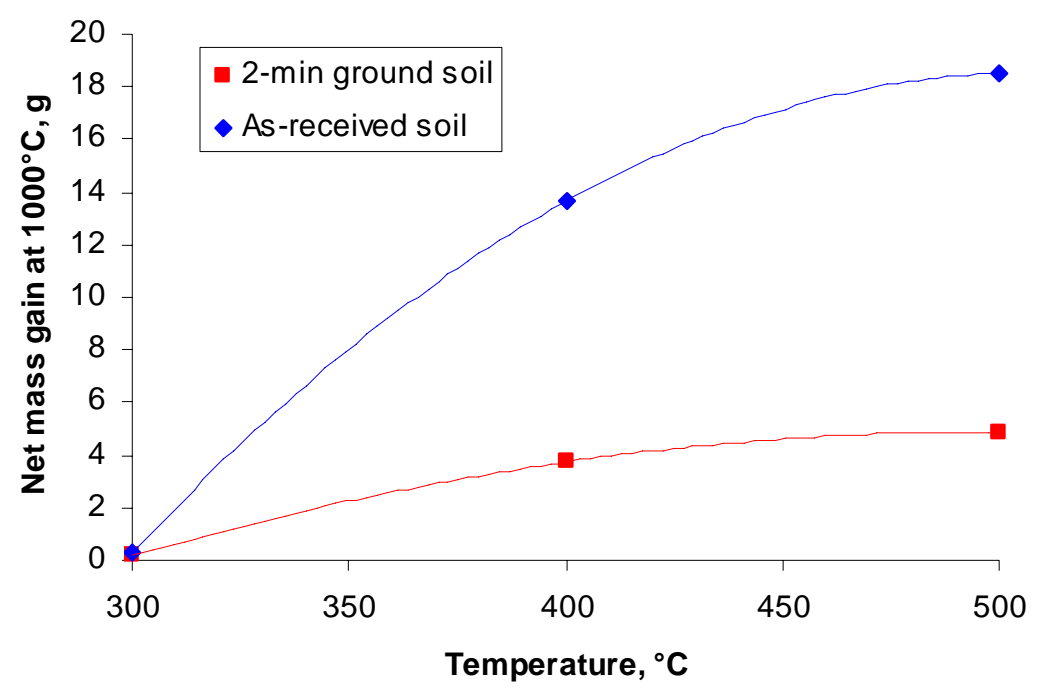

Figure J.1. Effect of Temperature on MIS Penetration to CRB from Feed with S-109 Simulant

Figures J.2 through J.4 present photographic documentation of the crucible appearance. Note that MIS from the feed with as-received soil penetrated to the outer wall of the crucible heated to $500^{\circ} \mathrm{C}$ (Figures J.2 and J.4). No visible penetration of MIS occurred at $300^{\circ} \mathrm{C}$ (Figure J.2), though some presence of MIS is visible on the crucible surface in the meniscus area (Figure J.4). 
Temperature

${ }^{\circ} \mathrm{C}$

As-received

soil

300

400

500

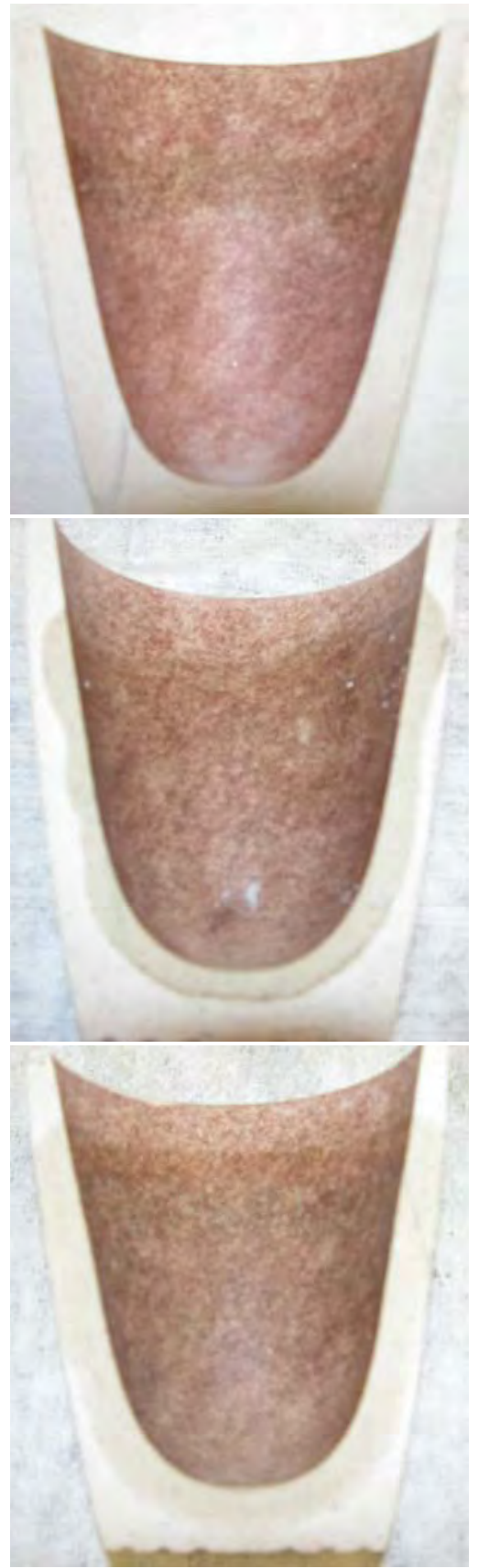

2-min ground

soil
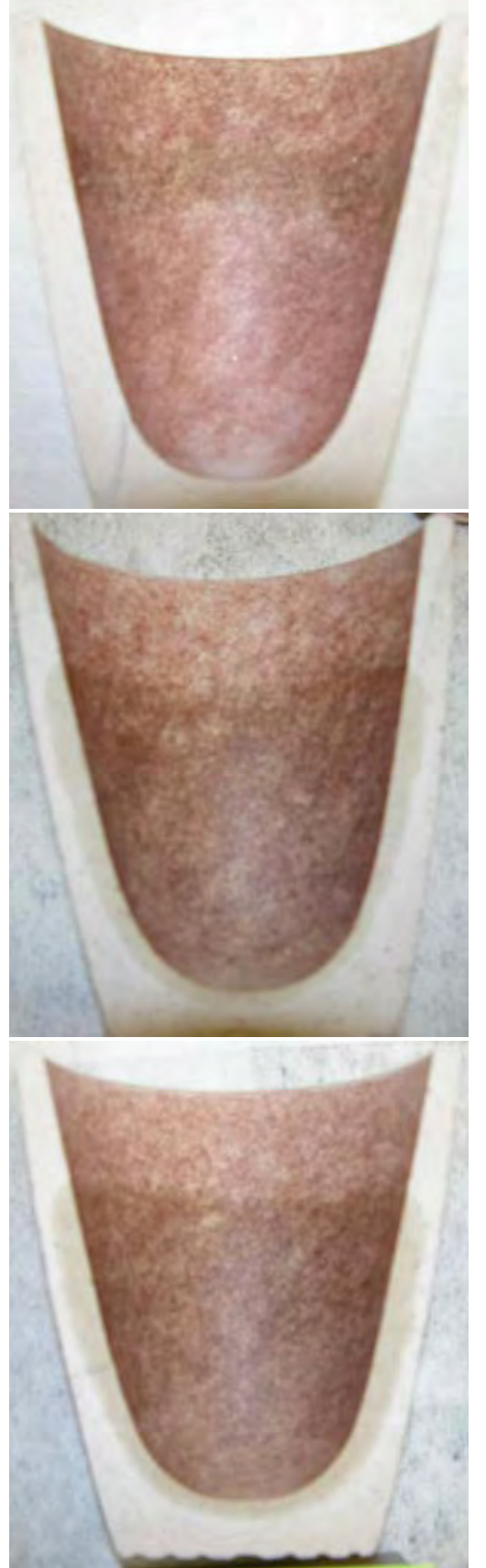

Figure J.2. Sections Through Silica Crucibles in Which Feed Containing S-109 LAW Simulant and Soil (as-received and 2-min ground) Was Heated for 30 Minutes at Temperatures of $300^{\circ} \mathrm{C}, 400^{\circ} \mathrm{C}$, and $500^{\circ} \mathrm{C}$. The feed was removed, and crucibles were heated for $60 \mathrm{~min}$ at $1000^{\circ} \mathrm{C}$ before sectioning. 


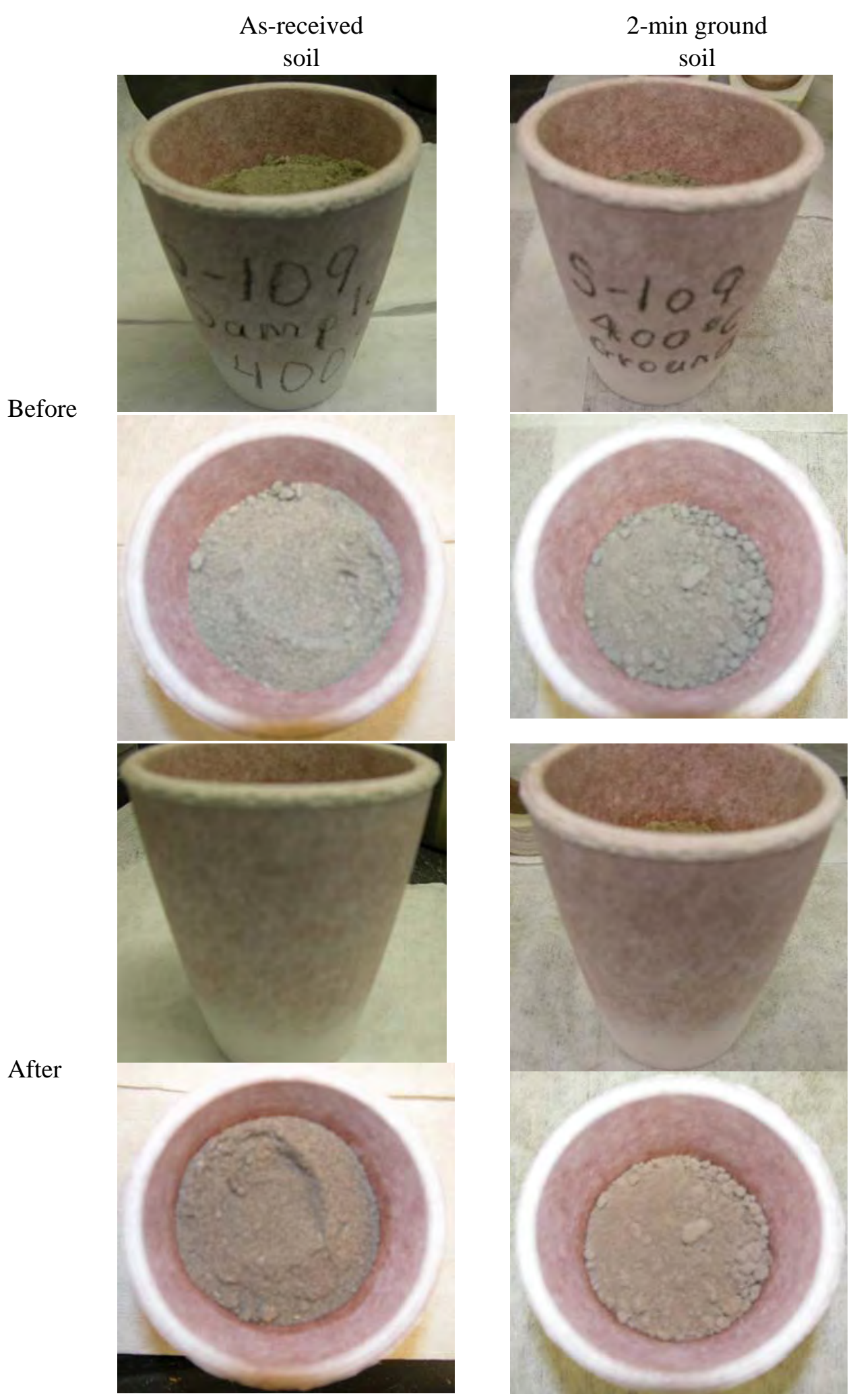

Figure J.3. Silica Crucibles with Feed Containing S-109 LAW Simulant and Soil (as-received and 2-min ground) Before and After 30-Minute Heat-Treatment at $400^{\circ} \mathrm{C}$ 
Temperature

${ }^{\circ} \mathrm{C}$

300

400

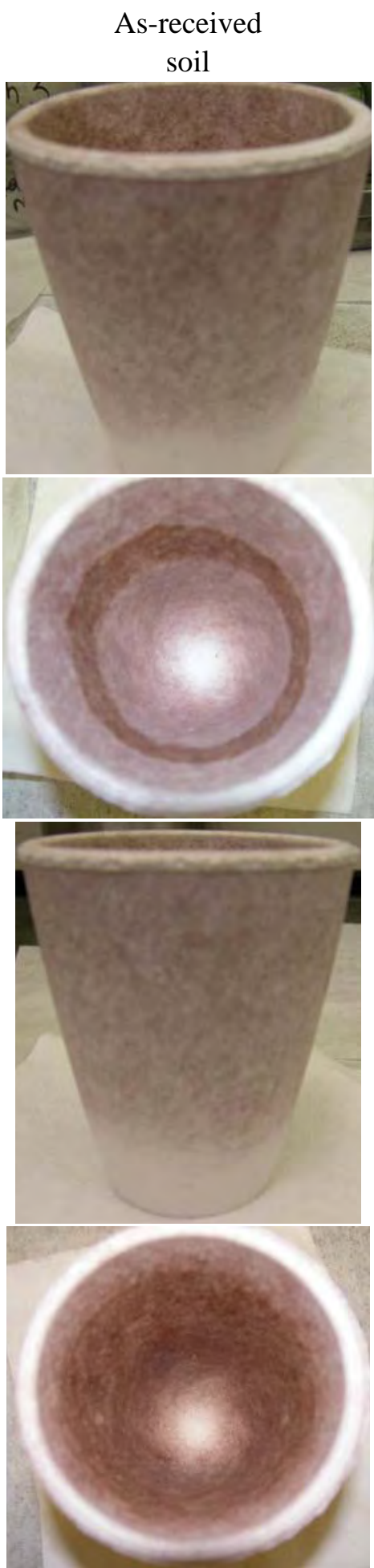

2-min ground

soil
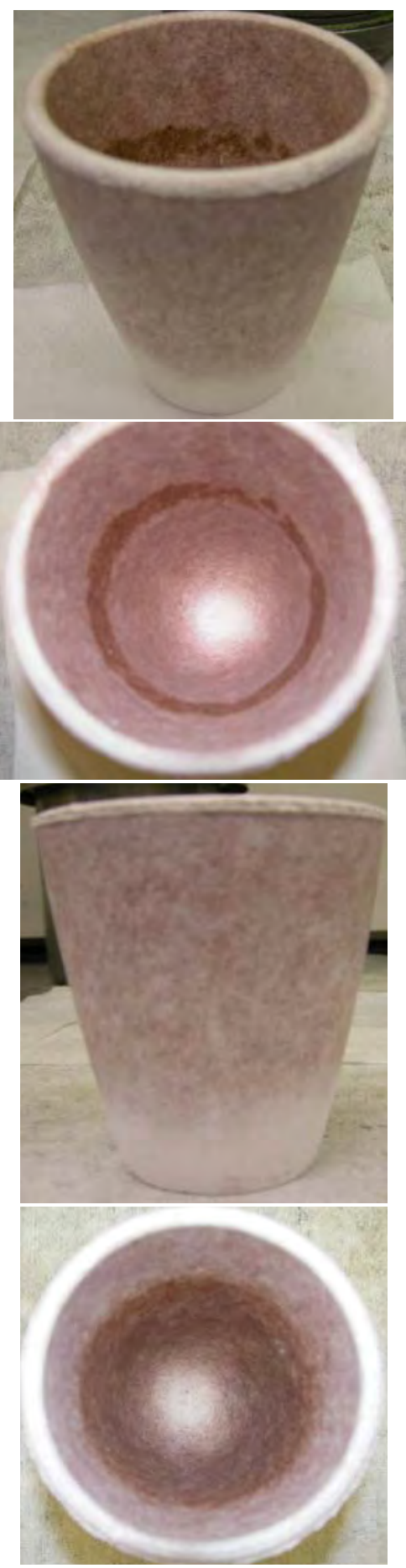

Figure J.4. Appearance of Silica Crucibles in Which Feed Containing S-109 LAW Simulant and Soil (as-received and 2-min ground) Was Heated for 30 Minutes at Temperatures of $300^{\circ} \mathrm{C}, 400^{\circ} \mathrm{C}$, and $500^{\circ} \mathrm{C}$. The feed was removed, and crucibles were heated for $60 \mathrm{~min}$ at $1000^{\circ} \mathrm{C}$. 


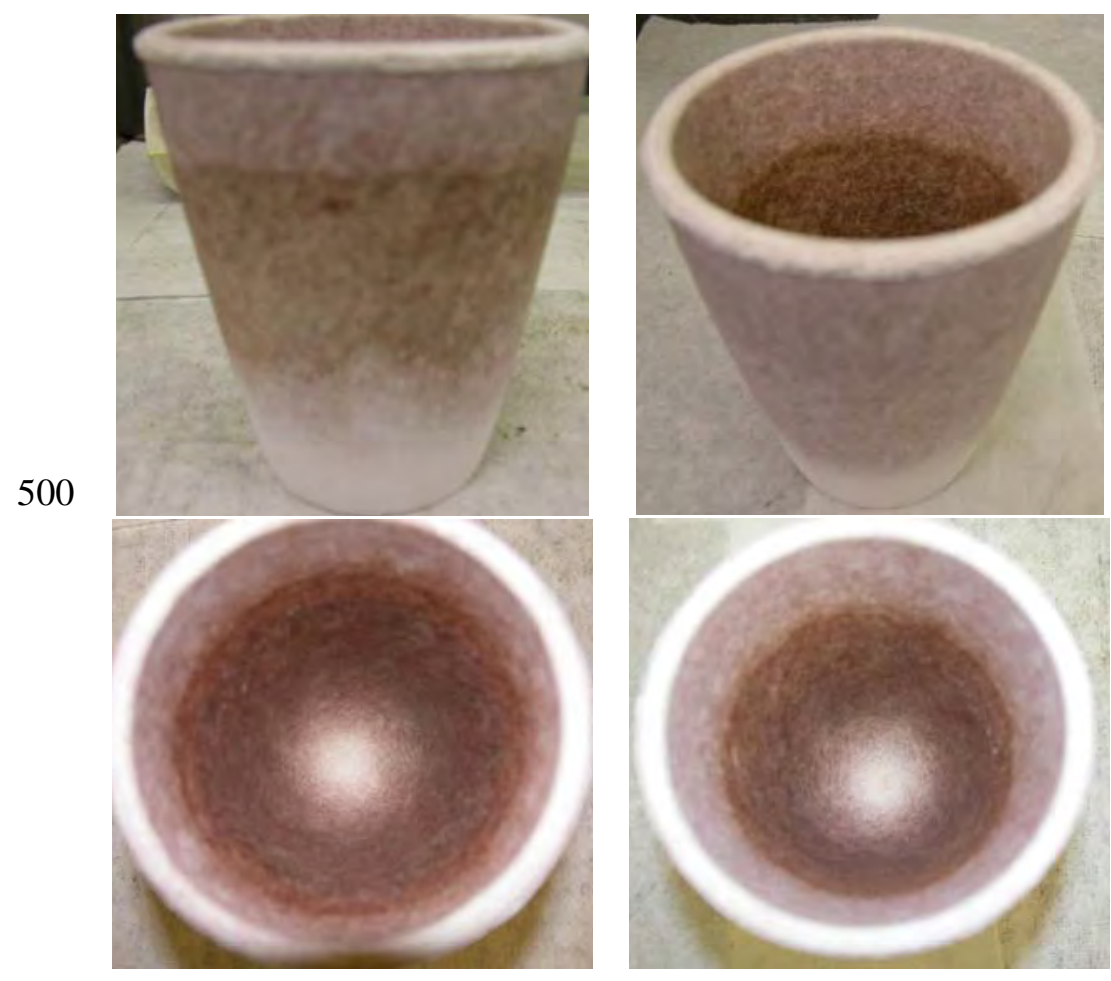

Figure J.4 (Contd) 
Appendix K

Effects of Soil Grinding, Fine-Grained Materials, and Carbohydrate Addition on MIS Migration for Feeds with S-109 Simulant 


\section{Appendix K: Effects of Soil Grinding, Fine-Grained Materials, and Carbohydrate Addition on MIS Migration for Feeds with S-109 Simulant}

The baseline feed contained S-109 simulant, HRTS05 soil, $\mathrm{ZrO}_{2}$, and $\mathrm{B}_{2} \mathrm{O}_{3}$. Feeds were also prepared with additions of diatomaceous earth, bentonite, formic acid, and sucrose. Table K.1 lists selected batch sheets. Feeds were prepared with soil as-received or ground for 2 min in 20-g batches.

Table K.1. Batch Sheets for Feeds Containing S-109 Simulant

\begin{tabular}{|c|c|c|c|c|c|c|}
\hline Materials $^{\text {(a) }}$ & " Baseline & 15\% DE & $20 \% \mathrm{DE}$ & 5\% B & $25 \% \mathrm{~S}$ & $50 \% \mathrm{~S}$ \\
\hline Target glass mass & 500 & 150 & 250 & 150 & 150 & 150 \\
\hline Liquid S-109 simulant & 725.9 & 217.8 & 363.0 & 217.8 & 217.7 & 217.7 \\
\hline HRTS05 soil & 351.0 & 82.3 & 124.4 & 97.6 & 105.3 & 105.3 \\
\hline Diatomaceous earth & & 22.9 & 51.0 & & & \\
\hline Bentonite & & & & 7.75 & & \\
\hline Sucrose & & & & & 6.55 & 13.1 \\
\hline $\mathrm{ZrO}_{2}$ & 35.0 & 10.5 & 17.5 & 10.5 & 10.5 & 10.5 \\
\hline $\mathrm{B}_{2} \mathrm{O}_{3}$ & 26.2 & 7.9 & 13.1 & 7.9 & 7.9 & 7.9 \\
\hline $\begin{array}{l}\text { (a) Mass of each componen } \\
\mathrm{DE}=\text { diatomaceous earth, } \mathrm{B} \\
\% \mathrm{DE} \text { and } \% \mathrm{~B} \text { indicate the } \mathrm{f}\end{array}$ & $\begin{array}{l}\text { is in g. } \\
=\text { bentonite, } \mathrm{S} \\
\text { action of glas }\end{array}$ & $\begin{array}{l}\text { = sucrose } \\
\text { componer }\end{array}$ & . & חס & B. & \\
\hline
\end{tabular}

Feeds were prepared by adding solids to liquid simulant during continuous stirring and drying. Sucrose was dissolved in liquid simulant before the solids were added. Feeds were added to silica crucibles and heated at $5^{\circ} \mathrm{C} / \mathrm{min}$ to $500^{\circ} \mathrm{C}$ with 30 -min hold. Feeds were then removed from crucibles and the crucibles were heated for 1 hour at $1000^{\circ} \mathrm{C}$. Initial masses of feeds and crucible and masses after each heattreatment were obtained. After the $1000^{\circ} \mathrm{C}$ heat treatment, crucibles were vertically sectioned. Sections of crucibles are compared in Figures K.1 through K.7. The results of measurements are listed in Tables K.2 through K.4 and displayed in Figures K.8 through K.11. The values listed in "Feed RT" column in Table K.4 represent feed mass without sucrose or formic acid. The percentage of sucrose (stoichiometric\%) is defined as the atomic ratio of carbon from sucrose to nitrogen in MIS multiplied by 100. 


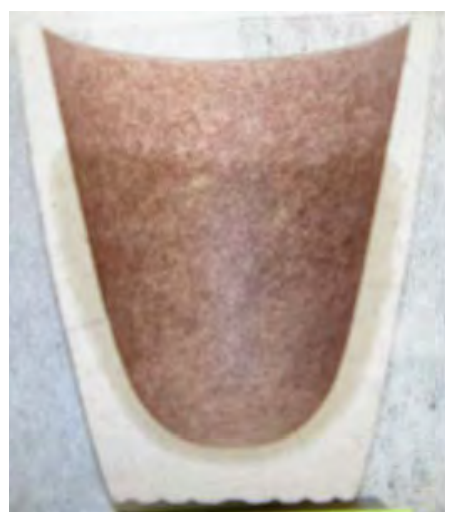

S-109

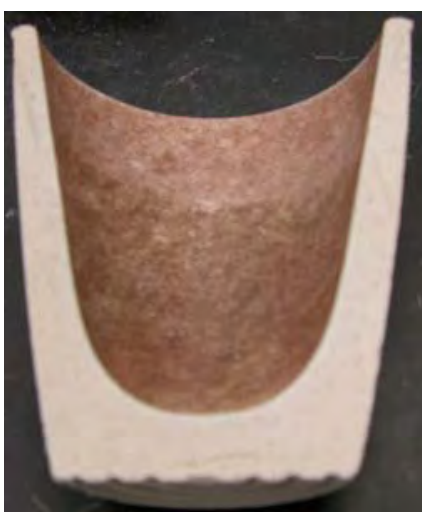

six-tank

Figure K.1. MIS Penetration into Silica Crucible; Feeds Were Prepared from Hanford Soil Ground for 2-Minutes and Two Types of LAW Simulant
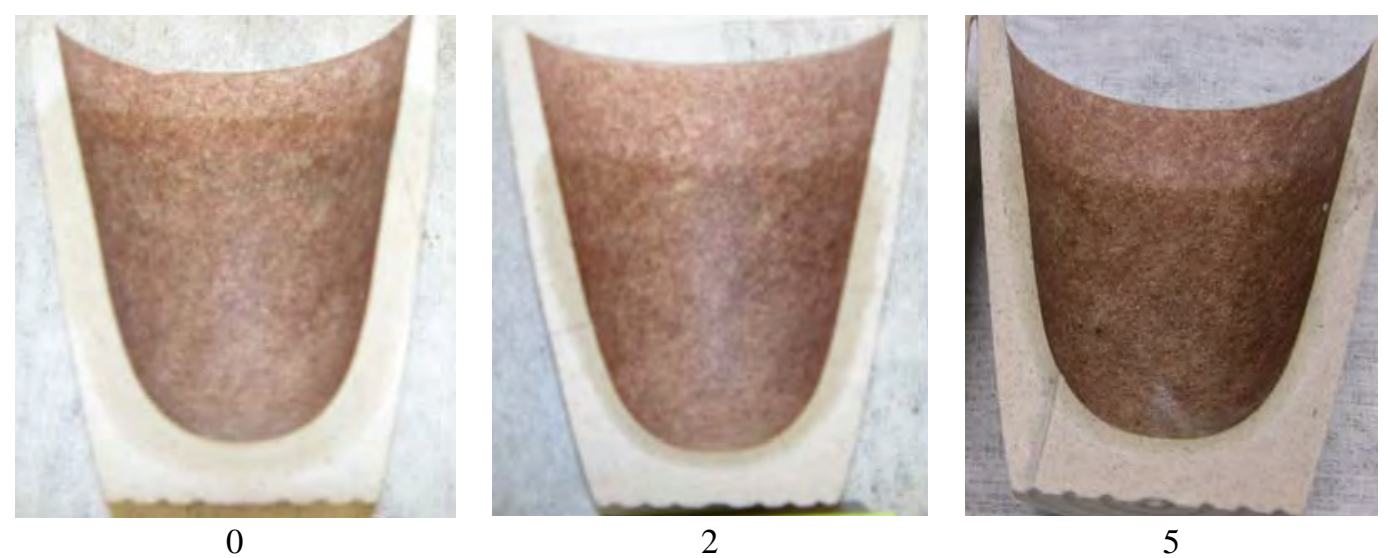

Figure K.2. MIS Penetration into Silica Crucible from Feeds with s-109 Simulant; Hanford Soil Was Ground for 0, 2, and 5 min 


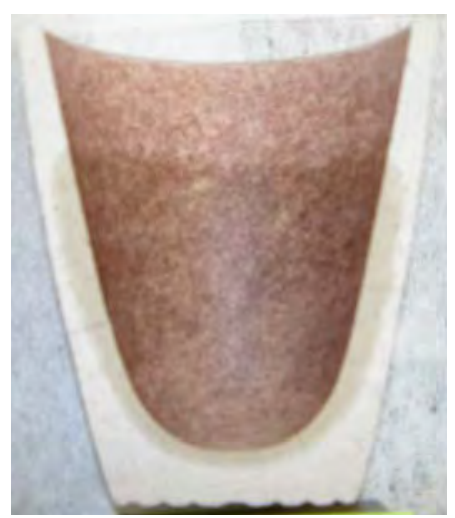

No addition

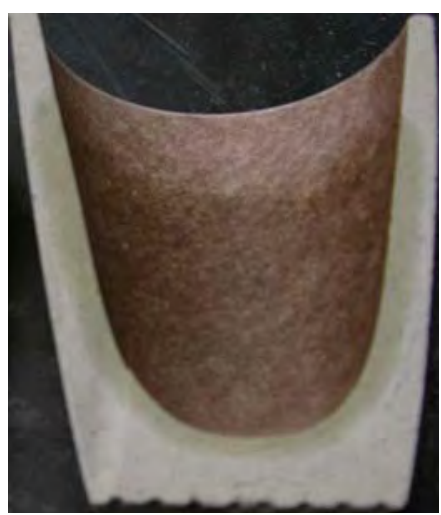

5 mass\% bentonite

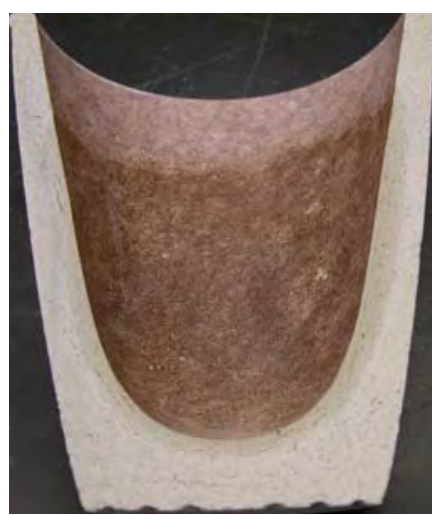

5 mass $\%$ DE

Figure K.3. Effect of 5-Mass\% Bentonite and 5-Mass\% Diatomaceous Earth Additions on MIS Penetration into Silica Crucible from Feeds with s-109 Simulant and 2-Minute Ground Hanford Soil

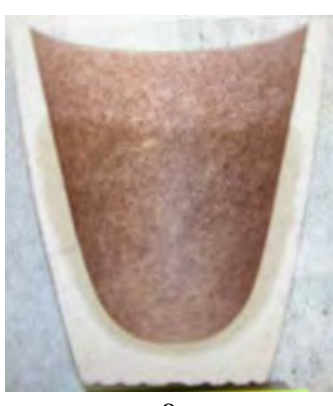

0

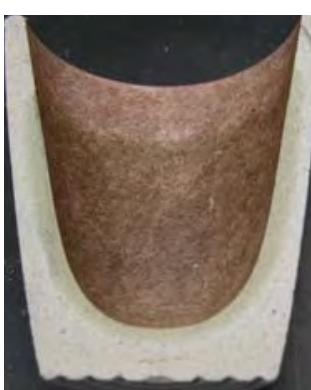

8.7

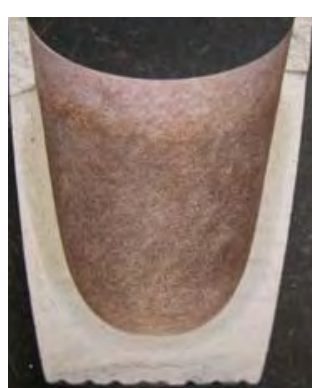

15

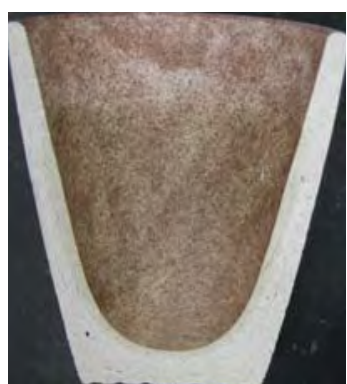

20

Figure K.4. Effect of Diatomaceous Earth Addition (in mass\%) on MIS Penetration into Silica Crucible from Feeds with s-109 Simulant and 2-Minute Ground Hanford Soil

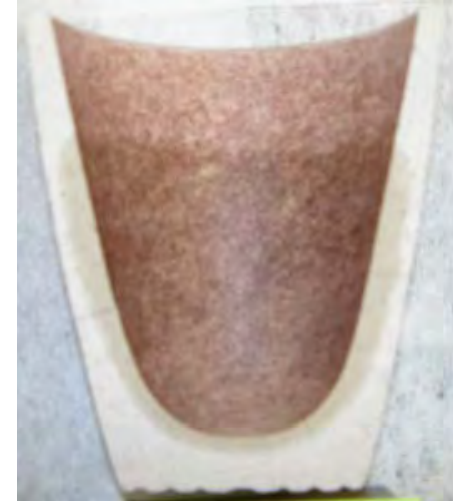

No addition

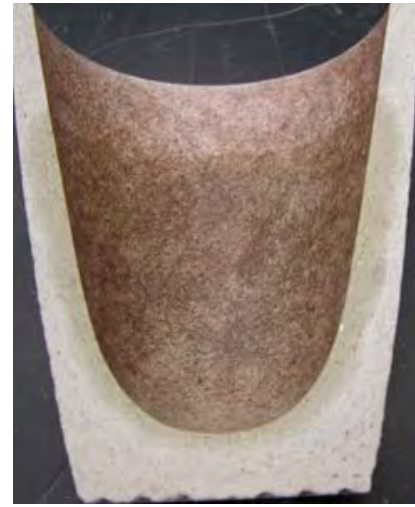

Formic acid

Figure K.5. Effect of Formic Acid Addition on MIS Penetration into Silica Crucible from Feeds with s-109 Simulant and 2-Minute Ground Hanford Soil 


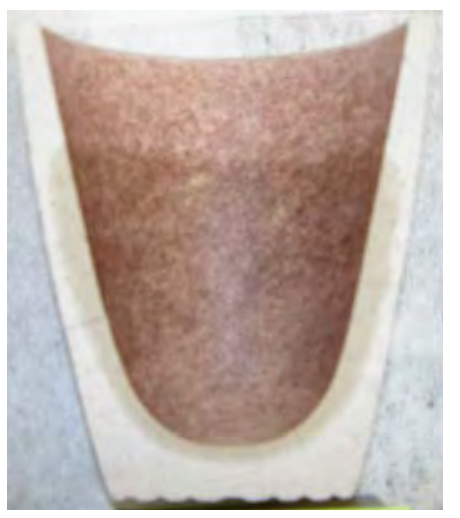

0

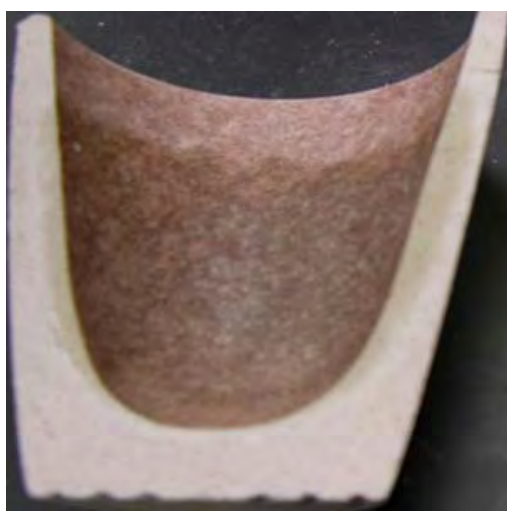

25

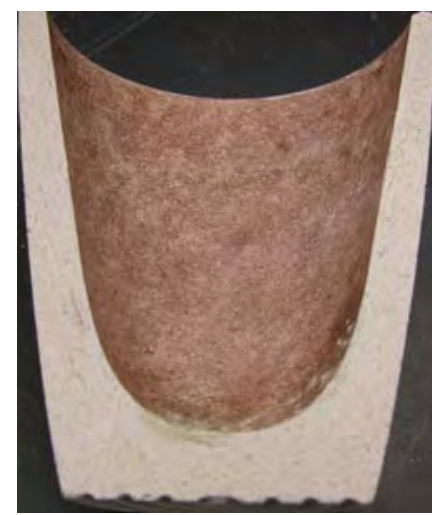

50

Figure K.6. Effect of Sucrose Addition (in \% of the stoichiometric amount) on MIS Penetration into Silica Crucible from Feeds with s-109 Simulant and 2-Minute Ground Hanford Soil

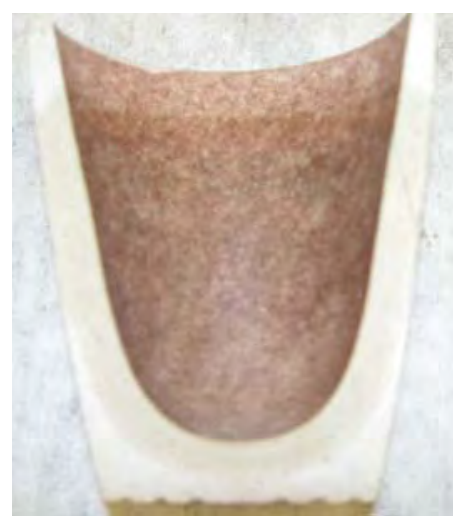

No addition

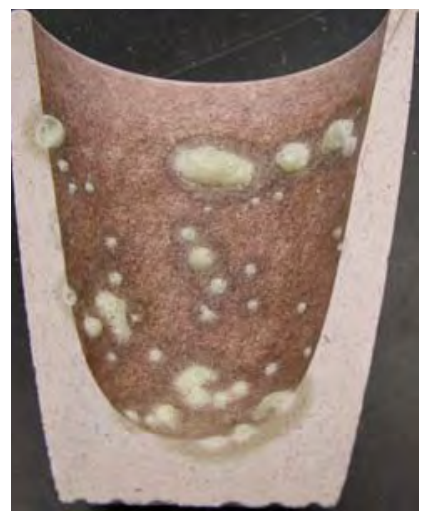

$50 \%$ sucrose

Figure K.7. Effect of Sucrose Addition (in \% of the stoichiometric amount) on MIS Penetration into Silica Crucible from Feeds with s-109 Simulant and As-Received Hanford Soil

Table K.2. Effect of Grinding Time on MIS Penetration into Silica Crucibles: Basic Data

\begin{tabular}{|c|c|c|c|c|c|c|c|c|c|}
\hline \multirow{2}{*}{$\begin{array}{c}\text { Grinding } \\
\text { Time } \\
\text { (min) }\end{array}$} & \multirow{2}{*}{$\begin{array}{c}\text { BET Specific } \\
\text { Surface Area } \\
\left(\mathrm{m}^{2} / \mathrm{g}\right)\end{array}$} & \multicolumn{3}{|c|}{ Crucible Mass (g) } & \multicolumn{2}{|c|}{ Migrated MIS (g) $^{(\mathrm{a})}$} & \multicolumn{2}{|c|}{ Feed Mass (g) } & \multirow{2}{*}{$\begin{array}{c}\text { Feed Mass } \\
\text { Change } \\
\text { (g) }\end{array}$} \\
\hline & & RT & $500^{\circ} \mathrm{C}$ & $1000^{\circ} \mathrm{C}$ & $500^{\circ} \mathrm{C}$ & $1000^{\circ} \mathrm{C}$ & RT & $500^{\circ} \mathrm{C}$ & \\
\hline 0 & 5.74 & 356.26 & 385.36 & 367.12 & 29.76 & 11.61 & 165.70 & 126.17 & 39.53 \\
\hline 2 & 9.75 & 355.33 & 367.11 & 359.47 & 12.44 & 4.89 & 172.17 & 142.88 & 29.29 \\
\hline 5 & 10.78 & 360.38 & 367.78 & 362.97 & 8.06 & 3.34 & 172.16 & 154.63 & 17.53 \\
\hline
\end{tabular}


Table K.3. Effect of Diatomaceous Earth and Bentonite Additions on MIS Penetration into Silica Crucibles from Feeds with 2-Minute Ground Soil: Basic Data

\begin{tabular}{|c|c|c|c|c|c|c|c|c|c|}
\hline & \multirow{2}{*}{$\begin{array}{c}\text { Addition } \\
\text { Mass \% }\end{array}$} & \multicolumn{3}{|c|}{ Crucible Mass (g) } & \multicolumn{2}{|c|}{ Migrated MIS (g) } & \multicolumn{2}{|c|}{ Feed Mass (g) } & \multirow{2}{*}{$\begin{array}{l}\text { Feed Mass } \\
\text { Change (g) }\end{array}$} \\
\hline & & RT & $500^{\circ} \mathrm{C}$ & $1000^{\circ} \mathrm{C}$ & $500^{\circ} \mathrm{C}$ & $1000^{\circ} \mathrm{C}$ & RT & $500^{\circ} \mathrm{C}$ & \\
\hline & 0 & 356.26 & 385.36 & 367.12 & 29.76 & 11.61 & 165.70 & 126.17 & 39.53 \\
\hline $\mathrm{DE}$ & 8.7 & 358.15 & 368.42 & 361.91 & 10.93 & 4.51 & 172.25 & 151.11 & 21.14 \\
\hline $\mathrm{DE}$ & 15 & 360.5 & 368.04 & 363.08 & 8.20 & 3.33 & 172.18 & 153.72 & 18.46 \\
\hline$\overline{\mathrm{DE}}$ & 20 & 354.54 & 361.16 & 357.01 & 7.28 & 3.22 & 172.18 & 153.15 & 19.03 \\
\hline $\mathrm{DE}^{(\mathrm{b})}$ & 20 & 359.38 & 372.37 & 363.99 & 13.65 & 5.36 & 172.39 & 148.01 & 24.38 \\
\hline B & 5 & 356.71 & 369.15 & 364.6 & 13.10 & 8.64 & 172.18 & 146.61 & 25.57 \\
\hline
\end{tabular}

Table K.4. Effect of Sucrose and Formic Acid Additions on MIS Penetration into Silica Crucibles from Feeds: Basic Data

\begin{tabular}{|c|c|c|c|c|c|c|c|c|c|}
\hline & \multirow[b]{2}{*}{ Addition $^{(f)}$} & \multicolumn{3}{|c|}{ Crucible Mass (g) } & \multicolumn{2}{|c|}{ Migrated MIS (g) $^{(\mathbf{a})}$} & \multicolumn{2}{|c|}{ Feed Mass (g) } & \multirow{2}{*}{$\begin{array}{c}\text { Feed Mass } \\
\text { Change (g) } \\
500^{\circ} \mathrm{C}\end{array}$} \\
\hline & & RT & $500^{\circ} \mathrm{C}$ & $1000^{\circ} \mathrm{C}$ & $500^{\circ} \mathrm{C}$ & $1000^{\circ} \mathrm{C}$ & RT & $500^{\circ} \mathrm{C}$ & \\
\hline & 0 & 356.26 & 385.36 & 367.12 & 29.76 & 11.61 & 165.70 & 126.17 & 39.53 \\
\hline $\mathrm{S}^{(\mathrm{b})}$ & 31 & 358.00 & 367.70 & 361.71 & 10.36 & 4.46 & 169.03 & 139.58 & 29.45 \\
\hline $\mathrm{S}^{(\mathrm{b})}$ & 63 & 359.25 & 359.14 & 358.93 & 0.55 & 0.43 & 165.89 & 136.05 & 29.84 \\
\hline $\mathrm{F}^{(\mathrm{c})}$ & 1.5 & 357.81 & 371.39 & 362.98 & 14.24 & 5.92 & 172.24 & 144.44 & 27.80 \\
\hline $\mathrm{S}^{(\mathrm{d})}$ & 63 & 359.40 & 363.53 & 361.09 & 4.79 & 2.44 & 166.37 & 139.50 & 26.87 \\
\hline $\mathrm{T}^{(\mathrm{e})}$ & 0 & 361.05 & 361.32 & 360.75 & 0.93 & 0.45 & 172.75 & 157.52 & 15.23 \\
\hline $\begin{array}{l}\text { (a) } \\
\text { (b) } \\
\text { (c) } \\
\text { (d) } \\
\text { (e) } \\
\text { (f) } \\
\mathrm{S}=\mathrm{s}\end{array}$ & $\begin{array}{l}\text { Mass gain is c } \\
\text { Sucrose dissol } \\
\text { Formic acid ac } \\
\text { Sucrose added } \\
\text { Six-tank LAW } \\
\text { Sucrose additi } \\
\text { (63\% correspo } \\
\text { sucrose, F = for }\end{array}$ & $\begin{array}{l}\text { rrected for } \\
\text { ed in liqui } \\
\text { led to liqu } \\
\text { o liquid si } \\
\text { simulant u } \\
\mathrm{n} \text { in } 100 \mathrm{C} \\
\text { ds to } 13.1 \\
\text { nic acid, T }\end{array}$ & $\begin{array}{l}\text { crucible L } \\
\text { d simulant } \\
\text { d simulant } \\
\text { nulant and } \\
\text { sed instead } \\
\mathrm{N}(\mathrm{C}: \mathrm{N}= \\
\text { g per } 296 \\
=\text { six-tank }\end{array}$ & $\begin{array}{l}\text { OI. } \\
\text { and soil gr } \\
\text { and soil g } \\
\text { soil used } \\
\text { of S-109 a } \\
\text { atoms fro } \\
\text {-g dry feec } \\
\text { composite }\end{array}$ & $\begin{array}{l}\text { und for } 2 \\
\text { ound for } 2 \\
\text { s-received } \\
\text { hd soil gro } \\
\text { n sucrose } \\
\text { and form } \\
\text { LAW sim }\end{array}$ & $\begin{array}{l}\text { grinding). } \\
\text { for } 2 \text { min. } \\
\mathrm{N} \text { atoms fro } \\
\text { cid in } \mathrm{mL} \mathrm{pe} \\
\mathrm{t}\end{array}$ & $\begin{array}{l}\text { ( MIS) } \\
\text { 200-g dr }\end{array}$ & feed. & \\
\hline
\end{tabular}




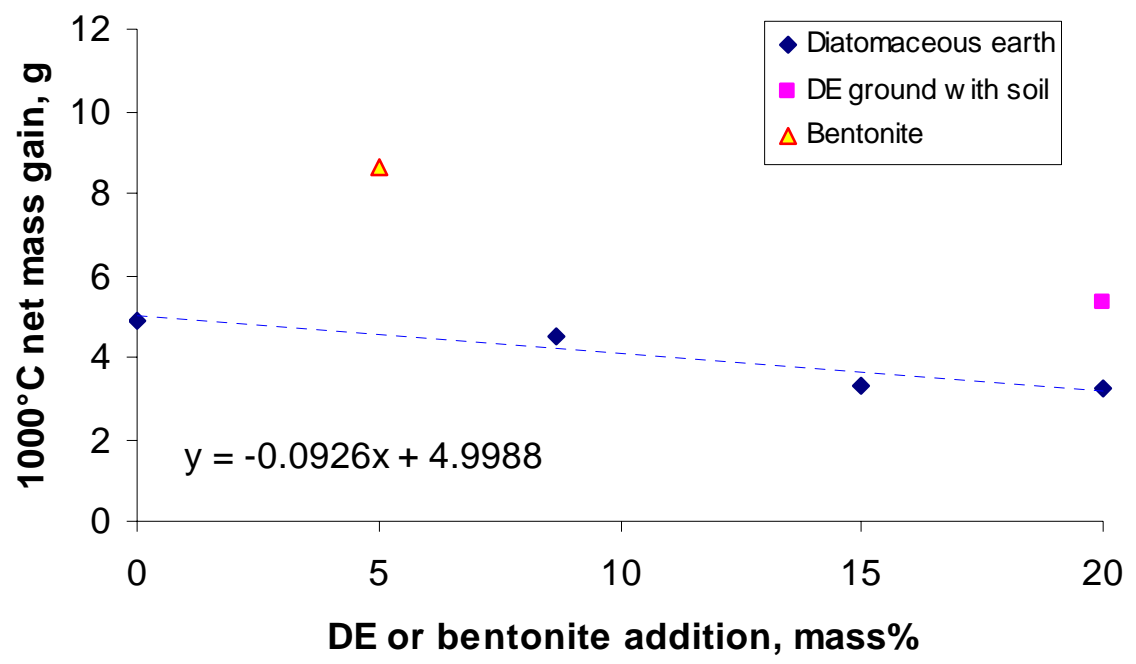

Figure K.8. Effect of Diatomaceous Earth and Bentonite Additions on MIS Migration into Silica Crucible

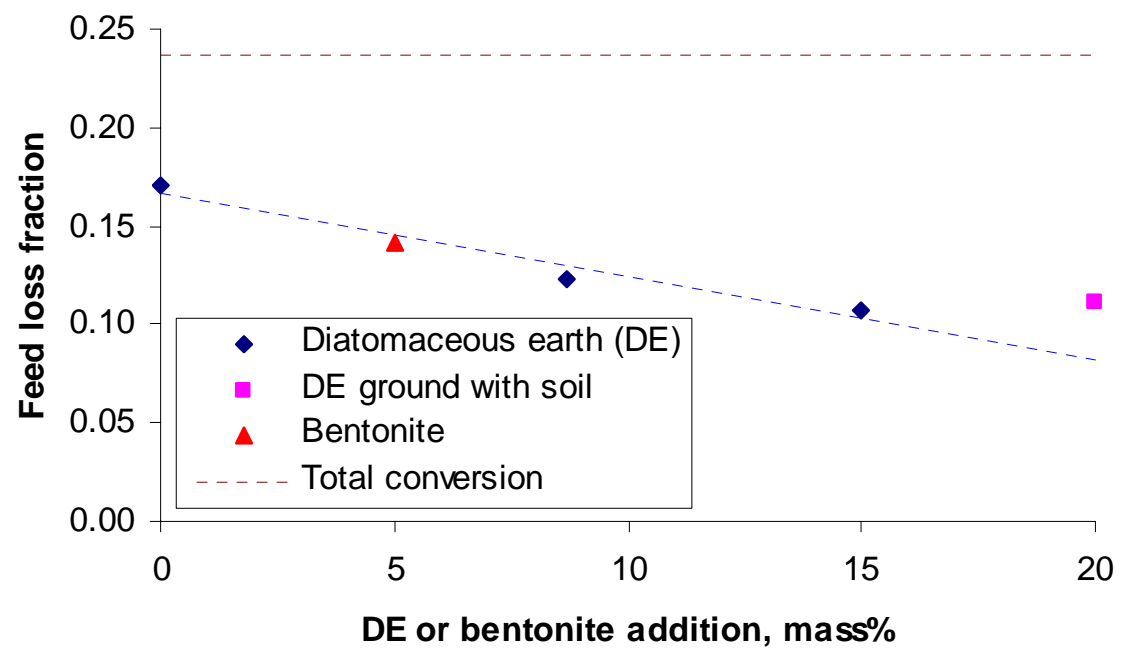

Figure K.9. Effect of Diatomaceous Earth and Bentonite Additions on MIS Decomposition in Feed 


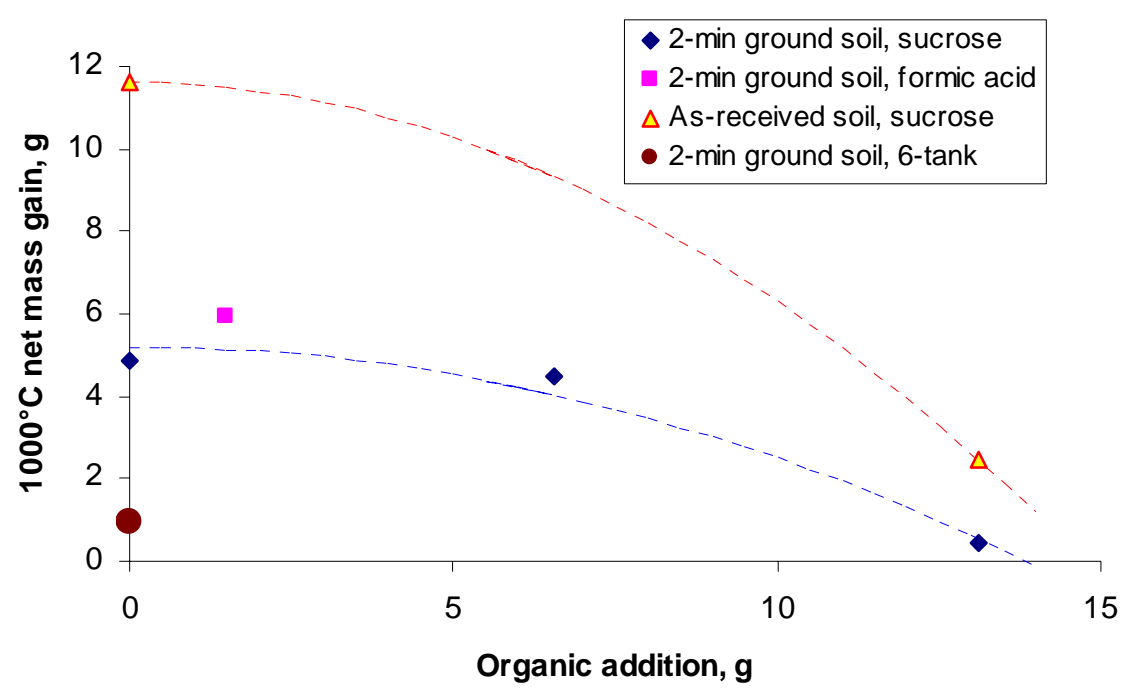

Figure K.10. Effect of Sucrose and Formic Acid Additions on MIS Migration into Silica Crucible

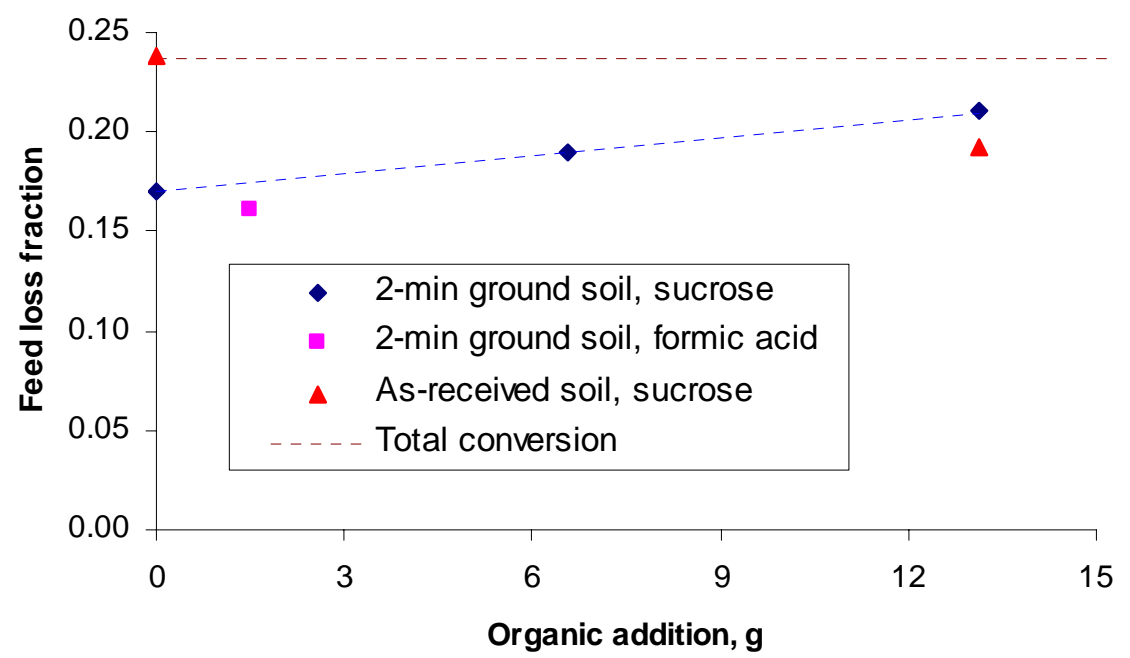

Figure K.11. Effect of Sucrose and Formic Acid Additions on MIS Decomposition in Feed

As Figures K.8 and K.9 show, adding diatomaceous earth slightly decreased MIS migration into the silica crucible and slowed feed conversion reactions. One can speculate that, although diatomaceous earth held MIS inside the fractal-like silica particles, it reacted with MIS at a slower rate than the soil did. Grinding diatomaceous earth with the soil had no effect on MIS behavior. A bentonite addition increased MIS migrations. One can speculate that bentonite was present in the form of agglomerates that were not wetted by MIS, thus increasing the MIS fraction in soil and promoting its mobility.

Figures K.10 and K.11 show that sucrose addition decreased MIS migration and appeared to increase the extent of feed reactions. 
Figure K.12 shows a linear relationship between the specific surface area of soil and the extent of MIS migration. By extrapolation, MIS migration from S-109 LAW simulant would stop at $a=12.8 \mathrm{~m}^{2} / \mathrm{g}$.

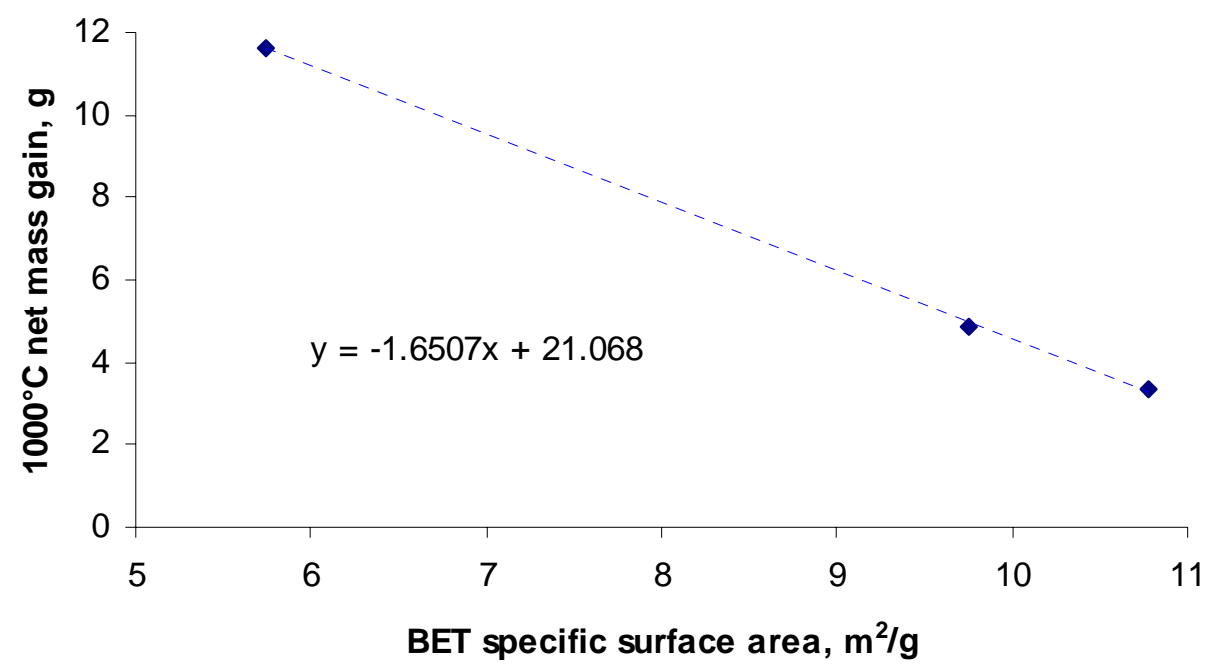

Figure K.12. Effect of the Specific Surface Area of Soil on MIS Migration into Silica Crucible

Compared to the baseline feed, 2-min grinding reduced MIS migration by 58\% and 5-min grinding by $71 \%$. Adding 63\% stoichiometric sucrose (no grinding) reduced MIS migration by $79 \%$. If the soil was also ground for 2 min, adding 63\% stoichiometric sucrose reduced MIS migration by 96\%. This matches the $96 \%$ reduction of 2-min grinding of feed containing six-tank composite LAW simulant without any additions.

Compared to the baseline feed, 2-min grinding plus 20\% addition of diatomaceous earth reduced MIS migration by $72 \%$, whereas 2-min grinding alone reduced MIS migration by $58 \%$. Hence, $20 \%$ addition of diatomaceous earth is less effective than $63 \%$ stoichiometric sucrose.

To understand the effect of feed alterations on MIS migrations, several processes need to be considered:

- LAW simulant forms MIS gradually, starting from low-temperature eutectics. Therefore, MIS composition changes until all LAW simulant becomes liquid.

- The organic and inorganic components in LAW simulant undergo oxidation-reduction reactions even at a solid state and continue as MIS is being formed.

- MIS reacts with feed components as it migrates through feed. It also reacts with crucible materials as it penetrates into crucible pores.

To obtain a better insight into the MIS migration mechanism, calculations were performed as described below.

The mass balance of MIS was deduced from data listed in Tables K2 through K4 as follows.

Disregarding the solid-state reactions, the initial mass fraction of MIS in dry feed was estimated from the 
feed composition as $m_{0}=0.3343$ for S-109 LAW simulant. Accordingly, a typical feed mass $F_{0}=170 \mathrm{~g}$ contains $M_{0}=F_{0} m_{0}=170 \times 0.3343=57 \mathrm{~g}$ of MIS. As the temperature increased to $500^{\circ} \mathrm{C}$, this initial MIS partly migrated to the crucible $\left(M_{\mathrm{C}}\right)$, was partly decomposed by feed conversion reactions $\left(M_{\mathrm{A}}\right)$, and partly remained unreacted in the feed at the end of the experiment $\left(M_{\mathrm{R}}\right)$.

When all $\mathrm{NO}_{\mathrm{x}}, \mathrm{CO}_{\mathrm{x}}$, and $\mathrm{H}_{2} \mathrm{O}$ are released from MIS, the remaining components are assumed to become a part of the glass. Their mass fraction in the MIS is $f_{\mathrm{S}}=0.4023$ for S-109 simulant and 0.4538 for six-tank simulant (hence, $f_{\mathrm{A}}=0.5977$ and 0.5462 eventually escapes from MIS in the form of $\mathrm{NO}_{\mathrm{x}}, \mathrm{CO}_{\mathrm{x}}$, and $\mathrm{H}_{2} \mathrm{O}$ ).

A part of the total MIS in the feed migrates into the crucible $\left(m_{\mathrm{C}}=M_{\mathrm{C}} / M_{0}\right)$. For simplicity, we disregarded the fact that MIS in the crucible may have had a slightly different composition than the MIS that remained in feed. We can safely assume that all $\mathrm{NO}_{\mathrm{x}}, \mathrm{CO}_{\mathrm{x}}$, and $\mathrm{H}_{2} \mathrm{O}$ escaped from the MIS during heating to $1000^{\circ} \mathrm{C}$. The amount of molten MIS that migrated into the crucible can then be estimated. For example, a hypothetical $1000^{\circ} \mathrm{C}$-net mass gain of $M_{\mathrm{C} 2}=10 \mathrm{~g}$ (the crucible mass at $1000^{\circ} \mathrm{C}$ minus the mass of empty crucible corrected for LOI) corresponds to $M_{\mathrm{C}}=M_{\mathrm{C} 2} / f_{\mathrm{S}}=10 / 0.4023=25 \mathrm{~g}$ of S-109 MIS that migrated from the feed into the crucible. Hence, out of the $M_{0}=57 \mathrm{~g}$ of MIS initially present, $M_{\mathrm{A}}+$ $M_{\mathrm{R}}=M_{0}-M_{\mathrm{C}}=57-25=32 \mathrm{~g}$ of MIS has been left in the feed at $500^{\circ} \mathrm{C}$; or, the faction $m_{\mathrm{C}}=M_{\mathrm{C}} / M_{0}=$ 0.44 of MIS migrated to the crucible, and $m_{\mathrm{A}}+m_{\mathrm{R}}=1-m_{\mathrm{C}}=0.56$ remained in the feed.

The MIS that remained in the feed partially decomposed because of feed conversion reactions. If this MIS decomposed fully by releasing all $\mathrm{NO}_{\mathrm{x}}, \mathrm{CO}_{\mathrm{x}}$, and $\mathrm{H}_{2} \mathrm{O}$, the remaining mass would be $D_{1 \mathrm{R}}=\left(M_{\mathrm{A}}+M_{\mathrm{R}}\right) / f_{\mathrm{S}}=32 \times 0.4023=13 \mathrm{~g}$, and $D_{1 \mathrm{~A}}=32-13=19 \mathrm{~g}$ would escape in the form of gas. The total mass loss of the feed would be $D_{1}=D_{1 \mathrm{~A}}+D_{1 \mathrm{R}}=25+13=38 \mathrm{~g}$. In terms of mass fractions, out of $m_{\mathrm{A}}+m_{\mathrm{R}}=0.56$ of MIS that remained in the feed, a fraction of $m_{\mathrm{A}}=D_{1 \mathrm{~A}} / M_{0}=13 / 57=0.33$ would escape in the form of gas, and $m_{\mathrm{R}}=D_{1 \mathrm{R}} / M_{0}=0.23$ would remain in the feed.

Though possible, it is unlikely that $100 \%$ of the MIS would decompose at $500^{\circ} \mathrm{C}$. Suppose that the measured feed lost during the heat-treatment was $M_{1}=M_{\mathrm{C}}+M_{\mathrm{A}}=30 \mathrm{~g}$. Of this amount, $M_{\mathrm{C}}=25 \mathrm{~g}$ migrated to the crucible. Hence, only $M_{\mathrm{A}}=5 \mathrm{~g}$ escaped to the atmosphere instead of the maximum theoretical amount of $19 \mathrm{~g}$. Of the total amount of initial MIS, $M_{\mathrm{R}}=M_{0}-M_{1}=57-30=27 \mathrm{~g}$ of MIS material remained in the feed instead of the theoretical amount of $13 \mathrm{~g}$ if all the MIS decomposed. In terms of mass fractions, $m_{\mathrm{C}}=0.44$ migrated to the crucible, $m_{\mathrm{A}}=0.09$ escaped in the form of gas, and $m_{\mathrm{R}}$ $=0.47$ remained in the feed.

Results of these calculations are shown for each experiment in Tables K.5 through K.7. In terms of mass fractions, mass balances of MIS are listed in Tables K.8 through K.10 and displayed in Figure K.13. 
Table K.5. Effect of Grinding Time on MIS Penetration into Silica Crucibles: MIS Migration and Reaction

\begin{tabular}{|c|c|c|c|c|c|}
\hline $\begin{array}{c}\text { Grinding } \\
\text { Time } \\
\text { (min) }\end{array}$ & $\begin{array}{c}\text { BET Specific } \\
\text { Surface Area } \\
\left(\mathrm{m}^{2} / \mathrm{g}\right)\end{array}$ & $\begin{array}{c}\text { Initial } \\
\text { Mass } \\
M_{0}(\mathrm{~g})\end{array}$ & $\begin{array}{c}\text { Migrated } \\
\text { to crucible } \\
M_{\mathrm{C}}(\mathrm{g})\end{array}$ & $\begin{array}{c}\text { Loss by feed } \\
\text { Reactions } \\
M_{\mathrm{A}}(\mathrm{g})\end{array}$ & $\begin{array}{c}\text { Remained } \\
\text { in feed } \\
M_{\mathrm{R}}(\mathrm{g})\end{array}$ \\
\hline 0 & 5.74 & 55.39 & 28.86 & 10.67 & 15.86 \\
\hline 2 & 9.75 & 57.56 & 12.16 & 17.13 & 28.27 \\
\hline 5 & 10.78 & 57.55 & 8.30 & 9.23 & 40.02 \\
\hline
\end{tabular}

Table K.6. Effect of Diatomaceous Earth and Bentonite Additions on MIS Penetration into Silica Crucibles: MIS Migration and Reaction

\begin{tabular}{|c|c|c|c|c|c|}
\hline & $\begin{array}{c}\text { Addition } \\
\text { Mass \% }\end{array}$ & $\begin{array}{c}\text { Initial } \\
\text { Mass } \\
M_{0}(\mathrm{~g})\end{array}$ & $\begin{array}{c}\text { Migrated } \\
\text { to crucible } \\
M_{\mathrm{C}}(\mathrm{g})\end{array}$ & $\begin{array}{c}\text { Loss by feed } \\
\text { Reactions } \\
M_{\mathrm{A}}(\mathrm{g})\end{array}$ & $\begin{array}{c}\text { Remained } \\
\text { in feed } \\
M_{\mathrm{R}}(\mathrm{g})\end{array}$ \\
\hline & 0 & 57.56 & 12.16 & 17.13 & 28.27 \\
\hline $\mathrm{DE}$ & 8.7 & 57.58 & 11.21 & 9.93 & 36.44 \\
\hline$\overline{\mathrm{DE}}$ & 15 & 57.56 & 8.28 & 10.18 & 39.10 \\
\hline$\overline{D E}$ & 20 & 57.56 & 8.00 & 11.03 & 38.53 \\
\hline $\mathrm{DE}^{(\mathrm{a})}$ & 20 & 57.63 & 13.32 & 11.06 & 33.25 \\
\hline $\mathrm{B}$ & 5 & 57.56 & 21.48 & 4.09 & 31.99 \\
\hline \multicolumn{6}{|c|}{ (a) $\mathrm{DE}$ and soil ground together } \\
\hline
\end{tabular}

Table K.7. Effect of Sucrose and Formic Acid Additions on MIS Penetration into Silica Crucibles: MIS Migration and Reaction

\begin{tabular}{|c|c|c|c|c|c|}
\hline & Addition $^{(\mathrm{e})}$ & $\begin{array}{c}\text { Initial } \\
\text { Mass } \\
M_{0} \text { (g) }\end{array}$ & $\begin{array}{c}\text { Migrated } \\
\text { to Crucible } \\
M_{\mathrm{C}}(\mathrm{g})\end{array}$ & $\begin{array}{c}\text { Loss by feed } \\
\text { Reactions } \\
M_{\mathrm{A}}(\mathrm{g})\end{array}$ & $\begin{array}{c}\text { Remained } \\
\text { in feed } \\
M_{\mathrm{R}}(\mathrm{g})\end{array}$ \\
\hline & 0 & 57.56 & 12.16 & 17.13 & 28.27 \\
\hline $\mathrm{S}^{(\mathrm{a})}$ & 31 & 56.51 & 11.09 & 21.60 & 23.82 \\
\hline $\mathrm{S}^{(\mathrm{a})}$ & 63 & 55.46 & 1.07 & 35.14 & 19.25 \\
\hline $\mathrm{F}^{(\mathrm{b})}$ & 1.5 & 57.58 & 14.72 & 13.08 & 29.78 \\
\hline $\mathrm{S}^{(\mathrm{c})}$ & 63 & 55.62 & 6.07 & 27.18 & 22.37 \\
\hline $\mathrm{T}^{(\mathrm{d})}$ & 0 & 57.75 & 0.99 & 14.24 & 42.52 \\
\hline \multicolumn{6}{|c|}{$\begin{array}{l}\text { (a) Sucrose dissolved in liquid simulant and soil ground for } 2 \text { min. } \\
\text { (b) Formic acid added to liquid simulant and soil ground for } 2 \text { min. } \\
\text { (c) Sucrose added to liquid simulant and soil used as-received (no grinding). } \\
\text { (d) Six-tank LAW simulant used instead S-109 and soil ground for } 2 \text { min. } \\
\text { (e) Sucrose addition in } 100 \mathrm{C} / \mathrm{N} \text { (C:N = C atoms from sucrose per } \mathrm{N} \text { atoms from MIS) } \\
\text { (63\% corresponds to } 13.13 \text { g per } 296 \text {-g dry feed) and formic acid in mL per } 200 \text {-g dry feed. } \\
\text { S = sucrose, F = formic acid, } \mathrm{T}=\text { six-tank composite LAW simulant }\end{array}$} \\
\hline
\end{tabular}


Table K.8. Effect of Grinding Time on MIS Penetration into Silica Crucibles: MIS Migration and Reaction (in mass fractions)

\begin{tabular}{||c|c|c|c|c||}
\hline $\begin{array}{c}\text { Grinding } \\
\text { Time } \\
(\mathbf{m i n})\end{array}$ & $\begin{array}{c}\text { BET Specific } \\
\text { Surface Area } \\
\left(\mathbf{m}^{\mathbf{2}} / \mathbf{g}\right)\end{array}$ & $\begin{array}{c}\text { Migrated } \\
\text { to Crucible } \\
\left(\boldsymbol{m}_{\mathbf{C}}\right)\end{array}$ & $\begin{array}{c}\text { Loss by feed } \\
\text { Reactions } \\
\left(\boldsymbol{m}_{\mathbf{A}}\right)\end{array}$ & $\begin{array}{c}\text { Remained } \\
\text { in Feed } \\
\left(\boldsymbol{m}_{\mathbf{R}}\right)\end{array}$ \\
\hline 0 & 5.74 & 0.521 & 0.193 & 0.286 \\
\hline 2 & 9.75 & 0.211 & 0.298 & 0.491 \\
\hline 5 & 10.78 & 0.144 & 0.160 & 0.695 \\
\hline
\end{tabular}

Table K.9. Effect of Diatomaceous Earth and Bentonite Additions on MIS Penetration into Silica Crucibles: MIS Migration and Reaction (in mass fractions)

\begin{tabular}{||l|c|c|c|c||}
\hline \hline & $\begin{array}{c}\text { Addition } \\
\text { Mass } \%\end{array}$ & $\begin{array}{c}\text { Migrated } \\
\text { to Crucible } \\
\left(\boldsymbol{m}_{\mathbf{C}}\right)\end{array}$ & $\begin{array}{c}\text { Loss by feed } \\
\text { Reactions } \\
\left(\boldsymbol{m}_{\mathbf{A}}\right)\end{array}$ & $\begin{array}{c}\text { Remained } \\
\text { in Feed } \\
\left(\boldsymbol{m}_{\mathbf{R}}\right)\end{array}$ \\
\hline & 0 & 0.211 & 0.298 & 0.491 \\
\hline $\mathrm{DE}$ & 8.7 & 0.195 & 0.172 & 0.633 \\
\hline $\mathrm{DE}$ & 15 & 0.144 & 0.177 & 0.679 \\
\hline $\mathrm{DE}$ & 20 & 0.139 & 0.192 & 0.669 \\
\hline $\mathrm{DE}$ & 20 & 0.231 & 0.192 & 0.577 \\
\hline $\mathrm{B}$ & 5 & 0.373 & 0.071 & 0.556 \\
\hline $\begin{array}{l}\text { (a) } \mathrm{DE} \\
\mathrm{DE}=\text { diatomaceous earth; B bentonite }\end{array}$ \\
\hline
\end{tabular}

Table K.10. Effect of Sucrose and Formic Acid Additions on MIS Penetration into Silica Crucibles: MIS Migration and Reaction (in mass fractions)

\begin{tabular}{|c|c|c|c|c|}
\hline & Addition $^{(e)}$ & $\begin{array}{c}\text { Migrated } \\
\text { to Crucible } \\
\left(m_{\mathrm{C}}\right)\end{array}$ & $\begin{array}{c}\text { Loss by feed } \\
\text { Reactions } \\
\left(m_{\mathrm{A}}\right)\end{array}$ & $\begin{array}{c}\text { Remained } \\
\text { in Feed } \\
\left(m_{R}\right)\end{array}$ \\
\hline & 0 & 0.211 & 0.298 & 0.491 \\
\hline $\mathrm{S}^{(\mathrm{a})}$ & 31 & 0.196 & 0.325 & 0.479 \\
\hline $\mathrm{S}^{(\mathrm{a})}$ & 63 & 0.019 & 0.519 & 0.462 \\
\hline $\mathrm{F}^{(\mathrm{b})}$ & 1.5 & 0.256 & 0.227 & 0.517 \\
\hline $\mathrm{S}^{(\mathrm{c})}$ & 63 & 0.109 & 0.374 & 0.517 \\
\hline $\mathrm{T}^{(\mathrm{d})}$ & 0 & 0.017 & 0.247 & 0.736 \\
\hline \multicolumn{5}{|c|}{$\begin{array}{l}\text { (a) Sucrose dissolved in liquid simulant and soil ground for } 2 \text { min. } \\
\text { (b) Formic acid added to liquid simulant and soil ground for } 2 \text { min. } \\
\text { (c) Sucrose added to liquid simulant and soil used as-received (no grinding). } \\
\text { (d) Six-tank LAW simulant used instead S-109 and soil ground for } 2 \text { min. } \\
\text { (e) Sucrose addition in } 100 \mathrm{C} / \mathrm{N} \text { ) (C:N = C atoms from sucrose per } \mathrm{N} \text { atoms from MIS) } \\
\text { (63\% corresponds to } 13.13 \mathrm{~g} \text { per } 296 \text {-g dry feed) and formic acid in mL per } 200 \text {-g dry feed. } \\
\mathrm{S}=\text { sucrose, } \mathrm{F}=\text { formic acid, } \mathrm{T}=\text { six-tank composite LAW simulant }\end{array}$} \\
\hline
\end{tabular}

Figure K.13 shows that grinding decreased MIS penetration into crucible walls, but also increased the fraction of unreacted MIS in the feed. Adding diatomaceous earth had a similar influence as grinding, both on MIS penetration and reactions, but was less effective than grinding. Sucrose addition decreased 
the fraction of unreacted MIS, but 31\% addition had little effect on MIS penetration, whereas 63\% addition had a dramatic effect.

The last bar on the right in Figure K.14 shows the theoretical fractions of gas and solids from totally decomposed MIS; 40 mass $\%\left(f_{\mathrm{S}}=0.4023\right)$ was the minimum fraction of MIS material after $\mathrm{NO}_{\mathrm{x}}, \mathrm{CO}_{\mathrm{x}}$, and $\mathrm{H}_{2} \mathrm{O}$ gases escaped. The actual fraction was higher in all feeds except one feed containing $63 \%$ of sucrose addition, in which the remaining MIS was essentially fully decomposed.

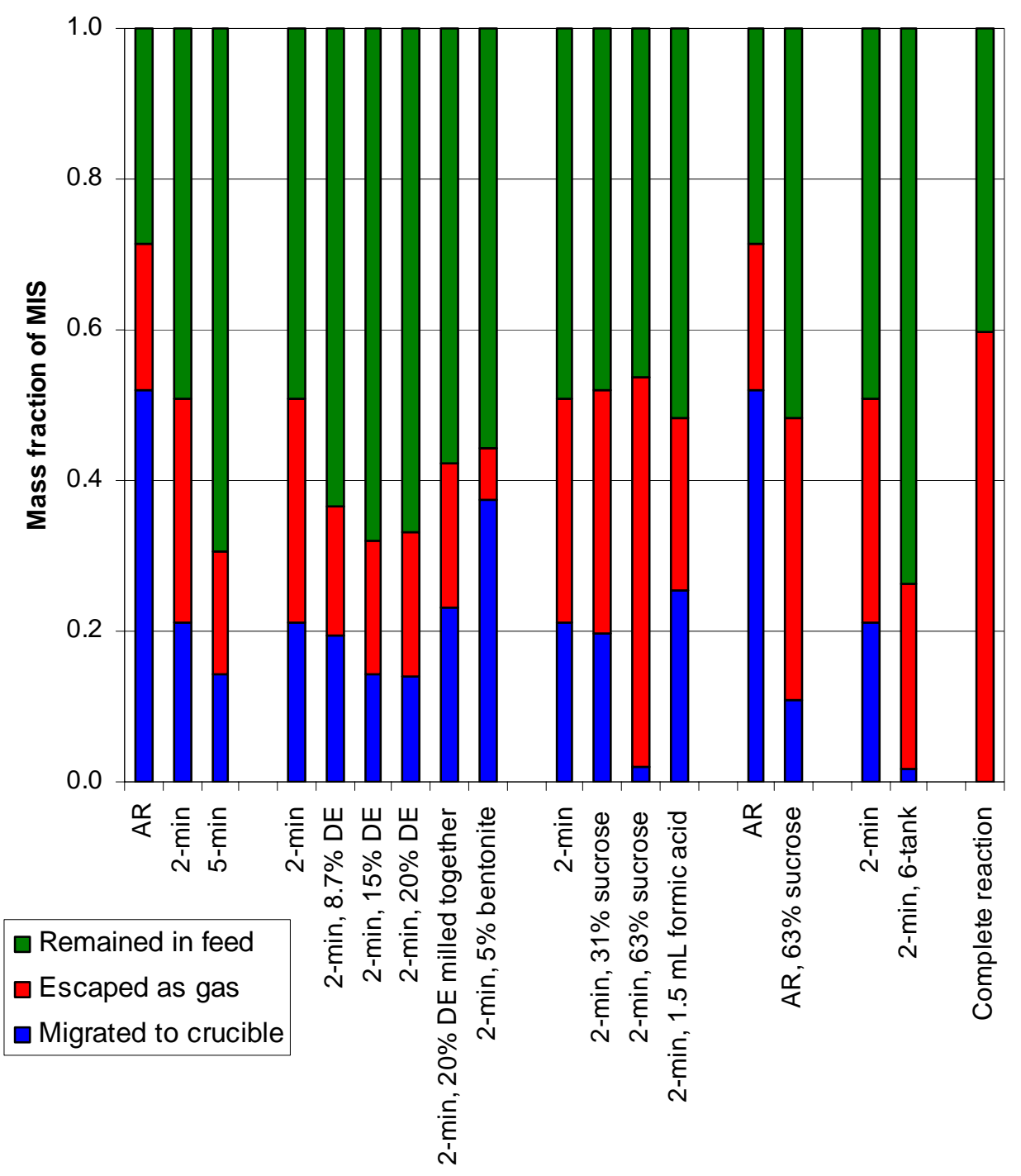

Figure K.13. Mass Balances for MIS in Various Feeds 


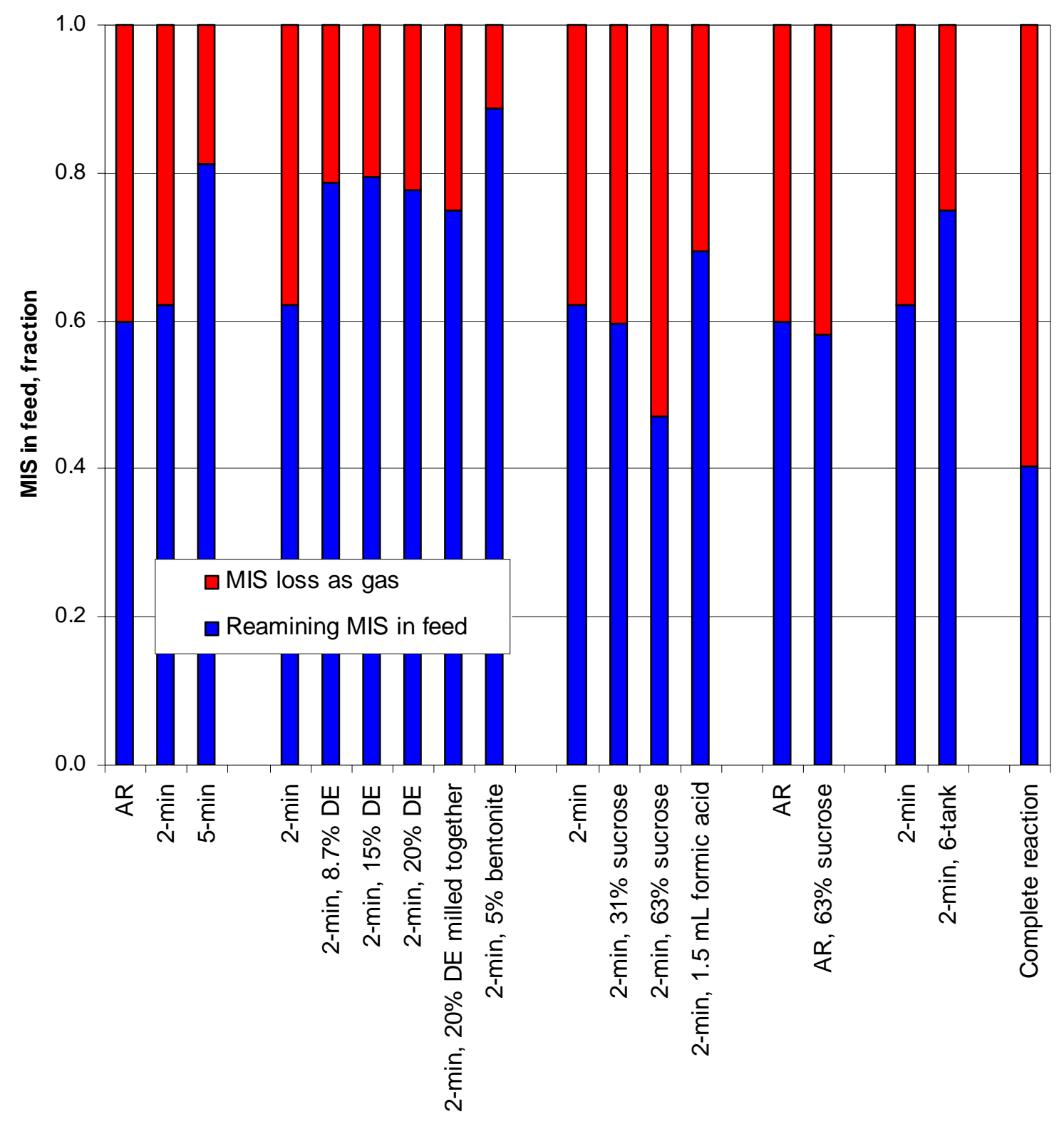

Figure K.14. Decomposition of MIS that Remained at $500^{\circ} \mathrm{C}$

Tables K.11 through K.13 list the measured amounts of MIS present in crucibles after $500^{\circ} \mathrm{C}$ heattreatment (the difference between the crucible mass after the feed was removed from it and the empty crucible before the heat-treatment, corrected for the LOI of the crucible material). The tables also list the amounts of total MIS that migrated to the crucible, as shown in Tables K.5 through K.7, where it was determined from the mass losses of crucible after $1000^{\circ} \mathrm{C}$ heat-treatment, based on the assumption that the miss in the pores had the same composition as the LAW simulant.

The amount present after a $500^{\circ} \mathrm{C}$ heat-treatment $\left(M_{\mathrm{C} 1}\right)$ is expected to be smaller than or equal to the total amount obtained from the mass of material found in crucible walls at $1000^{\circ} \mathrm{C}$, i.e., $M_{\mathrm{C} 1} / M_{\mathrm{C}}<1$. The numbers in the last columns of the tables below (also seen in Figure K.15) are up to 3\% larger than 1 for feeds with as-received soil and 2-min ground soil with no additions. This indicates that MIS that 
migrated into the crucible walls from these feeds contained an increased proportion of components with large fractions of volatiles (i.e., $\mathrm{NO}_{\mathrm{x}}, \mathrm{CO}_{\mathrm{x}}$, and $\mathrm{H}_{2} \mathrm{O}$ ). Experiments with the baseline feeds heated to temperatures from $350^{\circ} \mathrm{C}$ to $500^{\circ} \mathrm{C}$ showed that in the absence of additions the MIS components with a high content of gaseous constituents migrate to crucible walls first (see Table E.4).

Feed composition and granularity can only influence MIS before it migrates to the crucible. Inside the crucible pores, MIS reacts with the silica crucible material. If the volatiles partly decompose in the feed at temperatures lower that $300^{\circ} \mathrm{C}$, as in the case of sucrose addition, molten salts that migrates to crucible walls contains a reduced fraction of volatiles. How bentonite addition could produce a similar effect is unclear.

Table K.11. Effect of Grinding Time on MIS Penetration into Silica Crucibles

\begin{tabular}{||c|c|c|c|c||}
\hline $\begin{array}{c}\text { Grinding } \\
\text { Time }\end{array}$ & $\begin{array}{c}\text { BET Specific } \\
\text { Surface Area } \\
(\mathbf{a})\end{array}$ & $\begin{array}{c}\text { Migrated } \\
\text { to crucible } \\
\left.\mathbf{( M}_{\mathbf{C}}\right)\end{array}$ & \multicolumn{2}{|c||}{$\begin{array}{c}\text { Present } \\
\text { in crucible } \\
\left.\text { at } \mathbf{5 0 0} \mathbf{C}^{\circ} \mathbf{( \mathbf { M } _ { \mathbf { C } 1 }}\right)\end{array}$} \\
\hline$(\mathrm{min})$ & $\left(\mathrm{m}^{2} / \mathrm{g}\right)$ & $(\mathrm{g})$ & $(\mathrm{g})$ & $($ fraction$)$ \\
\hline 0 & 5.74 & 28.86 & 29.76 & 1.031 \\
\hline 2 & 9.75 & 12.16 & 12.44 & 1.023 \\
\hline 5 & 10.78 & 8.30 & 8.06 & 0.971 \\
\hline
\end{tabular}

Table K.12. Effect of Diatomaceous Earth and Bentonite Additions on MIS Penetration into Silica Crucibles (soil 2-min ground)

\begin{tabular}{|c|c|c|c|c|}
\hline & \multirow[t]{2}{*}{$\begin{array}{c}\text { Addition } \\
\text { Mass\% }\end{array}$} & \multirow{2}{*}{$\begin{array}{c}\begin{array}{c}\text { Migrated } \\
\text { to crucible } \\
\left(\mathbf{M}_{\mathrm{C}}\right)\end{array} \\
(\mathrm{g})\end{array}$} & \multicolumn{2}{|c|}{$\begin{array}{c}\text { Present } \\
\text { in crucible } \\
\text { at } 500^{\circ} \mathrm{C}\left(\mathrm{M}_{\mathrm{C} 1}\right)\end{array}$} \\
\hline & & & (g) & (fraction) \\
\hline & 0 & 12.16 & 12.44 & 1.023 \\
\hline$\overline{\mathrm{DE}}$ & 8.7 & 11.21 & 10.93 & 0.975 \\
\hline $\mathrm{DE}$ & 15 & 8.28 & 8.2 & 0.991 \\
\hline$\overline{\mathrm{DE}}$ & 20 & 8.00 & 7.28 & 0.910 \\
\hline $\mathrm{DE}^{(\mathrm{a})}$ & 20 & 13.32 & 13.65 & 1.025 \\
\hline B & 5 & 21.48 & 13.1 & 0.610 \\
\hline \multicolumn{5}{|c|}{ (a) DE and soil ground together } \\
\hline
\end{tabular}


Table K.13. Effect of Sucrose and Formic Acid Additions on MIS Penetration into Silica Crucibles

\begin{tabular}{|c|c|c|c|c|}
\hline & \multirow[t]{2}{*}{ Addition $^{(e)}$} & \multirow{2}{*}{$\begin{array}{c}\begin{array}{c}\text { Migrated } \\
\text { to crucible } \\
\left(\mathrm{M}_{\mathrm{C}}\right)\end{array} \\
(\mathrm{g})\end{array}$} & \multicolumn{2}{|c|}{$\begin{array}{c}\text { Present } \\
\text { in crucible } \\
\text { at } 500^{\circ} \mathrm{C}\left(\mathrm{M}_{\mathrm{C} 1}\right)\end{array}$} \\
\hline & & & (g) & (fraction) \\
\hline & 0 & 12.16 & 12.44 & 1.023 \\
\hline$S^{(a)}$ & 31 & 11.09 & 10.36 & 0.934 \\
\hline $\mathrm{S}^{(\mathrm{a})}$ & 63 & 1.07 & 0.55 & 0.515 \\
\hline $\mathrm{F}^{(\mathrm{b})}$ & 1.5 & 14.72 & 14.24 & 0.968 \\
\hline $\mathrm{S}^{(\mathrm{c})}$ & 63 & 6.07 & 4.79 & 0.790 \\
\hline $\mathrm{T}^{(\mathrm{d})}$ & 0 & 0.99 & 0.93 & 0.938 \\
\hline \multicolumn{5}{|c|}{$\begin{array}{l}\text { (a) Sucrose dissolved in liquid simulant and soil ground for } 2 \text { min. } \\
\text { (b) Formic acid added to liquid simulant and soil ground for } 2 \text { min. } \\
\text { (c) Sucrose added to liquid simulant and soil used as-received (no grinding). } \\
\text { (d) Six-tank LAW simulant used instead S-109 and soil ground for } 2 \text { min. } \\
\text { (e) Sucrose addition in } 100 \mathrm{C} / \mathrm{N}) \text { (C:N = C atoms from sucrose per } \mathrm{N} \\
\text { atoms from MIS) (63\% corresponds to } 13.13 \text { g per 296-g dry feed) } \\
\text { and formic acid in mL per } 200 \text {-g dry feed } \\
\text { S = sucrose, F = formic acid, T = six-tank composite LAW simulant }\end{array}$} \\
\hline
\end{tabular}




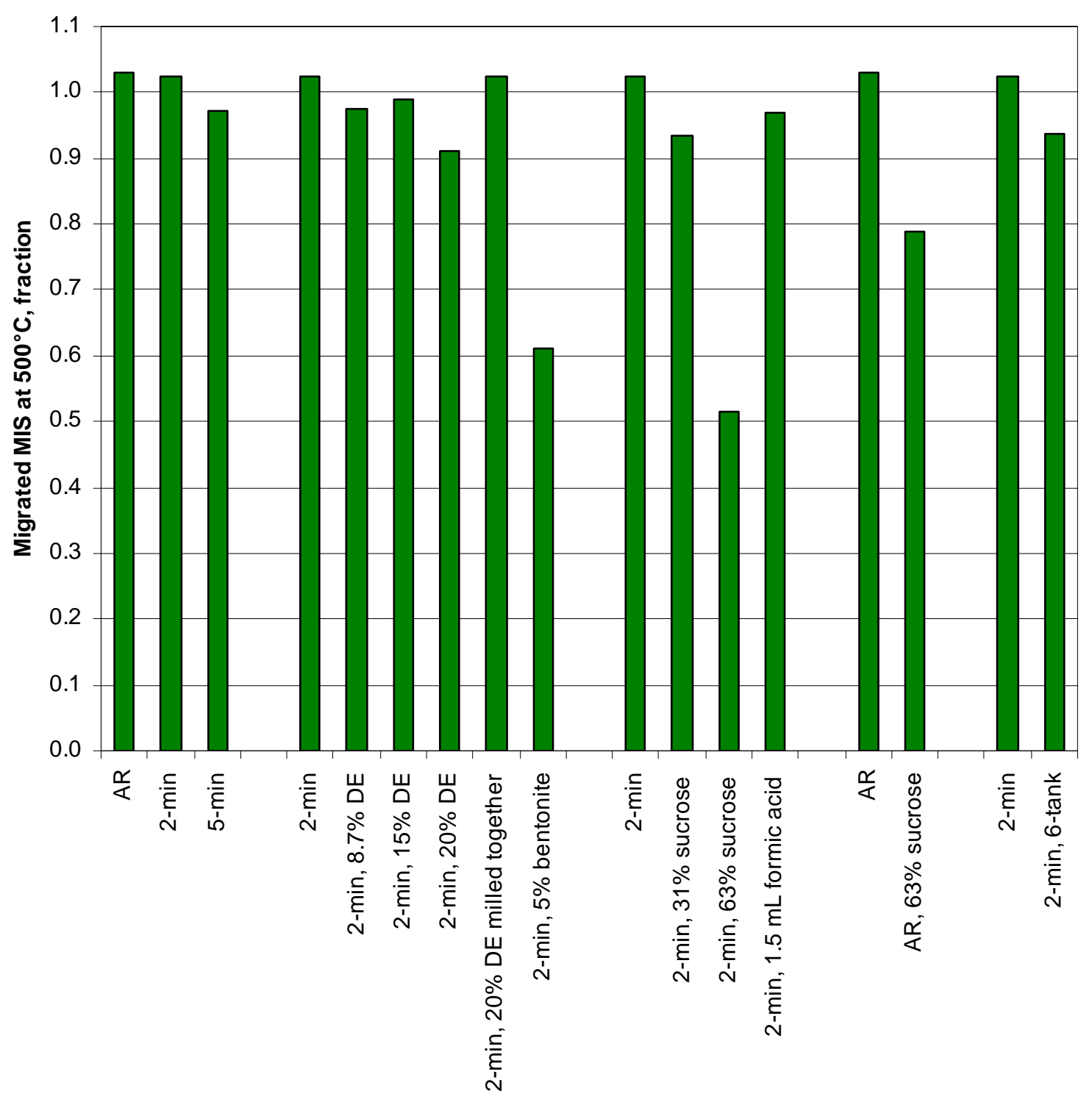

Figure K.15. MIS Present in Crucible Walls at $500^{\circ} \mathrm{C}$, Fraction of Total Estimated from $1000^{\circ} \mathrm{C}$ Data 


\section{Appendix L}

Experiments with Ground Soil and Sucrose Addition 


\section{Appendix L: Experiments with Ground Soil and Sucrose Addition}

Tables L.1 to L.3 list measured data obtained for feeds containing soil, S-109 simulant, and sucrose. Soil was used as-received or ground. The specific surface area of soil, as-received and ground using various methods, is listed in Table L.1. Table L.2 summarizes measured data for the initial series of experiments. Sucrose addition is indicated in terms of $\mathrm{C} / \mathrm{N}$ values, a ratio of $\mathrm{C}$ atoms from sucrose to $\mathrm{N}$ atoms in MIS; $\mathrm{C} / \mathrm{N}=1$ corresponds to $61.0 \mathrm{~g}$ of sucrose per $1 \mathrm{~kg}$ of sucrose-free feed. Table L.3 provides the distribution of MIS into three fractions: 1) MIS that migrated to crucible walls, 2) MIS components that escaped from feed as gas, and 3) MIS components that remained in feed. It also shows, in the last two columns, what fraction of MIS that did not penetrate to the crucible walls turned to gas and what fraction remained in feed.

Table L.1. Specific Surface Area of Soil Measured by the BET Method

\begin{tabular}{||l|r|}
\hline & $\mathbf{~ m}^{\mathbf{2}} / \mathbf{g}$ \\
\hline As-received & 5.74 \\
\hline Plate ground and milled by Praxair & 8.00 \\
\hline Milled by Praxair, & 8.80 \\
\hline Tungsten-carbide milled for 2 min & 9.75 \\
\hline Tungsten-carbide milled for 5 min & 10.78 \\
\hline
\end{tabular}


Table L.2. Effect of Grinding and Sucrose Addition on MIS Penetration into Silica Crucibles: Basic Data

\begin{tabular}{|c|c|c|c|c|c|c|c|c|c|}
\hline \multirow{2}{*}{\multicolumn{3}{|c|}{ Sucrose Addition }} & \multirow{3}{*}{$\begin{array}{c}\text { Soil } \\
\text { Specific } \\
\text { Surface } \\
\text { Area } \\
\mathrm{m}^{2} / \mathrm{g} \\
\end{array}$} & \multicolumn{3}{|c|}{ Crucible Mass } & \multicolumn{2}{|c|}{ Feed, RT } & \multirow{3}{*}{$\begin{array}{c}\text { Feed } \\
500^{\circ} \mathrm{C} \\
\mathrm{g}\end{array}$} \\
\hline & & & & \multirow{2}{*}{$\begin{array}{c}\text { RT } \\
\mathrm{g}\end{array}$} & \multirow{2}{*}{$\frac{500^{\circ} \mathrm{C}}{\mathrm{g}}$} & \multirow{2}{*}{$\frac{1000^{\circ} \mathrm{C}}{\mathrm{g}}$} & \multirow{2}{*}{$\begin{array}{c}\text { With } \\
\text { Sucrose }\end{array}$} & \multirow{2}{*}{$\begin{array}{c}\text { Without } \\
\text { Sucrose }\end{array}$} & \\
\hline & $\mathrm{C} / \mathrm{N}$ & $\mathrm{g}$ & & & & & & & \\
\hline & 0 & 0.00 & 5.74 & 356.26 & 385.36 & 367.12 & 165.70 & 165.70 & 126.17 \\
\hline & 0 & 0.00 & 8.00 & 353.30 & 370.11 & 359.52 & 172.33 & 172.33 & 145.33 \\
\hline & 0 & 0.00 & 8.80 & 349.66 & 365.61 & 355.54 & 172.35 & 172.35 & 145.76 \\
\hline & 0 & 0.00 & 9.75 & 355.33 & 367.11 & 359.47 & 172.17 & 172.17 & 142.88 \\
\hline & 0 & 0.00 & 10.78 & 360.38 & 367.78 & 362.97 & 172.16 & 172.16 & 154.63 \\
\hline $\mathrm{D}$ & 0.31 & 3.24 & 9.75 & 358.00 & 367.70 & 361.71 & 172.27 & 169.03 & 139.58 \\
\hline $\mathrm{D}$ & 0.38 & 3.89 & 9.75 & 330.35 & 337.15 & 332.99 & 172.71 & 168.82 & 140.05 \\
\hline $\mathrm{D}$ & 0.44 & 4.52 & 9.75 & 330.35 & 335.00 & 332.06 & 172.74 & 168.22 & 139.59 \\
\hline $\mathrm{D}$ & 0.5 & 5.13 & 9.75 & 327.06 & 327.76 & 326.98 & 172.30 & 167.17 & 142.26 \\
\hline $\mathrm{D}$ & 0.63 & 6.38 & 5.74 & 359.40 & 363.53 & 361.09 & 172.75 & 166.37 & 139.50 \\
\hline $\mathrm{G}$ & 0.63 & 6.85 & 5.74 & 325.78 & 335.43 & 329.91 & 185.47 & 178.62 & 144.49 \\
\hline $\mathrm{P}$ & 0.63 & 6.83 & 5.74 & 330.10 & 341.98 & 335.30 & 184.68 & 177.85 & 139.19 \\
\hline $\mathrm{G}$ & 0.63 & 6.86 & 5.74 & 328.64 & 335.69 & 331.24 & 185.57 & 178.71 & 151.63 \\
\hline $\mathrm{D}$ & 0.63 & 6.37 & 8.00 & 353.48 & 353.62 & 353.21 & 172.32 & 165.95 & 136.42 \\
\hline $\mathrm{D}$ & 0.63 & 6.37 & 8.80 & 355.33 & 355.30 & 354.97 & 172.34 & 165.97 & 139.43 \\
\hline $\mathrm{D}$ & 0.63 & 6.37 & 9.75 & 359.25 & 359.14 & 358.93 & 172.26 & 165.89 & 136.05 \\
\hline $\mathrm{D}$ & 0.75 & 7.59 & 9.75 & 330.11 & 329.61 & 329.63 & 172.36 & 164.77 & 131.22 \\
\hline $\mathrm{D}$ & 0.94 & 9.38 & 5.74 & 330.48 & 334.19 & 332.32 & 172.36 & 162.98 & 121.83 \\
\hline $\mathrm{D}$ & 1.25 & 12.28 & 5.74 & 327.09 & 326.62 & 326.12 & 172.31 & 160.03 & 110.80 \\
\hline
\end{tabular}


Table L.3. Effect of Grinding and Sucrose Addition on MIS Penetration into Silica Crucibles MIS Migration and Reaction

\begin{tabular}{|c|c|c|c|c|c|c|c|c|}
\hline \multirow{2}{*}{\multicolumn{3}{|c|}{ Sucrose Addition }} & \multirow{3}{*}{$\begin{array}{c}\text { Soil } \\
\text { Specific } \\
\text { Surface } \\
\text { Area } \\
\mathbf{m}^{2} / \mathbf{g}\end{array}$} & \multicolumn{3}{|c|}{ "MIS Mass Balance } & \multicolumn{2}{|c|}{ MIS in Feed } \\
\hline & & & & $\begin{array}{c}\text { Migrated } \\
\text { to Crucible } \\
\left(m_{\mathrm{C}}\right)\end{array}$ & $\begin{array}{c}\text { Escaped as Gas } \\
\text { from Feed } \\
\left(m_{\mathrm{A}}\right)\end{array}$ & $\begin{array}{c}\text { Remained } \\
\text { in Feed } \\
\left(m_{\mathrm{R}}\right)\end{array}$ & $\begin{array}{c}\text { Escaped } \\
\text { as Gas } \\
\left(m_{\mathrm{FA}}\right)\end{array}$ & $\begin{array}{c}\text { Remained } \\
\text { in Feed } \\
\left(m_{\mathrm{FR}}\right)\end{array}$ \\
\hline & $\mathbf{C} / \mathbf{N}$ & g & & \multicolumn{3}{|c|}{ Mass fraction } & \multicolumn{2}{|c|}{ Mass fraction } \\
\hline & 0 & 0.00 & 5.74 & 0.5210 & 0.1926 & 0.2864 & 0.4022 & 0.5978 \\
\hline & 0 & 0.00 & 8.00 & 0.3007 & 0.1679 & 0.5313 & 0.2402 & 0.7598 \\
\hline & 0 & 0.00 & 8.80 & 0.2860 & 0.1755 & 0.5385 & 0.2458 & 0.7542 \\
\hline & 0 & 0.00 & 9.75 & 0.2112 & 0.2977 & 0.4911 & 0.3774 & 0.6226 \\
\hline & 0 & 0.00 & 10.78 & 0.1443 & 0.1603 & 0.6954 & 0.1874 & 0.8126 \\
\hline $\mathrm{D}$ & 0.31 & 3.24 & 9.75 & 0.1962 & 0.3249 & 0.4789 & 0.4042 & 0.5958 \\
\hline $\mathrm{D}$ & 0.38 & 3.88 & 9.75 & 0.1493 & 0.3605 & 0.4902 & 0.4238 & 0.5762 \\
\hline $\mathrm{D}$ & 0.44 & 4.53 & 9.75 & 0.1087 & 0.4004 & 0.4909 & 0.4492 & 0.5508 \\
\hline $\mathrm{D}$ & 0.5 & 5.18 & 9.75 & 0.0298 & 0.4159 & 0.5543 & 0.4287 & 0.5713 \\
\hline $\mathrm{D}$ & 0.63 & 6.47 & 5.74 & 0.1091 & 0.3740 & 0.5169 & 0.4198 & 0.5802 \\
\hline G & 0.63 & 6.47 & 5.74 & 0.2031 & 0.3684 & 0.4285 & 0.4623 & 0.5377 \\
\hline $\mathrm{P}$ & 0.63 & 6.47 & 5.74 & 0.2488 & 0.4015 & 0.3497 & 0.5345 & 0.4655 \\
\hline G & 0.63 & 6.47 & 5.74 & 0.1394 & 0.3139 & 0.5467 & 0.3648 & 0.6352 \\
\hline $\mathrm{D}$ & 0.63 & 6.47 & 8.00 & 0.0215 & 0.5108 & 0.4677 & 0.5220 & 0.4780 \\
\hline $\mathrm{D}$ & 0.63 & 6.47 & 8.80 & 0.0175 & 0.4609 & 0.5217 & 0.4691 & 0.5309 \\
\hline $\mathrm{D}$ & 0.63 & 6.47 & 9.75 & 0.0193 & 0.5189 & 0.4619 & 0.5291 & 0.4709 \\
\hline $\bar{D}$ & 0.75 & 7.76 & 9.75 & 0.0122 & 0.5969 & 0.3909 & 0.6043 & 0.3957 \\
\hline $\mathrm{D}$ & 0.94 & 9.71 & 5.74 & 0.1182 & NA & NA & NA & NA \\
\hline $\mathrm{D}$ & 1.25 & 12.94 & 5.74 & 0.0036 & NA & NA & NA & NA \\
\hline
\end{tabular}

Table L.2 is an augmented Table K.4 and Table L.3 an augmented Table K.10. The symbols $m_{\mathrm{C}}, m_{\mathrm{A}}$, and $m_{\mathrm{R}}$ were defined in Appendix K. The values of the MIS in feed, remained and escaped as gas, are defined as $m_{\mathrm{FA}}=m_{\mathrm{A}} /\left(m_{\mathrm{A}}+m_{\mathrm{R}}\right)$ and $m_{\mathrm{FR}}=1-m_{\mathrm{FA}}$.

Figure L.1 shows that the relationship between MIS migration and the soil surface area is linear. This implies that the MIS retention is directly proportional to the internal surface of the solids in feed, as has been expected. A linear relationship is likely to exist also between MIS migration and sucrose addition as seen in Figure L.2. 


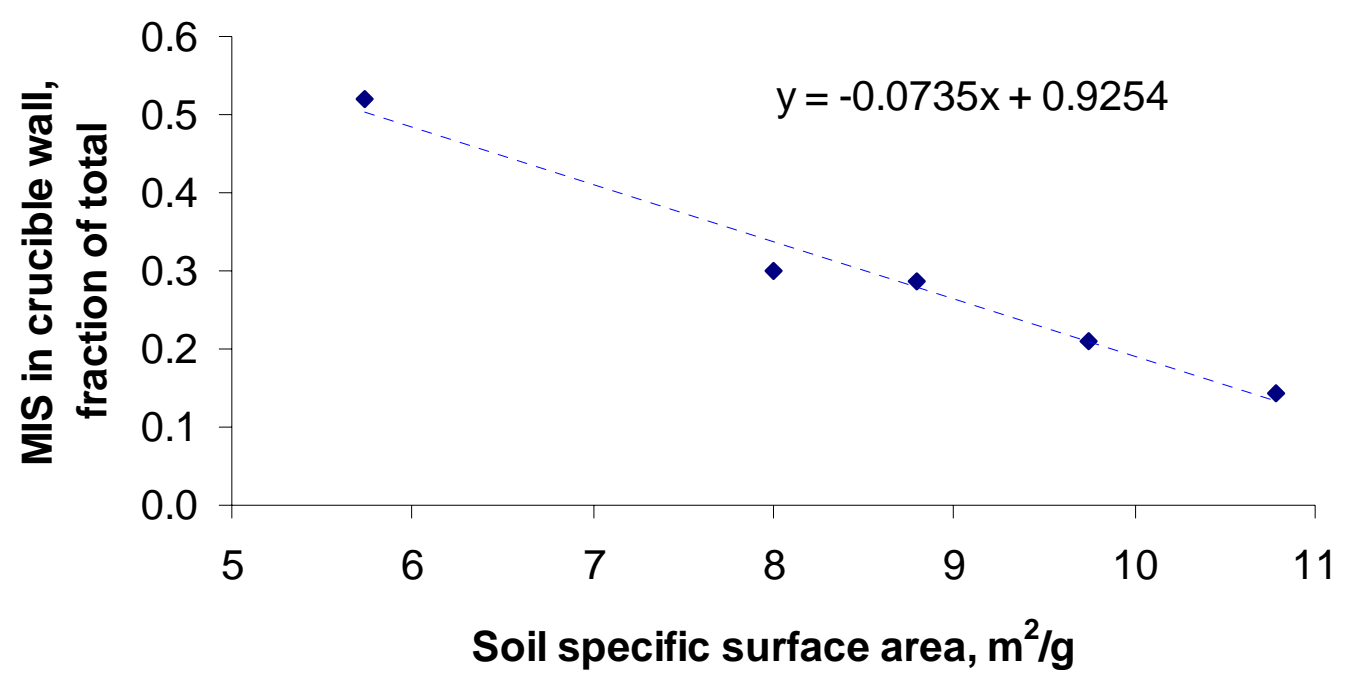

Figure L.1. Effect of Soil Specific Surface Area (by BET) on the Fraction of MIS Lost from Feed to Crucible Walls (no sucrose has been added to feed)

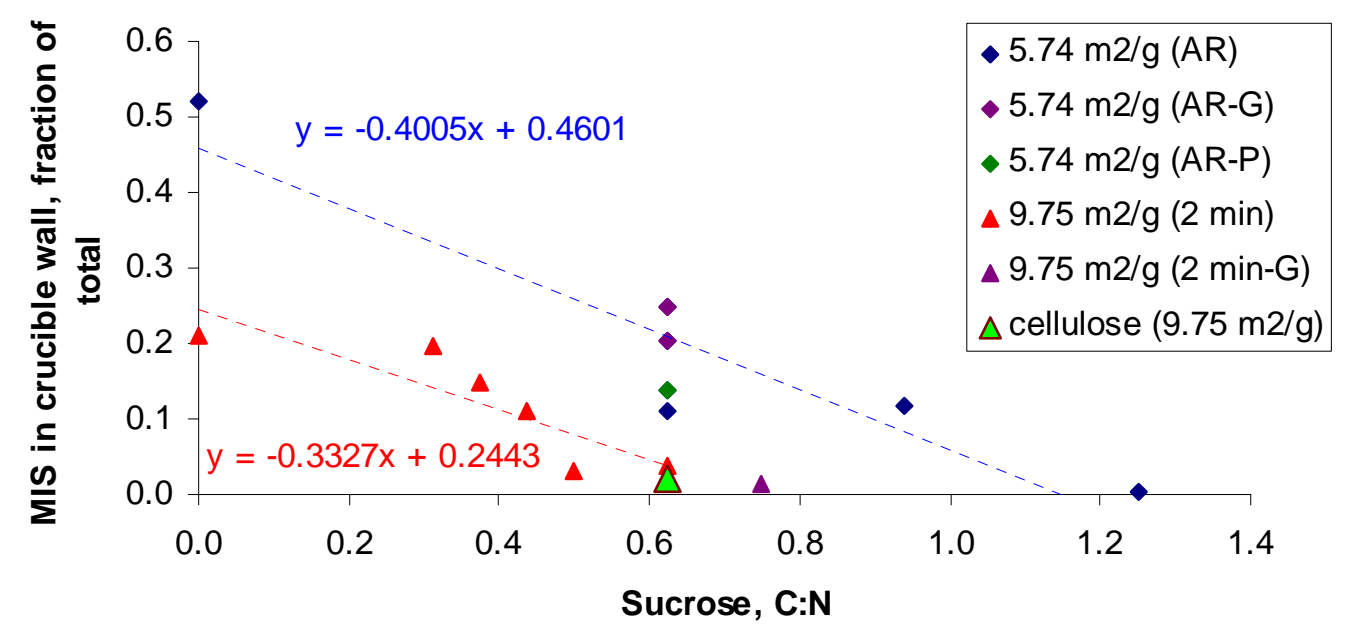

Figure L.2. Effect of Sucrose Addition (in stoichiometric \%) on the Fraction of MIS Lost from Feed with Soil As-Received $\left(5.74 \mathrm{~m}^{2} / \mathrm{g}\right.$ specific surface area) and Ground $\left(9.75 \mathrm{~m}^{2} / \mathrm{g}\right.$ specific surface area) to Crucible Walls (G granular sucrose added to dry feed, $P$ powdered sucrose added to dry feed; sucrose was dissolved in liquid LAW simulant for all other data points)

The fraction of MIS that migrated outside the feed is a linear function of the soil specific surface area for a constant sucrose addition and appears to be close to linear as a function of the sucrose addition for a constant soil surface area. This linearity suggests that linear regression can be applied to all data listed in Table L.2. The result can be expressed in the form of the relationship 


$$
m_{\mathrm{C}}=m_{\mathrm{C} 0}+m_{\mathrm{Ca}} a+m_{\mathrm{Cc}} C
$$

where $m_{\mathrm{C}}$ is the MIS fraction migrated to crucible walls, $a$ is the soil specific surface area in BET $\mathrm{m}^{2} / \mathrm{g}, c$ is the carbohydrate addition (in terms of $\mathrm{C} / \mathrm{N}$ ratio), and the values of coefficients are

$$
\begin{gathered}
m_{\mathrm{C}}=0.648 \pm 0.056 \\
m_{\mathrm{Ca}}=(-4.38 \pm 0.55) \times 10^{-2} \mathrm{~g} / \mathrm{m}^{2} \\
m_{\mathrm{Cc}}=-0.311 \pm 0.029
\end{gathered}
$$

Figure L.3 displays calculated $m_{\mathrm{C}}$ versus measured values.

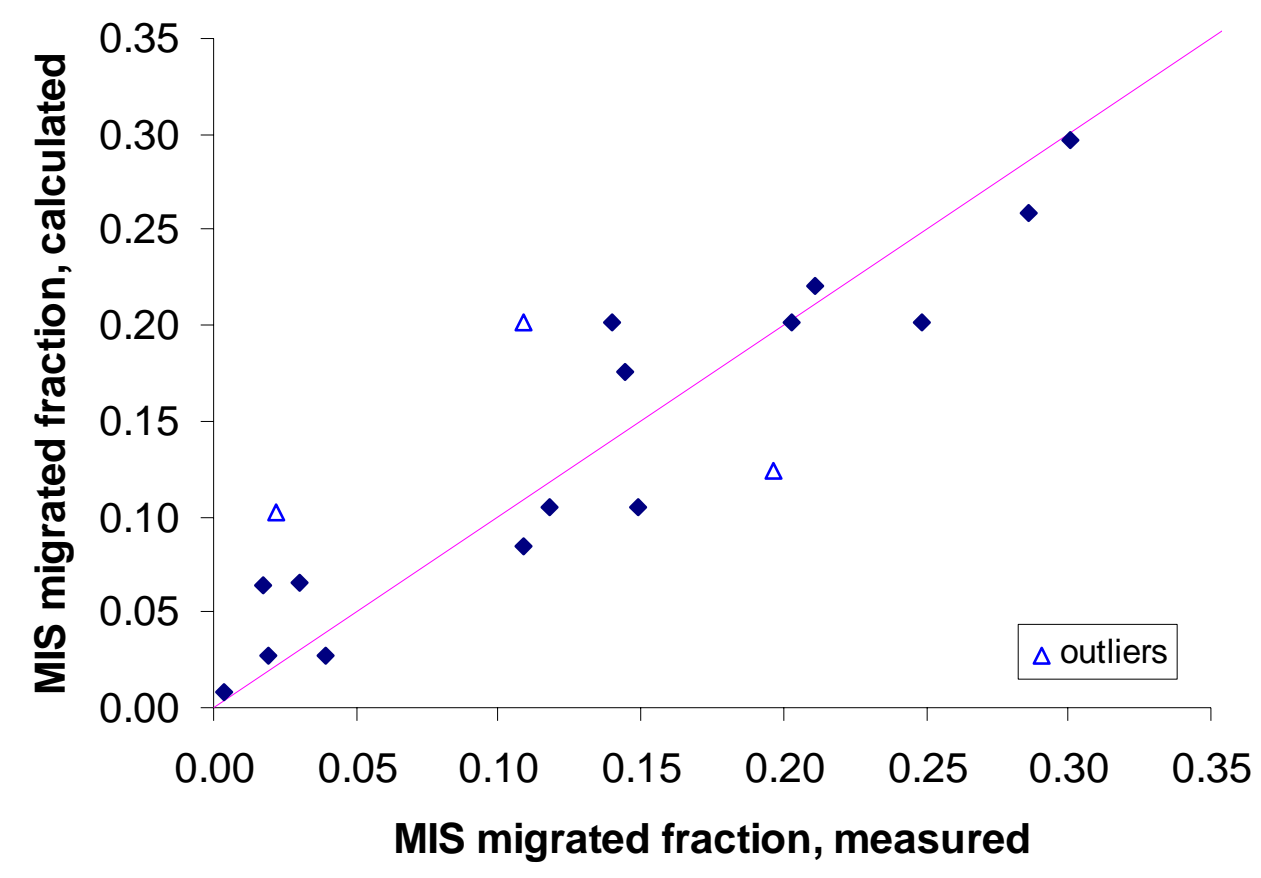

Figure L.3. Calculated Versus Measured Fraction of MIS Migrated into Crucible Walls

The linear fit allows, by setting $m_{\mathrm{C}}=0$, separating the $c$ - $a$ plain into a region were MIS migrates and where it does not migrate outside the feed. This is diagrammatically shown in Figure L.4. 


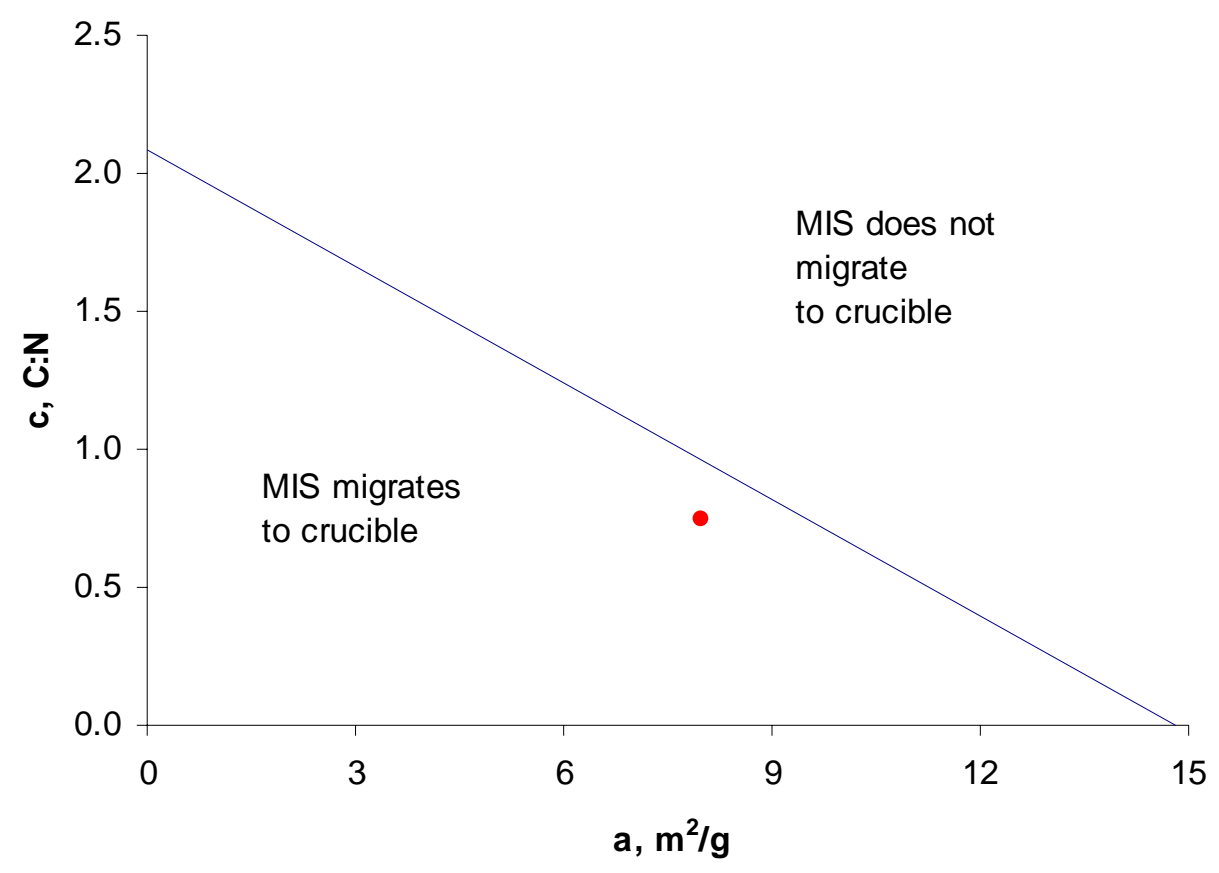

Figure L.4. Preventable MIS Migration Diagram

Accordingly, MIS does not migrate outside the feed if

$$
\mathrm{C} / \mathrm{N} \geq 2.08-0.141 a\left(\mathrm{BET} \mathrm{m}^{2} / \mathrm{g}\right)
$$

or

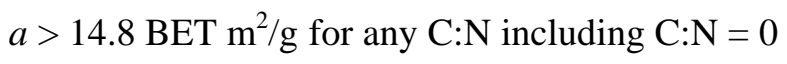

The plot in Figure L.4 is based on a rather small number of data points and only demonstrates the methodology. Also, it is based on the assumption that soil can effectively be ground to the required level and that dried feeds can be prepared with sucrose at any level without risking potential reactions in feed before it is charged into the BV melter. For example, if the maximum acceptable C:N is 0.75 and soil grinding to a specific surface area of only $8 \mathrm{~m}^{2} / \mathrm{g}$ is practicable (see the red point in the figure), MIS would not be $100 \%$ immobilized in the feed.

Plotting the mass fraction of gas evolved from MIS that remained in the feed against carbohydrate (sucrose or cellulose) addition (Figure L.5) shows that sucrose promotes early nitrate decomposition. The trendline indicates that complete decomposition at $500^{\circ} \mathrm{C}$ is achieved with $\mathrm{C} / \mathrm{N}=0.86$; if no sucrose is added, an increase in specific surface area exhibits a mild tendency to decrease the gas release at $500^{\circ} \mathrm{C}$ (Figure L.6). 


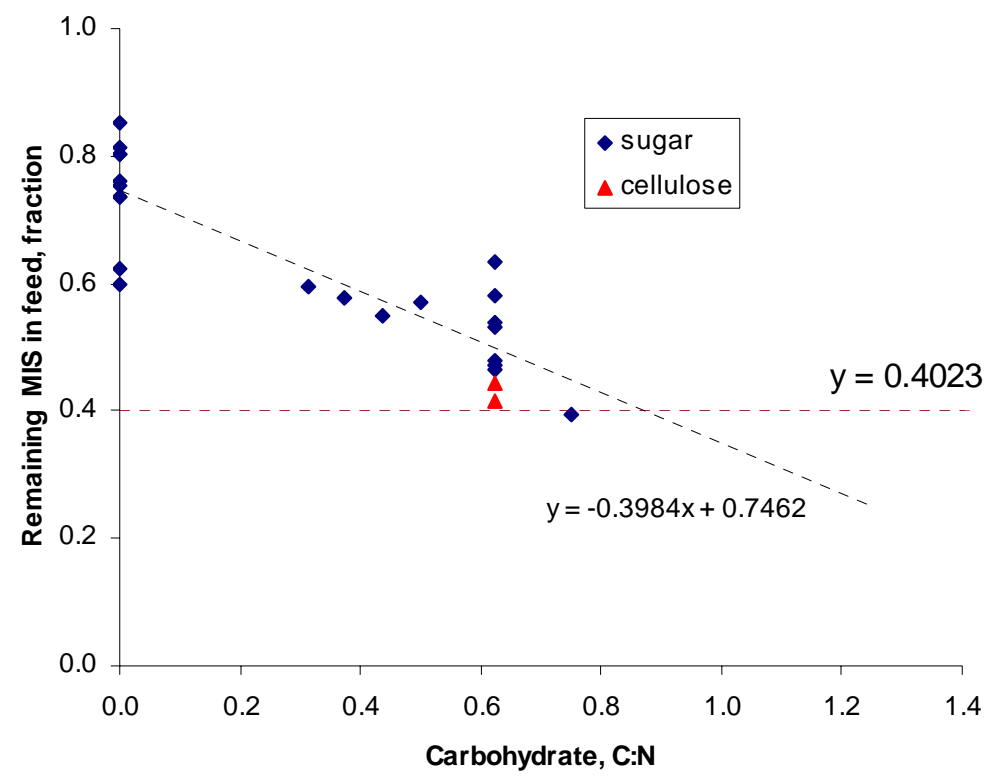

Figure L.5. Effect of Carbohydrate Addition (as C/N ratio) on the Fraction of MIS Remaining in Feed (the horizontal line represents complete decomposition)

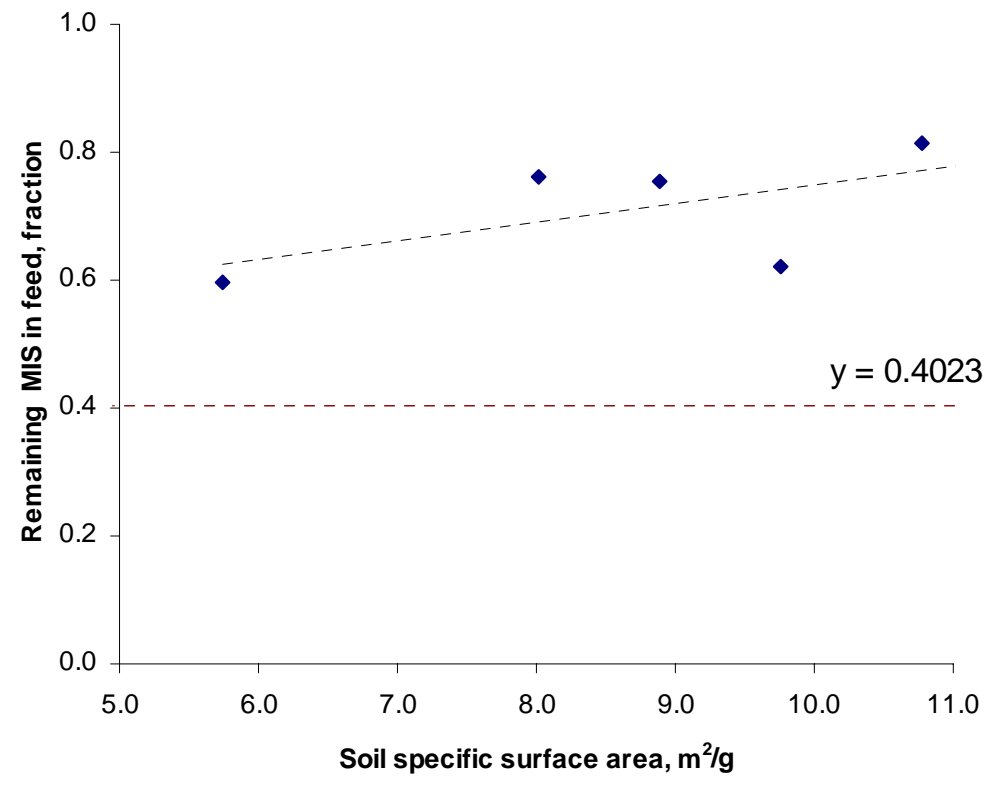

Figure L.6. Effect of Specific Surface Area of Soil on the Fraction of MIS Remaining in Feed (the horizontal line represents complete decomposition) 
Appendix M

\section{Temperature Evolution Data}




\section{Appendix M: Temperature Evolution Data}

Table M1 lists acronyms used in Table M2. Gray-shaded areas indicate the appearance of black smoke.

Table M1. Acronyms Used in Table M2

\begin{tabular}{||l|l||}
\hline TC-M & Thermocouple middle \\
\hline TC-T & Thermocouple top \\
\hline FC & Furnace controller thermocouple \\
\hline FO & Furnace over-temperature controller thermocouple \\
\hline AR & As received soil \\
\hline C & Cellulose \\
\hline S & Sucrose \\
\hline
\end{tabular}

Table M2. Temperature Evolution Data (temperatures are in ${ }^{\circ} \mathrm{C}$ )

\begin{tabular}{|c|c|c|c|c|c|c|c|c|c|c|c|c|}
\hline \multicolumn{2}{|c|}{ AR, 100\%C 13\% S } & \multicolumn{2}{|c|}{ AR, 113\% C } & \multicolumn{2}{|c|}{ AR, 125\% C } & \multicolumn{3}{|c|}{ AR, 113\% C13\% S } & \multicolumn{4}{|c|}{ AR, 88 \% C, repeated } \\
\hline FC & TC-M & FC & TC-M & FC & TC-M & FC & TC-M & TC-T & FC & FO & TC-M & TC-T \\
\hline 272 & 122 & 213 & 59.1 & 189 & 51.5 & 168 & 50.8 & 158 & 175 & 252 & 77.3 & 214 \\
\hline 279 & 200 & 220 & 68.2 & 200 & 64 & 175 & 60.6 & 161 & 199 & 251 & 109.6 & 247 \\
\hline 294 & 400 & 230 & 80.9 & 210 & 76.9 & 190 & 80.4 & 170 & 205 & 253 & 115.7 & 249 \\
\hline 295 & 350 & 240 & 93.5 & 220 & 88 & 200 & 89.9 & 190 & 218 & 256 & 127.3 & 253 \\
\hline 301 & 353 & 250 & 107.1 & 230 & 96.4 & 210 & 97.8 & 194 & 226 & 261 & 134 & 257 \\
\hline 304 & 338.4 & 260 & 112.6 & 240 & 103.6 & 220 & 103.8 & 197 & 235 & 264 & 144 & 261 \\
\hline 306 & 330.2 & 272 & 113.6 & 250 & 110.1 & 230 & 109.4 & 200 & 246 & 268 & 152 & 265 \\
\hline 310 & 321.4 & 280 & 113.8 & 260 & 112.8 & 240 & 114.4 & 209 & 250 & 273 & 155 & 268 \\
\hline 312 & 319 & 290 & 114.2 & 266 & 112 & 250 & 115.2 & 212 & 260 & 282 & 163 & 276 \\
\hline 314 & 315.6 & 288 & 111.3 & 282 & 114.9 & 262 & 117.9 & 220 & 266 & 290 & 166 & 410 \\
\hline 315 & 314.2 & 290 & 112.7 & 274 & 325 & 270 & 125 & 328 & 267 & 288 & 168 & 445 \\
\hline 318 & 311.4 & 293 & 110.9 & 275 & 354 & 280 & 127.8 & 429 & 268 & 289 & 171 & 454 \\
\hline 321 & 308.4 & 295 & 113.7 & 277 & 376.7 & 281 & 125.7 & 420 & 269 & 292 & 196 & 457 \\
\hline 325 & 305.9 & 300 & 115.1 & 278 & 379.4 & 282 & 129.9 & 428 & 270 & 294 & 226 & 454 \\
\hline 350 & 398.8 & 310 & 115.2 & 279 & 377.9 & 275 & 131.8 & 459 & 273 & 298 & 278 & 449 \\
\hline 358 & 405.1 & 320 & 115.5 & 280 & 377.1 & 273 & 134.2 & 461 & 274 & 298 & 310 & 446 \\
\hline 377 & 421.4 & 330 & 116.6 & 285 & 377.2 & 272 & 135.6 & 459 & 275 & 299 & 394 & 442 \\
\hline 401 & 444 & 340 & 118.9 & 288 & 374 & 273 & 138 & 451 & 277 & 300 & 350 & 438 \\
\hline 421 & 463 & 350 & 285 & 290 & 373 & 274 & 285 & 375 & 278 & 302 & 327 & 433 \\
\hline 448 & 489.7 & 351 & 452 & 300 & 371.6 & 277 & 440 & 446 & 280 & 304 & 310 & 427 \\
\hline 458 & 500 & 352 & 414 & 310 & 374.6 & 277 & 385 & 440 & 281 & 305 & 306 & 419 \\
\hline 475 & 516.8 & 353 & 408 & 320 & 380.3 & 278 & 360 & 431 & 283 & 307 & 394 & 415 \\
\hline 500 & 538.6 & 355 & 410.9 & 330 & 387.3 & 282 & 390 & 423 & 286 & 309 & 395 & 411 \\
\hline & & 360 & 418.7 & 340 & 395.1 & 284 & 415.7 & 417 & 288 & 312 & 390 & 406 \\
\hline & & 371 & 429.8 & 350 & 404.1 & 286 & 410 & 411 & 289 & 314 & 381 & 401 \\
\hline & & 380 & 437.1 & 360 & 412.8 & 288 & 400 & 406 & 290 & 315 & 376.4 & 399 \\
\hline
\end{tabular}


Table M.2 (Contd)

\begin{tabular}{|c|c|c|c|c|c|c|c|c|c|c|c|c|}
\hline \multicolumn{2}{|c|}{ AR, $100 \% C$ 13\% S } & \multicolumn{2}{|c|}{ AR, $113 \% \mathrm{C}$} & \multicolumn{2}{|c|}{ AR, 125\% C } & \multicolumn{3}{|c|}{ AR, $113 \%$ C13\% S } & \multicolumn{4}{|c|}{ AR, 88 \% C, repeated } \\
\hline FC & TC-M & FC & TC-M & FC & TC-M & FC & TC-M & TC-T & FC & FO & TC-M & TC-T \\
\hline & & 390 & 444.4 & 370 & 422.2 & 291 & 390 & 400 & 293 & 317 & 369 & 395 \\
\hline & & 400 & $\begin{array}{l}453.8 \\
\end{array}$ & 390 & 451.3 & 292 & 386.1 & 397 & 297 & 321 & 361 & 391 \\
\hline & & 410 & 463.6 & 400 & 471.4 & 294 & 382.6 & 396 & 303 & 326 & 354 & 384 \\
\hline & & 420 & 472.2 & 420 & 492.1 & 295 & 380.3 & 395 & 310 & 333 & 349 & 381 \\
\hline & & 430 & 482.6 & 442 & 509.9 & 296 & 376.7 & 393 & 322 & 346 & 343 & 381 \\
\hline & & 440 & 491.6 & 460 & 533.4 & 298 & 373.8 & 392 & 330 & 354 & 342 & 383 \\
\hline & & 450 & 501.2 & 483 & 544 & 300 & 370.6 & 390 & 340 & 365 & 341 & 388 \\
\hline & & 460 & 510.9 & 500 & 545 & 303 & 365.5 & 388 & 355 & 380 & 343.4 & 398 \\
\hline & & 470 & 520.6 & & & 310 & 358.1 & 386 & 365 & 391 & 346 & 406 \\
\hline & & 480 & 531.3 & & & 322 & 352.1 & 387 & 375 & 399 & 350 & 412 \\
\hline & & 490 & 540 & & & 336 & 351.2 & 391 & 500 & 519 & 509 & 533 \\
\hline & & 500 & 545.1 & & & 340 & 351.8 & 392 & & & & \\
\hline & & & & & & 350 & 354.4 & 397 & & & & \\
\hline & & & & & & 360 & 357.7 & 402 & & & & \\
\hline & & & & & & 370 & 360.8 & 408 & & & & \\
\hline & & & & & & 380 & 369.5 & 423 & & & & \\
\hline & & & & & & 390 & 375.1 & 432 & & & & \\
\hline & & & & & & 400 & 388.9 & 449 & & & & \\
\hline & & & & & & 420 & 405.9 & 467 & & & & \\
\hline & & & & & & 440 & 424.9 & 487 & & & & \\
\hline & & & & & & 460 & 443.4 & 505 & & & & \\
\hline & & & & & & 500 & 467.8 & 528 & & & & \\
\hline
\end{tabular}

Table M.2 (Contd)

\begin{tabular}{||r|r|r|r|r|r|r|r|r|r|r|r||}
\hline \multicolumn{4}{||c|}{ GFM, 75\% C as bran } & \multicolumn{4}{|c|}{ GFM, 75\% C as bran } & \multicolumn{3}{|c|}{ Praxair Dryer, 75\% C } \\
\hline FC & FO & TC-M & TC-T & FC & FO & TC-M & TC-T & FC & FO & TC-M & TC-T \\
\hline 255 & 276 & 158 & 190 & 183 & 251 & 82 & 210 & 203 & 259 & 107 & 181 \\
\hline 260 & 281 & 161 & 193 & 207 & 255 & 108 & 226 & 217 & 262 & 120 & 191 \\
\hline 270 & 287 & 165 & 198 & 226 & 262 & 122 & 230 & 234 & 269 & 137 & 206 \\
\hline 275 & 292 & 169 & 203 & 236 & 266 & 126 & 233 & 244 & 272 & 144 & 212 \\
\hline 280 & 298 & 173 & 208 & 241 & 267 & 128 & 243 & 257 & 279 & 154 & 222 \\
\hline 285 & 308 & 177 & 225 & 255 & 274 & 135 & 253 & 270 & 291 & 165 & 235 \\
\hline 290 & 304 & 180 & 332 & 265 & 283 & 141 & 258 & 283 & 304 & 178 & 250 \\
\hline 295 & 311 & 185 & 421 & 270 & 289 & 144 & 523 & 300 & 322 & 187 & 405 \\
\hline 300 & 315 & 189 & 440 & 280 & 300 & 150 & 509 & 302 & 326 & 188 & 487 \\
\hline 305 & 322 & 200 & 431 & 281 & 294 & 153 & 472 & 303 & 325 & 195 & 484 \\
\hline 310 & 326 & 204 & 417 & 285 & 299 & 156 & 440 & 305 & 326 & 203 & 483 \\
\hline 315 & 335 & 299 & 402 & 290 & 306 & 170 & 432 & 307 & 326 & 267 & 478 \\
\hline 320 & 340 & 290 & 409 & 292 & 309 & 206 & 424 & 308 & 327 & 297 & 471 \\
\hline 325 & 345 & 283 & 415 & 294 & 311 & 203 & 409 & 309 & 327 & 294 & 466 \\
\hline 330 & 350 & 284 & 415 & 298 & 315 & 268 & 404 & 311 & 330 & 289 & 457 \\
\hline
\end{tabular}


Table M.2 (Contd)

\begin{tabular}{||r|r|r|r|r|r|r|r|r|r|r|r||}
\hline \multicolumn{4}{||c|}{ GFM, 75\% C as bran } & \multicolumn{4}{|c|}{ GFM, 75\% C as bran } & \multicolumn{3}{|c|}{ Praxair Dryer, 75\% C } \\
\hline FC & FO & TC-M & TC-T & FC & FO & TC-M & TC-T & FC & FO & TC-M & TC-T \\
\hline 335 & 358 & 477 & 414 & 300 & 317 & 480 & 400 & 315 & 335 & 458 & 444 \\
\hline 340 & 366 & 430 & 412 & 301 & 318 & 493 & 398 & 316 & 336 & 453 & 439 \\
\hline 345 & 370 & 414 & 410 & 302 & 320 & 485 & 395 & 318 & 338 & 431 & 434 \\
\hline 350 & 374 & 408 & 408 & 304 & 321 & 464 & 393 & 323 & 344 & 399 & 423 \\
\hline 355 & 379 & 404 & 408 & 305 & 322 & 450 & 392 & 325 & 346 & 393 & 421 \\
\hline 360 & 383 & 402 & 408 & 307 & 324 & 440 & 389 & 329 & 354 & 381 & 414 \\
\hline 365 & 388 & 399 & 408 & 310 & 328 & 426 & 389 & 333 & 357 & 383 & 411 \\
\hline 370 & 394 & 397 & 408 & 312 & 333 & 414 & 388 & 342 & 367 & 384 & 405 \\
\hline 375 & 399 & 396 & 409 & 316 & 337 & 401 & 387 & 350 & 376 & 382 & 402 \\
\hline 380 & 403 & 396 & 410 & 320 & 342 & 392 & 386 & 362 & 390 & 381 & 403 \\
\hline & & & & 325 & 348 & 384 & 386 & 382 & 407 & 381 & 408 \\
\hline & & & & 330 & 353 & 380 & 386 & 410 & 438 & 389 & 423 \\
\hline & & & & 340 & 364 & 372 & 388 & 431 & 459 & 400 & 437 \\
\hline & & & & 350 & 374 & 368 & 392 & 445 & 472 & 409 & 448 \\
\hline & & & & 365 & 390 & 364 & 402 & 475 & 503 & 433 & 474 \\
\hline & & & & 385 & 410 & 366 & 415 & 492 & 519 & 448 & 489 \\
\hline & & & & 410 & 437 & 377 & 436 & 500 & 527 & 455 & 497 \\
\hline & & & & 432 & 459 & 392 & 456 & & & & \\
\hline & & & & 461 & 489 & 412 & 483 & & & & \\
\hline & & & & 486 & 513 & 438 & 506 & & & & \\
\hline & & & & 490 & 517 & 441 & 510 & & & & \\
\hline & & & & 500 & 526 & 450 & 519 & & & & \\
\hline
\end{tabular}

M.3 
PNNL-16773

\section{Distribution}

No. of

Copies

OFFSITE

3 AMEC

L. Thompson

B2-67

K. Witwer

J. R. Biggs

ONSITE

8 CH2M HILL

P. K. Brockman

F. R. Miera

R. E. Raymond

D. Shuford

A. R. Tedeschi

J. Van Beek (3)
H6-03

B2-67

B2-67

H6-03

S7-83

T4-67

S7-67

R3-47
No. of

Copies

\section{ONSITE}

3 U.S. Department of Energy/Office of River Protection
B. J. Harp
H6-60
D. H. Irby
H6-60
B. M. Mauss
H6-60

22 Pacific Northwest National Laboratory

L. M. Bagaasen (10) K6-28

T. M. Brouns K9-69

W. C. Buchmiller K6-24

M. L. Elliott K9-09

P. Hrma (2) K6-24

A. B. Gallegos K6-24

D.-S. Kim K6-24

J. Matyas K6-24

C. P. Rodriguez K6-24

M. J. Schweiger K6-24

R. W. Shimskey P7-27

S. T. Yokuda K7-15

Distr. 1 